
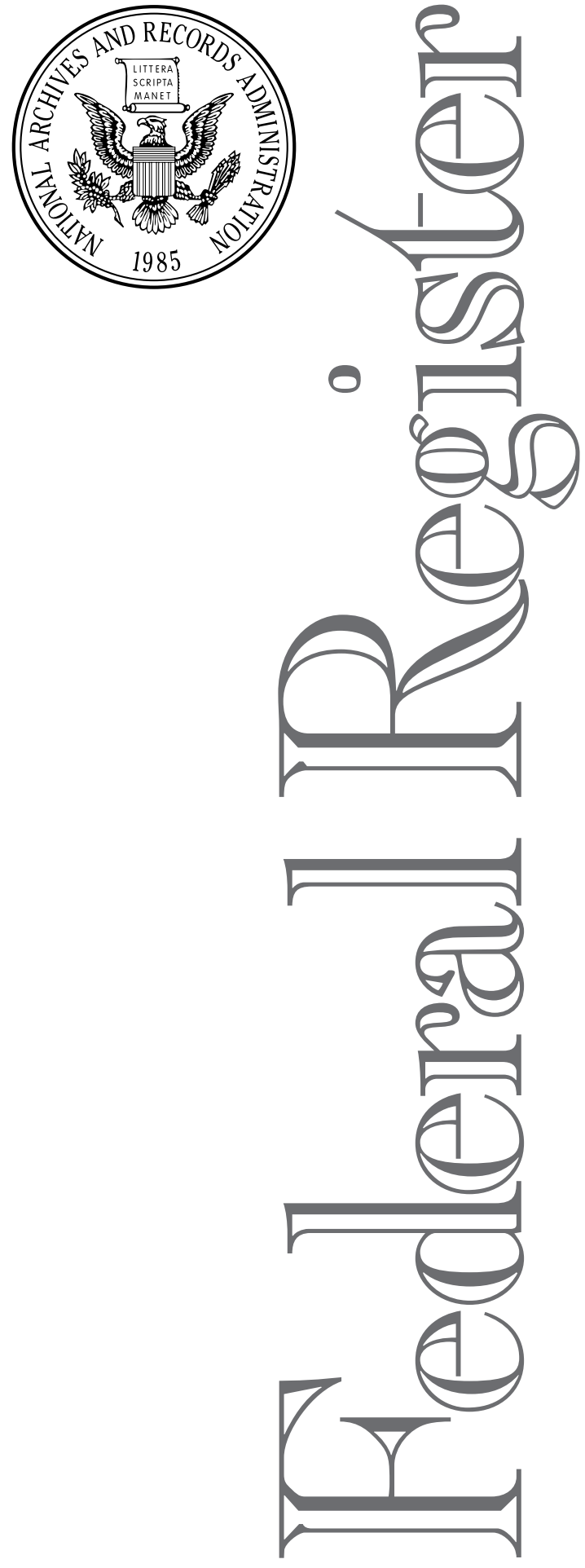

Wednesday,

July 28, 2010
Part II

\section{Department of Health and Human Services}

Centers for Medicare \& Medicaid Services

42 CFR Parts 412, 413, 422 et al. Medicare and Medicaid Programs; Electronic Health Record Incentive Program; Final Rule 


\section{DEPARTMENT OF HEALTH AND HUMAN SERVICES}

\section{Centers for Medicare \& Medicaid Services}

42 CFR Parts 412, 413, 422, and 495

[CMS-0033-F]

\section{RIN 0938-AP78}

\section{Medicare and Medicaid Programs; Electronic Health Record Incentive Program}

AGENCY: Centers for Medicare \& Medicaid Services (CMS), HHS.

ACTION: Final rule.

SUMmARY: This final rule implements the provisions of the American Recovery and Reinvestment Act of 2009 (ARRA) (Pub. L. 111-5) that provide incentive payments to eligible professionals (EPs), eligible hospitals and critical access hospitals (CAHs) participating in Medicare and Medicaid programs that adopt and successfully demonstrate meaningful use of certified electronic health record (EHR) technology. This final rule specifiesthe initial criteria EPs, eligible hospitals, and CAHs must meet in order to qualify for an incentive payment; calculation of the incentive payment amounts; payment adjustments under Medicare for covered professional services and inpatient hospital services provided by EPs, eligible hospitals and CAHs failing to demonstrate meaningful use of certified EHR technology; and other program participation requirements. Also, the Office of the National Coordinator for Health Information Technology (ONC) will be issuing a closely related final rule that specifies the Secretary's adoption of an initial set of standards, implementation, specifications, and certification criteria for electronic health records. ONC has also issued a separate final rule on the establishment of certification programs for health information technology.

DATES: Effective Date: These regulations are effective on September 27, 2010.

FOR FURTHER INFORMATION CONTACT:

Elizabeth Holland, (410) 786-1309, EHR incentive program issues.

Edward Gendron, (410) 786-1064, Medicaid incentive payment issues.

Jim Hart, (410) 786-9520, Medicare fee for service payment issues.

Bob Kuhl or Susan Burris, (410) 7865594, Medicare CAH payment and charity care issues.

Frank Szeflinski, (303) 844-7119, Medicare Advantage issues.

SUPPLEMENTARY INFORMATION:

\section{Acronyms}

ARRA American Recovery and

Reinvestment Act of 2009

AAC Average Allowable Cost (of certified

EHR technology)

AIU Adopt, Implement, Upgrade (certified EHR technology)

CAH Critical Access Hospital

CAHPS Consumer Assessment of

Healthcare Providers and Systems

CCN CMS Certification Number

CFR Code of Federal Regulations

CHIP Children's Health Insurance Program

CHIPRA Children's Health Insurance

Program Reauthorization Act of 2009

CMS Centers for Medicare \& Medicaid

Services

CPOE Computerized Physician Order Entry

CY Calendar Year

EHR Electronic Health Record

EP Eligible Professional

EPO Exclusive Provider Organization

FACA Federal Advisory Committee Act

FFP Federal Financial Participation

FFY Federal Fiscal Year

FFS Fee-For-Service

FQHC Federally Qualified Health Center

FTE Full-Time Equivalent

FY Fiscal Year

HEDIS Healthcare Effectiveness Data and

Information Set

HHS Department of Health and Human Services

HIE Health Information Exchange

HIT Health Information Technology

HIPAA Health Insurance Portability and Accountability Act of 1996

HITECH Health Information Technology for Economic and Clinical Health Act

HMO Health Maintenance Organization

HOS Health Outcomes Survey

HPSA Health Professional Shortage Area

HRSA Health Resource and Services Administration

IAPD Implementation Advance Planning Document

ICR Information Collection Requirement

IHS Indian Health Service

IPA Independent Practice Association

IT Information Technology

MA Medicare Advantage

MAC Medicare Administrative Contractor

MAO Medicare Advantage Organization

MCO Managed Care Organization

MITA Medicaid Information Technology Architecture

MMIS Medicaid Management Information Systems

MSA Medical Savings Account

NAAC Net Average Allowable Cost (of certified EHR technology)

NCQA National Committee for Quality Assurance

NCVHS National Committee on Vital and Health Statistics

NPI National Provider Identifier

NPRM Notice of Proposed Rulemaking

ONC Office of the National Coordinator for Health Information Technology

PAHP Prepaid Ambulatory Health Plan

PAPD Planning Advance Planning Document

PFFS Private Fee-For-Service

PHO Physician Hospital Organization

PHS Public Health Service

PHSA Public Health Service Act
PIHP Prepaid Inpatient Health Plan

POS Place of Service

PPO Preferred Provider Organization

PQRI Physician Quality Reporting Initiative

PSO Provider Sponsored Organization

RHC Rural Health Clinic

RHQDAPU Reporting Hospital Quality Data for Annual Payment Update

RPPO Regional Preferred Provider

Organization

SMHP State Medicaid Health Information

Technology Plan

TIN Tax Identification Number

Table of Contents

I. Background

A. Overview of the HITECH Programs

Created by the American Recovery and Reinvestment Act of 2009

B. Statutory Basis for the Medicare \& Medicaid EHR Incentive Programs

II. Provisions of the Proposed Regulations and Response and Analysis of Comments

A. Definitions Across the Medicare FFS,

Medicare Advantage, and Medicaid Programs

1. Definitions

a. Certified Electronic Health Record (EHR) Technology

b. Qualified Electronic Health Record

c. Payment Year

d. First, Second, Third, Fourth, Fifth and Sixth Payment Year

e. EHR Reporting Period

f. Meaningful EHR User

2. Definition of Meaningful Use

a. Considerations in Defining Meaningful Use

b. Common Definition of Meaningful Use Under Medicare and Medicaid

c. Stage 1 Criteria for Meaningful Use

3. Sections 4101(a) and 4102(a)(1) of HITECH Act: Reporting on Clinical Quality Measures Using EHR by EPs, Eligible Hospitals and CAHs

a. General

b. Requirements for the Submission of Clinical Quality Measures by EPs, Eligible Hospitals and CAHs

c. Statutory Requirements and Other Considerations for the Selection of Clinical Quality Measures for Electronic Submission by EPs, Eligible Hospitals and CAHs

(1) Statutory Requirements for the Selection of Clinical Quality Measures for Electronic Submission by EPs, Eligible Hospitals and CAHs

(2) Other Considerations for the Selection of Clinical Quality Measures for Electronic Submission by EPs, Eligible Hospitals and CAHs

d. Clinical Quality Measures for EPs

e. Clinical Quality Measures Reporting Criteria for EPs

f. Clinical Quality Measures for Electronic Submission by Eligible Hospitals

g. Potential Measures for EPs, Eligible Hospitals and CAHs in Stage 2 and Subsequent Years

h. Reporting Method for Clinical Quality Measures for 2011 and Beginning With the 2012 Payment Years

(1) Reporting Method for 2011 Payment Year

(2) Reporting Method Beginning in 2012 
i. Alternative Reporting Methods for Clinical Quality Measures

j. Reporting Period for Reporting Clinical Quality Measures

4. Demonstration of Meaningful Use

a. Common Methods of Demonstration in Medicare and Medicaid

b. Methods for Demonstration of the Stage 1 Criteria of Meaningful Use

5. Data Collection for Online Posting, Program Coordination, and Accurate Payments

a. Online Posting

b. Program Election Between Medicare FFS/MA and Medicaid for EPs

c. Data To Be Collected

6. Hospital-Based Eligible Professionals

7. Interaction With Other Programs

B. Medicare Fee-for-Service Incentives

1. Incentive Payments for Eligible

Professionals

a. Definitions

b. Incentive Payment Limits

c. Increase in Incentive Payment for EPs Who Predominantly Furnish Services in a Geographic Health Professional Shortage Area

d. Form and Timing of Payment

e. Payment Adjustment Effective in CY 2015 and Subsequent Years for EPs Who Are Not Meaningful Users of Certified EHR Technology

2. Incentive Payments for Hospitals

a. Definition of Eligible Hospital for Medicare

b. Incentive Payment Calculation for Eligible Hospitals

c. Medicare Share

d. Charity Care

e. Transition Factor

f. Duration and Timing of Incentive Payments

g. Incentive Payment Adjustment Effective in Federal FY 2015 and Subsequent Years for Eligible Hospitals Who Are Not Meaningful EHR Users

3. Incentive Payments for Critical Access Hospitals

a. Definition of CAHs for Medicare

b. Current Medicare Payment of Reasonable Cost for CAHs

c. Changes Made by the HITECH Act

d. Incentive Payment Calculation for CAHs

e. Reduction of Reasonable Cost Payment in FY 2015 and Subsequent Years for CAHs That Are Not Meaningful EHR Users

4. Process for Making Incentive Payments Under the Medicare FFS Program

a. Incentive Payments to EPs

b. Incentive Payments to Eligible Hospitals

c. Incentive Payments to CAHs

d. Payment Accounting Under Medicare

C. Medicare Advantage Organization Incentive Payments

1. Definitions

a. Qualifying MA Organization

b. Qualifying MA Eligible Professional

c. Qualifying MA-Affiliated Eligible Hospital

2. Identification of Qualifying MA Organizations, MA EPs, and MAAffiliated Eligible Hospitals

3. Computation of Incentives to Qualifying MA Organizations for MA EPs and Hospitals
4. Timeframe for Payment

5. Avoiding Duplicate Payment

6. Meaningful User Attestation

7. Posting Information on the CMS Web site

8. Limitation on Review

9. Conforming Changes

10. Payment Adjustment and Future Rulemaking

D. Medicaid Incentives

1. Overview of Health Information

Technology in Medicaid

2. General Medicaid Provisions

3. Identification of Qualifying Medicaid EPs and Eligible Hospitals

a. Overview

b. Program Participation

1. Acute Care Hospitals

2. Children's Hospitals

c. Medicaid Professionals Program Eligibility

d. Calculating Patient Volume Requirements

e. Entities Promoting the Adoption of Certified EHR Technology

4. Computation of Amount Payable to Qualifying Medicaid EPs and Eligible Hospitals

a. Payment Methodology for EPs

(1) General Overview

(2) Average Allowable Costs

(3) Net Average Allowable Costs

(4) Payments for Medicaid Eligible Professionals

(5) Basis for Medicaid EHR Incentive Program First Payment Year and Subsequent Payment Years

(i) Medicaid EP Who Begins Adopting, Implementing or Upgrading Certified EHR Technology in the First Year

(ii) Medicaid EP Who Has Already Adopted, Implemented or Upgraded Certified EHR Technology and Meaningfully Uses EHR Technology

b. Payment Methodology for Eligible Hospitals

c. Alternative and Optional Early State Implementation To Make Incentive Payments for Adopting, Implementing or Upgrading Certified EHR Technology

d. Process for Making and Receiving

Medicaid Incentive Payments

e. Avoiding Duplicate Payment

f. Flexibility To Alternate Between Medicare and Medicaid EHR Incentive Programs One Time

g. One State Selection

5. Single Provider Election Repository and State Data Collection

6. Collection of Information Related to the Eligible Professional's National Provider Identifier (NPI) and the Tax

Identification Number (TIN)

7. Activities Required To Receive Incentive Payments

a. General Overview

b. Definitions Related to Certified EHR

Technology and Adopting, Implementing or Upgrading Such Technology

(1) Certified EHR Technology

(2) Adopting, Implementing or Upgrading

c. Other General Terminology

d. Quality Measures

8. Overview of Conditions for States To Receive Federal Financial Participation (FFP) for Incentive Payments and Implementation Funding
9. Financial Oversight, Program Integrity and Provider Appeals

III. Collection of Information Requirements

A. ICRs Regarding Demonstration of Meaningful Use Criteria ( $\$ 495.8$ )

B. ICRs Regarding Participation Requirements for EPs, Eligible Hospitals, and Qualifying CAHs (§ 495.10)

C. ICRs Regarding Identification of Qualifying MA Organizations, MA-EPs and MA-Affiliated Eligible Hospitals (§ 495.202)

D. ICRs Regarding Incentive Payments to Qualifying MA Organizations for MAEPs and Hospitals (§ 495.204)

E. ICRs Regarding Meaningful User Attestation (§ 495.210)

F. ICRs Regarding Incentive Payments to Qualifying MA Organizations for MAEligible Professionals and Hospitals (§ 495.220)

G. ICRs Regarding Process for Payments (§ 495.312)

H. ICRs Regarding Activities Required To Receive an Incentive Payment (§ 495.314)

I. ICRs Regarding State Monitoring and Reporting Regarding Activities Required To Receive an Incentive Payment (§ 495.316)

J. ICRs Regarding State Responsibilities for Receiving FFP (§ 495.318)

K. ICRs Regarding Prior Approval Conditions ( $\$ 495.324$ )

L. ICRs Regarding Termination of Federal Financial Participation (FFP) for Failure To Provide Access to Information (§ 495.330)

M. ICRs Regarding State Medicaid Agency and Medicaid EP and Hospital Activities (§ 495.332 Through § 495.338)

N. ICRs Regarding Access to Systems and Records (§ 495.342)

O. ICRs Regarding Procurement Standards (§ 495.344)

P. ICRs Regarding State Medicaid Agency Attestations (§ 495.346)

Q. ICRs Regarding Reporting Requirements (§ 495.348)

R. ICRs Regarding Retroactive Approval of FFP With an Effective Date of February 18,2009 (§ 495.358)

S. ICRs Regarding Financial Oversight and Monitoring Expenditures (§ 495.362)

T. ICRs Regarding Appeals Process for a Medicaid Provider Receiving Electronic Health Record Incentive Payments (§ 495.366)

IV. Regulatory Impact Analysis

A. Overall Impact

B. Regulatory Flexibility Analysis

C. Small Rural Hospitals

D. Unfunded Mandates Reform Act

E. Federalism

F. Anticipated Effects

G. HITECH Impact Analysis

H. Accounting Statement

\section{Background}

A. Overview of the HITECH Programs Created by the American Recovery and Reinvestment Act of 2009

The American Recovery and

Reinvestment Act of 2009 (ARRA) (Pub. L. 111-5) was enacted on February 17, 
2009. Title IV of Division B of ARRA amends Titles XVIII and XIX of the Social Security Act (the Act) by establishing incentive payments to eligible professionals (EPs), eligible hospitals, and critical access hospitals (CAHs), and Medicare Advantage Organizations to promote the adoption and meaningful use of interoperable health information technology (HIT) and qualified electronic health records (EHRs). These provisions, together with Title XIII of Division A of ARRA, may be cited as the "Health Information Technology for Economic and Clinical Health Act" or the "HITECH Act." These incentive payments are part of a broader effort under the HITECH Act to accelerate the adoption of HIT and utilization of qualified EHRs.

On January 13, 2010 we published a proposed rule (75 FR 1844), entitled "Medicare and Medicaid Programs; Electronic Health Record Incentive Program" to implement the provisions of ARRA that provide incentive payments to EPs, eligible hospitals, and CAHs participating in Medicare and Medicaid programs that adopt and successfully demonstrate meaningful use of "certified EHR technology," and incentive payments to certain Medicare

Advantage Organizations for their affiliated EPs and eligible hospitals that meaningfully use certified EHR technology. Through this final rule, we are developing the incentive programs which are outlined in Division B, Title IV of the HITECH Act. This final rule sets forth the definition of "meaningful use of certified EHR technology."

Section 13101 of the HITECH Act adds a new section 3000 to the Public Health Service Act (PHSA), which defines "certified EHR technology" as a qualified EHR that has been properly certified as meeting standards adopted under section 3004 of the PHSA. CMS and ONC have been working closely to ensure that the definition of meaningful use of certified EHR technology and the standards for certified EHR technology are coordinated. In the interim final rule published on January 13, 2010 (75 FR 2014) entitled "Health Information Technology: Initial Set of Standards, Implementation Specifications, and Certification Criteria for Electronic Health Record Technology," ONC defined the term "certified EHR technology," identified the initial set of standards and implementation specifications that such EHR technology would need to support the achievement of the proposed meaningful use Stage 1, as well as the certification criteria that will be used to certify EHR technology. ONC is also issuing a final rule on the standards, implementation specifications, and certification criteria elsewhere in this issue of the Federal Register.

In a related proposed rule published on March 10, 2010, (75 FR 11328) entitled "Proposed Establishment of Certification Programs for Health Information Technology" ONC proposed the establishment of two certification programs for purpose of testing and certifying health information

technology. In the June 24, 2010 Federal Register (75 FR 36157), ONC published a final rule to establish a temporary certification program whereby the National Coordinator would authorize organizations to test and certify complete EHRs and EHR Modules, and plans to issue a separate final rule to establish a permanent certification program to replace the temporary certification program. Specifically, this final rule will ensure that the definition of meaningful use of certified EHR technology does not require EPs, eligible hospitals, and CAHs to perform functions for which standards have not been recognized or established. Similarly, the functionality of certified EHR technology should enable and advance the definition of meaningful use.

We urge those interested in this final rule to also review the ONC interim final rule on standards and implementation specifications for certified EHR technology and the related final rule as well as the final rule on the establishment of a temporary

certification program. Readers may also visit http://healthit.hhs.gov and http:// www.cms.hhs.gov/Recovery/11 HealthIT.asp\#TopOfPage for more information on the efforts at the Department of Health and Human Services (HHS) to advance HIT initiatives.

\section{B. Statutory Basis for the Medicare $\mathcal{E}$ Medicaid EHR Incentive Programs}

Section 4101(a) of the HITECH Act adds a new subsection (o) to section 1848 of the Act. Section 1848(o) of the Act establishes incentive payments for demonstration of meaningful use of certified EHR technology by EPs participating in the original Medicare program (hereinafter referred to as the Medicare Fee-for-Service (FFS) program) beginning in calendar year (CY) 2011. Section 4101(b) of the HITECH Act also adds a new paragraph (7) to section 1848(a) of the Act. Section 1848(a)(7) of the Act provides that beginning in CY 2015, EPs who do not demonstrate that they are meaningful users of certified EHR technology will receive an adjustment to their fee schedule for their professional services of 99 percent for 2015 (or, in the case of an eligible professional who was subject to the application of the payment adjustment under section 1848(a)(5) of the Act, 98 percent for 2014), 98 percent for 2016, and 97 percent for 2017 and each subsequent year. Section 4101(c) of the HITECH Act adds a new subsection (l) to section 1853 of the Act to provide incentive payments to certain Medicare

Advantage (MA) organizations for their affiliated EPs who meaningfully use certified EHR technology and meet certain other requirements, and requires a downward adjustment to Medicare payments to certain MA organizations for professional services provided by any of their affiliated EPs who are not meaningful users of certified EHR technology, beginning in 2015. Section 1853(l) of the Act also requires us to establish a process that ensures that there are no duplicate payments made to MA organizations under section 1853(l) of the Act and to their affiliated EPs under the FFS EHR incentive program established under section 1848(o)(1)(A) of the Act.

Section 4102(a) of the HITECH Act adds a new subsection (n) to section 1886 of the Act. Section 1886(n) of the Act establishes incentives payments for demonstration of meaningful use of certified EHR technology by subsection (d) hospitals, as defined under section 1886(d)(1)(B) of the Act, participating in the Medicare FFS program beginning in Federal fiscal year (FFY) 2011. Section 4102(b)(1) of the HITECH Act amends section 1886(b)(3)(B) of the Act to provide that, beginning in FY 2015, subsection (d) hospitals that are not meaningful users of certified EHR technology will receive a reduced annual payment update for their inpatient hospital services. Section 4102(a)(2) of the HITECH Act amends section 1814(l) of the Act to provide an incentive payment to critical access hospitals (CAHs) who meaningfully use certified EHR technology based on the hospitals' reasonable costs for the purchase of certified EHR technology beginning in FY 2011. In addition, section 4102(b)(2) of the HITECH Act amends section 1814(l) of the Act to provide for a downward payment adjustment for hospital services provided by CAHs that are not meaningful users of certified EHR technology for cost reporting periods beginning in FY 2015. Section 4102(c) of the HITECH Act adds a new subsection (m) to section 1853 of the Act to provide incentive payments to qualifying MA organizations for certain affiliated hospitals that meaningfully 
use certified EHR technology to make a downward adjustment to payments to certain MA organizations for inpatient hospital services provided by its affiliated hospitals that are not meaningful users of certified EHR technology beginning in FY 2015. Section $1853(\mathrm{~m})$ of the Act also requires us to establish a process that ensures that there are no duplicate payments made to MA organizations under section $1853(\mathrm{~m})$ of the Act and to their affiliated hospitals under the FFS EHR incentive program established under section 1886(n) of the Act.

Section 4103 of the HITECH Act provides for implementation funding for the EHR incentives program under Medicare.

Section 4201 of the HITECH Act amends section 1903 of the Act to provide 100 percent Federal financial participation (FFP) to States for incentive payments to certain eligible providers participating in the Medicaid program to purchase, implement, operate (including support services and training for staff) and meaningfully use certified EHR technology and 90 percent FFP for State administrative expenses related to the program outlined in 1903(t) of the Act. Section 4201(a)(2) of the HITECH Act adds a new subsection (t) to section 1903 of the Act to establish a program with input from the States to provide incentives for the adoption and subsequent meaningful use of certified EHR technology for providers participating in the Medicaid program.

\section{Provisions of the Proposed Rule and Analysis of and Responses to Public Comments}

We proposed to add a new part 495 to title 42 of the Code of Federal Regulations to implement the provisions of Title IV of Division B of ARRA providing for incentive payments to EPs, eligible hospitals, CAHs and certain Medicare Advantage organizations for the adoption and demonstration of meaningful use of certified EHR technology under the Medicare program or the Medicaid program.

The HITECH Act creates incentives under the Medicare Fee-for-Service (FFS), Medicare Advantage (MA), and Medicaid programs for EPs, eligible hospitals and CAHs to adopt and demonstrate meaningful use of certified EHR technology, and payment adjustments under the Medicare FFS and MA programs for EPs, eligible hospitals, and CAHs who fail to adopt and demonstrate meaningful use of certified EHR technology. The three incentive programs contain many common elements and certain provisions of the HITECH Act encourage avoiding duplication of payments, reporting, and other requirements, particularly in the area of demonstration of meaningful use of certified EHR technology. Eligible hospitals and CAHs may participate in both the Medicare program and the Medicaid program, assuming they meet each program's eligibility requirements, which vary across the two programs. In certain cases, the HITECH Act has used nearly identical or identical language in defining terms that are used in the Medicare FFS, MA, and Medicaid programs, including such terms as "hospital-based EPs" and "certified EHR technology." For these reasons, we seek to create as much commonality between the three programs as possible and have structured this final rule, as we did the proposed rule, based on the premise by beginning with those provisions that cut across the three programs before moving on to discuss the provisions specific to Medicare FFS, MA and Medicaid.

A. Definitions Across the Medicare FFS, $M A$, and Medicaid Programs

Title IV, Division B of ARRA establishes incentive payments under the Medicare and Medicaid programs for certain professionals and hospitals that meaningfully use certified EHR technology, and for certain MA organizations whose affiliated EPs and hospitals meaningfully use certified EHR technology. We refer to the incentive payments made under the original Medicare program to EPs, eligible hospitals, and CAHs as the Medicare FFS EHR incentive program, the incentive payments made to qualifying MA organizations as the MA EHR incentive program, and the incentive payments made under Medicaid to eligible professionals and eligible hospitals as the Medicaid EHR incentive program. When referring to the Medicare EHR incentive program, we are generally referring to both the Medicare FFS EHR and the MA EHR incentive programs.

\section{Definitions}

Sections 4101, 4102, and 4201 of the HITECH Act use many identical or similar terms. In this section of the preamble, we discuss terms for which we are finalizing uniform definitions for the Medicare FFS, MA, and Medicaid EHR incentive programs. These definitions are set forth in part 495 subpart A of the regulations. For definitions specific to an individual program, the definition is set forth and discussed in the applicable EHR incentive program section.
The incentive payments are available to EPs which are non-hospital-based physicians, as defined in section 1861(r) of the Act, who either receive reimbursement for services under the Medicare FFS program or have an employment or contractual relationship with a qualifying MA organization meeting the criteria under section 1853(1)(2) of the Act; or healthcare professionals meeting the definition of "eligible professional" under section 1903(t)(3)(B) of the Act as well as the patient-volume and non-hospital-based criteria of section 1903(t)(2)(A) of the Act and eligible hospitals which are subsection (d) hospitals as defined under subsection 1886(d)(1)(B) of the Act that either receive reimbursement for services under the Medicare FFS program or are affiliated with a qualifying MA organization as described in section 1853(m)(2) of the Act; critical access hospitals (CAHs); or acute care or children's hospitals described under section 1903(t)(2)(B) of the Act.

a. Certified Electronic Health Record (EHR) Technology

Under all three EHR incentive programs, EPs, eligible hospitals, and CAHs must utilize "certified EHR technology" if they are to be considered eligible for the incentive payments. In the Medicare FFS EHR incentive program this requirement for EPs is found in section 1848(o)(2)(A)(i) of the Act, and for eligible hospitals and CAHs in section 1886(n)(3)(A)(i) of the Act. In the MA EHR incentive program this requirement for EPs is found in section 1853(1)(1) of the Act, and for eligible hospitals and CAHs, in section 1853(m)(1) of the Act. In the Medicaid EHR incentive program this requirement for EPs and Medicaid eligible hospitals is found throughout section 1903(t) of the Act, including in section 1903(t)(6)(C) of the Act. Certified EHR technology is a critical component of the EHR incentive programs, and the Secretary has charged ONC, under the authority given to her in the HITECH Act, with developing the criteria and mechanisms for certification of EHR technology. Therefore, we finalize our proposal to use the definition of certified EHR technology adopted by ONC. ONC issued an interim final rule with comment for the standards and certification criteria for certified EHR technology at the same time our proposed rule was issued. After reviewing the comments they received and to address changes made in this final rule, ONC will be issuing a final rule in conjunction with this final rule. When we refer to the ONC final rule, we are referring to this final rule titled 
"Health Information Technology: Initial Set of Standards, Implementation Specifications, and Certification Criteria for Electronic Health Record Technology. When we refer to the ONC IFR, we are referring to the interim final rule with comment period published in the Federal Register on January 13, 2010.

Comment: Several commenters asked for clarification on the definition of certified EHR technology. Currently, hospitals utilize multiple systems to operate electronically. For example, some electronic operating systems feed EHR data and some systems pull EHR data. Data from the two systems are then extracted and manipulated to create a quality measure calculation. The commenters' inquired as to how these systems can continue to be utilized even though, independently, these systems will not meet all certification standards. Some commenters expressed concern the ONC IFR did not include generation of the data needed to demonstrate meaningful use as a certification requirement and that certified EHR technology requirements should also include compliance with HIPAA standards as well as all relevant state statutes for the state or states where it is installed. Commenters recommended various approaches to defining certified technology especially in the early stages of the program. Some suggestions included, grandfathering existing systems for a period of three years as long as the provider could meet specific meaningful use objectives while requiring all upgrades to existing systems to be certified, allowing all EHR products certified by the Certification Commission for Health Information Technology (CCHIT) at the criteria established for 2008 or later be deemed as meeting Stage 1 certification requirements or alternatively CMS provide a process that can verify compliance of required features at no cost to providers or vendors as is done now with Enterprise Data Interchange (EDI) claims processing. Some commenters also offered other thoughts on potential unintended consequences of defining the EHR certification software process to include certifying agencies that charge for the process. The commenters believed this could result in continued new and revised requirements to justify the certifying entities' existence and increase its revenue.

Response: We have referred those comments to ONC who addresses them in their final rule.

We are adopting the ONC definition of certified EHR technology at 45 CFR 170.102 in this final rule.

\section{b. Qualified Electronic Health Record}

In order for an EHR technology to be eligible for certification, it must first meet the definition of a Qualified Electronic Health Record. This term was defined by ONC in its in its IFR and finalized by ONC in their final rule, and we are finalizing our proposal to use the definition of qualified electronic health record adopted by ONC in their final rule to be published concurrently with this rule.

Comment: We received a few comments on the definition of qualified EHR technology. Commenters expressed concerns regarding perceived gaps in defining an EHR as qualified such as a lack of the requirement for a narrative text for physicians (also known as progress note). Another comment requested further clarification regarding the requirement for a qualified EHR to "capture and query information relevant to health care quality" and "exchange electronic health information with and integrate such information from other sources." For example, some might believe that these requirements apply strictly to information contained within the EHR or closed proprietary hospital systems and not to information that would have to be obtained from outside the four walls of the practice or the extended (but closed) system.

Response: We have referred those comments to ONC who addresses them in their final rule.

We are adopting the ONC definition of Qualified Electronic Health Record at 45 CFR 170.102.

\section{c. Payment Year}

As discussed in the proposed rule, under section 1848(o)(1)(A)(i) of the Act the Medicare FFS EHR incentive payment is available to EPs for a "payment year." Section 1848(o)(1)(E) of the Act defines the term "payment year" as a year beginning with 2011. While the Act does not use the term, "payment year," for the Medicaid EHR incentive program, it does use the term "year of payment" throughout section 1903(t) of the Act, for example, at sections 1903(t)(3)(C), 1903(t)(4)(A), and 1903(t)(6)(C) of the Act. For all EPs in the Medicare and Medicaid EHR incentive programs, we are proposing a common definition for both "payment year" and "year of payment," as "any calendar year beginning with 2011" at $\S 495.4$. In the proposed rule, we explained that this definition, which is consistent with the statutory definition of "payment year" under Medicare FFS, would simplify the EHR incentive programs for EPs. As discussed later in this preamble, EPs will have the opportunity to participate in either the Medicare or Medicaid incentive programs, and once an EP has selected a program, they are permitted to make a one-time switch from one program to the other. A common definition will allow EPs to more easily understand both incentive programs, and inform their decisions regarding participation in either program.

Under section 1886(n)(1) of the Act, the Medicare FFS EHR incentive payment is available to eligible hospitals and CAHs for a "payment year." Section 1886(n)(2)(G) of the Act defines the term "payment year" as a fiscal year beginning in 2011. As hospitals are paid based on the 12month Federal fiscal year, we interpret the reference to a "fiscal year" means the fiscal year beginning on October 1 of the prior calendar year and extending to September 30 of the relevant year. Again, for the Medicaid EHR incentive program, the HITECH Act uses the term, "year of payment" (see section 1903(t)(5)(D)(ii) of the Act), rather than "payment year." For the same reasons expressed in the proposed rule and summarized above for proposing a common definition of "payment year" for EPs, and because hospitals will have the opportunity to simultaneously participate in both the Medicare and Medicaid EHR incentive programs, we propose a common definition of "payment year" and "year of payment" for both programs.

For purposes of the incentive payments made to eligible hospitals and CAHs under the Medicare FFS, MA and Medicaid EHR incentive programs, we proposed to define payment year and year of payment at $\S 495.4$, consistent with the statutory definition, as "any fiscal year beginning with 2011.”

Comment: A commenter asked CMS to identify the first possible payment year for EPs, and hospitals and CAHs.

Response: The first payment year for EPs is any calendar year (CY) beginning with CY 2011 and for eligible hospitals and CAHs is any fiscal year (FY) beginning with 2011.

Comment: The majority of commenters favored our definition of "payment year" based on the different existing fiscal periods for eligible professionals and hospitals. Additional support was received from some commenters whom explained that they participated in performance-based initiatives, which define a payment year the same as the proposed rule.

Response: After consideration of the public comments received, we are adopting our proposed definition of "payment year" in the Medicare and 
Medicaid EHR incentive programs as described above.

Comment: The majority of comments received regarding the definition of a payment year asked whether payment years must be consecutive for an $\mathrm{EP}$ or eligible hospital to receive all years of incentive payments.

Response: In the proposed rule, we defined the second, third, fourth, fifth, and sixth payment year, respectively, to mean "the second, third, fourth, fifth, and sixth calendar or Federal fiscal year, respectively, for which an EP or eligible hospital receives an incentive payment." However, section 1848(o)(1)(E) of Act defines the second through fifth payment years for an EP as each successive year immediately following the first payment year for such professional for the Medicare FFS and MA EHR incentive programs. Similarly, section 1886(n)(2)(G)(ii) of the Act defines the second through fourth payment years for an eligible hospital or $\mathrm{CAH}$ as requiring the years to be "successive" and "immediately following" the prior year. This requirement, that each payment year "immediately follow" the prior year, means that every year subsequent to the first payment year is a payment year regardless of whether an incentive payment is received by the EP, eligible hospital or CAH. For example, if a Medicare EP receives an incentive in CY 2011, but does not successfully demonstrate meaningful use or otherwise fails to qualify for the incentive in CY 2012, CY 2012 still counts as one of the EP's five payment years and they would only be able to receive an incentive under the Medicare EHR incentive program for three more years as CY 2013 would be there third payment year. In this example, the maximum incentive payment that would apply for this Medicare EP not practicing predominately in a health professional shortage area (HPSA) would be $\$ 18,000$ in 2011 , and $\$ 8,000$ in 2013 as outlined in section 1848(o)(1)(B) of the Act. The EP would have qualified for a maximum incentive payment of $\$ 12,000$ in 2012 , but did not qualify as a meaningful user for this year. No incentives may be made under the Medicare EHR incentive program after 2016.

The same rule, however, does not apply to the Medicaid EHR incentive program. For that program, payments may generally be non-consecutive. If an $\mathrm{EP}$ or eligible hospital does not receive an incentive payment for a given $\mathrm{CY}$ or FY then that year would not constitute a payment year. For example, if a Medicaid EP receives incentives in CY 2011 and CY 2012, but fails to qualify for an incentive in CY 2013, they would still be eligible to receive incentives for an additional four payment years. For hospitals, however, starting with FY 2017 payments must be consecutive. This rule is required by section 1903(t)(5)(D) of the Act, which states that after 2016, no Medicaid incentive payment may be made to an eligible hospital unless "the provider has been provided payment * * * for the previous year." As a result, Medicaid eligible hospitals must receive an incentive in FY 2016 to receive an incentive in FY 2017 and later years. Starting in FY 2016, incentive payments must be made every year in order to continue participation in the program. In no case may any Medicaid EP or eligible hospital receive an incentive after 2021. We have revised our regulations at $\$ 495.4$ to incorporate these statutory requirements.

Comment: Some commenters requested that CMS clarify the impact on EPs when they change practices in the middle of the incentive payment program; in other words, if an EP leaves a practice in year two of the incentive payment program and goes to another practice, does that EP forfeit the ability to continue collecting incentive payments for years 3 through 5 ?

Response: A qualifying EP that leaves one practice for another may still be eligible to receive subsequent incentive payments if the EP is a meaningful EHR user in the new practice. The incentive payment is tied to the individual EP, and not to his or her place of practice.

d. First, Second, Third, Fourth, Fifth, and Sixth Payment Year

In accordance with sections 1848(o)(1)(A)(ii), 1886(n)(2)(E), 1814(l)(3)(A), 1903(t)(4)(B), and 1903(t)(5)(A) of the Act, for EPs, eligible hospitals, and CAHs that qualify for EHR incentive payments in a payment year, the amount of the payment will depend in part on whether the EP or hospital previously received an incentive payment and, if so (for the Medicare EHR incentive program) when the EP or hospital received his or her first payment. We proposed to define the first payment year to mean the first $\mathrm{CY}$ or Federal fiscal year (FY) for which an EP, eligible hospital, or CAH receives an incentive payment. Likewise, we proposed to define the second, third, fourth, fifth, and sixth payment year, respectively, to mean the second, third, fourth, fifth, and sixth CY or FY, respectively, for which an EP, eligible hospital, or CAH receives an incentive payment.
Comment: As stated above, many commenters requested clarification on non-consecutive payment.

Response: This comment is addressed above.

Comment: A commenter requested CMS to clarify the consequences for a hospital that originally qualified and received incentive payments the first year, but in a subsequent year failed to qualify as a meaningful user of certified EHR technology.

Response: Meaningful use will be assessed on a year-by-year basis as we establish different Stages of meaningful use criteria for different years. If an EP or an eligible hospital including a CAH has failed to demonstrate meaningful use of certified EHR technology for a certain payment year, the EP, eligible hospital, or CAH will not be qualified for incentive payments for that payment year. However, upon successful demonstration as a meaningful EHR user in subsequent years, an EP, eligible hospital or CAH may be eligible to receive an incentive payment. As discussed above, however, for the Medicare program, the failure of the eligible hospital or $\mathrm{CAH}$ to demonstrate meaningful use in the subsequent year, will affect the total payments that hospital is eligible to receive, as, pursuant to the statute, the hospital is treated as skipping a payment year. Payment adjustments apply to Medicare providers who are unable to demonstrate meaningful use starting in 2015.

Comment: One commenter asked if CMS could apply the same Medicaid EP's first year incentive eligibility requirements of adopting, implementing or upgrading to certified EHR technology to Medicare physicians instead of demonstration of meaningful use.

Response: The HITECH Act allows Medicaid EPs and eligible hospitals to receive an incentive for the adoption, implementation, or upgrade of certified EHR technology in their first participation year. In subsequent years, these EPs and eligible hospitals must demonstrate that they are meaningful users. There are no parallel provisions under the Medicare EHR incentive program that would authorize us to make payments to Medicare EPs, eligible hospitals, and CAHs for the adoption, implementation or upgrade of certified EHR technology. Rather, in accordance with sections 1848(o)(2), 1886(n)(3)(A), and 1814(l)(3)(A) of the Act, Medicare incentive payments are only made to EPs, eligible hospitals, and CAHs for the demonstration of meaningful use of certified EHR technology. 
After consideration of the public comments received, we are finalizing the definitions of First payment year as proposed. For the Medicare EHR incentive programs, we are modifying the definitions of second, third, fourth, fifth payment year to make clear that these years are "each successive year following the first payment year." For the Medicaid EHR incentive program, we included definitions of first, second, third, fourth, fifth and sixth payment year that make clear that these are the years for which payment is received. The regulations can now be found at $\S 495.4$ of our regulations.

\section{e. EHR Reporting Period}

In the proposed rule, we proposed a definition of EHR Reporting Period for purposes of the Medicare and Medicaid incentive payments under sections 1848(o), 1853(l)(3), 1886(n), 1853(m)(3), 1814(l) and 1903(t) of the Act. For these sections, we proposed that the EHR reporting period would be any continuous 90-day period within the first payment year and the entire payment year for all subsequent payment years. In our proposed rule, we did not make any proposals regarding the reporting period that will be used for purposes of the payment adjustments that begin in 2015. We intend to address this issue in future rulemaking, for purposes of Medicare incentive payment adjustments under sections 1848(a)(7), 1853(l)(4), 1886(b)(3)(B)(ix), 1853(m)(4), and 1814(l)(4) of the Act.

For the first payment year only, we proposed to define the term EHR reporting period at $\S 495.4$ of our regulations to mean any continuous 90day period within a payment year in which an EP, eligible hospital or CAH successfully demonstrates meaningful use of certified EHR technology. The EHR reporting period therefore could be any continuous period beginning and ending within the relevant payment year. Starting with the second payment year and any subsequent payment years for a given EP, eligible hospital or $\mathrm{CAH}$, we proposed to define the term EHR reporting period at $\S 495.4$ to mean the entire payment year. In our discussion of considerations in defining meaningful use later in this section we discuss how this policy may be affected by subsequent revisions to the definition of meaningful use.

For the first payment year, we stated in the proposed rule our belief that giving EPs, eligible hospitals and CAHs flexibility as to the start date of the EHR reporting period is important, as unforeseen circumstances, such as delays in implementation, higher than expected training needs and other unexpected hindrances, may cause an $\mathrm{EP}$, eligible hospital, or CAH to potentially miss a target start date.

Comment: Some commenters supported the 90-day reporting period proposed for the first payment year. One commenter requested that exceptions, per the provider request, be considered individually in cases of compliance for less than the 90 days (for example, 85 days). Commenters preferred the 90-day reporting period overall and many suggested it be used for subsequent years as well. We also received comments questioning why Medicaid providers would need to conform to the 90-day reporting period in order to adopt, implement or upgrade certified EHR technology.

Response: We do believe that for program integrity it is crucial to maintain a consistent reporting period. Basing the incentive payments on meaningful use implies a minimum level of use in order to receive the incentive payment. The timeframe is part of the determination of whether use is meaningful and therefore requires a minimum as well. Given the short time period as compared to the entire year, we do not believe an exception process is needed. However, we agree with commenters that an EHR reporting period for demonstrating adoption, implementation or upgrading certified EHR technology by Medicaid EPs and eligible hospitals is unnecessary and are removing it for the final rule in this instance. Similarly, Medicaid EPs and eligible hospitals who are demonstrating meaningful use for the first time in their second payment year, will have a 90day reporting period to maintain parity with Medicare providers' first meaningful use payment year. We do not believe that after successfully demonstrating meaningful use, a 90-day period is appropriate for subsequent years. The reasons for using the 90-day period instead of the full year are based on potential delays in implementing certifying EHR technology. Once certified EHR technology is implemented these are no longer applicable.

After consideration of the public comments received and with the clarification described above for adopting, implementing or upgrading, we are finalizing the 90-day reporting period for the first payment year based on meaningful use as proposed for Medicare EPs, eligible hospitals and CAHs and full year EHR reporting periods for subsequent payment years. For Medicaid EPs and eligible hospitals, the EHR reporting period will be a 90day period for the first year a Medicaid
EP or eligible hospital demonstrates meaningful use and full year EHR reporting periods for subsequent payment years.

\section{f. Meaningful EHR User}

Section 1848(o)(1)(A)(i) of the Act, limits incentive payments under the Medicare FFS EHR incentive program to an EP who is a "meaningful EHR user." Similarly, section 1886(n)(1) and 1814(l) of the Act, limits incentive payments under the Medicare FFS EHR incentive program to an eligible hospital or CAH, respectively, who is a "meaningful EHR user." Section 1903(t)(6)(C)(i)(II) of the Act limits incentive payments for payment years other than the first payment year to a Medicaid EP or eligible hospital who "demonstrates meaningful use of certified EHR technology." We proposed to define at $\S 495.4$ the term "meaningful EHR user" as an EP, eligible hospital, or CAH who, for an EHR reporting period for a payment year, demonstrates meaningful use of certified EHR technology in the form and manner consistent with our standards (discussed below).

Comment: Several commenters indicated there is a need to align measures and programs, to avoid having to report similar measure standards to different Federal, State and other entities.

Response: We concur with the goal of alignment to avoid redundant and duplicative reporting and seek to accomplish this to the extent possible now and in future rulemaking.

Comment: Several commenters suggested that CMS considers EPs, eligible hospitals, and CAHs who are participating in certain existing programs as meaningful EHR users. The commenters contended that the standards followed by participants in these programs are equivalent to those we proposed to adopt for purposes of demonstrating meaningful use. The programs recommended by commenters are-

- Qualified Health Information Exchange Networks; and

- Medicare Electronic Health Record Demonstration Program.

Response: We do not agree that participation in these programs would be the equivalent to demonstrating meaningful use in accordance with the criteria under the EHR incentive programs. Most of these programs place a heavy focus on one of the five priorities of meaningful use discussed in the next section such as reporting clinical quality measures or the exchange of health information, tailored to the individual program's goals. For example, the goal of the Medicare 
Electronic Health Record Demonstration Program, for example, which was started in 2009 and pre-dates passage of the HITECH Act, is to reward delivery of high-quality care supported by the adoption and use of electronic health records in physician small to mediumsize primary care practices. The purpose of this program is to encourage adoption and increasingly sophisticated use of EHRs by small to medium-sized primary care practices. While this goal is similar to the overall objective of the HITECH Act, the requirements for the demonstration are not as broad-based as that of the HITECH Act, and payment incentives are based on the level of use over the duration of the program, which will vary by practice. Therefore, it is not appropriate to deem practices participating in the EHR Demonstration as meaningful users for purposes of the HITECH Act. The HITECH Act also requires use certified EHR technology as defined by ONC to qualify for incentive payments. While CCHIT has certified EHR technology in the past, the ONC regulation "Establishment of the Temporary Certification Program for Health Information Technology; Final Rule" (see 75 FR 36157) which establishes a temporary certifying body has yet to be established. Where possible, we have aligned the criteria required to demonstrate meaningful use with existing programs like PQRI and RHQDAPU as discussed in section II.A.3 of this final rule. After consideration of the public comments received, we are finalizing our definition of a meaningful EHR user as proposed.

\section{Definition of Meaningful Use}

\section{a. Considerations in Defining Meaningful Use}

In sections 1848(o)(2)(A) and 1886(n)(3)(A) of the Act, the Congress identified the broad goal of expanding the use of EHRs through the term meaningful use. In section 1903(t)(6)(C) of the Act, Congress applies the definition of meaningful use to Medicaid eligible professionals and eligible hospitals as well. Certified EHR technology used in a meaningful way is one piece of a broader HIT

infrastructure needed to reform the health care system and improve health care quality, efficiency, and patient safety. HHS believes this ultimate vision of reforming the health care system and improving health care quality, efficiency and patient safety should drive the definition of meaningful use consistent with the applicable provisions of Medicare and Medicaid law.
In the proposed rule we explained that in defining meaningful use we sought to balance the sometimes competing considerations of improving health care quality, encouraging widespread EHR adoption, promoting innovation, and avoiding imposing excessive or unnecessary burdens on health care providers, while at the same time recognizing the short timeframe available under the HITECH Act for providers to begin using certified EHR technology.

Based on public and stakeholder input received prior to publishing the proposed rule, we consider a phased approach to be most appropriate. Such a phased approach encompasses reasonable criteria for meaningful use based on currently available technology capabilities and provider practice experience, and builds up to a more robust definition of meaningful use, based on anticipated technology and capabilities development. The HITECH Act acknowledges the need for this balance by granting the Secretary the discretion to require more stringent measures of meaningful use over time. Ultimately, consistent with other provisions of law, meaningful use of certified EHR technology should result in health care that is patient centered, evidence-based, prevention-oriented, efficient, and equitable.

Under this phased approach to meaningful use, we intend to update the criteria of meaningful use through future rulemaking. We refer to the initial meaningful use criteria as "Stage 1." We currently anticipate two additional updates, which we refer to as Stage 2 and Stage 3, respectively. We expect to update the meaningful use criteria on a biennial basis, with the Stage 2 criteria by the end of 2011 and the Stage 3 criteria by the end of 2013. The stages represent an initial graduated approach to arriving at the ultimate goal.

- Stage 1: The Stage 1 meaningful use criteria, consistent with other provisions of Medicare and Medicaid law, focuses on electronically capturing health information in a structured format; using that information to track key clinical conditions and communicating that information for care coordination purposes (whether that information is structured or unstructured, but in structured format whenever feasible); implementing clinical decision support tools to facilitate disease and medication management; using EHRs to engage patients and families and reporting clinical quality measures and public health information. Stage 1 focuses heavily on establishing the functionalities in certified EHR technology that will allow for continuous quality improvement and ease of information exchange. By having these functionalities in certified EHR technology at the onset of the program and requiring that the EP, eligible hospital or CAH become familiar with them through the varying levels of engagement required by Stage 1, we believe we will create a strong foundation to build on in later years. Though some functionalities are optional in Stage 1, as outlined in discussions later in this rule, all of the functionalities are considered crucial to maximize the value to the health care system provided by certified EHR technology. We encourage all EPs, eligible hospitals and CAHs to be proactive in implementing all of the functionalities of Stage 1 in order to prepare for later stages of meaningful use, particularly functionalities that improve patient care, the efficiency of the health care system and public and population health. The specific criteria for Stage 1 of meaningful use are discussed at section II.2.c of this final rule.

- Stage 2: Our goals for the Stage 2 meaningful use criteria, consistent with other provisions of Medicare and Medicaid law, expand upon the Stage 1 criteria to encourage the use of health IT for continuous quality improvement at the point of care and the exchange of information in the most structured format possible, such as the electronic transmission of orders entered using computerized provider order entry (CPOE) and the electronic transmission of diagnostic test results (such as blood tests, microbiology, urinalysis, pathology tests, radiology, cardiac imaging, nuclear medicine tests, pulmonary function tests, genetic tests, genomic tests and other such data needed to diagnose and treat disease). For the final rule, we elaborate on our plans for Stage 2. We expect that stage two meaningful use requirements will include rigorous expectations for health information exchange, including more demanding requirements for eprescribing and incorporating structured laboratory results and the expectation that providers will electronically transmit patient care summaries to support transitions in care across unaffiliated providers, settings and EHR systems. Increasingly robust expectations for health information exchange in stage two and stage three will support and make real the goal that information follows the patient. We expect that Stage 2 will build upon Stage 1 by both altering the expectations of the functionalities in Stage 1 and likely adding new functionalities which 
are not yet ready for inclusion in Stage 1 , but whose provision is necessary to maximize the potential of EHR technology. As discussed later in this final rule, we are making some objectives of the Stage 1 of meaningful use optional and other required. We will consider every objective that is optional for Stage 1 to be required in Stage 2 as well as revaluate the thresholds and exclusions of all the measures both percentage based and those currently a yes/no attestation. Additionally, we may consider applying the criteria more broadly to all outpatient hospital settings (not just the emergency department).

- Stage 3: Our goals for the Stage 3 meaningful use criteria are, consistent with other provisions of Medicare and Medicaid law, to focus on promoting improvements in quality, safety and efficiency leading to improved health outcomes, focusing on decision support for national high priority conditions, patient access to self management tools, access to comprehensive patient data through robust, patient-centered health information exchange and improving population health.

We did not include regulatory provisions for Stage 2 or Stage 3 in our proposal and with one exception discussed under the CPOE objective, we are not finalizing Stage 2 or Stage 3 requirements at this time. However, we plan to build upon Stage 1 by increasing the expectations of the functionalities in Stage 1 and adding new objectives for Stage 2. In our next rulemaking, we currently intend to propose that every objective in the menu set for Stage 1 (as described later in this section) be included in Stage 2 as part of the core set. While allowing providers flexibility in setting priorities for EHR

implementation takes into account their unique circumstances, we maintain that all the objectives are crucial to building a strong foundation for health IT and to meeting the statutory objectives of the Act. In addition, as indicated in our proposed rule, we anticipate raising the threshold for these objectives in both Stage 2 and 3 as the capabilities of HIT infrastructure increases. For Stage 2, we intend to review the thresholds and measures associated with all Stage 1 objectives considering advances in technology, changes in standard practice, and changes in the marketplace (for example, wider adoption of information technology by pharmacies) and propose, as appropriate, increases in these requirements.

We recognize that the thresholds included in the final regulation are ambitious for the current state of technology and standards of care. However, we expect the delivery of health care to evolve through the inception of the HITECH incentive programs and implementation of the Affordable Care Act prior to finalizing Stage 2. Furthermore, data collected from the initial attestations of meaningful use will be used to ensure that the thresholds of the measures that accompany the objectives in Stage 2 are continue to aggressively advance the use of certified EHR technology. Finally, we continue to anticipate redefining our objectives to include not only the capturing of data in electronic format but also the exchange (both

transmission and receipt) of that data in increasingly structured formats. As appropriate, we intend to propose the addition of new objectives to capture new functions that are necessary to maximize the potential of EHR technology, but were not ready for Stage 1. For instance, we would consider adding measures related to CPOE orders for services beyond medication orders. The intent and policy goal for raising these thresholds and expectations is to ensure that meaningful use encourages patient-centric, interoperable health information exchange across provider organizations.

We will continue to evaluate the progression of the meaningful use definition for consistency with the HITECH ACT and any future statutory requirements relating to quality measurement and administrative simplification. As the purpose of these incentives is to encourage the adoption and meaningful use of certified EHR technology, we believe it is desirable to account for whether an EP, eligible hospital or CAH is in their first, second, third, fourth, fifth, or sixth payment year when deciding which definition of meaningful use to apply in the

beginning years of the program. The HIT Policy Committee in its public meeting on July 16, 2009 also voiced its approval of this approach. However, such considerations are dependent on future rulemaking, so for this final rule Stage 1 criteria for meaningful use are valid for all payments years until updated by future rulemaking.

We proposed that Medicare EPs, eligible hospitals, and CAHs whose first payment year is 2011 must satisfy the requirements of the Stage 1 criteria of meaningful use in their first and second payment years (2011 and 2012) to receive the incentive payments. We anticipate updating the criteria of meaningful use to Stage 2 in time for the 2013 payment year and therefore anticipate for their third and fourth payment years (2013 and 2014), an EP, eligible hospital, or CAH whose first payment year is 2011 would have to satisfy the Stage 2 criteria of meaningful use to receive the incentive payments. We proposed that Medicare EPs, eligible hospitals, and CAHs whose first payment year is 2012 must satisfy the Stage 1 criteria of meaningful use in their first and second payment years (2012 and 2013) to receive the incentive payments. We anticipate updating the criteria of meaningful use to Stage 2 in time for the 2013 payment year and anticipate for their third payment year (2014), an EP, eligible hospital, or CAH whose first payment year is 2012 would have to satisfy the Stage 2 criteria of meaningful use to receive the incentive payments. We discussed in the proposed rule that Medicare EPs, eligible hospitals, and CAHs whose first payment year is 2013 must satisfy the Stage 1 criteria of meaningful use in their first payment year (2013) to receive the incentive payments. We anticipate updating the criteria of meaningful use to Stage 2 in time for the 2013 payment year and therefore anticipate for their second payment year (2014), an EP, eligible hospital, or CAH whose first payment year is 2013 would have to satisfy the Stage 2 criteria of meaningful use to receive the incentive payments. We discussed in the proposed rule that Medicare EPs, eligible hospitals, and CAHs whose first payment year is 2014 must satisfy the Stage 1 criteria of meaningful use in their first payment year (2014) to receive the incentive payments. In the proposed rule, we discussed the idea that alignment of stage of meaningful use and payment year should synchronize for all providers in 2015, and requested comment on the need to create such alignment. After reviewing public comment on this issue, our goal remains to align the stages of meaningful use across all providers in 2015. However, we acknowledge the concerns regarding the different Medicare and Medicaid incentive timelines, as well as concerns about whether Stage 3 would be appropriate for an EP's, eligible hospital's or CAH's first payment year at any point in the future and believe the issue needs additional review and discussion before we lay out a clear path forward for 2015 and beyond. Therefore, we have decided to remove language in the final rule discussing our possible directions for any year beyond 2014. We will address the years beyond 2014 in later rulemaking. Table 1 outlines how we anticipate applying the respective criteria of meaningful use in the first years of the program, and how we anticipate applying such criteria for 
subsequent payment years, through 2014. Please note that nothing in this discussion restricts us from requiring additional stages of meaningful use (beyond stage 3) through future rulemaking. In addition, as we expect to engage in rulemaking to adopt the criteria that will accompany Stages 2 and 3 of meaningful use, stakeholders should wait for those rulemakings to determine what will be required for those Stages and should not view the discussions in this preamble or final rule as binding the agency to any specific definition for those future stages.

\section{TABLE 1: Stage of Meaningful Use Criteria by Payment Year}

\begin{tabular}{|c|c|c|c|c|c|}
\hline \multirow{2}{*}{$\begin{array}{c}\text { First } \\
\text { Payment Year }\end{array}$} & $\mathbf{2 0 1 1}$ & $\mathbf{2 0 1 2}$ & $\mathbf{2 0 1 3}$ & $\mathbf{2 0 1 4}$ & $\mathbf{2 0 1 5}$ \\
\cline { 2 - 6 } & Ptage 1 & Stage 1 & Stage 2 & Stage 2 & TBD \\
\hline 2011 & & Stage 1 & Stage 1 & Stage 2 & TBD \\
\hline 2012 & & & Stage 1 & Stage 1 & TBD \\
\hline 2013 & & & & Stage 1 & TBD \\
\hline 2014 & & & &
\end{tabular}

Please note that each of the EHR incentive programs has different rules regarding the number of payment years available, the last year for which incentives may be received, and the last payment year that can be the first payment year for an EP, eligible hospital, or CAH. The applicable payment years and the incentive payments available for each program are also discussed in section II.C. of this final rule for the Medicare FFS EHR incentive program, in section II.D. of this final rule for the MA EHR incentive program, and in section II.E. of this final rule for the Medicaid EHR incentive program.

Comment: Numerous commenters noted that it is inappropriate to align the Medicaid EHR incentive payment program with the Medicare program due to the lack of penalties in the Medicaid program and due to the option for Medicaid providers to participate in their first year by adopting, implementing, or upgrading certified EHR technology.

Response: This was not the only reason for having all EPs, eligible hospitals, and CAHs align by 2015 . However, as we are not addressing stages of meaningful use beyond 2014 in this final rule, potential alignment is not discussed. We will reconsider this comment in future rulemaking.

The stages of criteria of meaningful use and how they are demonstrated are described further in this final rule and will be updated in subsequent rulemaking to reflect advances in HIT products and infrastructure. We note that such future rulemaking might also include updates to the Stage 1 criteria.

We invited comment on our alignment between payment year and the criteria of meaningful use particularly in regards to the need to create alignment across all EPs, eligible

hospitals, and CAHs in all EHR incentive programs in 2015.

Comment: Many commenters requested that if there continued to be a year where all EPs, eligible hospitals and CAHs must meet the same stage of meaningful use that that year be 2017, rather than 2015 as we had discussed in the proposed rule. These commenters asserted that EPs, eligible hospitals, and CAHs whose first payment year is after 2011 might not have sufficient time to reach the Stage 3 of meaningful use criteria by 2015. Some commenters pointed out that while the HITECH Act states that 2015 is the first year of payment adjustments, it provides for escalation of the payment adjustments so that they do not reach their full levels until 2017.

Response: As we explained in the proposed rule, equity in the level of meaningful use across all EPs, eligible hospitals, and CAHs subject to the payment adjustment was not the only reason for our plan that all EPs, eligible hospitals, and CAHs satisfy the Stage 3 criteria for either the Medicare or Medicaid EHR incentive programs. The achievement of many of the ultimate goals of meaningful use of certified EHR technology are dependent on a critical mass of EPs, eligible hospitals, and CAHs all being meaningful EHR users. Exchange of health information is most valuable when it is so robust that it can be relied upon to provide a complete or nearly complete picture of a patient's health. For example, robust Stage 3 meaningful use by an EP does not assist that EP in avoiding ordering a duplicative test, if the EP with information on the original test is only a Stage 1 meaningful EHR user and is not yet exchanging that information. This dependency is key to the need to get to Stage 3 for all providers. Another reason for alignment at Stage 3 in 2015 is that many of the barriers to functionalities of EHRs that exist today as may no longer exist in 2015 . The existence of these barriers today is one of the primary reasons for having a staged approach as opposed to requiring more robust meaningful use at the beginning of the program. Providers, developers of EHRs, government and non-governmental organizations are all working to remove these barriers. We believe it is likely there will be success in removing many of these barriers, which would make many of the compromises made in Stage 1 no longer necessary by 2015 . However, due to the many comments on alignment starting in 2015 and our plan to engage in additional more rounds of rulemaking, we are removing discussion of actual alignment between the first payment year of an EP, eligible hospital, or CAH and the Stage of meaningful use they will be expected to meet for all years after 2014. Our policies for 2015 and subsequent years will be determined through future rulemaking.

Comment: Several commenters requested that CMS base the payment adjustments on Stage 1 of meaningful use regardless of the EP, eligible hospital, or CAH's prior participation in the incentive program.

Response: We thank commenters for the thoughtful comments received, and will take their input into consideration when in future rulemaking when we consider whether to require that EPs, eligible hospitals, and CAHs satisfy the stage 3 definition of meaningful use in order to avoid reduced payments under Medicare for their professional services and inpatient hospital services beginning 2015. We reiterate, however, that in this final rule we are only adopting criteria that we expect will 
apply in 2011 and 2012. We have also outlined the expected progression of stages of meaningful use criteria until 2014. However, we are not in this rule finalizing regulations that address the meaningful use standards that apply in 2015 and thereafter.

Comment: Numerous commenters requested that we specifically propose objectives and measures for Stage 2 and 3 . We also received recommendations on what those objectives and, in rare cases, measures should be. We discussed some of these objectives in the proposed rule and discuss them again in this final rule in section II.d. Others are highly related to existing objectives, while still others were not discussed in any way in the proposed rule. The suggested objectives and measures for Stages 2 and 3 include the following:

- Use of evidence-based order sets.

- Electronic medication

administration record (eMAR).

- Bedside medication administration support (barcode/RFID).

- Record nursing assessment in EHR.

- Record nursing plan of care in EHR.

- Record physician assessment in EHR.

- Record physician notes in EHR.

- Multimedia/Imaging integration.

- Generate permissible discharge

prescriptions electronically.

- Contribute data to a PHR.

- Record patient preferences

(language, etc).

- Provide electronic access to patientspecific educational resources.

- Asking patients about their experience of care.

Response: With one exception discussed under the CPOE objective, we continue to believe that finalizing specific objectives and measures for later stages is inappropriate. One of the greatest benefits of the phased stage approach is the ability to consider the impact and lessons of the prior stage when formulating a new stage. Many commenters supported our discussion of later stages for this very reason. In addition, we do not believe it is appropriate to finalize objectives for any stage of meaningful use that were not specifically discussed in the proposed rule, as doing so would deprive the public the opportunity to comment on the objective in question. Nevertheless, we thank commenters for the thoughtful comments received, and expect to take their input into consideration when in future rulemaking we consider additional or revised criteria and measures to adopt for the stage 2 and stage 3 definitions of meaningful use.

Comment: A commenter indicated that attestation is an insufficient means to hold providers accountable for the expenditure of public funds and to protect against fraud and abuse.

Response: We likewise are concerned with the potential fraud and abuse. However, Congress for the HITECH Act specifically authorized submission of information as to meaningful use through attestation. CMS is developing an audit strategy to ameliorate and address the risk of fraud and abuse.

b. Common Definition of Meaningful

Use Under Medicare and Medicaid

Under sections 1848(o)(1)(A)(i), 1814(1)(3)(A), and 1886(n)(1) of the Act, an EP, eligible hospital or CAH must be a meaningful EHR user for the relevant EHR reporting period in order to qualify for the incentive payment for a payment year in the Medicare FFS EHR incentive program. Sections 1848(o)(2)(A) and 1886(n)(3)(A) of the Act provide that an $\mathrm{EP}$ and an eligible hospital shall be considered a meaningful EHR user for an EHR reporting period for a payment year if they meet the following three requirements: (1) Demonstrates use of certified EHR technology in a

meaningful manner; (2) demonstrates to the satisfaction of the Secretary that certified EHR technology is connected in a manner that provides for the electronic exchange of health information to improve the quality of health care such as promoting care coordination, in accordance with all laws and standards applicable to the exchange of information; and (3) using its certified EHR technology, submits to the Secretary, in a form and manner specified by the Secretary, information on clinical quality measures and other measures specified by the Secretary. The HITECH Act requires that to receive a Medicaid incentive payment in the initial year of payment, an EP or eligible hospital may demonstrate that they have engaged in efforts to "adopt, implement, or upgrade certified EHR technology." Details, including special timeframes, on how we define and implement "adopt, implement, and upgrade" are in section II.D.7.b.2 of this final rule. For subsequent payment years, or the first payment year if an EP or eligible hospital chooses, section

1903(t)(6)(C)(i)(II) of the Act, prohibits receipt of an incentive payment, unless "the Medicaid provider demonstrates meaningful use of certified EHR technology through a means that is approved by the State and acceptable to the Secretary, and that may be based upon the methodologies applied under section 1848(o) or 1886(n)." (Sections 1848(o) and 1886(n) of the Act refer to the Medicare EHR incentive programs for EPs and eligible hospitals/CAHs respectively.) Under section 1903(t)(8) of the Act to the maximum extent practicable, we are directed to avoid duplicative requirements from Federal and State governments to demonstrate meaningful use of certified EHR technology. Provisions included at section 1848(o)(1)(D)(iii) of the Act also contain a Congressional mandate to avoid duplicative requirements for meaningful use, to the extent practicable. Finally, section 1903(t)(8) of the Act allows the Secretary to deem satisfaction of the requirements for meaningful use of certified EHR

technology for a payment year under Medicare to qualify as meaningful use under Medicaid.

We stated in the proposed rule that we believe that given the strong level of interaction on meaningful use encouraged by the HITECH Act, there would need to be a compelling reason to create separate definitions for Medicare and Medicaid. We declared in the proposed rule that we had found no such reasons for disparate definitions in our internal or external discussions. To the contrary, stakeholders have

expressed strong preferences to link the Medicare and Medicaid EHR incentive programs wherever possible. Hospitals are entitled to participate in both programs, and we proposed to offer EPs an opportunity to switch between the Medicare and Medicaid EHR incentive programs. Therefore, we proposed to create a common definition of meaningful use that would serve as the definition for EPs, eligible hospitals and CAHs participating in the Medicare FFS and MA EHR incentive program, and the minimum standard for EPs and eligible hospitals participating in the Medicaid EHR incentive program. We clarified that under Medicaid this proposed common definition would be the minimum standard. We proposed to allow States to add additional objectives to the definition of meaningful use or modify how the existing objectives are measured; the Secretary would not accept any State alternative that does not further promote the use of EHRs and healthcare quality or that would require additional functionality beyond that of certified EHR technology. See section II.D.8. of this final rule for further details.

For hospitals, we proposed to exercise the option granted under section 1903(t)(8) of the Act and deem any Medicare eligible hospital or CAH who is a meaningful EHR user under the Medicare EHR incentive program and is otherwise eligible for the Medicaid incentive payment to be classified as a meaningful EHR user under the Medicaid EHR incentive program. This 
is applicable only to eligible hospitals and CAHs, as EPs cannot simultaneously receive an incentive payment under both Medicare and Medicaid.

We solicited comments as to whether there are compelling reasons to give the States additional flexibility in creating disparate definitions beyond what was proposed. In addition, if commenting in favor of such disparate definitions, we also asked interested parties to comment on whether the proposal of deeming meeting the Medicare definition as sufficient for meeting the Medicaid definition remains appropriate under the disparate definitions. This is applicable only to hospitals eligible for both the Medicare and Medicaid incentive programs. Furthermore, if a State has CMS-approved additional meaningful use requirements, hospitals deemed as meaningful users by Medicare would not have to meet the State-specific additional meaningful use requirements in order to qualify for the Medicaid incentive payment.

Comment: Most commenters believe that States should not be allowed the option to add to or change the meaningful use requirements for the Medicaid EHR incentive program. The commenters' main reason for standardizing the meaningful use requirements for both Medicare and Medicaid is to eliminate administrative burden on both providers and EHR vendors to accommodate programming and reporting using different technical specifications for the same or similar measures.

Response: After consideration of the comments received, we are finalizing the provisions regarding possible differences in the definition of meaningful use between Medicare and Medicaid with the following revisions. We believe that over time the option to add to or change the floor definition of meaningful use might represent an important policy tool for States and therefore CMS plans to review and adjudicate these requests over the duration of the program. For Stage 1 of meaningful use, we have revised the definition of meaningful use in response to the many comments and are requiring an overall lower bar and an approach that is more flexible. On the other hand, we wish to support the ability for States to reinforce their public health priorities and goals based upon their existing public health infrastructure and maturity. For that reason, we, for Stage 1, will only entertain States' requests to tailor the Stage 1 meaningful use definition as it pertains specifically to public health objectives and data registries. For purposes of the Medicaid
EHR incentive program during Stage 1 of meaningful use, these are limited to:

Objective: Generate lists of patients by specific conditions to use for quality improvement, reduction of disparities, research, or outreach.

Measure: Generate at least one report listing patients of the EP or eligible hospital with a specific condition.

Example: Generate lists of patients with the following conditions: depression, diabetes, obesity, etc. This would not be for reporting to the State but to draw EPs' or eligible hospitals' attention in order to better manage their patient population. States would also be permitted to request CMS approval to include this in the core set for all EPs and/or eligible hospitals.

Objective: Capability to submit electronic data to immunization registries of Immunization Information Systems and actual submission in accordance with applicable law and practice.

Measure: Performed at least one test of certified EHR technology's capacity to submit electronic data to immunization registries and follow up submission if the test is successful (unless none of the immunization registries to which the EP or eligible hospital submits such information have the capacity to received the information electronically).

Example: State could point to a specific immunization registry that supports standards-based transmission of data and dictate how that information is transmitted. States would also be permitted to request CMS approval to include this objective in the core list for all EPs and eligible hospitals. The justification for this request in their State Medicaid HIT Plan, should address any potential barriers for providers in achieving this objective.

Objective: Capability to submit electronic data on reportable (as required by state or local law) lab results to public health agencies and actual submission in accordance with applicable law and practice.

Measure: Performed at least one test of certified EHR technology's capacity to submit electronic data on reportable lab results to public health agencies and follow-up submission if the test is successful (unless none of the public health agencies to which an eligible hospital submits such information have the capacity to receive the information electronically).

Example: State could specify the standards-based means of transmission and/or the destination of this data. States would also be permitted to request CMS approval to include this objective in the core list for all and eligible hospitals. The justification for this request in their State Medicaid HIT Plan, should address any potential barriers for providers in achieving this objective.

Objective: Capability to submit electronic syndromic surveillance data to public health agencies and actual transmission according to applicable law and practice.

Measure: Performed at least one test of certified EHR technology's capacity to submit electronic syndromic

surveillance data to public health agencies and follow-up submission if the test is successful (unless none of the public health agencies to which an EP or eligible hospital submits such information have the capacity to receive the information electronically).

Example: State could specify the standards-based means of transmission and/or the destination of this data. States would also be permitted to request CMS approval to include this objective in the core list for all EPs and eligible hospitals. The justification for this request in their State Medicaid HIT Plan, should address any potential barriers for providers in achieving this objective.

We reiterate that we will not approve any requests that would require EHR functionality above and beyond that included in the ONC EHR certification criteria as finalized for Stage 1 of meaningful use.

Comment: Several commenters requested that CMS affirm the ability of States to require additional meaningful use criteria for all eligible professionals and hospitals (pursuant to $\S$ 495.316(a), 495.316(d)(2)), regardless of whether those entities were deemed eligible through Medicare.

Response: Section 1903(t)(8) provides authority for the Secretary to "deem satisfaction of requirements for * * * meaningful use for a payment year under title XVIII to be sufficient to qualify as meaningful use under [1903(t)]." We continue to believe that allowing deeming ensures that hospitals eligible for both programs are able to focus on only one set of measures, without requiring duplication of effort or confusion regarding meaningful use standards. Thus, hospitals eligible for both Medicare and Medicaid incentive payments will be deemed for Medicaid if they have met the meaningful use definition through Medicare, even if a State has an approved State-specific definition of meaningful use. States cannot withhold a Medicaid EHR incentive payment from dually eligible hospitals if they have met all the eligibility criteria for Medicaid, and have met the Medicare definition for meaningful use. 
Because of this comment, we are revising section $\S 495.4$ of our regulations to indicate that eligible hospitals who are meaningful users under the Medicare EHR incentive payment program are deemed as meaningful users under the Medicaid EHR incentive payment program, and need not meet additional criteria imposed by the State. While this is not a new requirement, it was not previously listed in regulations.

Comment: A commenter asked that CMS adopt and affirm the deeming approach in its final rule and ensure that the regulatory language reflects this approach.

Response: We agree and have included in the final rule regulation language that hospitals that are meaningful users under the Medicare EHR Incentive Program are deemed meaningful users under the Medicaid EHR Program.

Comment: Several commenters requested that CMS not deem hospitals having met the meaningful use requirements for the Medicare EHR Incentive Payment, as having fulfilled the meaningful use requirements for the State's Medicaid EHR Incentive

Payment. The commenters noted that if a State sought for acute care hospitals to participate in their statewide health information exchange and yet those hospitals did not have to do so in order to qualify for both the Medicare and Medicaid EHR Incentive Payments, then they would have no motivation to do so. The commenters would like acute care hospitals eligible for both the Medicare and Medicaid EHR Incentive Program to have to comply with any State-specific meaningful use requirements, in addition to the Medicare floor definition.

Response: In consideration of the comments received, CMS adopts its proposed preamble language about deeming hospitals and adds the corresponding regulation text. This is necessary for Stage 1 of meaningful use in particular, where we believe it is crucial to prevent additional burden on providers and foster eligible hospitals' path to successful EHR adoption and meaningful use. In addition, as already noted, for Stage 1, we will not entertain States' requests to alter the floor definition of meaningful use as codified in this final rule except for specific public health objectives. That thereby reduces the possible differences between the Medicare and Medicaid definitions of meaningful use. As part of Stage 2 of meaningful use, CMS might consider States requests to tailor meaningful use as it pertains to health information exchange, for example.
Further details about this policy option will be included in future rulemaking and subject to public comment.

\section{c. Stage 1 Criteria for Meaningful Use}

In the proposed rule we proposed that to qualify as a meaningful EHR user for 2011, EPs, eligible hospitals or CAHs must demonstrate that they meet all of the objectives and their associated measures as set forth in proposed $\S 495.6$. We further proposed and finalize in this final rule that except where otherwise indicated, each objective and its associated measure must be satisfied by an individual EP as determined by unique National Provider Identifiers (NPIs) and an individual hospital as determined by unique CMS certification numbers (CCN).

Discussion of Whether an EP, Eligible Hospital or CAH Must Meet All Stage 1 Meaningful Use Objectives and Their Associated Measures

Comment: Commenters almost unanimously said that requiring an EP, eligible hospital or CAH to meet all of the objectives and their associated measures in order to qualify as a meaningful EHR user was too ambitious given the current state of EHR technology, adoption levels, the timeline for certification of EHR technologies, the realities of implementing EHR technology and the timeline proposed for Stage 1 of meaningful use in our proposed rule.

Most of the commenters suggested alternatives that they believed would support the health care policy priorities of Stage 1. Several different alternatives were proposed. The first alternative would be to require a specified percentage of the Stage 1 meaningful use objectives and associated measures, with an EP, eligible hospital or $\mathrm{CAH}$ free to select which of the objectives and associated measures it would satisfy. For example under our proposed objectives and associated measures, if an EP were required to meet 20 percent, then an EP would be considered a meaningful EHR user if he or she satisfied any five of the proposed twenty-five objectives and associated measures. Most commenters suggesting this alternative envisioned that later stages of meaningful use would require that EPs, eligible hospitals, and CAHs satisfy a higher of the percentage of the objectives and associated measures. For example if 20 percent of the objectives and associated measures were required for Stage 1, then 50 percent might be required in Stage 2.

After a fixed percentage, the suggestion next favored by commenters, including the HIT Policy Committee and
MedPAC, was to divide the meaningful use objectives into two categories, a "core set" of objectives and "menu set" of objectives. To be a considered a meaningful user under this approach, an $\mathrm{EP}$, eligible hospital, or CAH would be required to satisfy (1) all core set of objectives, and (2) a specified percentage of the menu set of objectives, with the EP, eligible hospital, or CAH free to select which of the menu set of objectives it would satisfy. For example, if five objectives were in the core set all EPs, eligible hospitals, and CAHs would have to meet those objectives. If twenty objectives were in the menu set, then EPs, eligible hospitals, and CAHs would not have to meet one or more of those objectives. Commenters varied widely as to which objectives should be included in the core set of objectives, as well as the percentage of menu set objectives an EP, eligible hospital, or CAH must satisfy.

Some commenters suggested that we simply reduce the number of objectives required for Stage 1 of meaningful use. Recommendations in this regard varied from reducing the required objectives to only just a few (the lowest number being three), limiting the required objectives to only to those objectives that affect health outcomes of individual patients, to targeted elimination of a few objectives.

Finally, some commenters suggested that we eliminate all of the measures associated with the Stage 1 meaningful use objectives and only require that EPs, eligible hospitals, and CAHs attest that they have attempted to meet each of the objectives.

Response: After reviewing the comments, we agree that requiring that EPs, eligible hospitals, and CAHs satisfy all of the objectives and their associated measures in order to be considered a meaningful EHR user would impose too great a burden and would result in an unacceptably low number of EPs, eligible hospitals, and CAHs being able to qualify as meaningful EHR users in the first two years of the program. In considering an alternative approach, we have sought to develop an alternative that is responsive to some degree to all the concerns raised by the commenters. We have tried to reduce the requirements both in number required and in the thresholds of the associated measures and provide some flexibility as well. At the same time, however, we must be mindful of the relevant statutory requirements. Sections 1848 (o)(2)(A) and 1886(n)(3) of the Act, specify three requirements for meaningful use: (1) Use of certified EHR technology in a meaningful manner (for example, electronic prescribing); (2) that 
the certified EHR technology is connected in a manner that provides for the electronic exchange of health information to improve the quality of care; and (3) that, in using certified EHR technology, the provider submits to the Secretary information on clinical quality measures and such other measures selected by the Secretary. We believe that each EP, eligible hospital, and $\mathrm{CAH}$ must meet at least one objective within each of the three requirements for meaningful use. We are concerned that if we were to give EPs, eligible hospitals, and CAHs full discretion to select which meaningful use objectives they will satisfy, some providers would not choose one or more objectives within each of the three statutory requirements for meaningful use. Furthermore, we are concerned that affording EPs, eligible hospitals, and CAHs such flexibility as to which meaningful use objectives to meet would delay many of the goals outlined for meaningful use in section II.a.2. of this final rule. If in choosing what objectives to defer, one provider chooses to focus on improving processes to improve healthcare quality, another chooses to focus on being able to exchange health information and yet another on engaging patients and families it is possible that we would fail to accomplish any of these goals at a population level. For these reasons, we do not believe it would be appropriate to afford providers the unlimited flexibility to select which of the meaningful use objectives they will meet. Rather, as explained below, we believe providers at a minimum should have to satisfy a core set of objectives in order to qualify as meaningful EHR users.

Similarly, while we agree that merely reducing the number of objectives would make meaningful use easier to achieve for most providers, we believe that this reduction does not afford the same flexibility to all providers to account for their individual difficulties in meeting meaningful use that some of the other alternatives do as allowing a provider to choose certain objectives to defer. Due to any number of circumstances such as EHR adoption level, availability of health information exchange network, size of practice or hospital, etc., an objective that is easy for one EP to achieve might be very difficult for another EP. Under this alternative, no allowance is made for those differences. Finally, we disagree that meaningful use should be limited to improving the health outcomes of individual patients. There are significant gains that meaningful use can achieve in the areas of public health, privacy and security, engagement of patients and their families and efficiency of care that may not improve health outcomes, but have significant other benefits such as engaging patients more fully in decisions affecting their health and reducing costs through increased efficiency of care. We believe that all of these have a significant impact on health outcome priorities. Therefore, we do not categorically reduce the number of objectives for Stage 1 definition of meaningful use. We consider requests to defer an objective to later stages of the meaningful use criteria or eliminate a specific objective below in our discussion of each objective.

Comment: Another alternative that was recommended by a significant number of commenters was that we base the incentive payment amount on the number of stage 1 meaningful use objectives satisfied by an EP or eligible hospital, with those satisfying more objectives eligible for a higher incentive payment amount. While some commenters varied in the specifics or did not provide specifics, generally we take this to mean that if an EP, eligible hospital, or CAH met half of the objectives then they would receive half of the incentive payment they would have received had they met all the objectives.

Response: The HITECH Act does not give us the authority to award partial payments. As discussed elsewhere in this final rule, sections 1848(o)(1)(A) of the Act specifies the payment incentive amount to which an EP who is a meaningful EHR user is entitled. Similarly, section 1886(n)(2) of the Act sets forth a formula for calculation of incentive payment amount to which an eligible hospital that is a meaningful EHR user is entitled. Similarly, section 1814(l)(3)(A) of the Act sets forth a formula for calculation of incentive payment amount to which an eligible hospital that is a meaningful EHR user is entitled. Similarly, section 1903(t)(4)(B) of the Act sets parameters for determining the Medicaid EHR incentive for Medicaid EP. None of these parameters are related to meaningful use. Similarly, section 1903(t)(5)(A) of the Act sets forth a formula for calculation of the incentive payment amount to which a Medicaid eligible hospital is entitled. As we do not have the authority to alter these statutory formulas for calculating the incentive payment amounts under Medicare and Medicaid, we cannot pro rate the incentive payment amount based on the number of meaningful use objectives satisfied by an EP, eligible hospital, or $\mathrm{CAH}$.

After consideration of the public comments received, we are establishing a core set of objectives with associated measures and a menu set of objectives with associated measures. In order to qualify as a meaningful EHR user, an EP, eligible hospital, or CAH must successfully meet the measure for each objective in the core set and all but five of the objectives in the menu set. With one limitation, an EP, eligible hospital, or CAH may select any five objectives from the menu set to be removed from consideration for the determination of qualifying as a meaningful EHR user. Further discussion of the objectives, including additional details about their inclusion in the core set, can be found at each objective.

We believe that establishing both a core and a menu set adds flexibility and allows the minimum statutory set to be met. In determining the objectives to include in the core set, we looked at all comments, especially those of the HIT Policy Committee and other commenters who recommended some required and optional elements. The HITECH Act requires the use of health information technology in improving the quality of health care, reducing medical errors, reducing health disparities, increasing prevention and improving the continuity of care among health care settings. In defining the core set of meaningful use objective, we believe the most crucial aspect to consider is meeting the three statutory guidelines provided in the HITECH Act and discussed in section II.A.2.a of this final rule. Second is to identify those objectives that are most crucial to laying the foundation for obtaining value from meaningful use of certified EHR technology. Third, we believe that meaningful use should be patientcentered so we focus on getting the most value to the patient. We believe the recommendation of the HIT Policy Committee accomplishes third criteria, but falls short of the first and second. To accomplish the first criteria, we add the objective of submitting clinical quality measures to CMS or the States and the objective of exchanging key clinical information among providers of care and patient authorized entities. To accomplish the second, we add several additional objectives to the core set of measures as critical elements pertinent to the management of patients. We have received a number of comments in support of these particular measures as critical to the management of patients (maintaining an up-to-date problem list, active medication list, active allergy list, smoking history and incorporate clinical 
lab tests into EHR as structured data) in comparison to other requirements. The addition of two other functional objectives (drug-drug and drug-allergy features) as core measures are for improved patient-safety. All of the listed elements are integral to the initial or ongoing management of a patient's current or future healthcare. While each element is important in the management of patients in and of itself, the aggregate of the elements elevates the importance of clinical information to not only the primary provider but for all members of the interdisciplinary team involved in the patient's care. The HITECH Act statutorily requires the use of health information technology in improving the quality of health care, reducing medical errors, reducing health disparities, increasing prevention, and improving the continuity of care among health care settings. These core set of measures are also foundational and aligned with each other. For example, electronic copies of health information given to patient will be useless if it does not contain basic information such as a problem list, medication list or allergy list. Exchange of information to other members of the health care team across settings will depend on having structured data of these elements. Therefore, in support of the HITECH Act in meeting the statutory requirements, we have expanded the core set of measures to include these fundamental elements to improve patient care. Below we list the objectives included in the core set of meaningful use objectives.

-Use CPOE

- Implement drug to drug and drug allergy interaction checks

-E-Prescribing (EP only)

-Record demographics

-Maintain an up-to-date problem list

-Maintain active medication list

-Maintain active medication allergy list

— Record and chart changes in vital signs

- Record smoking status

-Implement one clinical decision support rule

-Report CQM as specified by the Secretary

-Electronically exchange key clinical information

-Provide patients with an electronic copy of their health information

-Provide patients with an electronic copy of their discharge instructions (Eligible Hospital/CAH Only)

-Provide clinical summaries for patients for each office visit (EP Only)

-Protect electronic health information created or maintained by certified EHR
In addition, achieving Stage 1 meaningful use means demonstration of progress in each of the five healthcare outcome priorities outlined in the proposed rule and discussed again later in this section. Only one of these priorities is not represented in the core set, population and public health. As we have discussed in this section we do not want any priority to be overlooked due to the flexibility we have added to Stage 1 of meaningful use; therefore, all EPs and hospitals must choose at least one of the population and public health measures to demonstrate as part of the menu set. This is the only limitation placed on which five objectives can be deferred from the menu set.

\section{Discussion on Whether Certain EP, Eligible Hospital or CAH Can Meet all Stage 1 Meaningful Use Objectives Given Established Scopes of Practice}

In the proposed rule, we specifically encouraged comments on whether certain providers may have difficulty meeting one or more of the objectives due to their provider type or chosen specialties

Comment: We received many comments, both general and specific, that certain providers or specialists may not be able to comply with certain objectives because they are beyond the scope of their licensing authority or because they are outside the scope of their standard of practice. For example, chiropractors do not have prescribing authority and thus may not make use of an EHR technology's e-prescribing function and rheumatologists may not require information on vital signs. While comments on this potential nonapplicability primarily focused on EPs, we did receive comments that some objectives may not be relevant to smaller or specialized eligible hospitals as well.

Response: We believe the division of the meaningful use objectives into a core set and a menu set may minimize the impact of including among the meaningful use objectives one or more objectives that certain providers or specialists may be unable to satisfy as the EP, eligible hospital, or CAH can defer five objectives from the menu set. However, if the EP, eligible hospital or $\mathrm{CAH}$ has an insurmountable barrier to meeting an objective in the core set or a significant number in the menu set then the problem remains. For example, without any consideration on an EP, eligible hospital or CAH's capability to meet the measure associated with a core objective any EP that could not order medications requiring a prescription would not be able to become a meaningful EHR user as e-prescribing is a core set objective. Similarly, any eligible hospital or CAH that did not have any requests for electronic copy of discharge instructions would not be able to become a meaningful EHR user. In addition, if this were to occur for a significant number of menu set objectives, the flexibility for the EP, eligible hospital, or CAH to use the five objectives to account for other concerns such as implementation struggles or workflow process redesign would be curtailed. To account for this possibility, we have modified each objective and measure to indicate when there is an option for an EP, eligible hospital, or $\mathrm{CAH}$ to report that the objective/measure is inapplicable to them, because they have no patients or no or insufficient number of actions that would allow calculation of the meaningful use measure. This will allow an EP, eligible hospital, or $\mathrm{CAH}$ to qualify as a meaningful EHR user without being required to meet objectives we have specified as potentially inapplicable. We note that the exclusions to meaningful use objectives/measures are specific to each objective/measure. In our discussion of each specific objective/measure (which occurs later in this preamble), we have identified specific exclusions where they exist. Providers wishing to claim that an objective/measure is inapplicable to them would need to meet the criteria of such an exception.

After consideration of the public comments received, we have identified, for each meaningful use objective, whether the EP, eligible hospital, or CAH may attest that they did not have any patients or insufficient actions on which to base a measurement of a meaningful use for the EHR reporting period. For objectives in the core set, such an attestation would remove the objective from consideration when determining whether an EP, eligible hospital, or CAH is a meaningful EHR user. In other words, the EP, eligible hospital, or CAH could satisfy the core set objectives by satisfying all remaining objectives included in the core set. For objectives in the menu set, such an attestation would also remove the objective from consideration when determining whether an EP, eligible hospital, or CAH is a meaningful EHR user. For example, if for one objective included in the menu set an EP attests that he or she did not have any patients or insufficient actions during the EHR reporting period on which to base a measurement of a meaningful use objective, rather than satisfy 5 of the 10 meaningful use objectives included in the menu set for EPs, the EP need only 
satisfy 4 of the 9 remaining meaningful use objectives included in the menu set for EPs

\section{EPs Practicing in Multiple Practices}

Another situation where flexibility may be needed in order for an EP to become a meaningful EHR user is the situation where an EP may provide care in multiple practices or multiple locations. We proposed a policy to account for EPs practicing in multiple practices and settings. We discussed in the proposed rule that we believe it is unlikely for an EP to use one record keeping system for one patient population and another system for another patient population at one location. We are concerned about the application of the measures associated with the meaningful use objectives for EPs who see patients in multiple practices or multiple locations. If an EP does not have certified EHR technology available at each location/practice where they see patients it could become impossible for the EP to successfully become a meaningful EHR user based on the measures associated with the meaningful use objectives. We do not seek to exclude EPs who meaningfully use certified EHR technology when it is available because they also provide care in another practice where certified EHR technology is not available. Therefore, we proposed that all measures be limited to actions taken at practices/ locations equipped with certified EHR technology. A practice is equipped if certified EHR technology is available at the beginning of the EHR reporting period for a given geographic location. Equipped does not mean the certified EHR technology is functioning on any given day during the EHR reporting period. Allowances for downtime and other technical issues with certified EHR technology are made on an objective-by-objective basis as discussed later in this section. We are concerned that seeing a patient without certified EHR technology available does not advance the health care policy priorities of the definition of meaningful use. We are also concerned about possible inequality of different EPs receiving the same incentive, but using certified EHR technology for different proportions of their patient population. We believe that an EP would have the greatest control of whether certified EHR technology is available in the practice in which they see the greatest proportion of their patients. We proposed that to be a meaningful EHR user an EP must have 50 percent or more of their patient encounters during the EHR reporting period at a practice/location or practices/locations equipped with certified EHR technology. An EP for who does not conduct 50 percent of their patient encounters in any one practice/location would have to meet the 50 percent threshold through a combination of practices/locations equipped with certified EHR technology. For example, if the EP practices at both a Federally Qualified Health Center (FQHC) and within his or her individual practice, we would include in our review both of these locations and certified EHR technology would have to be available at the location where the EP has at least 50 percent of their patient encounters.

Comment: Some commenters recommended that 50 percent or more of the patient encounters must occur at the practice location that receives the incentive payment.

Response: As discussed in section II.A.4 of this final rule, an EP may assign their incentive payment to other practices. We do not believe that limiting practices and EPs to only considering the location that receives an incentive payment provides advantages to the program. The requirement suggested by commenters would potentially cause some EPs not to meet the 50 percent threshold even if through a combination of practices they may use certified EHR technology for far more than 50 percent of their patient encounters.

Comment: Some commenters requested clarification of our proposed statement "Therefore, we proposed that all measures be limited to actions taken at practices/locations equipped with certified EHR technology"

Response: We mean this statement to be that as long as an EP has certified EHR technology available for 50 percent or more of their patient encounters during the EHR reporting period they only have to include those encounters where certified EHR technology is available at the start of the EHR reporting period. We discuss the measures later in this section of the final rule, but an illustrative example would be the objective of maintain an up-todate problem list. The measure associated with this objective is "More than $80 \%$ of all unique patients seen by the EP or admitted to the eligible hospital's or CAH's inpatient or emergency department (POS 21 or 23) have at least one entry or an indication that no problems are known for the patient recorded as structured data." Therefore, if an EP only practices at one location or has certified EHR technology available at all practice locations then the denominator would be all unique patients seen during the EHR reporting period. However, if an EP practices at multiple locations and only has certified EHR technology for 80 percent of their patient encounters, then the denominator is only those unique patients seen at locations where certified EHR technology is available. We reiterate that this is not to account for certified EHR technology downtime, Certified EHR technology is available at a location if it is available at the start of the EHR reporting period regardless of its actual availability for any given day during the EHR reporting period.

After consideration of the comments received, we are finalizing this requirement as proposed.

\section{Discussion of the Burden Created by the Measures Associated With the Stage 1 Meaningful Use Objectives}

Comment: Many commenters expressed concerns about the difficulties of capturing the denominators for the measures that are expressed as percentages. They pointed out that the formulas in the proposed rule would require providers to conduct labor-intensive counts of paper documents such as prescriptions or laboratory results in order to compute the denominators of the percentage based measures. Some commenters suggested that we adopt alternative measurement mechanisms, for example establishing simple counts of electronic occurrences, while others proposed that denominators be computed utilizing only data collected in the certified EHR technology.

Response: We acknowledge that the percentage-based measures, as expressed in the proposed rule, would create a reporting burden for EPs, eligible hospitals, and CAHs, and we examined a number of alternatives that potentially reduce the burden of reporting.

In the proposed rule, we discussed the option of counts instead of percentages and due to comments received have reassessed this option in the final rule. This approach clearly has the advantage of simplifying the process. For example, rather than counting the number of prescriptions transmitted electronically and then dividing by the total number of prescriptions, the EP would simply need to count the number of electronically transmitted prescriptions until a benchmark number is passed. If the benchmark number is exceeded, then the provider meets the measure. However, there are several shortcomings to this approach. First, we received little input from commenters as to where the benchmark numbers for the various objectives should be set and any benchmark set now would not benefit 
from public comment without significantly delaying the Medicare and Medicaid EHR incentive programs. (One exception was that a number of commenters suggested using the PQRI measure for e-prescribing, which is the generation of at least one $\mathrm{eRx}$ associated with a patient visit on 25 or more unique events during the reporting period.) Setting the limit too high would disadvantage small providers, since they would have smaller patient populations, while setting the limit too low would create requirements for larger providers that would be so limited as to be meaningless. A larger provider could implement the functionality for a much shorter period than the EHR reporting period and meet the count. In either case, it would be difficult to establish a trajectory in later stages that would result in meaningful progress being made by both small and large providers.

We then assessed the option of limiting the occurrences counted in the denominator to those included in the provider's certified EHR technology. As an example, if an EP captures 1,000 prescriptions as structured data in certified EHR technology, and electronically transmits 500 of these prescriptions, the EP's certified EHR technology generated score would be 50 percent. This approach does simplify the computation process, since this approach does not have to take into account whether some prescriptions were not included or included as unstructured data in the certified EHR technology. However, it does not demonstrate the extent to which the provider has used the certified EHR technology. For example, a provider that has captured only 10 prescriptions in the certified EHR technology as structured data, but writes 1,000 prescriptions because the provider achieved only a limited use of their certified EHR technology would also score 50 percent by electronically transmitting only 5 prescriptions according to an automatic report from the certified EHR technology. Again, this methodology does not lead providers toward an upward trajectory of both certified EHR technology deployment and accomplishment of meaningful use.

We selected a third option, which we believe addresses the shortcomings of the second option while still preserving much of the simplicity of that approach. In our approach, we focus on those measures whose denominator is not based on all patients, but rather a subset of patients or actions such as the ordering of a lab test or the recording of a patient's request for an electronic copy of their discharge instructions. We believe that it is reasonable to require an EP, eligible hospital, or CAH to know how many unique patients they care for in the EHR reporting period and therefore maintain that denominator where it applies. The maintenance of measures using the patient as the denominator as encompassing all patients ensures a certain level of utilization of certified EHR technology by the EP, eligible hospital, or CAH. If a measure encompassing all patients has a threshold of 80 percent, then at least 80 percent of the patients' records must be maintained using certified EHR technology otherwise the EP, eligible hospital or CAH could not possibly meet the threshold. We note a number of measures included in the core set (such as "Record Demographics" and "Maintain an Up-to-Date Problem List") require an analysis of all unique patients, and not just patients whose records are maintained in certified EHR technology As discussed later the thresholds for maintaining an up-to-date problem list, medication list and medication allergy list are set at $\mathbf{8 0}$ percent. We believe these thresholds will create a baseline that ensures that EPs, eligible hospitals and CAHS are maintain a minimum percentage of patient records in certified EHR technology, and allows the provider community to advance toward the longer-term objective of capturing all patient data in certified EHR technology. For those measures that focus on the recording of actions or subset of patients to generate the denominator, we limit the measures to the information for patients whose records are maintained in certified EHR technology. We offer the following examples that relate to the e-prescribing and the provision of electronic copy of a patient's health information:

E-Prescribing Example: An EP orders 1,000 prescriptions for patients whose records are maintained in their certified EHR technology and 500 of those are transmitted electronically. The EP's denominator is 1,000 prescriptions, the numerator is 500 prescriptions, and their score is 50 percent. If the EP captures all 1,000 prescriptions as structured data the calculation could be automated by the certified EHR technology. If the EP does not capture all 1,000 prescriptions as structured data than more manual review may be required. We would define "records maintained in the certified EHR technology" to include any patient for which sufficient data was entered in the certified EHR technology to allow the record to be saved, and not rejected due to incomplete data. This may be a more limited set of data, but an EP, eligible hospital, or CAH would still have to have sufficient information in certified EHR technology to meet the measures associated with Stage 1 of meaningful use. For example, an EP might be able to save a record with just a patient's name, but as the record would lack any information this patient would count in the denominator, but not the numerator for many objectives. Electronic Copy of a Patient's Health Information Provided upon Request Example: An EP

maintains 1,000 patient records in their certified EHR technology. Of those patients, fifty make requests for electronic copies of their health information. The EP provides all of the electronic copies within three business days. The denominator is 50 , the numerator is 50, and the EP's percentage is 100 percent. If the EP captures requests for information as structured data, the calculation could be automated by the certified EHR technology. If the $\mathrm{EP}$ does not capture all the requests as structured data then more manual review may be required. We will likely revisit the methodology in Stage 2, where we would expect that at least basic EHR functionality has been implemented throughout the provider enterprise.

After consideration of public comments, we are limiting the following objectives and their associated measures to patients whose records are maintained using certified EHR technology. Specific information on how to determine inclusion in the denominator and numerator is discussed in the full discussion of each objective later in this final rule.

- Use CPOE

- Generate and transmit permissible prescriptions electronically (eRx)

- Record and chart changes in vital signs

- Record smoking status for patients 13 years old or older

- Record advance directives for patients 65 years old or older

- Incorporate clinical lab-test results into certified EHR technology as structured data

- Provide patients with an electronic copy of their health information (including diagnostic test results, problem list, medication lists, medication allergies), upon request

- Provide patients with an electronic copy of their discharge instructions at time of discharge, upon request

- Provide clinical summaries for patients for each office visit

- Send reminders to patients per patient preference for preventive/ follow-up care 
- Perform medication reconciliation at relevant encounters and each transition of care

- Provide summary care record for each transition of care and referral

\section{Discussion on Meaningful Use Relationship to Certified EHR Technology}

Comment: We received several comments requesting more specific information of how certified EHR technology will accomplish meaningful use. Some commenters expressed concern that patient clinical outcome measurement and improvement was not addressed explicitly in the requirements of certified EHR technology, but rather the requirements focused data entry and provision of data electronically.

Response: One of the main purposes of certifying EHR technology is to provide the EP, eligible hospital, or $\mathrm{CAH}$ with confidence that the technology will not be the limiting factor in the achievement of meaningful use. As such, all questions of how or will certified EHR technology be able to accomplish meaningful use broadly or at a specific objective level are best answered by ONC. CMS and ONC have worked closely since the enactment of the HITECH Act to ensure certification fully supports meaningful use. We explicitly link each meaningful use objective to certification criteria for certified EHR technology. The capabilities and standards that are certified are those that are used to meet the Stage 1 objectives of meaningful use. This way we ensure that certified EHR technology can accomplish meaningful use and meaningful use has the intended consequences of improving the healthcare priorities that make up meaningful use.

Discussion on the Relationship Between a Stage 1 Meaningful Use Objective and Its Associated Measure

Comment: Many commenters pointed out gaps between what they believed were the anticipated results from an objective and the results that are measured by the associated measure. A particular concern of some of these commenters is cases where the certification criteria supports the measure, but in their view fell short of supporting the objective.

Response: In the proposed rule, we attempted to draw a clear distinction between the objective and the associated measure. The objectives represent a wide range of activities some of which are commonplace for EPs, eligible hospitals, and CAHs using EHRs today, while others are ambitious goals even for the most sophisticated EHR user of today. For some objectives, all aspects of the objective are within the control of the EP, eligible hospital, or CAH. Other objectives rely on electronic exchange with partners or external infrastructure over which EPs, eligible hospitals and CAHs may have little influence and no control. We have attempted to accommodate these differences when we select the Stage 1 measure for a given objective. The measure more accurately reflects our view of what is feasible for Stage 1 than the objective itself. The certification criteria necessarily reflect more on the measure than the objective, as full compliance with an objective is beyond the scope of what can be accomplished for a significant number of EPs, eligible hospitals or CAHs in our timeframe for Stage 1. This rationale was our assertion in the proposed rule as the justification for measures that represent less than full achievement of their objective. This is further supported by some of the comments received although for any given objective the comments addressing that objective were a small fraction of the total number of comments received and views on how much a measure should allow for less than full achievement varied widely among those commenting. Although we received over 2,000 public comments, the number of specific comments addressing an individual objective were relatively small ranging from 40 to 200 . We reviewed those comments and made specific changes to measures in the discussion of each objective. We reiterate that achievement of the measure always equates to achievement of the objective for Stage 1 of meaningful use. We also reiterate that certified EHR technology will always be able to support achievement of the measure by including the necessary functionalities. However, as with any technology, certified EHR technology is only as good as the information it contains and getting information into certified EHR technology is heavily dependent on processes developed by the EP, eligible hospital, or CAH. It is for this reason that all measures, even those for objective whose aspects are fully under the control of the EP, eligible hospital, or $\mathrm{CAH}$, represent less than full fulfillment of the objective to varying degrees. As stated, for demonstrating meaningful use and any follow up review by CMS or the States, successfully meeting the associated measure always equates to successfully meeting the objective. Updated information on the associated measures including the numerator, denominator, thresholds and exclusions are as discussed in the following section. More detailed specifications and guidance on calculating the measures will be issued soon after the publication of this final rule.

As we described in the proposed rule, in discussing the objectives that constitute the Stage 1 criteria of meaningful use, we adopted a structure derived from recommendations of the HIT Policy Committee of grouping the objectives under care goals, which are in turn grouped under health outcomes policy priorities. We believe this structural grouping provides context to the individual objectives; however, the grouping is not itself an aspect of meaningful use. The criteria for meaningful use are based on the objectives and their associated measures.

We will now review the comments for each objective and measure and make changes to our original proposal or finalize as proposed.

(1) Objectives and Their Associated Measures

The HIT Policy Committee identified as its first health outcomes policy priority improving quality, safety, efficiency and reducing health disparities. The HIT Policy Committee also identified the following care goals to address this priority:

- Provide access to comprehensive patient health data for patient's healthcare team.

- Use evidence-based order sets and CPOE.

- Apply clinical decision support at the point of care.

- Generate lists of patients who need care and use them to reach out to those patients.

- Report information for quality improvement and public reporting. As we explained in the proposed rule, for the last care goal, the HIT Policy Committee proposed the goal as "Report to patient registries for quality improvement, public reporting, etc." We have modified this care goal, because we believe that patient registries are too narrow a reporting requirement to accomplish the goals of quality improvement and public reporting. We note that the HIT Policy Committee's recommended objectives include the reporting of quality measures to CMS. We do not believe that CMS would normally be considered a "patient registry". We also removed the phrase "etc." We believe that the level of ambiguity created by "etc" is not appropriate for Federal regulations.

NPRM EP Objective: Use CPOE.

NPRM Eligible Hospital Objective: Use CPOE for orders (any type) directly 
entered by the authorizing provider (for example, MD, DO, RN, PA, NP).

In the proposed rule, we described CPOE as entailing the provider's use of computer assistance to directly enter medical orders (for example,

medications, consultations with other providers, laboratory services, imaging studies, and other auxiliary services) from a computer or mobile device. The order is also documented or captured in a digital, structured, and computable format for use in improving safety and organization. We said that for Stage 1 criteria, it will not include the electronic transmittal of that order to the pharmacy, laboratory, or diagnostic imaging center.

Comment: A majority of commenters recommended that EPs, eligible hospitals, and CAHs be allowed to defer CPOE for varying lengths of time ranging from 2012 to 2017 . The commenters cited various reasons for deferment including that CPOE is an advanced clinical function that typically is the last process to be implemented due to the need to build the entire infrastructure to support the CPOE process. Other commenters noted an increased burden as if the orders cannot be transmitted, then duplicate paper orders will have to be produced which can lead to patient safety risks. Commenters also noted that CPOE appears in the latter stages of the Certification Commission for Healthcare Information Technology (CCHIT) EHR implementation process. A minority, but significant number of comments encouraged CMS to maintain CPOE for 2011. Those commenters in favor of retaining CPOE in 2011 believed that CPOE is a basic EHR feature that should be a standard offering of a certified EHR technology and is critical to improving quality of care through audit trails and alerting of delinquent order and/or delinquent deferred orders.

Response: We have determined that CPOE should be included in the core set of measures for Stage 1 in order to advance meaningful use. CPOE is a foundational element to many of the other objectives of meaningful use including exchange of information and clinical decision support. Many commenters, including several physician associations, the HIT Policy Committee and members of Congress through their endorsement of the HIT Policy Committee's recommendation, recommended that CPOE be required in Stage 1. CPOE has been a major initiative of US hospitals for over a decade and is a foundational functionality to many of the activities that further the health care policy priorities of meaningful use. For example, entering a medication order using CPOE allows the EHR to provide feedback on whether the medication may have adverse reactions with other medications the patient is taking. Another benefit of CPOE is that greatly simplifies the workflow process of inputting information into certified EHR technology in a structured way to populate the patient record.

Comment: Several commenters asked that we further specify who could enter the order using CPOE. Some commenters stated that only the ordering provider should be permitted to enter the order. These commenters stated that the ordering professional needs to be presented with clinical decision support at the time of entry and that the relay of an order to another individual is a source of potential error. Other commenters recommended that any licensed healthcare professional or indeed any individual (licensed or not) who receives the order from the ordering provider be permitted to perform the CPOE. The most common argument presented by these commenters is that this is currently how CPOE is handled in practice and a shift to entry by only the ordering provider would be too disruptive to workflow.

Response: We agree with those commenters who recommend allowing any licensed healthcare professional to enter orders using CPOE. We further refine this recommendation to be that any licensed healthcare professional can enter orders into the medical record per state, local and professional guidelines. While we understand that this policy may decrease opportunities for clinical decision support and adverse interaction, we believe it balances the potential workflow implications of requiring the ordering provider to enter every order directly, especially in the hospital setting. We disagree with commenters that anyone should be allowed to enter orders using CPOE. This potentially removes the possibility of clinical decision support and advance interaction alerts being presented to someone with clinical judgment, which negates many of the benefits of CPOE.

Comment: We received requests for clarification of this objective and what types of orders would meet this requirement.

Response: Our intent in the proposed rule was to capture orders for medications, laboratory or diagnostic imaging.

However, after careful consideration of the comments, we are adopting an incremental approach by only requiring medication orders for Stage 1. First, this supports the objectives of e-prescribing, drug-drug and drug-allergy checks.
Second, this requirement will improve patient-safety because of the alignment of ordering medications in a structured data format will enable providers to create registries of patients for potential medical recalls, participate in surveillance for potential sentinel events and life-threatening side effects of new medications. Third, other measures involving transitions of care documents and summary of care document will require the entry of an active medication list. After consideration of the public comments received, we are finalizing the meaningful use objective for EPs at 495.6(d)(1)(i) and for eligible hospitals, and CAHs at 495.6(f)(1)(i) as "Use CPOE for medication orders directly entered by any licensed healthcare professional who can enter orders into the medical record per state, local and professional guidelines".

NPRM EP Measure: CPOE is used for at least 80 percent of all orders.

NPRM Eligible Hospital or $C A H$

Measure: For eligible hospitals, CPOE is used for 10 percent of all orders.

In the proposed rule under CPOE, we discussed several concepts related to any associated measure of any objective that relies on a percentage calculation. These are the use of a percentage versus a count; setting a threshold for measures not requiring the electronic exchange of information; EPs practicing in multiple locations, some of which may not have certified EHR technology available, and the patient population to which the measure would apply. All except the last of these received extensive comments and are addressed in comment and response sections earlier in this section. In the proposed rule, we said that we would base the measures associated with the objectives on both the Medicare/Medicaid patient population and all other patients as well. We said that we believe it is unlikely that an EP would use one record keeping system for one patient population and another system for another patient population at one location and that requiring reporting differences based on payers would actually increase the burden of meeting meaningful use. We received very few comments on this aspect of our proposed rule and those that were received were generally supportive of this proposal. Therefore, we are finalizing the policy that all meaningful use measures be calculated based on the eligible provider's entire patient population (except where otherwise noted).

Comment: Nearly every commenter who commented on CPOE objected to our proposal to limit this measure to the 
inpatient department (Place of Service Code 21) for the eligible hospital or CAH. Commenters stated that this limitation was inappropriate given the manner in which hospitals use EHR technology. To account for current practice, the commenters recommended the measures be expanded to include the emergency department (ED) (POS 23). Other reasons cited by commenters were that orders begin in the ED and remain open as the patient transitions to inpatient (for example, infusions), transitioning from paper documentation in the ED to electronic for subsequent care is unsafe as it can result in missed information, and/or transcription errors as the initial allergies and medications are entered into the system, significant data collection occurs in the ED that would not be included in the system, the exclusion of the ED creates disincentives to adoption and that the ED is a hybrid of temporal and functional services that are neither purely ambulatory nor inpatient.

Response: We agree with the commenters, and therefore are expanding this objective and its associated measure to the emergency room (POS 23). More information on place of service codes is available at http://www.cms.gov/

PlaceofServiceCodes/. Furthermore, given the revision to the HITECH Act that changed hospital based eligible professionals to include only the setting of inpatient and emergency departments and all of the benefits of integration of these two departments spelled out by commenters we will adopt both departments when considering the measure of eligible hospitals or CAHs unless we find there are unique circumstances of an objective and its associated measure that would preclude the inclusion of the emergency department for meaningful use. This change does not affect the incentive payment calculation described in section II.B. of this final rule

Comment: We received several recommendations from commenters that the requirement of a percentage measurement for determining whether an EP, eligible hospital or CAH meets this objective should be replaced with a numerical count for CPOE and many other measures associated with percentage thresholds. The two main reasons given for switching to numerical counts are the burden of calculating the percentage if it cannot be done automatically using certified EHR technology and the assertion that if an $\mathrm{EP}$, eligible hospital, or CAH does something a specific number of times it can be assumed that it is done often enough to constitute meeting the objective for Stage 1 of meaningful use. Response: We have previously discussed the merits of a percentage based measure over a count based measure earlier in this section under the discussion of the burden created by the measures associated with the Stage 1 meaningful use objectives. However, we do try to seek a balance reducing the burden on providers while still ensuring the progression of meaningful use of certified EHR technology. In the next comment/response, we discuss changes to this measure that respond to concerns regarding burden.

Comment: Many commenters representing EPs as well as other commenters recommended lowering the CPOE threshold for EPs. Those commenters representing EPs generally recommended parity with eligible hospitals at 10 percent, while other commenters recommending a reduction generally recommended 50 percent.

Response: With CPOE, we had a unique situation of disparate thresholds between EPs and hospitals. This was due to recommendations prior to the proposed rule by the HIT Policy Committee. Eligible hospitals were granted an even lower threshold for this particular requirement. The reason given for this recommendation was that CPOE is one of the last functionalities to be implemented in the hospital setting. Commenters point out that holds true for EPs as well. As discussed above, given the limitations we are placing on the numerator and denominator for calculating the CPOE percentage, we no longer see a compelling reason to maintain disparate thresholds for the EPs and the eligible hospital/CAH.

Comment: Commenters have suggested that our proposal to count an action per unique patients could be applied to the measure for CPOE as well through a revised measure of "[a]t least $10 \%$ of unique patients seen by the EP or admitted to the eligible hospital or $\mathrm{CAH}$ have at least one order entered using CPOE." Commenters also pointed to CPOE as an example of a case where adequate lead time is necessary to implement certified EHR technology.

Response: At the heart of this new basis for this measure is the assumption that every patient would have at least one order that could be entered using CPOE. We believe this is a reasonable assumption for EPs, eligible hospitals and CAHs. According to analysis of 25,665 office-based visits in the 2005 National Ambulatory Medical Care Survey, 31 percent of visits included a new medication order, and 44 percent included at least one refill; 66 percent had any type of medication order. However, whether a medication order is appropriate for every practice could vary significantly by scope of practice; therefore, for the final rule, we are further limiting the denominator to patients with at least one medication listed in their medication list. We believe that this limitation will reduce providers' burden as compared to accounting for all orders. To further reduce the burden on providers, we also will limit the numerator to unique patients with at least one medication order entered using CPOE. Because we have reduced provider burden by limiting the denominator and numerator as discussed above, we believe that a corresponding increase in the CPOE threshold is appropriate for hospitals and CAHs. For stage 1, we are finalizing a threshold for CPOE of 30 percent for EPs, eligible hospitals, and CAHS. We believe this relatively low threshold, in combination with the limitation to only medication orders, will allow hospitals and EPs to gain experience with CPOE. However, as providers gain greater experience with CPOE, we believe it is reasonable to expect greater use of the function. As explained above, we also believe CPOE is foundational to many other objectives of meaningful use. For these reasons, we believe it is reasonable to expect providers to move to a 60 percent threshold at Stage 2 of meaningful use. Thus, for this measure, we are finalizing, for Stage 2 of meaningful use, that EPs, eligible hospitals and CAHs must meet a 60 percent threshold for CPOE. Therefore, we are finalizing a Stage 2 measure for CPOE at $\S 495.6(\mathrm{~h})$ for EPs and $\S 495.6$ (i) for eligible hospitals and CAHs as "More than 60 percent of all unique patients with at least one medication in their medication list seen by the EP or admitted to the eligible hospital's or CAH's inpatient or emergency department (POS 21 or 23) during the EHR reporting period have at least medication one order entered using CPOE".

Comment: We received several comments asking for clarification of the term unique patient in response to various objectives.

Response: In the proposed rule, we state, "the reason we propose to base the measure on unique patients as opposed to every patient encounter, is that a problem list would not necessarily have to be updated at every visit." To further describe the concept of "unique patient" we mean that if a patient is seen by an EP or admitted to an eligible hospital's or CAH's inpatient or emergency department (POS 21 or 23) more than once during the EHR reporting period 
then for purposes of measurement they only count once in the denominator for the measure. All the measures relying on the term "unique patient" relate to what is contained in the patient's medical record. Not all of this information will need to be updated or even be needed by the provider at every patient encounter. This is especially true for patients whose encounter frequency is such that they would see the same provider multiple times in the same EHR reporting period. Measuring by every patient encounter places an undue burden on the EPs, eligible hospitals and CAHs and may have unintended consequences of affecting the provision of care to patients merely to comply with meaningful use. Given the emphasis placed on the reporting burden by commenters as described in the beginning of this section, we believe that our concerns about the burden of measurement were well founded. We also continue to believe that the use of patient encounters could have unintended consequences on the provision of care by providers.

Comment: Some commenters asked whether the CPOE objective and associated measure require transmission of the order. Most of these commenters were opposed to such transmission in Stage 1 for various reasons such as the cost of developing interfaces between EHRs and laboratory and radiology service providers, the volume of transmissions would outpace the capacity to connect, HIE infrastructure is not yet mature enough and the lack of the requirement for non-eligible entities to participate (for example, laboratory vendors, pharmacies). Some commenters supported the inclusion of the transmission of the order as they believed this would provide better outcomes than if the transmission was not required.

Response: In the proposed rule, we stated, "For Stage 1 criteria, we propose that it will not include the electronic transmittal of that order to the pharmacy, laboratory, or diagnostic imaging center." While a few commenters recommended that this objective be changed to require transmission, given the large opposition to the objective and measure as proposed and the reasons commenters presented against transmission, it would not be responsive to the vast majority of commenters to expand this objective beyond our proposal. We agree with the commenters that said the HIE

infrastructure is still being developed in most parts of the country. Furthermore, we note that in the hospital setting, most medication orders would not require transmission outside of the certified EHR technology of the hospital. For EPs, we already address transmission of the medication order in a separate objective for e-prescribing. Therefore, we finalize the proposal that the transmission of the order is not included in the objective or the associated measure for Stage 1.

After consideration of the public comments received, we are modifying the meaningful use measure for EPs at 495.6(d)(1)(ii) of our regulations and for eligible hospitals, and CAHs at

$\S 495.6(f)(1)(i i)$ of our regulations to "More than 30 percent of all unique patients with at least one medication in their medication list seen by the EP or admitted to the eligible hospital's or CAH's inpatient or emergency department (POS 21 or 23) during the EHR reporting period have at least medication one order entered using CPOE".

We further specify that in order to meet this objective and measure, an EP, eligible hospital, or CAH must use the capabilities Certified EHR Technology includes as specified and standards at 45 CFR 170.304(a) for EPs and 45 CFR 170.306(a) for eligible hospitals and CAHs. The ability to calculate the measure is included in certified EHR technology. Thus, for example, an EP, eligible hospital or CAH must use a certified functionality in entering the medication order, and could not use a functionality that has been added by the EHR vendor, but that is outside the scope of the certification. We believe this rule is necessary to ensure that the $\mathrm{EP}$, eligible hospital, or CAH is actually making meaningful use of "certified" EHR technology, and is not using noncertified technology. In addition, requiring providers to use functionalities that are certified will ensure the interoperability of information maintained in the EHR as providers will be able to operate according to consistent standards. We believe this standardization and consistency is key to realizing the goal of using EHR technology to improve health care.

As noted previously in this section under our discussion of the burden created by the measures associated with the Stage 1 meaningful use objectives, the only patients that are included in the denominator are those patients whose records are maintained using certified EHR technology.

To calculate the percentage, CMS and ONC have worked together to define the following for this objective:

- Denominator: Number of unique patients with at least one medication in their medication list seen by the EP or admitted to an eligible hospital's or
CAH's inpatient or emergency department (POS 21 or 23) during the EHR reporting period

- Numerator: The number of patients in the denominator that have at least one medication order entered using CPOE.

- Threshold: The resulting percentage must be more than 30 percent in order for an EP, eligible hospital or CAH to meet this measure.

Exclusion: If an EP's writes fewer than one hundred prescriptions during the EHR reporting period they would be excluded from this requirement as described previously in this section in our discussion whether certain EP, eligible hospital or CAH can meet all Stage 1 meaningful use objectives given established scopes of practices. We do not believe that any eligible hospital or CAH would have less than one hundred prescriptions written for patients admitted to their inpatient and emergency departments during the EHR reporting period.

NPRM EP/Eligible Hospital Objective: Implement drug-drug, drug-allergy, drug-formulary checks

In the proposed rule, we did not elaborate on this objective.

Comment: Many commenters requested clarification as to what formulary the checks would be conducted against.

Response: Ideally, this check would be performed against any formulary that may affect the patient's welfare, inform the provider as to the best drug to prescribe or provide the patient and provider information on the drug's cost to both the patient and any third party payer. We recognize, however, that not every available third party payer, pharmacy benefit management, preferred drug list is standardized and made available for query through certified EHR technology. As we cannot through this regulation impose such a requirement on every developer of a formulary, we do not require that an EP/ eligible hospital/CAH would have to accommodate every formulary in their implementation. However, at a minimum an EP/eligible hospital/CAH must have at least one formulary that can be queried. This may be an internally developed formulary or an external formulary. The formularies should be relevant for patient care during the prescribing process. To further address this, we expect that this measure will be expanded to be counted on a transactional basis for future stages.

Comment: Commenters suggested separating the objective into one objective for the clinical checks (drugdrug and drug-allergy) and a second objective for the administrative check 
(drug-formulary). The rationale stated for the division was that clinical measures are focused on preventing medication errors versus encouraging consideration of cost when prescribing medications. In addition, the two types involve connections to different kinds of resources (drug safety information versus formulary information).

Response: We agree that these should be separate objectives for the reasons stated by the commenters and split them accordingly.

Comment: We received comments that these functions were really part of CPOE and electronic prescribing. Commenters most commonly noted that the drug formulary is part of electronic prescribing, as is currently the case under the Medicare e-Prescribing program.

Response: While we agree that the drug-drug, drug-allergy, drug-formulary checks, CPOE, e-prescribing meaningful use objectives all serve the same broader goal of ensuring accurate ordering and prescribing that takes into account all available information about the patient the functions and their readiness for Stage 1 of meaningful use are distinct. In terms of functions, CPOE and eprescribing could be performed without the drug to drug, drug-allergy or drugformulary checks. Similarly, it is not necessary for CPOE or e-Prescribing to take place in order for a drug to drug allergy check to occur. In terms of readiness and ability to measure progress for Stage 1 of meaningful use, CPOE and e-prescribing both are percentage based measures of a distinct activity that creates a record even in today's EHR's and paper patient records. The viewing and consideration of information presented to the provider on possible drug interactions is not a similarly distinct activity and does not currently create a record. So while the goal of these functionalities is similar, we believe drug-drug, drug-allergy, drug-formulary checks create unique concerns for implementation and demonstration of meaningful use, and therefore we maintain them as separate objectives.

Comments: Several commenters expressed concern of "alert fatigue" occurring with drug-drug interaction checks. Alert fatigue or otherwise known as "pop-up" fatigue is a commonly perceived occurrence with electronic medical records and clinical decision support tools in which alerts are presented to the user when a potential safety issue is identified by the system (for example, drug to drug interaction). The alerts, while beneficial in some cases, can result in a type of "fatigue" whereby the provider, after receiving too many alerts, begins to ignore and/or override the alerts. Receiving too many alerts can result in slowing the provider down rendering the alert useless. Commenters recommended some changes to the objective and associated measure to mitigate the risk of "alert fatigue" such as limiting the checks for interactions to only the most critical medications or allowing for adjustment of risk levels rather than an on/off functionality.

Response: We recognize "alert fatigue" is a potential occurrence with drug-drug and drug-allergy checks. However, meaningful use seeks to utilize the capabilities of certified EHR technology and any means to address alert fatigue requires a critical evaluation of each alert. We believe this is beyond the scope of the definition of meaningful use. We believe these checks are valuable and improve patient care and therefore do not remove them to address alert fatigue.

Comment: Commenters recommended food allergies be included in the drugallergy check as some drugs contain ingredients that are contraindicated in individuals with certain allergies.

Response: We certainly agree that some allergies other than drug can interact with drugs; however, as we stated under our discussion of the objective "Medication Allergy List", the ability to identify other types of allergies in a useful way are not yet available to the extent necessary to require them in Stage 1 of meaningful use. This certainly does not preclude any EP, eligible hospital, or CAH from working with the designers of their certified EHR technology to include this functionality.

Comment: A commenter requested clarification as to whether the drugdrug, drug-allergy and drug-formulary checks are required for contrast media and imaging agents used by radiologists.

Response: We do not link the checks

to specific drugs or agents. However, we note that is common practice in radiology to identify a patient's past drug and food allergies and take appropriate interventions if necessary. Therefore, the drug-drug, drug-allergy and drug-formulary checks would be appropriate prior to administration of contrast media and imaging agents to patients

After consideration of the public comments received, we are finalizing the meaningful use objective for EPs at $\S 495.6(d)(2)$ (i) and for eligible hospitals and CAHs at $\S 495.6$ (f)(2)(i) as

"Implement drug-drug and drug-allergy checks." We include this objective in the core set as it is integral to the initial or on-going management of a patient's current or future healthcare and would give providers the necessary information to make informed clinical decisions for improved delivery of patient care.

In addition, we are finalizing the meaningful use objective at for EPs at $\S 495.6(\mathrm{e})(1)(\mathrm{i})$ and for eligible hospitals and CAHs at $\S 495.6(\mathrm{~g})(1)(\mathrm{i})$ of our regulations as "Implement drugformulary checks."

NPRM EP/Eligible Hospital Measure: The EP/eligible hospital/CAH has enabled the drug-drug, drug-allergy, and drug-formulary check functionality

In the proposed rule we discussed that the capability of conducting automated drug-drug, drug-allergy, and drug-formulary checks is included in the certification criteria for certified EHR technology. This automated check provides information to advise the EP, eligible hospital, or CAH's decisions in prescribing drugs to a patient. The only action taken by the EP, eligible hospital, or $\mathrm{CAH}$ is to consider this information. Many current EHR technologies have the option to disable these checks and the certification process does not require the removal of this option. Therefore, in order to meet this objective, an EP, eligible hospital, or $\mathrm{CAH}$ would be required to enable this functionality and ensure they have access to at least one drug formulary. While this does not ensure that an EP, eligible hospital or $\mathrm{CAH}$ is considering the information provided by the check, it does ensure that the information is available.

After consideration of the public comments received on the objective, we believe the measure as proposed requires more clarity on the length of time for which the functionality must be enabled, which we clarify to be the entire EHR reporting period. Therefore, we are modifying the meaningful use measure for "Implement drug-drug and drug-allergy checks for the entire EHR reporting period" for EPs at $\S 495.6(\mathrm{~d})(2)(\mathrm{ii})$ and for eligible hospitals and CAHs at $\S 495.6(f)(2)(i i)$ of our regulations to "The EP/eligible hospital/CAH has enabled this functionality for the entire EHR reporting period."

We further specify that in order to meet this objective and measure, an EP, eligible hospital, or CAH must use the capabilities Certified EHR Technology includes as specified and standards at 45 CFR 170.302(a). The ability to calculate the measure is included in certified EHR technology.

As this objective only requires that functionalities of certified EHR technology be enabled, we do not believe that any EP, eligible hospital or 
CAH would need an exclusion for this objective and its associated measure.

After consideration of the public comments received on the objective, we are modifying the meaningful use measure for "Implement drug-formulary checks" for EPs at § 495.6(e)(1)(ii) and for eligible hospitals and CAHs at $\S 495.6(\mathrm{~g})(1)(\mathrm{ii})$ of our regulations to "The EP/eligible hospital/CAH has enabled this functionality and has access to at least one internal or external formulary for the entire EHR reporting period."

We further specify that in order to meet this objective and measure, an EP, eligible hospital, or CAH must use the capabilities Certified EHR Technology includes as specified and standards at 45 CFR 170.302(b). The ability to calculate the measure is included in certified EHR technology.

The consideration of whether a drug is in a formulary or not only applies when considering what drug to prescribe. Therefore, we believe that any EP who writes fewer than one hundred prescriptions during the EHR reporting period should be excluded from this objective and associated measure as described previously in our discussion of whether certain EP, eligible hospital or CAH can meet all Stage 1 meaningful use objectives given established scopes of practices.

NPRM EP/Eligible Hospital Objective: Maintain an up-to-date problem list of current and active diagnoses based on ICD-9-CM-CM or SNOMED CT ${ }^{\circledR}$

In the proposed rule, we described the term "problem list" as a list of current and active diagnoses as well as past diagnoses relevant to the current care of the patient.

Comment: Several commenters noted that the coding of problem lists at the point of care is outside the normal workflow process and would be disruptive.

Response: We did not and do not intend that coding of the diagnosis be done at the point of care. This coding could be done later and by individuals other than the diagnosing provider.

Comment: Commenters suggested including ICD-10-CM, the Diagnostic and Statistical Manual of Mental Disorders and explicitly allowing subsets of SNOMED CT ${ }^{\circledR}$.

Response: We have removed the references to specific standards, as we believe specifying the relevant standards falls within the purview of ONC. For ONC's discussion of this functionality and the relevant standards including response to the above comment, we refer readers to ONC's final rule.
After consideration of the public comments received, we are modifying the meaningful use objective for EPs at $\S 495.6(d)(3)(i)$ and for eligible hospitals at $\$ 495.6$ (f)(3)(i) of our regulations to "Maintain an up-to-date problem list of current and active diagnoses".

We include this objective in the core set as it is integral to the initial or ongoing management of a patient's current or future healthcare and would give providers the necessary information to make informed clinical decisions for improved delivery of patient care.

NPRM EP/Eligible Hospital Measure: At least 80 percent of all unique patients seen by the EP or admitted to the eligible hospital or CAH have at least one entry or an indication of none recorded as structured data.

In the proposed rule, we introduced the concept of "unique patients" in the discussion of this objective. We received many comments requesting clarification of this term and address those in the comment and response section under our discussion of the CPOE measure.

Comment: A few commenters stated that "None" is not a clinically relevant term and should be replaced with no known problem or no problem.

Response: Our intent is not to dictate the exact wording of the specific value. Rather we are focused on the overall goal of making a distinction between a blank list because a patient does not have known problems and a blank list because either no inquiry of the patient has been made, or problems have been recorded through other means. As long as the indication accomplishes this goal and is structured data, we do not believe it is necessary to prescribe the exact terminology, thus leaving that level of detail to the designers and users of certified EHR technology.

Comment: Commenters requested clarification of the term "up-to-date".

Response: The term "up-to-date" means the list is populated with the most recent diagnosis known by the EP, eligible hospital, or CAH. This knowledge could be ascertained from previous records, transfer of information from other providers, or querying the patient. However, not every EP has direct contact with the patient and therefore has the opportunity to update the list. Nor do we believe that an EP, eligible hospital, or CAH should be required through meaningful use to update the list at every contact with the patient. There is also the consideration of the burden that reporting places on the EP, eligible hospital, or CAH. The measure, as finalized, ensures the EP, eligible hospital, or CAH has a problem list for patients seen during the EHR reporting period, and that at least one piece of information is presented to the EP, eligible hospital, or CAH. The EP, eligible hospital, or CAH can then use their judgment in deciding what further probing or updating may be required given the clinical circumstances.

Comment: Commenters stated that this measure should be replaced with either a simple attestation of yes, the problem list exists or the percentage of the measure should be replaced with a count. Alternatively, that the percentage should be maintained, but that the threshold should be lowered.

Commenters generally supported this lowering of the threshold for one or all of the following reasons: It may require a change in traditional workflow; implementation and rollout of certified EHR technology creates unforeseeable system downtimes, complications, and the required clinical classification

systems are not geared toward clinical information.

Response: For reasons discussed earlier in this section under our discussion of the burden created by the measures associated with the Stage 1 meaningful use objectives, we believe a percentage is a more appropriate measure than those suggested by comments. As this objective relies solely on a capability included as part of certified EHR technology and is not, for purposes of Stage 1 criteria, reliant on the electronic exchange of information, we believe it is appropriate to set a high percentage threshold. In the proposed rule, we set the percentage required for successful demonstration at 80 percent. Though full compliance (that is, 100 percent) is the ultimate goal, 80 percent seemed an appropriate standard for Stage 1 meaningful use as it creates a high standard, while still allowing room for technical hindrances and other barriers to reaching full compliance. We proposed 80 percent for every measure with a percentage that met the criteria of relying solely on a capability included as part of certified EHR technology and are not, for purposes of Stage 1 meaningful use criteria, reliant on the electronic exchange of information. Commenters generally agreed with this alignment; however, they disagreed that 80 percent sufficiently allows for "technical hindrances and other barriers". Commenters have highlighted numerous barriers towards successfully meeting an 80 percent threshold including technical barriers, barriers to implementation, applicability to all patients and all provider types eligible for the EHR incentives, patient requested exclusions and others. We address some of these with specific exclusions from the measure as 
discussed previously in this section under our discussion of whether certain EP, eligible hospital or CAH can meet all Stage 1 meaningful use objectives given established scopes of practices. Although some technical issues exist, recording an up-to-date problem list remains largely within the individual provider's control and does not rely to a large degree on some external sender or receiver of structured electronic health data. In addition, there is a standard of practice for collecting the elements required for an up-to-date problem list. Although the commenters may be right that some clinical workflow needs to change, that is an integral part of meaningful use of EHRs. Although we do not expect all clinical workflow to adapt in Stage 1, there is an expectation that the clinical workflow necessary to support the Stage 1 priority of data capture and sharing will be in place in order to effectively advance meaningful use of EHRs. In addition, given the wide range of activities that must occur for meaningful use, we believe that most EPs, eligible hospitals and CAHs will have fully rolled out the capabilities required by this objective and the others with an 80 percent threshold prior to the start of the EHR reporting period thereby reducing the likelihood of unexpected system downtime and other implementation complications.

For situations in which there is an existing standard of practice and complying is fundamentally within the provider's control and where the objective relies solely on a capability included as part of certified EHR technology and is not, for purposes of Stage 1 criteria, reliant on the electronic exchange of information, for the final rule, we adopt, the reasonably high threshold of 80 percent. We believe existing infrastructure and expectations support this relatively high target. This foundational step of structured data capture is a prerequisite for many of the more advanced functionalities (for example, clinical decision support, clinical quality measurement, etc.) for which a solid evidence base exists for improved quality, safety and efficiency of care. Without having most of a provider's up-to-date problem lists in structured, electronic data, that provider will have major challenges in building more advanced clinical processes going forward.

For other situations, where the objective may not be fundamentally within the provider's control and is not an existing standard of practice, but where objective continues to rely solely on a capability that is included as part of certified EHR technology and is not reliant on electronic exchange of information, we are setting the percentage at 50 percent. This was the most commonly recommended percentage for these objectives that rely solely on a capability included as part of certified EHR technology and do not rely on the electronic exchange of information.

After consideration of the public comments received, we are modifying the meaningful use measure for EPs at $\S 495.6(d)(3)(i)$ and for eligible hospitals at $\S 495.6(f)(3)(i)$ of our regulations to "More than 80 percent of all unique patients seen by the EP or admitted to the eligible hospital's or CAH's inpatient or emergency departments (POS 21 or 23) have at least one entry or an indication that no problems are known for the patient recorded as structured data".

We further specify that in order to meet this objective and measure, an EP, eligible hospital, or CAH must use the capabilities Certified EHR Technology includes as specified and standards at 45 CFR 170.302(c). The ability to calculate the measure is included in certified EHR technology.

To calculate the percentage, CMS and ONC have worked together to define the following for this objective:

- Denominator: Number of unique patients seen by the EP or admitted to an eligible hospital's or CAH's inpatient or emergency department (POS 21 or 23) during the EHR reporting period.

- Numerator: The number of patients in the denominator who have at least one entry or an indication that no problems are known for the patient recorded as structured data in their problem list.

- Threshold: The resulting percentage must be more than 80 percent in order for an EP, eligible hospital, or CAH to meet this measure.

We do not believe that any EP, eligible hospital, or $\mathrm{CAH}$ would be in a situation where they would not need to know at least one active diagnosis for a patient they are seeing or admitting to their hospital. Therefore, there are no exclusions for this objective and its associated measure.

NPRM EP Objective: Generate and transmit permissible prescriptions electronically (eRx).

Comment: Some commenters requested clarification of the term "permissible prescription."

Response: As discussed in the proposed rule, the concept of only permissible prescriptions refers to the current restrictions established by the Department of Justice on electronic prescribing for controlled substances in Schedule II. (The substances in
Schedule II can be found at http:// www.deadiversion.usdoj.gov/schedules/ orangebook/e_cs_sched.pdf). Any prescription not subject to these restrictions would be permissible. We note that the Department of Justice recently released a notice of proposed rulemaking that would allow the electronic prescribing of these substances; however, given the already tight timeframe for Stage 1 of meaningful use we are unable to incorporate any final changes that may result from that proposed rule.

Therefore, the determination of whether a prescription is a "permissible prescription" for purposes of the eRx meaningful use objective should be made based on the guidelines for prescribing Schedule II controlled substances in effect when the notice of proposed rulemaking was published on January 13, 2010. We define a prescription as the authorization by an EP to a pharmacist to dispense a drug that the pharmacist would not dispense to the patient without such authorization. We do not include authorizations for items such as durable medical equipment or other items and services that may require EP authorization before the patient could receive them. These are excluded from the numerator and the denominator of the measure.

Comment: Some commenters recommended combining this objective and measure with other meaningful use objectives such as CPOE or the drugdrug, drug-allergy, drug-formulary checks

Response: We addressed these comments under our discussion of the CPOE objective.

After consideration of the public comments received, we are finalizing the meaningful use objective at 495.6(d)(4)(i) as proposed.

We have also included this objective in the core set. Section 1848(o)(2)(A)(i) of the Act specifically includes electronic prescribing in meaningful use for eligible professionals. This function is the most widely adopted form of electronic exchange occurring and has been proven to reduce medication errors. We included this objective in the core set based on the combination of the maturity of this objective, the proven benefits and its specific mention as the only example provided in the HITECH Act for what is meaningfully using certified EHR technology.

NPRM EP Measure: At least 75 percent of all permissible prescriptions written by the EP are transmitted electronically using certified EHR technology. 
In the proposed rule, we said that while this measure does rely on the electronic exchange of information based on the public input previously discussed and our own experiences with e-prescribing programs, we believe this is the most robust electronic exchange currently occurring and proposed 75 percent as an achievable threshold for the Stage 1 criteria of meaningful use. Though full compliance (that is, 100 percent) is the ultimate goal, 75 percent seemed an appropriate standard for Stage 1 meaningful use as it creates a high standard, while still allowing room for technical hindrances and other barriers to reaching full compliance.

Comment: A majority of commenters commenting on this measure believe the 75 percent threshold is too high. Several issues were raised to explain why the commenters believe the threshold is too high. The first is that barriers to eprescribing exist at the pharmacies and they must be brought into the process to ensure compliance on the receiving end. The second represents the most common barrier cited by commenters and that is patient preference for a paper prescription over e-prescribing. A patient could have this preference for any number of reasons cited by commenters such as the desire to shop for the best price (especially for patients in the Part D "donut hole"), the ability to obtain medications through the VA, lack of finances, indecision to have the prescription filled locally or by mail order and desire to use a manufacturer coupon to obtain a discount. Other barriers mentioned by individual commenters were the limited functionality of current e-prescribing systems such as the inability to distinguish refills from new orders. Suggestions for addressing these difficulties were either to lower the threshold (alternatives recommended ranged from ten to fifty percent) or replacing the percentage with a numerical count of 25 to align with the 2010 Medicare e-Prescribing program. Of the comments received that requested a specific lower threshold, about half of them suggested a 50 percent threshold, and about half suggested a threshold of 25 percent to 30 percent.

Response: We are finalizing the use of a percentage threshold for the reasons discussed previously in this section under our discussion of the burden created by the measures associated with the Stage 1 meaningful use objectives. In the proposed rule, we pointed out that we "believe this is the most robust electronic exchange currently occurring" to justify a high threshold of 75 percent given that this objective relies on electronic exchange. While we continue to believe this is the case, two particular issues raised by commenters caused us to reconsider our threshold. The first is the argument to include pharmacies in the Medicare and Medicaid EHR incentive programs to ensure compliance on the receiving end. Nonparticipation by pharmacies was presented by commenters as a major barrier to e-Prescribing. The second is patient preference for a paper prescription. In regards to the first argument, we do not have the ability to impose requirements on pharmacies through the HITECH legislation.

However, prescriptions transmitted electronically have been growing at an exponential rate. The number of prescriptions sent electronically increased by 181 percent from 2007 to 2008 according to comments received. The number of pharmacies is also increasing rapidly. Yet this growth is uneven across the country and we wish to accommodate all EPs and do lower the threshold based on this argument. In regards to the second argument, we also have neither the ability nor the desire to limit patient preference. We considered allowing an EP to exclude from the denominator those instances where a patient requested a paper prescription. However, the burden of tracking when this occurs, the disincentive it would create for EPs to work with patients on establishing a relationship with a pharmacy and the hindrance to moving forward with e-prescribing lead us to address this through further reduction of the threshold as opposed to an exclusion. To address these concerns we are lowering the threshold for the eprescribing measure to 40 percent. As pointed out by commenters, e-prescribing it is not yet standard of practice and there may be important external barriers beyond the provider's control. In particular, for e-prescribing, providers are dependent upon an external receiver of electronic health data, and there are significant variations depending on where the provider practices.

After consideration of the public comments received, we are modifying the meaningful use measure at $\S 495.6(\mathrm{~d})(4)$ (ii) of our regulations to "More than 40 percent of all permissible prescriptions written by the EP are transmitted electronically using certified EHR technology".

We further specify that in order to meet this objective and measure, an EP, eligible hospital, or CAH must use the capabilities Certified EHR Technology includes as specified and standards at 45 CFR 170.304(b). The ability to calculate the measure is included in certified EHR technology.

As noted previously in this section under our discussion of the burden created by the measures associated with the Stage 1 meaningful use objectives, the prescriptions in the denominator are only those for patients whose records are maintained using certified EHR technology.

To calculate the percentage, CMS and ONC have worked together to define the following for this objective:

- Denominator: Number of prescriptions written for drugs requiring a prescription in order to be dispensed other than controlled substances during the EHR reporting period.

- Numerator: The number of prescriptions in the denominator generated and transmitted electronically.

- Threshold: The resulting percentage must be more than 40 percent in order for an EP, eligible hospital, or CAH to meet this measure.

As addressed in other objectives and in comment response, this objective and associated measure do not apply to any EP who writes fewer than one hundred prescriptions during the EHR reporting period, as described previously in this section under our discussion of whether certain EP, eligible hospital or CAH can meet all Stage 1 meaningful use objectives given established scopes of practices.

NPRM EP/Eligible Hospital Objective: Maintain active medication list.

Comment: Commenters requested clarification of the term "active medication list."

Response: We define an active medication list as a list of medications that a given patient is currently taking.

After consideration of the public comments received, we are finalizing this objective for EPs at $\S 495.6(d)(5)(i)$ and for eligible hospitals and CAHs at $\S 495.6$ (f)(4)(i) of our regulations as proposed.

We include this objective in the core set as it is integral to the initial or ongoing management of a patient's current or future healthcare and would give providers the necessary information to make informed clinical decisions for improved delivery of patient care.

NPRM EP/Eligible Hospital Measure: At least 80 percent of all unique patients seen by the EP or admitted by the eligible hospital have at least one entry (or an indication of "none" if the patient is not currently prescribed any medication) recorded as structured data.

As with the objective of maintaining a problem list, we clarify that the indication of "none" should distinguish between a blank list that is blank 
because a patient is not on any known medications and a blank list because no inquiry of the patient has been made. As long as the indication accomplishes this goal and is structured data, we do not believe it is necessary to prescribe the exact terminology, preferring to leave that level of detail to the designers and users of certified EHR technology.

Comment: Commenters stated that the measure should be replaced with a numerical count or attestation and that the threshold was too high for reasons including the lack of current electronic exchange of information, difficulty capturing information as structured data and lack of readiness of HIE infrastructure.

Response: We are finalizing the use of a percentage for the reasons discussed previously in this section under our discussion of the burden created by the measures associated with the Stage 1 meaningful use objectives. For the same reasons we explained under the discussion of up-to-date problem list, medication list is a functionality for which there is an existing standard of practice, it is foundational data capture function to make more advanced clinical processes possible, and complying is fundamentally within the provider's control. Therefore, we maintain the reasonably high threshold of 80 percent because the existing infrastructure and expectations support this target.

Comment: Commenters requested clarification as to whether the measure is limited to patients seen during the EHR reporting period.

Response: Yes, the measure applies to all unique patients seen by the EP or admitted to the eligible hospital's or CAH's inpatient or emergency departments (POS 21 or 23) during the EHR reporting period.

Comment: A few commenters expressed concern regarding the requirement that the entry must be recorded as "structured data." The commenters state that there may not be a code for over the counter,

homeopathic or herbal products and that would penalize the provider even though the data is collected and recorded.

Response: The distinction between structured data and unstructured data applies to all types of information. Structured data is not fully dependent on an established standard. Established standards facilitate the exchange of the information across providers by ensuring data is structured in the same way. However, structured data within certified EHR technology merely requires the system to be able to identify the data as providing specific information. This is commonly accomplished by creating fixed fields within a record or file, but not solely accomplished in this manner. For example, in this case for it to be structured, if the patient is on aspirin, then that information should be in the system so that it can be automatically identified as a medication and not as an order, note, or anything else. An example of unstructured data would be the word aspirin, but no ability of the system to identify it as a medication.

Comment: A few commenters pointed out their current health information system vendor does not utilize RxNorm as its standard.

Response: This is a certification issue best addressed in the ONC final rule. We therefore have referred these comments to ONC for their consideration.

Comment: We received comments suggesting that this requirement could create additional privacy/security concerns for patients who do not want all physicians and their clinical staff to have access to their entire medication history. Examples provided included antidepressant, antipsychotic or erectile dysfunction medications.

Response: We are only concerned with medications that are known to the provider through querying the patient, their own records and the transfer of records from other providers.

Meaningful use cannot address situations where the information is withheld from the EP, eligible hospital, or CAH by the patient or by other providers. We understand that some patients would prefer not to have their entire medical history available to all physicians and clinical staff. We also understand that laws in some states restrict the use and disclosure of information (including that related to medication) that may reveal that a patient has a specific health condition (for example, HIV). Recording data in a structured manner will facilitate the implementation of these preferences and policies in an electronic environment. It is easier to identify and potentially withhold specific data elements that have been recorded in a structured format than information recorded as free text.

After consideration of the public comments received, we are modifying the meaningful use measure for EPs at $\S 495.6(d)(5)(i i)$ and for eligible hospitals at $\S 495.6$ (f)(4)(ii) of our regulations to "More than 80 percent of all unique patients seen by the EP or admitted to the eligible hospital's or CAH's inpatient or emergency departments (POS 21 or 23) have at least one entry (or an indication that the patient is not currently prescribed any medication) recorded as structured data”.

We further specify that in order to meet this objective and measure, an EP, eligible hospital, or CAH must use the capabilities Certified EHR Technology includes as specified and standards at 45 CFR 170.302(d). The ability to calculate the measure is included in certified EHR technology.

To calculate the percentage, CMS and ONC have worked together to define the following for this objective:

- Denominator: Number of unique patients seen by the EP or admitted to an eligible hospital's or CAH's inpatient or emergency departments (POS 21 or 23) during the EHR reporting period. A definition of unique patient is discussed under the objective of CPOE.

- Numerator: The number of patients in the denominator who have a medication (or an indication that the patient is not currently prescribed any medication) recorded as structured data.

- Threshold: The resulting percentage must be more than 80 percent in order for an EP, eligible hospital, or CAH to meet this measure. Detailed discussion of the more than 80 percent threshold can be found under the objective of maintaining an up-to-date problem list. We do not believe that any EP, eligible hospital or $\mathrm{CAH}$ would be in a situation where they would not need to know whether their patients are taking any medications. Therefore, there are no exclusions for this objective and its associated measure.

NPRM EP/Eligible Hospital Objective: Maintain active medication allergy list.

Comment: We received comments that limiting this list to medication allergies instead of all allergies was not consistent with efficient workflow and that all allergies should be housed in the same location within the EHR.

Commenters also highlighted that lack of knowledge of other allergies such as latex and food allergies could lead to significant harm to the patient.

Response: We agree that information on all allergies, including nonmedication allergies, provide relevant clinical quality data. However, while we agree that collecting all allergies would be an improvement, current medication allergy standards exists in a structured data format that may be implemented in Stage 1. We hope to expand this measurement to include all allergies as the standards evolve and expand to include non-medication allergies. We believe EP/eligible hospitals/CAHs should continue to document all allergies, regardless of origin, consistent with standard of care practice for that EP/eligible hospital/CAH. We encourage 
them to work with the designers of their certified EHR technology to make this documentation as efficient and structured as possible.

Comment: A commenter inquired why the Substance Registration System Unique Ingredient Identifier (UNII) was not indicated for use until 2013 yet the measure requires the information to be recorded as structured data.

Response: Any standards for the structured vocabulary for medication allergies or other aspects of meaningful use are included in ONC final rule. Structured data does not require an established standard as discussed under the objective of maintaining a medication list.

Comment: We received a few comments requesting a definition of "allergy."

Response: We adopt the commonly held definition of an allergy as an exaggerated immune response or reaction to substances that are generally not harmful. The definition is derived from Medline Plus, a service of the U.S. National Library of Medicine and the National Institutes of Health.

After consideration of the public comments received, we are finalizing the meaningful use objective for EPs at 495.6(d)(6)(i) and for eligible hospitals and CAHs at 495.6(f)(5)(i) as proposed.

We include this objective in the core set as it is integral to the initial or ongoing management of a patient's current or future healthcare and would give providers the necessary information to make informed clinical decisions for improved delivery of patient care.

NPRM EP/Eligible Hospital Measure: At least 80 percent of all unique patients seen by the EP or admitted to the eligible hospital have at least one entry (or an indication of "none" if the patient has no medication allergies) recorded as structured data.

Comment: Multiple commenters noted that "none" is not a typical value to describe the absence of allergies in medical documentation and should be replaced with "no known allergies (NKA)," "no known drug allergies (NKDA)" or "no known medication allergies (NKMA)."

Response: Our intent is not to dictate the exact wording of the specific value. Rather we are focused on the overall goal of making a distinction between a blank list that is blank because a patient does not have known allergies and a blank list because no inquiry of the patient has been made or no information is available from other sources. As long as the indication accomplishes this goal and is structured data, we do not believe it is necessary to prescribe the exact terminology, preferring to leave that level of detail to the designers and users of certified EHR technology.

Comment: Given that the measure is only a one time check for a single entry, one commenter questioned whether this measure truly constitutes maintenance of an "active" list.

Response: We agree that this measure does not ensure that every patient under the care of every EP, eligible hospital, or $\mathrm{CAH}$ has an active or up-to-date medication list. However, not every EP comes in contact with the patient, and therefore has the opportunity to update the list. Nor do we believe that an EP, eligible hospital, or CAH should be required through meaningful use to update the list at every contact with the patient. There is also the consideration of the burden that reporting places on the EP, eligible hospital, or CAH. The measure as finalized ensures that the EP, eligible hospital, or CAH has not ignored having a medication allergy list for patients seen during the EHR reporting period and that at least one piece of information on medication allergies is presented to the EP, eligible hospital, or CAH. The EP, eligible hospital, or CAH can then use their judgment in deciding what further probing or updating may be required given the clinical circumstances at hand. Therefore, we are maintaining the measure of a one-time check for a single entry.

Comment: Several commenters recommended eliminating the percentage measurement and allowing the provider to attest that active medication lists are maintained in the certified EHR technology.

Response: We are retaining a percentage for the reasons discussed previously in this section under our discussion of the burden created by the measures associated with the Stage 1 meaningful use objectives. For the same reasons we explained under the discussion of up-to-date problem list, medication-allergy list is a functionality for which there is an existing standard of practice, it is foundational data capture function to make more advanced clinical processes possible, and complying is fundamentally within the provider's control. Therefore, we maintain the reasonably high threshold of 80 percent because the existing infrastructure and expectations support this target.

After consideration of the public comments received, we are modifying the meaningful use measure for EPs at $\S 495.6(d)(6)(i i)$ and for eligible hospitals at $\S 495.6$ (f)(5)(ii) of our regulations to "More than 80 percent of all unique patients seen by the EP or admitted to the eligible hospital's or
CAH's inpatient or emergency departments (POS 21 or 23) have at least one entry (or an indication that the patient has no known medication allergies) recorded as structured data".

We further specify that in order to meet this objective and measure, an EP, eligible hospital, or CAH must use the capabilities Certified EHR Technology includes as specified and standards at 45 CFR 170.302(e). The ability to calculate the measure is included in certified EHR technology.

To calculate the percentage, CMS and ONC have worked together to define the following for this objective:

- Denominator: Number of unique patients seen by the EP or admitted to an eligible hospital's or CAH's inpatient or emergency departments (POS 21 or 23) during the EHR reporting period. The definition of "a unique patient" is provided under the objective of CPOE.

- Numerator: The number of unique patients in the denominator who have at least one entry (or an indication that the patient has no known medication allergies) recorded as structured data in their medication allergy list.

- Threshold: The percentage must be more than 80 percent in order for an EP, eligible hospital, or CAH to meet this measure. Detailed discussion of the rationale more than 80 percent threshold can be found at under the objective of maintain an up-to-date problem list.

We do not believe that any EP, eligible hospital or CAH would be in a situation where they would not need to know whether their patients have medication allergies and therefore do not establish an exclusion for this measure.

NPRM EP Objective: Record the following demographics: Preferred language, insurance type, gender, race and ethnicity, and date of birth.

NPRM Eligible Hospital Objective: Record the following demographics: Preferred language, insurance type, gender, race and ethnicity, date of birth, and date and cause of death in the event of mortality.

In the proposed rule, we noted that race and ethnicity codes should follow current federal standards published by the Office of Management and Budget (http://www.whitehouse.gov/omb/ inforeg_statpolicy/\#dr). We maintain that proposal for the final rule.

Comment: Some commenters requested clarification of whether all of the demographics are required and under what circumstances no indication might be acceptable. Examples of acceptable circumstances from commenters include patient unwillingness to report, language 
barriers, and requirement to report ethnicity and/or race contrary to some state laws.

Response: In general, we do require that all demographic elements that are listed in the objective be included in a patient's record in certified EHR technology. However, we do not desire, nor could we require, that a patient provide this information if they are otherwise unwilling to do so. Similarly, we do not seek to preempt any state laws prohibiting EPs, eligible hospitals, or CAHs from collecting information on a patient's ethnicity and race. Therefore if a patient declines to provide the information or if capturing a patient's ethnicity or race is prohibited by state law, such a notation entered as structured data would count as an entry for purposes of meeting the measure.

Comment: Several commenters asked for clarity on the definition of preferred language. Commenters also indicated that standards are in development (ISO 639 and ANSIX12N Claim/Reporting Transaction). Some commenters also requested that we include the requirement that the $\mathrm{EP}$, eligible hospital or CAH also communicate with the patient in their preferred language.

Response: Preferred language is the language by which the patient prefers to communicate. This is just a record of the preference. We do not have the authority under the HITECH Act to require providers to actually communicate with the patient in his or her preferred language, and thus do not require EPs, eligible hospitals, and CAHs to do so in order to qualify as a meaningful EHR user as suggested by some commenters. In regards to standards, those would be adopted under the ONC final rule.

Comment: Some commenters also requested clarity on the definition of race and ethnicity. Some commenters noted an Institute of Medicine report entitled "Race, Ethnicity and Language Data: Standardization for Health Care Quality Improvement”, which makes recommendations for how to ask questions to collect information and builds on the OMB Standards for language, race and ethnicity. Some commenters were also concerned about situations where the available choices were not granular enough, did not properly account for mixed race and ethnicity, and when the patient did not know their ethnicity.

Response: In the proposed rule, we said that EPs, eligible hospitals and CAHs, should use the race and ethnicity codes that follow current federal standards published by the Office of Management and Budget (http:// www.whitehouse.gov/omb/inforeg statpolicy/\#dr). We continue to believe that these standards should be applied for purposes of implementing the Stage 1 meaningful use objectives, but will consider whether alternative standards or additional clarification would be appropriate for future stages of meaningful use criteria. We believe it is beyond the scope of the definition of meaningful use to provide additional definitions for race and ethnicity beyond what is established by OMB. In regards to patients who do not know their ethnicity, EPs, eligible hospitals, and CAHs should treat these patients the same way as patients who decline to provide the race or ethnicity, that is, they should identify in the patient record that the patient declined to provide this information.

Comment: Some commenters requested additional clarity on insurance type and others recommended the elimination of insurance type due to the complexity of insurance coverage, the function of the EHR as a medical tool and not a financial one, the volatility of this information due to patients frequently changing plans and concerns that information on a patient's insurance status will have a possible behavioral influence on the providers if this information were presented.

Response: Classifying insurance involves two distinctions-the source of coverage and insurance design. Source of coverage refers to the type of funding, such as public, private or self-pay. The design of the insurance program, such as health maintenance program (HMO), preferred provider organization (PPO), high-deductible consumer directed plan, fee-for-service, etc. Although not specified in the proposed rule, by insurance type we were referring to the first distinction-the source of funding for the insurance. We found two initiatives that could provide clarity on type. The first is the "Source of Payment Typology" developed by the Public Health Data Standards Consortium (http://www.phdsc.org/standards/payertypology.asp). The consortium is currently in the process of working with States to implement this typology. The other initiative is established in the Uniform Data Set (UDS) collected by HRSA (http://www.hrsa.gov/datastatistics/health-center-data/ index.html). The information in the UDS contains several caveats, however, that make it difficult to be used by all EPs, eligible hospitals and CAHs, and it does not accommodate patients with multiple types of insurance such as those dually eligible for Medicare and Medicaid or for those with both Medicare and MediGap coverage. Many
EHRs that currently report on HRSA UDS Insurance Type standards account for multiple types of insurance by maintaining separate Reporting Insurance Groups and deriving the Insurance Type data from the primary insurance company on the encounter and mappings to that Insurance Type Reporting Group. This information is documented at the patient demographic level or the patient encounter/progress note. Given the complexity of defining insurance type and attributing it to patients in an agreed upon way, we are eliminating "insurance type" from this meaningful use objective.

Comment: A minority of commenters commenting on this objective recommended that CMS remove cause of death from the objective for eligible hospitals. The most common rationale is that the coroner or medical examiner officially determines cause of death when the case is referred to them. By law, the hospital cannot declare a cause of death in these cases.

Response: When a patient expires, in the routine hospital workflow, a clinician evaluates the patient to pronounce the patient's death. The clinician typically documents in the patient's chart, the sequence of events leading to the patient's death, conducts the physical exam and makes a preliminary assessment of the cause of death. We are requiring that eligible hospitals record in the patient's EHR the clinical impression and preliminary assessment of the cause of death, and not the cause of death as stated in any death certificate issued by the Department of Health or the coroner's office.

Comment: A few commenters requested inclusion of Advanced Directives under this objective as recommended by the HIT Policy Committee.

Response: We discuss advance directives separately in this final rule under its own objective.

Comment: Several commenters recommended requiring the submission of the demographic data to CMS.

Response: Stage 1 of meaningful use seeks to ensure certified EHR technology has the capability to record demographic information and that those capabilities are utilized. We believe the information recorded for this measure is for provider use in the treatment and care of their patients and therefore should not be submitted to CMS at this time.

Comment: Commenters suggested requiring the use of the demographic data from this measure to stratify clinical quality measure reporting and 
the generation of reports for patient outreach and quality initiatives.

Response: While we encourage all providers and EHR developers to work together to develop reporting from the EHR system for use in the improvement of population and public health, for purposes of becoming a meaningful EHR user in Stage 1, we only require the recording of the specified demographics.

After consideration of the public comments received, we are modifying meaningful use objective at $\S 495.6(d)(7)(i)$ of our regulations for EPs to "Record the following demographics: Preferred language, gender, race and ethnicity, and date of birth".

After consideration of the public comments received, we are modifying meaningful use objective at $\S 495.6$ (f)(6)(i) of our regulations for eligible hospitals and CAHs to "Record the following demographics: Preferred language, gender, race and ethnicity, date of birth, and date and preliminary cause of death in the event of mortality in the eligible hospital or CAH".

We include this objective in the core set as it is integral to the initial or ongoing management of a patient's current or future healthcare, recommended by the HIT Policy Committee and would give providers the necessary information to make informed clinical decisions for improved delivery of patient care.

NPRM EP/Eligible Hospital Measure: At least 80 percent of all unique patients seen by the EP or admitted to the eligible hospital have demographics recorded as structured data.

Comment: Commenters said that this should be replaced with a count or attestation or alternatively that the threshold was too high.

Response: We are maintaining a percentage for the reasons discussed previously in this section under our discussion of the burden created by the measures associated with the Stage 1 meaningful use objectives. However, we do reduce the threshold to over 50 percent as this objective meets the criteria of relying solely on a capability included as part of certified EHR technology and is not, for purposes of Stage 1 criteria, reliant on the electronic exchange of information. In contrast to our discussion of maintaining an up-todate problem list/medication list/ medication allergy list, we believe that some demographic elements (especially race, ethnicity and language) are not as straightforward to collect as objective data elements and therefore the standard of practice for demographic data is still evolving. As we believe this measure may not be within current standard of practice, we are adopting the lower threshold of 50 percent (rather than 80 percent).

After consideration of the public comments received, we are modifying the meaningful use measure for EPs at $\S 495.6(d)(7)(i i)$ and for eligible hospitals at $\S 495.6$ (f)(6)(ii) of our regulations to "More than 50 percent of all unique patients seen by the EP or admitted to the eligible hospital's or CAH's inpatient or emergency department (POS 21 or 23) have demographics recorded as structured data".

We further specify that in order to meet this objective and measure, an EP, eligible hospital, or CAH must use the capabilities Certified EHR Technology includes as specified and standards at 45 CFR 170.304(c) for EPs and 45 CFR 170.304(b) for eligible hospitals and CAHs. The ability to calculate the measure is included in certified EHR technology.

To calculate the percentage, CMS and ONC have worked together to define the following for this objective:

- Denominator: Number of unique patients seen by the EP or admitted to an eligible hospital's or CAH's inpatient or emergency departments (POS 21 or 23) during the EHR reporting period. A unique patient is discussed under the objective of CPOE.

- Numerator: The number of patients in the denominator who have all the elements of demographics (or a specific exclusion if the patient declined to provide one or more elements or if recording an element is contrary to state law) recorded as structured data.

- Threshold: The resulting percentage must be more than 50 percent in order for an EP, eligible hospital or CAH to meet this measure. Most EPs and all eligible hospitals and CAHs would have access to this information through direct patient access. Some EPs without direct patient access would have this information communicated as part of the referral from the EP who identified the service as needed by the patient. Therefore, we did not include an exclusion for this objective and associated measure.

NPRM EP/Eligible Hospital Objective: Record and chart changes in the following vital signs: height, weight and blood pressure and calculate and display body mass index (BMI) for ages 2 and over; plot and display growth charts for children 2-20 years, including BMI.

In the proposed rule, we described why we included growth charts in this objective. The reason given was that BMI was not a sufficient marker for younger children.
Comment: Over two thirds of the commenters commenting on this objective expressed concern about the applicability of the listed vital signs to all provider types and care settings.

Response: While this objective could be met by receiving this information from other providers or non-provider data sources, we recognize that the only guaranteed way for a provider to obtain this information is through direct patient interaction and that this information is not always routinely provided from the EP ordering a service because of a direct patient interaction. EPs who do not see patients 2 years or older would be excluded from this requirement as described previously in this section under our discussion of whether certain EP, eligible hospital or $\mathrm{CAH}$ can meet all Stage 1 meaningful use objectives given established scopes of practices. We would also allow an EP who believes that measuring and recording height, weight and blood pressure of their patients has no relevance to their scope of practice to so attest and be excluded.

Comment: Several commenters stated this objective should be removed in favor of clinical quality measures addressing BMI and blood pressure as these measures serve the same purpose and to require both is to require duplicative reporting.

Response: We disagree that these two measures serve the same purpose and therefore that the measure should be eliminated in favor of clinical quality measures addressing BMI and blood pressure. The objective included here seeks to ensure that information on height, weight and blood pressure and the extractions based on them are included in the patient's record. Furthermore, the objective seeks to ensure that the data is stored in a structured format so that it can be automatically identified by certified EHR technology for possible reporting or exchanging. We also note that the clinical quality measure focuses on a smaller subset of the patient population.

After consideration of the public comments received, we are finalizing the objective for EPs at 495.6(d)(8)(i) and for eligible hospitals and CAHs at 495.6(f)(7)(i) as proposed.

We include this objective in the core set as it is integral to the initial or ongoing management of a patient's current or future healthcare and would give providers the necessary information to make informed clinical decisions for improved delivery of patient care.

NPRM EP/Eligible Hospital Measure: For at least 80 percent of all unique patients age 2 and over seen by the EP or admitted to the eligible hospital, 
record blood pressure and BMI; additionally, plot growth chart for children age 2 to 20 .

Comment: Commenters suggested replacement of the percentage measurement with a count or attestation or alternatively that that the threshold was too high.

Response: We are retaining a percentage for the reasons discussed previously in this section under our discussion of the burden created by the measures associated with the Stage 1 meaningful use objectives. However, we did reduce the threshold from 80 percent to greater than 50 percent as this objective meets the criteria of relying solely on a capability included as part of certified EHR technology and is not, for purposes of Stage 1 criteria, reliant on the electronic exchange of information. In addition, in contrast to the measures associated with maintaining an up-to-date problem list, an active medication list, and an active medication-allergy list, we believe that for many specialties, the current practice on vital signs may not be as well-established. We believe there may not be the same level of consensus regarding the relevance to patient care of vital signs for many specialties and the frequency with which such vital signs should be collected. Thus, for this measure, we adopt a percentage of 50 percent, rather than 80 percent.

Comment: Commenters requested clarification of the frequency and methods of recording the vital signs included in the measure.

Response: As discussed in the objective, the EP/eligible hospital/CAH is responsible for height, weight and blood pressure so we will focus our discussion on those items. First, we do not believe that all three must be updated by a provider at every patient encounter nor even once per patient seen during the EHR reporting period. For this objective we are primarily concerned that some information is available to the EP/eligible hospital/ $\mathrm{CAH}$, who can then make the determination based on the patient's individual circumstances as to whether height, weight and blood pressure needs to be updated. The information can get into the patient's medical record as structured data in a number of ways. Some examples include entry by the EP/ eligible hospital/CAH, entry by someone on the EP/eligible hospital/CAH's staff, transfer of the information electronically or otherwise from another provider or entered directly by the patient through a portal or other means. The measure hinges on access of the information. Therefore, any EP/eligible hospital/CAH that sees/admits the patient and has access to height, weight and blood pressure information on the patient can put that patient in the numerator.

Comment: Some commenters requested clarification regarding the role of both the EP/eligible hospital/CAH and the certified EHR technology for the calculation of BMI and the plotting and displaying of growth charts. Other commenters recommended the exclusion of growth charts for certain patients and care settings. Another commenter also expressed the desire for the exclusion of growth charts for patients over the age of 18 , inpatient care settings and more specifically, nonpediatric inpatient care settings.

Response: We believe a clarification is in order about which of the listed vital signs are data inputs to be collected by the EP/eligible hospital/CAH and which are calculations made by the certified EHR technology. The only information required to be inputted by the provider is the height, weight and blood pressure of the patient. The certified EHR technology will calculate BMI and the growth chart if applicable to patient based on age. As this requirement imposes no duty or action on the provider, we see no reason to limit its availability to any EP, eligible hospital, or CAH based on setting or other consideration. Concerns on presentation and interface are best left to designers of certified EHR technology and users. Finally, as certified EHR technology is able to automatically generate BMI and the growth chart if height and weight are entered as structured data we see no reason to include BMI and growth chart in the measure. We therefore will limit the final measure to data requiring provider data entry points.

Comment: A few commenters suggested that "reported height" by the patient should be acceptable when measurement is not appropriate such as in the case of severe illness.

Response: We agree and would allow height self-reported by the patient to be used.

After consideration of the public comments received, we are modifying the meaningful use measure for EPs at 495.6(d)(8)(ii) and for eligible hospitals $\S 495.6$ (f)(7)(ii) of our regulations to "For more than 50 percent of all unique patients age 2 and over seen by the EP or admitted to eligible hospital's or CAH's inpatient or emergency department (POS 21 or 23), height, weight and blood pressure are recorded as structured data".

We further specify that in order to meet this objective and measure, an EP, eligible hospital, or CAH must use the capabilities Certified EHR Technology includes as specified and standards at
45 CFR 170.302(f). The ability to calculate the measure is included in certified EHR technology.

As noted previously in this section under our discussion of the burden created by the measures associated with the Stage 1 meaningful use objectives, the percentage is based on patient records that are maintained using certified EHR technology. To calculate the percentage, CMS and ONC have worked together to define the following for this objective:

- Denominator: Number of unique patients age 2 or over seen by the EP or admitted to an eligible hospital's or CAH's inpatient or emergency

department (POS 21 or 23) during the EHR reporting period. A unique patient is discussed under the objective of CPOE.

- Numerator: The number of patients in the denominator who have at least one entry of their height, weight and blood pressure are recorded as structure data.

- Threshold: The resulting percentage must be more than 50 percent in order for an EP, eligible hospital, or CAH to meet this measure. As addressed in other objectives and in comment response, an EP who sees no patients 2 years old or younger would be excluded from this requirement as described previously in this section under our discussion of whether certain EP, eligible hospital or CAH can meet all Stage 1 meaningful use objectives given established scopes of practices. We would also allow an EP who believes that all three vital signs of height, weight and blood pressure have no relevance to their scope of practice to so attest and be excluded. However, we believe this attestation and exclusion from recording height, weight, and blood pressure does not hold for other patient specific information collection objectives, like maintaining an active medication allergy list. We do not believe that any EP would encounter a situation where the patient's active medication and allergy list is not pertinent to care and therefore would be outside of the scope of work for an EP. We believe the exclusion based on EP determination of their scope of practice for the record vital signs objective, as written in Stage 1, should be studied for relevance in further stages. We do not believe eligible hospitals or CAHs would ever only have a patient population for patients 2 years old or younger or that these vital signs would have no relevance to their scope of practice. Therefore, we do not include an exclusion for eligible hospitals or CAHs. 
NPRM EP/Eligible Hospital Objective: Record smoking status for patients 13 years old or older

In the proposed rule, we explained that we believe it is necessary to add an age restriction to this objective as we do not believe this objective is applicable to patients of all ages and there is no consensus in the health care community as to what the appropriate cut off age may be. We encouraged comments on whether this age limit should be lowered or raised. We received many comments on the age limit and address them below.

Comment: Several commenters requested a different age limitation. Commenters suggested ages anywhere between 5 years old up to 18 years old.

Response: For the purposes of this objective and for meaningful use, our interest is focused on when a record of smoking status should be in every patient's medical record. Recording smoking status for younger patients is certainly not precluded. We do believe there would be situations where an EP/ eligible hospital/CAH's knowledge about other risk factors would indicate that they should inquire about smoking status if it is unknown for patients under 13 years old. However, in order to accurately measure and thereby assure meaningful use, for this objective we believe that the age limit needs to be high enough so that the inquiry is appropriate for all patients. Therefore, we are maintaining the age limitation at 13 years old or older.

Comment: Some commenters suggested expanding smoking status to any type of tobacco use.

Response: While we agree that an extended list covering other types of tobacco use may provide valuable insight for clinical care for certified EHR technology ONC has adopted the CDC's NHIS standard recodes for smoking status. This will provide a standard set of questions across providers and standardize the data. The extended list does not make the collection of multiple survey questions clear. For example, a patient may be a current tobacco user as well as a smoker. For these reason in Stage 1 we will use the standards adopted by ONC for certified EHR technology at 45 CFR 170.302(g). For future stages, we will review this measure for possible inclusion of other questions. This is a minimum set. We do not intend to limit developers of EHR technology from creating more specific fields or to limit EPs/eligible hospitals/ CAHs from recording more specific information.

Comment: We also received comments requesting that second-hand smoking be included in the objective for children and adolescents.

Response: Including second-hand smoking introduces much more variability into the objective as to what constitutes a level of exposure and difficulty in measuring it successfully with different age limits to different aspects. For instance, how much exposure is acceptable for a given age and how is such exposure determined? How would these differing requirements be accounted for by certified EHR technology? As with the change from smoking status to tobacco use, we believe this introduces an unacceptable level of complexity for Stage 1 of meaningful use. For Stage 1 of meaningful use we are not adding second hand smoke exposure to this objective. However, we remind EPs, eligible hospitals and CAHs that nothing about the criteria for meaningful use prevents them from working with their EHR developer to ensure that their EHR system meets their needs and the needs of their patient population. We encourage all EPs, eligible hospitals and CAHs to critically review their implementation in light of their current and future needs both to maximize their own value and to prepare for future stages of meaningful use.

Comment: We received comments asking at what frequency the information must be recorded and whether the information can be collected by support staff.

Response: We clarify that this is a check of the medical record for patients 13 years old or older. If this information is already in the medical record available through certified EHR technology, we do not intend that an inquiry be made every time a provider sees a patient 13 years old or older. The frequency of updating this information is left to the provider and guidance is provided already from several sources in the medical community. The information could be collected by any member of the medical staff.

Comment: We received a number of comments recommending either removing this objective to record smoking status from the HIT

functionality objectives or removing the smoking measure from the core clinical quality measures as these measures serve the same purpose and to require both is to require duplicative reporting.

Response: We disagree that these two measures serve the same purpose and therefore only one should be included. The objective included here seeks to ensure that information on smoking status is included in the patient's record. Furthermore, that the information is stored in a structured format so that it can automatically be identified by certified EHR technology as smoking status for possible reporting or exchanging. We also note that the clinical quality measure only focuses on patients 18 years or older, while the objective focuses on patients 13 years or older. In addition, many quality measures related to smoking are coupled with follow-up actions by the provider such as counseling. We consider those follow-up actions to be beyond the scope of what we hope to achieve for this objective for Stage 1 of meaningful use.

After consideration of the public comments received, we are finalizing the meaningful use objective for EPs at $\S 495.6(d)(9)$ (i) and for eligible hospitals at $\S 495.6$ (f)(8)(i) of our regulations as proposed.

We include this objective in the core set as it is integral to the initial or ongoing management of a patient's current or future healthcare and would give providers the necessary information to make informed clinical decisions for improved delivery of patient care.

NPRM EP/Eligible Hospital Measure: At least 80 percent of all unique patients 13 years old or older seen by the EP or admitted to the eligible hospital have "smoking status" recorded.

In the proposed rule, discussion of this measure referenced other sections exclusively.

Comment: We received comments recommending alternative thresholds for this measure. Commenters provided thresholds ranging from anything greater than zero to 60 percent in stage 1.

Response: In the proposed rule, we established a consistent threshold for measures not requiring the exchange of information. For the final rule, (other than up-to-date problem list, active medication list and active medicationallergy list), we have lowered the threshold associated with these measures to 50 percent. In our discussion of the objective, we noted many concerns by commenters over the appropriate age at which to inquire about smoking status. There were also considerable differences among commenters as to what the appropriate inquiry is and what it should include. Due to these concerns, we do not believe this objective and measure fit into the threshold category described under up-to-date problem lists and therefore we adopt a 50 percent (rather than an 80 percent) threshold for this measure. After consideration of the public comments received, we are modifying the meaningful use measure for EPs at $\S 495.6$ (d)(9)(ii) and for eligible hospitals at $\$ 495.6$ (f)(8)(ii) of 
our regulations to "More than 50 percent of all unique patients 13 years old or older seen by the EP or admitted to the eligible hospital's or CAH's inpatient or emergency departments (POS 21 or 23) have smoking status recorded as structured data”.

We further specify that in order to meet this objective and measure, an EP, eligible hospital, or CAH must use the capabilities Certified EHR Technology includes as specified and standards at 45 CFR 170.302(g). The ability to calculate the measure is included in certified EHR technology.

As noted previously in this section under our discussion of the burden created by the measures associated with the Stage 1 meaningful use objectives, the percentage is based on patient records that are maintained using certified EHR technology.

To calculate the percentage, CMS and ONC have worked together to define the following for this objective:

- Denominator: Number of unique patients age 13 or older seen by the EP or admitted to an eligible hospital's or CAH's inpatient or emergency departments (POS 21 or 23) during the EHR reporting period. A unique patient is discussed under the objective of maintaining an up-to-date problem list.

- Numerator: The number of patients in the denominator with smoking status recorded as structured data.

- Threshold: The resulting percentage must be more than 50 percent in order for an EP, eligible hospital, or CAH to meet this measure. As addressed in other objectives, EPs, eligible hospitals or CAHs who see no patients 13 years or older would be excluded from this requirement as described previously in this section under our discussion of whether certain EP, eligible hospital or $\mathrm{CAH}$ can meet all Stage 1 meaningful use objectives given established scopes of practices. Most EPs and all eligible hospitals and CAHs would have access to this information through direct patient access. Some EPs without direct patient access would have this information communicated as part of the referral from the EP who identified the service as needed by the patient. Therefore, we did not include an exclusion based on applicability to scope of practice or access to the information for this objective and associated measure.

NPRM EP/Eligible Hospital Objective: Record advance directives.

In the proposed rule, we discussed this objective, but did not propose it as a requirement for demonstrating meaningful use, for a number of reasons, including: (1) It was unclear whether the objective would be met by indicating that an advance directive exists or by including the contents of the advance directive; (2) the objective seems relevant only to a limited and undefined patient population when compared to the patient populations to which other objectives of Stage 1 of meaningful use apply; and (3) we believe that many EPs would not record this information under current standards of practice. Dentists, pediatricians, optometrists, chiropractors, dermatologists, and radiologists are just a few examples of EPs who would require information about a patient's advance directive only in rare circumstances.

Comment: We received several comments including a comment from the HIT Policy Committee that we should include advance directives in the final rule. The HIT Policy Committee clarified that this would be an indication of whether a patient has an advanced directive. Furthermore, they recommend limiting this measure to patients 65 and older. We received other comments that said this should be a requirement for eligible hospitals. Other commenters reported that having this information available for the patient would allow eligible hospitals to make decisions that were better aligned with the patient's expressed wishes.

Response: In the proposed rule, we said that confusion as to whether this objective would require an indication of the existence of an advanced directive or the contents of the advance directive itself would be included in certified EHR technology was one of the reasons for not including the objective in Stage 1 of meaningful use. We expressed concerns that the latter would not be permissible in some states under existing state law. As commenters have clarified that advance directives should be just an indication of existence of an advance directive and recommended a population to apply the measure to, we reinstate this objective for eligible hospitals and CAHs. We believe that the concern over potential conflicts with state law are alleviated by limiting this to just an indication. We also believe that a restriction to a more at risk population is appropriate for this measure. By restricting the population to those 65 years old and older, we believe we focus this objective appropriately on a population likely to most benefit from compliance with this objective and its measure. This objective is in the menu set so if an eligible hospital or $\mathrm{CAH}$ finds they are unable to meet it then can defer it. However, we believe many EPs would not record this information under current standards of practice. Dentists, pediatricians, optometrists, chiropractors, dermatologists, and radiologists are just a few examples of EPs who would only require information about a patient's advance directive in rare circumstances. For other meaningful use objectives, we have focused our exclusions on rare situations, which would not be the case for this objective. Therefore, we do not include this objective for EPs.

After consideration of the public comments received, we are including this meaningful use objective for eligible hospitals and CAHs at $\S 495.6(\mathrm{~g})(2)$ (i) of our regulations as "Record whether a patient 65 years old or older has an advanced directive as structured data".

NPRM EP/Eligible Hospital Measure: N/A.

While we did not receive specific percentage recommendations from commenters, this objective is the recording of a specific data element as structured data in the patient record. This is identical to other objectives with established measures such as, recording vital signs, recording demographics and recording smoking status. Therefore, we adopt the measure format and the lower threshold (50 percent) from those objectives. We also believe that this information is a level of detail that is not practical to collect on every patient admitted to the eligible hospital's or CAH's emergency department, and therefore, have limited this measure only to the inpatient department of the hospital.

In the final rule, this meaningful use measure for eligible hospitals at $\S 495.6(\mathrm{~g})(2)(\mathrm{ii})$ of our regulations: "More than 50 percent of all unique patients 65 years old or older admitted to the eligible hospital's or CAH's inpatient department (POS 21) have an indication of an advance directive status recorded as structured data".

We further specify that in order to meet this objective and measure, an EP, eligible hospital, or CAH must use the capabilities Certified EHR Technology includes as specified and standards at 45 CFR 170.306(h). The ability to calculate the measure is included in certified EHR technology.

As noted previously in this section under our discussion of the burden created by the measures associated with the Stage 1 meaningful use objectives, the percentage is based on patient records that are maintained using certified EHR technology.

To calculate the percentage, CMS and ONC have worked together to define the following for this objective:

- Denominator: Number of unique patients age 65 or older admitted to an eligible hospital's or CAH's inpatient department (POS 21) during the EHR 
reporting period. A unique patient is discussed under the objective of CPOE.

- Numerator: The number of patients in the denominator with an indication of an advanced directive entered using structured data.

- Threshold: The resulting percentage must be more than 50 percent in order for eligible hospital or CAH to meet this measure. An exclusion, as described previously in this section under our discussion of whether certain EP, eligible hospital or CAH can meet all Stage 1 meaningful use objectives given established scopes of practices, would apply to an eligible hospital or $\mathrm{CAH}$ who admits no patients 65 years old or older during the EHR reporting period.

NPRM EP/Eligible Hospital Objective: Incorporate clinical lab-test results into EHR as structured data.

In the proposed rule, we defined structured data as data that has a specified data type and response categories within an electronic record or file. We have revised that definition for the final rule as discussed below.

Comment: Some commenters requested clarification on what constitutes structured data.

Response: The distinction between structured data and unstructured data applies to all types of information. Structured data is not fully dependent on an established standard. Established standards facilitate the exchange of the information across providers by ensuring data is structured in the same way. However, structured data within certified EHR technology merely requires the system to be able to identify the data as providing specific information. This is commonly accomplished by creating fixed fields within a record or file, but not solely accomplished in this manner.

After consideration of the public comments received, we finalize the meaningful use objective or EPs at $\S 495.6(\mathrm{e})(2)(\mathrm{i})$ and eligible hospitals and CAHs at $\S 495.6(\mathrm{~g})(3)(\mathrm{i})$ as proposed.

NPRM EP/Eligible Hospital Measure: At least 50 percent of all clinical lab tests results ordered by the EP or by an authorized provider of the eligible hospital during the EHR reporting period whose results are either in a positive/negative or numerical format are incorporated in certified EHR technology as structured data.

In the proposed rule, we identified this objective and associated measure as dependent on electronic exchange and therefore requiring special consideration in establishing the threshold. We said that we are cognizant that in most areas of the country, the infrastructure necessary to support such exchange is still being developed. Therefore, we stated our belief that 80 percent is too high a threshold for the Stage 1 criteria of meaningful use. As an alternative, we proposed 50 percent as the threshold based on our discussions with EHR vendors, current EHR users, and laboratories. We then invited comment on whether 50 percent is feasible for the Stage 1 criteria of meaningful use.

Finally, we indicated that we anticipate raising the threshold in future stages of meaningful use as the capabilities of HIT infrastructure increase. We received several comments on the appropriateness of this 50 percent threshold and discuss them in the comment and response section below.

Comment: Commenters requested clarification as to whether the measure includes only electronic exchange of information with a laboratory or if it also includes manual entry.

Response: We encourage every EP, eligible hospital and CAH to utilize electronic exchange of the results with the laboratory based on the certification and standards criteria in the 45 CFR 170.302(h). If results are not received in this manner, then they are presumably received in another form such as fax, telephone call, mail, etc. These results then must be incorporated into the patient's medical record in some way. We encourage that this way use structured data; however, that raises the concerns about the possibility of recording the data twice; for example scanning the results and then entering the results as structured data.

Telephoned results could be entered directly. We also recognize the risk of entry error, which is why we highly encourage the electronic exchange of the results with the laboratory, instead of manual entry through typing, option selecting, scanning or other means. Reducing the risk of entry error is one of the primary reasons we lowered the measure threshold for Stage 1 during which providers are changing their workflow processes to accurately incorporate information into EHRs through either electronic exchange or manual entry. However, for this measure, we do not limit the EP, eligible hospital or CAH to only counting structured data received via electronic exchange, but count in the numerator all structured data. By entering these results into the patient's medical record as structured data, the EP, eligible hospital or $\mathrm{CAH}$ is accomplishing a task that must be performed regardless of whether the provider is attempting to demonstrate meaningful use or not. We believe that entering the data as structured data encourages future exchange of information.
Comment: A majority of commenters commenting on this measure believe the proposed 50 percent threshold is too high. Suggestions for alternative thresholds ranged from more than zero to eighty percent. Some commenters suggested that the percentage calculation be replaced with a numeric count.

Response: We are finalizing a percentage calculation for the reasons discussed previously in this section under our discussion of the burden created by the measures associated with the Stage 1 meaningful use objectives. We based the 50 percent threshold in the proposed rule on our discussions with EHR vendors, current EHR users, and laboratories and specifically requested comment on whether the 50 percent threshold was feasible. While only a small number of commenters commented on this objective, those that did were overwhelming in favor of either a count or a lower threshold. EPs especially were concerned with our inability to impose any requirements on laboratory vendors. Based on the comments received, we have modified our assessment of the current environment for incorporating lab results into certified EHR technology, and believe that a threshold lower than fifty percent is warranted. We want to create a threshold that encourages, but does not require, the electronic exchange of this information and commenters indicated that 50 percent was too high given the current state of electronic exchange of lab results. Therefore, we lower the threshold to 40 percent.

Comment: Commenters requested clarification on what types of laboratories could generate the lab results.

Response: The focus of this objective is to get as many lab results as possible into a patient's electronic health record as structured data. Limiting the objective to a specific type of laboratory would not further this objective so therefore we leave it open to all lab tests and laboratories.

Comment: Several commenters expressed concern regarding the financial burden of establishing lab interfaces, especially for smaller hospitals and practices.

Response: The ability to exchange information is a critical capability of certified EHR technology. Exchange between lab and provider and provider to provider of laboratory results reduces errors in recording results and prevents the duplication of testing. Therefore, we continue to include this objective within Stage 1 of meaningful use although as noted above the measure 
does not rely on the electronic exchange of information between the lab and the provider.

Comment: We received comments requesting a listing of laboratory tests with results that are in a numerical or positive/negative format.

Response: We consider it impractical to develop an exhaustive list of such tests. Moreover, we believe further description of these tests is unnecessary. It should be self-evident to providers when a test returns a positive or negative result or a result expressed in numeric characters. In these case, the results should be incorporated into a patient's EHR as structured data.

Comment: Several commenters pointed out that many current EHR vendors do not support the use of LOINC ${ }^{\circledR}$ codes and there is no federal regulatory requirement for labs to transmit using this code set or for that matter, any structured code set.

Response: Standards such as LOINC $^{\circledR}$ codes are included in the ONC final rule. However, this measure requires incorporation of lab test results as structured data, but does not include a requirement for transmission or electronic receipt of the results using certified EHR technology.

After consideration of the public comments received, we are modifying the meaningful use measure for EPs at $\S 495.6(\mathrm{e})(2)(\mathrm{ii})$ and eligible hospitals at $\S 495.6$ (g)(3)(ii) of our regulations to "More than 40 percent of all clinical lab tests results ordered by the EP or by an authorized provider of the eligible hospital or $\mathrm{CAH}$ for patients admitted to its inpatient or emergency department (POS 21 or 23) during the EHR reporting period whose results are in either in a positive/negative or numerical format are incorporated in certified EHR technology as structured data".

We further specify that in order to meet this objective and measure, an EP eligible hospital, or CAH must use the capabilities Certified EHR Technology includes as specified and standards at 45 CFR 170.302(h). The ability to calculate the measure is included in certified EHR technology.

As noted previously in this section under our discussion of whether certain EP, eligible hospital or CAH can meet all Stage 1 meaningful use objectives given established scopes of practices, the percentage is based on labs ordered for patients whose records are maintained using certified EHR technology.

To calculate the percentage, CMS and ONC have worked together to define the following for this objective:

- Denominator: Number of lab tests ordered during the EHR reporting period by the EP or authorized providers of the eligible hospital or $\mathrm{CAH}$ for patients admitted to an eligible hospital's or CAH's inpatient or emergency department (POS 21 \& 23) whose results are expressed in a positive or negative affirmation or as a number.

- Numerator: The number of lab test results whose results are expressed in a positive or negative affirmation or as a number which are incorporated as structured data.

- Threshold: The resulting percentage must be more than 40 percent in order for an EP, eligible hospital, or CAH to meet this measure.

If an EP orders no lab tests whose results are either in a positive/negative or numeric format during the EHR reporting period they would be excluded from this requirement as described previously in this section under our discussion of whether certain EP, eligible hospital or CAH can meet all Stage 1 meaningful use objectives given established scopes of practices. We do not believe any eligible hospital or CAH would order no lab tests whose results are either in a positive/negative or numeric format during the EHR reporting period.

NPRM EP/Eligible Hospital Objective: Generate lists of patients by specific conditions to use for quality improvement, reduction of disparities, research, and outreach.

Comment: A few commenters recommended eliminating this requirement because they believe it is redundant of clinical quality reporting.

Response: We disagree that this is redundant of clinical quality reporting. Clinical quality reporting does not guarantee usability for all the purposes in the objective. One example of such a use is a provider could not only generate list of patients with specific conditions, but could stratify the output using other data elements in the certified EHR technology that are entered as structured data. The lists could also be utilized at an aggregate level for purposes of research into disparities, which could result in targeted outreach efforts.

Comment: Some commenters requested that if we finalize our proposal to only require one report that we change the "and" in the objective to "or".

Response: We are finalizing our measurement of only requiring one report for Stage 1 of meaningful use and will change "and" to "or". However, we note that all measures will be reconsidered in later stages of meaningful use and multiple reports could be required in those stages.
Comment: We received a few comments requesting the removal of the terms "reduction of disparities" and "outreach" as there are no actionable items or measures associated with the term. We also received comments that the measurement should include the requirement that the lists be stratified by race, ethnicity, preferred language, and gender for initiatives targeted at reducing disparities.

Response: We disagree that actions to reduce disparities or conduct outreach could not be guided by this report, especially if stratified and aggregated reports of many providers are combined within large organizations or among organizations. While we do not require such stratification or aggregation or specify specific uses, that does not preclude them.

Comment: Some commenters requested clarification of the term specific condition.

Response: Specific conditions are those conditions listed in the active patient problem list.

After consideration of the public comments received, we are modifying the meaningful use objective for EPs at $\S 495.6(e)(3)(i)$ and for eligible hospitals at $\S 495.6(\mathrm{~g})(4)(\mathrm{i})$ of our regulations to "Generate lists of patients by specific conditions to use for quality improvement, reduction of disparities, research, or outreach".

NPRM EP/Eligible Hospital Measure: Generate at least one report listing patients of the EP or eligible hospital with a specific condition.

In the proposed rule, we said that an EP or eligible hospital is best positioned to determine which reports are most useful to their care efforts. Therefore, we do not propose to direct certain reports be created. However, in order to ensure the capability can be utilized we proposed to require EPs and hospitals to attest to the ability of the EP or eligible hospital to create a report listing patients by specific condition and to attest that they have actually done so at least once. We received comments on this and address them and any revisions to the proposed rule in the comment and response section below.

Comment: Commenters requested clarification that only one report per EHR reporting period is required to meet the measure.

Response: Yes, only one report in required for any given EHR reporting period. The report could cover every patient whose records are maintained using certified EHR technology or a subset of those patients at the discretion of the EP, eligible hospital or CAH.

Comment: A few commenters suggested the measure should be 
expanded to require submission of the report to CMS or the States or to the local health department.

Response: Submission raises many questions about what types of information can be sent to different entities, how the information is used, patient consent for sending the information, and many of the issues, which add considerable complexity to this meaningful use objective.

Therefore, we are not requiring submission of the report to CMS, the States or local health departments for Stage 1 of meaningful use. We do note that this is one of the objectives for which a State can submit modifications to CMS for approval.

Comment: Several commenters requested a list of condition categories, a model report or the core data elements required to satisfy the measure.

Response: As stated in the rule, we believe an EP, eligible hospital, or $\mathrm{CAH}$ is best positioned to determine which reports are most useful to their care efforts. Therefore, we do not propose to direct certain reports be created.

Comment: For eligible hospitals, commenters stated that the analysis of patient data is derived from postdischarge coding of diagnosis and procedures and not problem lists.

Response: We do not specify that the list is limited to being generated from the data problem list; rather, for the definition of conditions we refer providers to those conditions contained in the problem list.

Comment: One commenter stated that for privacy and confidentiality reasons, patients should be allowed to opt out of any provider outreach initiatives.

Response: Stage 1 of meaningful use does not require the submission of these reports to other entities; rather, we require that the provider generate these reports for their own use. We therefore do not believe the generation of such reports raises privacy and confidentiality concerns. We understand, however, that some patients may have concerns about such lists being exchanged with others and will consider such concerns should future meaningful use requirements focus on exchange of these reports.

After consideration of the public comments received, we are finalizing the meaningful use measure for EPs at $\S 495.6(\mathrm{e})(3)(\mathrm{ii})$ and for eligible hospitals and CAHs at $\S 495.6(\mathrm{~g})(4)(\mathrm{ii})$ of our regulations as proposed.

We further specify that in order to meet this objective and measure, an EP, eligible hospital, or CAH must use the capabilities Certified EHR Technology includes as specified and standards at 45 CFR 170.302(i). The ability to calculate the measure is included in certified EHR technology.

As this measure relies on data contained in certified EHR technology the list would only be required to include patients whose records are maintained using certified EHR technology as discussed previously in this section under our discussion of the burden created by the measures associated with the Stage 1 meaningful use objectives.

We do not believe anything included in this objective or measure limit any $\mathrm{EP}$, eligible hospital or $\mathrm{CAH}$ from completing the measure associated with this objective, therefore, we do not include an exclusion.

NPRM EP Objective: Report ambulatory quality measures to CMS (or, for EPs seeking the Medicaid incentive payment, the States).

Specific comments on the quality measures are discussed in section II.A.3 of this final rule.

We are finalizing this meaningful use objective at $\S 495.6(d)(10)(i)$ of our regulations "Report ambulatory clinical quality measures to CMS (or, for EPs seeking the Medicaid incentive payment, the States)" to better align with the descriptions in section II.A.3.

In response to our revised requirements for meeting meaningful use, we are including this objective in the core set. Section 1848 (o)(2)(A)(iii) of the Act specifically includes submitting clinical quality measures in meaningful use for EPs. Section 1903(t)(6)(D) of the Act also anticipates that the demonstration of meaningful use may include quality reporting to the States for the Medicaid program.

NPRM Eligible Hospital Objective: Report ambulatory quality measures to CMS (or, for eligible hospitals seeking the Medicaid incentive payment, the States)

We make a technical correction to this objective from the proposed rule to ensure that it is clear to the public that we were referring to hospital quality measures.

Specific comments on the quality measures are discussed in section II.A.3 of this final rule.

After consideration of the public comments received, we are finalizing this meaningful use objective at $\S 495.6(d)(9)(i)$ to account for our technical correction and to better align with the descriptions in section II.A.3 as "Report hospital clinical quality measures to CMS (or, for eligible hospitals seeking the Medicaid incentive payment, the States)".

In response to our revised requirements for meeting meaningful use, we are including this objective in the core set. Section 1886 (n)(3)(A)(iii) of the Act specifically includes submitting clinical quality measures in meaningful use for eligible hospitals and CAHs. Section 1903(t)(6)(D) of the

Act also anticipates that the demonstration of meaningful use may include quality reporting to the States for the Medicaid program.

NPRM EP Measure: For 2011, an EP would provide the aggregate level data for the numerator, denominator, and exclusions through attestation as discussed in section II.A.3 of this final rule. For 2012, an EP would

electronically submit the measures that are discussed in section II.A.3. of this final rule.

Specific comments on the quality measures themselves are discussed in section II.A.3 of this final rule.

After consideration of the public comments received, we are finalizing this meaningful use objective at $\S 495.6(\mathrm{~d})(10)(\mathrm{ii})$ as proposed.

NPRM Eligible Hospital Measure: For 2011, an eligible hospital or CAH would provide the aggregate level data for the numerator, denominator, and exclusions through attestation as discussed in section II.A.3 of this final rule. For 2012, an eligible hospital or CAH would electronically submit the measures as discussed in section II.A.3. of this final rule. Specific comments on the quality measures are discussed in section II.A.3 of this final rule. After consideration of the public comments received, we are finalizing this meaningful use objective at 495.6(f)(9)(ii) as proposed.

NPRM EP Objective: Send reminders to patients per patient preference for preventive/follow-up care.

In the proposed rule, we described patient preference as the patient's choice between internet based delivery or delivery not requiring internet access. We are revising that description based on comments as discussed below.

Comment: Commenters have pointed out that requirements to accommodate reasonable requests by individuals to receive communications by means other than the means preferred by the provider already exist under HIPAA at 45 CFR 164.522(b).

Response: As we stated in the proposed rule, patient preference refers to the patient's preferred means of transmission of the reminder from the provider to the patient, and not inquiries by the provider as to whether the patient would like to receive reminders. In the proposed rule, we had proposed that patient preference be limited to the choice between internet based or non-internet based. In order to avoid unnecessary confusion and duplication of requirements, EPs meet 
the aspect of "per patient preference" of this objective if they are accommodating reasonable requests as outlined in 45 CFR 164.522(b), which are the guidance established under HIPAA for accommodating patient requests.

After consideration of the public comments received, we are finalizing the meaningful use objective at $\S 495.6(\mathrm{e})(4)(\mathrm{i})$ of our regulations as proposed.

NPRM EP Measure: Reminder sent to at least 50 percent of all unique patients seen by the EP or admitted to the eligible hospital that are 50 and over.

For the final rule, we are changing the measure to recognize that this is an EP only objective. Therefore, we make the technical correction of striking "or admitted to the eligible hospital".

Comment: Commenters indicated that "practice management systems" or "patient management systems" are commonly used for this function and that integrating them into certified EHR technology would be expensive and time consuming for little value in return.

Response: While we disagree with commenters who suggest there is little to no value in having information about reminders sent to patients available across all the systems used by the provider, we do not assert that such integration of systems must be in place to meet this measure. ONC provides for a modular approach that would allow these systems to be certified as part of certified EHR technology.

Comment: Some commenters pointed out that many patients seen during an EHR reporting period will not be sent a reminder during that same period. Commenters said this is especially true for the 90-day EHR reporting period, but for some services could be true of the full year EHR reporting period as well. Other commenters also pointed out that reminders are not limited to the older population and that children especially may require many reminders on immunizations.

Response: We agree with commenters that many patients not seen during the EHR reporting period would benefit from reminders. As the action in this objective is the sending of reminders, we base the revised measure on that action. This focus is supported by numerous public comments, including those by the HIT Policy Committee. Therefore, we are changing the requirement to account for all patients whose records are maintained using certified EHR technology regardless of whether they were seen by the EP during the EHR reporting period. This greatly expanded denominator caused us to reconsider both our threshold and the age limit. In order to increase the probability that a patient whose records are maintained in certified EHR technology will be eligible for a reminder we change the age limit of the population to 65 years old or older or 5 years old or under. We believe that older patient populations are more likely to have health statuses that will indicate the need for reminders to be sent and this segment of the population is have higher rates of chronic diseases which will require coordination in preventive care such as vaccine reminders. Likewise, the 5 years old and under population will require a multitude of childhood vaccinations such as influenza and will benefit from reminders. However, we do not believe that changing the age limit of the affected population will result in 50 percent of every patient whose records maintained in certified EHR technology requiring a reminder during the EHR reporting period. This is especially true for the first payment year when the EHR reporting period is only 90 days. We are also concerned about the variability among specialists' scopes of practice that may affect the number of patients in the denominator for which a reminder is appropriate. Therefore, we lower the threshold to 20 percent. The $\mathrm{EP}$ has the discretion to determine the frequency, means of transmission and form of the reminder limited only by the requirements of 45 CFR 164.522(b) and any other applicable federal, state or local regulations that apply to them. After consideration of the public comments received, we are modifying the meaningful use measure at $\S 495.6(\mathrm{e})(4)(\mathrm{ii})$ to "More than 20 percent of all patients 65 years or older or 5 years old or younger were sent an appropriate reminder during the EHR reporting period".

We further specify that in order to meet this objective and measure, an EP must use the capabilities Certified EHR Technology includes as specified and standards at 45 CFR $170.304(d)$. The ability to calculate the measure is included in certified EHR technology.

As noted previously in this section under our discussion of the burden created by the measures associated with the Stage 1 meaningful use objectives, the denominator is based on patients whose records are maintained using certified EHR technology.

To calculate the percentage, CMS and ONC have worked together to define the following for this objective:

- Denominator: Number of unique patients 65 years old or older or 5 years older or younger.
- Numerator: The number of patients in the denominator who were sent the appropriate reminder.

- Threshold: The resulting percentage must be more than 20 percent in order for an EP to meet this measure.

As addressed in other objectives and in comment responses, if an EP has no patients 65 years old or older or 5 years old or younger with records maintained using certified EHR technology that EP is excluded from this requirement as described previously in this section under our discussion of whether certain EP, eligible hospital or CAH can meet all Stage 1 meaningful use objectives given established scopes of practices.

NPRM EP/Eligible Hospital Objective: Document a progress note for each encounter. In the proposed rule, we discussed this objective, but did not propose it for Stage 1 of meaningful use. We noted our belief that documentation of progress notes is a medical-legal requirement and a component of basic EHR functionality, and is not directly related to advanced processes of care or improvements in quality, safety, or efficiency.

Comment: We received a limited number of comments regarding our decision not to include documentation of progress notes as an objective. The commenters generally fell into three categories: Those who supported inclusion of this objective in the final rule, those who supported its inclusion only if certain caveats are met and those who supported our proposal not to include it as an objective for Stage 1 of meaningful use. Concerns raised by those supporting the inclusion of this objective included the possibility that an EP may keep paper progress notes in conjunction with use of certified EHR technology as prescribed by Stage 1 of meaningful use and that such a choice by EPs would create the possibility of handwriting illegibility, loss of information and reduced access to health information by both patients and other providers. Another concern raised is that if the objective is not included in the criteria for the definition of meaningful use designers of EHR technology will not include the function in their products. The advocates in the second category agree with the above, but only support inclusion with certain caveats. Some of these caveats include preserving the option of transcription, voice recognition software, and direct entry by an EP or any combination of these. Another caveat is that progress notes not be required to be entered as structured data. The third category supports exclusion of progress notes as an objective for two fundamentally different reasons. Some commenters 
supported exclusion because they believe that the volume of objectives was already too high for Stage 1 of meaningful use and therefore opposed anything that would increase the volume.

Other commenters agree with our proposal that progress notes is already a fundamental part of current EHR products and did not represent a move that advances the use of EHRs.

Response: We predicated our discussion in the proposed rule on the assumption that progress notes are a component of basic EHR functionality. We still believe this is the case and have not received evidence to the contrary. However, we failed to clearly articulate the ramifications of our belief. Our view continues to be that an EP who incorporates the use of EHRs into a practice and complies with meaningful use criteria is unlikely to maintain separate paper progress notes outside of the EHR system. We believe that the potential disruption in workflow of the efforts to merge paper progress notes with the other records in certified EHR technology in order to have a complete medical record far outweighs the burden of electronically capturing progress notes. Moreover, we continue to believe this is a highly unlikely scenario. As with any meaningful use objective, it is important to have clear, definitive definitions. However, our observations of discussions held in public forums by the medical community and review of literature have led us to conclude that it not possible to provide a clear, definitive definition of a progress note at this time. We note that commenters recommending the documentation of a progress note be included as an objective did not attempt to define the term. Nor did commenters suggest an associated measure. We continue to believe that there is insufficient need and upon review believe there is insufficient consensus regarding the term progress note to include this objective for Stage 1 of meaningful use.

After consideration of the public comments received, we do not include this meaningful use objective in the final rule.

NPRM EP/Eligible Hospital Measure: N/A.

NPRM EP Objective: Implement five clinical decision support rules relevant to specialty or high clinical priority, including for diagnostic test ordering, along with the ability to track compliance with those rules.

NPRM Eligible Hospital Objective: Implement 5 clinical decision support rules related to a high priority hospital condition, including diagnostic test ordering, along with the ability to track compliance with those rules.

First, we make a technical correction. On page 1856 of the proposed rule, we described this objective for eligible hospitals as "Implement five clinical decision support rules relevant to specialty or high clinical priority, including for diagnostic test ordering, along with the ability to track compliance with those rules." The underlined language was

inappropriately carried over from the EP objective in this instance and in the regulation text. The table contained our intended language of "Implement 5 clinical decision support rules related to a high priority hospital condition, including diagnostic test ordering, along with the ability to track compliance with those rules." Many commenters pointed this discrepancy out to us and we appreciate their diligence.

Comment: Nearly half of the commenters mentioning clinical decision support suggested that the term needed additional clarification. Some commenters said that the term was too vague and open to interpretation while others said it was too specific. Other commenters provided recommendations on what a clinical decision support rule should mean or which elements it should include. These were evidencebased medicine templates, decision trees, reminders, linked online resources, scientific evidence, and consensus.

Response: In the proposed rule, we described clinical decision support as HIT functionality that builds upon the foundation of an EHR to provide persons involved in care processes with general and person-specific information intelligently filtered and organized, at appropriate times, to enhance health and health care. We purposefully used a description that would allow a provider significant leeway in determining the clinical decision support rules that are more relevant to their scope of practice and benefit their patients in the greatest way. In the proposed rule, we asked providers to relate the rules they select to clinical priorities and diagnostic test ordering. We do not believe that adding a more limiting description to the term clinical decision support would increase the value of this objective. We believe that this determination is best left to the provider taking into account their workflow and patient population.

Comment: Several commenters objected to the requirement of five clinical decision support rules when the HIT Policy Committee only recommended one. Others disagreed with our proposed assertion that most
EPs would report on at least five clinical quality measures from section II.A.3 of the proposed rule and eligible hospitals will all report on at least five.

Response: We accept the argument that there is value in focusing initial CDS efforts on a single CDS rule in order to get it right the first time and lay the foundation for future, broader CDS implementation. This will help to prevent the unintended negative consequences associated with poorly implemented CDS systems when providers have attempted to do too much too soon.

We agree that the appropriate balance is to require some degree of meaningful use of CDS in Stage 1 without

overburdening providers with too many areas to focus on at once. Since CDS is one area of health IT in which significant evidence exists that it can have a substantial positive impact on the quality, safety and efficiency of care delivery, it is important that it be included as a core objective with this more limited expectation. That requirement will assure that all meaningful users have taken the first steps in CDS implementation but allow them to focus as necessary on a single high-priority area at the outset in order to ensure that they can devote the appropriate level of attention to their first CDS priority. We anticipate that this will set the foundation for much more expansive CDS support in the near future.

Comment: A commenter inquired if modification of the clinical decision support tool negates the EHR's certification status.

Response: We believe this is a question on certification status and is outside of the scope of this rule. ONC discusses what would affect Certified EHR Technology's certified status in their final rule (75 FR 36157) entitled "Establishment of the Temporary Certification Program for Health Information Technology".

After consideration of the public comments received, we are modifying the meaningful use objective for EPs at 495.6(d)(11)(i) to "Implement one clinical decision support rule relevant to specialty or high clinical priority along with the ability to track compliance with that rule."

After consideration of public comments received, we are modifying the meaningful use objective for eligible hospitals and CAHs at $\S 495.6(f)(10)(i)$ of our regulations as "Implement one clinical decision support rule related to a high priority hospital condition along with the ability to track compliance with that rule." 
We believe that clinical decision support is one of the most common tools that uses the information collected as structured data included in the core set and therefore also include clinical decision support in the core as the information needed to support it are already included in the core set.

NPRM EP/Eligible Hospital Measure: Implement five clinical decision support rules relevant to the clinical quality metrics the EP/Eligible Hospital is responsible for as described further in section II.A.3. of this final rule.

In the proposed rule, we said that clinical decision support at the point of care is a critical aspect of improving quality, safety, and efficiency. Research has shown that decision support must be targeted and actionable to be effective, and that "alert fatigue" must be avoided. Establishing decision supports for a small set of high priority conditions, ideally linked to quality measures being reported, is feasible and desirable. Meaningful use seeks to ensure that those capabilities are utilized.

Comment: Commenters, both in the requests for clarification of the term clinical decision support and explicitly in response to this measure, expressed concern about the linkage to a particular quality measure.

Response: We agree that such linkage puts constraints on the provider and eliminates many types of clinical decision support rules that may be beneficial. Therefore, we revise this measure to require that at least one of the five rules be related to a clinical quality measure, assuming the EP, eligible hospital or CAH has at least one clinical quality measure relevant to their scope of practice. However, we strongly encourage EPs, eligible hospitals and CAHs to consider the clinical quality measures as described in section II.A.3 when deciding which additional rules to implement for this measure.

Comment: Several commenters, including the HIT Policy Committee, recommended that we focus at least one clinical decision support rule on efficiency of care.

Response: In light of decision to limit the objective to one clinical decision support rule, we do not believe that it is appropriate to further to link that rule to specific requirements and therefore give the $\mathrm{EP}$, eligible hospital or $\mathrm{CAH}$ discretion on what to focus the clinical decision support rule used to satisfy this measure.

Comment: A few commenters asked for clarification of how the "** * with the ability to track compliance with those rules" language of the proposed objective for clinical decision support rules relates to the associated measures.

Response: While an integral part of the objective and certified EHR technology, we did not include this aspect of the objective in the measure for Stage 1 of meaningful use. An EP, eligible hospital, or CAH is not required to demonstrate to CMS or the States its compliance efforts with the CDS recommendations or results for Stage 1 either at initial attestation or during an subsequent review of that attestation.

After consideration of the public comments received, we are modifying the meaningful use measure for EPs at $\S 495.6(\mathrm{e})(11)(\mathrm{ii})$ and for eligible hospitals and CAHs at \$495.6(g)(10)(ii) to "Implement one clinical decision support rule.

We further specify that in order to meet this objective and measure, an EP, eligible hospital, or CAH must use the capabilities Certified EHR Technology includes as specified and standards at 45 CFR 170.304(e) for EPs and 45 CFR 170.306(c). The ability to calculate the measure is included in certified EHR technology.

Given the added flexibility added to this measure in the final rule, we do not believe that any EP, eligible hospital, or $\mathrm{CAH}$ would be in a situation where they could not implement one clinical decision support rules as described in the measure. Therefore, there are no exclusions for this objective and its associated measure.

NPRM EP/Eligible Hospital Objective: Submit claims electronically to public and private payers.

Comment: Over three quarters of those commenting on this objective recommended that it be eliminated for various reasons. The majority of the other commenters requested a modification. Reasons given are:

-Electronic claims submission is already covered under HIPAA;

-Electronic claims submission is not part of traditional EHR technology;

- Billing systems would have to be certified adding to cost and burden of compliance with meaningful use even though when electronic claims submission for Medicare is already in place for all by the very smallest of providers;

-Electronic claims submission falls outside of the scope of the statutory mandate given by Congress to implement the HITECH legislation to improve care delivery through broad scale adoption and utilization of Electronic Health Record technologies. This function does not impact the quality of care delivered and relies on product components that are traditionally part of practice management systems;

-Private payers may customize the HIPAA-recognized standard transactions, which limits the ability of practices to obtain accurate information prior to receiving an Explanation of Benefits based on the actual services provided and negates many of the benefits of having standardized transactions;

-Workers' compensation and auto insurers do not accept electronic claims; and

-Many providers use clearinghouses and they requested that the burden of electronic submission be shifted to the clearinghouse.

Response: In our proposed rule, we specifically cite that the existence of standard transactions available under HIPAA for submitting claims as a reason for including this objective as a meaningful use objective for Stage 1. We also disagree that this objective is outside the scope of meaningful use as defined by the HITECH legislation. The HITECH legislation states the Secretary shall seek to improve not only health care quality, but also the use of electronic health records. In addition, we note that sections 1848(o)(2)(A) and 1886(n)(3)(A) of the Act provide that to be considered a meaningful EHR user, an EP, eligible hospital, or CAH must demonstrate use of certified EHR technology in a meaningful manner as defined by the Secretary. In the Medicaid context, any demonstration of meaningful use must be "acceptable to the Secretary" under 1903(t)(6). We believe this language gives us broad discretion to require the use of certified EHR technology in a manner that not only improves health care quality, but results in gains in efficiency, patient engagement and enhances privacy and security. Under the broad definition of electronic health record established by ONC in their final rule, electronic exchange of eligibility information and claims submission could certainly improve the use of electronic health records.

We believe that inclusion of administrative simplification in meaningful use is an important longterm policy goal for several reasons. First, administrative simplification can improve the efficiency and reduce unnecessary costs in the health care system as a whole; the small percentage of paper claims submitted represent a disproportionate administrative cost for health plans; the reconciliation of billing charges for services not eligible for payment creates a significant burden for providers, health plans, and most 
significantly, for patients. Second, the integration of administrative and clinical information systems is necessary to support effective management and coordinated care in physician practices. The ability to leverage clinical documentation in support of appropriate charge capture (for example, for preventive counseling, or immunizations provided), the ability to link lists of patients needing clinical reminders with patient contact information, the ability to stratify quality measures by patient demographic factors (for example, race/ ethnicity) and insurer status (for example, Medicare beneficiaries), are examples.

In addition, there are important benefits to the inclusion of administrative transactions in criteria and standards for the certification of EHR technologies. The option of modular certification provides an opportunity for eligible professionals and hospitals to use practice management systems or clearinghouses that provide these functions as components of their certified EHR technologies. However, we recognize there is not current agreement as to which systems constitute an EHR and that many entities may view their billing system to be outside their EHR and that the vendors of some practice management systems that provide these functionalities in doctors' offices today may not be prepared to seek certification for these legacy products in $2010 / 2011$. We also recognize that the introduction of the X12 5010 standards in January 2012 would further complicate the certification process for stage 1 . We also acknowledge that we do not have the ability to impose additional requirements on third-party payers or clearinghouses to participate in this exchange beyond what is required by HIPAA. Based on these considerations, we are not including this objective in the final rule for Stage 1 of meaningful use.

However, the introduction of these new X12 5010 standards, and the coming introduction of ICD-10 in 2013 provides an opportunity for change in Stage 2 of meaningful use. In order to meet these and other administrative simplification provisions, most providers will have to upgrade their practice management systems or implement new ones. This provides an important opportunity to achieve alignment of capabilities and standards for administrative transactions in EHR technologies with the administrative simplification provisions that the Affordable Care Act provides for health plans and health plan clearinghouses.
We therefore intend to include administrative transactions as a part of Stage 2 of meaningful use, and expect providers and vendors to take this into consideration in their decisions leading up to 2013.

Comment: Commenters focusing on how meaningful use would translate into the Medicare Advantage program said that the measure of checking eligibility electronically and submitting claims electronically for 80 percent of patients seen would not be possible. They explained that for most of their visits, there is no insurance company with which to check, and there is no insurance company to whom to submit claims. They described themselves as a capitated system and for most of the patient visits, the concept of checking eligibility and submitting claims in not relevant.

Response: This comment illustrates the difficulties in adopting FFS Medicare meaningful use measures for qualifying MA organizations, MAaffiliated hospitals and MA EPs. For purposes of determining meaningful use in a Medicare Advantage environment, we agree that submitting claims

electronically is not a useful standard in a capitated environment where virtually all patients are members of the same insurance plan.

After consideration of the public comments received, we are not finalizing the objective "Submit claims electronically to public and private payers".

NPRM EP/Eligible Hospital Measure: At least 80 percent of all claims filed electronically by the EP or the eligible hospital.

We received many comments on the difficulty in calculating this measure. However, as all measures are tied to objectives and we do not finalize this objective we also do not finalize the measure.

NPRM EP/Eligible Hospital Objective: Check insurance eligibility electronically from public and private payers.

Comment: Over three quarters of those commenting on this objective recommended that it be eliminated for various reasons. Some of the most common reasons for elimination are:

—Electronic eligibility checks are already covered under HIPAA; —Electronic eligibility checks are not part of traditional EHR technology; -Billing and practice management systems that are used for electronic eligibility checks would have to be certified as certified EHR technology adding to cost and burden;

-Electronic eligibility checks is outside of the scope of the mandate given by
Congress to implement the HITECH legislation in such a way as to improve care delivery through broad scale adoption and utilization of Electronic Health Record technologies. This function does not impact the quality of care delivered and relies on product components that are traditionally part of practice management systems;

-Information returned on typical electronic eligibility checks is of little use to providers-as responses are usually a yes/no answer on coverage, but not the specificity of coverage;

- The current poor adoption rate of the use of electronic eligibility verification is indicative of the deficiencies in current methods;

-Once eligibility checking becomes easy to use and reliable, no incentive will be required as providers will adopt the process readily;

-Payers do not guarantee their eligibility results;

-Many payers are still not in compliance with the HIPAA 270/271 electronic eligibility standard.

Therefore the objective should only be required if compliance with the standard by health plans can be guaranteed; and

-Private payers may customize the HIPAA-recognized standard transactions, which limits the ability of practices to obtain accurate information prior to receiving an Explanation of Benefits based on the actual services provided and negates many of the benefits of having standardized transactions.

Response: In our proposed rule, we specifically cite the existence of the standard transaction for eligibility checks available under HIPAA as an enabling factor for the inclusion this objective. As with the electronic claims submission objective discussed above, we disagree that this objective is outside the scope of meaningful use as defined by the HITECH legislation. The HITECH legislation requires the Secretary to seek to improve not only health care quality, but also the use of electronic health records. Under the broad definition of electronic health record established by ONC in their final rule, electronic exchange of eligibility information could certainly improve the use of electronic health records. However, we recognize there is not current agreement as to which systems constitute an EHR and that many entities may view their practice management system to be outside their EHR. We also acknowledge that we do not have the ability to impose additional requirements on third-party payers to participate in this 
exchange beyond what is required by HIPAA. Third-party payers can provide simple yes/no responses, modify the standard transactions and do not have to guarantee their results. We agree with commenters that this significantly devalues the results of this objective. However, we do believe that as electronic records and exchange based on this and considerations that commenters nearly universally considered this to not be a function of EHR, we are not including this objective in the final rule for Stage 1 of meaningful use. However, we do believe that inclusion of a robust system to check insurance eligibility electronically is an important long term policy goal for meaningful use of certified EHR technology and we intend to include this objective as well as electronic claims submission Stage 2.

After consideration of the public comments received, we are not finalizing the objective to "Check insurance eligibility electronically from public and private payers" or any modification thereof. Given that we are not finalizing the objective, we also are not finalizing the associated EP and eligible hospital/CAH measures.

The second health outcomes policy priority identified by the HIT Policy Committee is to engage patients and families in their healthcare. The following care goal for meaningful use addresses this priority:

- Provide patients and families with timely access to data, knowledge, and tools to make informed decisions and to manage their health.

As explained in the proposed rule, we do not intend to preempt any existing Federal or State law regarding the disclosure of information to minors, their parents, or their guardians in setting the requirements for meaningful use. For this reason, we defer to existing Federal and State laws as to what is appropriate for disclosure to the patient or their family. For purposes of all objectives of the Stage 1 criteria of meaningful use involving the disclosure of information to a patient, a disclosure made to a family member or a patient's guardian consistent with Federal and State law may substitute for a disclosure to the patient.

Comment: Several commenters requested that all objectives under the health care policy priority be combined, as they are redundant.

Response: We disagree that they are redundant and believe each serves a unique purpose. We will more fully describe those purposes in the discussion of each objective.

NPRM EP Objective: Provide patients with an electronic copy of their health information (including diagnostics test results, problem list, medication lists, allergies) upon request.

NPRM Eligible Hospital Objective: Provide patients with an electronic copy of their health information (including diagnostic test results, problem list, medication lists, allergies, discharge summary, procedures), upon request

The purpose of this objective is to provide a patient's health information to them electronically and in a human readable format and in accordance with the standards specified in the ONC final rule subject to its availability to the provider electronically and any withholding under regulations related to the HIPAA Privacy Act at 45 CFR 164.524, Access of individuals to protected health information.

In the proposed rule, we indicated that electronic copies may be provided through a number of secure electronic methods (for example, personal health record (PHR), patient portal, CD, USB drive). We have changed this description in response to comments to that when responding to patient requests for information, the $\mathrm{EP}$, eligible hospital, or CAH should accommodate patient requests in accordance with 45 CFR 164.524, Access of individuals to protected health information. The objective provides additional criteria for meeting meaningful use concerning the electronic copy or provision of information that the EP, eligible hospital or CAH maintains in or can access from the certified EHR technology and is maintained by or on behalf of the EP, eligible hospital or $\mathrm{CAH}$.

Comment: We received requests for clarification that only information that the EP, eligible hospital, or CAH has available electronically must be provided to the patient.

Response: Yes, we limit the information that must be provided electronically to that information that exists electronically in or accessible from the certified EHR technology and is maintained by or on behalf of the EP, eligible hospital or CAH. We believe it is impractical to require information maintained on paper to be transmitted electronically. Furthermore, given the other criteria of Stage 1 of meaningful use, we believe sufficient information will be available through certified EHR technology, especially given the inclusion of many of the foundational objectives that were included in the core set.

Comment: Commenters pointed out that the HIPAA Privacy Rule permits licensed healthcare professionals to withhold certain information if its disclosure would cause substantial harm to the patient or another individual.

Response: As previously discussed for patient preference, we do not seek to conflict with or override HIPAA through meaningful use requirements. Therefore, an EP, eligible hospital, or CAH may withhold information from the electronic copy of a patient's health information in accordance with the regulations at 45 CFR 164.524, Access of individuals to protected health information.

Comment: Commenters requested clarification of the term "health information" or alternatively a list of elements required to satisfy the objective.

Response: Subject to the withholding described above, an EP, eligible hospital, or CAH should provide a patient with all of the health information they have available electronically. At a minimum, this would include the elements listed in the ONC final rule at 45 CFR 170.304(f) for EPs and 45 CFR 170.306(d) for eligible hospitals and CAHs as required for EHR technology to become certified.

Comment: Several commenters indicated that a provider should be allowed to charge a fee for providing an electronic copy of a patient's health information.

Response: We do not have the authority under the HITECH Act to regulate fees in this manner. Rather, the charging of fees for this information is governed by the HIPAA Privacy Rule at 45 CFR 164.524(c)(4) (which only permits HIPAA covered entities to charge an individual a reasonable, costbased fee for a copy of the individual's health information). We would expect these costs to be very minimal considering that the ability to generate the copy is included in certified EHR technology. Additional clarification on the fee that a HIPAA covered entity may impose on an individual for an electronic copy of the individual's health information will be addressed in upcoming rulemaking.

Comment: Commenters pointed out that the general term "allergies" is inconsistent with other objectives of Stage 1 and with the capabilities mandated by certification under the ONC IFR, which address only medication allergies.

Response: As we have stated on several other objectives, we encourage all EPs, eligible hospitals, and CAHs to work with their EHR technology designers to make capabilities most relevant to their individual practices of care. However, we have maintained that at a minimum the capabilities that are part of certification should be included 
and those should be the basis for meaningful use so we do modify this objective to medication allergies to align it with other objectives and certification

After consideration of the public comments received, we are modifying the meaningful use objective for EPs at $\S 495.6(d)(12)(i)$ of our regulations to "Provide patients with an electronic copy of their health information (including diagnostics test results, problem list, medication lists, medication allergies) upon request" and for eligible hospitals and CAHs at $\S$ 495.6(f)(11)(i) of our regulations to "Provide patients with an electronic copy of their health information (including diagnostic test results, problem list, medication lists, medication allergies, discharge summary, procedures), upon request".

We include this objective in the core set as it is integral to involving patients and their families in their provision of care and was recommended by the HIT Policy Committee for inclusion in the core set.

NPRM EP/Eligible Hospital Measure: At least 80 percent of all patients who request an electronic copy of their health information are provided it within 48 hours.

In the proposed rule, we pointed out that all patients have a right under ARRA to an electronic copy of their health information. We said that our purpose for including it in meaningful use was to ensure that this requirement in met in a timely fashion. We also said that providing patients with an electronic copy of their health information demonstrates one of the many benefits health information technology can provide and we believe that it is an important part of becoming a meaningful EHR user. We received requests for clarifications on what must be provided and in what timeframe. We address those requests in the comment and response section below. We note here that participation in the Medicare and Medicaid EHR incentive programs is voluntary. Nothing in the Stage 1 criteria of meaningful use supersedes or exempts an EP, eligible hospital or $\mathrm{CAH}$ from complying with otherwise applicable requirements to provide patients with their health information.

Comment: An overwhelming majority of commenters commenting on this objective indicated that the 48-hour time frame is too short and inconsistent with the HIPAA Privacy Rule.

Response: We discuss the reasoning for the time frame in the proposed rule. We state that this measure seeks to ensure that a patient's request is met in a timely fashion. Providing patients with an electronic copy of their health information demonstrates one of the many benefits health information technology can provide. We also believe that certified EHR technology will provide EPs, eligible hospitals, and CAHs more efficient means of providing copies of health information to patients, which is why we proposed that a request for an electronic copy be provided to the patient within 48 hours.

In the final rule, we further point out that this objective is limited to health information maintained and provided electronically while HIPAA can require the retrieval, copying and mailing of paper documents. For this reason, we do not believe the timeframes under this meaningful use objective and the HIPAA Privacy Rule must be aligned. However, we appreciate that the 48hour timeframe may be burdensome for some providers, particularly for those providers who do not operate $24 / 7$. We therefore are lengthening the timeframe to three business days. Business days are defined as Monday through Friday excluding federal or state holidays on which the EP, eligible hospital, or $\mathrm{CAH}$ or their respective administrative staffs are unavailable. As an example if a patient made a request for an electronic copy of their health information on Monday then the EP, eligible hospital, or CAH would have until the same time on Thursday to provide the information assuming there were no intervening holidays. If provision of the copy involves the mailing of physical electronic media, then it would need to be mailed on the Thursday.

Comment: Some commenters believed the 80 percent threshold was too high or introduced examples of extraordinary circumstances such as natural disasters or system crashes that would indicate a lower threshold is needed to accommodate them.

Response: We reduce the threshold to over 50 percent as this objective meets the criteria of relying solely on a capability included as part of certified EHR technology and is not, for purposes of Stage 1 criteria, reliant on the electronic exchange of information, as explained under our discussion of the objective of maintain an up-to-date problem list. As this is a relatively new capability that was not available to either providers or patients before the introduction of EHRs, we do not believe it meets the same standard of practice as maintaining an up-to-date problem list and therefore adopt a threshold of 50 percent (rather than 80 percent).

Comment: We received comments that were concerned about the reporting burden of this requirement.

Response: We believe that as long as the request by the patient is accurately recorded in the certified EHR technology then the certified EHR technology should be able to calculate the measure. Recording patient requests for certain actions should be part of the expectations of meaningful use of certified EHR technology. If the EP, eligible hospital, or CAH records the requests using certified EHR technology, certified EHR technology will be able to assist in calculating both the numerator and denominator. If the requests are recorded by another means at the choice of the provider, the provider would be responsible for determining the denominator.

Comment: Commenters inquired if third-party requests for information are included in the denominator.

Response: Only specific third party requests for information are included in the denominator. As we stated in the opening discussion for this health care priority, providing the copy to a family member or patient's authorized representative consistent with federal and state law may substitute for a disclosure of the information to the patient and count in the numerator. A request from the same would count in the denominator. All other third party requests are not included in the numerator or the denominator.

Comment: Commenters inquired if asking the patient to register for their own personal health record (PHR) satisfies the intent of the objective.

Response: EPs, eligible hospitals and CAHs are to provide the information pursuant to the reasonable accommodations for patient preference under 45 CFR164.522(b). To be included in this measure, the patient has already requested an electronic method. While having a third party PHR certainly would be one method, assuming the provider could populate the PHR with all the information required to meet this objective. The provider should provide the same level of assistance to the patient that would be provided as if they maintained their own patient portal.

Comments: Comments were received requesting the format and media for the provision of the health information.

Response: As this is for use by the patient, the form and format should be human readable and comply with the HIPAA Privacy Rule, as specified at 45 CFR 164.524(c). In addition, efforts should be made to make it easily understandable to the patient. The media could be any electronic form such as patient portal, PHR, CD, USB fob, etc. As stated in the previous response, EPs, eligible hospitals and CAHs are expected to make reasonable 
accommodations for patient preference as outlined in 45 CFR $164.522(\mathrm{~b})$.

After consideration of the public comments received, we are modifying the meaningful use measure for EPs at §495.6(d)(12)(i) and for eligible hospitals at $\$ 495.6(f)(11)(i)$ of our regulations to "More than 50 percent of all patients of the EP or the inpatient or emergency departments of the eligible hospital or CAH (POS 21 or 23) who request an electronic copy of their health information are provided it within 3 business days."

We further specify that in order to meet this objective and measure, an EP, eligible hospital, or CAH must use the capabilities Certified EHR Technology includes as specified and standards at 45 CFR 170.304(f) for EPs and 45 CFR 170.306(d) for eligible hospitals and CAHs. The ability to calculate the measure is included in certified EHR technology.

As the provision of the electronic copy is limited to the information contained within certified EHR technology, this measure is by definition limited to patients whose records are maintained using certified EHR technology as described previously in this section under our discussion of the burden created by the measures associated with the Stage 1 meaningful use objectives.

To calculate the percentage, CMS and ONC have worked together to define the following for this objective:

- Denominator: The number of patients of the EP or eligible hospital's or CAH's inpatient or emergency departments (POS 21 or 23) who request an electronic copy of their electronic health information four business days prior to the end of the EHR reporting period.

- Numerator: The number of patients in the denominator who receive an electronic copy of their electronic health information within three business days.

- Threshold: The resulting percentage must be more than 50 percent in order for an EP, eligible hospital, or CAH to meet this measure. As addressed in other objectives and in comment response, if the EP, eligible hospital, or $\mathrm{CAH}$ has no requests from patients or their agents for an electronic copy of patient health information during the EHR reporting period they would be excluded from this requirement as described previously in this section under our discussion of whether certain $\mathrm{EP}$, eligible hospital or CAH can meet all Stage 1 meaningful use objectives given established scopes of practices.

NPRM Eligible Hospital Objective: Provide patients with an electronic copy of their discharge instructions and procedures at time of discharge, upon request.

The purpose of this objective is to provide the option to patients to receive their discharge instructions electronically. Discharge instructions would not necessarily be included in a copy of health information and it is unlikely that a patient would request a copy of their health information at every discharge. This objective is unique to eligible hospitals and CAHs.

Comment: We received several comments suggesting that we eliminate or clarify the term "procedures."

Response: As we believe the terms "instructions" and "procedures" are interchangeable as used in this objective, we are removing the term "procedures" from the objective. We left this term in the provision of electronic copy of health information as the term "instructions" is not in that objective. We clarify that the term "instructions" means any directions that the patient must follow after discharge to attend to any residual conditions that need to be addressed personally by the patient, home care attendants, and other clinicians on an outpatient basis.

Comment: Commenters pointed out that the HIPAA Privacy Rule permits licensed healthcare professionals to withhold certain information if its disclosure would cause substantial harm to the patient or another individual.

Response: We reiterate that it is not our intent for the meaningful use objectives to conflict or override the HIPAA Privacy Rule through meaningful use requirements. Therefore an EP, eligible hospital, or CAH may withhold information from the electronic copy to the extent they are permitted or required to do so in accordance with the regulations at 45 CFR 164.524.

Comment: Some commenters recommended that hospitals should be required to either provide every patient an electronic copy of their discharge instructions or at least inform them of the option to receive it electronically.

Response: We believe it would be too burdensome to provide every patient an electronic copy of his or her discharge instructions. Furthermore, we anticipate that many, if not most, patients will prefer a paper copy during the years of Stage 1 . While we certainly encourage eligible hospitals to inform their patients of the option to receive their discharge instructions electronically, we do not see requiring this as within the scope of meaningful use of certified EHR technology for Stage 1.

Comment: Comments were received requesting a clarification of the data that should be included in the discharge instructions.

Response: This objective simply refers to the option of the electronic provision of instructions that would be provided to the patient. We believe eligible hospitals are the appropriate entity to determine the information that should be included in the discharge

instructions.

Comment: Comments were received requesting the format and media for the discharge instructions.

Response: As this is for use by the patient, the form and format should be human readable and comply with the HIPAA Privacy Rule, as specified at 45 CFR 164.524(c). In addition, efforts should be made to make it easily understandable to the patient. The media could be any electronic form such as patient portal, PHR, CD, USB fob, etc. EPs, eligible hospitals and CAHs are expected to make reasonable accommodations for patient preference as outlined in 45 CFR 164.522(b).

After consideration of the public comments received, we are finalizing the objective at 495.6(f)(12)(i) of our regulations as proposed.

We include this objective in the core set as it is integral to involving patients and their families in their provision of care and was recommended by the HIT Policy Committee for inclusion in the core set.

NPRM Eligible Hospital Measure: At least 80 percent of all patients who are discharged from an eligible hospital and who request an electronic copy of their discharge instructions and procedures are provided it.

Comment: Some commenters believed the 80 percent threshold was too high or introduced examples of extraordinary circumstances that would indicate that a lower threshold is needed to accommodate them.

Response: We reduce the threshold to over 50 percent as this objective meets the criteria of relying solely on a capability included as part of certified EHR technology and is not, for purposes of Stage 1 criteria, reliant on the electronic exchange of information. However, as this is a relatively new capability that was not available to either providers or patients before the introduction of EHRs we do not believe it meets the same standard of practice as maintaining an up-to-date problem list and therefore adopt a threshold of 50 percent (rather than 80 percent).

Comment: Some commenters expressed concern about the reporting burden imposed by this requirement.

Response: We believe that as long as the request by the patient is accurately recorded in the certified EHR 
technology then the certified EHR technology should be able to calculate the measure. We believe that recording patient requests for certain actions that involve the use of certified EHR technology should be part of EPs, eligible hospitals and CAHs standard practice. If the eligible hospital or $\mathrm{CAH}$ records the requests using certified EHR technology, certified EHR technology will be able to assist in calculating both the numerator and denominator. If the requests are recorded by another means at the choice of the provider, the provider would be responsible for determining the denominator.

Comment: Several of the comments requested clarification of the timeframe in which the discharge instructions should be provided to the patient.

Response: As discussed previously, this objective simply refers to the option of the electronic provision of instructions that would be provided to the patient at the time of discharge. Therefore, we believe for the information to be useful to the patient, the instructions themselves or instructions on how to access them electronically should be furnished at the time of discharge from the eligible hospital or CAH.

Comment: Some comments expressed concern that providing an electronic copy of discharge instructions to the patient at the time of discharge would disrupt workflows and lengthen the discharge process resulting in reduced bed turnover in emergency departments.

Response: As discussed previously, this objective simply refers to the option of the electronic provision of instructions that would be provided to the patient at the time of discharge. We do not believe the provision of an electronic copy of the discharge instructions, upon request, at the time of discharge alters current workflow or lengthens the discharge process. A patient could be provided instructions on how to access an Internet Web site where they can get the instructions or asked to provide an e-mail address or simply be handed electronic media instead of or in addition to a paper copy.

After consideration of the public comments received, we are modifying the meaningful use measure at $\S 495.6$ (f)(12)(ii) of our regulations to "More than 50 percent of all patients who are discharged ${ }^{1}$ from an eligible

\footnotetext{
${ }^{1}$ Please note that although the final rule meaningful use measures refer to patients discharged from an emergency department, such emergency room releases are not eligible hospital discharges for purpose of determining hospital payment incentives under section 1886(n) of the Act. Section 1886(n) payments are only with
}

hospital's or CAH's inpatient or emergency department (POS 21 or 23) and who request an electronic copy of their discharge instructions are provided it."

We further specify that in order to meet this objective and measure, an EP, eligible hospital, or CAH must use the capabilities Certified EHR Technology includes as specified and standards at 45 CFR 170.306(e). The ability to calculate the measure is included in certified EHR technology.

As with the previous objective, the provision of the electronic copy of the discharge summary is limited to the information contained within certified EHR technology; therefore this measure is by definition limited to patients whose records are maintained using certified EHR technology as described previously in this section under our discussion of the burden created by the measures associated with the Stage 1 meaningful use objectives.

To calculate the percentage, CMS and ONC have worked together to define the following for this objective:

- Denominator: Number of patients discharged from an eligible hospital's or CAH's inpatient or emergency department (POS 21 or 23) who request an electronic copy of their discharge instructions and procedures during the EHR reporting period.

- Numerator: The number of patients in the denominator who are provided an electronic copy of discharge

instructions.

- Threshold: The resulting percentage must be more than 50 percent in order for an EP, eligible hospital, or CAH to meet this measure.

As addressed in other objectives and in comment response, if the eligible hospital or $\mathrm{CAH}$ has no requests from patients or their agents for an electronic copy during the EHR reporting period they would be excluded from this requirement as described previously in this section under our discussion of whether certain EP, eligible hospital or CAH can meet all Stage 1 meaningful use objectives given established scopes of practices.

NPRM EP Objective: Provide patients with timely electronic access to their health information (including lab results, problem list, medication lists, and allergies) within 96 hours of the information being available to the EP.

In the proposed rule, we described timely as within 96 hours of the information being available to the EP through either the receipt of final lab results or a patient interaction that

respect to "inpatient" hospital services pursuant to section 1886(n)(1)(A) of the Act. updates the EP's knowledge of the patient's health. We said we judged 96 hours to be a reasonable amount of time to ensure that certified EHR technology is up to date and welcomed comment on if a shorter or longer time is advantageous. We did receive comments on the time frame and have revised it as discussed below in the comment and response section.

Comment: We received comments recommending that "access" be clarified to determine whether this is online access as indicated in the ONC certification criteria for certified EHR technology or just electronic access.

Response: We believe we inadvertently created confusion by listing the examples of electronic media (CD or USB drive) in which this access could be provided. As many commenters inferred, it was our intention that this be information that the patient could access on demand such as through a patient portal or PHR. We did not intend for this to be another objective for providing an electronic copy of health information upon request.

Comment: Several commenters requested that all objectives included in the health care policy priority "engage patients and their families" be combined, as they are redundant.

Response: We disagree that they are redundant and believe each serves a unique purpose. We regret any confusion created by the inclusion of CD or USB drive as examples of electronic media caused in the intent of this measure. The difference between electronic access and an electronic copy is that a patient with electronic access can access the information on demand at anytime while a patient must affirmatively request an electronic copy from the EP, eligible hospital or $\mathrm{CAH}$ at a specific time and the information in the copy is current only as of the time that the copy is transferred from the provider to the patient.

Comment: Some commenters asserted that some results and other sensitive information are best communicated at a face-to-face encounter.

Response: We agree that there may be situations where a provider may decide that electronic access of a portal or Personal Health Record is not the best forum to communicate results. Within the confines of laws governing patient access to their medical records, we would defer to EP's, eligible hospital or CAH's judgment as to whether to hold information back in anticipation of an actual encounter between the provider and the patient. Furthermore just as in the provision of electronic copy, an EP may withhold information from being 
accessible electronically by the patient in accordance with regulations at 45 CFR 164.524. Any such withholding would not affect the EP's, eligible hospital's or CAH's ability to meet this objective as that information would not be included. We do not believe there would be a circumstance where all information about an encounter would be withheld from the patient and therefore no information would be eligible for uploading for electronic access. If nothing else, the information that the encounter occurred can be provided. Please note that providers must comply with all applicable requirements under the HIPAA Privacy Rule, including 45 CFR 164.524.

Comment: We received several comments stating that the time frame of 96 hours is too burdensome for EPs.

Response: While we believe that 96 hours is sufficient, most EPs do not operate $24 / 7$. Therefore, we will limit the timeframe to business days, in effect changing the timeframe from 96 hours in the proposed rule to four business days. Business days are defined as Monday through Friday excluding federal or state holidays on which the EP, eligible hospital or CAH or their respective administrative staffs are unavailable.

Comment: Commenters pointed out that allergies is inconsistent with other objectives of Stage 1 and with the capabilities mandated by certification under the ONC final rule.

Response: As we have stated on several other objectives, we encourage all EPs, eligible hospitals, and CAHs to work with their EHR technology designers to make capabilities as relevant to their individual practices of care as possible. However, we maintain that at a minimum the capabilities that are part of certification should be included in certified EHR technology so we do modify this objective to medication allergies to align it with other objectives and certification.

After consideration of the public comments received, we are modifying the objective for EPs at $\S 495.6(d)(6)(i)$ of our regulations to "Provide patients with timely electronic access to their health information (including lab results, problem list, medication lists, medication allergies) within four business days of the information being available to the EP".

NPRM EP Measure: At least 10 percent of all unique patients seen by the EP are provided timely electronic access to their health information.

In the proposed rule, we said that we recognize that many patients may not have internet access, may not be able or interested to use a patient portal. Health systems that have actively promoted such technologies have been able to achieve active use by over 30 percent of their patients, but this may not be realistic for many practices in the short term. We received comments on this justification for the threshold and requests for clarification, which are addressed in the comment and response section below.

Comment: Some commenters expressed concern about the calculation of the percentage and expressed the preference to use an absolute count instead of a percentage.

Response: We acknowledge there are unique concerns about calculating this percentage as it involves determining the timeliness of the information.

Certified EHR technology would be able to ascertain the time from when the information was entered into its system to when the information was available for electronic access. As certified EHR technology can provide the access, any perceivable delay or requirement for affirmative action would be built in by the user to allow for review of the information before posting. Certified EHR technology could not be distinguish the difference in time when the information was available to the provider and when it was entered into certified EHR technology. However, we see no reasonable way to track this time frame that does not impose a heavy burden on the EP. Therefore, for the measure, we define the four business days time frame as the time frame when the information is updated in the certified EHR technology to when it is available electronically to the patient, unless the provider indicates that the information should be withheld. It is acceptable for a provider to set an automated withhold on certain information at their discretion. As we have discussed previously in this section, we do not believe absolute counts are an adequate substitute for percentage calculations.

Comment: We received comments requesting clarification on what data must be made available.

Response: Certified EHR technology must be able to make certain data available according to the ONC final rule. At a minimum, the data specified in the ONC final rule at 45 CFR $170.304(\mathrm{~g})$ must be available subject to the ability of the provider to withhold it discussed previously.

Comment: Commenters suggested that some EPs might not have 10 percent of their patient population who desire or could utilize such access.

Response: We agree that this is a possibility. We stated in the proposed rule that "we recognize that many patients may not have internet access, may not be able or interested in the use of a patient portal." Health systems that have actively promoted such technologies have been able to achieve active use by over 30 percent of their patients. However, this 30 percent threshold may not be realistic for many practices in the short term and therefore serves justification for the 10 percent threshold. However, the objective and measure focus on the availability of the access and the timeliness of the data in it, not its utilization. Therefore, we focus on the fact that more than 10 percent of unique patients seen during the EHR reporting period could access it and that the information is timely.

The EP is not responsible for ensuring that 10 percent request access or have the means to access. However, we encourage EPs to make the availability of electronic access known to their patients.

Comment: A commenter inquired about the provider's liability versus the EHR technology vendor for a security breach of the system.

Response: Depending on the facts surround the security breach, the provider may be liable for a violation under the HIPAA Privacy and Security Rules, as well as under any other applicable federal or state laws. Additionally, there may be circumstances where the EHR technology vendor acted as a business associate and may potentially have liability under the HIPAA Privacy and Security Rules. The issue of business associate liability under the HIPAA Privacy and Security Rules will be addressed in upcoming rulemaking.

After consideration of the public comments received, we are modifying the meaningful use measure for EPs at $\S 495.6(\mathrm{~d})(6)(\mathrm{ii})$ of our regulations to "At least 10 percent of all unique patients seen by the EP are provided timely (available to the patient within four business days of being updated in the certified EHR technology) electronic access to their health information subject to the EP's discretion to withhold certain information".

We further specify that in order to meet this objective and measure, an EP, eligible hospital, or CAH must use the capabilities Certified EHR Technology includes as specified and standards at 45 CFR $170.304(\mathrm{~g})$. The ability to calculate the measure is included in certified EHR technology.

To calculate the percentage, CMS and ONC have worked together to define the following for this objective:

- Denominator: Number of unique patients seen by the EP during the EHR 
reporting period. A unique patient is discussed under the objective of CPOE.

- Numerator: The number of patients in the denominator who have timely (available to the patient within four business days of being updated in the certified EHR technology) electronic access to their health information online.

- Threshold: The resulting percentage must be at least 10 percent in order for an EP to meet this measure.

As addressed in other objectives and in comment response, if an EP neither orders nor creates any of the information listed in the ONC final rule 45 CFR 170.304(g) and therefore included in the minimum data for this objective during the EHR reporting period they would be excluded from this requirement as described previously in this section under our discussion of whether certain EP, eligible hospital or CAH can meet all Stage 1 meaningful use objectives given established scopes of practices.

NPRM EP Objective: Provide clinical summaries for patients for each office visit.

In the proposed rule, we discussed why we were basing the objective on office visits rather than encounters. We said that we did want encounter to be construed to mean every time a provider interacts with the patient. We received comments requesting that we further define office visit and address those in the comment and response section below. In discussing the measure in the proposed rule, we also said that the clinical summary can be provided through a PHR, patient portal on the web site, secure email, electronic media such as CD or USB fob, or printed copy. The after-visit clinical summary contains an updated medication list, laboratory and other diagnostic test orders, procedures and other instructions based on clinical discussions that took place during the office visit.

Comment: We received requests for clarification as to what constitutes an "office visit".

Response: An office visit is defined as any billable visit that includes: (1) Concurrent care or transfer of care visits, (2) Consultant visits and (3) Prolonged Physician Service without Direct (FaceTo-Face) Patient Contact (tele-health). A consultant visit occurs when a provider is asked to render an expert opinion/ service for a specific condition or problem by a referring provider.

Comment: Some commenters believed the requirement for the provision of a clinical summary at an office visit should be linked to the type or purpose of the office visit. Samples of the suggested visits are-

—Level 4 or level 5 evaluation and management services;

- Visits conducted at the conclusion of an episode of care;

—Visits conducted at each transition of care;

—Visits relevant to specific conditions such as asthma; and

-Provider to patient face-to-face visits. Response: We believe that a clinical summary should be provided at all office visits included in the definition of office visit as defined in this final rule. We believe all of the office visits described in our definition result in the EP rendering a clinical judgment that should be communicated to the patient.

Comment: Commenters requested CMS define "clinical summary" and offered several specific data elements that should be included in the definition such as patient name, provider name, date of visit, location of visit, reason for visit, updated medication list, laboratory orders, diagnostic orders, patient instructions based on discussions with the provider and a nutrition care management plan.

Response: After reviewing the comments we define clinical summary as an after-visit summary that provides a patient with relevant and actionable information and instructions containing, but not limited to, the patient name, provider's office contact information, date and location of visit, an updated medication list and summary of current medications, updated vitals, reason(s) for visit, procedures and other instructions based on clinical discussions that took place during the office visit, any updates to a problem list, immunizations or medications administered during visit, summary of topics covered/considered during visit, time and location of next appointment/ testing if scheduled, or a recommended appointment time if not scheduled, list of other appointments and testing patient needs to schedule with contact information, recommended patient decision aids, laboratory and other diagnostic test orders, test/laboratory results (if received before 24 hours after visit), and symptoms.

Comment: Commenters pointed out that the HIPAA Privacy Rule permits licensed healthcare professionals to withhold certain information if its disclosure would cause substantial harm to the patient or another individual.

Response: As the EP is proactively providing this information to the patient, 45 CFR 164.524 of the HIPAA Privacy rule does not apply to this situation. However, we still believe that an EP should be able to withhold information if its disclosure would cause substantial harm to the patient or another individual. Therefore, if in their judgment substantial harm may arise from the disclosure of particular information, an EP may choose to withhold that particular information from the clinical summary

Comment: Most commenters noted that other than "at the time of the visit", there was no specific time period given in which to comply with this objective. If CMS intended "at the time of the visit" to mean before the patient leaves the building or upon the patient's request, neither are possible due to workflow and review processes. Most commenters assumed we would associate the 48 hours related to the 'copy' requirement or the 96 hours related to the 'access' requirement to address this comment and stated that both were too short a period for a clinical visit summary. Others recommended the 30-day timeframe for the provision information set forth under the HIPAA Privacy Rule.

Response: We agree that our proposed objective lacked specificity about the time to comply. To provide such specificity, we adopt the timeframe of three business days from our objective of providing electronic health information to the patient. That is three business days following the day of the visit excluding holidays as described in the providing electronic health information to the patient objective.

Comment: Several commenters requested changes to the media through which this information could be provided. Differing commenters recommended eliminating the paper option, while others recommended only the paper option.

Response: We believe that more options give the EP needed flexibility. The EP could choose any of the listed means from the proposed rule of PHR, patient portal on a Web site, secure email, electronic media such as CD or USB fob, or printed copy. If the EP chooses an electronic media, they would be required to provide the patient a paper copy upon request. Both forms can be and should be produced by certified EHR technology.

Comment: Several commenters indicated that a provider should be allowed to charge a fee for providing the copy.

Response: As this is a proactive requirement on the part of the $\mathrm{EP}$ and not a response to a request from the patient, we do not believe it is appropriate to charge the patient a fee for this copy. We note that we give the EP considerable flexibility in the 
manner in which the copy is provided including the provision of a paper copy. The only accommodation an EP is required to make is the provision of a paper copy that can be automatically generated certified EHR technology. We therefore believe that costs of this will be negligible.

Comment: A number of commenters expressed concern regarding whether the current available technology could produce a summary of the required information in a standardized format, the use of clinical nomenclature rather than lay terms and the fact that some providers use multiple modules to document the care of the patient.

Response: We believe it is appropriate to leave the design of EHR technology systems and their outputs to the system developers and the EHR technology users. However, we note that the capability to meet this objective is included in the ONC final rule at 45 CFR 170.304(h) as a criteria for certified EHR technology and we are confident that vendors will be able to produce certified EHR technologies.

After consideration of the public comments received, we are finalizing the objective for EPs at $\S 495.6(d)(13)(i)$ of our regulations as proposed.

We include this objective in the core set as it is integral to involving patients and their families in their provision of care and was recommended by the HIT Policy Committee for inclusion in the core set.

NPRM EP Measure: Clinical summaries provided to patients for at least 80 percent of all office visits.

Comment: Some commenters believed the threshold was too high or should be replaced with a numerical count or attestation.

Response: We reduce the threshold to over 50 percent as this objective meets the criteria of relying solely on a capability included as part of certified EHR technology and is not, for purposes of Stage 1 criteria, reliant on the electronic exchange of information. Also, as this is a relatively new capability that was not available to either providers or patients before the introduction of EHRs, we do not believe it meets the same standard of practice as maintaining an up-to-date problem list and therefore adopt a threshold of 50 percent (rather than 80 percent).

After consideration of the public comments received, we are modifying the meaningful use measure for EPs at $\S 495.6(d)(13)(i i)$ of our regulation to "Clinical summaries provided to patients for more than 50 percent of all office visits within 3 business days".

We further specify that in order to meet this objective and measure, an EP, eligible hospital, or CAH must use the capabilities Certified EHR Technology includes as specified and standards at 45 CFR 170.304(h). The ability to calculate the measure is included in certified EHR technology.

As with the previous objective, the provision of the clinical summary is limited to the information contained within certified EHR technology; therefore this measure is by definition limited to patients whose records are maintained using certified EHR technology as described previously in this section under our discussion of the burden created by the measures associated with the Stage 1 meaningful use objectives.

To calculate the percentage, CMS and ONC have worked together to define the following for this objective:

- Denominator: Number of unique patients seen by the EP for an office during the EHR reporting period. A unique patient is discussed under the objective of using CPOE.

- Numerator: Number of patients in the denominator who are provided a clinical summary of their visit within three business days.

- Threshold: The resulting percentage must be more than 50 percent in order for an EP, eligible hospital, or CAH to meet this measure.

As addressed in other objectives, EPs who have no office visits during the EHR reporting period would be excluded from this requirement as described previously in this section under our discussion of whether certain EP, eligible hospital or CAH can meet all Stage 1 meaningful use objectives given established scopes of practices.

NPRM EP/Eligible Hospital Objective: "Provide access to patient-specific education resources upon request."

In the proposed rule, we discussed this objective, but did not propose it. We stated that there was a paucity of knowledge resources that are integrated with EHR, and that also are widely available. We also noted that the ability to provide education resources in multiple languages might be limited. We stated our intent to further explore the objective in subsequent stages of meaningful use.

Comment: We received many comments, including comments from both the HIT Policy Committee and MedPAC, to include this measure in the final rule. These commenters disagreed with our assertion in the proposed rule that "there is currently a paucity of knowledge resources that are integrated within EHRs, that are widely available, and that meet these criteria, particularly in multiple languages." Specific examples of the availability of knowledge resources integrated with current EHRs were provided. The HIT Policy Committee amended their recommendation in their comments on the proposed rule to:

-EPs and hospitals should report on the percentage of patients for whom they use the EHR to suggest patientspecific education resources.

Other recommended language for the objective includes:

-Provide patients educational information that is specific to their health needs as identified by information contained in their EHR technology such as diagnoses and demographic data, and

- The original HIT Policy Committee objective of "Provide access to patientspecific education resources upon request."

Response: We are convinced by commenters that the availability of education resources linked to EHRs is more widely available than we had indicated in the proposed rule. Therefore, for the final rule we will include this objective for the Stage 1 of meaningful use. We note that the new recommendation of the HIT Policy

Committee is a hybrid of a measure and an objective, whereas in developing the meaningful use criteria we consistently identify both an objective and associated measure. However, we agree with the HIT Policy Committee and others that the objective and associated measure should make clear that the EP, eligible hospital or CAH should utilize certified EHR technology in a manner where the technology suggests patientspecific educational resources based on the information stored in the certified EHR technology. Therefore, we are including a revised version of this objective in the final rule for Stage 1 of meaningful use.

We also believe it is necessary to state what level of EP, eligible hospital and $\mathrm{CAH}$ discretion is available when deciding whether to provide education resources identified by certified EHR technology to the patient. Therefore, we include the phrase "if appropriate", which allows the EP or the authorized provider in the eligible hospital or $\mathrm{CAH}$ final decision on whether the education resource is useful and relevant to a specific patient.

After consideration of the public comments received, we are including this meaningful use objective for EPs at $\S 495.6(e)(6)(i)$ and eligible hospitals and CAHs at $\S 495.6(\mathrm{~g})(5)(\mathrm{i})$ of our regulations as "Use certified EHR technology to identify patient-specific education resources and provide those resources to the patient if appropriate". 
NPRM EP/Eligible Hospital Measure: Not applicable.

Comment: CMS received a comment requesting an 80 percent threshold of appropriate patients and/or caregivers receiving patient-specific educational materials. In addition, the HIT Policy Committee's revised objective suggests a patient based percentage.

Response: As with the addition of the recording of advance directives, we are able to relate this measure to one that is based on patients and can be accomplished solely using certified EHR technology. As this objective requires more than just the recording of information in certified EHR technology, we adopt a lower threshold of 10 percent.

After consideration of the public comments received, we are including this meaningful use measure for EPs at § 495.6(e)(6)(ii) and eligible hospitals at $\S 495.6$ (g)(5)(ii) of our regulations as "More than 10 percent of all unique patients seen by the EP or admitted to the eligible hospital's or CAH's inpatient or emergency department (POS 21 or 23) are provided patientspecific education resources".

We further specify that in order to meet this objective and measure, an EP, eligible hospital, or CAH must use the capabilities Certified EHR Technology includes as specified and standards at 45 CFR 170.302(m). The ability to calculate the measure is included in certified EHR technology.

To calculate the percentage, CMS and ONC have worked together to define the following for this objective:

- Denominator: Number of unique patients seen by the EP or admitted to the eligible hospital's or CAH's inpatient or emergency department (POS 21 or 23) during the EHR reporting period. A unique patient is discussed under the CPOE objective.

- Numerator: Number of patients in the denominator who are provided patient education specific resources.

- Threshold: The resulting percentage must be more than 10 percent in order for an EP, eligible hospital, or CAH to meet this measure.

We do not believe that any EP, eligible hospital, or CAH will not have more than 10 percent of their patients eligible to receive patient specific education resources and therefore do not believe an exclusion is necessary for this objective.

The third health outcomes policy priority identified by the HIT Policy Committee is to improve care coordination. The HIT Policy Committee recommended the following care goals to address this priority:
- Exchange meaningful clinical information among professional health care team

NPRM EP Objective: Capability to exchange key clinical information (for example, problem list, medication list, allergies, and diagnostic test results), among providers of care and patient authorized entities electronically.

NPRM Eligible Hospital Objective: Capability to exchange key clinical information (for example, discharge summary, procedures, problem list, medication list, allergies, diagnostic test results), among providers of care and patient authorized entities electronically.

In the proposed rule, we defined the term "diagnostic test results" as all data needed to diagnose and treat disease, such as blood tests, microbiology, urinalysis, pathology tests, radiology, cardiac imaging, nuclear medicine tests, and pulmonary function tests. We maintain this description for the final rule. We said that when the information was available in a structured format we expected that it be transferred in a structured format. However, if it was unavailable in a structured format, that the transmission of unstructured data was permissible. We provide additional information on structured data in the comment and response section, but maintain for the final rule the concept that the exchange can be of structured or unstructured data.

Comment: Commenters requested clarification of the term "key clinical information."

Response: By "clinical information", we mean all data needed to diagnose and treat disease, such as blood tests, microbiology, urinalysis, pathology tests, radiology, cardiac imaging, nuclear medicine tests, and pulmonary function tests. We leave it to the provider's clinical judgment as to identifying what clinical information is considered key clinical information for purposes of exchanging clinical information about a patient at a particular time with other providers of care. The examples we provided in the proposed rule and the final rule below are not intended to be exhaustive. ONC in their final rule provides a minimum set of information that certified EHR technology must be able to exchange in order to be certified. A provider's determination of key clinical information could include some or all of this information as well as information not included in the ONC final rule at 45 CFR 170.304(i) for EPs and 45 CFR 170.306(f) for eligible hospitals and CAHs.
Comment: Commenters requested clarification of the term "patient authorized entities."

Response: By "patient authorized entities", we mean any individual or organization to which the patient has granted access to their clinical information. Examples would include an insurance company that covers the patient, an entity facilitating health information exchange among providers or a personal health record vendor identified by the patient. A patient would have to affirmatively grant access to these entities.

Comment: Commenters requested clarification of the term "exchange."

Response: We expect that this information, when exchanged electronically, would be exchanged in structured electronic format when available (for example, drug and clinical lab data). However, where the information is available only in unstructured electronic formats (for example, free text and scanned images), we would allow the exchange of unstructured information. We believe that the electronic exchange of information is most efficient when it is exchanged from a provider's certified EHR technology to another certified EHR technology either directly or through an entity facilitating health information exchange using structured data that can be automatically identified by the receiving system and integrated into the receiver's records. However, we know that much information cannot currently be, and may never be, transmitted in the way we just described.

Comment: Commenters requested clarification of the term "structured data."

Response: This distinction between structured data and unstructured data applies to all types of information. We have previously defined structured data in this section. To ensure that certified EHR technology has a certain level of functionality, ONC at 45 CFR 170.304(i) for EPs and 45 CFR 170.306(f) for eligible hospitals and CAHs specified certain types of information that a certified EHR technology must be able to exchange to become certified. ONC also provided standards to support this exchange. These standards do not preclude a vendor of EHR technology from enabling its product to exchange additional types of information nor limit the provider's discretion (either in exchanging more or less) in deciding what information is key and should be exchanged about a given patient at a given time.

Comment: Commenters expressed concern that the exchange of key 
clinical information via certified EHR systems requires a unique or national patient identifier to ensure accurate exchange.

Response: While such an identifier could facilitate an exchange, it need only be unique to the parties involved in the exchange and need not be national in scope, nor is a specific unique identifier necessary for successful exchanges. Many current health information exchanges have had success identifying patients by a combination of several elements of information without a separate independent identifier.

Comment: Commenters pointed out that the general term "allergies" is inconsistent with other objectives of Stage 1 and with the capabilities mandated by certification under the ONC final rule, which uses the term "medication allergies".

Response: As we have stated on several other objectives, we encourage all EPs, eligible hospitals, and CAHs to work with their certified EHR technology designers to make capabilities most relevant to their individual practices of care. However, we have maintained that at a minimum the capabilities that are part of certification should be included so we modify the example to change allergies to medication allergies to align it with other objectives and certification.

After consideration of the public comments received, we are modifying the meaningful use objective for EPs at $\S 495.6(d)(14)(i)$ of our regulations to "Capability to exchange key clinical information (for example, problem list, medication list, medication allergies, and diagnostic test results), among providers of care and patient authorized entities electronically" and for eligible hospitals and CAHs at $\S 495.6(f)(13)(i)$ to "Capability to exchange key clinical information (for example, discharge summary, procedures, problem list, medication list, medication allergies, diagnostic test results), among providers of care and patient authorized entities electronically".

In response to our revised requirements for meeting meaningful use, we included this objective in the core set. Section 1848 (o)(2)(A)(ii) of the Act specifically includes electronic exchange of health information in meaningful use for eligible professionals.

NPRM EP/Eligible Hospital Measure:

Performed at least one test of certified EHR technology's capacity to electronically exchange key clinical information.

In the proposed rule, we identified this objective as reliant on the electronic exchange of information. We said that we are aware that in most areas of the country, the infrastructure necessary to support such exchange is still being developed. Therefore, for the Stage 1 criteria of meaningful use we proposed that EPs and eligible hospitals test their ability to send such information at least once prior to the end of the EHR reporting period. We proposed that the testing could occur prior to the beginning of the EHR reporting period. We also said that if multiple EPs are using the same certified EHR technology in a shared physical setting, the testing would only have to occur once for a given certified EHR technology, as we do not see any value to running the same test multiple times just because multiple EPs use the same certified EHR technology. Finally, we attempted to define an "exchange" as the clinical information must be sent between different clinical entities with distinct certified EHR technology and not between organizations that share a certified EHR. We received many comments requesting further clarification on these concepts and we attempt to provide additional information in the comment and response section below.

Comment: Commenters expressed concern that the receiving entities are not required to have the same capabilities as meaningful users of certified EHR technology.

Response: The HITECH Act does not provide us the authority to require any entity (medical provider or otherwise) to conform to certain standards and criteria unless they seek to become a meaningful EHR user. The Act also limits the entities that are eligible to become meaningful EHR users. In developing the associated measure for this objective, we have ensured that eligible providers will be able to meet this objective as long as there is one other entity with which they can test their capability. As electronic exchange is not constrained by distance, we are confident that every provider seeking to test their system will be able to find another entity with which to conduct such test.

Comment: Commenters asked whether the test needs to be "live" or if it could be a "simulation."

Response: As specified in the proposed rule, this test must involve the actual submission of information to another provider of care with distinct certified EHR technology or other system capable of receiving the information.

Comment: Commenters asked whether the use of "test" or "dummy" data is permissible.
Response: While the use of test patient information may increase the risk that the system will not be testing to its full capability, given the privacy and security concerns surrounding the transmission of actual patient

information we do not require it for the purposes of a test. Therefore, the use of test information about a fictional patient that would be identical in form to what would be sent about an actual patient would satisfy this objective.

Comment: Commenters suggested deferring the measure to a later stage due to the lack of a mature HIE infrastructure and/or to emulate the Health Information and Management System Society (HIMSS) EMR Adoption Model.

Response: We agree that many areas of the country currently lack the infrastructure to support the electronic exchange of information. As the goal of this meaningful use objective is to ensure that certified EHR technology has the capability to electronically exchange key clinical information, we only require a single test.

After consideration of the public comments received, we are finalizing the meaningful use measure at $\S 495.6(d)(14)(i i)$ and $\S 495.6(f)(13)(i i)$ of our regulations as proposed.

We further specify that in order to meet this objective and measure, an EP, eligible hospital, or CAH must use the capabilities Certified EHR Technology includes as specified and standards at 45 CFR 170.304(i) for EPs and 45 CFR 170.306(f) for eligible hospitals and CAHs. The ability to calculate the measure is included in certified EHR technology. EPs, eligible hospitals, and CAHs should attempt to identify one other entity with whom to conduct a test of the submission of electronic data. This test must include the transfer of either actual or "dummy" data to the chosen other entity. The testing could occur prior to the beginning of the EHR reporting period, but must occur prior to the end of the EHR reporting period and every payment year would require its own, unique test as infrastructure for health information exchange is expected to mature over time. Therefore, if an eligible hospital or CAH were to become a meaningful EHR user in 2011 for their first payment year, they would have to conduct another, unique test to become a meaningful EHR user in 2012 for their second payment year. If multiple EPs are using the same certified EHR technology in a shared physical setting, the testing would only have to occur once for a given certified EHR

technology, as we do not see any value to running the same test multiple times just because multiple EPs use the same 
certified EHR technology. To be considered an "exchange" for this objective and measure the clinical information must be sent between different legal entities with distinct certified EHR technology or other system that can accept the information and not between organizations that share certified EHR technology. CMS will accept a yes/no attestation to verify all of the above for EPs, eligible

hospitals, and CAHs.

As the measure already accounts for the possibility of a failed test and we are confident that everyone will be identify an entity with which to conduct a test, we do not believe an exception is required for EPs, eligible hospitals or CAHs.

NPRM EP/Eligible Hospital Objective: Perform medication reconciliation at relevant encounters and each transition of care.

In the proposed rule, we described "medication reconciliation" as the process of identifying the most accurate list of all medications that the patient is taking, including name, dosage, frequency and route, by comparing the medical record to an external list of medications obtained from a patient, hospital or other provider. We maintain this description for the final rule. We also described "relevant encounter" and "transition of care"; however, as we received comments requested additional clarification of these terms we address them in the comment and response section below.

Comment: Several commenters requested that this objective be deferred until it can be conducted using the exchange of electronic information between certified EHR technology. Other commenters believed that the process is not one for avoiding medication errors, but a human workflow process supported by the EHR, and not an automated EHR process.

Response: We certainly look forward to a time when most medication reconciliation occurs as an automated process within the EHR reconciling information that has been exchanged. However, it is unlikely that an automated process within the EHR will fully supplant the medication reconciliation conducted between the provider and the patient. In order for this automated reconciliation process to occur and be useful, the relevant structured data exchanged needs to be as accurate as possible. Requiring medication reconciliation as part of meaningful use in Stage 1 lays the groundwork for future reliable electronic exchange. We therefore do not believe this objective should be deferred to a later stage.

Comment: Commenters requested additional clarity of the term "relevant encounter." Only a few suggestions on such clarity were provided by commenters. Two examples of commenters' recommendations are "when a prescription is generated" and "a significant change in the patient's condition that resulted in change in medication regimen which could include significant change in dosing of more than 1 medication, identification of a new medical condition, decline in functional status or change in advanced directive."

Response: We finalize our proposal by defining "relevant encounter" as an encounter during which the EP, eligible hospital or CAH performs a medication reconciliation due to new medication or long gaps in time between patient encounters or for other reasons determined appropriate by the EP, eligible hospital or CAH. Essentially an encounter is relevant if the EP, eligible hospital, or CAH judges it to be so. This flexibility has implications for the measure that were not fully considered in the proposed rule. We will discuss those below in connection with our discussion of the associated measure.

Comment: Commenters requested additional clarity of the term "transition of care." A few suggestions were provided by commenters including expanding the description to include all transfers to different settings within a hospital or revising the definition to "the movement of a patient from one setting of care (hospital, ambulatory primary care practice, ambulatory specialty care practice, long-term care, home health, rehabilitation facility) to another".

Response: In the proposed rule we clarified "transition of care" as the transfer of a patient from one clinical setting (inpatient, outpatient, physician office, home health, rehab, long-term care facility, etc.) to another or from one $\mathrm{EP}$, eligible hospital, or CAH (as defined by CCN) to another. We believe that different settings within one hospital using certified EHR technology would have access to the same information so reconciliation would not be necessary. We modify our clarification to account for some of the revisions provided. We clarify "transition of care" as the movement of a patient from one setting of care (hospital, ambulatory primary care practice, ambulatory specialty care practice, long-term care, home health, rehabilitation facility) to another. We also clarify that the receiving eligible hospital or EP would conduct the medication reconciliation.
Comment: Some commenters requested clarification on which EP, eligible hospital or $\mathrm{CAH}$ would conduct the medication reconciliation. The one to whom the patient is transferred or the one who transfers the patient.

Response: When conducting medication reconciliation during a transfer of care, we believe that it is the $\mathrm{EP}$, eligible hospital or CAH that receives the patient into their care that should conduct the medication reconciliation. It is for this provider that the information is most crucial, as they will be making the future clinical judgments regarding the patient. Therefore, we revise this objective and its associated measure to reflect this clarification.

Comment: Commenters requested a standard list be defined for the process including prescription and non prescription medications, herbal products, dietary supplements, prescriber, drug name, regimen and allergies.

Response: We believe the information included in the process of medication reconciliation is appropriately determined by the provider and patient.

After consideration of the public comments received, we are modifying the meaningful use objective for EPs at $\S 495.6(\mathrm{e})(7)(\mathrm{i})$ and for eligible hospitals and CAHs at $\S 495.6(\mathrm{~g})(6)(\mathrm{i})$ of our regulations to "The EP, eligible hospital or $\mathrm{CAH}$ who receives a patient from another setting of care or provider of care or believes an encounter is relevant should perform medication reconciliation".

NPRM EP/Eligible Hospital Measure: Perform medication reconciliation for at least 80 percent of relevant encounters and transitions of care.

Comment: Commenters believed it was an unjustifiable burden to record which encounters were relevant and which were not given our flexible definition of "relevant encounter".

Response: We agree that the inclusion of relevant encounters creates a burden that one commenter described as "nonvalue-added work". We also believe that when the EP, eligible hospital, or $\mathrm{CAH}$ identifies the encounter as relevant, it is unlikely that the EP, eligible hospital, or CAH would then not carry out the medication reconciliation. For these reasons, we are removing relevant encounters from the measure for this objective.

Comment: Commenters said the percent measurements should be replaced with a numerical count or an attestation the objective has been met or the demonstration of the capability by performing one test of certified EHR technology's capacity to present 
providers with patient medication information that supports the reconciliation of medications at time of admission and discharge. Other commenters stated the proposed 80 percent threshold was too high.

Response: We are maintaining a percentage for the reasons discussed previously in this section. However, we do reduce the threshold to over 50 percent as this objective meets the criteria of relying solely on a capability included as part of certified EHR technology and while not absolutely reliant on electronic exchange of information, it does involve the exchange of information between providers and therefore we adopt a threshold of 50 percent (rather than 8 percent).

Comment: Commenters requested we align this objective with The Joint Commission National Patient Safety Goal on medication reconciliation (Goal 8 ) in order to decrease confusion, prevent the slowing of adoption of best practices and match current hospital reconciliation processes.

Response: CMS understands the commenters' concerns regarding possible confusion if the meaningful use medication reconciliation requirement differs from The Joint Commission's requirement for those facilities accredited by that organization. However, currently there is no finalized Joint Commission standard as the Commission is currently in the process of re-evaluating their National Patient Safety Goal 8 (Accurately and completely reconcile medications across the continuum of care) given the difficulties that many organizations are having in meeting the complex requirements. In the absence of a definitive Joint Commission standard to take into consideration, this is not possible.

Comment: Some commenters expressed the desire to expand the scope of the measure to include the clinical decision making and patient counseling and education by a pharmacist.

Response: We believe that is both beyond the scope of meaningful use as pharmacists are not eligible professionals for the EHR incentive programs and that the provision of patient counseling is more aligned with the objectives of clinical quality measures. Information from the medication reconciliation could be used for the basis of clinical decision support rules, but is not in and of itself a clinical decision.

After consideration of the public comments received, we are modifying the meaningful use measure for EPs at
§ 495.6(e)(7)(ii) and for eligible hospitals and CAHs at $\S 495.6(\mathrm{~g})(6)$ (ii) of our regulations to "The EP, eligible hospital or $\mathrm{CAH}$ performs medication reconciliation for more than 50 percent of transitions of care in which the patient is transitioned into the care of the EP or admitted to the eligible hospital's or CAH's inpatient or emergency department (POS 21 or 23)”.

We further specify that in order to meet this objective and measure, an EP, eligible hospital, or CAH must use the capabilities Certified EHR Technology includes as specified and standards at 45 CFR 170.302(j). The ability to calculate the measure is included in certified EHR technology.

As discussed previously in this section under our discussion of the burden created by the measures associated with the Stage 1 meaningful use objectives, we only include in the denominator transitions of care related to patients whose records are maintained using certified EHR technology. To calculate the percentage, CMS and ONC have worked together to define the following for this objective:

- Denominator: Number of transitions of care during the EHR reporting period for which the EP or eligible hospital's or CAH's inpatient or emergency department (POS 21 to 23) was the receiving party of the transition.

- Numerator: The number of transitions of care in the denominator where medication reconciliation was performed.

- Threshold: The resulting percentage must be more than 50 percent in order for an EP, eligible hospital, or CAH to meet this measure. If an EP was not on the receiving end of any transition of care during the EHR reporting period they would be excluded as previously discussed in this section under our discussion of whether certain EP, eligible hospital or CAH can meet all Stage 1 meaningful use objectives given established scopes of practices. We do not believe that any eligible hospital or CAH would be in a situation where they would not need to know the precise medications their patients are taking.

NPRM EP/Eligible Hospital Objective: Provide summary care record for each transition of care or referral.

In the proposed rule, we pointed out that this objective was not explicitly included in the HIT Policy Committee's recommended objectives, but that they did include a measure for the "percent of transitions in care for which summary care record is shared." We said that we believe that in order for a measure to be relevant it must correspond to an objective in the definition of meaningful use. Therefore, we proposed to add this objective in order to be able to include the recommended measure. Furthermore, we add referrals because the sharing of the patient care summary from one provider to another communicates important information that the patient may not have been able to provide, and can significantly improve the quality and safety of referral care, and reduce unnecessary and redundant testing. We received support for this inclusion from commenters and include this objective in the final rule for the reasons outlined in the proposed rule. We did receive comments requesting clarifications around this objective and address them in the comment and response section below.

Comment: We received several comments that requested clarification as to the purpose of this objective.

Response: The purpose of this objective is to ensure a summary of care record is provided to the receiving provider when a patient is transitioning to a new provider or has been referred to another provider while still remaining under the care of the referring provider. If the provider to whom the referral is made or to whom the patient is transitioned to has access to the medical record maintained by the referring provider then the summary of care record would not need to be provided. The most common example cited by commenters was a referral during which patient remains an inpatient of the hospital. Finally, unlike with medication reconciliation, where the receiving party of the transfer conducts the action, the transferring party would provide the summary care record to the receiving party.

Comment: Commenters requested additional clarity of the term "transition of care". A few suggestions were provided by the commenters including expanding the description to include all transfers to different settings within a hospital or revising the definition to "the movement of a patient from one setting of care (hospital, ambulatory primary care practice, ambulatory, specialty care practice, long-term care, home health, rehabilitation facility) to another".

Response: In the proposed rule we clarified that the term transition of care means a transfer of a patient from one clinical setting (inpatient, outpatient, physician office, home health, rehab, long-term care facility, etc.) to another or from one EP, eligible hospital, or $\mathrm{CAH}$ (as defined by CMS Certification Number (CCN) to another. We believe that different settings within a hospital using certified EHR technology would have access to the same information so 
providing a clinical care summary would not be necessary. We further clarify transition of care as the movement of a patient from one setting of care (hospital, ambulatory primary care practice, ambulatory, specialty care practice, long-term care, home health, rehabilitation facility) to another.

Comment: Some commenters requested clarification on which $\mathrm{EP}$, eligible hospital or CAH should provide the summary of care document; the one to whom the patient is transferred or referred or the one who transfers or refers the patient.

Response: We believe that it is the EP, eligible hospital or CAH that transfers or refers the patient to another setting of care or provider that should provide the summary of care document. It is for this provider that has the most recent information on the patient that may be crucial to the provider to whom the patient is transferred or referred. Therefore, we revise this objective and its associated measure to reflect this clarification.

Comment: Commenters asked for clarification on how the summary of care record should be transferred.

Response: The goal is to get the summary care record into the next provider's possession. While we highly encourage all EPs, eligible hospitals, and CAHs to explore ways to accomplish the transfer using electronic exchange, we realize that this capability is still in the development stages. Therefore, an EP, eligible hospital, or CAH could send an electronic or paper copy of the summary care record directly to the next provider or could provide it to the patient to deliver to the next provider, if the patient can reasonably expected to do so. Certified EHR technology would be used to generate the summary of care record and to document that it was provided to the patient or receiving provider.

After consideration of the public comments received, we are modifying the meaningful use objective for EPs at $\S 495.6(\mathrm{e})(8)(\mathrm{i})$ and for eligible hospitals and CAHs at $\S 495.6(\mathrm{~g})(7)(\mathrm{i})$ of our regulations to "The EP, eligible hospital or $\mathrm{CAH}$ who transitions their patient to another setting of care or provider of care or refers their patient to another provider of care should provide summary care record for each transition of care or referral".

NPRM EP/Eligible Hospital Measure: Provide summary of care record for at least 80 percent of transitions of care and referrals.

Comment: Commenters said that this should be replaced with a count and that the threshold was too high.
Response: We are maintaining a percentage for the reasons discussed previously in this section. However, we do reduce the threshold to over 50 percent as this objective meets the criteria of relying solely on a capability included as part of certified EHR technology and while not absolutely reliant on electronic exchange of information, it does involve the exchange of information between providers and therefore we adopt a threshold of 50 percent (rather than 80 percent).

Comment: There were concerns about the ability of certified EHR technology to calculate this measure. As long as an $\mathrm{EP}$, eligible hospital, or CAH records the order for a referral or transfer as structured data and a record is made that the summary care record was provided then certified EHR technology will be able to calculate this measure.

After consideration of the public comments received, we are modifying the meaningful use measure for EPs at $\S 495.6(\mathrm{e})(8)(\mathrm{ii})$ and for eligible hospitals and CAHs at $\S 495.6(\mathrm{~g})(7)$ (ii) of our regulations to "The EP, eligible hospital or CAH who transitions or refers their patient to another setting of care or provider of care provides a summary of care record for more than 50 percent of transitions of care and referrals".

We further specify that in order to meet this objective and measure, an EP, eligible hospital, or CAH must use the capabilities Certified EHR Technology included as specified and standards at 45 CFR 170.304(i) for EPs and 45 CFR 170.306(f) for eligible hospitals and CAHs. The ability to calculate the measure is included in certified EHR technology.

As discussed previously in this section under our discussion of the burden created by the measures associated with the Stage 1 meaningfu use objectives, we only include in the denominator transitions of care and referrals related to patients whose records that are maintained using certified EHR technology. To calculate the percentage, CMS and ONC have worked together to define the following for this objective:

- Denominator: Number of transitions of care and referrals during the EHR reporting period for which the EP or eligible hospital's or CAH's inpatient or emergency department (POS 21 to 23) was the transferring or referring provider.

- Numerator: The number of transitions of care and referrals in the denominator where a summary of care record was provided.

- Threshold: The percentage must be more than 50 percent in order for an EP, eligible hospital, or CAH to meet this measure.

As addressed in other objectives and in comment response, if an EP does not transfer a patient to another setting or refer a patient to another provider during the EHR reporting period then they would have a situation of a null denominator as described would be excluded from this requirement as described previously in this section under our discussion of whether certain EP, eligible hospital or CAH can meet all Stage 1 meaningful use objectives given established scopes of practices. We do not believe that any eligible hospital or $\mathrm{CAH}$ would be in a situation where they would never transfer a patient to another care setting or make a referral to another provider.

The fourth health outcomes policy priority identified by the HIT Policy Committee is improving population and public health. The HIT Policy Committee identified the following care goal to address this priority:

- The patient's health care team communicates with public health agencies.

The goal as recommended by the HIT Policy Committee is "communicate with public health agencies." In the proposed rule, we explained that we found this goal to be somewhat ambiguous, as it does not specify who must communicate with public health agencies. We propose to specify "the patient's health care team" as the individuals who would communicate with public health agencies.

NPRM EP/Eligible Hospital Objective: Capability to submit electronic data to immunization registries and actual submission where required and accepted.

In the proposed rule, we did not elaborate on this objective.

Comment: Some commenters suggested out that not every EP, eligible hospital, or CAH administers immunization. Therefore, as proposed, this objective and its associated measure would require an EP, eligible hospital, or CAH to implement and test a capability that they would not use.

Response: We acknowledge that this objective is not relevant to all EPs, eligible hospitals or CAHs. Therefore, in this final rule, we clarify that this objective and its associated measure apply only to EPs, eligible hospitals or CAHs that administer one or more immunizations during the EHR reporting period.

Comment: Some commenters recommended revising the language of the immunization objective to be consistent with the language of the syndromic surveillance objective by 
replacing "where required and accepted" with "according to applicable law and practice."

Response: First, we make a technical correction. The objective listed for EPs on page 1858 of the proposed rule listed this objective as "Capability to submit electronic data to immunization registries and actual submission where possible and accepted." The objective was intended to be "Capability to submit electronic data to immunization registries and actual submission where required and accepted" for EPs, eligible hospitals, and CAHs. It is written as such in every other instance in the proposed rule including the regulation text. Second, in response to the comment that "where required and accepted" be replaced with "according to applicable law and practice", we see little distinction between the two in terms of requirement as applicable law and practice would be the things imposing a requirement. Therefore, we adopt the proposed language, but modify the language slightly to "in accordance with applicable law and practice". We do note however, that applicable law and practice do not guarantee every receiving entity will be able to accept it electronically. Our measure for meeting this objective is one test of electronic data submission and if the test is successful follow up submission to that one entity. We do not seek to enforce through meaningful use every law and practice that may require submission of immunization data. We also make another consistency change to the objectives under the health care policy goal of improving population and public health. In this objective, we describe the capability as submitting electronic data. In the other objectives under this goal we describe the capability as providing electronic data. We believe that functionally these terms are interchangeable, but to avoid any confusion we adopt the same term of "submit" electronic data across all three objectives.

Comment: Some commenters suggested that the term "Immunization Information Systems (IIS)" has replaced the term "registry" and is referred to as such by the Centers for Disease Control (CDC).

Response: We modified the objective to account for both terms. After consideration of the public comments received, we are modifying the meaningful use objective for EPs at $\S 495.6(\mathrm{e})(9)(\mathrm{i})$ and for eligible hospitals and CAHs at $\S 495.6(\mathrm{~g})(8)(\mathrm{i})$ of our regulations to Capability to submit electronic data to immunization registries or Immunization Information Systems and actual submission in accordance with to applicable law and practice.

NPRM EP/Eligible Hospital Measure:

Performed at least one test of certified EHR technology's capacity to submit electronic data to immunization registries (unless none of the immunization registries to which the EP, eligible hospital, or CAH submits such information have the capacity to receive the information electronically)

In the proposed rule, we identified

this as an objective where more stringent requirements may be established for EPs and hospitals under the Medicaid program in states where this capability exists. This is just one example of a possible State proposed modification to meaningful use in the Medicaid EHR incentive program. This ability for the States is also included in our final rule.

Comment: As with the objective of exchanging key clinical information, some commenters asked whether the test needs to be "live" or if it could be a "simulation". Some commenters suggested that a simulation where the ability was tested without being transmitted to another party should be sufficient. Others suggested that the test needs to include transmission or difficulties in actual sending information might not be uncovered.

Response: As specified in the proposed rule, this test must involve the actual submission of information to a registry or immunization information system, if one exists that will accept the information.

Comment: Commenters asked whether the use of "test" or "dummy" data is permissible.

Response: While the use of test patient information may increase the risk that the system will not be testing to its full capability, given the privacy and security concerns surrounding the transmission of actual patient information we do not require it for the purposes of a test. Therefore, the use of test information about a fictional patient that would be identical in form to what would be sent about an actual patient would satisfy this objective. However, we note that this is one of the objectives that a State may modify in accordance with the discussion in II.A.2.c. of the proposed rule. Therefore, more stringent requirements may be established for EPs and eligible hospitals under the

Medicaid program in states where this capability exists.

Comment: Commenters expressed concern about the burden of multiple requirements for submission from Federal, State, and local government agencies or non-governmental registries. They also raised the issue of lack of standardization of means and form of submission.

Response: Standards for content exchange and vocabulary are established in the ONC final rule at 45 CFR 170.302(k). As meaningful use seeks to utilize certified EHR technology for purposes of the test and subsequent submission (if test was successful) these are the standards that should be utilized. While we encourage all providers and registries to work together to develop efficient, electronic submission of immunization information to all registries where it can be used to improve population and public health, for purposes of becoming a meaningful EHR user, we only require a single test and follow up submission if that test is successful.

Comment: Commenters suggested deferring the measure to a later stage due to the lack of a mature HIE infrastructure.

Response: We agree that many areas of the country currently lack the infrastructure to support the electronic exchange of information. As meaningful use seeks to ensure certified EHR technology has the capability to submit electronic data to registries, we only require a single test if a receiving entity is available and follow up submission only if that test is successful. If none of the immunization registries to which the EP, eligible hospital or CAH submits information has the capacity to receive the information electronically, then this objective would not apply.

Comment: Commenters requested clarification whether on a failed attempted test satisfies the criteria of this measure and whether EPs in a group setting using identical certified EHR technology would only need to conduct a single test, not one test per EP.

Response: A failed attempt would meet the measure. We highly encourage EPs, eligible hospitals, and CAHs to work with their vendor and the receiving entity with whom they tested to identify the source of the failure and develop remedies, but for Stage 1 of meaningful use a failed attempt would meet the requirements. We had indicated in the proposed rule that only one test is required for EPs practicing in a group setting that shares the same certified EHR technology. We maintain that proposal for the final rule.

Comment: Commenters recommended the inclusion of electronically reporting to other types of registries in addition to immunization registries such as diseasespecific registries such as the Cystic Fibrosis Registry.

Response: While we encourage all providers and registries to work together 
to develop efficient, electronic submission of information to all registries where it can be used to improve population and public health, for purposes of becoming a meaningful EHR user, we only require a single test utilizing immunization data and follow up submission if that test is successful.

After consideration of the public comments received, we are modifying the meaningful use measure for EPs at $\S 495.6(\mathrm{e})(9)(\mathrm{ii})$ and for eligible hospitals and CAHs at $\$ 495.6(\mathrm{~g})(8)$ (ii) of our regulations to "Performed at least one test of certified EHR technology's capacity to submit electronic data to immunization registries and follow up submission if the test is successful (unless none of the immunization registries to which the $\mathrm{EP}$, eligible hospital, or CAH submits such information have the capacity to receive the information electronically)".

We further specify that in order to meet this objective and measure, an EP, eligible hospital, or CAH must use the capabilities Certified EHR Technology includes as specified and standards at 45 CFR 170.302(k). The ability to calculate the measure is included in certified EHR technology. We require that an EP, eligible hospital, or CAH determine if they have given any immunizations during the EHR reporting period. Those that have not given any immunizations during the EHR reporting period are excluded from this measure according to the discussion of whether certain EP, eligible hospital or CAH can meet all Stage 1 meaningful use objectives given established scopes of practices. If they have given immunizations during the reporting period, they should then attempt to locate a registry or IIS with whom to conduct a test of the submission of electronic data. This test must include the transfer of either actual or "dummy" data to the chosen registry or IIS. The testing could occur prior to the beginning of the EHR reporting period, but must occur prior to the end of the EHR reporting period. EPs in a group setting using identical certified EHR technology would only need to conduct a single test, not one test per EP. If the test is successful, then the EP, eligible hospital, or CAH should institute regular reporting to that entity in accordance with applicable law and practice. CMS will accept a yes/no attestation to verify all of the above for EPs, eligible hospitals or CAHs that have administered immunizations during the EHR reporting period.

NPRM Eligible Hospital Objective: Capability to provide electronic submission of reportable (as required by state or local law) lab results to public health agencies and actual submission where it can be received.

In the proposed rule, we did not elaborate on this objective.

Comment: A few commenters requested this objective be applied to EPs as long as the EHR Certification requirements are met. A commenter remarked that electronic submission of reportable lab results should not put an additional burden on the providers as the EHR would be able to automate this process.

Response: We based the limitation on the recommendation of the HIT Policy Committee who in turn went through a considerable public development process. We do not believe that burden of reporting was the only limiting factor in keeping this objective from being applied to EPs; therefore, we maintain our proposal to limit this objective to eligible hospitals and CAHs. EPs usually send out lab test to other organizations on which reporting burdens may fall.

Comment: Commenters requested that the actual transmission of the

information be required.

Response: In the discussion of the reporting immunization data objective, we discussed at length the need to align the language for the three objectives included under the health care policy priority of improve population and public health, which is one of the five priorities of the Stage 1 definition of meaningful use. Our interpretation is that the three phrases result in the same outcome, but introduce confusion due to the varied wordings. As commenters strongly preferred the phrase "according to applicable law and practice", we will so modify this objective. We do note however that applicable law and practice does not guarantee every receiving entity will be able to accept it electronically. Our measure for meeting this objective is one test of electronic data submission and if the test is successful, a follow up submission to that one entity. We do not seek to enforce through meaningful use every law and practice that may require submission of lab results.

After consideration of the public comments received, we are modifying the meaningful use objective for eligible hospitals and CAHs at $\S 495.6(\mathrm{~g})(9)$ (i) of our regulations to "Capability to submit electronic data on reportable (as required by state or local law) lab results to public health agencies and actual submission in accordance with applicable law and practice".

NPRM Eligible Hospital Measure: Performed at least one test of certified EHR technology capacity to provide electronic submission of reportable lab results to public health agencies (unless none of the public health agencies to which eligible hospital submits such information have the capacity to receive the information electronically).

In the proposed rule, we identified this as an objective where more stringent requirements may be established for eligible hospitals under the Medicaid program in states where this capability exists. This is just one example of a possible State proposed modification to

Comment: Commenters asked whether the test needs to be "live" or if it could be a "simulation".

Response: As specified in the proposed rule, this test must involve the actual submission of information to a public health agency, if one exists that will accept the information.

Comment: Commenters asked whether the use of "test" or "dummy" data is permissible.

Response: While the use of test patient information may increase the risk that the system will not be testing to its full capability, given the privacy and security concerns surrounding the transmission of actual patient information we do not require it for the purposes of a test. Therefore, the use of test information about a fictional patient that would be identical in form to what would be sent about an actual patient would satisfy this objective. However, we note that this is one of the objectives that a State may modify as discussed previously in this section. Therefore, more stringent requirements may be established for EPs and eligible hospitals under the Medicaid program in states where this capability exists.

Comment: Commenters requested that one national standard be established for reporting lab results to public health agencies.

Response: Standards for content exchange and vocabulary are established in the ONC final rule at 45 CFR 170.306(g). While we encourage all providers and public health agencies to work together to develop efficient, electronic submission of reportable lab results to all public health agencies, for purposes of becoming a meaningful EHR user, we only require a single test and follow up submission if that test is successful.

Comment: Commenters suggested deferring the measure to a later stage due to the lack of a mature HIE infrastructure and lack of a clear standard for exchanging biosurveillance data.

Response: We agree that many areas of the country currently lack the infrastructure to support the electronic exchange of information. As meaningful use seeks to ensure certified EHR 
technology has the capability to submit electronic data to public health agencies, we only require a single test if a receiving entity is available and follow up submission only if that test is successful.

After consideration of the public comments received, we are modifying the meaningful use measure for eligible hospitals and CAHs at $\S 495.6(\mathrm{~g})(9)(\mathrm{ii})$ of our regulations to "Performed at least one test of certified EHR technology's capacity to provide electronic submission of reportable lab results to public health agencies and follow-up submission if the test is successful (unless none of the public health agencies to which eligible hospital or CAH submits such information have the capacity to receive the information electronically)".

We further specify that in order to meet this objective and measure, an EP, eligible hospital, or CAH must use the capabilities Certified EHR Technology includes as specified and standards at 45 CFR 170.306(g). The ability to calculate the measure is included in certified EHR technology. Eligible hospitals and CAHs should attempt to identify one public health agency with whom to conduct a test of the submission of electronic data. This test must include the transfer of either actual or "dummy" data to the chosen public health agency. The testing could occur prior to the beginning of the EHR reporting period, but must occur prior to the end of the EHR reporting period. If the test is successful, then the eligible hospital or CAH should institute regular reporting to that entity according to applicable law and practice. CMS will accept a yes/no attestation to verify all of the above for eligible hospitals and CAHs.

NPRM EP/Eligible Hospital Objective: Capability to provide electronic syndromic surveillance data to public health agencies and actual transmission according to applicable law and practice.

In the proposed rule, we did not elaborate on this objective.

Comment: Half of the commenters commenting on this objective recommended that the objective be deferred to Stage 2 or 3 as the objective is considered expensive, complex and imposes significant administrative burdens on EPs, eligible hospitals and CAHs unless the certified EHR technologies support the automate, electronic capture of the requisite data.

Response: The measure for this objective accounts for the possibility that such electronic exchange of syndromic data is not possible. Standards and certification for certified
EHR technologies are covered under the ONC final rule and do support the automatic identification of the requisite data and its electronic capture. This greatly limits the cost, complexity and burden of this objective.

Comment: Commenters requested that an actual transmission be required.

Response: In discussing the reporting immunization data objective, we focused on the need to align the language for the three objectives contained in under the health care policy priority of improving population and public health. Our interpretation is that the three phrases result in the same outcome, but introduce confusion with the current language. We adopted the language from this objective for the others. We do note however that applicable law and practice does not guarantee every receiving entity will be able to accept it electronically. Our measure for meeting this objective is one test of electronic data submission and if the test is successful, then follow up submission to that one entity based on the reporting requirements of that entity. We do not seek to enforce through meaningful use every law and practice that may require submission of lab results.

Comment: Some commenters requested a clarification of the term "public health agencies."

Response: A public health agency is an entity under the jurisdiction of the U.S. Department of Health and Human Services, tribal organization, State level and/or city/county level administration that serves a public health function.

Comment: Some commenters recommended that providers be required to satisfy either electronic submission to immunization registries or electronic submission of syndromic surveillance data to a public health agency, but not both.

Response: We disagree. We believe these are fundamentally different types of information. Each may impose unique requirements in terms of ability to exchange information on both the EP, eligible hospital, or $\mathrm{CAH}$ and the receiving entity. Therefore, a test for one does not prove or disprove the ability to exchange information for the other.

After consideration of the public comments received, we are modifying the meaningful use objective for EPs at $\S 495.6(\mathrm{e})(10)(\mathrm{i})$ and eligible hospitals and CAHs at $\S 495.6$ (g)(10(i) of our regulations to "Capability to submit electronic syndromic surveillance data to public health agencies and actual submission in accordance with applicable law and practice."

NPRM EP/Eligible Hospital Measure: Performed at least one test of certified
EHR technology's capacity to provide electronic syndromic surveillance data to public health agencies (unless none of the public health agencies to which an EP, eligible hospital, or CAH submits such information have the capacity to receive the information electronically).

In the proposed rule, we identified

this as an objective where more stringent requirements may be established for EPs and hospitals under the Medicaid program in states where this capability exists. This is just one example of a possible State proposed modification to meaningful use.

First, a technical correction, in the proposed rule we incorrectly stated that the capability to send electronic data to immunization registries was included in the certification standards for certified EHR technology. We intended for this data to be sent to public health agencies and ONC in their final rule at 45 CFR 170.304(l) correctly stated this capability as such.

Comment: Commenters asked whether the test needs to be "live" or if it could be a "simulation".

Response: As specified in the proposed rule, this test must involve the actual submission of information to a public health agency, if one exists that will accept the information.

Comment: Commenters asked whether the use of "test" or "dummy" data is permissible.

Response: While the use of test patient information may increase the risk that the system will not be testing to its full capability, given the privacy and security concerns surrounding the transmission of actual patient information we do not require it for the purposes of a test. Therefore, the use of test information about a fictional patient that would be identical in form to what would be sent about an actual patient would satisfy this objective. However, we note that this is one of the objectives that a State may modify in accordance with the discussion in II.A.2.c. of the proposed rule. Therefore, more stringent requirements may be established for EPs and eligible hospitals under the Medicaid program in states where this capability exists.

Comment: A few commenters expressed confusion as to the required ferquency of the test.

Response: As stated in the proposed rule, the required frequency of a test in Stage 1 for EPs, eligible hospitals, and CAHs is at least once prior to the end of the EHR reporting period. We further clarify that each payment year would require it own unique test.

Comment: Commenters requested that one national standard be established for 
reporting syndromic surveillance data to public health agencies.

Response: Standards for content exchange and vocabulary are established in the ONC final rule. While we encourage all providers and public health agencies to work together to develop efficient, electronic submission of syndromic surveillance data to all public health agencies, for purposes of becoming a meaningful EHR user, we only require a single test and follow up submission if that test is successful.

Comment: Commenters suggested deferring the measure to a later stage due to the lack of a mature HIE infrastructure.

Response: We agree that many areas of the country currently lack the infrastructure to support the electronic exchange of information. As meaningful use seeks to ensure certified EHR technology has the capability to submit electronic data to public entities, we only require a single test if a receiving entity is available and follow up submission only if that test is successful. We note that this measure only applies if there is a public health agency with the capacity to receive this information.

Comment: Commenters requested clarification on whether a failed attempted test satisfies the measure and whether EPs in a group setting using identical certified EHR technology would only need to conduct a single test, not one test per EP.

Response: A failed attempt would meet the measure. We highly encourage EPs, eligible hospitals, and CAHs to work with their vendor and the receiving entity with whom they tested to identify the source of the failure and develop remedies, but for Stage 1 of meaningful use a failed attempt would meet the requirements. We had indicated in the proposed rule that only on test is required for EPs practicing in a group setting that shares the same certified EHR technology. We maintain that proposal for the final rule.

After consideration of the public comments received, we are modifying the meaningful use measure for EPs at $\S 495.6(\mathrm{e})(10)(\mathrm{ii})$ and eligible hospitals and CAHs at $\S 495.6(\mathrm{~g})(10)(\mathrm{ii})$ of our regulations to "Performed at least one test of certified EHR technology's capacity to provide electronic syndromic surveillance data to public health agencies and follow-up submission if the test is successful (unless none of the public health agencies to which an EP, eligible hospital, or CAH submits such information have the capacity to receive the information electronically.)"
We further specify that in order to meet this objective and measure, an EP, eligible hospital, or CAH must use the capabilities Certified EHR Technology includes as specified and standards at 45 CFR 170.302(l). The ability to calculate the measure is included in certified EHR technology. EPs, eligible hospitals, and CAHs should attempt to identify one public health agency with whom to conduct a test of the submission of electronic data. This test must include the transfer of either actual or "dummy" data to the chosen public health agency. The testing could occur prior to the beginning of the EHR reporting period, but must occur prior to the end of the EHR reporting period. If the test is successful, then the EP, eligible hospital, or CAH should institute regular reporting to that entity according to applicable law and practice. CMS will accept a yes/no attestation to verify all of the above for eligible hospitals and CAHs.

If an EP does not collect any reportable syndromic information on their patients during the EHR reporting period, then they are excluded from this measure according to the discussion of whether certain EP, eligible hospital or $\mathrm{CAH}$ can meet all Stage 1 meaningful use objectives given established scopes of practices.

The fifth health outcomes policy priority is to ensure adequate privacy and security protections for personal health information. The following care goals for meaningful use address this priority:

- Ensure privacy and security protections for confidential information through operating policies, procedures, and technologies and compliance with applicable law.

- Provide transparency of data sharing to patient.

NPRM EP/Eligible Hospital Objective: Protect electronic health information created or maintained by the certified EHR technology through the implementation of appropriate technical capabilities.

In the proposed rule, we discussed how we were relating the objectives presented by the HIT Policy committee more tightly to the meaningful use of certified EHR technology as opposed to the broader success of the EP, eligible hospital or CAH in ensuring privacy and security. The primary reason we gave was that the proper vehicle for ensuring privacy and security is the HIPAA Privacy and Security Act and that we sought with this objective to ensure that certified EHR technology does not impede an EP's, eligible hospital's or CAH's ability to comply with HIPAA.
Comment: We received considerable support from many commenters who supported this objective and measure as proposed.

Response: We appreciate the support of these commenters for our proposed objective and measure.

Comment: Commenters requested clarification of appropriate technical capabilities.

Response: The ONC final rule specifies certain capabilities that must be in certified EHR technology. For the objective we simply mean that a technical capability would be appropriate if it protected the electronic health information created or maintained by the certified EHR technology. All of these capabilities could be part of the certified EHR technology or outside systems and programs that support the privacy and security of certified EHR technology. We could not develop an exhaustive list. Furthermore as we state in the proposed rule compliance with HIPAA privacy and security rules is required for all covered entities, regardless of whether or not they participate in the EHR incentive programs. Furthermore, compliance with the HIPAA Privacy and Security Rules constitutes a wide range of activities, procedures and infrastructure. We rephrased the objective to ensure that meaningful use of the certified EHR technology supports compliance with the HIPAA Privacy and Security Rules and compliance with fair sharing data practices outlined in the Nationwide Privacy and Security Framework (http://healthit.hhs.gov/ portal/server.pt/gateway/PTARGS_O 10731 848088_o_o_18/

NationwidePS Framework-5.pdf), but do not believe meaningful use of certified EHR technology is the appropriate regulatory tool to ensure such compliance with the HIPAA Privacy and Security Rules.

Comment: Several commenters urged CMS not to finalized requirements for the fair data sharing practices set forth in the Nationwide Privacy and Security Framework and to clarify the policies to which CMS is referring.

Response: While we stated in the proposed rule we rephrased the objective to ensure "compliance with fair sharing data practices outline in the Nationwide Privacy and Security Framework," we did not propose any practices or policies related to the Nationwide Privacy and Security Framework and do not finalize any in this final rule.

Comment: Several commenters requested the elimination of this objective as redundant to HIPAA. 
Response: We do not see meaningful use as an appropriate regulatory tool to impose different, additional, and/or inconsistent privacy and security policy requirements from those policies already required by HIPAA. With that said, we do feel it is crucial that EPs, eligible hospitals, and CAHs evaluate the impact certified EHR technology has on their compliance with HIPAA and the protection of health information in general. Therefore, we retain this objective and measure for meaningful use in the final rule.

Comment: We received hundreds of comments that requested the cancelation of the EHR incentive payment program due to the privacy and security risks imposed by the implementation and use of certified EHR technology.

Response: We are required by the ARRA to implement the EHR incentive programs and cannot cancel them. We seek to mitigate the risks to the security and privacy of patient information by requiring EPs, eligible hospitals, and CAHs to conduct or review a security risk analysis in accordance with the requirements under 45 CFR 164.308 (a)(1) and implement security updates as necessary.

After consideration of the public comments received, we are finalizing the meaningful use objective for EPs at $\S 495.6(d)(15)(i)$ and eligible hospitals and CAHs at $\S 495.6(f)(14)(i)$ of our regulations as proposed.

We include this objective in the core set. We believe maintaining privacy and security is crucial for every EP, eligible hospital or CAH that uses certified EHR technology and was recommended by the HIT Policy Committee for inclusion in the core set.

NPRM EP/Eligible Hospital Measure: Conduct or review a security risk analysis in accordance with the requirements under 45 CFR 164.308 (a)(1) and implement security updates as necessary.

In the proposed rule, we discussed the role of certified EHR technology in privacy and security. We said that while certified EHR technology provides tools for protecting health information, it is not a full protection solution. Processes and possibly tools outside the scope of certified EHR technology are required. Therefore, for the Stage 1 criteria of meaningful use we propose that EPs and eligible hospitals conduct or review a security risk analysis of certified EHR technology and implement updates as necessary at least once prior to the end of the EHR reporting period and attest to that conduct or review. The testing could occur prior to the beginning of the EHR reporting period. This is to ensure that the certified EHR technology is playing its role in the overall strategy of the EP or eligible hospital in protecting health information. We have maintained this discussion for the final rule, but modified the measure to account for requests discussed in the comment and response section below.

Comment: Some commenters requested clarification of the phrase "implement security updates as necessary".

Response: A security update would be required if any security deficiencies were identified during the risk analysis. A security update could be updated software for certified EHR technology to be implemented as soon as available, to changes in workflow processes, or storage methods or any other necessary corrective action that needs to take place in order to eliminate the security deficiency or deficiencies identified in the risk analysis. To provide better clarity on this requirement, we are modifying the measure.

After consideration of the public comments received, we are modifying the meaningful use measure for EPs at $\S$ 495.6(d)(15)(ii) and eligible hospitals and CAHs at $\S 495.6(f)(14)(i i)$ of our regulations "Conduct or review a security risk analysis per 45 CFR 164.308(a)(1) of the certified EHR technology, and implement security updates and correct identified security deficiencies as part of its risk management process."

BILLING CODE 4120-01-P 
Table 2: Stage 1 Meaningful Use Objectives and Associated Measures Sorted by Core and

\section{Menu Set}

\begin{tabular}{|c|c|c|c|}
\hline \multicolumn{4}{|c|}{ CORE SET } \\
\hline \multirow{2}{*}{$\begin{array}{c}\text { Health } \\
\text { Outcomes Policy } \\
\text { Priority }\end{array}$} & \multicolumn{2}{|c|}{ Stage 1 Objectives } & \multirow{2}{*}{ Stage 1 Measures } \\
\hline & Eligible Professionals & Eligible Hospitals and CAHs & \\
\hline \multirow[t]{5}{*}{$\begin{array}{l}\text { Improving } \\
\text { quality, safety, } \\
\text { efficiency, and } \\
\text { reducing health } \\
\text { disparities }\end{array}$} & $\begin{array}{l}\text { Use CPOE for medication } \\
\text { orders directly entered by any } \\
\text { licensed healthcare } \\
\text { professional who can enter } \\
\text { orders into the medical record } \\
\text { per state, local and } \\
\text { professional guidelines }\end{array}$ & $\begin{array}{l}\text { Use CPOE for medication } \\
\text { orders directly entered by any } \\
\text { licensed healthcare professional } \\
\text { who can enter orders into the } \\
\text { medical record per state, local } \\
\text { and professional guidelines }\end{array}$ & $\begin{array}{l}\text { More than } 30 \% \text { of unique } \\
\text { patients with at least one } \\
\text { medication in their } \\
\text { medication list seen by the } \\
\text { EP or admitted to the } \\
\text { eligible hospital's or } \\
\text { CAH's inpatient or } \\
\text { emergency department } \\
\text { (POS } 21 \text { or } 23 \text { ) have at } \\
\text { least one medication order } \\
\text { entered using CPOE }\end{array}$ \\
\hline & $\begin{array}{l}\text { Implement drug-drug and } \\
\text { drug-allergy interaction } \\
\text { checks }\end{array}$ & $\begin{array}{l}\text { Implement drug-drug and drug- } \\
\text { allergy interaction checks }\end{array}$ & $\begin{array}{l}\text { The EP/eligible } \\
\text { hospital/CAH has enabled } \\
\text { this functionality for the } \\
\text { entire EHR reporting } \\
\text { period }\end{array}$ \\
\hline & $\begin{array}{l}\text { Generate and transmit } \\
\text { permissible prescriptions } \\
\text { electronically }(\mathrm{eRx})\end{array}$ & & $\begin{array}{l}\text { More than } 40 \% \text { of all } \\
\text { permissible prescriptions } \\
\text { written by the EP are } \\
\text { transmitted electronically } \\
\text { using certified EHR } \\
\text { technology }\end{array}$ \\
\hline & $\begin{array}{ll}\text { Record demographics } \\
0 & \text { preferred language } \\
0 & \text { gender } \\
0 & \text { race } \\
0 & \text { ethnicity } \\
0 & \text { date of birth }\end{array}$ & $\begin{array}{l}\text { Record demographics } \\
0 \text { preferred language } \\
0 \text { gender } \\
0 \text { race } \\
0 \text { ethnicity } \\
0 \text { date of birth } \\
0 \text { date and preliminary cause } \\
\text { of death in the event of } \\
\text { mortality in the eligible hospital } \\
\text { or CAH }\end{array}$ & $\begin{array}{l}\text { More than } 50 \% \text { of all } \\
\text { unique patients seen by } \\
\text { the EP or admitted to the } \\
\text { eligible hospital's or } \\
\text { CAH's inpatient or } \\
\text { emergency department } \\
\text { (POS } 21 \text { or 23) have } \\
\text { demographics recorded as } \\
\text { structured data }\end{array}$ \\
\hline & $\begin{array}{l}\text { Maintain an up-to-date } \\
\text { problem list of current and } \\
\text { active diagnoses }\end{array}$ & $\begin{array}{l}\text { Maintain an up-to-date problem } \\
\text { list of current and active } \\
\text { diagnoses }\end{array}$ & $\begin{array}{l}\text { More than } 80 \% \text { of all } \\
\text { unique patients seen by } \\
\text { the EP or admitted to the } \\
\text { eligible hospital's or } \\
\text { CAH's inpatient or } \\
\text { emergency department } \\
\text { (POS } 21 \text { or } 23 \text { ) have at } \\
\text { least one entry or an } \\
\text { indication that no } \\
\text { problems are known for } \\
\text { the patient recorded as } \\
\text { structured data }\end{array}$ \\
\hline
\end{tabular}




\begin{tabular}{|c|c|c|}
\hline $\begin{array}{l}\text { Maintain active medication } \\
\text { list }\end{array}$ & Maintain active medication list & $\begin{array}{l}\text { More than } 80 \% \text { of all } \\
\text { unique patients seen by } \\
\text { the EP or admitted to the } \\
\text { eligible hospital's or } \\
\text { CAH's inpatient or } \\
\text { emergency department } \\
\text { (POS } 21 \text { or } 23 \text { )have at } \\
\text { least one entry (or an } \\
\text { indication that the patient } \\
\text { is not currently prescribed } \\
\text { any medication) recorded } \\
\text { as structured data }\end{array}$ \\
\hline $\begin{array}{l}\text { Maintain active medication } \\
\text { allergy list }\end{array}$ & $\begin{array}{l}\text { Maintain active medication } \\
\text { allergy list }\end{array}$ & $\begin{array}{l}\text { More than } 80 \% \text { of all } \\
\text { unique patients seen by } \\
\text { the EP or admitted to the } \\
\text { eligible hospital's or } \\
\text { CAH's inpatient or } \\
\text { emergency department } \\
\text { (POS } 21 \text { or } 23 \text { ) have at } \\
\text { least one entry (or an } \\
\text { indication that the patient } \\
\text { has no known medication } \\
\text { allergies) recorded as } \\
\text { structured data }\end{array}$ \\
\hline $\begin{array}{l}\text { Record and chart changes in } \\
\text { vital signs: } \\
\circ \text { Height } \\
\circ \text { Weight } \\
\circ \text { Blood pressure } \\
\circ \text { Calculate and display } \\
\\
\text { BMI } \\
\circ \quad \text { Plot and display } \\
\text { growth charts for } \\
\text { children 2-20 years, } \\
\text { including BMI }\end{array}$ & $\begin{array}{l}\text { Record and chart changes in } \\
\text { vital signs: } \\
\circ \text { Height } \\
\circ \text { Weight } \\
\circ \text { Blood pressure } \\
\circ \quad \text { Calculate and display } \\
\\
\text { BMI } \\
\circ \quad \text { Plot and display } \\
\\
\text { growth charts for } \\
\text { children 2-20 years, } \\
\text { including BMI }\end{array}$ & $\begin{array}{l}\text { For more than } 50 \% \text { of all } \\
\text { unique patients age } 2 \text { and } \\
\text { over seen by the EP or } \\
\text { admitted to eligible } \\
\text { hospital's or CAH's } \\
\text { inpatient or emergency } \\
\text { department (POS } 21 \text { or } \\
23 \text { ), height, weight and } \\
\text { blood pressure are } \\
\text { recorded as structured data }\end{array}$ \\
\hline $\begin{array}{l}\text { Record smoking status for } \\
\text { patients } 13 \text { years old or older }\end{array}$ & $\begin{array}{l}\text { Record smoking status for } \\
\text { patients } 13 \text { years old or older }\end{array}$ & $\begin{array}{l}\text { More than } 50 \% \text { of all } \\
\text { unique patients } 13 \text { years } \\
\text { old or older seen by the } \\
\text { EP or admitted to the } \\
\text { eligible hospital's or } \\
\text { CAH's inpatient or } \\
\text { emergency department } \\
\text { (POS } 21 \text { or } 23 \text { ) have } \\
\text { smoking status recorded } \\
\text { as structured data }\end{array}$ \\
\hline $\begin{array}{l}\text { Implement one clinical } \\
\text { decision support rule relevant } \\
\text { to specialty or high clinical } \\
\text { priority along with the ability } \\
\text { to track compliance that rule }\end{array}$ & $\begin{array}{l}\text { Implement one clinical decision } \\
\text { support rule related to a high } \\
\text { priority hospital condition along } \\
\text { with the ability to track } \\
\text { compliance with that rule }\end{array}$ & $\begin{array}{l}\text { Implement one clinical } \\
\text { decision support rule }\end{array}$ \\
\hline $\begin{array}{l}\text { Report ambulatory clinical } \\
\text { quality measures to CMS or } \\
\text { the States }\end{array}$ & $\begin{array}{l}\text { Report hospital clinical quality } \\
\text { measures to CMS or the States }\end{array}$ & $\begin{array}{l}\text { For } 2011 \text {, provide } \\
\text { aggregate numerator, } \\
\text { denominator, and } \\
\text { exclusions through } \\
\text { attestation as discussed in } \\
\text { section II(A)(3) of this }\end{array}$ \\
\hline
\end{tabular}




\begin{tabular}{|c|c|c|c|}
\hline & & & final rule \\
\hline & & & $\begin{array}{l}\text { For } 2012 \text {, electronically } \\
\text { submit the clinical quality } \\
\text { measures as discussed in } \\
\text { section II(A)(3) of this } \\
\text { final rule }\end{array}$ \\
\hline $\begin{array}{l}\text { Engage patients } \\
\text { and families in } \\
\text { their health care }\end{array}$ & $\begin{array}{l}\text { Provide patients with an } \\
\text { electronic copy of their health } \\
\text { information (including } \\
\text { diagnostic test results, } \\
\text { problem list, medication lists, } \\
\text { medication allergies), upon } \\
\text { request }\end{array}$ & $\begin{array}{l}\text { Provide patients with an } \\
\text { electronic copy of their health } \\
\text { information (including } \\
\text { diagnostic test results, problem } \\
\text { list, medication lists, medication } \\
\text { allergies, discharge summary, } \\
\text { procedures), upon request }\end{array}$ & $\begin{array}{l}\text { More than } 50 \% \text { of all } \\
\text { patients of the EP or the } \\
\text { inpatient or emergency } \\
\text { departments of the eligible } \\
\text { hospital or CAH (POS } 21 \\
\text { or } 23 \text { ) who request an } \\
\text { electronic copy of their } \\
\text { health information are } \\
\text { provided it within } 3 \\
\text { business days }\end{array}$ \\
\hline & & $\begin{array}{l}\text { Provide patients with an } \\
\text { electronic copy of their } \\
\text { discharge instructions at time of } \\
\text { discharge, upon request }\end{array}$ & $\begin{array}{l}\text { More than } 50 \% \text { of all } \\
\text { patients who are } \\
\text { discharged from an } \\
\text { eligible hospital or CAH's } \\
\text { inpatient department or } \\
\text { emergency department } \\
\text { (POS } 21 \text { or 23) and who } \\
\text { request an electronic copy } \\
\text { of their discharge } \\
\text { instructions are provided it }\end{array}$ \\
\hline & $\begin{array}{l}\text { Provide clinical summaries for } \\
\text { patients for each office visit }\end{array}$ & & $\begin{array}{l}\text { Clinical summaries } \\
\text { provided to patients for } \\
\text { more than } 50 \% \text { of all } \\
\text { office visits within } 3 \\
\text { business days }\end{array}$ \\
\hline $\begin{array}{l}\text { Improve care } \\
\text { coordination }\end{array}$ & $\begin{array}{l}\text { Capability to exchange key } \\
\text { clinical information (for } \\
\text { example, problem list, } \\
\text { medication list, medication } \\
\text { allergies, diagnostic test } \\
\text { results), among providers of } \\
\text { care and patient authorized } \\
\text { entities electronically }\end{array}$ & $\begin{array}{l}\text { Capability to exchange key } \\
\text { clinical information (for } \\
\text { example, discharge summary, } \\
\text { procedures, problem list, } \\
\text { medication list, medication } \\
\text { allergies, diagnostic test } \\
\text { results), among providers of } \\
\text { care and patient authorized } \\
\text { entities electronically }\end{array}$ & $\begin{array}{l}\text { Performed at least one test } \\
\text { of certified EHR } \\
\text { technology's capacity to } \\
\text { electronically exchange } \\
\text { key clinical information }\end{array}$ \\
\hline $\begin{array}{l}\text { Ensure adequate } \\
\text { privacy and } \\
\text { security } \\
\text { protections for } \\
\text { personal health } \\
\text { information }\end{array}$ & $\begin{array}{l}\text { Protect electronic health } \\
\text { information created or } \\
\text { maintained by the certified } \\
\text { EHR technology through the } \\
\text { implementation of appropriate } \\
\text { technical capabilities }\end{array}$ & $\begin{array}{l}\text { Protect electronic health } \\
\text { information created or } \\
\text { maintained by the certified } \\
\text { EHR technology through the } \\
\text { implementation of appropriate } \\
\text { technical capabilities }\end{array}$ & $\begin{array}{l}\text { Conduct or review a } \\
\text { security risk analysis per } \\
45 \text { CFR } 164.308 \text { (a)(1) } \\
\text { and implement security } \\
\text { updates as necessary and } \\
\text { correct identified security } \\
\text { deficiencies as part of its } \\
\text { risk management process }\end{array}$ \\
\hline \multicolumn{4}{|c|}{ MENU SET } \\
\hline Health Outcomes & \multicolumn{2}{|l|}{ Stage 1 Objectives } & Measures \\
\hline
\end{tabular}




\begin{tabular}{|c|c|c|c|}
\hline Policy Priority & Eligible Professionals & $\begin{array}{l}\text { Eligible Hospitals and } \\
\text { CAHs }\end{array}$ & \\
\hline \multirow[t]{5}{*}{$\begin{array}{l}\text { Improving quality, } \\
\text { safety, efficiency, } \\
\text { and reducing } \\
\text { health disparities }\end{array}$} & $\begin{array}{l}\text { Implement drug- } \\
\text { formulary checks }\end{array}$ & $\begin{array}{l}\text { Implement drug-formulary } \\
\text { checks }\end{array}$ & $\begin{array}{l}\text { The EP/eligible hospital/CAH has } \\
\text { enabled this functionality and has } \\
\text { access to at least one internal or } \\
\text { external drug formulary for the } \\
\text { entire EHR reporting period }\end{array}$ \\
\hline & & $\begin{array}{l}\text { Record advance directives } \\
\text { for patients } 65 \text { years old or } \\
\text { older }\end{array}$ & $\begin{array}{l}\text { More than } 50 \% \text { of all unique } \\
\text { patients } 65 \text { years old or older } \\
\text { admitted to the eligible hospital's or } \\
\text { CAH's inpatient department (POS } \\
\text { 21) have an indication of an } \\
\text { advance directive status recorded }\end{array}$ \\
\hline & $\begin{array}{l}\text { Incorporate clinical lab- } \\
\text { test results into certified } \\
\text { EHR technology as } \\
\text { structured data }\end{array}$ & $\begin{array}{l}\text { Incorporate clinical lab-test } \\
\text { results into certified EHR } \\
\text { technology as structured } \\
\text { data }\end{array}$ & $\begin{array}{l}\text { More than } 40 \% \text { of all clinical lab } \\
\text { tests results ordered by the EP or by } \\
\text { an authorized provider of the } \\
\text { eligible hospital or CAH for } \\
\text { patients admitted to its inpatient or } \\
\text { emergency department (POS } 21 \text { or } \\
\text { 23) during the EHR reporting } \\
\text { period whose results are either in a } \\
\text { positive/negative or numerical } \\
\text { format are incorporated in certified } \\
\text { EHR technology as structured data }\end{array}$ \\
\hline & $\begin{array}{l}\text { Generate lists of patients } \\
\text { by specific conditions to } \\
\text { use for quality } \\
\text { improvement, reduction } \\
\text { of disparities, research } \\
\text { or outreach }\end{array}$ & $\begin{array}{l}\text { Generate lists of patients by } \\
\text { specific conditions to use } \\
\text { for quality improvement, } \\
\text { reduction of disparities, } \\
\text { research or outreach }\end{array}$ & $\begin{array}{l}\text { Generate at least one report listing } \\
\text { patients of the EP, eligible hospital } \\
\text { or CAH with a specific condition }\end{array}$ \\
\hline & $\begin{array}{l}\text { Send reminders to } \\
\text { patients per patient } \\
\text { preference for } \\
\text { preventive/ follow up } \\
\text { care }\end{array}$ & & $\begin{array}{l}\text { More than } 20 \% \text { of all unique } \\
\text { patients } 65 \text { years or older or } 5 \text { years } \\
\text { old or younger were sent an } \\
\text { appropriate reminder during the } \\
\text { EHR reporting period }\end{array}$ \\
\hline $\begin{array}{l}\text { Engage patients } \\
\text { and families in } \\
\text { their health care }\end{array}$ & $\begin{array}{l}\text { Provide patients with } \\
\text { timely electronic access } \\
\text { to their health } \\
\text { information (including } \\
\text { lab results, problem list, } \\
\text { medication lists, } \\
\text { medication allergies) } \\
\text { within four business } \\
\text { days of the information } \\
\text { being available to the }\end{array}$ & & $\begin{array}{l}\text { More than } 10 \% \text { of all unique } \\
\text { patients seen by the EP are provided } \\
\text { timely (available to the patient } \\
\text { within four business days of being } \\
\text { updated in the certified EHR } \\
\text { technology) electronic access to } \\
\text { their health information subject to } \\
\text { the EP's discretion to withhold } \\
\text { certain information }\end{array}$ \\
\hline
\end{tabular}




\begin{tabular}{|c|c|c|c|}
\hline & EP & & \\
\hline & $\begin{array}{l}\text { Use certified EHR } \\
\text { technology to identify } \\
\text { patient-specific } \\
\text { education resources and } \\
\text { provide those resources } \\
\text { to the patient if } \\
\text { appropriate }\end{array}$ & $\begin{array}{l}\text { Use certified EHR } \\
\text { technology to identify } \\
\text { patient-specific education } \\
\text { resources and provide those } \\
\text { resources to the patient if } \\
\text { appropriate }\end{array}$ & $\begin{array}{l}\text { More than } 10 \% \text { of all unique } \\
\text { patients seen by the EP or admitted } \\
\text { to the eligible hospital's or CAH's } \\
\text { inpatient or emergency department } \\
\text { (POS } 21 \text { or } 23 \text { ) are provided } \\
\text { patient-specific education resources }\end{array}$ \\
\hline $\begin{array}{l}\text { Improve care } \\
\text { coordination }\end{array}$ & $\begin{array}{l}\text { The EP, eligible hospital } \\
\text { or CAH who receives a } \\
\text { patient from another } \\
\text { setting of care or } \\
\text { provider of care or } \\
\text { believes an encounter is } \\
\text { relevant should perform } \\
\text { medication } \\
\text { reconciliation }\end{array}$ & $\begin{array}{l}\text { The EP, eligible hospital or } \\
\text { CAH who receives a } \\
\text { patient from another setting } \\
\text { of care or provider of care } \\
\text { or believes an encounter is } \\
\text { relevant should perform } \\
\text { medication reconciliation }\end{array}$ & $\begin{array}{l}\text { The EP, eligible hospital or CAH } \\
\text { performs medication reconciliation } \\
\text { for more than } 50 \% \text { of transitions of } \\
\text { care in which the patient is } \\
\text { transitioned into the care of the EP } \\
\text { or admitted to the eligible hospital's } \\
\text { or CAH's inpatient or emergency } \\
\text { department (POS } 21 \text { or } 23 \text { ) }\end{array}$ \\
\hline & $\begin{array}{l}\text { The EP, eligible hospital } \\
\text { or CAH who transitions } \\
\text { their patient to another } \\
\text { setting of care or } \\
\text { provider of care or } \\
\text { refers their patient to } \\
\text { another provider of care } \\
\text { should provide summary } \\
\text { of care record for each } \\
\text { transition of care or } \\
\text { referral }\end{array}$ & $\begin{array}{l}\text { The EP, eligible hospital or } \\
\text { CAH who transitions their } \\
\text { patient to another setting of } \\
\text { care or provider of care or } \\
\text { refers their patient to } \\
\text { another provider of care } \\
\text { should provide summary of } \\
\text { care record for each } \\
\text { transition of care or referral }\end{array}$ & $\begin{array}{l}\text { The EP, eligible hospital or CAH } \\
\text { who transitions or refers their } \\
\text { patient to another setting of care or } \\
\text { provider of care provides a } \\
\text { summary of care record for more } \\
\text { than } 50 \% \text { of transitions of care and } \\
\text { referrals }\end{array}$ \\
\hline $\begin{array}{l}\text { Improve } \\
\text { population and } \\
\text { public health }\end{array}$ & $\begin{array}{l}\text { Capability to submit } \\
\text { electronic data to } \\
\text { immunization registries } \\
\text { or Immunization } \\
\text { Information Systems } \\
\text { and actual submission in } \\
\text { accordance with } \\
\text { applicable law and } \\
\text { practice }\end{array}$ & $\begin{array}{l}\text { Capability to submit } \\
\text { electronic data to } \\
\text { immunization registries or } \\
\text { Immunization Information } \\
\text { Systems and actual } \\
\text { submission in accordance } \\
\text { with applicable law and } \\
\text { practice }\end{array}$ & $\begin{array}{l}\text { Performed at least one test of } \\
\text { certified EHR technology's capacity } \\
\text { to submit electronic data to } \\
\text { immunization registries and follow } \\
\text { up submission if the test is } \\
\text { successful (unless none of the } \\
\text { immunization registries to which } \\
\text { the EP, eligible hospital or CAH } \\
\text { submits such information have the } \\
\text { capacity to receive the information } \\
\text { electronically) }\end{array}$ \\
\hline
\end{tabular}

\footnotetext{
${ }^{2}$ Unless an EP, eligible hospital or CAH has an exception for all of these objectives and measures they must complete at least one as part of their demonstration of the menu set in order to be a meaningful EHR user.
} 


\begin{tabular}{|l|l|l|l|}
\hline & $\begin{array}{l}\text { Capability to submit } \\
\text { electronic data on } \\
\text { reportable (as required by } \\
\text { state or local law) lab } \\
\text { results to public health } \\
\text { agencies and actual } \\
\text { submission in accordance } \\
\text { with applicable law and } \\
\text { practice }\end{array}$ & $\begin{array}{l}\text { Performed at least one test of } \\
\text { certified EHR technology's } \\
\text { capacity to provide electronic } \\
\text { submission of reportable lab results } \\
\text { to public health agencies and } \\
\text { follow-up submission if the test is } \\
\text { successful (unless none of the } \\
\text { public health agencies to which } \\
\text { eligible hospital or CAH submits } \\
\text { such information have the capacity } \\
\text { to receive the information } \\
\text { electronically) }\end{array}$ \\
\cline { 2 - 4 } & $\begin{array}{l}\text { Capability to submit } \\
\text { electronic syndromic } \\
\text { surveillance data to } \\
\text { public health agencies } \\
\text { and actual submission in } \\
\text { accordance with } \\
\text { applicable law and } \\
\text { practice }\end{array}$ & $\begin{array}{l}\text { Capability to submit } \\
\text { electronic syndromic } \\
\text { surveillance data to public } \\
\text { health agencies and actual } \\
\text { submission in accordance } \\
\text { with applicable law and } \\
\text { practice }\end{array}$ & $\begin{array}{l}\text { Performed at least one test of } \\
\text { certified EHR technology's capacity } \\
\text { to provide electronic syndromic } \\
\text { surveillance data to public health } \\
\text { agencies and follow-up submission } \\
\text { if the test is successful (unless none } \\
\text { of the public health agencies to } \\
\text { which an EP, eligible hospital or } \\
\text { CAH submits such information } \\
\text { have the capacity to receive the } \\
\text { information electronically) }\end{array}$ \\
\hline
\end{tabular}


Table 3: Stage 1 Meaningful Use Objectives and Associated Measures Sorted by Method of

\section{Measure Calculation}

\begin{tabular}{|c|c|c|}
\hline \multicolumn{3}{|c|}{$\begin{array}{l}\text { Measures with a Denominator of Unique Patients Regardless of Whether the Patient's Records Are } \\
\text { Maintained Using Certified EHR Technology }\end{array}$} \\
\hline \multicolumn{2}{|c|}{ Stage 1 Objectives } & \multirow{2}{*}{ Stage 1 Measures } \\
\hline Eligible Professionals & Eligible Hospitals and CAHs & \\
\hline $\begin{array}{l}\text { Maintain an up-to-date problem } \\
\text { list of current and active } \\
\text { diagnoses }\end{array}$ & $\begin{array}{l}\text { Maintain an up-to-date problem } \\
\text { list of current and active } \\
\text { diagnoses }\end{array}$ & $\begin{array}{l}\text { More than } 80 \% \text { of all unique patients seen } \\
\text { by the EP or admitted to the eligible } \\
\text { hospital's or CAH's inpatient or } \\
\text { emergency department (POS } 21 \text { or } 23 \text { ) } \\
\text { have at least one entry or an indication } \\
\text { that no problems are known for the patient } \\
\text { recorded as structured data }\end{array}$ \\
\hline Maintain active medication list & Maintain active medication list & $\begin{array}{l}\text { More than } 80 \% \text { of all unique patients seen } \\
\text { by the EP or admitted to the eligible } \\
\text { hospital's or CAH's inpatient or } \\
\text { emergency department (POS } 21 \text { or } \\
\text { 23)have at least one entry (or an } \\
\text { indication that the patient is not currently } \\
\text { prescribed any medication) recorded as } \\
\text { structured data }\end{array}$ \\
\hline $\begin{array}{l}\text { Maintain active medication } \\
\text { allergy list }\end{array}$ & $\begin{array}{l}\text { Maintain active medication } \\
\text { allergy list }\end{array}$ & $\begin{array}{l}\text { More than } 80 \% \text { of all unique patients seen } \\
\text { by the EP or admitted to the eligible } \\
\text { hospital's or CAH's inpatient or } \\
\text { emergency department (POS } 21 \text { or } 23 \text { ) } \\
\text { have at least one entry (or an indication } \\
\text { that the patient has no known medication } \\
\text { allergies) recorded as structured data }\end{array}$ \\
\hline $\begin{array}{ll}\text { Record demographics } \\
\circ & \text { Preferred language } \\
\circ & \text { Gender } \\
\circ & \text { Race } \\
\circ & \text { Ethnicity } \\
\circ & \text { Date of Birth }\end{array}$ & $\begin{array}{ll}\text { Record demographics } \\
\circ & \text { Preferred language } \\
\circ & \text { Gender } \\
\circ & \text { Race } \\
\circ & \text { Ethnicity } \\
\circ & \text { Date of Birth } \\
\circ & \text { Date and preliminary cause of } \\
& \text { death in the event of } \\
& \text { mortality in the eligible } \\
& \text { hospital or CAH }\end{array}$ & $\begin{array}{l}\text { More than } 50 \% \text { of all unique patients seen } \\
\text { by the EP or admitted to the eligible } \\
\text { hospital's or CAH's inpatient or } \\
\text { emergency department (POS } 21 \text { or } 23 \text { ) } \\
\text { have demographics recorded as structured } \\
\text { data }\end{array}$ \\
\hline $\begin{array}{l}\text { Provide patients with timely } \\
\text { electronic access to their health } \\
\text { information (including lab results, } \\
\text { problem list, medication lists, } \\
\text { medication allergies) within four } \\
\text { business days of the information } \\
\text { being available to the EP }\end{array}$ & & $\begin{array}{l}\text { More than } 10 \% \text { of all unique patients seen } \\
\text { by the EP are provided timely (available } \\
\text { to the patient within four business days of } \\
\text { being updated in the certified EHR } \\
\text { technology) electronic access to their } \\
\text { health information subject to the EP's } \\
\text { discretion to withhold certain information }\end{array}$ \\
\hline
\end{tabular}


Use certified EHR technology to identify patient-specific education resources and provide those resources to the patient if appropriate
Use certified EHR technology to identify patient-specific education resources and provide those resources to the patient if appropriate
More than $10 \%$ of all unique patients seen by the EP or admitted to the eligible hospital's or CAH's inpatient or emergency department (POS 21 or 23) are provided patient-specific education resources

\begin{tabular}{|c|c|c|}
\hline \multicolumn{3}{|c|}{$\begin{array}{l}\text { Measures with a Denominator of Based on Counting Actions for Patients whose Records are Maintained } \\
\text { Using Certified EHR Technology }\end{array}$} \\
\hline \multicolumn{2}{|c|}{ Stage 1 Objectives } & \multirow{2}{*}{ Stage 1 Measures } \\
\hline Eligible Professionals & Eligible Hospitals and CAHs & \\
\hline $\begin{array}{l}\text { Use CPOE for medication orders } \\
\text { directly entered by any licensed } \\
\text { healthcare professional who can } \\
\text { enter orders into the medical } \\
\text { record per state, local and } \\
\text { professional guidelines }\end{array}$ & $\begin{array}{l}\text { Use CPOE for medication orders } \\
\text { directly entered by any licensed } \\
\text { healthcare professional who can } \\
\text { enter orders into the medical } \\
\text { record per state, local and } \\
\text { professional guidelines }\end{array}$ & $\begin{array}{l}\text { More than } 30 \% \text { of unique patients with at } \\
\text { least one medication in their medication } \\
\text { list seen by the EP or admitted to the } \\
\text { eligible hospital's or CAH's inpatient or } \\
\text { emergency department (POS } 21 \text { or } 23 \text { ) } \\
\text { have at least one medication order entered } \\
\text { using CPOE }\end{array}$ \\
\hline $\begin{array}{l}\text { Generate and transmit } \\
\text { permissible prescriptions } \\
\text { electronically }(\mathrm{eRx})\end{array}$ & & $\begin{array}{l}\text { More than } 40 \% \text { of all permissible } \\
\text { prescriptions written by the EP are } \\
\text { transmitted electronically using certified } \\
\text { EHR technology }\end{array}$ \\
\hline 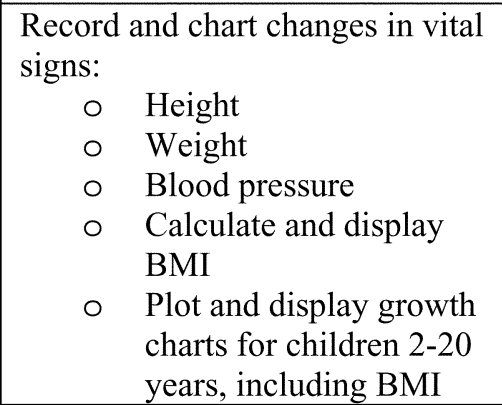 & 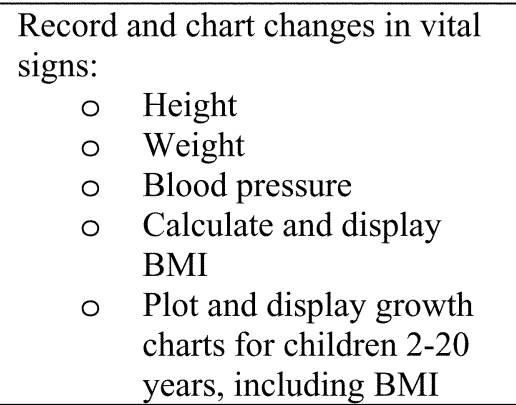 & $\begin{array}{l}\text { For more than } 50 \% \text { of all unique patients } \\
\text { age } 2 \text { and over seen by the EP or admitted } \\
\text { to eligible hospital's or CAH's inpatient } \\
\text { or emergency department (POS } 21 \text { or } 23 \text { ), } \\
\text { height, weight and blood pressure are } \\
\text { recorded as structured data }\end{array}$ \\
\hline \multirow[t]{2}{*}{$\begin{array}{l}\text { Record smoking status for } \\
\text { patients } 13 \text { years old or older }\end{array}$} & $\begin{array}{l}\text { Record smoking status for } \\
\text { patients } 13 \text { years old or older }\end{array}$ & $\begin{array}{l}\text { More than } 50 \% \text { of all unique patients } 13 \\
\text { years old or older seen by the EP or } \\
\text { admitted to the eligible hospital's or } \\
\text { CAH's inpatient or emergency department } \\
\text { (POS } 21 \text { or } 23 \text { ) have smoking status } \\
\text { recorded as structured data }\end{array}$ \\
\hline & $\begin{array}{l}\text { Record advance directives for } \\
\text { patients } 65 \text { years old or older }\end{array}$ & $\begin{array}{l}\text { More than } 50 \% \text { of all unique patients } 65 \\
\text { years old or older admitted to the eligible } \\
\text { hospital have an indication of an advance } \\
\text { directive status recorded }\end{array}$ \\
\hline $\begin{array}{l}\text { Incorporate clinical lab-test } \\
\text { results into certified EHR } \\
\text { technology as structured data }\end{array}$ & $\begin{array}{l}\text { Incorporate clinical lab-test results } \\
\text { into certified EHR technology as } \\
\text { structured data }\end{array}$ & $\begin{array}{l}\text { More than } 40 \% \text { of all clinical lab tests } \\
\text { results ordered by the EP or by an } \\
\text { authorized provider of the eligible hospital } \\
\text { or CAH for patients admitted to its } \\
\text { inpatient or emergency department (POS } \\
21 \text { or } 23 \text { ) during the EHR reporting period } \\
\text { whose results are either in a } \\
\text { positive/negative or numerical format are } \\
\text { incorporated in certified EHR technology } \\
\text { as structured data }\end{array}$ \\
\hline
\end{tabular}




\begin{tabular}{|c|c|c|}
\hline $\begin{array}{l}\text { Provide patients with an } \\
\text { electronic copy of their health } \\
\text { information (including diagnostic } \\
\text { test results, problem list, } \\
\text { medication lists, medication } \\
\text { allergies), upon request }\end{array}$ & $\begin{array}{l}\text { Provide patients with an } \\
\text { electronic copy of their health } \\
\text { information (including diagnostic } \\
\text { test results, problem list, } \\
\text { medication lists, medication } \\
\text { allergies, discharge summary, } \\
\text { procedures), upon request }\end{array}$ & $\begin{array}{l}\text { More than } 50 \% \text { of all patients of the EP or } \\
\text { the inpatient or emergency departments of } \\
\text { the eligible hospital or CAH (POS } 21 \text { or } \\
23 \text { ) who request an electronic copy of } \\
\text { their health information are provided it } \\
\text { within } 3 \text { business days }\end{array}$ \\
\hline & $\begin{array}{l}\text { Provide patients with an } \\
\text { electronic copy of their discharge } \\
\text { instructions at time of discharge, } \\
\text { upon request }\end{array}$ & $\begin{array}{l}\text { More than } 50 \% \text { of all patients who are } \\
\text { discharged from an eligible hospital or } \\
\text { CAH's inpatient department or emergency } \\
\text { department (POS } 21 \text { or } 23 \text { ) and who } \\
\text { request an electronic copy of their } \\
\text { discharge instructions are provided it }\end{array}$ \\
\hline $\begin{array}{l}\text { Provide clinical summaries for } \\
\text { patients for each office visit }\end{array}$ & & $\begin{array}{l}\text { Clinical summaries provided to patients } \\
\text { for more than } 50 \% \text { of all office visits } \\
\text { within } 3 \text { business days }\end{array}$ \\
\hline $\begin{array}{l}\text { Send reminders to patients per } \\
\text { patient preference for preventive/ } \\
\text { follow up care }\end{array}$ & & $\begin{array}{l}\text { More than } 20 \% \text { of all unique patients } 65 \\
\text { years or older or } 5 \text { years old or younger } \\
\text { were sent an appropriate reminder during } \\
\text { the EHR reporting period }\end{array}$ \\
\hline $\begin{array}{l}\text { The EP, eligible hospital or CAH } \\
\text { who receives a patient from } \\
\text { another setting of care or provider } \\
\text { of care or believes an encounter is } \\
\text { relevant should perform } \\
\text { medication reconciliation }\end{array}$ & $\begin{array}{l}\text { The EP, eligible hospital or CAH } \\
\text { who receives a patient from } \\
\text { another setting of care or provider } \\
\text { of care or believes an encounter is } \\
\text { relevant should perform } \\
\text { medication reconciliation }\end{array}$ & $\begin{array}{l}\text { The EP, eligible hospital or CAH } \\
\text { performs medication reconciliation for } \\
\text { more than } 50 \% \text { of transitions of care in } \\
\text { which the patient is transitioned into the } \\
\text { care of the EP or admitted to the eligible } \\
\text { hospital's or CAH's inpatient or } \\
\text { emergency department (POS } 21 \text { or } 23 \text { ) }\end{array}$ \\
\hline $\begin{array}{l}\text { The EP, eligible hospital or CAH } \\
\text { who transitions their patient to } \\
\text { another setting of care or provider } \\
\text { of care or refers their patient to } \\
\text { another provider of care should } \\
\text { provide summary of care record } \\
\text { for each transition of care or } \\
\text { referral }\end{array}$ & $\begin{array}{l}\text { The EP, eligible hospital or CAH } \\
\text { who transitions their patient to } \\
\text { another setting of care or provider } \\
\text { of care or refers their patient to } \\
\text { another provider of care should } \\
\text { provide summary of care record } \\
\text { for each transition of care or } \\
\text { referral }\end{array}$ & $\begin{array}{l}\text { The EP, eligible hospital or CAH who } \\
\text { transitions or refers their patient to another } \\
\text { setting of care or provider of care provides } \\
\text { a summary of care record for more than } \\
50 \% \text { of transitions of care and referrals }\end{array}$ \\
\hline \multicolumn{3}{|c|}{ Measures Requiring Only a Yes/No Attestation } \\
\hline \multicolumn{2}{|l|}{ Stage 1 Objectives } & \multirow{2}{*}{ Stage 1 Measures } \\
\hline Eligible Professionals & Hospitals & \\
\hline $\begin{array}{l}\text { Implement drug-drug and drug- } \\
\text { allergy interaction checks }\end{array}$ & $\begin{array}{l}\text { Implement drug-drug and drug- } \\
\text { allergy interaction checks }\end{array}$ & $\begin{array}{l}\text { The EP/eligible hospital/CAH has enabled } \\
\text { this functionality for the entire EHR } \\
\text { reporting period }\end{array}$ \\
\hline
\end{tabular}




\begin{tabular}{|c|c|c|}
\hline $\begin{array}{l}\text { Implement drug-formulary } \\
\text { checks }\end{array}$ & Implement drug-formulary checks & $\begin{array}{l}\text { The EP/eligible hospital/CAH has enabled } \\
\text { this functionality and has access to at least } \\
\text { one internal or external drug formulary for } \\
\text { the entire EHR reporting period }\end{array}$ \\
\hline $\begin{array}{l}\text { Generate lists of patients by } \\
\text { specific conditions to use for } \\
\text { quality improvement, reduction } \\
\text { of disparities, research or } \\
\text { outreach }\end{array}$ & $\begin{array}{l}\text { Generate lists of patients by } \\
\text { specific conditions to use for } \\
\text { quality improvement, reduction of } \\
\text { disparities, research or outreach }\end{array}$ & $\begin{array}{l}\text { Generate at least one report listing patients } \\
\text { of the EP, eligible hospital or CAH with a } \\
\text { specific condition }\end{array}$ \\
\hline $\begin{array}{l}\text { Implement one clinical decision } \\
\text { support rule relevant to specialty } \\
\text { or high clinical priority along } \\
\text { with the ability to track } \\
\text { compliance that rule }\end{array}$ & $\begin{array}{l}\text { Implement one clinical decision } \\
\text { support rule related to a high } \\
\text { priority hospital condition along } \\
\text { with the ability to track } \\
\text { compliance with that rule }\end{array}$ & $\begin{array}{l}\text { Implement one clinical decision support } \\
\text { rule }\end{array}$ \\
\hline $\begin{array}{l}\text { Capability to exchange key } \\
\text { clinical information (for example, } \\
\text { problem list, medication list, } \\
\text { medication allergies, diagnostic } \\
\text { test results), among providers of } \\
\text { care and patient authorized } \\
\text { entities electronically }\end{array}$ & $\begin{array}{l}\text { Capability to exchange key } \\
\text { clinical information (for example, } \\
\text { discharge summary, procedures, } \\
\text { problem list, medication list, } \\
\text { medication allergies, diagnostic } \\
\text { test results), among providers of } \\
\text { care and patient authorized } \\
\text { entities electronically }\end{array}$ & $\begin{array}{l}\text { Performed at least one test of certified } \\
\text { EHR technology's capacity to } \\
\text { electronically exchange key clinical } \\
\text { information }\end{array}$ \\
\hline \multirow[t]{2}{*}{$\begin{array}{l}\text { Capability to submit electronic } \\
\text { data to immunization registries or } \\
\text { Immunization Information } \\
\text { Systems and actual submission in } \\
\text { accordance with applicable law } \\
\text { and practice }\end{array}$} & $\begin{array}{l}\text { Capability to submit electronic } \\
\text { data to immunization registries or } \\
\text { Immunization Information } \\
\text { Systems and actual submission in } \\
\text { accordance with applicable law } \\
\text { and practice }\end{array}$ & $\begin{array}{l}\text { Performed at least one test of certified } \\
\text { EHR technology's capacity to submit } \\
\text { electronic data to immunization registries } \\
\text { and follow up submission if the test is } \\
\text { successful (unless none of the } \\
\text { immunization registries to which the EP, } \\
\text { eligible hospital or CAH submits such } \\
\text { information have the capacity to receive } \\
\text { the information electronically) }\end{array}$ \\
\hline & $\begin{array}{l}\text { Capability to submit electronic } \\
\text { data on reportable (as required by } \\
\text { state or local law) lab results to } \\
\text { public health agencies and actual } \\
\text { submission in accordance with } \\
\text { applicable law and practice }\end{array}$ & $\begin{array}{l}\text { Performed at least one test of certified } \\
\text { EHR technology capacity's to provide } \\
\text { electronic submission of reportable lab } \\
\text { results to public health agencies and } \\
\text { follow-up submission if the test is } \\
\text { successful (unless none of the public } \\
\text { health agencies to which eligible hospital } \\
\text { or CAH submits such information have } \\
\text { the capacity to receive the information } \\
\text { electronically) }\end{array}$ \\
\hline $\begin{array}{l}\text { Capability to submit electronic } \\
\text { syndromic surveillance data to } \\
\text { public health agencies and actual } \\
\text { submission in accordance with } \\
\text { applicable law and practice }\end{array}$ & $\begin{array}{l}\text { Capability to submit electronic } \\
\text { syndromic surveillance data to } \\
\text { public health agencies and actual } \\
\text { submission in accordance with } \\
\text { applicable law and practice }\end{array}$ & $\begin{array}{l}\text { Performed at least one test of certified } \\
\text { EHR technology's capacity to provide } \\
\text { electronic syndromic surveillance data to } \\
\text { public health agencies and follow-up } \\
\text { submission if the test is successful (unless } \\
\text { none of the public health agencies to } \\
\text { which an EP, eligible hospital or CAH } \\
\text { submits such information have the } \\
\text { capacity to receive the information } \\
\text { electronically) }\end{array}$ \\
\hline
\end{tabular}




\begin{tabular}{|l|}
\hline Protect electronic health \\
information created or maintained \\
by the certified EHR technology \\
through the implementation of \\
appropriate technical capabilities
\end{tabular}

Protect electronic health information created or maintained by the certified EHR technology through the implementation of appropriate technical capabilities
Conduct or review a security risk analysis per 45 CFR 164.308 (a)(1) and implement security updates as necessary and correct identified security deficiencies as part of its risk management process
BILLING CODE 4120-01-C

3. Sections 4101(a) and 4102(a)(1) of the HITECH Act: Reporting on Clinical Quality Measures Using EHRs by EPs, Eligible Hospitals, and CAHs ${ }^{3}$

\section{a. General}

As discussed in the meaningful use background in section II.A.2.a. there are three elements of meaningful use. In this section, we discuss the third requirement: using certified EHR technology, the EP, eligible hospital, or CAH submits to the Secretary, in a form and manner specified by the Secretary, information for the EHR reporting period on clinical quality measures and other measures specified by the Secretary. The submission of other measures is discussed in section II.A.2.C of this final rule. The two other elements of meaningful use are discussed in section II.A.2.d.1 of this final rule.

b. Requirements for the Submission of Clinical Quality Measures by EPs, Eligible Hospitals, and CAHs

Sections 1848(o)(2)(B)(ii) and 1886(n)(3)(B)(ii) of the Act provide that the Secretary may not require the electronic reporting of information on clinical quality measures unless the Secretary has the capacity to accept the information electronically, which may be on a pilot basis.

In the proposed rule, we stated that we do not anticipate that HHS will complete the necessary steps for us to have the capacity to electronically accept data on clinical quality measures from EHRs for the 2011 payment year. We believe that it is unlikely that by 2011 there will be adequate testing and demonstration of the ability to receive the required transmitted information on a widespread basis. The capacity to accept information on clinical quality measures also would depend upon the Secretary promulgating technical specifications for EHR vendors with respect to the transmission of information on clinical quality measures

${ }^{3}$ For purposes of this final rule, the term "eligible hospital" for the Medicaid EHR incentive program is inclusive of Critical Access Hospitals (CAHs) as defined in this final rule. sufficiently in advance of the EHR reporting period for 2011, so that adequate time has been provided either for such specifications to be certified, or for EHR vendors to code such specifications into certified systems. Therefore, for 2011, we proposed that Medicare EPs, eligible hospitals, and CAHs use an attestation methodology to submit summary information to us on clinical quality measures as a condition of demonstrating meaningful use of certified EHR technology, rather than electronic submission.

We proposed that from the Medicaid perspective, delaying the onset of clinical quality measures electronic reporting until 2012 addresses concerns about States having the ready infrastructure to receive and store clinical quality measures data before then. More importantly, we recognized that since Medicaid providers are eligible to receive incentive payments for adopting, implementing, or upgrading certified EHR technology, Medicaid providers may not be focused on demonstrating meaningful use until 2012 or later.

We stated that we anticipate that for the 2012 payment year we will have completed the necessary steps to have the capacity to receive electronically information on clinical quality measures from EHRs, including the promulgation of technical specifications for EHR vendors to use for obtaining certification of their systems. Therefore, for the Medicare EHR incentive program beginning in CY 2012 we proposed that an EP using a certified EHR technology or beginning in FY 2012 an eligible hospital or CAH using a certified EHR technology, as appropriate for clinical quality measures, must submit information on clinical quality measures electronically, in addition to submitting the other measures described in section II.2.d.2, in order for the EP, eligible hospital, or CAH to be a meaningful EHR user, regardless of whether CY 2012 is their first or second payment year. However, if the Secretary does not have the capacity to accept the information on clinical quality measures electronically in 2012, consistent with sections 1848(o)(2)(B)(ii) and
1886(n)(3)(B)(ii) of the Act, we will continue to rely on an attestation methodology for reporting of clinical quality measures as a requirement for demonstrating meaningful use of certified EHR technology for payment year 2012. We stated in the proposed rule that should we not have the capacity to accept information on clinical quality measures electronically in 2012, we would inform the public of this fact by publishing a notice in the

Federal Register and providing instructions on how this information should be submitted to us.

We also are finalizing in this final rule that States must identify for us in their State Medicaid HIT Plans how they plan to accept data from Medicaid providers who seek to demonstrate meaningful use by reporting on clinical quality measures, either via attestation or via electronic reporting, subject to our prior approval. If they initiate their program by accepting attestations for clinical quality measures, they must also describe how they will inform providers of their timeframe to accept submission of clinical quality measures electronically. We expect that States will have the capacity to accept electronic reporting of clinical quality measures by their second year implementing their Medicaid EHR incentive program.

For purposes of the requirements under sections 1848(o)(2)(A)(iii) and 1886(n)(3)(iii) of the Act, we defined "clinical quality measures" to consist of measures of processes, experience, and/ or outcomes of patient care,

observations or treatment that relate to one or more quality aims for health care such as effective, safe, efficient, patientcentered, equitable, and timely care. We noted that certain statutory limitations apply only to the reporting of clinical quality measures, such as the requirement discussed in the previous paragraph prohibiting the Secretary from requiring the electronic reporting of information on clinical quality measures unless the Secretary has the capacity to accept the information electronically, as well as other statutory requirements for clinical quality measures that are discussed below in 
section II.A.3.c.1 of this final rule. These limitations apply solely to the submission of clinical quality measures, and do not apply to other measures of meaningful EHR use. The clinical quality measures on which EPs, eligible hospitals, or CAHs will be required to submit information using certified EHR technology, the statutory requirements and other considerations that were used to select these measures, and the reporting requirements are described below.

With respect to Medicaid EPs and eligible hospitals, we noted that section 1903(t)(6) of the Act recognizes that the demonstration of meaningful use may also include the reporting of clinical quality measures to the States. We proposed that in the interest of simplifying the program and guarding against duplication of meaningful use criteria, the clinical quality measures adopted for the Medicare EHR incentive program, would also apply to EPs and eligible hospitals in the Medicaid EHR incentive program.

Despite the statutory limitation prohibiting the Secretary from requiring the electronic submission of clinical quality measures in the Medicare EHR incentive program, if HHS does not have the capacity to accept this information electronically, as previously discussed, the Secretary has broad discretion to establish requirements for meaningful use of certified EHR technology and for the demonstration of such use by EPs, eligible hospitals, and CAHs. Although we proposed to require the electronic submission of information on clinical quality measures in 2012, we stated that we do not desire this to delay the use of certified EHR technology by EPs, eligible hospitals, and CAHs to measure and improve clinical quality.

Specifically, we stated that using EHR functionalities that support measurement of clinical quality is critical to a central goal of the HITECH Act, improving health care quality. Measuring quality is a fundamental aspect of improving such quality, because it allows EPs, eligible hospitals, and CAHs to receive quantitative information upon which they can then act in order to improve quality.

Accordingly, although we did not propose under sections 1848(o)(2)(A)(iii) and 1886(n)(3)(A)(iii) of the Act to require that for 2011 EPs, eligible hospitals, and CAHs report clinical quality measures to us or States electronically, we proposed to require as an additional condition of demonstrating meaningful use of certified EHR technology under sections 1848(o)(2)(A)(i), 1886(n)(3)(A)(ii), and $1903(\mathrm{t})(6)$ of the Act that EPs and eligible hospitals use certified EHR technology to capture the data elements and calculate the results for certain clinical quality measures. Further, we proposed that EPs, eligible hospitals, and CAHs demonstrate that they have satisfied this requirement during the EHR reporting period for 2011 through attestation. We also proposed to require that Medicare EPs, eligible hospitals, and CAHs attest to the accuracy and completeness of the numerators and denominators for each of the applicable measures. Finally, in accordance with our authority under sections 1848(o)(C)(i)(V) and 1886(n)(3)(C)(i)(V) of the Act, which grants us broad discretion to specify the means through which EPs, eligible hospitals, and CAHs demonstrate compliance with the meaningful use criteria, we proposed that EPs, eligible hospitals, and CAHs demonstrate their use of certified EHR technology to capture the data elements and calculate the results for the applicable clinical quality measures by reporting the results to us for all applicable patients. For the Medicaid incentive program, we proposed that States may accept provider attestations in the same manner to demonstrate meaningful use in 2011. However, we indicated that we expect that most Medicaid providers will qualify for the incentive payment by adopting, implementing, or upgrading to certified EHR technology, and therefore will not need to attest to meaningful use of certified EHR technology in 2011, for their first payment year.

We stated that we recognize that considerable work needs to be done by measure owners and developers with respect to the clinical quality measures that we proposed. This includes completing electronic specifications for measures, implementing such specifications into EHR technology to capture and calculate the results, and implementing the systems, themselves. We also recognized that some measures are further developed than others, as discussed in the measures section (see 75 FR 1871) of the proposed rule.

Nevertheless we stated our belief that overall there is sufficient time to complete work on measures and measures specifications so as to allow vendors and EPs, eligible hospitals, and CAHs to implement such systems. We stated that it was our intention not to finalize those specific measures should the necessary work on measure specifications not be completed for particular measures according to the timetable we discuss below. As we discuss below, we finalize in this final rule only those clinical quality measures for which clearly defined electronic specifications have been finalized by the date of display of this final rule.

Finalized clinical quality measures are listed in Table 6 for EPs and Table 7 for eligible hospitals and CAHs. We also clarify that while States may not have the capacity to accept electronic reporting of clinical quality measures in 2011 or their first year implementing their Medicaid EHR incentive program, we expect that they will have such capacity by their second

implementation year. However, if they do not, as with the Federal government, the State would continue to rely on an attestation methodology for reporting clinical quality measures as a requirement for demonstrating meaningful use of certified EHR technology, subject to CMS prior approval via an updated State Medicaid HIT plan.

Comment: A few commenters requested that the definition of "clinical quality measures" be expanded to include "appropriate clinical prevention."

Response: We agree that appropriate clinical prevention is a pertinent topic for clinical quality measures, but we do not believe the definition of clinical quality measures needs to delineate every aspect of quality care included in the definition.

Comment: Several commenters said it will be difficult to develop the EHR capability to capture, integrate and train staff regarding measure specifications if the clinical quality measures are not posted with sufficient time to allow these activities. Other commenters said there is insufficient time allowed for vendors to retool their products and complete development of the reports and/or systems. Several commenters indicated that the clinical quality measures have not been tested, and reliability and validity testing should be performed. Other commenters indicated that standard, clearly defined electronic specifications do not exist and new specifications should be pilot tested and published for stakeholder/public comment. A commenter requested that CMS establish an explicit process for development and testing of evidence based electronically specified measures (eMeasure), and ensure adequate time for field testing.

Response: In general we agree with the desirability of having electronic specifications available, pilot tested, and published for stakeholder viewing sufficiently in advance so as to allow adequate time for modifications if necessary and vendors to incorporate them into certified EHR technology, and for EPs, eligible hospitals, and CAHs to 
integrate the measures into their operations and train staff on the measures. In this case, however, there is a process for certification of certified EHR technology which includes testing of the capability of the certified EHR. The final rule issued by ONC (found elsewhere in this issue of the Federal Register) provides that certified EHR technology must have the ability to calculate clinical quality measures as specified by us. We interpret this requirement to mean that certified EHR technology must have the capability to calculate those clinical quality measures selected in this final rule based on the specifications we select and post on the CMS Web site. In order to provide sufficient time for vendors to retool their products and complete development of the necessary reports and/or systems for calculation of the results for the required clinical quality measures, and for certifying bodies to test and certify that EHR technologies adequately do so, we are adopting only those electronic specifications that are posted on the CMS Web site as of the date of display of this final rule. We believe testing that is part of the process for certification of EHR technology will substitute for testing that might otherwise occur. Additionally, some of the selected measures have undergone various amounts of testing already. For example, the Emergency Department Throughput, Stroke and Venous Thromboembolism (VTE) measures mentioned by the commenter were tested during the January 2010 Connectathon and demonstrated at the Health Information and Management Systems Society (HIMSS) 2010 Interoperability Showcase which demonstrated the use of the measures by participating vendors. However, we expect the EHR certification process to carry out the necessary testing to assure that applicable certified EHR technology can calculate sufficient number of EP, eligible hospital and CAH clinical quality measures required to qualify for the meaningful use incentive program. In order to permit greater participation by EHR vendors, including specialty EHRs, the certification program (see ONC final rule found elsewhere in this issue of the Federal Register) will permit EHRs to be certified if they are able to calculate at a minimum three clinical quality measures in addition to the six core and alternative core measures. In addition, the fact that EPs, eligible hospitals, and CAHs can adopt an EHR reporting period toward the end of FY/CY 2011, we believe, will provide additional time for providers to implement and train staff on the measures we adopt in this final rule.

c. Statutory Requirements and Other Considerations for the Selection of Clinical Quality Measures for Electronic Submission by EPs, Eligible Hospitals, and CAHs

(1) Statutory Requirements for the Selection of Clinical Quality Measures for Electronic Submission by EPs, Eligible Hospitals, and CAHs

Sections 1848(o)(2)(B)(i)(II) and 1886(n)(3)(B)(i) of the Act require that prior to any clinical quality measure being selected, the Secretary will publish in the Federal Register such measure and provide for a period of public comment on such measure. The proposed clinical quality measures for EPs, eligible hospitals, and CAHs for 2011 and 2012 payment were listed in Tables 3 through 21 of the proposed rule (see 75 FR 1874 through 1900).

In the proposed rule, we noted that for purposes of selecting clinical quality measures on which EPs will be required to submit information using certified EHR technology, section 1848(o)(2)(B)(i)(I) of the Act, as added by section 4101 of the HITECH Act, states that the Secretary shall provide preference to clinical quality measures that have been endorsed by the entity with a contract with the Secretary under section 1890(a) of the Act, as added by section 183 of the Medicare

Improvement for Patients and Providers Act (MIPPA) of 2008. For submission of clinical quality measures by eligible hospitals and CAHs, section 1886(n)(3)(B)(i)(I) of the Act, as added by section 4102(a) of the HITECH Act, requires the Secretary to provide preference to those clinical quality measures that have been endorsed by the entity with a contract with the Secretary under section 1890(a) of the Act, as added by section 183 of the MIPPA, or clinical quality measures that have been selected for the purpose of applying section 1886(b)(3)(B)(viii) of the Act (that is, measures that have been selected for the Reporting Hospital Quality Data for Annual Payment Update (RHQDAPU) program).

On January 14, 2009, the U.S. Department of Health and Human Services awarded the contract required under section 1890(a) of the Act to the National Quality Forum (NQF).

Therefore, we explained in the proposed rule that when selecting the clinical quality measures EPs must report in order to demonstrate meaningful use of certified EHR technology in accordance with section 1848(o)(2)(B)(i)(I) of the Act, we will give preference to the clinical quality measures endorsed by the NQF, including NQF endorsed measures that have previously been selected for the Physician Quality Reporting Initiative (PQRI) program. Similarly, we stated that when selecting the clinical quality measures eligible hospitals and CAHs must report in order to demonstrate meaningful use of certified EHR technology in accordance with section 1886(n)(3)(B)(i)(I) of the Act, we will give preference to the clinical quality measures selected from those endorsed by the NQF or that have previously been selected for the RHQDAPU program. In some instances we proposed measures for EPs, eligible hospitals, and CAHs that are not currently NQF endorsed in an effort to include a broader set of clinical quality measures. In the proposed rule, we noted that the HITECH Act does not require the use of NQF endorsed measures, nor limit the measures to those included in PQRI or RHQDAPU. We stated that if we, professional societies, or other stakeholders identify clinical quality measures which may be appropriate for the EHR incentive programs, we will consider those measures even if they are not endorsed by the NQF or have not been selected for the PQRI or RHQDAPU programs, subject to the requirement to publish in the Federal Register such measure(s) for a period of public comment.

We proposed certain clinical quality measures for EPs, eligible hospitals, and CAHs, and listed these measures in Tables 3 through 21 of the proposed rule (see 75 FR 1874-1900) for use in the 2011 and 2012 payment years. We stated that no changes (that is, additions of clinical quality measures) would be made after publication of the final rule, except through further rulemaking. However, we stated that we may make administrative and/or technical modifications or refinements, such as revisions to the clinical quality measures titles and code additions, corrections, or revisions to the detailed specifications for the 2011 and 2012 payment year measures. We stated that the 2011 specifications for user submission of clinical quality measures would be available on our Web site when they are sufficiently developed or finalized. Specifications for the EHR incentive programs must be obtained only from the specifications documents for the EHR incentive program clinical quality measures.

Comment: Numerous comments were received regarding the criteria for selection of clinical quality measures. Some commenters noted the importance of scientific and medical evidence supporting the measure, as well as 
concerns regarding how the clinical quality measures are maintained. Many other commenters indicated that all clinical quality measures should be evidence-based and up-to-date with current medical standards. Several commenters communicated support for using NQF; Hospital Quality Alliance (HQA); Ambulatory care Quality Alliance (AQA); and the American Medical Association-Physician Consortium for Performance Improvement (AMA-PCPI) clinical quality measures. Another commenter suggested that measures that have a related U.S. Preventative Services Task Force (USPSTF) recommendation should follow the USPSTF guidelines and the regulations should allow for clinical quality measures to be updated as the evidence base changes. Another commenter indicated CMS should ensure that all clinical quality measures are endorsed through a stakeholder consensus process. Commenters also questioned why some clinical quality measures in the proposed rule do not have identifiers for example, NQF number and another commenter indicated some of the clinical quality measures titles were different in the clinical quality measure tables. Some commenters also stated that clinical quality measures should be phased in, implementing the clinical quality measures by clinically related sets, and that all CMS proposed clinical quality measures should be NQF endorsed.

Some commenters suggested that CMS should consult with other quality measure stakeholders, such as, NQF, the Hospital Quality Alliance (HQA), and the National Committee for Quality Assurance (NCQA), The Joint Commission (TJC), and Regional Health Improvement Collaboratives to verify the validity, reliability, and appropriateness of proposed clinical measures. In addition when developing, validating and recommending clinical quality measures for the pediatric population, a commenter suggested CMS include consultation with the Child Healthcare Corporation of America (CHCA) or the National Association of Children's Hospitals (NACHRI).

Response: The HITECH Act requires that we give preference to clinical quality measures that are NQF endorsed. NQF is the only organization that we are aware of which is in compliance with the requirements of National Technology Transfer and Advancement Act (NTTAA), to endorse quality measures through voluntary consensus standards. However, the HITECH Act does not require the exclusive use of NQF endorsed measures, nor limit the measures to those produced by any particular developer or adopted or supported by any particular organization, such as those suggested by the commenters. We gave preference to NQF endorsed clinical quality measures in this final rule. However, we do not adopt a policy that would restrict the Secretary's discretion of beyond what is required by the statute. Measures listed in the proposed rule that did not have an NQF identifying number were not NQF endorsed.

With respect to specific organizations, we have received broad input regarding clinical quality measures including from many organizations mentioned by commenters and have considered their comments in determining which clinical quality measures to finalize in this final rule. We also note that, for NQF endorsed measures, the NQF provides a venue for public and member input as a part of the endorsement process. With respect to commenters urging consideration of whether the scientific and medical evidence support the measure, whether the clinical quality measures are evidence-based and consistent with current medical standards, and how the clinical quality measures are maintained, we note that these factors are part of the NQF process, as well as standard measure development processes. We are committed to working with national, State and local associations to identify or develop additional electronically specified clinical quality measures, particularly for pediatric populations, for later stages of meaningful use.

In selecting clinical quality measures for the Medicare EHR incentive program, the Secretary is required to provide for notice in the Federal Register with public comment. This provides broad public input which we fully consider. However, as we stated in the proposed rule, we are finalizing the policy that technical specifications for clinical quality measures are developed and finalized through the sub-regulatory process. Further, this requirement does not pertain to the Medicaid EHR incentive program. We expect to develop a process in the future to solicit public input on Medicaid-specific clinical quality measures for future stages of meaningful use, if needed. However, because there are no such Medicaid-specific measures in this final rule, and all measures apply uniformly across both the Medicare and Medicaid EHR incentive program, we have not developed such a process in this final rule.

After consideration of the public comments received, the HITECH Act requires that we give preference to clinical quality measures that are NQF endorsed. However, it does not require the exclusive use of NQF endorsed measures, nor limit the measures to those produced by any particular developer nor be adopted by any particular organization. In this case, all clinical quality measures we are finalizing are NQF endorsed and have current electronic specifications as of the date of display of this final rule. Effective with the publication of this final rule, these specifications are final for clinical quality measure reporting under the HITECH Act beginning with 2011 and 2012. The detailed electronic specifications of the clinical quality measures for EPs, eligible hospitals, and CAHs are displayed on the CMS Web site at http://Www.cms.gov/

QualityMeasures/03_Electronic Specifications.asp\#TopOfPage.

Sections 1848(o)(2)(B)(iii) and 1886(n)(3)(B)(iii) of the Act requires that in selecting clinical quality measures, the Secretary shall seek to avoid redundant or duplicative reporting otherwise required, including reporting under section 1848(k)(2)(C) of the Act (the PQRI program) and eligible reporting under section

1886(b)(3)(B)(viii) of the Act (RHQDAPU program). For EPs, when the proposed rule was issued there was no statutory authority to provide PQRI incentive payments for services furnished for 2011 or subsequent years. Since then, the PQRI incentive payment for 2011 has been authorized. We acknowledge there is overlap within the clinical quality measure reporting for EPs in the EHR incentive program with the PQRI incentive program. However, the reporting periods in these two incentive programs are different. Currently, the PQRI has a six and a twelve month reporting period. The reporting period for the HITECH EHR incentive program for the first payment year is 90 days, which does not meet the PQRI reporting requirement of six or twelve month reporting period, as currently provided. However, in the second payment year of the HITECH EHR incentive program the reporting period is one year, and the PQRI reporting period, would be synchronous. The requirement for qualification for PQRI is subject to a separate regulation. Although there may be additional issues beyond the reporting periods, we anticipate efforts to avoid redundant and duplicative reporting in PQRI of the same clinical quality measures as required in the EHR incentive program. We envision a single reporting infrastructure for electronic 
submission in the future, and will strive to align the EHR incentive program and PQRI as we develop the reporting framework for clinical quality measures to avoid redundant or duplicative reporting. Further, we also note that the Affordable Care Act (Pub. L. 111-148) requires that the Secretary develop a plan to integrate the EHR incentive program and PQRI by January 1, 2012. In doing so we expect to further address the issue of redundant and duplicative reporting. For eligible hospitals and CAHs, for the EHR incentive program, we are finalizing one set of 15 clinical quality measures for both Medicare and Medicaid. For Stage 1 (for clinical quality measures Stage 1 is 2011 and beginning in 2012), none of the finalized 15 clinical quality measures for eligible hospitals and CAHs are currently included in the RHQDAPU program, and therefore there is no issue of redundant and duplicative reporting based upon the HITECH Act.

Nevertheless, clinical quality measures in the EHR incentive program for eligible hospitals and CAHs were electronically specified for use in the RHQDAPU program with the anticipation to place these measures in RHQDAPU once we have completed and implemented the mechanism to accept quality measures through electronic submission. For the future, we do not anticipate having one set of clinical quality measures for the EHR incentive program and another set for RHQDAPU. Rather, we anticipate a single set of hospital clinical quality measures, most of which we anticipate can be electronically specified. We note some of the RHQDAPU quality measures, for example HCAHPS experience of care measures, do not lend themselves to EHR reporting. Similarly, certain outcome quality measures, such as the current RQHDAPU readmission measures, are based on claims rather than clinical data. In the future, we anticipate hospitals that report RHQDAPU measures electronically would receive incentives from both the RHQDAPU and EHR incentive program, in addition to properly reporting any required quality measures that are not able to be derived from EHRs; this is however subject to future rulemaking. Further, in the future, for hospitals that do not report electronically we anticipate that they may only qualify for an incentive through the RHQDAPU program, and not through the EHR incentive program. Again this is subject to future rulemaking. We envision a single reporting infrastructure for electronic submission in the future, and will strive to align the hospital quality initiative programs to seek to avoid redundant and duplicative reporting of quality measures for eligible hospitals and CAHs.

Comment: Many commenters also suggested aligning clinical quality measure reporting across federal agencies (for example, HRSA, CMS) as well as across programs, (for example, PQRI, CHIP, Medicare and Medicaid) to avoid duplicative and redundant quality performance reporting. Additionally, several commenters suggested that similar clinical quality measures and/or quality data efforts included in the proposed rule are included in other clinical quality recognition programs and EPs who successfully report in these programs via a certified EHR should be deemed to have successfully reported in the EHR incentive program. Other commenters suggested using the PQRI reporting process to satisfy the meaningful use requirement under the EHR incentive program for EPs. Another commenter indicated that clinical quality measures employed by this program and others will be valuable if EPs using EHRs have an in-depth understanding of how to leverage the technology and the data they produce to improve care. A number of commenters requested that only clinical quality measures chosen for use in the RHQDAPU program should be considered for implementation in the EHR incentive program for eligible hospitals and CAHs that qualify for both incentives. Additionally, the commenters stated they would like the process for avoiding duplicative reporting clearly defined.

Response: The HITECH Act requires that the Secretary seek to avoid redundant and duplicative reporting, with specific reference to PQRI for EPs and RHQDAPU for eligible hospitals and CAHs. We have sought to avoid duplicative and redundant reporting in the implementation of the HITECH Act as discussed elsewhere in our responses to comments in this final rule. We will seek to align quality initiative programs in future rulemaking.

(2) Other Considerations for the Selection of Clinical Quality Measures for Electronic Submission by EPs, Eligible Hospitals, and CAHs

In addition to the requirements under sections 1848(o)(2)(B)(i)(I) and 1886(n)(3)(B)(i)(I) of the Act and the other statutory requirements described above, we also proposed applying the following considerations to the selection of the clinical quality measures for electronic submission under the
Medicare and Medicaid EHR incentive programs:

- Clinical quality measures that are included in, facilitate alignment with, or allow determination of satisfactory reporting in other Medicare (for example, PQRI or the RHQDAPU program), Medicaid, and Children's Health Insurance Program (CHIP) program priorities.

- Clinical quality measures that are widely applicable to EPs and eligible hospitals based on the services provided for the population of patients seen.

- Clinical quality measures that promote CMS and HHS policy priorities related to improved quality and efficiency of care for the Medicare and Medicaid populations that would allow us to track improvement in care over time. These current and long term priority topics include: prevention; management of chronic conditions; high cost and high volume conditions; elimination of health disparities; healthcare-associated infections and other conditions; improved care coordination; improved efficiency; improved patient and family experience of care; improved end-of-life/palliative care; effective management of acute and chronic episodes of care; reduced unwarranted geographic variation in quality and efficiency; and adoption and use of interoperable HIT.

- Clinical quality measures that address or relate to known gaps in the quality of care and measures that through the PQRI program, performed at low or highly variable rates.

- Clinical quality measures that have been recommended for inclusion in the EHR incentive by the HIT Policy

Committee.

We noted in the proposed rule that the Children's Health Insurance Program Reauthorization Act (CHIPRA) of 2009 (Pub. L. 111-3) Title IV, section 401 requires the Secretary to publish a core set of clinical quality measures for the pediatric population. We stated that, to the extent possible, we would align the clinical quality measures selected under the EHR incentive program with the measures selected under the CHIPRA core measure set. Included in the proposed clinical quality measures were nine clinical quality measures pertaining to pediatric providers. Four of these nine measures were on the list of CHIPRA initial core measures that were recommended to the Secretary by the Subcommittee to AHRQ's National Advisory Committee (SNAC). In our proposed rule, we noted that not all CHIPRA initial measures recommended to the Secretary were applicable to EHR technology or to the EHR incentive payment program. For example, some of 
the measures are population-based, survey-derived, or not yet NQF endorsed. We stated that new or additional measures for the next iteration of the CHIPRA core set would have EHR extractability as a priority.
Since the publication of the proposed rule, the CHIPRA core measure set has been published in a final rule (see $74 \mathrm{FR}$ 68846 through 68849). In this EHR incentive program final rule, there are four clinical quality measures that are also in the published CHIPRA initial core measure set. These clinical quality measures are shown below in Table 4:

\section{Table 4: Clinical Quality Measures in the EHR Incentive Program Final Rule that are also in the CHIPRA Initial Core Measure Set}

\begin{tabular}{|l|l|}
\hline Measure Number & \multicolumn{1}{c|}{ Clinical Quality Measure Title } \\
\hline NQF 0024 & Weight Assessment Counseling for Children and Adolescents \\
\hline NQF 0033 & Chlamydia Screening for Women \\
\hline NQF 0038 & Childhood Immunization Status \\
\hline $\begin{array}{l}\text { NQF 0002 } \\
\text { PQRI 66 }\end{array}$ & Appropriate Testing for Children with Pharyngitis \\
\hline
\end{tabular}

Due to the concurrent CHIPRA and ARRA HIT implementation activities, we believe there is an exciting opportunity to align the two programs and strive to create efficiencies for States and pediatric providers, where applicable. Similarly, the adult quality measures requirements enacted in the ACA will provide another opportunity for CMS to align its quality measures programs for consistency and to maximize use of electronic reporting. As these programs move forward, we will continue to prioritize consistency in clinical quality measure selection for providers when possible.

We solicited comments on the inclusion or exclusion of any clinical quality measure or measures proposed for the 2011 and 2012 payment years, and to our approach in selecting clinical quality measures.

We stated in the proposed rule that we do not intend to use notice and comment rulemaking as a means to update or modify clinical quality measure specifications. A clinical quality measure that has completed the consensus process through NQF has a designated party (usually, the measure developer/owner) who has accepted responsibility for maintenance of the clinical quality measure. In general, it is the role of the clinical quality measure owner, developer, or maintainer/ steward to make basic changes to a clinical quality measure in terms of the numerator, denominator, and exclusions. We proposed that the clinical quality measures selected for the 2011 and 2012 payment year be supplemented by our technical specifications for EHR submission. We proposed to post the complete clinical quality measures specifications including technical specifications to our
Web site and solicited comments on our approach.

We received various comments as to our proposed considerations for selection of clinical quality measures for submission by EPs, eligible hospitals, and CAHs.

Comment: One commenter said that there needs to be longer than nine months for the look back for capturing clinical quality measures data. Several commenters indicated that baseline measurements that have used the clinical quality measure in the past have not been performed. Commenters also recommended the linkage of clinical decision support to clinical quality measures to strengthen quality improvement efforts. A commenter supported our inclusion of measures that address both quality and resource use efficiency. Another commenter indicated support for the clinical quality measures as represented in the proposed rule.

Response: The look back for capturing clinical quality measures is the period of time for which data would be considered as applying to the measure calculation. The look back period for a clinical quality measure and the method of documentation of prior information is defined by the clinical quality measure specification. The clinical quality measures require reporting and not achievement on particular performance thresholds. We agree with the commenters regarding the benefits of linking clinical decision support tools to the clinical quality measures, and anticipate that as EHR technology evolves, many of the clinical quality measures will be supported by clinical decision support tools. We also agree with the benefits of efficiency measures and we expect that in future program years the scope and variety of measures that address these factors will expand.

Comment: Commenters requested a definition for "Eligible Provider and Non-Qualifying Eligible Provider" with respect to the provider's ability to meet meaningful use if there are no appropriate clinical quality measures to report, the application of financial penalties beginning in 2015, and the handling of exclusions. Another commenter stressed the need for detailed information regarding what is included and excluded in the numerator and denominator for each measure so as to ensure that certified EHR technology's programmed analytics capture all patients who meet the relevant criteria and to ensure that clinical quality measures are properly evaluated. Others indicated that reporting measures electronically will reduce administrative reporting costs. Other commenters supported the ability to report "N/A" for clinical quality measures where an insufficient denominator exists. Other commenters urged that CMS not include any clinical quality measures in Stage 1 of Meaningful Use because they believe Stage 1 should focus on the initial implementation of certified EHR systems and its use for patient care, and that EPs must gain experience with their certified EHR technology before attesting to the accuracy and completeness of numerators, denominators and quality calculations generated from these systems.

Response: While some commenters recommended we not include any clinical quality measures in Stage 1 (2011 and beginning in 2012), as previously described for Stage 1 EPs are required to attest to the clinical quality measures calculated results (numerator, 
denominator, and exclusions) as automatically calculated by the certified EHR technology. Given that the statutory requirement for clinical quality measures is an element of meaningful use, we believe that providing this information on clinical quality measures is appropriate for Stage 1 (2011 and beginning in 2012). We would expect that the patient for whom a clinical quality measure does not apply will not be included in the denominator of the clinical quality measure. If not appropriate for a particular EP we would expect that either patients would not appear in the denominator of the measure (a zero value) or an exclusion would apply. Therefore reporting "N/A" is not necessary. Exclusion parameters-that is, information on what is included and excluded in the numerator and denominator for a clinical quality measure-are included in the measure specifications. We agree that reporting measures electronically will reduce administrative reporting costs, however as discussed in this final rule we will not require electronic submission of clinical quality measures until 2012. Also discussed earlier in this final rule, we believe collecting clinical quality measure data is an important part of meaningful use.

Comment: A commenter indicated that CMS should take ownership of each of the EP clinical quality measures so that CMS can then adjudicate issues related to the clinical quality measures, instead of referring the EP to the measure owner. One commenter believes that EPs and their specialty societies should be the only owners of EP clinical quality measures.

Response: We are the owner/ developer for certain clinical quality measures. More commonly, we use the clinical quality measures developed and owned by others, who are then responsible for the clinical quality measure specifications as endorsed by NQF. Numerous measures have been developed over the years by various organizations and CMS, and therefore we do not believe that specialty societies should be the only owners of EP clinical quality measures. The HITECH Act does not suggest or require that we should be the sole owner/ developer of clinical quality measures.

Comment: A commenter questioned whether clinical quality measures would be updated during the bi-annual review process and how much lead time will be given.

Response: The measures for Stage 1 (2011 and beginning in 2012) of meaningful use are finalized in this final rule and will not change during that stage. Additionally, the electronic specifications, as posted on the CMS Web site at the time of publication of this final rule, are final. We intend to expand the clinical quality measures again for Stage 2 of meaningful use, which we anticipate will first be effective for the 2013 payment year. As required by the HITECH Act for the Medicare EHR incentive program, prior to selecting any new clinical quality measure(s) for Stage 2 of meaningful use, we will publish notice of the proposed measure(s) and request and consider public comments on the proposed measures. We note that the Medicaid EHR incentive program does not have the same statutory

requirement. If future stages of meaningful use include clinical quality measures specific for Medicaid providers, we will consider a process to receive public input on such measures.

Comment: One commenter suggested that only measures chosen for use in the pay-for-reporting program should be considered for implementation in the EHR incentive program.

Response: We selected clinical quality measures that are broadly applicable for the 2011 and 2012 EHR incentive program. Many clinical quality measures used in other Medicare payfor-reporting programs are not applicable to all Medicaid eligible providers, such as pediatricians, certified nurse-midwives, and children's hospitals.

Comment: Commenters suggested alignment between measures with vocabulary standards, in order to promote interoperability of clinical data Stage 1 allows alternative vocabularies for problems, drugs, and procedures; and measures should only be included if alternative specifications using all Stage 1 vocabularies are provided.

Commenters recommended incorporating HL7, LOINC, SNOMED, ICD-9, and ICD-10 for data exchange. Response: Standards for certified EHRs, including vocabulary standards, are included in ONC's final rule (found elsewhere in this issue of the Federal Register).

Comment: Commenter recommended that in the beginning stages of implementation of the EHR incentive programs, CMS should base its reporting initiatives on existing industry models to prevent delays, consumer mistrust, and potential legal issues.

Response: We have conducted extensive reviews of industry standards, employed the comments of industry experts and solicited public comments on all proposed processes.

Comment: Many commenters are concerned that there will not be adequate time to communicate and implement the electronic specification for 2011 clinical quality measure requirements. Additionally, one commenter expressed concern that the additional clinical quality measures required for 2011 reporting will not be posted by CMS in time for careful review and assessment, since currently there are only 15 measures

electronically specified and posted. Commenters requested clinical quality measures to be posted with

implementation guides for each quality reporting metric to ensure successful reporting.

Response: We have limited the requirements for clinical quality measure reporting for eligible hospitals and CAHs to the 15 measures that were electronically specified and posted at the time of publishing the proposed rule. All measures specifications for clinical quality measures selected are final effective upon publication of the EHR incentive program final rule.

\section{d. Clinical Quality Measures for EPs}

For the 2011 and 2012 EHR reporting periods, based upon the considerations for selecting clinical quality measures discussed above, we proposed certain clinical quality measures that were identified in the proposed rule (see 75 FR 1874-1889) for EPs. Tables 4 though 19 of the proposed rule divided the clinical quality measures identified in Table 3 into core measures and specialty group measures (see 75 FR 1890 through 1895). The concept of core measures and specialty group measures is discussed below.

We also stated that some measures were in a higher state of readiness than others, and requested comment on each measure's state of readiness for use in the EHR incentive programs. For those measures where electronic specifications did not, at the time of the proposed rule, exist, we solicited comment on how quickly electronic specifications could be developed, and the period of time required from final posting of the electronic specifications for final measures to ensure the effective implementation of the measures. We stated our intention to publish electronic specifications for the proposed clinical quality measures on the CMS Web site as soon as they become available from the measure developer(s). Electronic specifications may be developed concurrently with the development of measures themselves and potentially with the NQF endorsement processes. We stated that all of the proposed clinical quality measures included in Table 3 (see 75 FR 1874-1889) meet one or more of the 
criteria for the selection of clinical quality measures, discussed in the proposed rule. A large portion of these measures had been through notice and comment rulemaking for PQRI, and nearly all PQRI clinical quality measures are NQF endorsed.

Additionally, they have broad applicability to the range of Medicare designated specialties, and the services provided by EPs who render services to Medicare and Medicaid beneficiaries and many others. Further, nine of the proposed 90 clinical quality measures listed in Table 3 (see 75 FR 1874-1889) (PQRI numbers 1, 2, 3, 5, 7, 110, 111, 112 , and 113) had preliminary specifications for electronic submission that had already been developed for the purpose of testing the submission of clinical quality data extracted from an EHR for the PQRI program. The link to the preliminary electronic specifications for nine PQRI clinical quality measures was provided: http://www.cms.hhs.gov/ pqri.

We stated that in terms of CMS and HHS healthcare quality priorities, clinical quality PQRI measures numbered 1, 2, 3, 5, and 7 address high priority chronic conditions, namely diabetes, coronary artery disease, and heart disease. Clinical quality PQRI measures numbered 110, 111, 112, 113 , 114, 115, and 128 support prevention which is a high CMS and HHS priority. The PQRI clinical quality measure specifications for claims-based or registry-based submission of these clinical quality measures for the most current PQRI program year can be found on the PQRI section of the CMS Web site at http://www.cms.hhs.gov/PQRI/ 15_MeasuresCodes.asp\#TopOfPage. A description of the clinical quality measure, including the clinical quality measure's numerator and denominator, can be found in the PQRI clinical quality measure specifications.

We pointed out that the PQRI clinical quality measures that were proposed largely align with the recommendations of the HIT Standards Committee. However, in addition to proposed clinical quality measures that are currently included in PQRI, we also proposed certain other clinical quality measures that we stated are of high importance to the overall population. Those clinical quality measures are Ischemic Vascular Disease (IVD): Use of Aspirin or another Antithrombotic; IVD: Complete Lipid Profile; IVD: Low Density Lipoprotein (LDL-C) Control, and Blood Pressure Management. Finally, we proposed an array of other measures which address important aspects of clinical quality.
We stated our belief that the proposed clinical quality measures were broad enough to allow for reporting for EPs and addressed high priority conditions. We recognized the importance of integrating the measures into certified EHR technologies for calculation of measures results, and that not all measures would be feasible for 2011 and 2012. We invited comment on the advisability of including the measures for payment years 2011 and 2012. Although we recognized that there are many other important clinical quality measures of health care provided by EPs, we anticipated expanding the set of clinical quality measures in future years and listed a number of clinical quality measures for future consideration in section II.A.3.g of the proposed rule preamble, on which we also invited comment.

Comment: Many of the proposed clinical quality measures received favorable comments and support for inclusion in the final clinical quality measure set. A few examples of measures that were supported for inclusion were measures related to prevention and screening, and diabetes. It was stated by a commenter that the proposed rule includes some similar clinical quality measures. For example, the commenter indicated NQF 0059 and NQF 0575 both deal with hemoglobin A1c control. Others commented that some measures should be eliminated and not utilized in the final set of clinical quality measures for EPs. For example, a few commented that the following two measures should be eliminated, NQF 0052 and NQF 0513 were intended to be implemented at the administrator site level using outpatient hospital claims and not at the individual practitioner level. A number of commenters stated that the specifications for certain clinical quality measures, for example, NQF 0022, NQF 0031, NQF 0032, NQF 0033, NQF 0034, and NQF 0061 were not consistent with current clinical practice guidelines. Another commenter requested clarification for the specifications for NQF 0013 because blood pressures are not routinely monitored for 2-month-old patients. Many commenters provided suggestions for other clinical quality measures not included in the proposed rule.

Response: We appreciate all of the suggestions from the commenters. We are unable to add any clinical quality measures that were not identified in the proposed rule due to language in sections 1848(o)(2)(B)(i)(II) and 1886(n)(3)(B)(i) of the Act requiring a period of public comment for any finalized measures. This requirement does not pertain to the Medicaid EHR incentive program; we expect to develop a process in the future to solicit public input on Medicaid-specific clinical quality measures for future stages of meaningful use, if needed. However, we will consider those additional clinical quality measures recommended by commenters for future inclusion in the clinical quality measure sets.

In regard to suggested changes/ revisions and/or elimination of the proposed clinical quality measures, we considered these suggestions when finalizing clinical quality measures in this final rule. In regard to this, we considered these suggestions when evaluating the clinical quality measures for selection in this final rule. Of the clinical quality measures in the proposed rule that we are not finalizing, we removed the measures that do not have electronic specifications by the date of display of this final rule. Additionally, some of the proposed clinical quality measures were recommended for deletion or modification, and therefore were recommended to not be used in the final rule; this is delineated in other comments and responses in this final rule. Further, we are only finalizing clinical quality measures that are electronically specified the date of display of the final rule. The electronic specifications included in the final set of clinical quality measures for EPs are posted to the CMS Web site at: http:// www.cms.gov/QualityMeasures/03 ElectronicSpecifications.asp\# TopOfPage.

Comment: Numerous commenters were concerned about the burden

(economic and other) of reporting on the large number of clinical quality measures and the overall quality reporting burden this will add to EPs.

Some commenters stated that the use of numerators and denominators for some measures will require manual

calculation on the part of the EPs since there are no automated reports that can capture all of the information that must be tabulated. One commenter stated that there are insufficient resources to calculate the denominators of the required measures. Other commenters suggested using the PQRI requirements of reporting only three measures, and others suggested reporting on significantly smaller number of measures.

Response: In response to the many comments received regarding the undue burden associated with reporting on a large number of clinical quality measures, or measures that involve a manual process, we have finalized only those clinical quality measures that can 
be automatically calculated by a certified EHR technology. We further limited the measures to those for which electronic specifications are currently available, which we posted as final by the date of display of this final rule. This limitation significantly reduces the number of measures EPs are required to report in 2011 and 2012, thus reducing the EPs' reporting burden as well as addressing commenters' concerns about readiness. Although for 2011, Medicare EPs, eligible hospitals, and CAHs will still need to manually report (attest) to the results automatically calculated by their certified EHR technology, we believe that with the reduction in the number of measures that the burden is reasonable. Additionally, this provides for the reporting of clinical quality measures beyond simply the core clinical quality measures that EPs identify as suitable to report.
Table 5, below, shows the proposed clinical quality measures for submission by Medicare and Medicaid EPs for the 2011 and 2012 payment year as stated in the proposed rule (see 75 FR 18741889) for EPs, but that are not being finalized. Table 5 conveys the NQF measure number and PQRI implementation number (that is, the number used in the PQRI program to identify the measure as implemented in PQRI (for the 2010 PQRI measures list see https://www.cms.gov/PQRI/ Downloads/2010 PQRI_MeasuresList_111309.pdf)), clinical quality measure title and description, and clinical quality measure steward and contact information. The measures listed below in Table 5 do not have electronic specifications finished before the date of display of this final rule, thus we have eliminated these measures for this final rule and will consider the addition of these measures in future rulemaking. Also several measures listed below were only concepts at the time of publication of the proposed rule (that is, Hysterectomy rates, Appropriate antibiotic use for ear infections, Statin after Myocardial Infarction, 30 day Readmission Rate, 30 Readmission Rate following deliveries, and Use of CT Scans). These concept measures were not developed or electronically specified clinical quality measures, nor NQF endorsed; and there was not adequate time to consider these concepts for development for this final rule. Therefore, the concepts listed below will be considered in future rulemaking. Lastly, NQF 0026 has since been retired since publication of the proposed rule.

BILLING CODE 4120-01-P 
TABLE 5: Proposed Clinical Quality Measures for Submission by Medicare or Medicaid EPs for the 2011 and 2012 Payment Year; Included in the Proposed Rule (see 75 FR 1874 through 1889) and Not in the Final Rule

\begin{tabular}{|c|c|c|}
\hline $\begin{array}{c}\text { NQF Measure } \\
\text { Number \& PQRI } \\
\text { Implementation } \\
\text { Number }\end{array}$ & Clinical Quality Measure Title \& Description & $\begin{array}{l}\text { Clinical Quality Measure } \\
\text { Steward \& Contact } \\
\text { Information }\end{array}$ \\
\hline $\begin{array}{l}\text { NQF } 0246 \\
\text { PQRI } 10\end{array}$ & $\begin{array}{l}\text { Title: Stroke and Stroke Rehabilitation: } \\
\text { Computed Tomography (CT) or Magnetic } \\
\text { Resonance Imaging (MRI) Reports } \\
\text { Description: Percentage of final reports for CT or } \\
\text { MRI studies of the brain performed within } 24 \\
\text { hours of arrival to the hospital for patients aged } 18 \\
\text { years and older with either a diagnosis of ischemic } \\
\text { stroke or transient ischemic attack (TIA) or } \\
\text { intracranial hemorrhage or at least one documented } \\
\text { symptom consistent with ischemic stroke or TIA or } \\
\text { intracranial hemorrhage that includes } \\
\text { documentation of the presence or absence or each } \\
\text { of the following: hemorrhage and mass lesion and } \\
\text { acute infarction. }\end{array}$ & $\begin{array}{l}\text { AMA-PCPI/NCQA } \\
\text { Contact Information: } \\
\text { cpe@ama-assn.org } \\
\text { www.ncqa.org }\end{array}$ \\
\hline $\begin{array}{l}\text { NQF } 0270 \\
\text { PQRI } 20\end{array}$ & $\begin{array}{l}\text { Title: Perioperative Care: Timing of Antibiotic } \\
\text { Prophylaxis - Ordering Physician } \\
\text { Description: Percentage of surgical patients aged } \\
18 \text { years and older undergoing procedures with the } \\
\text { indications for prophylactic parenteral antibiotics, } \\
\text { who have an order for prophylactic antibiotic to be } \\
\text { given within one hour (if fluoroquinolone or } \\
\text { vancomycin, two hours), prior to the surgical } \\
\text { incision (or start of procedure when no incision is } \\
\text { required) }\end{array}$ & $\begin{array}{l}\text { AMA-PCPI/NCQA } \\
\text { Contact Information: } \\
\text { cpe@ama-assn.org } \\
\text { www.ncqa.org }\end{array}$ \\
\hline $\begin{array}{l}\text { NQF } 0268 \\
\text { PQRI } 21\end{array}$ & $\begin{array}{l}\text { Title: Perioperative Care: Selection of } \\
\text { Prophylactic Antibiotic - First OR Second } \\
\text { Generation Cephalosporin } \\
\text { Description: Percentage of surgical patients aged } \\
18 \text { years and older undergoing procedures with the } \\
\text { indications for a first OR second generation } \\
\text { cephalosporin prophylactic antibiotic, who had an } \\
\text { order for cefazolin OR cefuroxime for } \\
\text { antimicrobial prophylaxis }\end{array}$ & $\begin{array}{l}\text { AMA-PCPI/NCQA } \\
\text { Contact Information: } \\
\text { cpe@ama-assn.org } \\
\text { www.ncqa.org }\end{array}$ \\
\hline $\begin{array}{l}\text { NQF } 0271 \\
\text { PQRI } 22\end{array}$ & $\begin{array}{l}\text { Title: Perioperative Care: Discontinuation of } \\
\text { Prophylactic Antibiotics (Non-Cardiac Procedures) } \\
\text { Description: Percentage of non-cardiac surgical } \\
\text { patients aged } 18 \text { years and older undergoing } \\
\text { procedures with the indications for prophylactic } \\
\text { antibiotics AND who received a prophylactic } \\
\text { antibiotic, who have an order for discontinuation } \\
\text { of prophylactic antibiotics within } 24 \text { hours of } \\
\text { surgical end time }\end{array}$ & $\begin{array}{l}\text { AMA-PCPI/NCQA } \\
\text { Contact Information: } \\
\text { cpe@ama-assn.org } \\
\text { www.ncqa.org }\end{array}$ \\
\hline
\end{tabular}




\begin{tabular}{|c|c|c|}
\hline $\begin{array}{c}\text { NQF Measure } \\
\text { Number \& PQRI } \\
\text { Implementation } \\
\text { Number }\end{array}$ & Clinical Quality Measure Title \& Description & $\begin{array}{l}\text { Clinical Quality Measure } \\
\text { Steward \& Contact } \\
\text { Information }\end{array}$ \\
\hline $\begin{array}{l}\text { NQF } 0239 \\
\text { PQRI } 23\end{array}$ & $\begin{array}{l}\text { Title: Perioperative Care: Venous } \\
\text { Thromboembolism (VTE) Prophylaxis (When } \\
\text { Indicated in ALL Patients) } \\
\text { Description:Percentage of patients aged } 18 \text { years } \\
\text { and older undergoing procedures for which VTE } \\
\text { prophylaxis is indicated in all patients, who had an } \\
\text { order for Low Molecular Weight Heparin } \\
\text { (LMWH), Low-Dose Unfractionated Heparin } \\
\text { (LDUH), adjusted-dose warfarin, fondaparinux or } \\
\text { mechanical prophylaxis to be given within } 24 \\
\text { hours prior to incision time or within } 24 \text { hours after } \\
\text { surgery end time }\end{array}$ & $\begin{array}{l}\text { AMA-PCPI/NCQA } \\
\text { Contact Information: } \\
\text { cpe@ama-assn.org } \\
\text { www.ncqa.org }\end{array}$ \\
\hline $\begin{array}{l}\text { NQF } 0241 \\
\text { PQRI } 33\end{array}$ & $\begin{array}{l}\text { Title: Stroke and Stroke Rehabilitation: } \\
\text { Anticoagulant Therapy Prescribed for Atrial } \\
\text { Fibrillation at Discharge } \\
\text { Description: Percentage of patients aged } 18 \text { years } \\
\text { and older with a diagnosis of ischemic stroke or } \\
\text { transient ischemic attack (TIA) with documented } \\
\text { permanent, persistent, or paroxysmal atrial } \\
\text { fibrillation who were prescribed an anticoagulant } \\
\text { at discharge }\end{array}$ & $\begin{array}{l}\text { AMA-PCPI/NCQA } \\
\text { Contact Information: } \\
\text { cpe@ama-assn.org } \\
\text { www.ncqa.org }\end{array}$ \\
\hline $\begin{array}{l}\text { NQF } 0102 \\
\text { PQRI } 52\end{array}$ & $\begin{array}{l}\text { Title: Chronic Obstructive Pulmonary Disease } \\
\text { (COPD): Bronchodilator Therapy } \\
\text { Description: Percentage of patients aged } 18 \text { years } \\
\text { and older with a diagnosis of COPD and who have } \\
\text { an FEV1/FVC less than } 70 \% \text { and have symptoms } \\
\text { who were prescribed an inhaled bronchodilator }\end{array}$ & $\begin{array}{l}\text { AMA-PCPI } \\
\text { Contact Information: } \\
\text { cpe@ama-assn.org }\end{array}$ \\
\hline $\begin{array}{l}\text { NQF } 0069 \\
\text { PQRI } 65\end{array}$ & $\begin{array}{l}\text { Title: Treatment for Children with Upper } \\
\text { Respiratory Infection (URI): Avoidance of } \\
\text { Inappropriate Use } \\
\text { Description: Percentage of children aged } 3 \\
\text { months through } 18 \text { years with a diagnosis of URI } \\
\text { who were not prescribed or dispensed an antibiotic } \\
\text { prescription on or within } 3 \text { days of the initial date } \\
\text { of service }\end{array}$ & $\begin{array}{l}\text { NCQA } \\
\text { Contact Information: } \\
\text { www.ncqa.org }\end{array}$ \\
\hline $\begin{array}{l}\text { NQF } 0323 \\
\text { PQRI } 81\end{array}$ & $\begin{array}{l}\text { Title: End Stage Renal Disease (ESRD): Plan of } \\
\text { Care for Inadequate Hemodialysis in ESRD } \\
\text { Patients } \\
\text { Description: Percentage of calendar months } \\
\text { during the } 12 \text {-month reporting period in which } \\
\text { patients aged } 18 \text { years and older with a diagnosis } \\
\text { of ESRD receiving hemodialysis have a } \mathrm{Kt} / \mathrm{V} \geq 1.2 \\
\text { OR patients who have a Kt/V }<1.2 \text { with a } \\
\text { documented plan of care for inadequate } \\
\text { hemodialysis }\end{array}$ & $\begin{array}{l}\text { AMA-PCPI } \\
\text { Contact Information: } \\
\text { cpe } @ \text { ama-assn.org }\end{array}$ \\
\hline
\end{tabular}




\begin{tabular}{|c|c|c|}
\hline $\begin{array}{c}\text { NQF Measure } \\
\text { Number \& PQRI } \\
\text { Implementation } \\
\text { Number }\end{array}$ & Clinical Quality Measure Title \& Description & $\begin{array}{c}\text { Clinical Quality Measure } \\
\text { Steward \& Contact } \\
\text { Information }\end{array}$ \\
\hline $\begin{array}{l}\text { NQF } 0321 \\
\text { PQRI } 82\end{array}$ & $\begin{array}{l}\text { Title: End Stage Renal Disease (ESRD): Plan of } \\
\text { Care for Inadequate Peritoneal Dialysis } \\
\text { Description: Percentage of patients aged } 18 \text { years } \\
\text { and older with a diagnosis of ESRD receiving } \\
\text { peritoneal dialysis who have a Kt } / \mathrm{V} \geq 1.7 \mathrm{OR} \\
\text { patients who have a Kt/V }<1.7 \text { with a documented } \\
\text { plan of care for inadequate peritoneal dialysis at } \\
\text { least three times (every } 4 \text { months) during the } 12 \text { - } \\
\text { month reporting period }\end{array}$ & $\begin{array}{l}\text { AMA-PCPI } \\
\text { Contact Information: } \\
\text { cpe@ama-assn.org }\end{array}$ \\
\hline $\begin{array}{l}\text { NQF } 0397 \\
\text { PQRI } 86\end{array}$ & $\begin{array}{l}\text { Title: Hepatitis C: Antiviral Treatment Prescribed } \\
\text { Description: Percentage of patients aged } 18 \text { years } \\
\text { and older with a diagnosis of chronic hepatitis C } \\
\text { who were prescribed peginterferon and ribavirin } \\
\text { therapy within the } 12 \text {-month reporting period }\end{array}$ & $\begin{array}{l}\text { AMA-PCPI } \\
\text { Contact Information: } \\
\text { cpe@ama-assn.org }\end{array}$ \\
\hline $\begin{array}{l}\text { NQF } 0401 \\
\text { PQRI } 89\end{array}$ & $\begin{array}{l}\text { Title: Hepatitis C: Counseling Regarding Risk of } \\
\text { Alcohol Consumption } \\
\text { Description: Percentage of patients aged } 18 \text { years } \\
\text { and older with a diagnosis of hepatitis C who were } \\
\text { counseled about the risks of alcohol use at least } \\
\text { once within the 12-month reporting period }\end{array}$ & $\begin{array}{l}\text { AMA-PCPI } \\
\text { Contact Information: } \\
\text { cpe@ama-assn.org }\end{array}$ \\
\hline $\begin{array}{l}\text { NQF } 0103 \\
\text { PQRI } 106\end{array}$ & $\begin{array}{l}\text { Title: Major Depressive Disorder (MDD): } \\
\text { Diagnostic Evaluation } \\
\text { Description: Percentage of patients aged } 18 \text { years } \\
\text { and older with a new diagnosis or recurrent } \\
\text { episode of MDD who met the DSM-IV criteria } \\
\text { during the visit in which the new diagnosis or } \\
\text { recurrent episode was identified during the } \\
\text { measurement period }\end{array}$ & $\begin{array}{l}\text { AMA-PCPI } \\
\text { Contact Information: } \\
\text { cpe@ama-assn.org }\end{array}$ \\
\hline $\begin{array}{l}\text { NQF } 0104 \\
\text { PQRI } 107\end{array}$ & $\begin{array}{l}\text { Title: Major Depressive Disorder (MDD): Suicide } \\
\text { Risk Assessment } \\
\text { Description: Percentage of patients aged } 18 \text { years } \\
\text { and older with a new diagnosis or recurrent } \\
\text { episode of MDD who had a suicide risk } \\
\text { assessment completed at each visit during the } \\
\text { measurement period }\end{array}$ & $\begin{array}{l}\text { AMA-PCPI } \\
\text { Contact Information: } \\
\text { cpe@ama-assn.org }\end{array}$ \\
\hline $\begin{array}{l}\text { NQF } 0066 \\
\text { PQRI } 118\end{array}$ & $\begin{array}{l}\text { Title: Coronary Artery Disease (CAD): } \\
\text { Angiotensin-Converting Enzyme (ACE) Inhibitor } \\
\text { or Angiotensin Receptor Blocker (ARB) Therapy } \\
\text { for Patients with CAD and Diabetes and/or Left } \\
\text { Ventricular Systolic Dysfunction (LVSD) } \\
\text { Description: Percentage of patients aged } 18 \\
\text { years and older with a diagnosis of CAD who also } \\
\text { have diabetes mellitus and/or LVSD (LVEF < } \\
40 \% \text { ) who were prescribed ACE inhibitor or ARB } \\
\text { therapy }\end{array}$ & $\begin{array}{l}\text { AMA-PCPI } \\
\text { Contact Information: } \\
\text { cpe@ama-assn.org }\end{array}$ \\
\hline
\end{tabular}




\begin{tabular}{|c|c|c|}
\hline $\begin{array}{c}\text { NQF Measure } \\
\text { Number \& PQRI } \\
\text { Implementation } \\
\text { Number }\end{array}$ & Clinical Quality Measure Title \& Description & $\begin{array}{c}\text { Clinical Quality Measure } \\
\text { Steward \& Contact } \\
\text { Information } \\
\end{array}$ \\
\hline $\begin{array}{l}\text { PQRI } 121 \\
\text { Ambulatory Quality } \\
\text { Alliance (AQA) } \\
\text { adopted }\end{array}$ & $\begin{array}{l}\text { Title: Chronic Kidney Disease (CKD): } \\
\text { Laboratory Testing (Calcium, Phosphorus, Intact } \\
\text { Parathyroid Hormone (iPTH) and Lipid Profile) } \\
\text { Description: Percentage of patients aged } 18 \text { years } \\
\text { and older with a diagnosis of advanced CKD } \\
\text { (stage } 4 \text { or 5, not receiving Renal Replacement } \\
\text { Therapy [RRT]), who had the following laboratory } \\
\text { testing ordered within } 12 \text { months: serum levels of } \\
\text { calcium, phosphorus and intact PTH, and lipid } \\
\text { profile }\end{array}$ & $\begin{array}{l}\text { AMA-PCPI } \\
\text { Contact Information: } \\
\text { cpe@ama-assn.org }\end{array}$ \\
\hline $\begin{array}{l}\text { PQRI } 122 \\
\text { AQA adopted }\end{array}$ & $\begin{array}{l}\text { Title: Chronic Kidney Disease (CKD): Blood } \\
\text { Pressure Management } \\
\text { Description: Percentage of patient visits for } \\
\text { patients aged } 18 \text { years and older with a diagnosis } \\
\text { of advanced CKD (stage } 4 \text { or } 5 \text {, not receiving } \\
\text { Renal Replacement Therapy [RRT]), with a blood } \\
\text { pressure }<130 / 80 \mathrm{mmHg} \text { OR blood pressure } \geq \\
130 / 80 \mathrm{mmHg} \text { with a documented plan of care }\end{array}$ & $\begin{array}{l}\text { AMA-PCPI } \\
\text { Contact Information: } \\
\text { cpe@ama-assn.org }\end{array}$ \\
\hline $\begin{array}{l}\text { PQRI } 123 \\
\text { AQA adopted }\end{array}$ & $\begin{array}{l}\text { Title: Chronic Kidney Disease (CKD): Plan of } \\
\text { Care - Elevated Hemoglobin for Patients } \\
\text { Receiving Erythropoiesis-Stimulating Agents } \\
\text { (ESA) } \\
\text { Description: Percentage of calendar months } \\
\text { during the } 12 \text {-month reporting period in which } \\
\text { patients aged } 18 \text { years and older with a diagnosis } \\
\text { of advanced CKD (stage } 4 \text { or } 5 \text {, not receiving } \\
\text { Renal Replacement Therapy [RRT]), receiving } \\
\text { ESA therapy, have a hemoglobin }<13 \mathrm{~g} / \mathrm{dL} \mathrm{OR} \\
\text { patients whose hemoglobin is } \geq 13 \mathrm{~g} / \mathrm{dL} \text { and have a } \\
\text { documented plan of care }\end{array}$ & $\begin{array}{l}\text { AMA-PCPI } \\
\text { Contact Information: } \\
\text { cpe@ama-assn.org }\end{array}$ \\
\hline $\begin{array}{l}\text { NQF } 0416 \\
\text { PQRI } 127\end{array}$ & $\begin{array}{l}\text { Title: Diabetes Mellitus: Diabetic Foot and Ankle } \\
\text { Care, Ulcer Prevention - Evaluation of Footwear } \\
\text { Description: Percentage of patients aged } 18 \text { years } \\
\text { and older with a diagnosis of diabetes mellitus who } \\
\text { were evaluated for proper footwear and sizing }\end{array}$ & $\begin{array}{l}\text { American Podiatric } \\
\text { Medical Association } \\
\text { (APMA) } \\
\text { Contact Information: } \\
\text { http://www.apma.org/ }\end{array}$ \\
\hline $\begin{array}{l}\text { NQF } 0510 \\
\text { PQRI } 145\end{array}$ & $\begin{array}{l}\text { Title: Radiology: Exposure Time Reported for } \\
\text { Procedures Using Fluoroscopy } \\
\text { Description: Percentage of final reports for } \\
\text { procedures using fluoroscopy that include } \\
\text { documentation of radiation exposure or exposure } \\
\text { time }\end{array}$ & $\begin{array}{l}\text { AMA-PCPI/NCQA } \\
\text { Contact Information: } \\
\text { cpe@ama-assn.org } \\
\text { www.ncqa.org }\end{array}$ \\
\hline $\begin{array}{l}\text { NQF } 0508 \\
\text { PQRI } 146\end{array}$ & $\begin{array}{l}\text { Title: Radiology: Inappropriate Use of "Probably } \\
\text { Benign" Assessment Category in Mammography } \\
\text { Screening } \\
\text { Description: Percentage of final reports for } \\
\text { screening mammograms that are classified as } \\
\text { "probably benign" }\end{array}$ & $\begin{array}{l}\text { AMA-PCPI/NCQA } \\
\text { Contact Information: } \\
\text { cpe@ama-assn.org } \\
\text { www.ncqa.org }\end{array}$ \\
\hline
\end{tabular}




\begin{tabular}{|c|c|c|}
\hline $\begin{array}{c}\text { NQF Measure } \\
\text { Number \& PQRI } \\
\text { Implementation } \\
\text { Number } \\
\end{array}$ & Clinical Quality Measure Title \& Description & $\begin{array}{l}\text { Clinical Quality Measure } \\
\text { Steward \& Contact } \\
\text { Information }\end{array}$ \\
\hline $\begin{array}{l}\text { NQF } 0511 \\
\text { PQRI } 147\end{array}$ & $\begin{array}{l}\text { Title: Nuclear Medicine: Correlation with Existing } \\
\text { Imaging Studies for All Patients Undergoing Bone } \\
\text { Scintigraphy } \\
\text { Description: Percentage of final reports for all } \\
\text { patients, regardless of age, undergoing bone } \\
\text { scintigraphy that include physician documentation } \\
\text { of correlation with existing relevant imaging } \\
\text { studies (for example, x-ray, MRI, CT, etc.) that } \\
\text { were performed }\end{array}$ & $\begin{array}{l}\text { AMA-PCPI } \\
\text { Contact Information: } \\
\text { cpe@ama-assn.org }\end{array}$ \\
\hline $\begin{array}{l}\text { PQRI } 153 \\
\text { AQA adopted }\end{array}$ & $\begin{array}{l}\text { Title: Chronic Kidney Disease (CKD): Referral } \\
\text { for Arteriovenous (AV) Fistula } \\
\text { Description: Percentage of patients aged } 18 \text { years } \\
\text { and older with the diagnosis of advanced CKD } \\
\text { (stage } 4 \text { or } 5 \text {, not receiving Renal Replacement } \\
\text { Therapy [RRT]), who were referred for AV fistula } \\
\text { at least once during the 12-month reporting period }\end{array}$ & $\begin{array}{l}\text { AMA-PCPI } \\
\text { Contact Information: } \\
\text { cpe@ama-assn.org }\end{array}$ \\
\hline $\begin{array}{l}\text { NQF } 0399 \\
\text { PQRI } 183\end{array}$ & $\begin{array}{l}\text { Title: Hepatitis C: Hepatitis A Vaccination in } \\
\text { Patients with HCV } \\
\text { Description: Percentage of patients aged } 18 \text { years } \\
\text { and older with a diagnosis of hepatitis C who } \\
\text { received at least one injection of hepatitis A } \\
\text { vaccine, or who have documented immunity to } \\
\text { hepatitis A }\end{array}$ & $\begin{array}{l}\text { AMA-PCPI } \\
\text { Contact Information: } \\
\text { cpe@ama-assn.org }\end{array}$ \\
\hline $\begin{array}{l}\text { NQF } 0400 \\
\text { PQRI } 184\end{array}$ & $\begin{array}{l}\text { Title: Hepatitis C: Hepatitis B Vaccination in } \\
\text { Patients with HCV } \\
\text { Description: Percentage of patients aged } 18 \text { years } \\
\text { and older with a diagnosis of hepatitis C who } \\
\text { received at least one injection of hepatitis B } \\
\text { vaccine, or who have documented immunity to } \\
\text { hepatitis B }\end{array}$ & $\begin{array}{l}\text { AMA-PCPI } \\
\text { Contact Information: } \\
\text { cpe@ama-assn.org }\end{array}$ \\
\hline $\begin{array}{l}\text { PQRI } 185 \\
\text { AQA adopted }\end{array}$ & $\begin{array}{l}\text { Title: Endoscopy \& Polyp Surveillance: } \\
\text { Colonoscopy Interval for Patients with a History of } \\
\text { Adenomatous Polyps - Avoidance of Inappropriate } \\
\text { Use } \\
\text { Description: Percentage of patients aged } 18 \text { years } \\
\text { and older receiving a surveillance colonoscopy and } \\
\text { a history of colonic polyp(s) in a previous } \\
\text { colonoscopy, who had a follow-up interval of } 3 \text { or } \\
\text { more years since their last colonoscopy } \\
\text { documented in the colonoscopy report }\end{array}$ & $\begin{array}{l}\text { AMA-PCPI/NCQA } \\
\text { Contact Information: } \\
\text { cpe@ama-assn.org } \\
\text { www.ncqa.org }\end{array}$ \\
\hline $\begin{array}{l}\text { NQF } 0507 \\
\text { PQRI } 195\end{array}$ & $\begin{array}{l}\text { Title: Stenosis Measurement in Carotid Imaging } \\
\text { Reports } \\
\text { Description: Percentage of final reports for } \\
\text { carotid imaging studies (neck MR angiography } \\
\text { [MRA], neck CT angiography [CTA], neck duplex } \\
\text { ultrasound, carotid angiogram) performed for } \\
\text { patients aged } 18 \text { years and older with the diagnosis } \\
\text { of ischemic stroke or transient ischemic attack } \\
\text { (TIA) that include direct or indirect reference to } \\
\text { measurements of distal internal carotid diameter as } \\
\text { the denominator for stenosis measurement }\end{array}$ & $\begin{array}{l}\text { AMA-PCPI/NCQA } \\
\text { Contact Information: } \\
\text { cpe@ama-assn.org } \\
\text { www.ncqa.org }\end{array}$ \\
\hline
\end{tabular}




\begin{tabular}{|c|c|c|}
\hline $\begin{array}{c}\text { NQF Measure } \\
\text { Number \& PQRI } \\
\text { Implementation } \\
\text { Number }\end{array}$ & Clinical Quality Measure Title \& Description & $\begin{array}{l}\text { Clinical Quality Measure } \\
\text { Steward \& Contact } \\
\text { Information }\end{array}$ \\
\hline NQF 0022 & $\begin{array}{l}\text { Title: Drugs to be avoided in the elderly: a. } \\
\text { Patients who receive at least one drug to be } \\
\text { avoided, b. Patients who receive at least two } \\
\text { different drugs to be avoided. } \\
\text { Description: Percentage of patients ages } 65 \text { years } \\
\text { and older who received at least one drug to be } \\
\text { avoided in the elderly in the measurement year. } \\
\text { Percentage of patients } 65 \text { years of age and older } \\
\text { who received at least two different drugs to be } \\
\text { avoided in the elderly in the measurement year. }\end{array}$ & $\begin{array}{l}\text { NCQA } \\
\text { Contact Information: } \\
\text { www.ncqa.org }\end{array}$ \\
\hline NQF 0026 & $\begin{array}{l}\text { Title: Measure pair - a. Tobacco use prevention for } \\
\text { infants, children and adolescents, b. Tobacco use } \\
\text { cessation for infants, children and adolescents } \\
\text { Description: Percentage of patients' charts } \\
\text { showing either that there is no tobacco } \\
\text { use/exposure or (if a user) that the current use was } \\
\text { documented at the most recent clinic visit. } \\
\text { Percentage of patients with documented tobacco } \\
\text { use or exposure at the latest visit who also have } \\
\text { documentation that their cessation interest was } \\
\text { assessed or that they received advice to quit. }\end{array}$ & $\begin{array}{l}\text { Institute for Clinical } \\
\text { Systems Improvement } \\
\text { (ICSI) } \\
\text { Contact Information: } \\
\text { http://www.icsi.org/ }\end{array}$ \\
\hline NQF 0060 & $\begin{array}{l}\text { Title: Hemoglobin Alc test for pediatric patients } \\
\text { Description: Percentage of pediatric patients with } \\
\text { diabetes with a HBA1c test in a } 12 \text {-month } \\
\text { measurement period. }\end{array}$ & $\begin{array}{l}\text { NCQA } \\
\text { Contact Information: } \\
\text { www.ncqa.org }\end{array}$ \\
\hline NQF 0106 & $\begin{array}{l}\text { Title: Diagnosis of attention deficit hyperactivity } \\
\text { disorder (ADHD) in primary care for school age } \\
\text { children and adolescents } \\
\text { Description: Percentage of patients newly } \\
\text { diagnosed with attention deficit hyperactivity } \\
\text { disorder (ADHD) whose medical record contains } \\
\text { documentation of Diagnostic and Statistical } \\
\text { Manual of Mental Disorders, Fourth Edition } \\
\text { (DSM-IV) or Diagnostic and Statistical Manual for } \\
\text { Primary Care (DSM-PC) criteria being addressed. }\end{array}$ & $\begin{array}{l}\text { ICSI } \\
\text { Contact Information: } \\
\frac{\text { http://www.icsi.org/ }}{}\end{array}$ \\
\hline NQF 0107 & $\begin{array}{l}\text { Title: Management of attention deficit } \\
\text { hyperactivity disorder (ADHD) in primary care for } \\
\text { school age children and adolescents } \\
\text { Description: Percentage of patients diagnosed } \\
\text { with attention deficit hyperactivity disorder } \\
\text { (ADHD) and on first-line medication whose } \\
\text { medical record contains documentation of a } \\
\text { follow-up visit twice a year. }\end{array}$ & $\begin{array}{l}\text { ICSI } \\
\text { Contact Information: } \\
\text { http://www.icsi.org/ }\end{array}$ \\
\hline
\end{tabular}




\begin{tabular}{|c|c|c|}
\hline $\begin{array}{c}\text { NQF Measure } \\
\text { Number \& PQRI } \\
\text { Implementation } \\
\text { Number } \\
\end{array}$ & Clinical Quality Measure Title \& Description & $\begin{array}{c}\text { Clinical Quality Measure } \\
\text { Steward \& Contact } \\
\text { Information } \\
\end{array}$ \\
\hline NQF 0108 & $\begin{array}{l}\text { Title: ADHD: Follow-Up Care for Children } \\
\text { Prescribed Attention-Deficit/Hyperactivity } \\
\text { Disorder (ADHD) Medication. } \\
\text { Description: a. Initiation Phase: Percentage of } \\
\text { children } 6 \text { - } 12 \text { years of age as of the Index } \\
\text { Prescription Episode Start Date with an } \\
\text { ambulatory prescription dispensed for and ADHD } \\
\text { medication and who had one follow-up visit with a } \\
\text { practitioner with prescribing authority during the } \\
\text { 30-Day Initiation } \\
\text { Phase b. Continuation and Maintenance (C\&M) } \\
\text { Phase: Percentage of children } 6 \text { - } 12 \text { years of age } \\
\text { as of the Index Prescription Episode Start Date } \\
\text { with an ambulatory prescription dispensed for } \\
\text { ADHD medication who remained on the } \\
\text { medication for at least } 210 \text { days and who in } \\
\text { addition to the visit in the Initiation Phase had at } \\
\text { least two additional follow-up visits with a } \\
\text { practitioner within } 270 \text { days ( } 9 \text { months) after the } \\
\text { Initiation Phase ends. }\end{array}$ & $\begin{array}{l}\text { NCQA } \\
\text { Contact Information: } \\
\text { www.ncqa.org }\end{array}$ \\
\hline NQF 0110 & $\begin{array}{l}\text { Title: Bipolar Disorder and Major Depression: } \\
\text { Appraisal for alcohol or chemical substance use } \\
\text { Description: Percentage of patients with } \\
\text { depression or bipolar disorder with evidence of an } \\
\text { initial assessment that includes an appraisal for } \\
\text { alcohol or chemical substance use }\end{array}$ & $\begin{array}{l}\text { Center for Quality } \\
\text { Assessment and } \\
\text { Improvement in Mental } \\
\text { Health } \\
\text { Contact Information: } \\
\text { http://www.cqaimh.org/ } \\
\end{array}$ \\
\hline NQF 0299 & $\begin{array}{l}\text { Title: Surgical Site Infection Rate } \\
\text { Description: Percentage of surgical site infections } \\
\text { occurring within thirty days after the operative } \\
\text { procedure if no implant is left in place or with one } \\
\text { year if an implant is in place in patients who had } \\
\text { an NHSN operative procedure performed during a } \\
\text { specified time period and the infection appears to } \\
\text { be related to the operative procedure. }\end{array}$ & $\begin{array}{l}\text { Centers for Disease Control } \\
\text { and Prevention (CDC) } \\
\text { Contact Information: } \\
\mathrm{http}: / / \text { www.cdc.gov/ }\end{array}$ \\
\hline NQF 0471 & $\begin{array}{l}\text { Title: Cesarean Rate for low-risk first birth women } \\
\text { (aka NTSV CS rate) } \\
\text { Description: Percentage of low-risk first birth } \\
\text { women (aka NTSV CS rate: nulliparous, term, } \\
\text { singleton, vertex) with a Cesarean rate that has the } \\
\text { most variation among practicioners, hospitals, } \\
\text { regions and states. Unlike other cesarean measures, } \\
\text { it focuses attention on the proportion of cesarean } \\
\text { births that is affected by elective medical practices } \\
\text { such as induction and early labor admission. } \\
\text { Furthermore, the success (or lack thereof) of } \\
\text { management of the first labor directly impacts the } \\
\text { remainder of the woman's reproductive life } \\
\text { (especially given the current high rate of repeat } \\
\text { cesarean births). }\end{array}$ & $\begin{array}{l}\text { California Maternal } \\
\text { Quality Care Collaborative } \\
\text { (CMQCC) } \\
\text { Contact Information: } \\
\text { http://cmqcc.org/ }\end{array}$ \\
\hline
\end{tabular}




\begin{tabular}{|c|c|c|}
\hline $\begin{array}{c}\text { NQF Measure } \\
\text { Number \& PQRI } \\
\text { Implementation } \\
\text { Number }\end{array}$ & Clinical Quality Measure Title \& Description & $\begin{array}{l}\text { Clinical Quality Measure } \\
\text { Steward \& Contact } \\
\text { Information }\end{array}$ \\
\hline NQF 0513 & $\begin{array}{l}\text { Title: Use of Contrast: Thorax CT } \\
\text { Description: Thorax CT - Use of combined } \\
\text { studies (with and without contrast) }\end{array}$ & $\begin{array}{l}\text { CMS } \\
\text { Contact Information: } \\
\text { http://www.cms.hhs.gov/ }\end{array}$ \\
\hline NQF 0519 & $\begin{array}{l}\text { Title: Diabetic Foot Care and Patient Education } \\
\text { Implemented } \\
\text { Description: Percent of diabetic patients for } \\
\text { whom physician-ordered monitoring for the } \\
\text { presence of skin lesions on the lower extremities } \\
\text { and patient education on proper foot care were } \\
\text { implemented during their episode of care }\end{array}$ & $\begin{array}{l}\text { CMS } \\
\text { Contact Information: } \\
\text { http://www.cms.hhs.gov/ }\end{array}$ \\
\hline Not applicable & $\begin{array}{l}\text { Title: Hysterectomy rates } \\
\text { Description: }\end{array}$ & \\
\hline Not applicable & $\begin{array}{l}\text { Title: Appropriate antibiotic use for ear infections } \\
\text { Description: }\end{array}$ & \\
\hline Not applicable & $\begin{array}{l}\text { Title: Statin after Myocardial Infarction } \\
\text { Description: }\end{array}$ & \\
\hline Not Applicable & $\begin{array}{l}\text { Title: } 30 \text { day Readmission Rate } \\
\text { Description: }\end{array}$ & \\
\hline Not Applicable & $\begin{array}{l}\text { Title: } 30 \text { Readmission Rate following deliveries } \\
\text { Description: }\end{array}$ & \\
\hline Not applicable & $\begin{array}{l}\text { Title: Use of CT scans } \\
\text { Description: Number of repeat CT scans within } 60 \\
\text { days }\end{array}$ & \\
\hline
\end{tabular}

BILLING CODE 4120-01-C

Comment: Some commenters requested that CMS implement feedback reports early in the process that document whether EPs are successfully participating in the PQRI Program, the EHR incentive program, and the eprescribing program, and that the report communicate whether the information received by CMS for these programs was successfully submitted and received.

Response: As the PQRI and eprescribing programs are beyond the scope of this rule, we do not address suggestions that we implement feedback reports related to these programs. The criteria to qualify for the EHR incentive payments are based on results automatically calculated by EPs' certified EHR technology, as attested by the EPs. As such, we believe that the EP will be able to determine whether they have reported the required clinical quality measures to CMS or the State, rendering it unnecessary that CMS or the State provide the EP with a feedback report. We expect the system through which EPs, must submit information would indicate successful receipt beginning the first year of Stage 1 .

Comment: A commenter indicated that the clinical quality measure that addresses tobacco use and the measure that addresses smoking status apply to different age groups, and stated that they should be consistent. A number of commenters recommended removing smoking status as an objective from meaningful use section of this final rule, and only including it in the clinical quality measures in order to avoid confusion.

Response: We are in agreement that the meaningful use objective and the clinical quality measure address the same topic of smoking. The clinical quality measure requires measurement of a clinical action performed by the EP to address the negative consequences of smoking, whereas the meaningful use objective seeks to make sure smokers are identified. Additionally, the age for recording smoking status for meaningful use is 13 years and older, and the population addressed by the clinical quality measure is 18 years and older, thus they are different with respect to intent of the objective/measure and the age population. For the clinical quality measure, we are keeping the age range at 18 years and older because the measure is currently NQF endorsed with these specifications. We will consider merging these in the future to reconcile the age range.

Comment: Some commenters stated that reporting of ambulatory quality measures should remain voluntary for EPs, based on the view that many process measures do not correlate with outcomes and are not evidence based. A process measure focuses on a process which leads to a certain outcome, meaning that a scientific basis exists for believing that the process, when executed well, will increase the probability of achieving a desired outcome. A commenter stated that EPs serving needy patients, minorities, and populations with lower socioeconomic levels will experience lower performance on many clinical quality measures, and therefore will be deterred from participating in the EHR incentive program.

Response: The EHR incentive program is voluntary. Similar to other Medicare quality measure reporting programs, EPs are not required to satisfy minimum clinical quality performance levels in order to qualify for the EHR payment incentive, but rather merely report on their ambulatory quality measure results. Thus, as currently structured, we do not believe the requirement that EPs report clinical quality measures would deter EPs who serve minority patients or patients of lower socioeconomic status or otherwise disadvantaged from participating in the program.

After consideration of the public comments received, we are finalizing the basic requirement that EPs submit results for clinical quality measures. 
This requirement applies to both the 2011 and 2012 reporting periods (and will potentially continue to apply, until CMS issues a subsequent final rule that supplants this final rule). We are limiting the clinical quality measures to those for which electronic specifications are available (posted by CMS on the Web site at the time of display of this final rule.) These measures are listed in Table 6 of this final rule for EPs. They constitute the clinical quality measures "specified by CMS" for the purposes of the ONC final rule (found elsewhere in this issue of the Federal Register) and are the measures that certified EHRs are required to be able to calculate. Of these, nine EP measures have preliminary electronic specifications for which we provided links for in the proposed rule. The remaining 35 clinical quality measures for EPs were electronically specified more recently and posted on the CMS Web site by the date of display of this final rule. We are finalizing only those measures for which there are available electronic specifications as of the date of display of this final rule. Although we are not finalizing all of 90 proposed clinical quality measures that were proposed for EPs in Table 3 (see 75 FR 1874-1889) of the proposed rule, because of lack of electronic specifications, our intent is to include all of them in our proposed Stage 2 requirements, or to propose alternative measures following a transparent process that includes appropriate consultation with stakeholders and other interested parties. In addition, we plan to add new measures to fill gaps where measures were not previously proposed, such as in behavior and mental health (e.g., depression and alcoholism). Certified EHR technology must be able to calculate each measure numerators, denominators and exclusions for each of the clinical quality measures finalized for the EHR incentive program. Table 6 conveys the applicable NQF measure number and PQRI implementation number (that is, the number used in the PQRI program to identify the measure as implemented in PQRI (for the 2010 PQRI measures list see https:// www.cms.gov/PQRI/Downloads/2010 PQRI MeasuresList 111309.pdf)), titlē, description, the owner/steward, and a link to existing electronic specifications. The NQF number is an identifying number that is associated with the NQF endorsed measure number. All of the clinical quality measures in Table 6 are NQF endorsed and have broad applicability to the range of Medicare designated specialties, and the services provided by EPs who render services to Medicare and Medicaid beneficiaries and many others. In terms of CMS and HHS healthcare quality priorities, clinical quality PQRI measures numbered 1, 2, 3, 5, and 7 address high priority chronic conditions, namely diabetes, coronary artery disease, and heart disease. Clinical quality PQRI measures numbered 66, 110, 111, 112, $113,114,115$, and 128 support screening and prevention all of which is a high CMS and HHS priority.

BILLING CODE 4120-01-P 


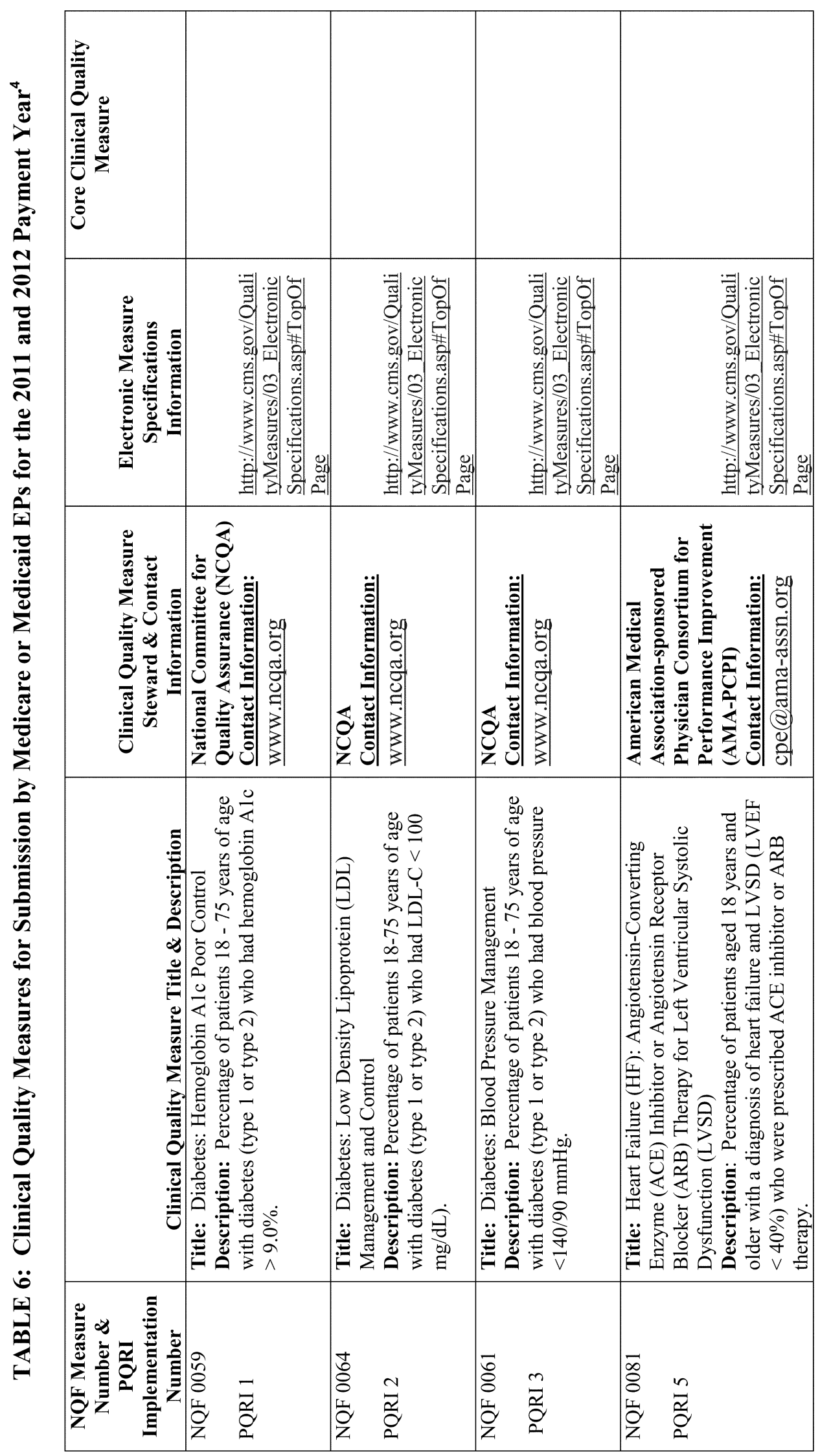

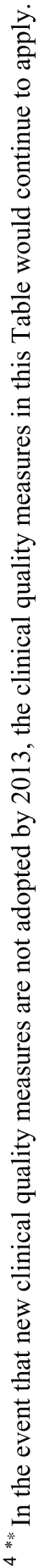




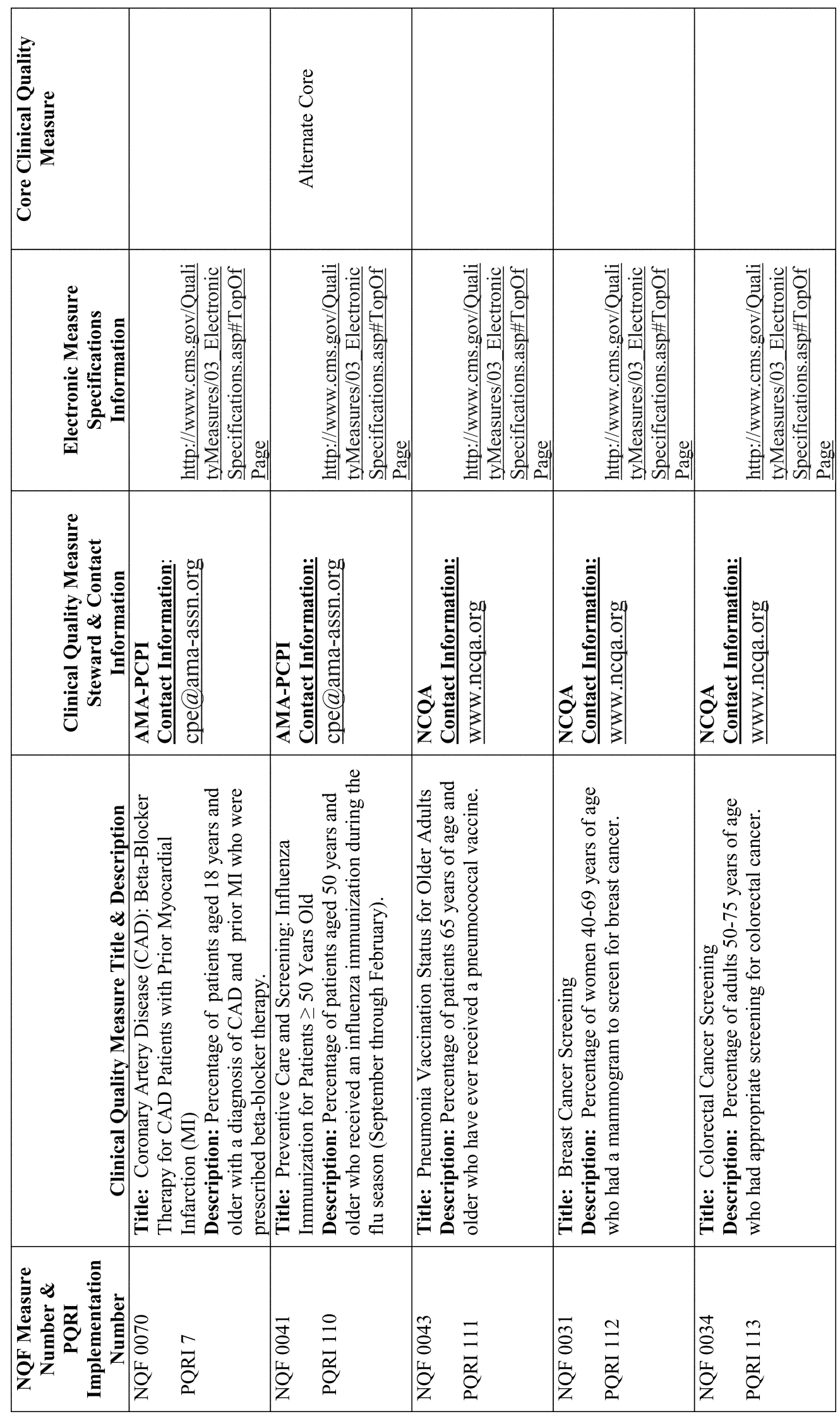




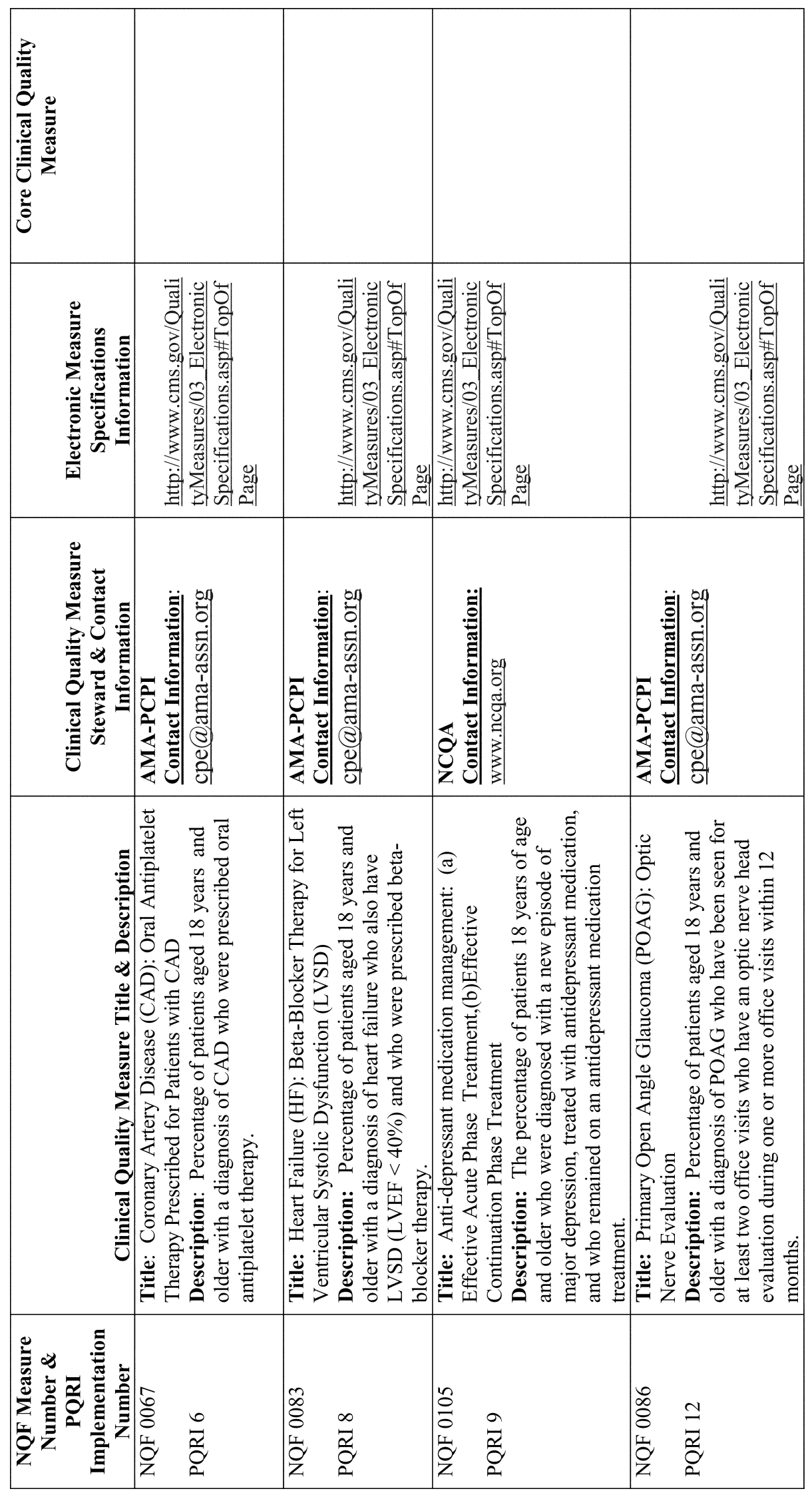




\begin{tabular}{|c|c|c|c|c|c|}
\hline 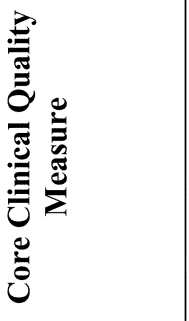 & & & & & \\
\hline 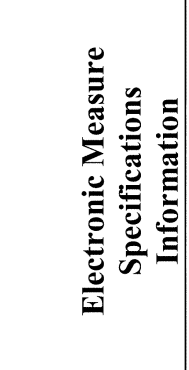 & & 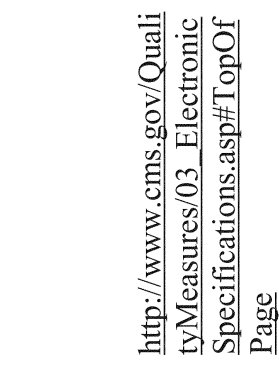 & 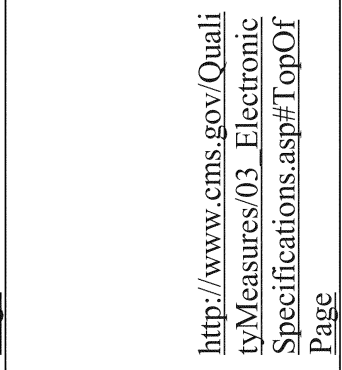 & 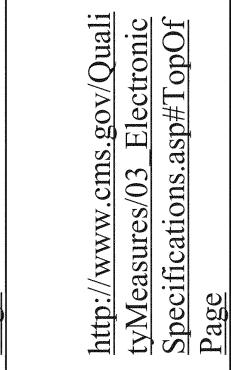 & 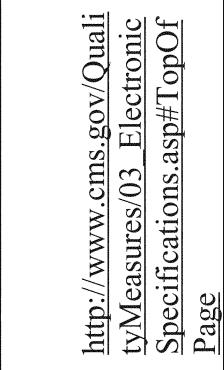 \\
\hline 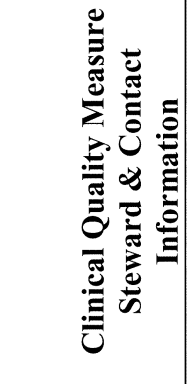 & 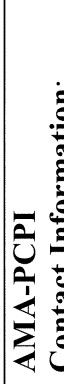 & : & 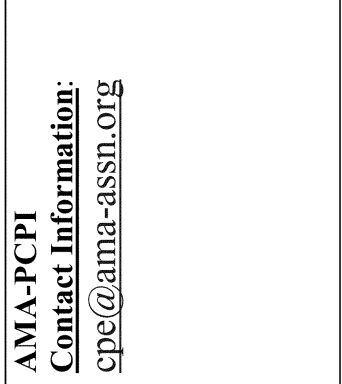 & 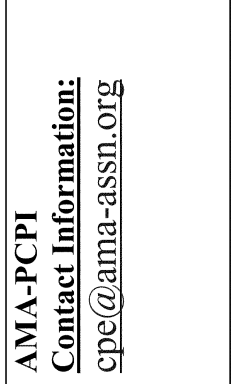 & 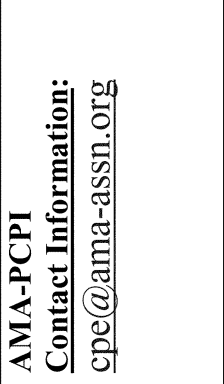 \\
\hline 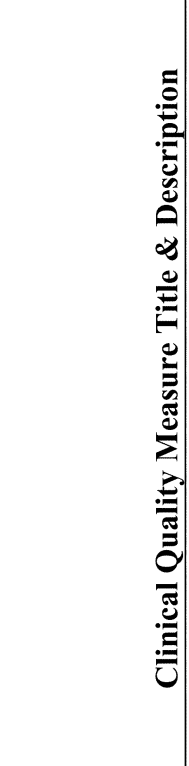 & & 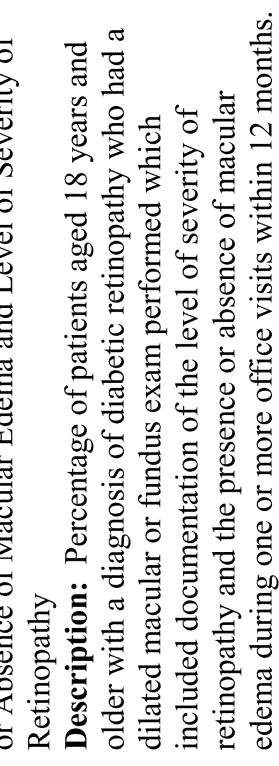 & 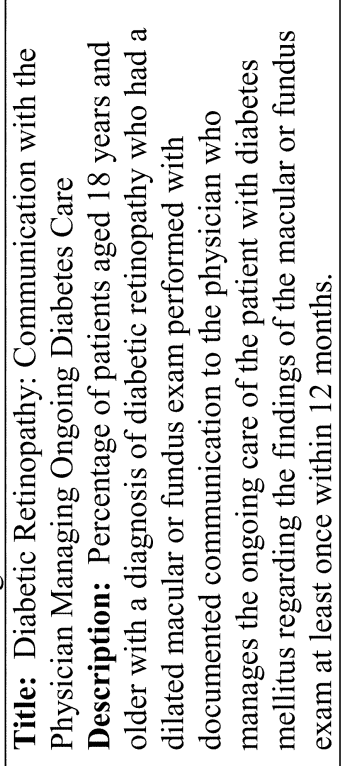 & 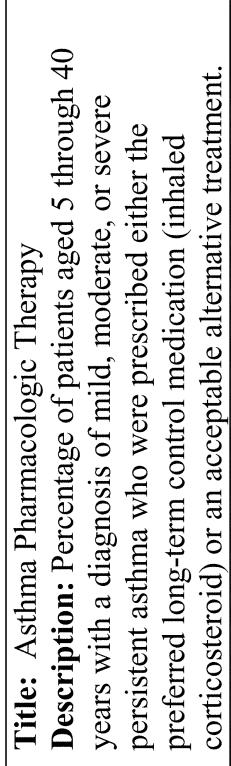 & 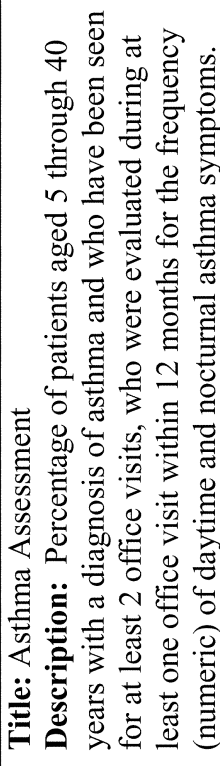 \\
\hline 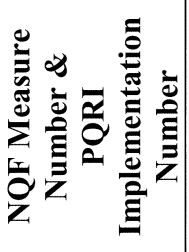 & & $\stackrel{\infty}{\approx}$ & 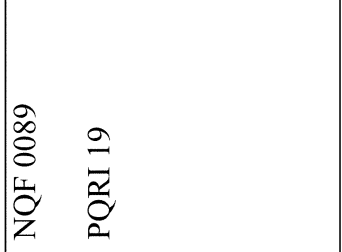 & 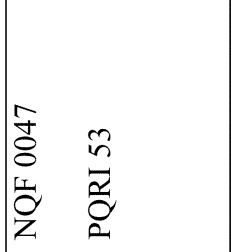 & 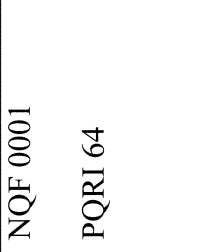 \\
\hline
\end{tabular}




\begin{tabular}{|c|c|c|c|c|c|c|c|c|}
\hline 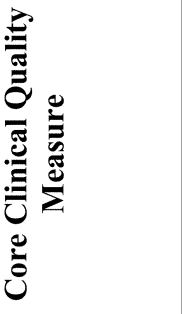 & \multirow{2}{*}{\multicolumn{2}{|c|}{ 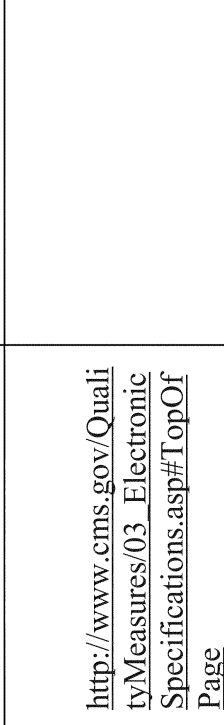 }} & \multirow{2}{*}{\multicolumn{2}{|c|}{ 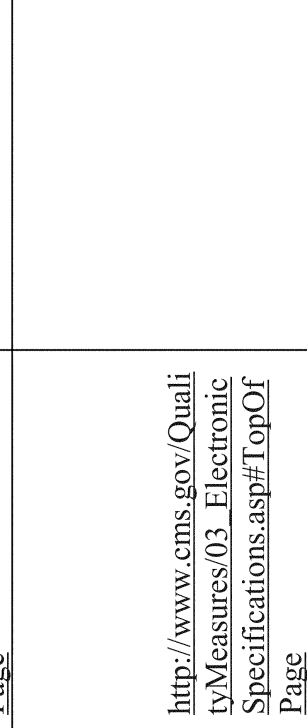 }} & \multirow{2}{*}{\multicolumn{2}{|c|}{ 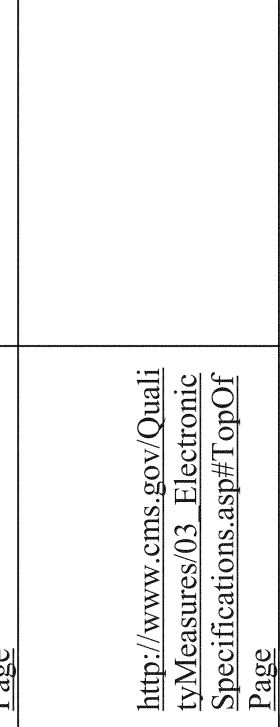 }} & \multirow{2}{*}{\multicolumn{2}{|c|}{ 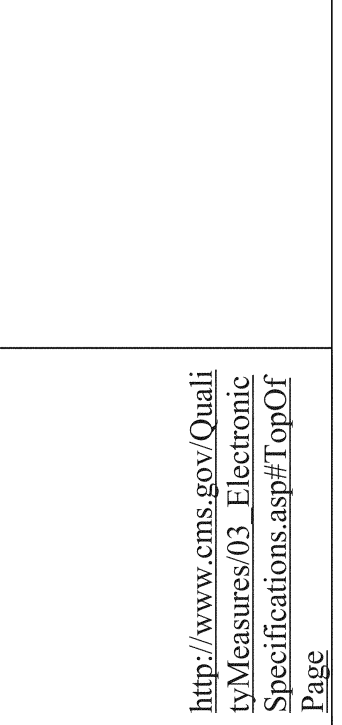 }} \\
\hline 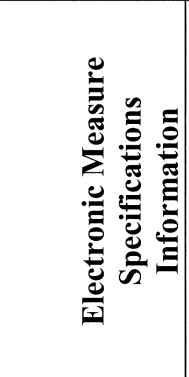 & & & & & & & & \\
\hline 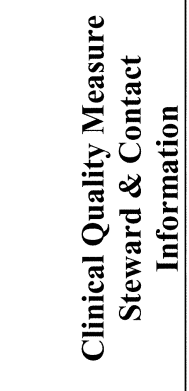 & \multicolumn{2}{|c|}{ 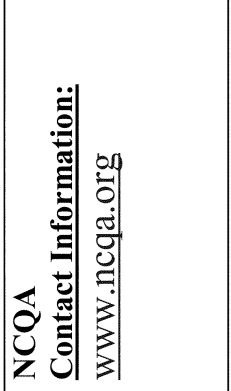 } & \multicolumn{2}{|c|}{ 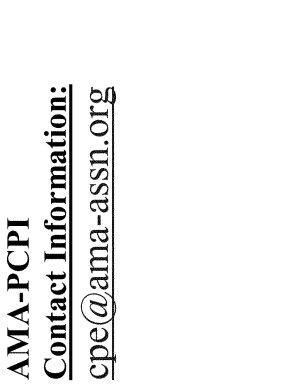 } & \multicolumn{2}{|c|}{ 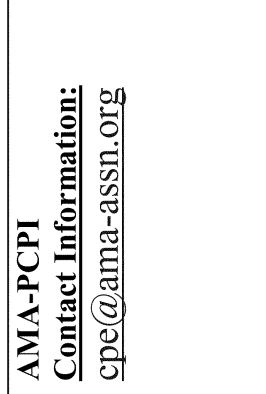 } & \multicolumn{2}{|l|}{ 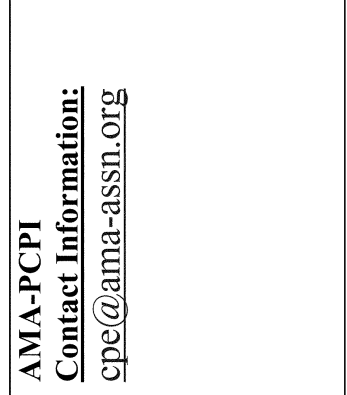 } \\
\hline 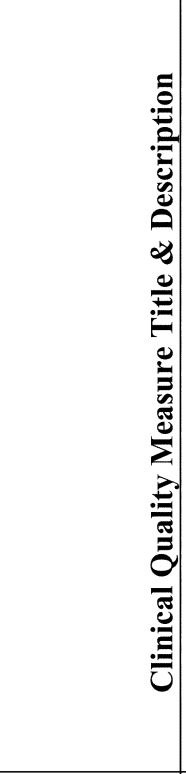 & \multicolumn{2}{|c|}{ 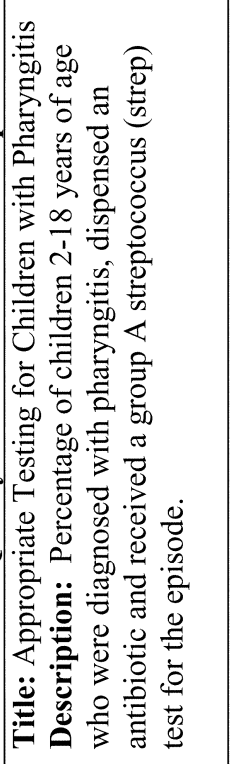 } & \multicolumn{2}{|c|}{ 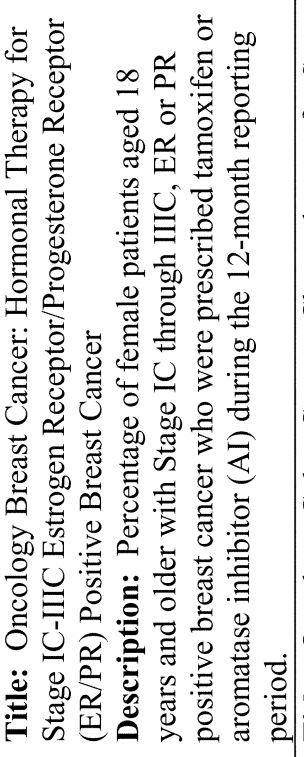 } & \multicolumn{2}{|c|}{ 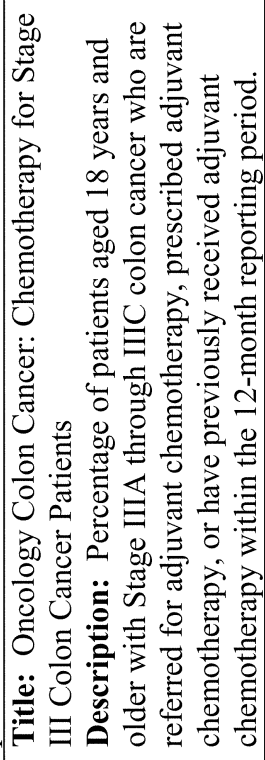 } & \multicolumn{2}{|c|}{ 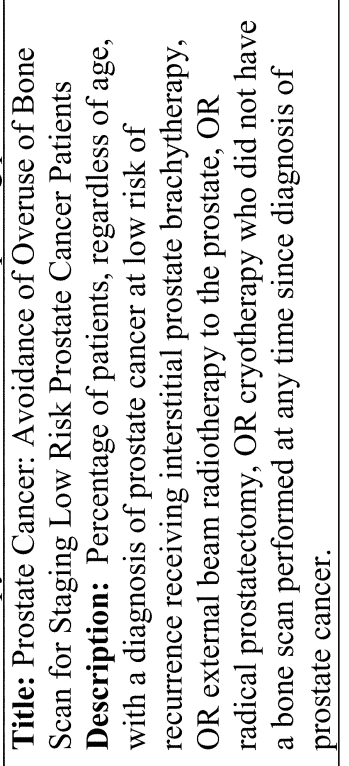 } \\
\hline 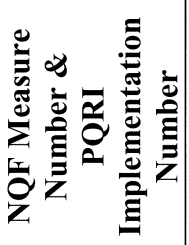 & 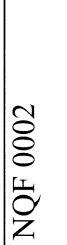 & $\begin{array}{l}\stackrel{8}{0} \\
\stackrel{\sigma}{\sigma} \\
\approx\end{array}$ & & 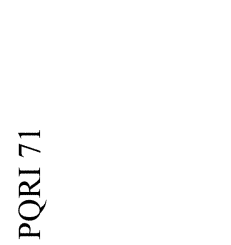 & $\begin{array}{l}\infty \\
0 \\
0 \\
\sigma \\
z\end{array}$ & $\stackrel{\approx}{\approx}$ & 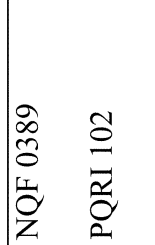 & \\
\hline
\end{tabular}




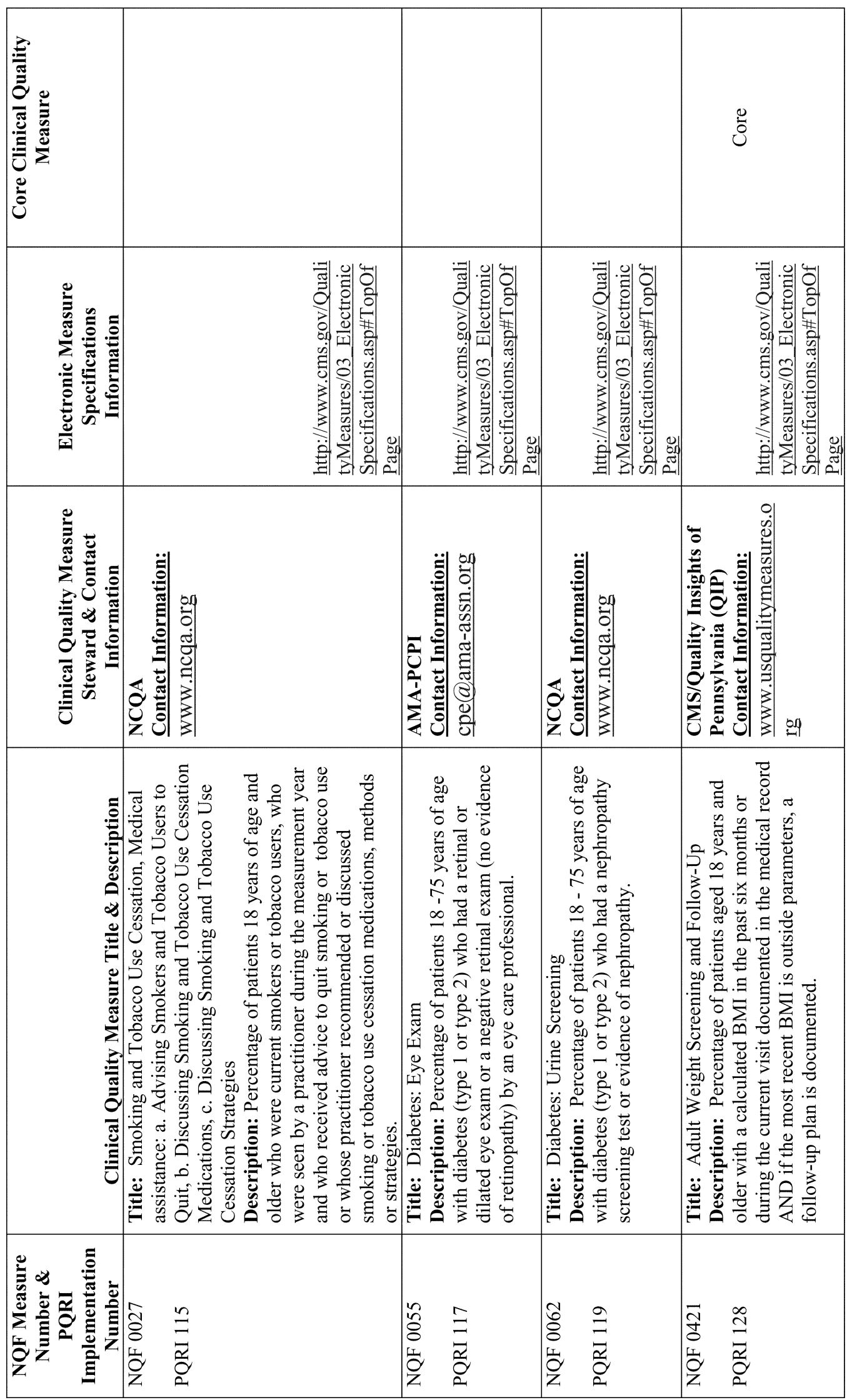




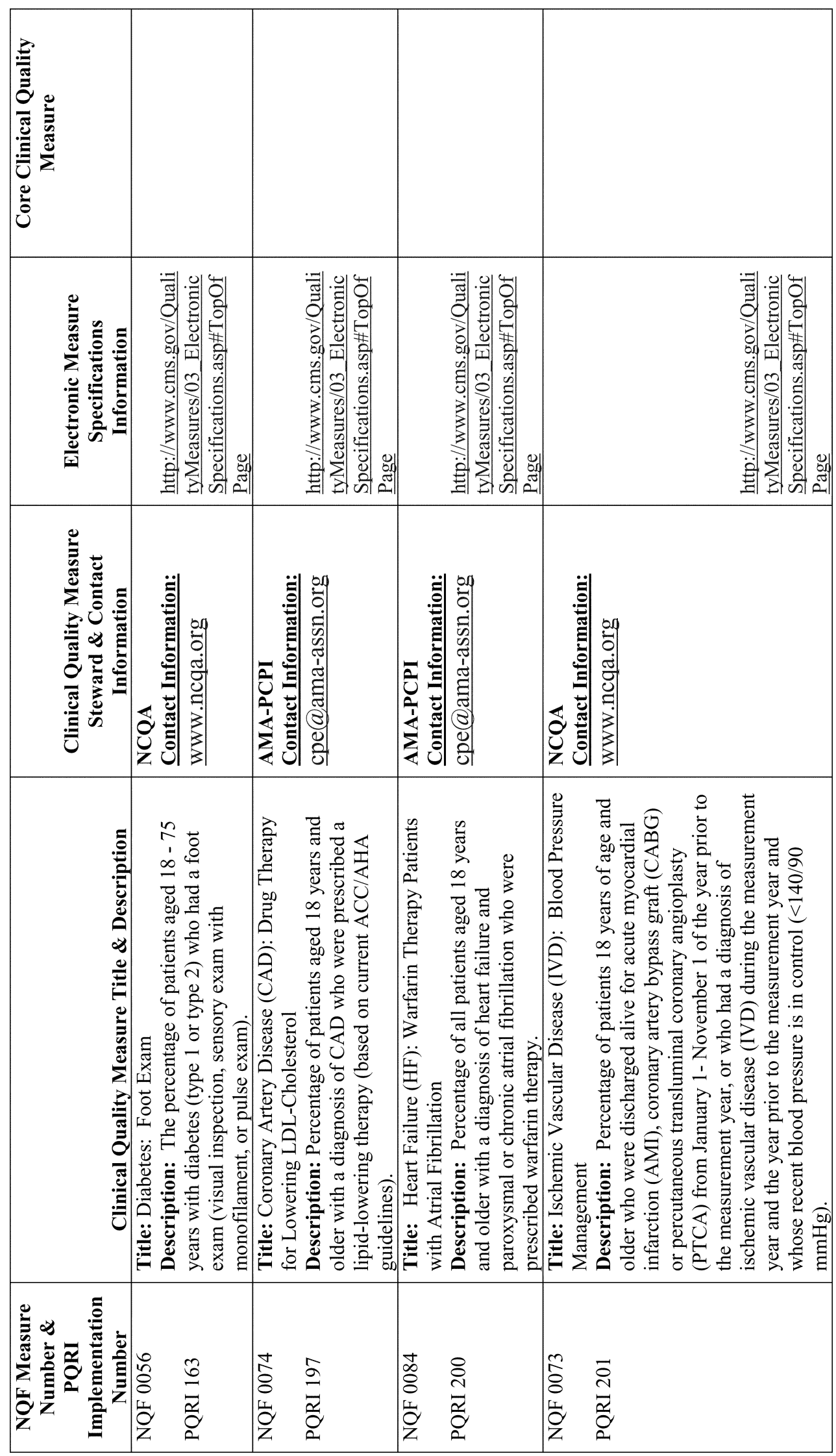




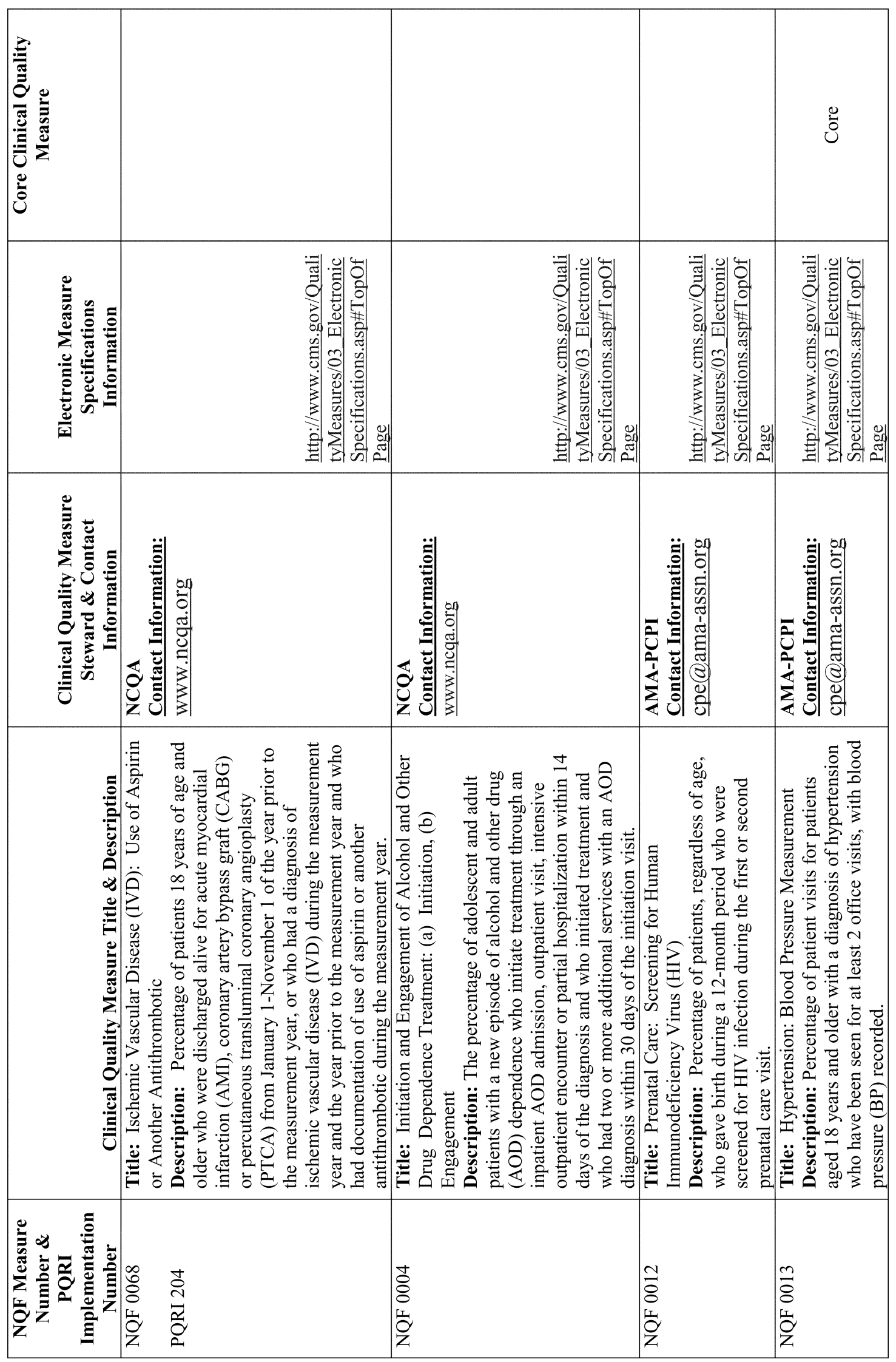




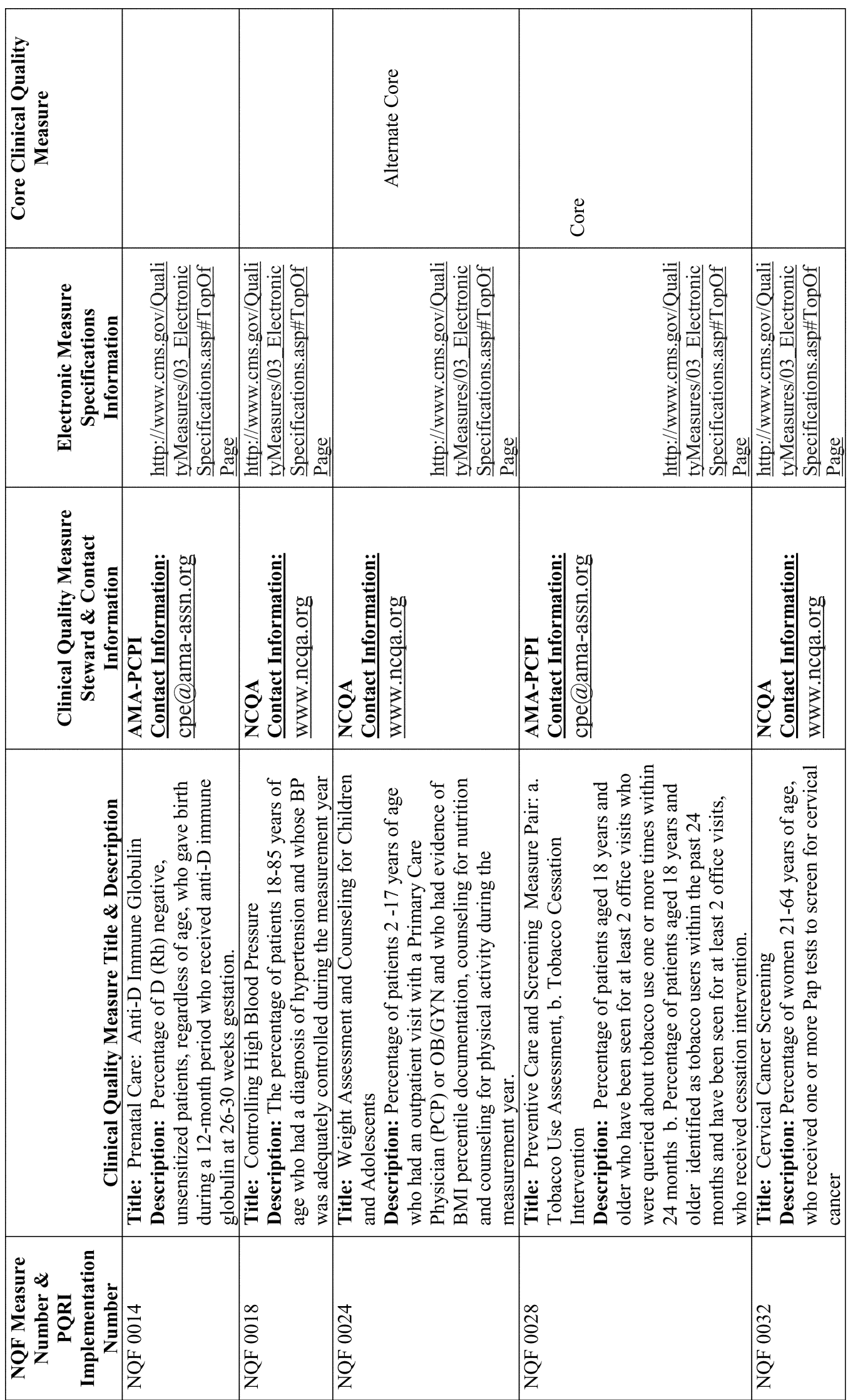




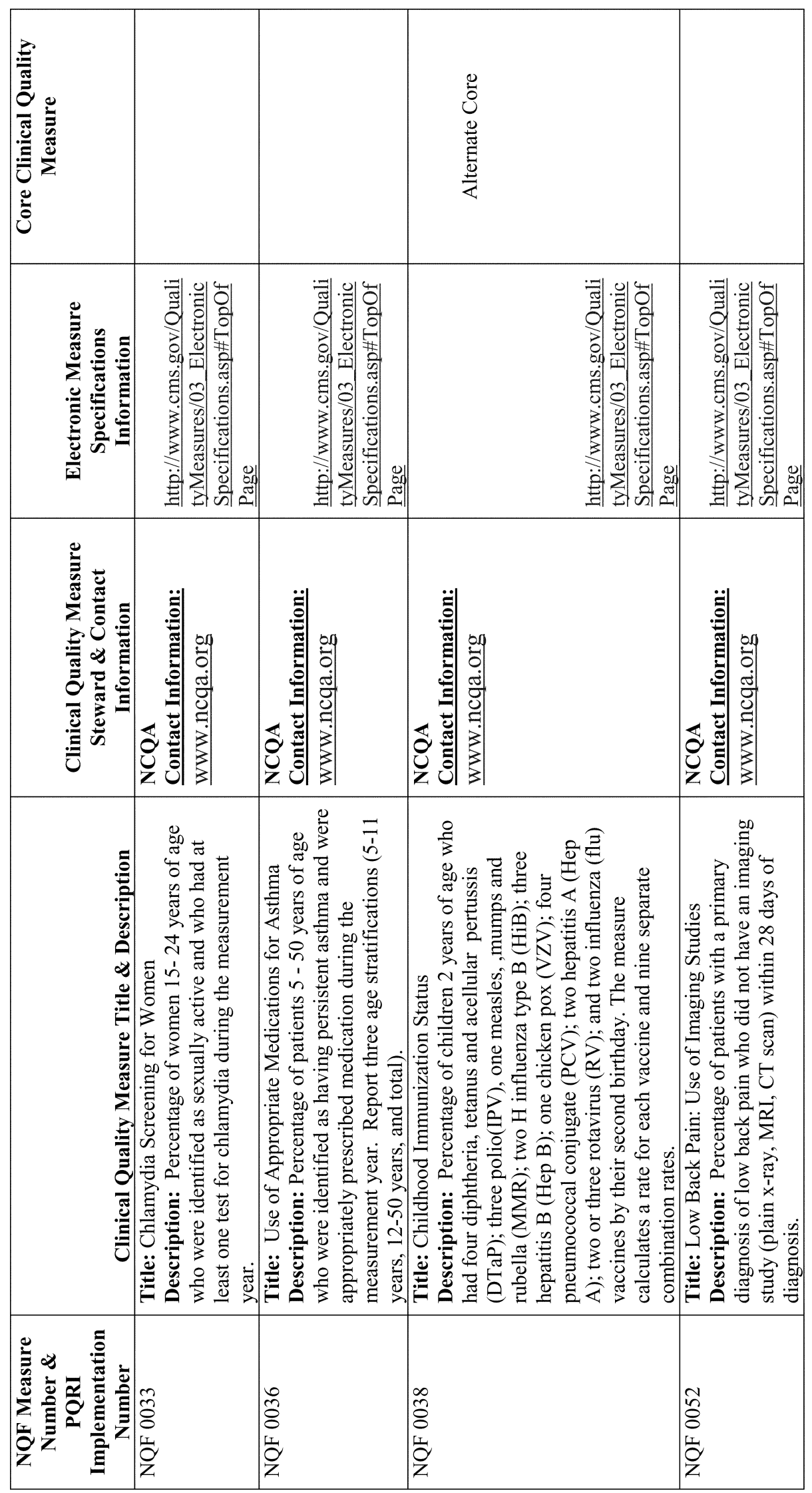




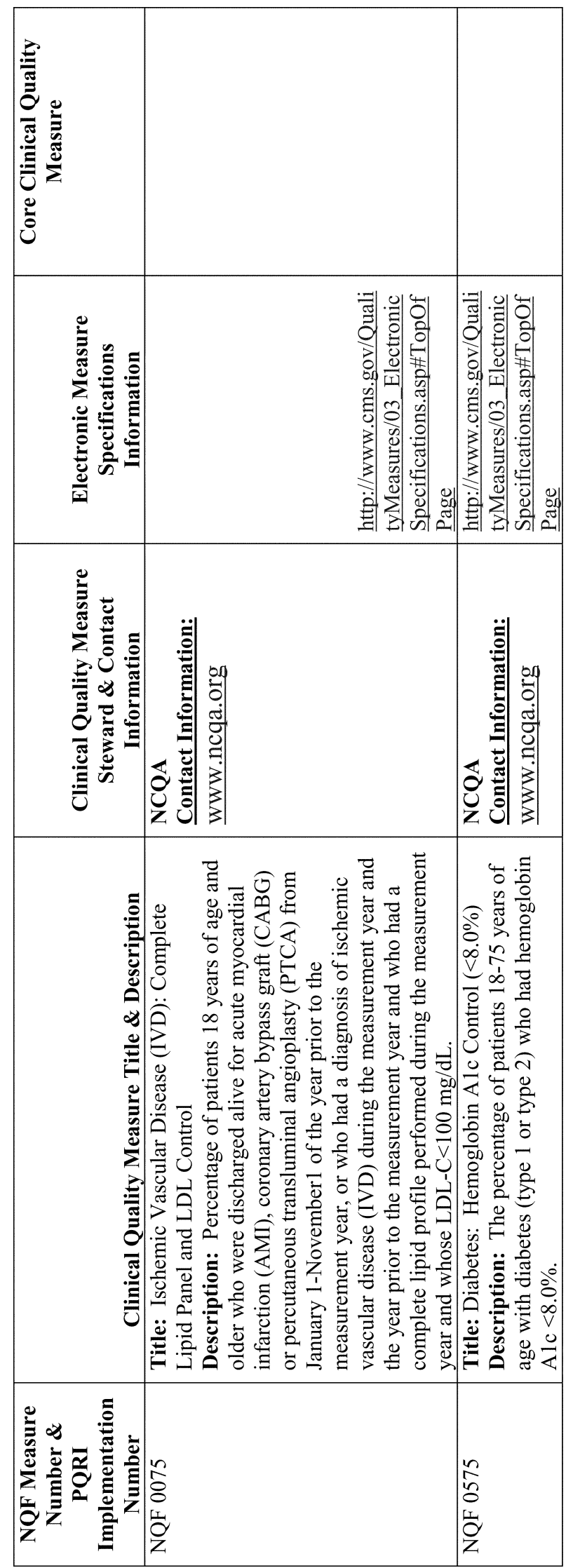


e. Clinical Quality Measures Reporting Criteria for EPs

For the 2011 and 2012 EHR reporting periods, to satisfy the requirements for reporting on clinical quality measures for Medicare under section 1848(o)(2)(A)(i) and (iii) of the Act and for Medicaid under section 1903(t)(6)(C) of the Act, we proposed to require that each EP submit information on two measure groups: a core measures group (Table 4 of the proposed rule see $75 \mathrm{FR}$ 1890), and the subset of clinical measures most appropriate given the EP's specialty (Tables 5 through 19 specialty group measures see $75 \mathrm{FR}$ 1891 through 1895). For the core measure group, we stated our belief that the clinical quality measures were sufficiently general in application and of such importance to population health; we would require that all EPs treating Medicare and Medicaid patients in the ambulatory setting report on all of the core measures as applicable for their patients.

We proposed that with the inclusion of measures applicable to targeting children and adolescents and the wide applicability of the measures like Blood Pressure Management, we believed the proposed core set of clinical quality measures and specialty measures was broad enough to enable reporting by all EPs. However, we encouraged commenters to identify the EPs in question and propose specific remedies if the public believed that other EPs would not have sufficient patients in the denominator of these core measures.

Comment: Several commenters requested clarification about the core measures group. Many comments were received regarding the inclusion of a core measure set for EPs. Some commenters favored the inclusion of one or more core measures (for example, preventive care) and others indicated core measures were essential for improving the quality of care. Conversely, numerous commenters suggested eliminating the core measure set for EPs. The primary reason offered by commenters for excluding core measures was that these clinical quality measures were outside their scope of practice and/or not relevant to their specific patient population. A commenter requested that the core set of clinical quality measures be better defined and/or increased for each reporting period. Many commenters indicated the clinical quality measures included in the core measure set are not appropriate to all EPs and specialists (for example, EPs that do not have direct physical access to the patients such as teleradioloists, EPs that do not routinely report blood pressure in patients with diagnosed hypertension, such as dermatologists) and they would not be able to report on these clinical quality measures. Many commenters supported reporting exclusions. A commenter recommended the use of PQRI 128/NQF 0421 Preventive Care and Screening: BMI Screening and Follow-up as a core clinical quality measure. Other commenters indicated these clinical quality measures were important for improving care and the core measure set should be expanded.

Response: After considering the comments, we agree there may be circumstances such that the core clinical quality measures are not applicable for specific patient populations and/or a specific EP's scope of practice. In such circumstances we anticipate that the patients will not appear in the denominator at all or will be excluded. We have defined the core measure set for EPs in Table 7 of this final rule, and these core measures will be required for Stage 1. We expanded the core measures set to include three alternate measures, as well as added PQRI 128/NQF0421 as a required core measure, based on commenters feedback. Although we require all EPs to report the core measures, there is no requirement that the $\mathrm{EP}$ have any particular number of patients in the denominator, which could be zero as calculated by the EHR. Therefore we have changed the reporting criteria to require EPs to report on all three core measures (as shown in Table 7, below) and three additional clinical quality measures selected from Table 6 (other than the core or alternate core measures listed in Table 6). The clinical quality measures included in this final rule reflect a subset of measures that were included in the proposed rule (see 75 FR 1874 through 1889). The clinical quality measures included in Table 6 of this final rule were selected from the Tables included in the proposed rule, based on having electronic specifications fully developed by the date of display of this final rule.

Comment: Many commenters indicated that NQF 0022 Drugs to be avoided in the elderly is an inappropriate clinical quality measure and should be removed. The rationale given for removal is that the numerator (at least one prescription for any drug to be avoided in the elderly in the measurement year or at least two different drugs to be avoided in the elderly in the measurement year) tends to be very small. Others considered poly-pharmacy a more significant problem in the elderly than avoidance of specific drugs. A number of commenters indicated this clinical quality measure should include a list of the drugs to be avoided.

Response: We agree with the concerns expressed by the commenters and have removed the measure NQF 0022.

Additionally, electronic specifications are not available for this measure by the date of display of this final rule making this measure impractical to use for Stage 1. We will consider this measure in future rulemaking.

After consideration of the public comments received, we are finalizing the requirement that all EPs must submit calculated results for three core measures using the certified EHR technology. However, we are finalizing only two of the clinical quality measures that were proposed as "core measures" in the proposed rule. The other core measures presented in Table 6 of this final rule were selected because they have broad applicability, support prevention, were recommended by commenters, and have electronic specifications by the date of display of this final rule. Insofar as a measure does not apply to patients treated by the EP, this will be reflected in the calculation of the clinical quality measure either by the patient not being included in the denominator for the measure or the patient being excluded. Therefore, it is not necessary for CMS to delineate for a particular specialty which measures may or not apply. We note that to qualify as a meaningful EHR user, EPs need only report the required clinical quality measures; they need not satisfy a minimum value for any of the numerator, denominator, or exclusions fields for clinical quality measures. The value for any or all of those fields, as reported to CMS or the States, may be zero if these are the results as displayed by the certified EHR technology. Thus, the clinical quality measure requirement for 2011 and beginning in 2012 is a reporting requirement and not a requirement to meet any particular performance standard for the clinical quality measure, or to in all cases have patients that fall within the denominator of the measure.

The three core measures that EPs will be required to report are: [NQF 0013: Hypertension: Blood Pressure Management; NQF 0028: Preventative Care and Screening Measure Pair: a. Tobacco Use Assessment b. Tobacco Cessation Intervention; and NQF0421/ PQRI 128: Adult Weight Screening and Follow-up]. Insofar as the denominator for one or more of the core measures is zero, EPs will be required to report results for up to three alternate core measures [NQF 0041/PQRI 110: Preventative Care and Screening: 
Influenza Immunization for Patients $\geq 50$ Years Old; NQF 0024: Weight Assessment and Counseling for Children and Adolescents; and NQF 0038: Childhood Immunization Status]. We believe this final set of core clinical quality measures provides EPs a greater opportunity for successful reporting. The EP will not be excluded from reporting any core or alternate clinical quality measure because the measure does not apply to the EPs scope of practice or patient population. The expectation is that the EHR will automatically report on each core clinical quality measure, and when one or more of the core measures has a denominator of zero then the alternate core measure(s) will be reported. If all six of the clinical quality measures in Table 7 have zeros for the denominators (this would imply that the EPs patient population is not addressed by these measures), then the EP is still required to report on three additional clinical measures of their choosing from Table 6 in this final rule. In regard to the three additional clinical quality measures, if the EP reports zero values, then for the remaining clinical quality measures in Table 6 (other than the core and alternate core measures) the EP will have to attest that all of the other clinical quality measures calculated by the certified EHR technology have a value of zero in the denominator, if the
EP is to be exempt from reporting any of the additional clinical quality measures (other than the core and alternate core measures) in Table 6. Thus, EPs are not penalized in the Stage 1 reporting years as long as they have adopted a certified EHR and that EHR calculates and the EP submits the required information on the required clinical quality measures, and other meaningful use requirements as defined in this final rule in section II.A.2.d.1 of this final rule.

Table 7, below, shows the core measure groups for all EPs for Medicare and Medicaid to report.

\section{TABLE 7: Measure Group: Core for All EPs, Medicare and Medicaid}

\begin{tabular}{|l|l|}
\hline $\begin{array}{c}\text { NQF Measure Number } \\
\text { \& PQRI } \\
\text { Implementation } \\
\text { Number }\end{array}$ & \multicolumn{1}{c|}{ Clinical Quality Measure Title } \\
\hline NQF 0013 & Title: Hypertension: Blood Pressure Measurement \\
\hline NQF 0028 & $\begin{array}{l}\text { Title: Preventive Care and Screening Measure Pair: a. Tobacco Use Assessment } \\
\text { b. Tobacco Cessation Intervention }\end{array}$ \\
\hline $\begin{array}{l}\text { NQF 0421 } \\
\text { PQRI 128 }\end{array}$ & Title: Adult Weight Screening and Follow-up \\
\hline NQF 0024 & Alternate Core Measures \\
\hline $\begin{array}{l}\text { NQF 0041 } \\
\text { PQRI 110 }\end{array}$ & $\begin{array}{l}\text { Title: Weight Assessment and Counseling for Children and Adolescents } \\
\text { Years Old }\end{array}$ \\
\hline NQF 0038 & Title: Childhood Immunization Status \\
\hline
\end{tabular}

We proposed that EPs were to submit calculated results on at least one of the sets listed in Tables 5 and 19 as specialty groups (see 75 FR 1891-1895). The specialty groups were Cardiology, Pulmonary Diseases, Endocrinology, Oncology, Proceduralist/Surgery, Primary Care Physicians, Pediatrics, Obstetrics and Gynecology, Neurology, Psychiatry, Ophthalmology, Podiatry, Radiology, Gastroenterology, and Nephrology.

We recognized that clinical quality measures as specified by measures developers and as endorsed by the NQF were not specific to particular specialties. Rather, the denominator of clinical quality measures and the applicability of a measure is determined by the patient population to whom the measure applies and the services rendered by the particular EP.

Nevertheless, we grouped the proposed measures according to the types of patients commonly treated and services rendered by EPs of various specialties. We did this for purposes similar to measures groups used in PQRI which, however, are based on clinical conditions, rather than specialty types. We proposed that the general purpose of each specialty measures grouping was to have standardized sets of measures, all of which must be reported by the EP for the self-selected specialty measures groups in order to meet the reporting requirements. We expected to narrow down each set to a required subset of three-five measures based on the availability of electronic measure specifications and comments received.
We also proposed to require for 2011 and 2012 that EPs would select a specialty measures group, on which to report on all applicable cases for each of the measures in the specialty group. We also proposed that the same specialty measures group selected for the first payment year would be required for reporting for the second payment year. We invited comment on whether there were EPs who believed no specialty group would apply to them. In accordance with public comments, we noted that we would specify in the final rule which EP specialties would be exempt from selecting and reporting on a specialty measures group. As stated, we proposed, EPs that are so-designated would be required to attest, to CMS or the States, to the inapplicability of any of the specialty groups and would not 
be required to report information on clinical quality measures from a specialty group for 2011 or 2012 , though the EP would still be required to report information on all of the clinical quality measures listed in the proposed core measure set (see 75 FR 1890).

Comment: Several commenters asked if certain specialties, such as chiropractors, audiologists, allergist and immunology, otolaryngologists, etc., could be exempt from having to report all specific clinical quality specialty measures. Many of these EPs indicated the clinical quality measures included in Table 3 were not relevant to their specific practice and/or patient population. Other commenters requested that specialty groups be created for specialties not included in the proposed rule measure groups, (for example, chiropractors, dentists, dermatologists, infectious disease, pediatric oncology, neurosurgery, interventional radiology, plastic \& reconstructive surgery, physical therapists, occupational therapists, eye care specialists, family planning, genetics, ear/nose/throat, and nutritionists providers, etc.). Other commenters indicated that specialty clinical quality measures were specific to a subset of patients, but were not broadly applicable to their specialty for treating other conditions within their specialty area. Other commenters asked that CMS reconsider allowing EPs to attest only and be exempt from reporting if no applicable clinical quality measures specialty group exists for them. Another commenter indicated support of specific measure sets for different clinical specialties. Many commenters supported the elimination of specialty groups altogether as a mandatory set and instead supported the reporting of a fixed number of relevant clinically quality measures regardless of the specialty group. A commenter asked for a definition of "specialist" which is not included in the proposed rule. Several commenters expressed concern about the large number of clinical quality measures in certain measure groups versus other measure groups (for example, the primary care, pediatric and ob/gyn measure groups) as well as the applicability of clinical quality measures assigned to primary care EPs when they do not manage conditions that are typically referred to a specialist for example, ischemic vascular disease. A commenter requested clarification and suggestions on how to select a clinical quality measure group. Several commenters wanted clarification on the proposed EP Specialty Measures Tables (see 75 FR 1874), and whether the EPs are accountable for only the clinical quality measures for their specialty. One comment indicated agreement with CMS regarding requiring EPs to report on the same specialty measure groups for 2011 and 2012 and another commenter indicated that CMS should not delay reporting of clinical quality measures as early adopters of EHRs will be ready to report. A few commenters suggested adding NQF 0033 Chlamydia screening in women to all other appropriate specialty clinical quality measure groups. A commenter indicated that PQRI \#112, 113, and NQF 0032 should be removed from the oncology clinical quality specialty measure group as oncologists do not perform routine cancer screenings.

Response: We are appreciative of the detail provided by commenters to the potential inapplicability of the proposed specialty measures groups to various practitioner types or to the inapplicability of certain measures within groups to the specialties designated. Our primary purpose, similar to the core measures, was to encourage a certain consistency in reporting of clinical quality measures by EPs. However, after consideration of the comments we do not believe that the proposed specialty measures groups are sufficient to have a robust set of specialty measures groups. Further, given the lack of electronic specifications or final development of many of these measures, requiring specialty measures groups becomes even more impractical. We expect that electronic specifications will be developed for measures which would allow for a broadly applicable set of specialty measures groups in the future. After consideration of the public comments received, we removed the requirement for EPs to report on specialty measures groups as proposed. We intend to reintroduce the proposed rule's specialty group reporting requirement in Stage 2 with at least as many clinical quality measures by specialty as we proposed for Stage 1 in the proposed rule. We expect to use a transparent process for clinical quality measure development that includes appropriate consultation with specialty groups and other interested parties, and we expect that electronic specifications will be developed for all of the measures that we originally proposed for Stage 1 or alternative related measures, which would allow for a broadly applicable set of specialty measures groups and promote consistency in reporting of clinical quality measures by EPs. Also, in consideration of public comments received, we are finalizing the requirement (in addition to the core measure requirement) that EPs must report on three measures to be selected by the EP from the set of 38 measures as shown in Table 6, above. As stated previously, in regard to the three additional clinical quality measures, if the EP reports zero values, then for the remaining clinical quality measures in Table 6 (other than the core and alternate core measures) the EP will have to attest that all of the other clinical quality measures calculated by the certified EHR technology have a value of zero in the denominator. In sum, EPs must report on six total measures, three core measures (substituting alternate core measures where necessary) and three additional measures (other than the core and alternate core measures) selected from Table 6.

We also proposed that although we do not require clinical quality measure reporting electronically until 2012, we would require clinical quality reporting through attestation in the 2011 payment year. We solicited comment on whether it may be more appropriate to defer some or all clinical quality reporting until the 2012 payment year. If reporting on some but not all measures in 2011 was feasible, we solicited comment on which key measures should be chosen for 2011 and which should be deferred until 2012 and why. We discuss comments received regarding the reporting method for clinical quality measures in section II.A.3.h. of this final rule.

f. Clinical Quality Measures for Electronic Submission by Eligible Hospitals and CAHs

Our proposed rule would have required eligible hospitals and CAHs to report summary data to CMS on the set of clinical quality measures identified in Table 20 and 21 of the proposed rule (see 75 FR 1896-1899), with eligible hospitals attesting to the measures in 2011 and electronically submitting these measures to CMS using certified EHR technology beginning in 2012. For hospitals eligible for only the Medicaid EHR incentive program, we proposed that reporting would be to the States. In the proposed rule, for eligible hospitals under both programs, we proposed that they would have to also report on the clinical quality measures identified in Table 21 of the proposed rule to meet the requirements for the reporting of clinical quality measures for the Medicaid program incentive (see 75 FR 1896 through 1900). Tables 20 and 21 of the proposed rule (see 75 FR 1896 through 1900) conveyed the clinical quality measure's title, number, owner/ 
developer and contact information, and a link to existing electronic specifications where applicable.

We included in the proposed hospital measures set several clinical quality measures which have undergone development of electronic specifications. These clinical quality measures have been developed for future RHQDAPU consideration. The electronic specifications were developed through an interagency agreement between CMS and ONC to develop interoperable standards for EHR electronic submission of the Emergency Department Throughput, Stroke, and Venous Thromboembolism clinical quality measures on Table 20 of the proposed rule (see 75 FR 1896 through 1899). We also proposed to test the submission of these clinical quality measures in Medicare (see 75 FR 43893). The specifications for the RHQDAPU clinical quality measures for eligible hospitals and CAHs that are being used for testing EHR-based submission of these clinical quality measures can be found at http:// www.hitsp.org/ConstructSet Details.aspx? $\mathcal{E}$ PrefixAlpha $=\overline{5} \mathcal{E}$ PrefixNumeric $=906$ (A description of the clinical quality measure, including the clinical quality measure's numerator and denominator, can be found here as well.) Other measures we proposed derived from the RHQDAPU program or were measures we considered important for measuring or preventing adverse outcomes. In addition to risk standardized readmission clinical quality measures, we proposed that nonrisk-adjusted readmission rates also be reported. For the proposed rule, we also considered HIT Standards Committee recommendations, including the Committee's recommendation to include a measure on Atrial Fibrillation Receiving Anticoagulation Therapy which was included on Table 20 of the proposed rule Our proposed rule noted that we did not propose one measure recommended by the HIT Standards Committee: Surgery patients who received Venous Thromboembolism prophylaxis within 24 hours period to surgery to 24 hours after surgery end time. We noted that the measure is a current clinical quality measure collected in the RHQDAPU program through chart abstraction for all applicable patients (SCIP-VTE-2), and that the VTE-2 clinical quality measure in Table 20 of the proposed rule (see 75 FR 1896 through 1899) was a parallel clinical quality measure to SCIP-VTE2. SCIP-VTE-2 includes surgical and non-surgical patients, and can be more easily implemented for the EHR incentive program because electronic specifications had been completed. We added SCIP-VTE-2 for future consideration.

Comment: Many commenters recommended reducing the number of eligible hospital clinical quality measures and indicated that such a large number of measures would pose a significant financial and administrative burden on hospitals. Commenters suggested a variety of solutions which include: Eliminating duplication between clinical quality measures and meaningful use objectives and associated measures, reducing the number of clinical quality measures for reporting and allowing organizations to select a limited number of clinical quality measures on which they would like to report.

We received comments supporting many of the measures in the proposed rule including Venous Thromboembolism, Emergency Department, Stroke, RHQDAPU, and measures that are evidence-based that could improve the quality of care. Others recommended additional clinical quality measures, changes to the specifications for clinical quality measures or the elimination of certain clinical quality measures such as risk adjusted re-admission measures or measures not applicable to CAHs. Many commenters supported the process through which the electronic

specifications were developed for the Emergency Department Throughput, Stroke and Venous Thromboembolism measures while also pointing out the length of time necessary to adequately develop electronic specifications and test the clinical quality measures. Many commented that the remaining measures had not been electronically specified or had otherwise not completed

development and would not be ready in time for the 2011-2012 implementation. Others stated their concerns about duplicate reporting systems and the belief that the HITECH Act reporting requirements should be based on the RHQDAPU program, similar to the conceptual framework of hospitals value-based purchasing plan. Others pointed to measures that are already currently reported in RHQDAPU and the statutory provision that clinical quality measure reporting required for the HITECH Act should seek to avoid duplicative and redundant reporting of measures reported under RHQDAPU.

Response: We are appreciative of the comments supporting many of the clinical quality measure sets and the process utilized for electronically specifying the Emergency Department Throughput, Stroke, and Venous
Thromboembolism sets. As we have discussed for the EP measures, we agree that we should limit the required clinical quality measures to those measures for where there are electronic specifications as of the date of display of this final rule. This will allow EHR vendors sufficient time to ensure that certified EHR technology will be able to electronically calculate the measures. Therefore, we are not finalizing those clinical quality measures that either have not been fully developed, are currently only specified for claims based calculation, or for which there are not fully developed electronic specifications as of the date of display of this final rule. Accordingly, we are only finalizing the 15 measures listed in Table 10 of this final rule. We note that none of these measures are duplicate measures which are currently required for reporting in the RHQDAPU program. We therefore do not need to address the issue of duplicate or redundant reporting. We will consider adding, changing, developing, and eliminating duplicative clinical quality measures and meaningful use objectives/ associated measures in future rulemaking.

Table 8, shows the proposed clinical quality measures for submission by Medicare and Medicaid Eligible Hospitals for the 2011 and 2012 payment year as stated in the proposed rule (see 75 FR 1896-1899) for EPs, but that are not being finalized. Table 9 , shows the proposed alternative Medicaid clinical quality measures for Medicaid eligible hospitals in the proposed rule (see 75 FR 1899-1900). Tables 8 and 9 convey the NQF measure number, clinical quality measure title and description, and clinical quality measure steward and contact information. The measures listed below in Tables 8 and 9 do not have electronic specifications finished before the date of display of this final rule, thus we have eliminated these measures for this final rule and will consider the addition of these measures in future rulemaking. Also several measures listed below were only concepts at the time of publication of the proposed rule (that is, Hospital Specific 30 day Rate following AMI admission, Hospital Specific 30 day Rate following Heart Failure admission, Hospital Specific 30 day Rate following Pneumonia admission, and All-Cause Readmission Index). These concept measures were not developed or electronically specified clinical quality measures, nor NQF endorsed; and there was not adequate time to consider these concepts for development for this final rule. Therefore, the concepts listed 
below will be considered in future

rulemaking.

BILLING CODE 4120-01-P

TABLE 8: Proposed Clinical Quality Measures for Submission by Medicare or Medicaid Eligible Hospitals for the 2011 and 2012 Payment Year; Included in the Proposed Rule (see 75 FR 1896 through 1899) and Not in the Final Rule

\begin{tabular}{|c|c|}
\hline $\begin{array}{c}\text { Measure } \\
\text { Number } \\
\text { Identifier }\end{array}$ & Measure Title, Description \& Measure Developer \\
\hline Emergency & Title: Emergency Department Throughput - discharged patients \\
\hline Department & Median Time from ED Arrival to ED Departure for Discharged ED \\
\hline (ED)-3 & Patients \\
\hline \multirow{3}{*}{ NQF 0496} & Description: Median Time from ED arrival to time of departure from the \\
\hline & ED for patients discharged from the ED \\
\hline & Measure Developer: CMS/OFMQ \\
\hline RHQDAPU & Title: Primary PCI Received Within 90 Minutes of Hospital Arrival \\
\hline AMI-8a & $\begin{array}{l}\text { Description: Acute myocardial infarction (AMI) patients with ST- } \\
\text { segment elevation or LBBB on the ECG closest to arrival time receiving }\end{array}$ \\
\hline \multirow[t]{3}{*}{ NQF 0163} & primary PCI during the hospital stay with a time from hospital arrival to \\
\hline & PCI of 90 minutes or less \\
\hline & Measure Developer: CMS/OFMQ \\
\hline RHQD & Title: Blood Cultures Performed in the Emergency Department Prior to \\
\hline $\mathrm{PN}-3 \mathrm{~b}$ & Initial Antibiotic Received in Hospital \\
\hline \multirow{4}{*}{ NQF 0148} & Description: Pneumonia patients whose initial emergency room blood \\
\hline & culture specimen was collected prior to first hospital dose of antibiotics. \\
\hline & $\begin{array}{l}\text { This measure focuses on the treatmer } \\
\text { Department patients prior to admissic }\end{array}$ \\
\hline & Measure Developer: CMS/OFMQ \\
\hline RHQDAPU & Title: Aspirin Prescribed at Discharge \\
\hline AMI-2 & $\begin{array}{l}\text { Description: Acute myocardial infarction (AMI) patients who are } \\
\text { prescribed aspirin at hospital discharge }\end{array}$ \\
\hline NQF & Measure Developer: CMS/OFMQ \\
\hline RHQDAPU & Title: Angiotensin Converting Enzyme Inhibitor(ACEI) or Angiotensin \\
\hline AMI-3 & $\begin{array}{l}\text { Receptor Blocker (ARB) for Left Ventricular Systolic } \\
\text { Dysfunction (LVSD) }\end{array}$ \\
\hline NQF 0137 & Description: Acute myocardial infarction (AMI) patients with left \\
\hline & ventricular systolic dysfunction (LVSD) who are prescribed an ACEI or \\
\hline & ARB at hospital discharge. For purposes of this measure, LVSD is defined \\
\hline & $\begin{array}{l}\text { as chart documentation of a left ventricular ejection fraction (LVEF) less } \\
\text { than } 40 \% \text { or a narrative description of left ventricular systolic (LVS) }\end{array}$ \\
\hline & $\begin{array}{l}\text { function consistent with moderate or severe systolic dysfunction. } \\
\text { Measure Developer: CMS/OFMQ }\end{array}$ \\
\hline RHQDAPU & Title: Beta-Blocker Prescribed at Discharge \\
\hline AMI-5 & $\begin{array}{l}\text { Description: Acute myocardial infarction (AMI) patients who are } \\
\text { prescribed a betablocker at hospital discharge }\end{array}$ \\
\hline NQF 0160 & Measure Developer: CMS/OFMQ \\
\hline
\end{tabular}




\begin{tabular}{|c|c|}
\hline $\begin{array}{l}\text { Measure } \\
\text { Number } \\
\text { Identifier }\end{array}$ & Measure Title, Description \& Measure Developer \\
\hline RHQDAPU & Title \&Description: Hospital Specific 30 day Risk-Standardized \\
\hline AMI-READ & Readmission Rate following AMI admission \\
\hline NQF 0505 & Measure Developer: CMS \\
\hline $\begin{array}{l}\text { Not } \\
\text { applicable }\end{array}$ & Title: Hospital Specific 30 day Rate following AMI admission \\
\hline RHQDAPU & Title \&Description: Hospital Specific 30 day Risk-Standardized \\
\hline HF-READ & Readmission Rate following Heart Failure admission \\
\hline NQF 0330 & Measure Developer: CMS/OFMQ \\
\hline $\begin{array}{l}\text { Not } \\
\text { applicable }\end{array}$ & Title: Hospital Specific 30 day Rate following Heart Failure admission \\
\hline $\begin{array}{l}\text { RHQDAPU } \\
\text { PNE-READ } \\
\text { NQF } 0506\end{array}$ & $\begin{array}{l}\text { Title \&Description: Hospital Specific } 30 \text { day Risk-Standardized } \\
\text { Readmission Rate following Pneumonia admission } \\
\text { Measure Developer: CMS }\end{array}$ \\
\hline $\begin{array}{l}\text { Not } \\
\text { applicable }\end{array}$ & Title: Hospital Specific 30 day Rate following Pneumonia admission \\
\hline NQF 0528 & $\begin{array}{l}\text { Title: Infection SCIP Inf-2 Prophylactic antibiotics consistent with } \\
\text { current recommendations } \\
\text { Description: Surgical patients who received prophylactic antibiotics } \\
\text { consistent with current guidelines (specific to each type of surgical } \\
\text { procedure). } \\
\text { Measure Developer : CMS/OFMQ }\end{array}$ \\
\hline NQF 0302 & $\begin{array}{l}\text { Title: Ventilator Bundle } \\
\text { Description: Percentage of intensive care unit patients on mechanical } \\
\text { ventilation at time of survey for whom all four elements of the ventilator } \\
\text { bundle are documented and in place. The ventilator bundle elements are: } \\
\text { •Head of bed (HOB) elevation } 30 \text { degrees or greater (unless medically } \\
\text { contraindicated); noted on } 2 \text { different shifts within a } 24 \text { hour period } \cdot \text { Daily } \\
\text { "sedation interruption" and daily assessment of readiness to extubate; } \\
\text { process includes interrupting sedation until patient follow commands and } \\
\text { patient is assessed for discontinuation of mechanical ventilation; } \\
\text { Parameters of discontinuation include: resolution of reason for intubation; } \\
\text { inspired oxygen content roughly } 40 \% \text {; assessment of patients ability to } \\
\text { defend airway after extubation due to heavy sedation; minute ventilation } \\
\text { less than equal to } 15 \text { liters/minute; and respiratory rate/tidal volume less } \\
\text { than or equal to } 105 / \text { min/L(RR/TV }<105) \cdot S U D \text { (peptic ulcer disease) } \\
\text { prophylaxis } \cdot D V T \text { (deep venous thrombosis) prophylaxis } \\
\text { Measure Developer: IHI }\end{array}$ \\
\hline
\end{tabular}




\begin{tabular}{|c|c|}
\hline $\begin{array}{l}\text { Measure } \\
\text { Number } \\
\text { Identifier }\end{array}$ & Measure Title, Description \& Measure Developer \\
\hline NQF 0298 & $\begin{array}{l}\text { Title: Central Line Bundle Compliance } \\
\text { Description: Percentage of intensive care patients with central lines for } \\
\text { whom all elements of the central line bundle are documented and in place. } \\
\text { The central line bundle elements include: } \bullet \text { Hand hygiene }, \cdot \text { Maximal } \\
\text { barrier precautions upon insertion } \bullet \text { Chlorhexidine skin antisepsis } \bullet \text { Optimal } \\
\text { catheter site selection, with subclavian vein as the preferred site for non- } \\
\text { tunneled catheters in patients } 18 \text { years and older } \cdot \text { Daily review of line } \\
\text { necessity with prompt removal of unnecessary lines } \\
\text { Measure Developer: IHI }\end{array}$ \\
\hline NQF 0140 & $\begin{array}{l}\text { Title: Ventilator-associated pneumonia for ICU and high-risk nursery } \\
\text { (HRN) patients } \\
\text { Description: Percentage of ICU and HRN patients who over a certain } \\
\text { amount of days have ventilator-associated pneumonia } \\
\text { Measure Developer: CDC }\end{array}$ \\
\hline NQF & $\begin{array}{l}\text { Title: Urinary catheter-associated urinary tract infection for intensive care } \\
\text { unit (ICU) patients } \\
\text { Description: Percentage of intensive care unit patients with urinary } \\
\text { catheter-associated urinary tract infections } \\
\text { Measure Developer: CDC }\end{array}$ \\
\hline NQF 0139 & $\begin{array}{l}\text { Title: Central line catheter-associated blood stream infection rate for ICU } \\
\text { and high-risk nursery (HRN) patients } \\
\text { Description: Percentage of ICU and high-risk nursery patients, who over } \\
\text { a certain amount of days acquired a central line catheter-associated blood } \\
\text { stream infections over a specified amount of line-days } \\
\text { Measure Developer: CDC }\end{array}$ \\
\hline NQF 0329 & $\begin{array}{l}\text { Title: All-Cause Readmission Index (risk adjusted) } \\
\text { Description: Overall inpatient } 30 \text {-day hospital readmission rate. } \\
\text { Measure Developer: United Health Group }\end{array}$ \\
\hline $\begin{array}{l}\text { Not } \\
\text { applical }\end{array}$ & $\begin{array}{l}\text { Title: All-Cause Readmission Index } \\
\text { Description: Overall inpatient } 30 \text {-day hospital readmission rate. }\end{array}$ \\
\hline
\end{tabular}


TABLE 9: Proposed Alternative Medicaid Clinical Quality Measures for Medicaid Eligible Hospitals; Included in the Proposed Rule (see 75 FR 1899-1900) and Not in the Final Rule

\begin{tabular}{|c|c|}
\hline $\begin{array}{l}\text { NQF } \\
\text { Measure } \\
\text { Number }\end{array}$ & Measure Title, Description \& Measure Developer \\
\hline 0341 & $\begin{array}{l}\text { Title: PICU Pain Assessment on Admission } \\
\text { Description: Percentage of PICU patients receiving: } \\
\text { a. Pain assessment on admission } \\
\text { b. Periodic pain assessment. } \\
\text { Measure Developer: Vermont Oxford Network }\end{array}$ \\
\hline 0348 & $\begin{array}{l}\text { Title: Iotrogenic pneumothorax in non-neonates (pediatric up to } 17 \text { years of } \\
\text { age) } \\
\text { Description: Percent of medical and surgical discharges, age under } 18 \text { years, } \\
\text { with ICD-9-CM-CM code of iatrogenic pneumothorax in any secondary } \\
\text { diagnosis field. } \\
\text { Measure Developer: AHRQ }\end{array}$ \\
\hline 0362 & $\begin{array}{l}\text { Title: Foreign body left after procedure, age under } 18 \text { years } \\
\text { Description: Discharges with foreign body accidentally left in during } \\
\text { procedure per } 1,000 \text { discharges } \\
\text { Measure Developer: AHRQ }\end{array}$ \\
\hline 0151 & $\begin{array}{l}\text { Title: Pneumonia Care PNE-5c Antibiotic } \\
\text { Description: Percentage of pneumonia patients } 18 \text { years of age and older who } \\
\text { receive their first dose of antibiotics within } 6 \text { hours after arrival at the hospital } \\
\text { Measure Developer: CMS/OFMQ }\end{array}$ \\
\hline 0147 & $\begin{array}{l}\text { Title: Pneumonia Care PN-6 Antibiotic selection } \\
\text { Description: Percentage of pneumonia patients } 18 \text { years of age or older selected } \\
\text { for initial receipts of antibiotics for community-acquired pneumonia (CAP). } \\
\text { Measure Developer: CMS/OFMQ }\end{array}$ \\
\hline 0356 & $\begin{array}{l}\text { Title: Pneumonia Care PN-3a Blood culture } \\
\text { Description: Percent of pneumonia patients, age } 18 \text { years or older, transferred } \\
\text { or admitted to the ICU within } 24 \text { hours of hospital arrival who had blood } \\
\text { cultures performed within } 24 \text { hours prior to or } 24 \text { hours after arrival at the } \\
\text { hospital. } \\
\text { Measure Developer: CMS/OFMQ }\end{array}$ \\
\hline 0527 & $\begin{array}{l}\text { Title: Infection SCIP Inf-1 Prophylactic antibiotic received within } 1 \text { hour prior } \\
\text { to surgical incision } \\
\text { Description: Surgical patients with prophylactic antibiotics initiated within one } \\
\text { hour prior to surgical incision. Patients who received vancomycin or a } \\
\text { fluoroquinolone for prophylactic antibiotics should have the antibiotics initiated } \\
\text { within two hours prior to surgical incision. Due to the longer infusion time } \\
\text { required for vancomycin or a fluoroquinolone, it is acceptable to start these } \\
\text { antibiotics within two hours prior to incision time. } \\
\text { Measure Developer: CMS/OFMQ }\end{array}$ \\
\hline
\end{tabular}




\begin{tabular}{|c|c|}
\hline $\begin{array}{c}\text { NQF } \\
\text { Measure } \\
\text { Number }\end{array}$ & Measure Title, Description \& Measure Developer \\
\hline 0529 & $\begin{array}{l}\text { Title: Infection SCIP Inf-3 Prophylactic antibiotics discontinued within } 24 \\
\text { hours after surgery end time } \\
\text { Description: Surgical patients whose prophylactic antibiotics were discontinued } \\
\text { within } 24 \text { hours after Anesthesia End Time. } \\
\text { Measure Developer: CMS/OFMO }\end{array}$ \\
\hline
\end{tabular}

BILLING CODE 4120-01-C

Comment: Commenters stated that current health information technology is not capable of electronically collecting or reporting on clinical quality measures. Commenters also stated we should not require reporting on clinical quality measures that cannot easily be derived from EHRs. Other commenters believed the timeline was unreasonable to obtain the functionality required in the EHR system to report on these clinical quality measures and were concerned that there were no vocabulary standards.

Response: We agree with the comment that eligible hospitals should only be required to submit information that can be automatically obtained from certified EHR technology. As we discussed elsewhere, ONC's final rule (found elsewhere in this issue of the Federal Register) requires that certified EHR technology must be able to calculate clinical quality measures specified by us in this final rule. Standards for certified EHRs, including vocabulary standards, are included in ONC's final rule (found elsewhere in this issue of the Federal Register).

Comment: Commenters recommended that CMS conduct a pilot test of the NQF endorsed HITSP electronic specifications of measures in the proposed rule for Stage 1 prior to their adoption. Commenters requested CMS publish results of the pilot and use this information to inform the setting of Stage 2 and 3 objectives and clinical quality measures. Commenters also requested allowing adequate time for implementation after the pilot test before such measures are considered for certification, and 24 months before requiring them for meaningful use. One commenter stated that the Emergency Department Throughput, Stroke, and Venous Thromboembolism have not yet been thoroughly tested for automated reporting and data element capture. Additional commenters recommended that the measures selected for the eligible hospitals incentive program should be comprehensively standardized and tested in the field to ensure that they are thoroughly specified, clinically valid when the data are collected through the eligible hospitals system, feasible to collect, and are regularly updated and maintained with a well established process.

Response: We agree with the commenters that it is important to allow adequate time for pilot testing and implementation before clinical quality measures should be considered for certification, as well as requiring these measures for meaningful use. Emergency Department 1, Emergency Department 2, and Stroke 3, clinical quality measures for eligible hospitals and CAHs that are included in this final rule, were tested during the January 2010 Connectathon and demonstrated at the HIMSS 2010 Interoperability Showcase. Additionally, as part of the process of certification of EHR technology it is expected that certifying bodies will test the ability of EHR technology to calculate the clinical quality measures finalized in this final rule.

After consideration of the public comments received, eligible hospitals and CAHs will be required to report on each of the 15 clinical quality measures, as shown in Table 10. Requiring eligible hospitals and CAHs to report on each of the 15 clinical quality measures in the EHR incentive program is consistent with the RHQDAPU program, which requires reporting on all applicable quality measures. Eligible hospitals and CAHs will report numerators, denominators, and exclusions, even if one or more values as displayed by their certified EHR is zero. We note that to qualify as a meaningful EHR user, eligible hospitals and CAHs need only report the required clinical quality measures; they need not satisfy a minimum value for any of the numerator, denominator, or exclusions fields for clinical quality measures. The value for any or all of those fields, as reported to CMS or the States, may be zero if these are the results as displayed by the certified EHR technology. Thus, the clinical quality measure requirement for 2011 and beginning with 2012 is a reporting requirement and not a requirement to meet any particular performance standard for the clinical quality measure, or to in all cases have patients that fall within the denominator of the measure. Further, the criteria to qualify for the EHR incentive payments are based on results automatically calculated by eligible hospitals or CAHs certified EHR technology, as attested by the eligible hospital or CAH. As such, we believe that the eligible hospitals or CAHs will be able to determine whether they have reported the required clinical quality measures to CMS or the State, rendering it unnecessary that CMS or the State provide the eligible hospital or $\mathrm{CAH}$ with a feedback report, which provides information to eligible hospitals and CAHs as to whether they have reported their required clinical quality measures. We expect successful receipt of Medicare eligible hospitals and CAHs' information, beginning the first year of Stage 1.

We are finalizing Table 10, which conveys the clinical quality measure's title, number, owner/steward and contact information, and a link to existing electronic specifications. BILLING CODE 4120-01-P 
TABLE 10: Clinical Quality Measures for Submission by Eligible Hospitals and CAHs for Payment Year 2011-2012

\begin{tabular}{|c|c|c|}
\hline $\begin{array}{c}\text { Measure Number } \\
\text { Identifier }\end{array}$ & Measure Title, Description \& Measure Steward & $\begin{array}{l}\text { Electronic Measure } \\
\text { Specifications } \\
\text { Information } \\
\end{array}$ \\
\hline $\begin{array}{l}\text { Emergency } \\
\text { Department (ED)-1 } \\
\text { NQF } 0495\end{array}$ & $\begin{array}{l}\text { Title: Emergency Department Throughput - admitted } \\
\text { patients Median time from ED arrival to ED departure for } \\
\text { admitted patients } \\
\text { Description: Median time from emergency department } \\
\text { arrival to time of departure from the emergency room for } \\
\text { patients admitted to the facility from the emergency } \\
\text { department } \\
\text { Measure Developer: CMS/Oklahoma Foundation for } \\
\text { Medical Quality (OFMQ) }\end{array}$ & $\begin{array}{l}\text { http://www.cms.gov/Q } \\
\text { ualityMeasures/03 El } \\
\text { ectronicSpecifications. } \\
\text { asp\#TopOfPage }\end{array}$ \\
\hline $\begin{array}{l}\text { ED-2 } \\
\text { NQF } 0497\end{array}$ & $\begin{array}{l}\text { Title: Emergency Department Throughput - admitted } \\
\text { patients } \\
\text { Admission decision time to ED departure time for admitted } \\
\text { patients } \\
\text { Description: Median time from admit decision time to time } \\
\text { of departure from the emergency department of emergency } \\
\text { department patients admitted to inpatient status } \\
\text { Measure Developer: CMS/OFMQ }\end{array}$ & $\begin{array}{l}\frac{\text { http://www.cms.gov/Q }}{\text { ualityMeasures/03 El }} \\
\text { ectronicSpecifications. } \\
\text { asp\#TopOfPage }\end{array}$ \\
\hline $\begin{array}{l}\text { Stroke-2 } \\
\text { NQF } 0435\end{array}$ & $\begin{array}{l}\text { Title: Ischemic stroke - Discharge on anti-thrombotics } \\
\text { Description: Ischemic stroke patients prescribed antithromboti } \\
\text { therapy at hospital discharge } \\
\text { Measure Developer: The Joint Commission }\end{array}$ & $\begin{array}{l}\text { http://www.cms.gov/Q } \\
\text { ualityMeasures/03 El } \\
\text { ectronicSpecifications. } \\
\text { asp\#TopOfPage }\end{array}$ \\
\hline $\begin{array}{l}\text { Stroke-3 } \\
\text { NQF } 0436\end{array}$ & $\begin{array}{l}\text { Title: Ischemic stroke - Anticoagulation for A-fib/flutter } \\
\text { Description: Ischemic stroke patients with atrial } \\
\text { fibrillation/flutter who are prescribed anticoagulation therapy } \\
\text { at hospital discharge. } \\
\text { Measure Developer: The Joint Commission }\end{array}$ & $\begin{array}{l}\text { http://www.cms.gov/Q } \\
\text { ualityMeasures/03 El } \\
\text { ectronicSpecifications. } \\
\text { asp\#TopOfPage }\end{array}$ \\
\hline $\begin{array}{l}\text { Stroke-4 } \\
\text { NQF } 0437\end{array}$ & $\begin{array}{l}\text { Title: Ischemic stroke - Thrombolytic therapy for patients } \\
\text { arriving within } 2 \text { hours of symptom onset } \\
\text { Description: Acute ischemic stroke patients who arrive at } \\
\text { this hospital within } 2 \text { hours of time last known well and for } \\
\text { whom IV t-PA was initiated at this hospital within } 3 \text { hours of } \\
\text { time last known well. } \\
\text { Measure Developer: The Joint Commission }\end{array}$ & $\begin{array}{l}\text { http://www.cms.gov/Q } \\
\text { ualityMeasures/03 El } \\
\text { ectronicSpecifications. } \\
\text { asp\#TopOfPage }\end{array}$ \\
\hline $\begin{array}{l}\text { Stroke-5 } \\
\text { NQF } 0438\end{array}$ & $\begin{array}{l}\text { Title: Ischemic or hemorrhagic stroke - Antithrombotic } \\
\text { therapy by day } 2 \\
\text { Description: Ischemic stroke patients administered } \\
\text { antithrombotic therapy by the end of hospital day } 2 \text {. } \\
\text { Measure Developer: The Joint Commission }\end{array}$ & $\begin{array}{l}\text { http://www.cms.gov/Q } \\
\text { ualityMeasures/03 El } \\
\text { ectronicSpecifications. } \\
\text { asp\#TopOfPage }\end{array}$ \\
\hline
\end{tabular}

\footnotetext{
5 * In the event that new clinical quality measures are not adopted by 2013 , the clinical quality measures in this Table would continue to apply.
} 


\begin{tabular}{|c|c|c|}
\hline $\begin{array}{c}\text { Measure Number } \\
\text { Identifier }\end{array}$ & Measure Title, Description \& Measure Steward & $\begin{array}{c}\text { Electronic Measure } \\
\text { Specifications } \\
\text { Information } \\
\end{array}$ \\
\hline $\begin{array}{l}\text { Stroke-6 } \\
\text { NQF } 0439\end{array}$ & $\begin{array}{l}\text { Title: Ischemic stroke - Discharge on statins } \\
\text { Description: Ischemic stroke patients with LDL } \geq 100 \\
\mathrm{mg} / \mathrm{dL} \text {, or LDL not measured, or, who were on a lipid- } \\
\text { lowering medication prior to hospital arrival are prescribed } \\
\text { statin medication at hospital discharge. } \\
\text { Measure Developer: The Joint Commission }\end{array}$ & $\begin{array}{l}\underline{\text { http://www.cms.gov/Q }} \\
\text { ualityMeasures/03 El } \\
\text { ectronicSpecifications. } \\
\text { asp\#TopOfPage }\end{array}$ \\
\hline $\begin{array}{l}\text { Stroke-8 } \\
\text { NQF } 0440\end{array}$ & $\begin{array}{l}\text { Title: Ischemic or hemorrhagic stroke - Stroke education } \\
\text { Description: Ischemic or hemorrhagic stroke patients or } \\
\text { their caregivers who were given educational materials during } \\
\text { the hospital stay addressing all of the following: activation of } \\
\text { emergency medical system, need for follow-up after } \\
\text { discharge, medications prescribed at discharge, risk factors } \\
\text { for stroke, and warning signs and symptoms of stroke. } \\
\text { Measure Developer: The Joint Commission }\end{array}$ & $\begin{array}{l}\text { http://www.cms.gov/Q } \\
\text { ualityMeasures/03 El } \\
\text { ectronicSpecifications. } \\
\text { asp\#TopOfPage }\end{array}$ \\
\hline $\begin{array}{l}\text { Stroke-10 } \\
\text { NQF } 0441\end{array}$ & $\begin{array}{l}\text { Title: Ischemic or hemorrhagic stroke - Rehabilitation } \\
\text { assessment } \\
\text { Description: Ischemic or hemorrhagic stroke patients who } \\
\text { were assessed for rehabilitation services. } \\
\text { Measure Developer: The Joint Commission }\end{array}$ & $\begin{array}{l}\text { http://www.cms.gov/Q } \\
\text { ualityMeasures/03 El } \\
\text { ectronicSpecifications. } \\
\text { asp\#TopOfPage }\end{array}$ \\
\hline $\begin{array}{l}\text { Venous } \\
\text { Thromboembolism } \\
\text { (VTE)-1 } \\
\text { NQF } 0371\end{array}$ & $\begin{array}{l}\text { Title: VTE prophylaxis within } 24 \text { hours of arrival } \\
\text { Description: This measure assesses the number of patients } \\
\text { who received VTE prophylaxis or have documentation why } \\
\text { no VTE prophylaxis was given the day of or the day after } \\
\text { hospital admission or surgery end date for surgeries that start } \\
\text { the day of or the day after hospital admission. } \\
\text { Measure Developer: The Joint Commission }\end{array}$ & $\begin{array}{l}\text { http://www.cms.gov/Q } \\
\text { ualityMeasures/03 El } \\
\text { ectronicSpecifications. } \\
\text { asp\#TopOfPage }\end{array}$ \\
\hline $\begin{array}{l}\text { VTE-2 } \\
\text { NQF } 0372\end{array}$ & $\begin{array}{l}\text { Title: Intensive Care Unit VTE prophylaxis } \\
\text { Description: This measure assesses the number of patients } \\
\text { who received VTE prophylaxis or have documentation why } \\
\text { no VTE prophylaxis was given the day of or the day after the } \\
\text { initial admission (or transfer) to the Intensive Care Unit } \\
\text { (ICU) or surgery end date for surgeries that start the day of or } \\
\text { the day after ICU admission (or transfer). } \\
\text { Measure Developer: The Joint Commission }\end{array}$ & $\begin{array}{l}\frac{\text { http://www.cms.gov/Q }}{\text { ualityMeasures/03 El }} \\
\text { ectronicSpecifications. } \\
\text { asp\#TopOfPage }\end{array}$ \\
\hline
\end{tabular}




\begin{tabular}{|c|c|c|}
\hline $\begin{array}{c}\text { Measure Number } \\
\text { Identifier }\end{array}$ & Measure Title, Description \& Measure Steward & $\begin{array}{c}\text { Electronic Measure } \\
\text { Specifications } \\
\text { Information } \\
\end{array}$ \\
\hline $\begin{array}{l}\text { VTE-3 } \\
\text { NQF } 0373\end{array}$ & $\begin{array}{l}\text { Title: Anticoagulation overlap therapy } \\
\text { Description: This measure assesses the number of patients } \\
\text { diagnosed with confirmed VTE who received an overlap of } \\
\text { parenteral (intravenous [IV] or subcutaneous [subcu]) } \\
\text { anticoagulation and warfarin therapy. For patients who } \\
\text { received less than five days of overlap therapy, they must be } \\
\text { discharged on both medications. Overlap therapy must be } \\
\text { administered for at least five days with an international } \\
\text { normalized ratio (INR) } \geq 2 \text { prior to discontinuation of the } \\
\text { parenteral anticoagulation therapy or the patient must be } \\
\text { discharged on both medications. } \\
\text { Measure Developer: The Joint Commission }\end{array}$ & $\begin{array}{l}\text { http://www.cms.gov/Q } \\
\text { ualityMeasures/03 El } \\
\text { ectronicSpecifications. } \\
\text { asp\#TopOfPage }\end{array}$ \\
\hline $\begin{array}{l}\text { VTE-4 } \\
\text { NQF } 0374\end{array}$ & $\begin{array}{l}\text { Title: Platelet monitoring on unfractionated heparin } \\
\text { Description: This measure assesses the number of patients } \\
\text { diagnosed with confirmed VTE who received intravenous } \\
\text { (IV) UFH therapy dosages AND had their platelet counts } \\
\text { monitored using defined parameters such as a nomogram or } \\
\text { protocol. } \\
\text { Measure Developer: The Joint Commission }\end{array}$ & $\begin{array}{l}\text { http://www.cms.gov/Q } \\
\text { ualityMeasures/03 El } \\
\text { ectronicSpecifications. } \\
\text { asp\#TopOfPage }\end{array}$ \\
\hline $\begin{array}{l}\text { VTE-5 } \\
\text { NQF } 0375\end{array}$ & $\begin{array}{l}\text { Title: VTE discharge instructions } \\
\text { Description: This measure assesses the number of patients } \\
\text { diagnosed with confirmed VTE that are discharged to home, } \\
\text { to home with home health, home hospice or } \\
\text { discharged/transferred to court/law enforcement on warfarin } \\
\text { with written discharge instructions that address all four } \\
\text { criteria: compliance issues, dietary advice, follow-up } \\
\text { monitoring, and information about the potential for adverse } \\
\text { drug reactions/interactions. } \\
\text { Measure Developer: The Joint Commission }\end{array}$ & $\begin{array}{l}\text { http://www.cms.gov/Q } \\
\text { ualityMeasures/03 El } \\
\text { ectronicSpecifications. } \\
\text { asp\#TopOfPage }\end{array}$ \\
\hline $\begin{array}{l}\text { VTE-6 } \\
\text { NQF } 0376\end{array}$ & $\begin{array}{l}\text { Title: Incidence of potentially preventable VTE } \\
\text { Description: This measure assesses the number of patients } \\
\text { diagnosed with confirmed VTE during hospitalization (not } \\
\text { present on arrival) who did not receive VTE prophylaxis } \\
\text { between hospital admission and the day before the VTE } \\
\text { diagnostic testing order date. } \\
\text { Measure Developer: The Joint Commission }\end{array}$ & $\begin{array}{l}\text { http://www.cms.gov/Q } \\
\text { ualityMeasures/03 El } \\
\text { ectronicSpecifications. } \\
\text { asp\#TopOfPage }\end{array}$ \\
\hline
\end{tabular}


We proposed that to satisfy the requirements of reporting on clinical quality measures under sections 1886(n)(3)(A)(iii) and 1903(t)(6)(C) of the Act for the 2011-2012 payment year, we would require eligible hospitals and CAHs to report on all EHR incentive clinical quality measures for which they have applicable cases, without regard to payer. We proposed that Medicare eligible hospitals and CAHs, who are also participating in the Medicaid EHR incentive program, will also be required to report on all Medicaid clinical quality measures for which the eligible hospital has applicable cases. We also proposed that to demonstrate an eligible hospital or CAH is a meaningful EHR user, the eligible hospital or CAH would be required to electronically submit information on each clinical quality measures for each patient to whom the clinical quality measure applies, regardless of payer, discharged from the hospital during the EHR reporting period and for whom the clinical quality measure is applicable. Although as proposed, we did not require clinical quality reporting electronically until 2012 , we would begin clinical quality reporting though attestation in the 2011 payment year. We solicited comment on whether it may be more appropriate to defer some or all clinical quality reporting until the 2012 payment year. If reporting on some but not all measures in 2011 was feasible, we solicited comment on which key measures should be chosen for 2011 and which should be deferred until 2012 and why.

Comment: We received numerous comments strongly opposed to requiring the reporting of clinical quality measures by eligible hospitals prior to 2013, although some comments favored the reporting in 2011 and 2012.

Comments in favor pointed to the importance of quality measurement to achieving improvement in healthcare quality. Those opposed to the reporting of clinical quality measures in 2011 and 2012 cited concerns as to the readiness of EHR technology for automated calculation and reporting of clinical quality measures as well as financial and administrative burden. Many commenters stated that measures should be fully automated and tested prior to implementation, and recommended the process for Emergency Department Throughput, Stroke, and Venous Thromboembolism measures where CMS developed the specifications and has in place a plan to test the submission of such measures for RHQDAPU. Commenters stated their expectation that the testing process would reveal important insights as to potential challenges of electronic submission. Numerous commenters opposed measures already in

RHQDAPU and not able to be calculated by the EHR technology. Many commenters stated that electronic data submission should be developed through the RHQDAPU program rather than have a separate quality measure reporting program, such as the EHR incentive program. Further, commenters stated that RHQDAPU should provide the foundation for migration to electronic reporting. Numerous commenters were opposed to having a temporary data collection and reporting process through attestation that would need to be updated or replaced once CMS has the appropriate infrastructure in place. Many commenters stated that requiring hospitals to report summary data through attestation, without the ability for CMS to receive the summary data electronically, creates a dual reporting burden for measures currently in RHQDAPU. Many commenters stated concerns as to the timing of the certification process for EHRs since having a certified EHR is an essential element for quality incentives.

Numerous commenters pointed out that only 15 of the proposed measures have electronic specifications currently available.

Response: We are sensitive to and appreciate the many comments urging us not to require the submission of clinical quality measures, through attestation or electronic submission, prior to 2013, based on lack of readiness of many of the proposed measures, fully automating and testing prior to implementation, burden, and the potential duplication of quality measures reporting requirements under the RHQDAPU and the EHR incentive payment programs. Having carefully considered these comments, we have sought to address them while still retaining the important goal of beginning the process of using the capacity of EHRs to promote improved quality of care in hospitals by providing calculated results of clinical quality measures. In terms of readiness, we are limiting the clinical quality measures to those measures having existing electronic specifications as of the date of display of this final rule. Additionally, as recommended by commenters, we will only require hospitals to submit that information that can be automatically calculated by their certified EHR technology. Thus we will require no separate data collection by the hospital, but require submission solely of that information that can be generated automatically by the certified EHR technology; that is, we only adopt those clinical quality measures where the certified EHR technology can calculate the results. Further, we are not adopting any measures which are already being collected and submitted in the RHQDAPU program. Therefore, we are imposing no duplicate reporting requirement on hospitals who participate in RHQDAPU. Through future rulemaking we will seek to align the EHR incentive program with RHQDAPU.

Comment: Some commenters stated that CMS contradicts itself, where the proposed rule states that Medicare eligible hospitals who are also participating in Medicaid EHR incentive program will need to report on all of the Medicaid clinical quality measures and where it says that Table 21 is an alternative set of clinical quality measures if the hospital does not have any patients in the denominators of the measures in Table 20. Many commenters requested clarification of the Medicare and Medicaid reporting.

Response: We agree that the description of the eligible hospital and $\mathrm{CAH}$ reporting requirements was unclear. To clarify, our proposal was that if a hospital could submit information on clinical quality measures sufficient to meet the requirements for Medicare that would also be sufficient for Medicaid. However, hospitals for which the Medicare measures did not reflect their patient populations could satisfy the Medicaid requirements by reporting the alternate Medicaid clinical quality measures. Reporting the alternate Medicaid measures would only qualify for the Medicaid program and would not qualify eligible hospitals as to the Medicare incentive program. In this final rule, this clarification is moot, however, because we removed the alternate Medicaid list of clinical quality measures listed in Table 21 (see 75 FR 1896 through 1900) of the proposed rule for eligible hospitals. This was based on the lack of electronic specifications for these measures available at the time of display of this final rule. Hospitals that report information on all 15 of the clinical quality measures, as applicable to their patient population, will qualify for both the Medicare and the Medicaid submission requirements for clinical quality measures. We recognize that many of the measures in the Medicare list would likely not apply to certain hospitals, such as children's hospitals. However, an eligible hospital would meet the clinical quality measure requirement by reporting values for the 15 clinical quality measures, including, 
values of zero for the denominator, if accurate. Some value is required for each of the 15 clinical quality measures for eligible hospitals and CAHs. Therefore, for example, a children's hospital would enter zero for the denominator for any of the 15 measures for which they do not have any patients as described in the measure.

After consideration of public comments received, we are finalizing 15 clinical quality measures that eligible hospitals and CAHs will be required to report for Stage 1 (2011 and beginning 2012), as applicable to their patient population. Those 15 clinical quality measures for eligible hospitals and CAHs can be found in Table 10 of this final rule.

g. Potential Measures for EPs, Eligible Hospitals, and CAHs in Stage 2 and Subsequent Years

We stated our expectation that the number of clinical quality measures for which EPs, eligible hospitals, and CAHs would be able to electronically submit information would rapidly expand in 2013 and beyond.

We plan to consider measures from the 2010 PQRI program. These clinical quality measures can be found at http://www.cms.hhs.gov/PQRI/05 StatuteRegulations

ProgramInstructions.asp. For future considerations of clinical quality measures for Stage 2 of meaningful use and beyond for eligible hospitals and CAHs, we also plan to consider other clinical quality measures from the RHQDAPU program which are identified in the FY 2010 IPPS final rule (75 FR 43868-43882). We invited comments on inclusion of clinical quality measures for the 2013 and beyond for the HITECH Act Medicare and Medicaid incentive program. We note that as with the other meaningful use objectives and measures, in the event that we have not promulgated clinical quality measures for the 2013 payment year, the measures for Stage 1 (beginning in 2011) would continue in effect.

For the Stage 2 of meaningful use, we indicated in the proposed rule that we are considering expanding the Medicaid EHR incentive program's clinical quality measure set for EPs and eligible hospitals to include clinical quality measures that address the following clinical areas, to address quality of care for additional patient populations, and facilitate alignment with Medicaid and CHIP programs:

- Additional pediatrics measures (such as completed growth charts, electronic prescriptions with weight- based dosing support and

documentation of newborn screening).

- Long-term care measures.

- Additional obstetrics measures.

- Dental care/oral health measures.

- Additional behavioral/mental health and substance abuse measures. The above list does not constitute a comprehensive list of all clinical quality measures that may be considered. We stated that specific measures for Stage 2 of meaningful use and beyond may be addressed by CMS in future notice and comment rulemaking. To assist us in identifying potential clinical quality measures for future consideration for Stage 2 of meaningful use and beyond, we solicited comments on the potential topics and/or clinical quality measures listed above as well as suggestions for additional clinical quality measure topics and/or specific clinical quality measures.

The following is a summary of comments received regarding the request for public comment on potential measures for EPs, eligible hospitals, and CAHs for Stage 2 of meaningful use and subsequent stages, and our responses.

Comment: A commenter suggested using newly adopted NQF Level 3 measures that incorporate common electronic administrative and clinical data that represent a better measure of the patient's condition. A commenter suggested adding long term care and post acute care measures in the next stage of meaningful use. A few commenters suggested future clinical quality measures be coordinated with Healthy People 2020. Another comment regarding measures included a request for medication measures that evaluate provider intervention. Other commenters indicated CMS should provide a more structured process for the adoption of clinical quality measures such that specialty EPs would have greater input into and ownership of the process. A commenter requested consideration that future clinical quality measures address both quality and resource use efficiency (for example potentially preventable Emergency Department visits and hospitalizations and inappropriate use of imaging MRI for acute low back pain). A commenter requested future clinical quality measures for the following areas: reduce hospital readmissions and to improve medication management, specifically safe and efficient management of heart disease, diabetes, asthma, mental health conditions and hospital procedures. A commenter requested clinical quality measures that will aid in increasing improved patient safety and reduce disparities. A commenter also recommended developing new clinical quality outcomes measures to address overuse and efficiency, care coordination, and patient safety. Some commenters requested the inclusion of HIV testing and reporting for preventive service quality measures. Some commenters stated that this would help to facilitate continued efforts to promote and implement the 2006 CDC Revised Recommendation on HIV testing, especially to non-HIV medical specialties. Some commenters recommended measure development in the areas of community mental health, home health, renal dialysis centers, long term care, post acute care, and nursing homes. A commenter recommended including 3 month treatment of pulmonary emboli (NQF 0593) and deep vein thrombosis (NQF 0434) for the next stage of meaningful use and beyond. A commenter requested including health disparity data in all clinical quality measure analyses. Some commenters also recommended future clinical quality measure development in the following areas: Diabetes, heart disease, asthma, disease screening, chronic disease management, patient safety, nursing sensitive measures, atrial fibrillation, and ethnic disparities. Commenters requested expanding pediatric measures to provide expanded focus on childhood diseases that require hospitalization such as asthma, developmental issues and weight-based medication dosage safety issues. Additional commenters requested measures for blood test for lead levels for children up to 1 year of age and between 1 and 2 years of age, co-morbid conditions and dental utilization. A commenter recommended that only one EP should be accountable for the quality intervention and clinical quality measure such as NQF 0323 Title: End Stage Renal Disease (ESRD): Plan of Care for Inadequate Hemodialysis in ESRD Patient. The commenter indicated that this type of measure could involve more than one provider, for example, nephrologist and a dialysis facility. Because provider clinical practices may vary, practice variations may independently influence patient outcomes. Some commenters suggested future development of measures foster greater use of the clinical information available in EHRs to improve clinical processes and evaluate patient outcomes and suggested use of outcomes measures instead of process measures.

Furthermore, commenters support the inclusion of outcomes measures rather than process measures and composite versus individual measures. Several commenters indicated support for the preventive care measures included in 
the proposed rule and suggested expanding the set of preventive care measures to include HIV and STD screening and eye care specialty measures. A commenter requested CMS provide information about their strategic plan for future Medicare clinical quality measurement selection, how they will improve care delivery, proposed stages of reporting, goals and metrics.

Response: We are appreciative of the many suggestions and acknowledge the breadth of interest in certified EHR technology being the vehicle for clinical quality measures reporting. We expect to consider these suggestions for future measure selection in the Medicare and Medicaid EHR incentive payment programs.

Comment: We received various comments pertaining to future clinical quality measures applicable principally to the Medicaid population. One commenter urged CMS to include clinical quality measures specific to newborn screening in Stage 1 of meaningful use for pediatric providers.

Response: We agree that newborn screening, both as a clinical quality measure, and from a data standards perspective, is a prime candidate for inclusion in the Stage 2 definition of meaningful use. We affirm our proposed statement about our commitment to work with the measure development community to fill noted gaps. We are appreciative of the many suggestions. We expect to consider these suggestions for immunizations, prenatal screening, infectious disease, etc. in measure selection in future rulemaking.

Comment: A commenter indicated CMS should make explicit the health goals and targets for the HITECH Act investments that are already implied by the proposed clinical measures. Making them explicit allows CMS to set national targets.

Response: In general, the goal with respect to clinical quality measures is to improve healthcare quality as measured by the clinical quality measures. We believe that specific quantitative targets are impractical at this stage given lack of established base level notes and no prior clinical quality measure reporting via certified EHR technology.

Comment: Several commenters asked how CMS plans to develop further measure specifications for clinical quality measures. Another commenter asked for an electronic source for ICD9 and CPT codes defining the specific conditions or diagnoses or treatments in order to maintain an up-to-date capability.

Response: For many clinical quality measures, clearly defined electronic specifications are not yet available. In general, CMS relies on the measures' stewards to both develop measures and to provide the specifications.

Nevertheless, we recognize that many existing measures, some of which are owned and maintained by us or its contractors, do not currently have electronic specifications. We are aware of work currently taking place to fill this gap. We expect to actively work in a collaborative way with measures developers and stewards to help assure the development of electronic specifications for clinical quality measures, but we also expect to engage a contractor to perform work developing electronic specifications which may or may not involve measure developers and stewards. As for CPT codes, these are copyrighted by and are available from the American Medical Association. The National Center for Health Statistics (NCHS) and CMS are the U.S. governmental agencies responsible for overseeing all changes and modifications to the ICD-9 codes.

Comment: Some commenters suggested specific new clinical quality measures which are listed below in Table 11. Several commenters suggested new or revised clinical quality measures or the use of existing measures from other programs.

BILLING CODE 4120-01-P 
Table 11: EP Proposed New Clinical Quality Measures

\begin{tabular}{|c|c|}
\hline Measure Number & $\begin{array}{c}\text { Clinical Quality Measure Title and/or } \\
\text { Description }\end{array}$ \\
\hline PQRI 27 & $\begin{array}{l}\text { Diabetes Mellitus: Diabetic foot and ankle care, } \\
\text { ulcer prevention evaluation of footwear; } \\
\text { preventive care and screening }\end{array}$ \\
\hline PQRI 30 & $\begin{array}{l}\text { Timely administration of prophylactic parenteral } \\
\text { antibiotics }\end{array}$ \\
\hline PQRI 76 & $\begin{array}{l}\text { Prevention of catheter related bloodstream } \\
\text { infections CBSI }\end{array}$ \\
\hline PQRI 124 & HIT: Adoption/use of medical records \\
\hline PQRI 126 & $\begin{array}{l}\text { Diabetes Mellitus: Diabetic foot and ankle care, } \\
\text { peripheral neuropathy neurological evaluation }\end{array}$ \\
\hline PQRI 128 & BMI Screening and follow-up \\
\hline PQRI 130 & $\begin{array}{l}\text { Documentation and Verification of Current } \\
\text { Medications in the Medical Record }\end{array}$ \\
\hline PQRI 131 & $\begin{array}{l}\text { Pain Assessment Prior to Initiation of Patient } \\
\text { Treatment }\end{array}$ \\
\hline PQRI 148 & Back Pain: Initial Visit \\
\hline PQRI 149 & Back Pain: Physical Exam \\
\hline PQRI 150 & Back Pain: Advice for Normal Activities \\
\hline PQRI 151 & Back Pain: Advice Against Bed Rest \\
\hline PQRI 154 & Falls: Plan of care \\
\hline PQRI 155 & Falls: Risk Assessment \\
\hline PQRI 159 & $\begin{array}{l}\text { HIV/AIDS: CD4 + Cell Count or CD4 + } \\
\text { Percentage }\end{array}$ \\
\hline PQRI 160 & $\begin{array}{l}\text { HIV/AIDS: Pneumocystis jirovecii Pneumonia } \\
\text { Prophylaxis }\end{array}$ \\
\hline PQRI 161 & $\begin{array}{l}\text { HIV/AIDS: Adolescent and Adult Patients with } \\
\text { HIV/AUDS who are Prescribed Potent } \\
\text { Antiretroviral Therapy }\end{array}$ \\
\hline PQRI 162 & $\begin{array}{l}\text { HIV/AIDS: HIV RNA Control After } 6 \text { Months of } \\
\text { Potent Antiretroviral Therapy }\end{array}$ \\
\hline PQRI 193 & Perioperative temperature management \\
\hline PQRI 205 & $\begin{array}{l}\text { HIV/AIDS: STDs, Chlamydia and Gonorrhea } \\
\text { Screenings }\end{array}$ \\
\hline PQRI 206 & $\begin{array}{l}\text { HIV/AIDS: Screening for High Risk Sexual } \\
\text { Behaviors }\end{array}$ \\
\hline PQRI 207 & HIV/AIDS: Screening for Injection Drug Use \\
\hline PQRI 208 & HIV/AIDS: STDs Syphilis Screening \\
\hline NQF 0021 & $\begin{array}{l}\text { Therapeutic Monitoring: Annual monitoring for } \\
\text { patients on persistent medications }\end{array}$ \\
\hline NQF 0039 & Flu Shots for Adults Ages 50-64 \\
\hline
\end{tabular}




\begin{tabular}{|c|c|}
\hline Measure Number & $\begin{array}{c}\text { Clinical Quality Measure Title and/or } \\
\text { Description }\end{array}$ \\
\hline NQF 0058 & $\begin{array}{l}\text { Inappropriate antibiotic treatment for adults with } \\
\text { acute bronchitis }\end{array}$ \\
\hline NQF 0071 & $\begin{array}{l}\text { Acute Myocardial Infarction: Persistence of Beta- } \\
\text { Blocker Treatment After a Heart Attack }\end{array}$ \\
\hline NQF 0082 & Heart Failure: Patient Education \\
\hline NQF 0111 & Bipolar Disorder: Appraisal for risk of suicide \\
\hline NQF 0116 & CABG: Anti-Platelet Medication at Discharge \\
\hline NQF 0117 & CABG: Beta Blockage at Discharge \\
\hline NQF 0118 & CABG: Anti-Lipid Treatment at Discharge \\
\hline NQF 0278 & Low Birth Weight \\
\hline NQF 0477 & Rate of Very Low Birth Weight Deliveries \\
\hline NQF 0309 & $\begin{array}{l}\text { LBP: Appropriate Use of Epidural Steroid } \\
\text { Injections }\end{array}$ \\
\hline NQF 0602 & $\begin{array}{l}\text { Migraine: Adults with frequent use of acute } \\
\text { medications that also received prophylactic } \\
\text { medications }\end{array}$ \\
\hline NQF 0613 & MI: Use of beta blocker therapy \\
\hline NQF 0632 & $\begin{array}{l}\text { Primary prevention of cardiovascular events in } \\
\text { diabetics (older than } 40 \text { yrs): Use of Aspirin or } \\
\text { Antiplatelet Therapy }\end{array}$ \\
\hline NQF EC-20-08 & Warfarin - INR Monitoring \\
\hline NQF EC-203-08 & $\begin{array}{l}\text { Hyperlipidemia (Primary Prevention) - Lifestyle } \\
\text { changes and/or lipid lowering therapy }\end{array}$ \\
\hline NQF EC-227-08 & $\begin{array}{l}\text { High Risk for Pneumococcal Disease - } \\
\text { Pneumococcal vaccination. }\end{array}$ \\
\hline NQF EC-231-08 & $\begin{array}{l}\text { Diabetes with LDL greater than } 100 \text { - Use of } \\
\text { lipid lowering agent }\end{array}$ \\
\hline NQF EC-232-08 & $\begin{array}{l}\text { Diabetes with Hypertension or Proteinuria - Use } \\
\text { of an ACE Inhibitor or ARB. }\end{array}$ \\
\hline NQF EC-238-08 & Non-diabetic Nephropathy \\
\hline NQF EC-252-08 & $\begin{array}{l}\text { Chronic Kidney Disease with LDL greater than } \\
130\end{array}$ \\
\hline NQF EC-256-08 & $\begin{array}{l}\text { Male Smokers or Family History of AAA } \\
\text { Screening for AAA }\end{array}$ \\
\hline NQF EC-262-08 & $\begin{array}{l}\text { Diabetes and elevated } \mathrm{HbA1c}-\text { Use of diabetes } \\
\text { medications }\end{array}$ \\
\hline NQF EC-272-08 & $\begin{array}{l}\text { Secondary Prevention of Cardiovascular Events - } \\
\text { Use of Aspirin or anti-platelet therapy }\end{array}$ \\
\hline NQF EC-274-08 & $\begin{array}{l}\text { Primary prevention of cardiovascular events in } \\
\text { diabetics older than } 40 \mathrm{yrs} \text { - Use of aspirin or anti } \\
\text { platelet therapy }\end{array}$ \\
\hline NQF EC-281-08 & $\begin{array}{l}\text { Osteopenia and Chronic Steroid Use - Treatment } \\
\text { to prevent Osteoporosis }\end{array}$ \\
\hline
\end{tabular}




\begin{tabular}{|c|c|}
\hline Measure Number & $\begin{array}{c}\text { Clinical Quality Measure Title and/or } \\
\text { Description }\end{array}$ \\
\hline NQF EC-285-08 & Chronic Liver Disease - Hepatitis A vaccination \\
\hline NQF EC-288-08 & $\begin{array}{l}\text { Atherosclerotic Disease and LDL greater than } \\
\text { 100-use of a Lipid Lowering Agent }\end{array}$ \\
\hline N/A & $\begin{array}{l}\text { Family Planning - Percent of sexually active } \\
\text { clients at risk for unintended pregnancy - } \\
\text { screened at least once annually for use of } \\
\text { contraceptive method at last intercourse. }\end{array}$ \\
\hline N/A & $\begin{array}{l}\text { Percent of patients for which EP retrieves and acts } \\
\text { on prescription refill data obtained through the e- } \\
\text { Rx system }\end{array}$ \\
\hline N/A & $\begin{array}{l}\text { Percent of patients for which a generic drug has } \\
\text { been prescribed }\end{array}$ \\
\hline N/A & $\begin{array}{l}\text { Provider follow-up on growth chart information } \\
\text { where clinically indicated }\end{array}$ \\
\hline N/A & Inappropriate Use of Antibiotics in Bronchitis \\
\hline $\mathrm{N} / \mathrm{A}$ & $\begin{array}{l}\text { Chronic Disease Self Management Goal: Percent } \\
\text { of Asthmatics, Diabetics, Diagnosed } \\
\text { Hypertension, or Other CVD-Related Illness with } \\
\text { a Self-Management Goal/Readiness Plan ( } 4 \\
\text { possible measures) }\end{array}$ \\
\hline N/A & Good glycemic control: A1C $<7$ \\
\hline N/A & Elective Preterm Induction Rate \\
\hline $\mathrm{N} / \mathrm{A}$ & $\begin{array}{l}\text { Diabetes Mellitus A1C Frequency: Percent of } \\
\text { patients with Diabetes Mellitus with two A1C } \\
\text { measures in most recent } 12 \text { month period }\end{array}$ \\
\hline N/A & $\begin{array}{l}\text { Pediatric Type I Diabetes Mellitus Diabetic } \\
\text { Retinopathy }\end{array}$ \\
\hline NA & $\begin{array}{l}\text { Performing a complete lipid panel to assess CVD } \\
\text { risk }\end{array}$ \\
\hline N/A & Adolescent Preventive Care \\
\hline N/A & Child Preventive Care \\
\hline N/A & $\begin{array}{l}\text { Preventive Screening Lipid Disorders: Percent of } \\
\text { male patients over age } 35 \text { who have been } \\
\text { screened for lipid disorders, percent of females } \\
\text { over age } 45 \text { screened if they have risk factors for } \\
\text { CAD }\end{array}$ \\
\hline N/A & $\begin{array}{l}\text { Preventive Care \& Screening: Screening for } \\
\text { Diabetes }\end{array}$ \\
\hline N/A & $\begin{array}{l}\text { Cervical Cancer Prevention: Percent of female } \\
\text { patients age 9-26 yrs who received three doses of } \\
\text { HPV vaccine }\end{array}$ \\
\hline
\end{tabular}


Federal Register/Vol. 75, No. 144/Wednesday, July 28, 2010/Rules and Regulations

\begin{tabular}{|l|l|}
\hline \multicolumn{1}{|c|}{ Measure Number } & \multicolumn{1}{c|}{$\begin{array}{c}\text { Clinical Quality Measure Title and/or } \\
\text { Description }\end{array}$} \\
\hline N/A & $\begin{array}{l}\text { Asthma Action Plan: Percent of asthma patients } \\
\text { with a documented asthma action plan that has } \\
\text { been developed or updated within the past } 6 \\
\text { months. }\end{array}$ \\
\hline N/A & $\begin{array}{l}\text { Asthma Assessment of Percent of asthma patients } \\
\text { who have a documented level of control at last } \\
\text { asthma visit }\end{array}$ \\
\hline N/A & $\begin{array}{l}\text { Asthma Assessment/Spirometry -Percent of } \\
\text { asthma patients ages 5 and older who received } \\
\text { spirometry in the past 12 months. }\end{array}$ \\
\hline N/A & $\begin{array}{l}\text { Asthma Assessment of Severity: Percent of } \\
\text { Patients who have a Documented Level of } \\
\text { Asthma Severity for the Last Asthma Visit }\end{array}$ \\
\hline
\end{tabular}

Response: Many of the proposed clinical quality measures are in the existing PQRI program or are NQF endorsed. Others are not. We are appreciative of these many specific suggestions and will retain the comments for future consideration. Prior to including measures in the
Medicare EHR incentive payment program, as required by the HITECH Act, we will publish the measures in the Federal Register and provide an opportunity for public comment. We will examine all options for soliciting public comment on future Medicaidspecific clinical quality measures, as the
Federal Register notice requirement does not apply to the Medicaid EHR incentive program.

Comment: Some commenters suggested the following new topics for clinical quality measure development for our program: 
Table 12: EP Proposed New Topics

\begin{tabular}{|l|l|}
\hline \multicolumn{1}{|c|}{ Measure Number } & \multicolumn{1}{c|}{ Proposed Clinical Quality Measure Topics } \\
\hline N/A & $\begin{array}{l}\text { Measures dealing with overuse e.g, antibiotics and epidural } \\
\text { injections and unwarranted procedures-spine surgery, PTCA, } \\
\text { hysterectomy, CT, polypharmacy }\end{array}$ \\
\hline N/A & History regarding new or changing moles \\
\hline N/A & Counseling on monthly skin self exam \\
\hline N/A & Melanoma patients entered into recall system \\
\hline N/A & Newborn Screening \\
\hline N/A & Preventing Eye Disease \\
\hline N/A & Epilepsy \\
\hline N/A & Health Disparities \\
\hline N/A & Long Term Care \\
\hline N/A & Mental Health \\
\hline N/A & Substance Abuse \\
\hline N/A & School Health Services for Children \\
\hline N/A & Newborn Hearing and Bloodspot Screening \\
\hline N/A & Children at Risk for Developmental Disabilities \\
\hline N/A & Children with Chronic Disabling Conditions \\
\hline N/A & Child Health-Related Quality of Life \\
\hline N/A & Child Specific Health Outcomes \\
\hline N/A & Lead Poisoning Screening for Children \\
\hline N/A & Hepatitis A (childhood immunization) \\
\hline N/A & $\begin{array}{l}\text { Hepatitis B and hepatitis immune globulin (for newborns of } \\
\text { mothers with chronic hepatitis) }\end{array}$ \\
\hline N/A & Functional Status \\
\hline N/A & Use of epidural injections \\
\hline N/A & Healthy Weight/Reduction in Obesity \\
\hline N/A & Population-level lipid test results \\
\hline N/A & Population-level Blood pressure results \\
\hline N/A & Population-level Aspirin therapy \\
\hline N/A & Pharmacologic Prescription for Tobacco Cessation \\
\hline N/A & Alcohol/Drug Misuse \\
\hline N/A & $\begin{array}{l}\text { Family History for Chronic Diseases } \\
\text { Sexually activity status (13+) to trigger screening for STDs }\end{array}$ \\
\hline N/A & Screening pregnant women for STDs \\
\hline N/A & Screening for infectious disease risk factors \\
\hline N/A & Vaccine Reminders \\
\hline N/A & STD HIV Screening \\
\hline N/A & $\begin{array}{l}\text { Central Line Placement-Related Pneumothorax for Pediatric } \\
\text { Population }\end{array}$ \\
\hline N/A & $\begin{array}{l}\text { Acute Otitis Externa-Topical Therapy, Pain assessment, and } \\
\text { systemic antimicrobial therapy }\end{array}$ \\
\hline & \\
\hline
\end{tabular}




\begin{tabular}{|l|l|}
\hline \multicolumn{1}{|c|}{ Measure Number } & \multicolumn{1}{c|}{ Proposed Clinical Quality Measure Topics } \\
\hline N/A & $\begin{array}{l}\text { Otitis media with effusion (OME)- diagnostic evaluation of } \\
\text { tympanic membrane mobility }\end{array}$ \\
\hline N/A & NQF Care Coordination Measures \\
\hline N/A & Additional new pediatric measures \\
\hline N/A & Radiation dose \\
\hline N/A & Dental measures/Oral Health \\
\hline N/A & $\begin{array}{l}\text { HRSA Clinical Measures for Health Center Grantee } \\
\text { Performance Reviews }\end{array}$ \\
\hline N/A & Patient centered quality measures \\
\hline N/A & Outcomes Measures \\
\hline N/A & Outpatient Measure core set (NQA/AQA/HQA) \\
\hline N/A & Nutrition-related measures \\
\hline N/A & Efficiency Measures \\
\hline N/A & Patient Engagement Measures \\
\hline N/A & Decision Support Measures \\
\hline N/A & New Radiation Oncology measures \\
\hline N/A & Tobacco Use Assessment \\
\hline N/A & Tobacco Use Treatment \\
\hline N/A & Tobacco Use Treatment at Discharge \\
\hline N/A & Tobacco Use Follow-up \\
\hline N/A & Preventive Screening: Tobacco Use \\
\hline N/A & Preventive Screening: Falls in Older Adults \\
\hline N/A & Preventive Counseling: Breastfeeding \\
\hline N/A & Preventive Counseling: Use of Folic Acid \\
\hline N/A & HRSA/BPHC Measures \\
\hline 75, 610, 120,355, 560, & CDS alert responses \\
79, & \\
\hline N84, 132, 566, 356 & Population health measures \\
\hline N/A & Identifying patients with paroxysmal atrial fibrillation \\
\hline N/A & Group practice measures \\
\hline N/A & Genetic Measures \\
\hline N/A & Ear, nose, throat measures \\
\hline N/A & Home health \\
\hline N/A & ESRD Center measures \\
\hline N/A & Adherence related measures by therapeutic class \\
\hline N/A & Medication dosing for certain disease states such as diabetes \\
\hline N/A & $\begin{array}{l}\text { Suboptimal treatment regimens for chronic disease such as } \\
\text { diabetes and asthma }\end{array}$ \\
\hline N/A & Absence of control therapy in persistent asthma patients \\
\hline N/A & HEDIS high risk medication use in the elderly measures \\
\hline N/A & Patient self report satisfaction \\
\hline N/A & \\
\hline &
\end{tabular}




\begin{tabular}{|l|l|}
\hline \multicolumn{1}{|c|}{ Measure Number } & \multicolumn{1}{c|}{ Proposed Clinical Quality Measure Topics } \\
\hline N/A & $\begin{array}{l}\text { Prescribing and monitoring of psychotropic medications for } \\
\text { children and adolescents with psychiatric illness }\end{array}$ \\
\hline N/A & Measure for treatment of ADD and other mood disorders \\
\hline N/A & $\begin{array}{l}\text { Measure immunizations for adolescents including TDaP, } \\
\text { HPV, and meningococcal. }\end{array}$ \\
\hline N/A & $\begin{array}{l}\text { Hepatitis B/immune globulin to newborns to mothers who } \\
\text { have chronic hepatitis B infection as recommended by CDC }\end{array}$ \\
\hline N/A & Underutilization of medication measures \\
\hline N/A & Improve active engagement of patients in their care \\
\hline N/A & Improved care coordination and reduce gaps in care \\
\hline
\end{tabular}

BILLING CODE 4120-01-C

Response: We appreciate the suggested measure topics submitted by commenters for potential new clinical quality measures. Any future clinical quality measures developed will be in consideration of the clinical practices particular to EPs and eligible hospitals. We have captured these recommendations and will have them available for consideration in future years.

h. Reporting Method for Clinical Quality Measures for 2011 and Beginning With the 2012 Payment Year

(1) Reporting Method for 2011 Payment Year

As we previously discussed, we proposed to use attestation as a means for EPs, eligible hospitals and CAHs, for purposes of the Medicare incentive program, to demonstrate the meaningful use requirement for the calculation and submission of clinical quality measure results to CMS.

Specifically, for 2011, we proposed to require that Medicare EPs and hospitals attest to the use of certified EHR technology to capture the data elements and calculate the results for the applicable clinical quality measures. State Medicaid HIT Plans submitted to CMS will address how States will verify use of certified EHR technology to capture and calculate clinical quality measures by Medicaid EPs and eligible hospitals.

Further, we proposed to require that Medicare EPs, eligible hospitals, and CAHs attest to the accuracy and completeness of the numerators, denominators, and exclusions submitted for each of the applicable measures, and report the results to CMS for all applicable patients. We expect that States will follow a similar strategy as Medicare for the Medicaid EHR incentive program.

We proposed that attestation will utilize the same system for other attestation for meaningful use objectives, and proposed we would require for Medicare EPs that they attest to the following:

- The information submitted with respect to clinical quality measures was generated as output of an identified certified EHR technology.

- The information submitted is accurate to the best of the knowledge and belief of the EP.

- The information submitted includes information on all patients to whom the clinical quality measure applies.

- The NPI and TIN of the EP submitting the information, and the specialty group of clinical quality measures that are being submitted.

- For an EP who is exempt from reporting each of the core measures, an attestation that one or more of the core measures do not apply to the scope of practice of the EP.

- For an EP who is exempt from reporting on a specialty group, an attestation that none of the specialty groups applies to the scope of practice of the EP.

- For an EP who does report on a specialty group, but is exempt from reporting on each of the clinical quality measures in the group, an attestation that the clinical quality measures not reported do not apply to any patients treated by the EP.

- The numerators, denominators, and exclusions for each clinical quality measure result reported, providing separate information for each clinical quality measure including the numerators, denominators, and exclusions for all patients irrespective of third party payer or lack thereof; for Medicare FFS patients; for Medicare Advantage patients; and for Medicaid patients.

- The beginning and end dates for which the numerators, denominators, and exclusions apply.

Again, State Medicaid Agencies will determine the required elements for provider attestations for clinical quality measure reporting, subject to CMS prior approval via the State Medicaid HIT Plan.

For eligible hospitals, we proposed to require that they attest to the following:

- The information submitted with respect to clinical quality measures was generated as output from an identified certified EHR technology.

- The information submitted to the knowledge and belief of the official submitting on behalf of the eligible hospital.

- The information submitted includes information on all patients to whom the measure applies.

- The identifying information for the eligible hospital.

- For eligible hospitals that do not report one or more measures an attestation that the clinical quality measures not reported do not apply to any patients treated by the eligible hospital during the reporting period.

- The numerators, denominators, and exclusions for each clinical quality measure result reported, providing separate information for each clinical quality measure including the numerators, denominators, and exclusions for all patients irrespective of third party payer or lack thereof; for Medicare FFS patients; for Medicare Advantage patients; and for Medicaid patients.

- The beginning and end dates for which the numerators, denominators, and exclusions apply.

The following is a summary of comments received regarding the proposed reporting method for clinical quality measures for the 2011 payment year, and our responses.

Comment: The majority of commenters were against requiring attestation for 2011, rather than suggesting modification of the specific attestation requirements. Others commented that reporting should not be delayed to realize quality improvements 
and better health outcomes for patients as soon as possible. Many commenters suggested deferral of clinical quality measures submission until CMS can electronically accept data. Commenters indicated that this is consistent with allowing delayed reporting by Medicaid providers until 2012 or beyond. A number of commenters suggested that attestation should be confined to attesting that the EP's had reviewed or selected relevant clinical quality measures.

Response: While we received many comments to delay attestation past 2011, we are finalizing our proposed requirement for EPs and eligible hospitals to attest to the numerators, denominators, and exclusions in their first payment year for the required clinical quality measures as described in section II.A.3.d through $\mathrm{f}$ of this final rule. Medicaid providers do not have "delayed reporting of clinical quality measures." The statute and this final rule allow Medicaid providers the option of receiving the EHR Incentive Payment for having adopted, implemented or upgraded to certified EHR technology, in lieu of meeting the meaningful use bar in their first participation year. We expect that most Medicaid providers would choose to adopt, implement or upgrade to certified EHR technology, rather than demonstrating they are meaningful EHR users in their first participation year.

Comment: Some commenters also suggested EPs should only have to attest that the EP is entering the required data elements for clinical quality measure reporting where those fields exist in the certified EHR technology and provide feedback to the vendor where structured data fields are not available. Other commenters indicated the burden of adding numerous new data elements is high and labor intensive.

Response: We considered the suggestion of only requiring attestation of documentation of clinical encounters. While we agree that this could be considered "information on clinical quality measures," however, we do not believe that such information is needed when including the submission of information on clinical quality measures, which is a required element of meaningful use. We also believe that submission of such information would be of limited value. We believe that by limiting the clinical quality measure submission requirement to those results calculated by certified EHR technology, we have limited the potential burden.

After consideration of the public comments received, we are requiring EPs, eligible hospitals, and CAHs to attest to the numerator, denominator, and exclusions for the payment year 2011 at $\S 495.8$. We are finalizing the following requirements for EPs in this final rule for reporting clinical quality measures:

- The information submitted with respect to clinical quality measures was generated as output of an identified certified electronic health record.

- The information submitted is accurate to the best of the knowledge and belief of the EP.

- The information submitted includes information on all patients to whom the clinical quality measure applies for all patients included in the certified EHR technology.

- The NPI and TIN of the EP submitting the information at $\S 495.10$.

- The numerators, denominators, and exclusions for each clinical quality measure result reported, providing separate information for each clinical quality measure including the numerators, denominators, and exclusions for all applicable patients contained in the certified EHR technology irrespective of third party payer or lack thereof.

- The beginning and end dates for which the numerators, denominators, and exclusions apply (the Medicare EHR reporting period in payment year 1 is 90 days as stated at $\S 495.4$, and for payment year 2 is the beginning and end date of the reporting period as stated at $\S 495.4$. For Medicaid providers, as there is no EHR reporting period for adopting, implementing or upgrading for their first payment year, it is in their second payment year/first year of demonstrating meaningful use that they have a 90-day EHR reporting period. Therefore, it is their 2nd year of demonstrating meaningful use that has a 12 month EHR reporting period. For eligible hospitals and CAHs, we are finalizing the following requirements in this final rule:

- The information submitted with respect to clinical quality measures was generated as output from an identified certified EHR technology.

- The information submitted is accurate to the best of the knowledge and belief of the official submitting on behalf of the eligible hospital or CAHs.

- The information submitted includes information on all patients to whom the measure applies for all patients included in the certified EHR technology.

- The identifying information for the eligible hospital and CAH at $\S 495.10$.

- The numerators, denominators, and exclusions for each clinical quality measure result reported, providing separate information for each clinical quality measure including the numerators, denominators, and exclusions for all applicable patients contained in the certified EHR technology irrespective of third party payer or lack thereof.

- The beginning and end dates for which the numerators, denominators, and exclusions apply (the Medicare EHR reporting period in payment year 1 is 90 days as stated at $\S 495.4$, and for payment year 2 is the beginning and end date of the reporting period as stated at $\S 495.4$. For Medicaid providers, as there is no EHR reporting period for adopting, implementing or upgrading for their first payment year, it is in their second payment year/first year of demonstrating meaningful use that they have a 90-day EHR reporting period. Therefore, it is their 2nd year of demonstrating meaningful use that has a 12 month EHR reporting period.

States must implement the same meaningful use requirements, including clinical quality measures, with the exceptions described in section II.A. of this final rule. Therefore, Medicaid EPs and eligible hospitals must submit the same required information described above for clinical quality measures. States will propose in their State Medicaid HIT Plans how they will accept provider attestations in the first year they implement their Medicaid EHR incentive program, and how they will accept electronic reporting of clinical quality measures from providers' certified EHR technology in their second and subsequent implementation years.

(2) Reporting Method Beginning in 2012

In our proposed rule, we proposed that for the 2012 payment year, the reporting method for clinical quality measures would be the electronic submission to CMS of summary information, (that is, information that is not personally identifiable) on the clinical quality measures selected by the Secretary using certified EHR technology. For Medicaid, we proposed that EPs and hospitals eligible only for the Medicaid EHR incentive program must report their clinical quality measures data to States. We proposed that States would propose to CMS how they plan to accept and validate Medicaid providers' clinical quality measures data in their State Medicaid HIT Plans, subject to CMS review and approval.

As we did for payment year 2011, for 2012, we also proposed reporting on all cases to which a clinical quality measures applies in order to accurately assess the quality of care rendered by the particular EP, eligible hospital, or CAH generally. Otherwise it would only 
be possible to evaluate the care being rendered for a portion of patients and lessen the ability to improve quality generally. We solicited comments on the impact of requiring the submission of clinical quality measures data on all patients, not just Medicare and Medicaid beneficiaries.

The following is a summary of comments received regarding the proposed reporting method beginning in 2012 in regard to the collection of aggregate level data on all patients.

Comment: Several commenters noted that it appears that EPs are supposed to submit clinical quality measures electronically to the States in 2012. The commenters noted that several States have aging Medicaid Management Information Systems that may not be capable of accepting this data/ information. The commenters requested clarification about whether CMS expects the States to utilize and report this data immediately.

Response: To clarify, States may propose to CMS in their State Medicaid HIT Plans (See Section 495.332) the means by which they want to receive providers' clinical quality measures, starting with States' second

implementation year of their Medicaid EHR incentive program. States are not obliged to receive this data using their MMIS but can consider other options such as but not limited to: An external data warehouse, registries or health information exchanges that include data repositories.

Comment: A commenter asked that we state the authority which provides us the ability to require EPs and hospitals to report on non-Medicare and Medicaid patients.

Response: Sections 1848(o)(A)(2)(iii) and 1886(n)(3)(A)(iii) of the Act broadly state that as a condition of demonstrating meaningful use of certified EHR technology, an EP, CAH or eligible hospital must "submit information" for the EHR reporting period on the clinical quality or other measures selected by the Secretary "in a form and manner specified by the Secretary." Likewise, section 1903(t)(6) of the Act states that demonstrating meaningful use may include clinical quality reporting to the States, and may be based upon the methodologies that are used in sections 1848(o) and 1886(n). This language does not limit us to collecting only that information pertaining to Medicare and Medicaid beneficiaries. Therefore, we believe that we have the authority to collect summarized clinical quality measures selected by the Secretary, with respect to all patients to whom the clinical quality measure applies, treated by the
EP, eligible hospital, or CAH. We believe that the quality of care of our EP, eligible hospitals, and CAHs, as well as the ability to demonstrate the meaningful use of certified EHR technology, is best reflected by the care rendered to all patients, not just Medicare or Medicaid beneficiaries.

Comment: Some commenters recommended patient level data for clinical quality measure reporting while others supported CMS' requirement to submit summary level data for EPs and hospitals. There were several commenters that indicated support for reporting clinical quality measure data on all patients rather than just on Medicare and Medicaid patients. Another commenter stated that CMS should not require hospitals to submit patient level data and that the data should be at the aggregated level for all payment years. Another commenter stated that it is well proven in other disciplines that aggregated clinical data on quality measures can drive improvements in outcomes. Another commenter recommended patient level data that would be useful to State health programs and link information to managed care organizations.

Response: We agree with the commenters that stated that reporting clinical quality measure data for all patients provides a more comprehensive measure of quality. We acknowledge that there are potential advantages to patient level data in measuring quality such as those stated by the commenter. However, for Stage 1 we have elected to require aggregate level data since the EHR standards as adopted by ONC's final rule (found elsewhere in this issue of the Federal Register) do not provide standards for the submission of patient level data.

Comment: The commenter requested that CMS should have a process in place to support end-users with on-going help desk support.

Response: We agree with the suggestion for the implementation of a help desk to respond to questions related to the various CMS related questions after implementation of the proposed rule. Information about how we will provide assistance to providers will occur outside this final rule.

Comment: A few commenters asked for clarification regarding the Stage 1 audit process to ensure accuracy for the reporting of clinical quality measures (for example, numerator, denominator, and exception data).

Response: EPs, eligible hospitals, and CAHs are required for 2011 to attest to results as automatically calculated by certified EHR technology. Beginning with 2012, such information will be submitted electronically with respect to these requirements; we expect our audit strategy would be based on verifying that the results submitted accord with how they were calculated by the certified EHR technology.

Comment: We received comments requesting that CMS require that eligible providers report their clinical quality measures data to not only States and CMS, but also to Regional Health Improvement Collaboratives, where such programs exist. The commenters believed that this represents an alternative means for data submission rather than attestation and would allow States and CMS to test this alternative in 2011 or 2012. A commenter requested that CMS interpret the statutory requirement (Sections 1848(o)(2)(B)(iii) and 1886(n)(3)(B)(iii)) to avoid redundant or duplicative reporting of quality measures to include not just other CMS reporting efforts but also to avoid duplicative and redundant reporting with State and/or regional quality measurement and reporting efforts. They therefore requested that for Medicaid, CMS require EPs and hospitals report their clinical quality measures to not only States/CMS but also to Regional Health Improvement Collaboratives, where such programs exist.

Response: Clinical quality measures need to be reported to CMS for the Medicare program. For 2011, we intend to provide a web based tool for attestation. Beginning with 2012 for Medicare, we will provide one or more alternative options for electronic submission which may include intermediaries. For Medicaid, information will go to the States as directed by the States. We believe it would go well beyond the purview of this provision to require additional reporting other than to CMS or the States. To clarify the issue raised by the commenter, sections 1848(o)(2)(B)(iii) and $1886(n)(3)(B)(i i i)$ are tied to the Secretary and Federally-required quality measures reporting programs. However, CMS agrees that State and regional redundancies could be very problematic. We therefore clarify our proposed policy. States must include in their State Medicaid HIT Plans an environmental scan of existing HIT and quality measure reporting activities related to Medicaid. We expect States to include details in their SMHP about how these other on-going efforts can be leveraged and supported under HITECH; and how HITECH will not result in duplicative and/or burdensome reporting requirements on the same providers or organizations. 
In the proposed rule, we proposed that Medicare EPs, eligible hospitals, and CAHs would be required to report the required clinical quality measures information electronically using certified EHR technology via one of three methods. The primary method we proposed would require the EP, eligible hospital, or CAH to log into a CMSdesignated portal. Once the EP, eligible hospital, or CAH has logged into the portal, they would be required to submit, through an upload process, data payload based on specified structures, such as Clinical Data Architecture (CDA), and accompanying templates produced as output from their certified EHR technology.

As an alternative to this data submission method, we proposed to permit Medicare EPs, eligible hospitals, and CAHs to submit the required clinical quality measures data using certified EHR technology through Health Information Exchange (HIE)/ Health Information Organization (HIO). This alternative data submission method would be dependent on the Secretary's ability to collect data through a HIE/HIO network and would require the EP, eligible hospital, or $\mathrm{CAH}$ who chooses to submit data via an HIE/ HIO network to be a participating member of the HIE/HIO network. Medicare EPs, eligible hospitals, and CAHs would be required to submit their data payload based on specified structures or profiles, such as Clinical Data Architecture (CDA), and accompanying templates. The EPs, eligible hospitals, or CAHs data payload would be an output from their respective certified EHR technologies, in the form and manner specified from their HIE/HIO adopted architecture into the CMS HIE/HIO adopted architecture.

As another potential alternative, we proposed to accept submission through registries dependent upon the development of the necessary capacity and infrastructure to do so using certified EHRs.

We stated in the proposed rule that we intended to post the technical requirements for portal submission and the alternative HIE/HIO submission, the $\mathrm{HIE} / \mathrm{HIO}$ participating member definition, and other specifications for submission on our Web site for Medicare EPs on or before July 1, 2011 and for Medicare eligible hospitals and CAHs on or before April 1, 2011 for EHR adoption and incorporation and to accommodate EHR vendors.

The following is a summary of comments received regarding the proposed reporting method for clinical quality measures beginning with the 2012 payment year, and our responses.
Comment: A commenter recommended that CMS test a range of reporting options for clinical quality measures to establish uniform and reliable rates of data transmission. Several commenters supported the three data submission methodologies listed in the proposed rule to allow flexibility in the quality reporting mechanisms. Many commenters requested reporting via registries.

Response: We agree with the desirability of considering the three transmission methodologies listed in the proposed rule. The submission through a portal is the only mechanism that is feasible and practical for 2012 electronic clinical quality measure submission. We plan to test HIE/HIO and registry submission for future possible implementation through HITECH. Comment: A commenter requested clarification as to when CMS would no longer accept data for 2012 for Medicare EPs.

Response: The specific technical mechanism for attestation and electronic submission will be posted on the CMS Web site, and through various educational products in development. We anticipate that the last date for attestation or electronic submission will be two-three months after the close of the applicable EHR reporting period for EPs, eligible hospitals, and CAHs respectively.

Comment: Several commenters requested that CMS continue programs that incentivize advanced patient care for providers who are not eligible for the EHR incentive program and/or who do not become meaningful users of certified EHR technology.

Response: CMS clarifies, based upon the comments, that our efforts to avoid duplicative quality reporting requirements do not necessarily mean the discontinuation of other quality reporting programs. CMS and State Medicaid agencies support several quality reporting programs that are legislatively mandated or approach quality measurement in ways that are not exclusively tied to HIT, or that, are voluntary and/or address emerging or developing quality measure focus areas. We are committed to determining where the EHR incentive program's quality measure reporting can support other quality objectives, where it cannot and how to best align our overall quality measurement efforts across programs.

Comment: Many commenters requested deferring quality measure reporting until 2012 and/or 2013, at which time all measures will be electronically specified and tested. Commenters believed that this was especially important for new clinical quality measures such as Emergency Department Throughput and Stroke, and recommended gradually phasing in or gradually increasing the number of reportable measures and measure sets over time to allow for sufficient testing and harmonization between programs. Some commenters suggested that for Stage 1, eligible hospitals should be required to report only on the 15 measures that have been electronically specified and those that are appropriate for that organization. One commenter requested clinical quality measure reporting should be optional. Also, commenters requested for 2011 and 2012 that hospitals continue to report clinical quality measures through the current pay-for-reporting (RHQDAPU and HOP QDRP) programs or on clinical quality measures that coincide with HEDIS reporting measures including HOS and CAHPS, using the existing approaches, while quality measurement specialists and vendors create valid, reliable, and field-tested e-measures for deployment in the eligible hospitals for 2013. Finally, commenters stated that the proposed timeline may negatively impact credibility of data produced and have potentially negative impact on patient safety.

Response: With respect to comments received regarding the timeline for implementation of the EHR incentive program, we are only finalizing clinical quality measures that are electronically specified by the date of display of this final rule. For eligible hospitals and CAHs, we are finalizing 15 clinical quality measures as listed in Table 10 of this final rule that will be required to report for 2011 and 2012, as applicable to their patient population. Although we understand the suggestion that reporting through RHQDAPU should suffice for the HITECH Act, the difficulty is that HITECH specifically requires that EPs, eligible hospitals, and CAHs use "certified EHR technology" in connection with the submission of clinical quality measures. Thus the HITECH Act introduces a requirement that at least some clinical quality measures be submitted in connection with the use of certified EHR

technology, whereas RHQDAPU has no such requirement. We have limited the measures to those that have been electronically specified and that are able to be automatically calculated by the certified EHR technology. These results will be reported by EPs, eligible hospitals, and CAHs. We will seek to align the EHR incentive program and quality reporting programs in future rulemaking.

Comment: A number of commenters urged CMS not to require submission of 
clinical quality measures data beyond what a certified EHR can produce. Specifically, commenters stated that no clinical quality measures required for submission in Stage 1 should require a manual chart review. Some commenters also requested allowing submission of clinical quality measures through other EHRs that are not certified.

Response: We have adopted the suggested approach for 2011 and 2012 that limits the required information on clinical quality measures results to that which can be automatically calculated by the certified EHR technology. As to non-certified EHR technology, the HITECH Act incentive program specifically requires the meaningful use of certified EHR technology.

Comment: Several commenters stated that currently the data required to be used in the calculation of clinical quality measures are obtained from EHR discrete fields, free text and paper records. Commenters recommended a uniform reporting structure.

Commenters questioned if they would be submitting raw data, numerators and denominators only, if there will be an intermediary file that will allow manual edits to the file prior to submission, and if not will validity be based entirely on discrete electronic data. Commenters asked if sampling will be permitted or if hospitals will be required to report on entire populations. Commenters supported the value of reporting clinical quality measures for all patients, not just Medicare and Medicaid patients, in order to see the whole picture of the patient population which will enhance quality improvement.

Response: As discussed elsewhere, the submission requirement is limited to calculated results of clinical quality measures from certified EHR technology, as specified in this final rule, and as is consistent with the ONC final rule (see 75 FR 2014) which requires certified EHR technology to be able to calculate clinical quality measures as specified by CMS.

Comment: Several commenters suggested the clinical quality measures requiring medication administration data be delayed for reporting because they require advanced features of EHR systems with implementation of the features, in particular Electronic Medication Administration Record (eMAR).

Response: The Department has adopted certification criteria for EHR Modules and Complete EHRs, as identified in the Health Information Technology: Initial Set of Standards, Implementation Specifications, and Certification Criteria for Electronic Health Record Technology; Interim
Final Rule (75 FR 2014). It has also proposed temporary and permanent certification programs for testing and certifying health information technology in a March 10, 2010 proposed rule (75 FR 11328). The certification of EHRs will assure functionality of the information system to obtain clinical quality data from the EHR.

After consideration of the public comments received, starting in payment year 2012, in addition to meeting requirements for measures on meaningful EHR use and other requirements, Medicare EPs, eligible hospitals, and CAHs will be required to electronically submit clinical quality measures results (numerators,

denominators, exclusions) as calculated by certified EHR technology at $\S 495.8$. Medicaid EPs will be required to do so in the State's second implementation year for their Medicaid EHR incentive program. The clinical quality measures will be for all patients, regardless of payer, and will be for the period of the EHR reporting period. Medicare EPs, eligible hospitals, and CAHs will be required to report the required clinical quality measures information electronically using certified EHR technology via one of three methods. The primary method will require the EP, eligible hospital, or CAH to log into a CMS-designated portal. Once the EP, eligible hospital, or CAH has logged into the portal, they will be required to submit, through an upload process, data payload based on specified structures, such as Clinical Data Architecture (CDA), and accompanying templates produced as output from their certified EHR technology.

As an alternative to this data submission method, contingent on feasibility, we will permit Medicare EPs, eligible hospitals, and CAHs to submit the required clinical quality measures data using certified EHR technology through a Health Information Exchange (HIE)/Health Information Organization (HIO). This alternative data submission method will be dependent on the Secretary's ability to collect data through a HIE/HIO network and would require the $\mathrm{EP}$, eligible hospital, or $\mathrm{CAH}$ who chooses to submit data via an HIE/ HIO network to be a participating member of the HIE/HIO network. Medicare EPs, eligible hospitals, and CAHs would be required to submit their data payload based on specified structures or profiles. The EPs, eligible hospitals, or CAHs data payload should be an output from their respective certified EHR technologies, in the form and manner specified from their HIE/ HIO adopted architecture into the CMS HIE/HIO adopted architecture.
As another alternative, we will also accept submission through registries dependent upon the development of the necessary capacity and infrastructure to do so using certified EHRs. Finally, qualifying Medicare Advantage organizations for their eligible Medicare Advantage EPs, as well as, Medicare Advantage-affiliated eligible hospitals and CAHs will continue to submit HEDIS, HOS and CAHPS data instead of the clinical quality measures results under this final rule in section II.C.6.

We will post the technical requirements for portal submission and the alternative HIE/HIO submission, the $\mathrm{HIE} / \mathrm{HIO}$ participating member definition, and other specifications for submission on our Web site for Medicare EPs on or before July 1, 2011 and for Medicare eligible hospitals and CAHs on or before April 1, 2011 for EHR adoption and to accommodate EHR vendors.

State Medicaid Agencies must follow the same requirements for meaningful use, including clinical quality measures, for example, across all payers and for the entire EHR reporting period for EPs and eligible hospitals. We expect that States will be able to accept the electronic reporting of clinical quality measures by their second year of implementing the EHR incentive program. States will include in their State Medicaid HIT Plan a description of how Medicaid providers will be able to electronically report clinical quality measures, subject to CMS prior approval.

\section{i. Alternative Reporting Methods for Clinical Quality Measures}

We proposed several alternative reporting methods to create a dataset of provider-submitted summary data. One such alternative we proposed is the development of a distributed network of EHRs where health information is retained locally in individual EP, eligible hospital, and CAH EHRs and only summary reports are submitted to CMS. Another alternative we proposed is the creation of databases of patientlevel EHR data stored at the state or regional level.

The following is a summary of comments received regarding the proposed alternative reporting methods for clinical quality measures and our responses.

Comment: A commenter recommends aggregate reporting necessary for clinical quality measures to be able to be completed in secondary systems such as data warehouses.

Response: For Medicare, we require that the data source be from certified EHR technology. EPs, eligible hospitals 
and CAHs may use intermediaries (data warehouses) to submit the EHRgenerated clinical quality measure if available, assuming all requirements are met. States may seek CMS prior approval via their State Medicaid HIT Plans for how they expect Medicaid providers to report the required meaningful use data, including clinical quality measures. For example, States may propose that the data, while it originates in the providers' certified EHR technology, may be reported using a health information exchange organization or registry as an intermediary.

Comment: A few commenters communicated that the calculation and submission of quality measures may depend on the use of health information technology systems beyond those used by the EP such as data warehouses or registries that have to manipulate the data received. They indicated the final rule should not exclude the use of additional non-certified EHR technology to assist EPs in satisfying the quality reporting requirements provided the EP uses certified EHR technology to capture the data and to calculate the results.

Response: Certified EHR technology will be required to calculate the clinical quality measure results for the CMS specified measures we finalize in this final rule and transmit under the PQRI Registry XML specification, as provided in the ONC final rule (found elsewhere in this issue of the Federal Register).

Comment: Several commenters recommended inclusion of QRDA with PQRI XML for reporting, thus allowing vendors the ability to bypass PQRI XML if they plan to ultimately implement QRDA. There is also concern that switching to QRDA from XML will require duplicative investments. They recommended attestation for 2011 and 2012 as well as allowing use of QRDA in 2012.

Response: Electronic specifications will need to utilize standards that the certified EHR can support. ONC's final rule (found elsewhere in this issue of the Federal Register) limits this to PQRI Registry XML specifications. There is no current requirement that a certified EHR be able to produce QRDA.

\section{j. Reporting Period for Reporting of Clinical Quality Measures}

Sections 1848(o)(A)(2)(iii) and 1886(n)(3)(A)(iii) of the Act state that to demonstrate meaningful use of certified EHR technology for an EHR reporting period, an EP, eligible hospital, and CAH must submit information "for such period" on the clinical quality measures and other measures selected by the Secretary. Therefore we proposed that the reporting period for the clinical quality measures selected by the Secretary be the EHR reporting period.

Another alternative we proposed was a fixed reporting period of four quarterly reporting periods, or two six-month reporting periods. In terms of practice and precedent for other Medicare clinical quality measure reporting programs, all of these programs submit data to us at specific reporting intervals.

The following is a summary of comments received regarding the proposed EHR reporting period for EPs, eligible hospitals, and CAHs.

Comment: Some commenters asked for clarification on whether the EP must continuously report during the "entire payment year" or whether the reporting period for clinical quality measures covers a 12-month period. Other commenters questioned the timing of the requirements associated with the measures-whether the specifications for Stage 1 payment year 1 apply to EPs regardless of when the EPs become first eligible or whether the clinical quality measure specifications follow the calendar year.

Response: The EP only needs to report clinical quality measures once a year, as described at $\S 495.4$. For Medicare EPs, eligible hospitals and CAHs, the EHR reporting period is 90 days for their first payment year. For Medicaid eligible providers, their first payment year in which they demonstrate meaningful use (which may be their second payment year, if they adopted, implemented or upgraded in their first payment year) also has a 90-day EHR reporting period. For Medicare EPs, eligible hospitals and CAHs, in their second payment year, the reporting period is 12 months. For Medicaid EPs and eligible hospitals, in their second payment year of demonstrating meaningful use, they also have a 12-month EHR reporting period. Related to the timing of the requirements, the final clinical quality measure specifications for 2011 and 2012 will be posted at the time of display of this final rule.

Comment: Some commenters requested clarification of the process for reporting in the entire payment year. A commenter requested clarification regarding whether the EP must continuously report during the entire payment year or whether the reporting period for clinical quality measures covers an entire 12-month period. Some commenters pointed out that reporting capability may not be available every day of the year due to information system availability.

Response: Technical requirements for electronic reporting will be posted on the CMS Web site prior to the reporting period. The reporting period refers to parameters of the data captured in the EHR or the services documented in the EHR, not the time when the submission of information regarding clinical quality measures is made. States will dictate for Medicaid EPs and eligible hospitals the timing of submission of their clinical quality measures data via electronic reporting. Submission could be as infrequent as once a year after the close of the reporting period. The reporting period beyond 2011 and 2012 for clinical quality measures will be determined in future rulemaking.

\section{Demonstration of Meaningful Use}

Section 1848(o)(3)(C) of the Act, as added by section 4101(a) of the HITECH Act, requires that as a condition of eligibility for the incentive payment, an EP must demonstrate meaningful use of certified EHR technology (other than the reporting on clinical quality and other measures) as discussed in section II.A.3 of this final rule in the manner specified by the Secretary, which may include the following: An attestation, the submission of claims with appropriate coding, a survey response, reporting of clinical quality or other measures, or other means. Similarly, section 1886(n)(3)(c) of the Act, as added by section 4102(a) of the HITECH Act, requires that hospitals seeking the incentive payment demonstrate meaningful use of certified EHR technology in the manner specified by the Secretary. Section 1903(t)(6)(C)(i)(II) of the Act, as added by section 4201(a)(2) under the HITECH Act, states that a Medicaid EP or eligible hospital must demonstrate meaningful use through a "means that is approved by the State and acceptable to the Secretary." In addition, pursuant to section 1903(t)(9) of the Act, a State must demonstrate to the satisfaction of the Secretary that the State is conducting adequate oversight, including the routine tracking of meaningful use attestations and reporting mechanisms.

a. Common Methods of Demonstration in Medicare and Medicaid

As proposed, in the final rule, we are adopting a common method for demonstrating meaningful use in both the Medicare and Medicaid EHR incentive programs, for the same reasons we have a uniform definition of meaningful use. The demonstration methods we adopt for Medicare would automatically be available to the States for use in their Medicaid programs. The Medicare methods are segmented into two parts, as discussed in section II.4.b of this final rule. States seeking to 
modify or propose alternative

demonstration methods must submit the proposed methods for prior CMS

approval. This process is discussed more fully in section II.D.7.b.2.c. of this final rule.

b. Methods for Demonstration of the

Stage 1 Criteria of Meaningful Use

Our final regulations, at $\S 495.8$, will require that for CY 2011, EPs demonstrate that they satisfy each of the fifteen objectives and their associated measures of the core set listed at $\S 495.6(\mathrm{~d})$ and five of the objectives and their associated measures from the menu set listed at $\S 495.6(\mathrm{e})$ unless excluded as described in $\S 495.6(a)(2)$. (An exclusion will reduce the number of objectives/measures the EP must satisfy by the number that is equal to the EP's exclusions. For example, an EP that can exclude two menu objectives/measures is required to satisfy only three of the objectives and associated measures from the menu set. Similarly, an exclusion will reduce the number of core objectives/measures that apply). We permit only those exclusions that are specifically indicated in the description of each objective and its associated measure ( $\$ 495.6(d)$ for the core set and $\S 495.6$ (e) for the menu set). If an exclusion exists and the EP meets the criteria for it, the EP would report to CMS or the States that fact rather than demonstrating that they satisfy the objective and associated measure. At $\S 495.8$, we will require that for FY 2011, eligible hospitals and CAHs demonstrate that they satisfy each of the fourteen objectives and their associated measures of the core set listed at $\S 495.6(\mathrm{f})$ and five of objectives and their associated measures from the menu set listed at $\S 495.6(\mathrm{~g})$ unless excluded as described in $\S 495.6(\mathrm{~b})(2)$. As with EPs, all exclusions are specifically indicated, in the description of the objective and associated measures ( $§ 495.6(f)$ for the core set and $\S 495.6$ (g) for the menu set) and an exclusion will reduce the number of objectives and associated measures an eligible hospital or $\mathrm{CAH}$ must satisfy (see above example for EPs). If an exclusion exists and the hospital meets the criteria for it, the eligible hospital or CAH would report to CMS or the States that fact rather than demonstrating that they satisfy the objective and associated measure. Finally, as specified in 495.316(d), for those participating in the Medicaid EHR incentive program, the State may alter the requirements for demonstrating that an EP or eligible hospital is a meaningful user, with regard to four specific objectives and measures. For these objectives and measures, the State may also choose to make a menu-set objective a core objective. Such State additions could increase the core or menu set objectives and measures that must be satisfied.

For payment years beginning in $\mathrm{CY}$ 2012 and subsequent years, our final regulations, at $\S 495.8$, will require that for Stage 1 of meaningful use, EPs demonstrate that they satisfy each of the 15 objectives and their associated measures of the core set listed at § 495.6(d), except § 495.6(d)(4) “Report ambulatory quality measures to CMS or, in the case of Medicaid EPs, the states" and 5 of the objectives and their associated measures from the menu set listed at $\S 495.6(\mathrm{e})$ unless excluded as described in $\S 495.6(a)(2)$. The form and mechanism for excluding an objective and its associated measure is the same for CY2012 and subsequent years as it is for CY2011. The ability for States to add certain requirements is the same for CY 2012 and subsequent years as it is for CY 2011. The EP must demonstrate that they satisfy the objective

"Submitting quality measure to CMS or the States" through electronic reporting of clinical quality measures to CMS or the States, as specified in section II.A.3 of this final rule. For payment years beginning in FY2012 and subsequent years, our final regulations, at $\S 495$.8, will require that eligible hospitals and CAHs demonstrate that they satisfy each of the fourteen objectives and their associated measures of the core set listed at $\S 495.6(f)$, except $\S 495.6$ (f)(3) "Report hospital quality measures to CMS or, in the case of Medicaid EPs, the states" and five of the objectives and associated measures from the menu set listed at $\S 495.6(\mathrm{~g})$ unless excluded as described in $\S 495.6(\mathrm{~b})(2)$. The form and mechanism for excluding an objective and its associated measure is the same for FY2012 and subsequent years as it is for FY2011. The ability for States to add certain requirements also is the same for FY 2012 and subsequent years as it is for FY 2011. The eligible hospital or CAH must demonstrate that they satisfy the objective "Submitting quality measure to CMS or the States" through electronic reporting of clinical quality measures to CMS or the States, as specified in section II.A.3 of this final rule.

Except for the clinical quality measures (for which we require electronic reporting in CY or FY 2012 and subsequent years as discussed above), satisfaction of meaningful use objectives and associated measures may be demonstrated through attestation. Specifically, we will require that EPs, eligible hospitals and CAHs attest through a secure mechanism, such as through claims based reporting or an online portal. For the Medicare FFS and MA EHR incentive programs, CMS will issue additional guidance on this mechanism. For the Medicaid EHR incentive program, the States will include additional information in the State Medicaid HIT plans they submit to CMS to implement the program. We will require that an EP, eligible hospital or $\mathrm{CAH}$ would, through a one-time attestation following the completion of the EHR reporting period for a given payment year, identify the certified EHR technology they are utilizing and the results of their performance on all the measures associated with the reported objectives of meaningful use. We would require attestation through a secure mechanism because we do not believe that HIT will advance enough from its current state to allow for more automated and/or documented options of demonstrating meaningful use. As HIT matures we expect to base demonstration more on automated reporting by certified EHR technologies, such as the direct electronic reporting of measures both clinical and non clinical and documented participation in HIE. The first example is to the move from attestation for clinical quality measures to direct reporting in 2012 and subsequent years for EPs, eligible hospitals and CAHs. As HIT advances we expect to move more of the objectives away from being demonstrated through attestation. However, given the current state of HIT, we believe that imposing such demonstration requirements for 2011 would pose significant barriers to participation in the EHR incentive programs.

We believe that the means by which EPs, eligible hospitals and CAHs demonstrate meaningful use should work for all provider types. We also believe that uniform means of demonstration for EPs, eligible hospitals and CAHs are preferred and that a greater burden should not be placed on one or the other. In addition, we do not believe that demonstration of meaningful use could require use of certified EHR technology beyond the capabilities certified according to the ONC FR.

In addition to requiring electronic reporting of clinical quality measures beginning in 2012 in Medicare and Medicaid, we also leave open the possibility for CMS and/or the States to test options to utilize existing and emerging HIT products and infrastructure capabilities to satisfy other objectives of the meaningful use definition. The optional testing could involve the use of registries or the direct 
electronic reporting of some measures associated with the objectives of the meaningful use definition. We do not require any $\mathrm{EP}$, eligible hospital or $\mathrm{CAH}$ to participate in this testing in either 2011 or 2012 in order to receive an incentive payment. The state of electronic exchange varies widely across the country and is dependent on numerous Federal, State, local, nonprofit and for-profit initiatives. Given this high state of flux, CMS and/or the States would have to issue considerable updated guidance to EPs, eligible hospitals and CAHs who wish to join in our efforts to explore the electronic exchange of information. Any testing should be based on the principle of electronic exchange of information from certified EHR technology either directly to the States or through an intermediary. For purposes of the programs in this final rule it would be counterproductive for an intermediary to collect information through paper abstraction.

We will issue further instructions on the specifics for submitting attestation through established outreach venues.

Comment: Several commenters submitted comments regarding the methods of demonstration for clinical quality measures.

Response: We summarize and respond to those comments in section II.A. 3 of this final rule.

Comment: A few commenters submitted comments regarding section 1848(o)(2)(A) of the Act, which provides discretion to the Secretary to provide for the use of alternative means for meeting the requirements of meaningful use in the case of an eligible professional furnishing covered professional services in a group practice. Some of these commenters suggested that CMS provide such an alternative means in the final rule, while others suggested we consider doing so in future rulemaking.

Response: We did not propose any alternative means in the proposed rule. Given the per EP basis for most of the objectives and their associated measures, we did not believe group reporting would provide an accurate reflection of meaningful use. In addition, as the incentives payments are calculated on a per EP basis it is unclear to us how variance of meaningful use among EPs within the group should be treated. We believe the possible reduction in burden of attesting once per group versus once per EP is outweighed by the less accurate reporting, increased possibility of duplicate payments and decreased transparency. We note that many of the measures rely on data which could easily be stored at a group level such as a patient's demographics or medication lists and any EP with access to that information about a patient in their certified EHR technology and who sees that same patient in the EHR reporting period would receive credit for that patient in their numerator and denominator. Other aspects such as the enabling of drug-drug, drug-allergy checks, using CPOE and eRx could vary widely from EP to EP within the same group. We would also be concerned with EPs in multi-specialty group practices some of whom might be eligible for an exclusion, while others would not be. As requested by commenters we will continue to review this option in future rulemaking, but for this final rule we do not include the option to demonstrate meaningful use at a group level.

While we did not make changes to the demonstration of meaningful use requirements based on the comments above, we did make modifications to other aspects of the Stage 1 definition of meaningful use that required the descriptions of how many and which objectives and their associated measure EPs, eligible hospitals and CAHs to be altered accordingly. These changes are to the first paragraph of this section (II.4.b).

5. Data Collection for Online Posting, Program Coordination, and Accurate Payments

As described below, the HITECH Act requires the Secretary to post online the names of Medicare EPs and eligible hospitals and CAHs who are meaningful EHR users for the relevant payment year. Section 1903(t)(2) of the Act also requires us to ensure that EPs do not receive an EHR incentive payment under both Medicare and Medicaid. To fulfill these mandates, we must collect several data elements from EPs and eligible hospitals. Beyond these two direct HITECH Act requirements, CMS and the States also require certain data in order to accurately calculate and distribute the incentive payments.

\section{a. Online Posting}

In the proposed rule, we said that section 1848(o)(3)(D) of the Act requires the Secretary to list in an easily understandable format the names, business addresses, and business phone numbers of the Medicare EPs and, as determined appropriate by the Secretary, of group practices receiving incentive payments for being meaningful EHR users under the Medicare FFS program on our Internet Web site. We will not post information on group practices because we will not base incentive payments at the group practice level. Section 1886(n)(4)(B) of the Act, as added by section 4102(c) of the HITECH Act, requires the Secretary to list in an easily understandable format the names and other relevant data, as she determines appropriate, of eligible hospitals and CAHs who are meaningful EHR users under the Medicare FFS program, on our Internet Web site. Eligible hospitals and CAHs will have the opportunity to review the list before the list is publicly posted. Sections 1853(m)(5) and 1853(l)(7) of the Act, as added by sections 4101(c) and 4102(c) of the HITECH Act, require the Secretary to post the same information for EPs and eligible hospitals in the MA program as would be required if they were in the Medicare FFS program. Additionally, the Secretary must post the names of the qualifying MA organizations receiving the incentive payment or payments. We would collect the information necessary to post the name, business address and business phone numbers of all EPs, eligible hospitals and CAHs participating in the Medicare FFS and MA EHR incentive programs, and to post this information on our Web site. The HITECH Act did not require Medicaid EPs and eligible hospitals to be identified online so we will not do so.

We did not receive any comments and we are finalizing these provisions as proposed.

b. Program Election Between Medicare FFS/MA and Medicaid for EPs

In the proposed rule, we said section 1903(t)(2) of the Act prohibits an EP from receiving incentive payments under the Medicaid program unless the EP has waived any rights to incentive payments under the Medicare FFS or MA programs. Furthermore, section 1903(t)(7) of the Act requires the Secretary to assure no duplication of funding with respect to the Medicaid program, and the physician and MA incentive payments under sections 1848(o) and 1853(l) of the Act. This waiver and non-duplication requirement applies only to EPs meeting both the Medicare FFS/MA and Medicaid EHR incentive programs eligibility criteria, and does not apply to hospitals (which, if eligible, could receive incentive payments from both Medicare and Medicaid simultaneously). Section 495.10 allows an EP meeting the eligibility criteria for both the Medicare FFS/MA and Medicaid programs to participate in either program. We would also allow an EP to change his or her election once during the life of the EHR incentive programs after making the initial election, for payment years 2014 and 
before. We believe this one-time election rule allows an EP whose patient volume no longer makes him or her eligible for the Medicaid program to nevertheless continue to receive incentive payments that would encourage the meaningful use of certified EHR technology. For example, an EP who moves to a different practice or geographically relocates practices may reduce his or her Medicaid patient volume, and therefore become ineligible for the Medicaid incentive payments. Allowing this EP to continue to receive incentive payments under Medicare (if eligible) continues the availability to the EP of the incentive for meaningfully using EHR technology, and would allow EPs a certain amount of flexibility in their operations. While allowing this flexibility creates administrative complexity, we believe a significant number of EPs could have their participation in the EHR incentive programs endangered due to changing circumstances unrelated to the EHR incentive programs.

In the proposed rule, we proposed at 495.10(e)(5), that an EP switching program is "placed in the payment year the EP would have been in, had the EP not switched programs." For example, if an EP decides to switch after receiving his or her Medicare FFS incentive payment for their second payment year, then the EP would be in its third payment year for purposes of the Medicaid incentive payments. For the final rule, we are clarifying that the EP is "placed in the payment year the EP would have been in had the EP begun in and remained in the program to which he or she has switched." We have modified 495.10(e)(5) accordingly.

We believe this clarification is necessary in order to address comments we received on non-consecutive payments. As outlined in II.A.1.c and d of this final rule, the definition of first, second, third, fourth, fifth, and sixth payment year differs across the Medicare and Medicaid programs. Section 1848(o)(1)(E)(ii) of the Act requires that the second Medicare payment year be successive to the first payment year and immediately follow it. Similarly, the third payment year must immediately follow the second, and so on. Thus, as explained in II.A.1.c., "if a Medicare EP receives an incentive in CY2011, but does not successfully demonstrate meaningful use or otherwise fails to qualify for the incentive in CY2012, CY2012 still counts as one of the EP's five payment years and they would only be able to receive an incentive under the Medicare EHR incentive program for three more years." The same rule, however, does not apply to the Medicaid EHR

incentive program. For that program, EP payments may generally be nonconsecutive. If an EP does not receive an incentive payment for a given $\mathrm{CY}$ or FY then that year would not constitute a payment year. For example, if a Medicaid EP receives incentives in CY2011 and CY2012, but fails to qualify for an incentive in CY 2013, they would still be potentially eligible to receive incentives for an additional four payment years.

The rules on consecutive payment, discussed above, govern how an EP should be treated after switching from the Medicaid to the Medicare EHR incentive program, or vice versa. As stated above, we believe that an EP that switches from the Medicaid to the Medicare program should be treated in the same manner as if such EP had started in the Medicare program. Payment years that are skipped in the Medicaid EHR incentive program thus become payment years that count against the EP's five years of payment in Medicare. For example, an EP that receives nonconsecutive payment under Medicaid for CYs 2011 and 2013 (but skips CY 2012), and then switches to the Medicare program in CY 2014, is in the fourth payment year in 2014, and is limited to that payment year's limit on incentive payments. Such an EP may receive only one more year of incentive payments under the Medicare EHR incentive program. We believe this rule is equitable, given that, had the EP started in the Medicare program, the EP would not have been able to benefit from non-consecutive payments available under the Medicaid EHR incentive program. We see no reason why EPs that switch from the Medicaid to the Medicare program should be treated differently from those who initially began in the Medicare program, and believe that any other rule might encourage gaming on the part of eligible professionals.

By the same token, an EP that switches from the Medicare to the Medicaid EHR incentive program will not be penalized for non-consecutive payment years accrued while in the Medicare program. For example, an EP that receives nonconsecutive payment under Medicare for CYs 2011 and 2013 (but skips CY 2012), and then switches to the Medicaid program in CY 2014, is in the third year of payment in 2014, and is potentially eligible to receive three additional years of payment under Medicaid (after 2014), for a total of six years of payment. Similar to our rationale described in the paragraph above, we do not believe an EP that switches to the Medicaid program should be treated differently from the EP that initially begins in the Medicaid program, as once the EP switches to the Medicaid program, there is no statutory requirement that the payment year ordering be consecutive.

We believe it is self-evident that an EP switching to a new program is subject to the requirements of such new program. Thus, for example, an EP switching from Medicaid to Medicare might be subject to a higher stage of meaningful use upon moving to the Medicare program. The EP also would be subject to fewer years of payment and to the requirement that no incentive payments may be made after 2016 .

Finally, even after lining up the payment years, it is possible for an EP to exceed the payment cap under Medicaid by switching programs at the right time. We do not believe that the Congress intended for the payment caps to be exceeded under any circumstance, and therefore proposed that no EP should receive more than the maximum incentive available to them under Medicaid, which is the higher of the two caps. The last year incentive payment would be reduced if awarding the EP the full amount would exceed the overall maximum available under Medicaid. This is possible if an EP receives their first two payment years from Medicare and then the last four from Medicaid, as the cap would be exceeded by $\$ 250$. If the EP receives the HPSA bonus available under the Medicare FFS EHR incentive program, this amount could be as much as $\$ 4,450$. An EP who switches from Medicaid to Medicare could potentially exceed the Medicare threshold in a number of circumstances; however, since they will not be allowed to exceed the Medicaid threshold under any circumstance, we would pay the incentive for which they are eligible for a given payment year in whichever program they are in for that payment year until they exceed the Medicaid threshold. No incentive payments will be made to any EP that would allow the EP to exceed the Medicaid threshold. We anticipate that this would result in a prorated final year incentive payment. Finally, we proposed that the last year for making an incentive payment program switch would be CY 2014. In making this proposal, we considered that it is both the last year an EP can enroll in the Medicare EHR incentive program, and also the last year before the payment adjustments under Medicare can begin.

Comment: We received comments requesting clarification on when an EP could make their one switch.

Response: As described in our example, the EP could make their one 
switch anytime after the receipt of an incentive payment under either the Medicare or Medicaid program. Since this policy would also apply to other program changes (for example, changing from one State to another, or updating registration data elements), we want to clarify when program registration changes can take place. An EP, eligible hospital or $\mathrm{CAH}$ sets into motion receipt of the incentive payment when they attempt to demonstrate meaningful use or demonstrate to the State efforts to adopt, implement, or upgrade to certified EHR technology. Therefore, prior to their first successful attempt to demonstrate meaningful use or demonstrate to the State efforts to adopt, implement, or upgrade to certified EHR technology, the EP could change their registration in either the Medicare or Medicaid EHR incentive program as many times as they wish. Furthermore, EPs and hospitals selecting the Medicaid incentive program may also switch freely prior to payment as described here. However, there may only be one payment from one State in any one payment year.

After consideration of the public comment received, we are modifying the provision at $\S 495.10(\mathrm{e})(2)$ to “(2) After receiving at least one EHR incentive payment, may switch between the two EHR incentive programs only one time, and only for a payment year before 2015". This modification better reflects our clarification in response to the comment received on the ability to switch between programs. For the final rule, we have made a few other technical changes to $\S 495.10$, in addition to the changes made to $\S 495.10(e)(2)$ and $(e)(5)$.

\section{c. Data To Be Collected}

In addition to information regarding the demonstration of meaningful use, in $\S 495.10$ of this final rule we would collect the following administrative data for the Medicare and Medicaid EHR incentive programs to fulfill our requirements of online posting, avoidance of duplication of incentive payments, and to ensure accurate and timely incentive payments:

- Name, NPI, business address, and business phone of each EP or eligible hospital.

- Taxpayer Identification Number (TIN) to which the EP or eligible hospital wants the incentive payment made. For Medicaid EPs this must be consistent with assignment rules at $\S 495.10$.

- For EPs, whether they elect to participate in the Medicare EHR incentive programs or the Medicaid EHR incentive program.
- For eligible hospitals and CAHs, their CCN.

To coordinate with the States to avoid duplication of payments, we would make available to the States through a single National Level Repository (NLR) the following additional data:

- Whether an EP or eligible hospital is a meaningful EHR user, and

- The remittance date and amount of any incentive payments made to an EP or eligible hospital.

- Other information as specified by CMS.

CMS, our contractors, and the States will have access to these data elements through the NLR maintained by CMS. The States will have to provide information to us on whether EPs or eligible hospitals are eligible for the Medicaid incentive program, whether EPs or eligible hospitals participating in the Medicaid program are meaningful EHR users, and when any Medicaid incentive payments are made and the amount of the payment. We will put in place processes for an EP or eligible hospital to change their information, including the one-time switch in EHR incentive program election by EPs.

Comment: We received comments that some EPs do not use TINs, but rather the EP's Social Security Number (SSN).

Response: In these cases the EP would submit a TIN, which is their SSN. An incorporated EP would have a TIN for the corporation that would be an EIN. The EP's own TIN remains his/her SSN.

Comment: Some commenters requested clarification on whether the business address is the physical location or the mailing address.

Response: We believe that the HITECH Act required reporting of this information to assist the public in identifying meaningful EHR users. We believe the practice location address serves this purpose better than the mailing address. However we will allow EPs to enter an alternate address for posting purposes but will not allow that address to be a post office box.

Comment: Commenters suggested that States would be allowed to determine the requirements associated with Medicaid provider TIN assignments.

Response: We discuss the requirements associated with TIN assignment in 495.10(f) and in the requirements associated with SMHPs in this preamble at section 495.332 SMHPs. States are responsible for making sure the providers are providing an acceptable TIN, consistent with the regulations at 495.10(f), which states that providers may only assign to certain TINs.
We clarified 495.10(f), to reflect this and other changes.

Comment: CMS received numerous comments about the schedule for and State's role in the national single repository where CMS will collect data elements on all registrants.

Response: The technological requirements and systems interfaces are outside this regulation and we look forward to providing additional guidance.

Comment: Some commenters recommended a shorter record retention period that the ten years proposed. Commenters recommended periods ranging from three to eight years. The reasons given for a shorter time period were the cost of record retention, no perceived need for a retention period longer than the incentive period, rapid changes in EHR technology and consistency with other unspecified retention requirements.

Response: After reviewing the comments, we agree with commenters that ten years is longer than necessary to ensure the integrity of the program. In considering a shorter retention period, we believe that there may be cause to look over the entire incentive period. As a Medicaid EP would be eligible for incentives over a six-year period if they successfully receive an incentive each year and that is the longest such period available to any participant in the Medicare and Medicaid EHR incentive programs, we adopt a new retention period of six years for this final rule.

Comment: We received a comment suggesting that Medicare adopt an appeals process similar to the one proposed for Medicaid.

Response: We expect to address Medicare appeals in future guidance.

6. Hospital-Based Eligible Professionals

Section 1848(o)(1)(C)(i) of the Act, as added by section 4101(a) of the HITECH Act, states that hospital-based EPs are not eligible for the Medicare incentive payments. Similarly, the majority of hospital-based EPs will not be eligible for Medicaid incentive payments under 1903(t)(2)(A) of the Act (the only exception to this rule is for those practicing predominantly in an FQHC or RHC). Sections 4101(a) and 4201(a) of the HITECH Act originally defined the term "hospital-based eligible professional" to mean an EP, such as a pathologist, anesthesiologist, or emergency physician, who furnishes substantially all of his or her Medicarecovered professional services during the relevant EHR reporting period in a hospital setting (whether inpatient or outpatient) through the use of the 
facilities and equipment of the hospital, including the hospital's qualified EHRs. Following publication of our proposed rule, Congress modified the definition of hospital-based EPs. More specifically, on April 15, 2010, President Obama signed into law the Continuing Extension Act of 2010 (Pub. L. 111-157) which, in Section 5, made the following changes to the Social Security Act as it applies to both the Medicare and Medicare EHR incentives for EPs:

(1) Medicare-Section

1848(o)(1)(C)(ii) of the Social Security Act (42 U.S.C. $1395 \mathrm{w}-4(\mathrm{o})(1)(\mathrm{C})(\mathrm{ii}))$ is amended by striking 'setting (whether inpatient or outpatient)' and inserting 'inpatient or emergency room setting'.

(2) Medicaid-Section 1903(t)(3)(D) of the Social Security Act (42 U.S.C. $1396 \mathrm{~b}(\mathrm{t})(3)(\mathrm{D}))$ is amended by striking 'setting (whether inpatient or outpatient)' and inserting 'inpatient or emergency room setting'.

These amendments were effective as if included in the enactment of the HITECH Act.

The above sections indicate that the determination of whether an EP is a hospital-based EP shall be made on the basis of the site of service, as defined by the Secretary, and without regard to any employment or billing arrangement between the EP and any other provider. For example, the hospital-based determination for an EP would not be affected by whether the EP is an employee of the hospital, under a contractual relationship with the hospital, or with respect to whether he or she has made a reassignment to the hospital for Part B billing purposes.

In addition, as discussed below, section 1848(a)(7)(D) of the Act, as added by section 4101(b) of the HITECH Act, exempts hospital-based EPs from the downward payment adjustment applied under section 1848(a)(7)(A)(i) of the Act to covered professional services provided during a payment year by EPs who are not meaningful EHR users for the relevant payment year beginning in 2015.

Based on section 4101(a) of the HITECH Act (and prior to the amendments in the Continuing Extension Act of 2010), we proposed that an EP would be a hospital-based EP and therefore ineligible to receive a Medicare or Medicaid EHR incentive payment if more than 90 percent of their services are provided in the following place of service (POS) codes for HIPAA standard transactions: 21-Inpatient Hospital, 22-Outpatient Hospital, 23Emergency Room.

In addition, because of concerns that some primary care EPs who provide services to Medicare and Medicaid beneficiaries would be ineligible for the incentive payments under this proposed definition, in the proposed rule, we asked for comments on whether we should use another method for defining hospital-based EPs. We estimated that under this proposal, 12-13 percent of family practitioners under Medicare would be considered hospital-based. We did not have corresponding data for Medicaid EPs.

Comment: Many congressional representatives, hospital associations, individual providers and other commenters indicated that they believed that the proposal would inappropriately exclude from receiving EHR incentive payments EPs practicing in ambulatory settings such as those that practice in hospital provider-based departments (referred to by most commenters as "outpatient centers and clinics"). They indicated these centers and clinics provide services similar to services furnished by EPs in private offices. Many suggested that this definition may inhibit hospital investments in their outpatient primary care sites. Commenters believe the absence of any EP incentive payment in these settings may discourage hospitals from adopting EHR in ambulatory settings, particularly if doing so requires the purchase of an ambulatory-based EHR system (or an ambulatory component to be added to the hospital's EHR system). This is because the hospital's total incentive payment is based on total inpatient services. A hospital with a large outpatient department will not receive a higher incentive payment as a result of their outpatient services. These commenters indicated that ambulatory care EHRs are very different from inpatient EHRs because of the inherent differences between the types of care provided in each setting. Commenters differed somewhat to the extent that they provided specific alternatives. Some commenters went so far as to suggest that all EPs should be eligible to receive EHR incentive payments, regardless of where they practice.

Response: The changes to the hospital-based definition that are included in the Continuing Extension Act of 2010 (Pub. L. 111-157) discussed above address commenters concerns about ambulatory settings. These changes have been incorporated into the final rule. An EP will be a hospitalbased EP and therefore ineligible to receive a Medicare (or Medicaid) EHR incentive payment if more than 90 percent of their Medicare (or Medicaid) services are provided in the following two place of service (POS) codes for HIPAA standard transactions: 21-
Inpatient Hospital, 23-Emergency Room.

Comment: Some commenters argued that the proposed rule failed to make a critical distinction between hospitalbased EPs who primarily use an EHR paid for and maintained by the hospital and those that did not. Some

commenters suggested that an EP should be eligible for an EHR incentive payment if he or she had contributed 15 percent or more toward the cost of acquiring or maintaining the certified EHR. Some commenters requested that CMS change the definition of a hospitalbased EP to read: "An EP who furnishes 90 percent or more of his or her covered professional services in the CY preceding the payment year in a hospital setting and primarily through the use of the qualified electronic health records of the hospital." The commenters believed that Congress's intent was to exclude only those EPs using qualified EHRs of the hospital, and that their approach would allow separate EHR incentive payments for EPs who have developed cutting-edge, patient centered EHR modules, thereby allowing for a clinical specificity not currently available in more generalized, hospital-wide EHR systems.

Commenters stated that these EHR technologies are currently used in hospital settings and interoperate with hospital systems, but are paid for and primarily maintained by physician groups who see patients in hospital settings. The commenters indicate that these physician groups continue to invest in their EHRs through improvements, ongoing maintenance, and support staff employed to ensure optimal use of such technology. The commenters indicated that many early health IT champions, including hospital-based anesthesiologists, radiologists, pathologists, hospitalists, emergency medicine physicians, and neonatal physicians would be negatively affected by the proposal. These comments would apply to EP services provided in all hospital settings, including inpatient, outpatient, and emergency rooms.

Response: The statute, as now amended, indicates that hospital-based EPs are those who furnish substantially all their services in an inpatient or emergency room setting, such as a pathologist, anesthesiologist, or emergency physician, and who do so using the facility and equipment, including qualified electronic health care records, of the hospital. While commenters focused on the statutory language: “* * * including qualified electronic health care records of the hospital", they did not address the 
broader meaning of the section which also includes the requirement that hospital-based EPs are those who furnish services "using the facility and equipment", including qualified electronic health care records of the hospital. We believe both phrases together are intended to provide an explanation of why hospital-based EPs are to be excluded from receiving EHR incentive payments (that is, that they would typically use the facilities and equipment, including the EHR, of the hospital and that therefore it would represent double payment if both hospitals and hospital-based EPs were to be paid incentives). We do not believe that the intent of this language was to require CMS to evaluate each EP as to whether they are using the EHR of the hospital. Further, the commenters did not address the significance of the next sentence of the statute, which clearly indicates that: "The determination of whether an eligible professional is a hospital-based eligible professional shall be made on the basis of the site of service * * *". Since Congress directed that site of service must be the determinant of whether an EP is hospital-based, we could not use individualized determinations of whether an EP is using the EHR of the hospital to deliver his or her services. Also, the subsequent legislation in the Continuing Extension Act of 2010 is consistent with the interpretation that the determination of whether an EP is hospital-based is based on the place where the EP furnishes services, as that subsequent legislation further limited hospital-based to those EPs providing substantially all services in the emergency room or inpatient hospital settings. Furthermore, our final policy is that eligible hospitals must demonstrate meaningful use based upon all applicable cases in the inpatient (21) and emergency department (23) site of service codes. Therefore, there would be duplication in measuring meaningful use for the purposes of making EHR incentive payments in the scenario proposed by these commenters.

The HITECH Act does not define the term "hospital" for purposes of establishing a definition of hospitalbased EPs for Medicare and Medicaid. However, section 1861(e) of the Act defines the term a "hospital" to mean an institution that "is primarily engaged in providing, by or under the supervision of physicians, to inpatients (A) diagnostic services and therapeutic services for medical diagnosis, treatment, and care of injured, disabled, or sick persons, or (B) rehabilitation services for the rehabilitation of injured, disabled, or sick persons.” Therefore, clearly EPs that practice primarily in inpatient hospital settings, as referenced in section 1861(e) of the Act, would be considered hospital-based EPs.

We will consider the use of place of service (POS) codes on physician claims to determine whether an EP furnishes substantially all of their professional services in a hospital setting and is, therefore, hospital-based. This code set is required for use in the implementation guide adopted as the national standard for electronic transmission of professional health care claims under the provisions of the Health Insurance Portability and Accountability Act of 1996 (HIPAA). HIPAA directed the Secretary of HHS to adopt national standards for electronic transactions. These standard transactions require all health plans and providers to use standard code sets to populate data elements in each transaction. The Transaction and Code Set Rule (65 FR 50312) adopted the ASC X12N-837 Health Care Claim:

Professional, volumes 1 and 2, version 4010 , as the standard for electronic submission of professional claims. This standard names the POS code set currently maintained by CMS as the code set to be used for describing sites of service in such claims and is available at http://www4.cms.gov/ PlaceofServiceCodes/Downloads/ posdatabase110509.pdf.

From this code set, we would consider the use of the following POS codes to determine whether an EP is a hospital-based eligible professional for Medicare:

- 21-Inpatient Hospital-is a facility, other than psychiatric, which primarily provides diagnostic, therapeutic (both surgical and nonsurgical), and rehabilitation services by, or under, the supervision of physicians, to patients admitted for a variety of medical conditions.

- 23-Emergency Room, Hospital-is a portion of a hospital where emergency diagnosis and treatment of illness or injury is provided.

Comment: Most commenters were supportive of the proposal to define "substantially all" of his or her covered professional services in a hospital setting as EPs who furnish at least 90 percent of his/her services in a hospital setting. However, some commenters expressed concerns that this threshold will be too high starting in 2015 when the time comes to determine which EPs should be subject to penalties for failure to become meaningful users of certified EHR technology. A few commenters misunderstood the proposal and requested that a hospital-based EP be defined as one who provides at least 90 percent of his or her services, defined as encounters and not as charges.

Response: The statutory definition of hospital-based EP provides that to be considered a hospital-based EP, the EP must provide "substantially all" of his or her covered professional services in a hospital setting. Therefore, we must identify the minimum percentage of an EP's covered professional services that must be provided in a hospital setting in order for the EP to be considered as providing "substantially all" of his or her covered professional services in a hospital setting. Consistent with the statute, we proposed to make this determination on the basis of services performed by each EP, not the charges for each EP. We are finalizing the proposed definition of "substantially all" as furnishing at least 90 percent of services in a hospital setting. We believe a 90 percent threshold certainly would qualify as "substantial."

Comment: Representatives of surgeons asked that CMS make an accommodation to the hospital-based definition to account for services paid under a global fee.

Response: The determination of whether or not an EP is hospital-based is determined individually for each EP. A global fee is a single payment for a bundle of services, some of which could be performed in a hospital such as major surgery or hospital visits, whereas some could be performed in an office such as follow-up visits, CMS does not have data, for the place of service for services performed by individual EPs when the services are paid as part of a global fee. We considered possibilities for using national level estimates for individual services typically performed under global fees as proxies for services provided by individual EPs. However, this would add significant additional operational complexity to the determination of hospital-based status and we have not pursued this approach.

Comment: Some commenters requested that CMS establish a process by which EPs could know in advance of a payment year whether CMS considered them as being hospital-based and therefore ineligible for an incentive payment.

Response: To the extent practical, we intend on establishing a process whereby the EP would know his/her hospital-based status during the registration period. We plan to provide information to EPs regarding their hospital-based status as early as possible (that is, no later than early in each payment year). As indicated in the proposed rule, we will make a determination for Medicare incentive 
payment purposes, as to whether or not an EP is hospital-based by annually analyzing an EP's claims history from the prior year. In the proposed rule we indicated that we would use claims data from the prior calendar year to make hospital-based determinations for EPs. However, in order to provide information regarding the hospitalbased status of each EP at the beginning of each payment year, we will need to use claims data from an earlier period. Therefore, we will use claims data from the prior fiscal year (October through September). Under this approach, the hospital-based status of each EP would be reassessed each year, using claims data from the fiscal year preceding the payment year. The hospital-based status will be available for viewing beginning in January of each payment year. For Medicaid purposes, State Medicaid agencies will make the determination about whether or not an EP is hospitalbased by analyzing an EP's Medicaid claims data, or in the case of EPs who deliver care via Medicaid managed care programs, by analyzing either encounter data or other equivalent data sources, at the State's option. For purposes of making this determination, States would be permitted to use data either from the prior fiscal or calendar year.

After consideration of the public comments received, we are revising the definition of hospital based EPs in this final rule. An EP will be defined as being hospital-based and therefore ineligible to receive an EHR incentive payment under either Medicare or Medicaid, regardless of the type of service provided, if more than 90 percent of their services are identified as being provided in places of service classified under two place of service codes 21 (Inpatient Hospital) or 23 Emergency Room, Hospital. We plan to reassess the hospital-based status of each EP for Medicare purposes each year, using claims data from the fiscal year immediately preceding the payment year. Based on preliminary claims data from the first 9 months of 2009, CMS currently estimates that, under this final definition of hospitalbased EPs, about 14 percent of Medicare EPs (physicians) would be considered hospital-based and thus not eligible to receive any incentive payments. We do not have any data on Medicaid practitioners.

\section{Interaction With Other Programs}

In the proposed rule, we described how the HITECH Act addresses interactions between the Medicare EHR incentive program and the E-prescribing Incentive Program authorized by MIPPA. Under section 1848(m)(2)(D) of the Act, as added by section 4101(f)(2)(B) of the HITECH Act, if a Medicare FFS or MA EP receives an incentive payment from the Medicare EHR incentive program, the EP (or group practice) is not eligible to also receive the incentive payment under the E-prescribing Incentive Program created by MIPPA. Given the payment timelines in this final rule for the Medicare EHR incentive program and the existing payment timeline for the E-prescribing Incentive Program, we will know whether an EP received a Medicare EHR incentive payment before the eprescribing Incentive Program payment is calculated. Thus we will exclude those EPs (or group practices) who accept a Medicare EHR incentive payment for a given year from being eligible for the e-prescribing Incentive Program payment for that same year. EPs receiving a Medicaid EHR incentive payment would remain eligible for the Medicare MIPAA E-Prescribing Incentive Program payment.

As the HITECH Act does not specify any other restrictions on participation in other programs and participation in the Medicare and Medicaid EHR incentive programs, we do not propose any other restrictions. There may be opportunities to avoid duplication of reporting requirements among our various programs. In section II.A.3. of this final rule, we discuss how we will avoid duplication of reporting requirements for clinical quality measures.

Comment: Some commenters requested more information on efforts to avoid duplication of requirements and highly encouraged CMS to do everything it could in this regard.

Response: We address comments on the avoidance of duplication of requirements in several other areas of this rule where more specifics can be provided.

Comment: Commenters generally supported our proposal to only apply the limitation of participation in multiple programs to the limitation outlined in the HITECH Act.

Response: We continue to believe that providers should be able to participate in every program for which they are statutorily eligible and therefore are maintaining our proposal to only limit Medicare EPs from receiving either the Medicare EHR incentive payment or the Medicare E-Prescribing incentive payment.

\section{B. Medicare Fee for Service Incentives}

1. Incentive Payments for Eligible Professionals (EP)

Section 1848(o)(1)(A) of the Act, as amended by section 4101(a) of the
HITECH Act, provides for incentive payments to EPs who are meaningful users of certified EHR technology during the relevant EHR reporting periods. Section 1848(o)(1)(A)(i) of the Act provides that EPs who are meaningful EHR users during the relevant EHR reporting period are entitled to an incentive payment amount, subject to an annual limit, equal to 75 percent of the Secretary's estimate of the Medicare allowed charges for covered professional services furnished by the EP during the relevant payment year. Under section 1848(o)(1)(B)(ii)(VI) of the Act, an EP is entitled to an incentive payment for up to 5 years. In addition, in accordance with section

1848(o)(1)(A)(ii) of the Act, there shall be no incentive payments made with respect to a year after 2016. The incentive payments would be disbursed from the Federal Supplementary Medical Insurance Trust Fund, as provided for under section 1848(o)(1)(A)(i) of the Act. As noted in section II.A. of this final rule, EPs who qualify for both the Medicare and Medicaid incentive payments must elect to receive payments from one program or the other.

\section{a. Definitions}

In accordance with section 1848(o)(5)(C) of the Act, we will add a definition of the term "eligible professional" in our regulations at $\S 495.100$ to mean a physician as defined under section 1861(r) of the Act. Section 1861(r) of the Act defines the term "physician" to mean the following five types of professionals, each of which must be legally authorized to practice their profession under state law: a doctor of medicine or osteopathy, a doctor of dental surgery or dental medicine, a doctor of podiatric medicine, a doctor of optometry, or a chiropractor. As discussed in section II.B.1.a of this final rule, in accordance with section 1848(o)(1)(C) of the Act, hospital-based EPs are not eligible for an incentive payment.

Section 1848(o)(5)(A) of the Act defines covered professional services as having the same meaning as in section $1848(\mathrm{k})(3)$ of the Act, that is, services furnished by an eligible professional for which payment is made under, or is based on, the Medicare physician fee schedule.

In accordance with section 1848(a)(1) of the Act, the Medicare allowed charge for covered professional services is the lesser of the actual charge or the Medicare physician fee schedule amount established in section 1848 the Act. As specified under section 1848(o)(1)(A)(i) of the Act, the 
Secretary's estimate of allowed charges is based on claims submitted to Medicare no later than 2 months following the end of the relevant payment year. We proposed to codify these specifications and definitions in our regulations at 495.102 .

Comment: The commenters who expressed concerns about the EP definition under the Medicare program had one overall theme. It is that the definition is too narrow and that it should be more inclusive of other health professionals in order to serve the goals of the HITECH Act. The commenters stated that they believe that the intent of the electronic health records (EHR) legislation is to encompass a wide range of health professionals to incorporate efficient and effective EHR technology. Specifically, these commenters stated that the Medicare EP definition should be expanded to include nonphysician practitioners and health professionals such as physician assistants (PAs), nurse practitioners (NPs), clinical nurse specialists (CNSs), certified nursemidwives (CNMs), clinical psychologists (CPs), clinical social workers (CSWs), certified registered nurse anesthetists (CRNAs), registered nurses (RNs), occupational therapists (OTs), and credentialed pedorthists who make shoes for diabetic patients.

Additionally, we received a comment that the Medicare EP definition should recognize health professionals who provide health support services as members of an interdisciplinary health care team such as a team consisting of diabetes nurse educators, NPs, pharmacists, PAs, dieticians, and case managers.

Representatives of rural health clinics (RHCs), Federally qualified health centers (FQHCs), ambulatory surgical centers (ASCs), outpatient clinics and dialysis facilities commented that their providers should also be included under the Medicare EP definition to qualify for Medicare incentive payments. These providers believe that they are a key set of contributors that will implement and meaningfully utilize electronic health care record program modules that directly benefit their patient populations. Alternatively, one of these commenters recommended that provider eligibility should be determined by type of service provided rather than by location of service and should include non-physician clinicians and providers.

The sub-theme of the comments that we received on the Medicare EP definition is that the definition of an "eligible provider" that qualifies for EHR incentive payments should be a common definition for the Medicare and
Medicaid programs. The commenters believe that a uniform definition of an EP would be more administratively efficacious for the Medicare and Medicaid programs considering that EPs are permitted to switch participation between the Medicare and Medicaid incentive programs one-time after the initial payment year.

An organization representing pathologists expressed concern that the Medicare EP definition, as currently drafted would subject certain pathologists to payment incentive penalties for not being meaningful EHR users if the pathologists performed less than 90 percent of their professional services in any inpatient or outpatient setting in the prior year. All EPs have to report on all Core Measures and a subset of clinical measures that pathologists could not meet in their day-to-day practice given the nature of pathology's scope of practice. Accordingly, this organization recommended that CMS ensure that pathologists who are currently defined as Medicare EPs be considered as "non-qualifying" EPs, that are exempt from future meaningful user penalties.

Response: While we appreciate the comments that we received on the Medicare EP definition, we are unable to expand or alter this statutory definition or consolidate it with the Medicaid program EP definition as suggested by the commenters. Under the EHR incentive payment program, the law provided a separate Medicare EP definition rather than giving the Secretary authority or discretion to determine who is a Medicare EP or, who is an EP for both the Medicare and Medicaid programs.

Comment: A commenter requested clarification of the method used for determining Medicare incentives for EPs practicing in a rural health clinic.

Response: The amount of the EHR incentive payment is based on the estimated allowed charges for all covered professional services furnished by an EP during the payment year, subject to the maximum payment amount for the payment year for the EP. For EPs that practice in an RHC, EHR incentive payments are based on the amount of covered professional services that are not part of the RHC package of services and are billed by the EP through the physician fee schedule.

Comment: A commenter suggested that the definition of allowable charges be amended to include the RHC schedule of services, or allow providers who use UB92 and HCFA 1500 forms to be eligible for the EHR incentive payment.
Response: The allowed charge is the amount that Medicare determines to be reasonable payment for a provider or service under Part B, including coinsurance and deductibles. RHC services furnished by an EP are not considered covered professional services for purposes of the Medicare EHR because they are not billed or paid under the physician fee schedule.

After consideration of the public comments received on the term, "eligible professional" for the Medicare program, we are adopting the Medicare EP definition in our regulations at $\S 495.100$ that state that a Medicare EP is a physician as defined under $\S 1861(\mathrm{r})$ of the Social Security Act. That is, a Medicare EP is a doctor of medicine or osteopathy, a doctor of dental surgery or dental medicine, a doctor of podiatric medicine, a doctor of optometry, or a chiropractor and a doctor who is legally authorized to practice their profession under State law.

\section{b. Incentive Payment Limits}

Section 1848(o)(1)(B)(i) of the Act sets forth the annual limits on the EHRrelated incentive payments to EPs. Specifically, section 1848(o)(1)(B) of the Act provides that the incentive payment for an EP for a given payment year shall not exceed the following amounts:

- For the EP's first payment year, for such professional, $\$ 15,000$ (or, $\$ 18,000$ if the EP's first payment year is 2011 or 2012).

- For the EP's second payment year, $\$ 12,000$

- For the EP's third payment year, $\$ 8,000$.

- For the EP's fourth payment year, $\$ 4,000$.

- For the EP's fifth payment year, $\$ 2,000$.

- For any succeeding year, $\$ 0$.

Under section 1848(o)(1)(B)(iv) of the Act, for EPs who predominantly furnish services in a geographic HPSA (as designated by the Secretary under section 332(a)(1)(A) of the Public Health Service (PHS) Act), the incentive payment limitation amounts for each payment year are increased by 10 percent. Section 1848(o)(1)(B)(iii) of the Act also provides for a phased reduction in payment limits for EPs who first demonstrate meaningful use of certified EHR technology after 2013. Specifically, if the EP's first payment year is after 2013, then the annual limit on the incentive payment equals the annual limit applicable to an EP whose first payment year is 2013. Accordingly, if the EP's first payment year is 2014, the EP's maximum incentive payment will be $\$ 12,000$ in $2014, \$ 8,000$ in 2015 , and $\$ 4,000$ in 2016. Section 1848(o)(1)(B)(v) 
of the Act provides that if the EP's first payment year is after 2014, then the applicable incentive payment limit for such year and any subsequent year shall be $\$ 0$. In other words, an EP who does not qualify to receive an EHR-related incentive payment prior to 2015 will not receive any of these incentive payments.

Comment: One commenter believes that the methodology for determining the incentive payments under the incentive program does not offer each EP an equal incentive, despite being held to the same standards of adoption and implementation.

Response: We are uncertain why the commenter believes that the methodology for determining the incentive payments under the incentive program does not offer each EP an equal incentive to adopt EHR technology. However, the payment methodology in the statute for EPs (as well as the methodologies for hospitals and CAHs) is quite prescriptive, and offers no discretion for us to adopt revisions designed to enhance incentives for adoption. For EPs, the HITECH Act defines the incentive payment amount as, "an amount equal to 75 percent of the Secretary's estimate * * * of the allowed charges under this part of all such covered professional services furnished by the eligible professional during such year."

c. Increase in Incentive Payment for EPs Who Predominantly Furnish Services in a Geographic Health Professional Shortage Area (HPSA)

Section 1848(o)(1)(B)(iv) of the Act provides that the amount of the annual incentive payment limit for each payment year be increased by 10 percent for EPs who predominantly furnish services in an area that is designated by the Secretary (under section 332(a)(1)(A) of the PHS Act) as a geographic health professional shortage area (HPSA). This section of the PHS Act refers to geographic HPSAs, which are areas that have been designated by the Secretary as having a shortage of health professionals, based on the population-to-provider ratio and other factors. HPSAs are located in every State, and in both rural and urban areas.

Geographic HPSAs are defined in 42 CFR Part 5 and include primary medical care, dental, and mental health HPSAs. In accordance with the statute, we will increase the limits per payment year by 10 percent for EHR-related incentive payments to EPs who predominantly furnish covered professional services in a geographic primary medical care, dental, or mental health HPSA.
We proposed that for an EP to be considered as "predominantly" furnishing covered professional services in a geographic HPSA, more than 50 percent of the EP's covered professional services must be furnished in a geographic HPSA. We stated that using "more than 50 percent" as the criterion to define "predominantly" is consistent with how the term is defined in general parlance as well as how the definition is used for purposes of other aspects of the Medicare program. Our data indicates that most physicians furnishing services in a HPSA furnish 100 percent of their covered services in a HPSA, and only very few furnish services in both HPSA and non-HPSA areas.

To determine whether an EP has furnished more than 50 percent of his/ her covered professional services in a geographic HPSA, we proposed to utilize frequency of services provided over a 1-year period from January 1 to December 31, rather than basing it on the percentage of allowed charges. We proposed to make the incentive payment to the EP based on an EP's estimated allowed charges for the relevant payment year.

We proposed that once we compile a full year of data, we would determine eligibility for the EHR HPSA payment limit increase for the payment year based on whether the EP provided more than 50 percent of his/her services in a geographic HPSA during the payment year. The determination would be made based on claims submitted not later than 2 months after the end of the year. If we determine that the EP provided more than 50 percent of his/her services in a geographic HPSA and is therefore eligible for the EHR HPSA payment limit increase, we would then make an additional lump sum payment to reflect that increased limit amount based on the estimated allowable charges for that EP for the prior year. The additional amount would be paid no later than 120 days after the end of the prior year for which the EP was eligible for the 10 percent EHR HPSA payment limit increase.

Most physicians furnishing services in a HPSA furnish 100 percent of their covered services in a HPSA. Section 1848(o)(1)(B)(iv) of the Act also authorizes us to apply the provisions of sections $1833(\mathrm{~m})$ and $(\mathrm{u})$ of the Act in implementing this 10 percent EHR HPSA payment limit increase, as the Secretary determines appropriate. Section $1833(\mathrm{~m})$ of the Act establishes the HPSA bonus program, which provides a 10 percent bonus to physicians who furnish Medicare covered professional services in a geographic HPSA.

Section 1833(m)(1) of the Act provides that physicians who furnish covered professional services in a year in an area that is designated as a geographic HPSA prior to the beginning of the year are eligible to receive the HPSA bonus for services furnished during the current year. We have interpreted this to mean that bonus payments should continue throughout the current year, even if the area loses its designation as a geographic HPSA during the current year. Physicians furnishing Medicare-covered professional services in an area that is not designated as a geographic HPSA by December 31 of the prior year are not eligible to receive the HPSA bonus for the current year, even if the area is subsequently designated as a geographic HPSA during the current year. We will apply these same rules for the 10 percent EHR HPSA payment limit increase provided under section 1848(o)(1)(B)(iv) of the Act.

Section $1833(\mathrm{~m})(2)$ of the Act also provides that geographic HPSAs that consist of an entire county be identified and the bonus paid automatically. We publish a list annually of the zip codes that are in these areas on our Web site at http://www.cms.hhs.gov/ HPSAPSAPhysicianBonuses/ 01 Overview.asp\#TopOfPage.

$\bar{P}$ hysicians furnishing Medicarecovered professional services in a zip code that is on this list automatically receive the HPSA bonus payment. Physicians furnishing Medicare covered professional services in a zip code that is not on this list but that was designated as a geographic HPSA as of December 31 of the prior year must use a modifier when submitting a Medicare claim in order to receive the HPSA bonus.

Comment: We received a comment stating that many EPs who work in a HPSA do so only on a part-time basis and that most would not qualify for the 10 percent increase in the payment limit based on the proposed threshold of furnishing more than 50 percent of his/ her covered professional services in a geographic HPSA. The commenter suggested that an EP should be able to qualify for the ten percent increase in the payment limit if at least 25 percent of his/her covered services during an EHR reporting period are furnished in a HPSA.

Response: The statute states that the annual payment limit be increased by ten percent for EPs who predominantly furnish services in a geographic HPSA. We continue to believe that "more than fifty percent" correctly reflects the 
meaning of the word "predominantly" as used in this statute. As noted above, our data also indicate that most physicians furnish all of their services either in a HPSA or outside of a HPSA, and only very few furnish services in both HPSA and non-HPSA areas.

Comment: Several commenters requested that Federally Qualified Health Centers (FQHCs) be eligible to receive the ten percent increase in the payment limit for EPs who predominantly furnish services in a HPSA since the FQHC is a legal entity that bills Medicare and receives payment for services provided by physicians.

Response: The 10 percent increase in the payment limit applies to EPs who predominantly furnish services in a geographic HPSA. FQHCs and RHCs are not eligible for the ten percent increase in the payment limit because they do not meet the definition of EP as specified in section 1848(o)(5)(C) of the Act. Please see others sections of the regulation that discuss the criteria to be considered an EP. Additionally, we wish to restate that FQHCs are not entitled to any Medicare or Medicaid incentive payments under this program.

Comment: A commenter suggested that "predominantly" be defined as the location where the EP provides the most services, so that an EP who sees patients in more than two locations could receive the increase in the payment limit if he/she provided more care in the HPSA location than any other location. The commenter also suggested that if this is too difficult to administer, we should accept an attestation from the EP.

Response: We are aware that many physicians, especially in rural areas, furnish services in more than one location, and appreciate the commenter's interest in making the HPSA payment limit increase available to these EPs. If we were to accept this recommendation, then an EP who worked in three locations at forty percent, thirty percent, and thirty percent time respectively, would be eligible for the HPSA payment limit increase if the first location was in a geographic HPSA. If the EP worked in four locations at thirty percent, twentyfive percent, twenty five percent, and twenty percent time respectively, he/she would be eligible for the HPSA payment limit increase if the first location was in a geographic HPSA. We considered this suggestion and concluded that lowering the threshold for services furnished in a HPSA would be inconsistent with the intent of the HPSA payment limit increase, which is to provide an incentive to promote the use of EHR by
EPs who practice predominantly in HPSAs. Also, if an EP who worked in more than two locations and furnished services in a HPSA only thirty or forty percent of his/her time was eligible for the HPSA payment limit increase, this would be unfair to an EP who worked in two locations and spent forty-five percent of his/her time in a HPSA and fifty-five percent time in a non-HPSA, because this EP would not be eligible for the HPSA payment limit increase even though he/she spent more total time in a HPSA.

Comment: A commenter stated that the proposed HPSA payment limit increase was being applied inconsistently because an EP would still get the payment limit increase if the designation was removed mid-year, and would not get the payment limit increase if the designation was added mid-year.

Response: Section 1848(o)(1)(B)(iv) of the Act authorizes us to apply the provisions of the HPSA bonus program to the implementation of the EHR HPSA payment limit increase. The HPSA bonus is paid to physicians who furnish Medicare-covered professional services in an area that is designated as a geographic HPSA as of December 31 of the prior year. They are authorized to receive the HPSA bonus throughout the current year, even if the area loses its designation as a geographic HPSA during the current year. Physicians furnishing Medicare-covered professional services in an area that is not designated as a geographic HPSA as of December 31 of the prior year are not eligible to receive the HPSA bonus for the current year, even if the area is subsequently designated as a geographic HPSA during the current year. We proposed to use the same methodology for the HPSA EHR program, and believe that this is consistent with the statute.

After consideration of the public comments received, we are finalizing these provisions as proposed.

\section{d. Form and Timing of Payment}

Section 1848(o)(1)(D)(i) of the Act, as amended by section 4101(a) of the HITECH Act, provides that the incentive payments may be disbursed as a single consolidated payment or in periodic installments as the Secretary may specify. We proposed to make a single, consolidated, annual incentive payment to EPs. Payments would be made on a rolling basis, as soon as we ascertained that an EP had demonstrated meaningful use for the applicable reporting period (that is, 90 days for the first year or a calendar year for subsequent years), and reached the threshold for maximum payment.
Section 1848(o)(1)(A) of the Act provides that "with respect to covered professional services provided by an eligible professional," the incentive payment "shall be paid to the eligible professional (or to an employer or facility in the cases described in clause (A) of section 1842(b)(6))." Section 1842(b)(6)(A) of the Act allows for reassignment to an employer or entity with which the physician has a valid contractual arrangement allowing the entity to bill for the physician's services. Therefore, we proposed that EPs would be allowed to reassign their incentive payment to their employer or an entity which they have a valid employment agreement or contract providing for such reassignment, consistent with all rules governing reassignments. We proposed to preclude an EP from reassigning the incentive payment to more than one employer or entity. To implement this requirement, we proposed to use the EP's Medicare enrollment information to determine whether an EP belongs to more than one practice (that is, whether the EP's National Provider Identifier (NPI) is associated with more than one practice). In cases where the EP was associated with more than one practice, we proposed that EPs would select one tax identification number to receive any applicable EHR incentive payment.

As mentioned above, we proposed that payments would be made on a rolling basis, as soon as we ascertain that an EP has demonstrated meaningful use for the applicable reporting period (that is, 90 days for the first year or a calendar year for subsequent years), and reached the threshold for maximum payment. We proposed to add a new part 495.10(e) and (f) to permit reassignment of the incentive payment with certain limitations. The following is a summary of the comments we received and our responses.

Comment: Several commenters, including one representing Rural Health Clinics, requested clarification of the statement in the proposed rule (75 FR 1910) that an eligible professional (EP) is allowed to reassign his/her EHR incentive payment to an employer or other entity to which the EP has reassigned his/her payments for Medicare covered services. The commenters believe that the HITECH Act requires in such cases that any Medicare EHR incentive for which the EP qualifies must be paid to such employer or other entity. The commenters reference the phrases from the HITECH Act, "shall be paid" to an eligible professional (or to an employer or facility in cases described in the reassignment provisions of the Social 
Security Act). In addition, the commenters referenced the phrase regarding the transfer of an EP's Medicaid EHR incentive which states that "such incentives are paid directly to such provider (or to an employer or facility to which such provider has assigned payments)". The commenters interpret these phrases to mean that an EP's EHR incentive payments (both Medicare and Medicaid) must be paid to an employer or other entity to which the $\mathrm{EP}$ has reassigned payments for his/her services.

Response: We do not agree with the commenters' conclusions regarding to whom the payments must be made. As we stated in the proposed rule, Section 1842(b)(6) of the Act allows, but does not require reassignment to an employer or entity with which the physician has a valid contractual arrangement allowing the employer or entity to bill for the physician's services. The HITECH Act provisions cited by the commenter similarly do not require that the EHR incentive payment be made pursuant to a reassignment, but provide that the payment may be made directly to the EP or to the employer or other entity. A physician reassigns payment based on the scope of his or her employment or contractual arrangement. Based upon our interpretation of the applicable provisions, we are finalizing our proposal at $\S 495.10(f)$ to permit EPs to reassign their incentive payments to their employer or to an entity with which they have a contractual arrangement, consistent with all rules governing reassignments including part 424, subpart F.

We are taking this opportunity to remind the public that if the EP wishes to reassign his or her incentive payment to the employer or entity with which the EP has a contractual arrangement, the parties should review their existing contract(s) to determine whether the contract(s) currently provides for reassignment of the incentive payment or if the contract(s) needs to be revised. Reassignment of the incentive payment must be consistent with applicable Medicare laws, rules, and regulations, including, without limitation, those related to fraud, waste, and abuse. For Medicaid, a discussion of reassignment of the incentive payment is found in section II.D.3.e of this final rule "Entities Promoting the Adoption of Certified EHR technology."

Comment: Several commenters stated that the rationale and objectives of the HITECH Act provisions regarding transfer of the EP's EHR incentives are merely to align EHR incentives and EHR costs. Therefore, they believe that the
HITECH Act provisions support their view that Congressional intent was to prevent windfall EHR incentives to EPs who incur no EHR-related costs. The commenters also asserted that CMS's failure to address this issue will require entities that employ or contract with EPs to enter into negotiations and a separate agreement transferring the EP's EHR incentive payments to the employer or other entity.

Response: We do not agree with the commenters' statement that the Congress intended to prevent windfall EHR incentives to EPs who incur no EHR-related costs. Title IV, Division B of the HITECH Act establishes incentive payments under the Medicare and Medicaid programs for certain professionals and hospitals that meaningfully use certified EHR technology. The provisions are not focused solely upon the costs associated with the EHR technology. Rather, as we stated in the proposed rule (75 FR 1849), it focuses upon the adoption, implementation, upgrade, or meaningful use of the technology.

However, we do agree that some entities may have to review and/or negotiate current contractual arrangements to address the transfer of the incentive payments. The first payment year for the incentive payment is CY 2011, which we believe should afford parties sufficient time to reach a new agreement. For Medicaid, a discussion of reassignment of the incentive payment is found in section II.D.3.e of this final rule "Entities Promoting the Adoption of Certified EHR technology.”

Comment: Several commenters supported our proposal that if an EP has reassigned his or her payments for services to more than one employer or entity, that only one of those employers or entities should receive the EP's EHR incentive payments for a particular EHR Reporting Period (75 FR 1910). The commenters do not believe that EPs should decide which employer or entity should receive his or her EHR incentive payment. Rather, the commenters stated that such payments should

automatically be paid to the employer or entity that has received for the reporting period the largest percentage of the EP's Medicare or Medicaid payments for services.

Response: We are not persuaded to adopt the commenters' suggestion. We believe that the suggestion by the commenters would create administrative complexities for both CMS and EPs with little benefit. Many of these obstacles would be similar to those described in the proposed rule when discussing the possibility of making proportional EHR incentive payments (75 FR 1911). Therefore, we are finalizing our proposal to revise $\S 495.10(\mathrm{e})$ to preclude an EP from reassigning the incentive payment to more than one employer or entity. In cases where the EP is associated with more than one practice, EPs must select one TIN to receive any applicable EHR incentive payment.

Comment: The commenters also state that if an EP has incurred out-of-pocket costs in connection with an EHR provided by an employer or other entity to which the EP has reassigned payments for his or her services, the EP should be permitted to keep an amount of his or her EHR incentives equal to the amount of such costs incurred.

Response: The statute does not address this issue. It simply provides that the incentive payments are to be made directly to the EP or to an employer or other entity to which the EP has reassigned the incentive payment. Reassignment of the incentive payment must be consistent with applicable Medicare laws, rules, and regulations, including, without limitation, those related to fraud, waste, and abuse. We believe that any costsharing or subsequent distribution of the incentive payment, such as in the manner described by the commenter, should be resolved between the parties.

Comment: Several commenters urged CMS to clarify that any reassignment of the EP's EHR incentive payment should not constitute a financial arrangement within the meaning of the physician self-referral law, or remuneration within the meaning of the federal anti-kickback statute.

Response: The physician self-referral law prohibits a physician from making a referral for designated health services to an entity with which the physician or a member of the physician's immediate family has a financial relationship, unless an exception applies. For purposes of the physician self-referral law, a financial arrangement includes ownership or investment interests and compensation arrangements. The statute defines a "compensation arrangement" to mean any arrangement involving remuneration, direct or indirect, overt or covert, in cash or in kind. A reassignment of an EP's EHR payment would constitute remuneration, and we note that reassignment generally occurs in the context of an existing compensation arrangement (for example, employment). There are many potentially applicable exceptions for compensation arrangements that involve a physician's reassignment of Medicare payments. 
Similarly, with respect to the antikickback statute, absent compliance with a safe harbor, a determination of whether a reassignment constitutes prohibited remuneration would be made on a case-by-case basis and we therefore decline to issue any statement regarding the application of the anti-kickback statute to a reassignment. For additional information regarding the anti-kickback statute, please refer to the OIG's Web site at http://oig.hhs.gov.

Comment: One commenter representing American Indian and Alaska Native health providers urged CMS to require that the HITECH/EHR Meaningful Use provider incentive payments be reassigned to the Tribal outpatient clinics, because the Tribal clinics developed the infrastructure not the EPs themselves, and purchased electronic medical record systems to complement the current Registration Patient Management Systems (RPMS) of the Indian Health Service. In addition, the commenter noted that many tribal outpatient clinics have employment contracts with their EPs. Thus, the commenters urged CMS to require that incentive EHR payments should be included in employment contracts to help protect the EP as employee and the Tribe as the employer.

Response: As stated above, section 1848(o)(1)(A) of the Act provides that the EP's incentive payment shall be paid to the eligible professional (or to an employer or other entity with which the physician has a valid contractual arrangement allowing the employer or other entity to bill for the physician's services). We recognize that some tribes purchased EHR systems based upon criteria established by the Indian Health Service. However, after careful consideration, we believe that the same standards concerning the incentive payments should apply. The EP and the Tribal outpatient clinic should jointly resolve whether the EP's EHR incentive payment will be reassigned to the Tribal outpatient clinic or made directly to the EP. Similarly, any decision by the Tribal outpatient clinic concerning whether to include language in its employment contract (or in the alternative, whether any pre-existing contract already requires reassignment of the payment), is a matter of contract interpretation that should be resolved by the parties themselves. This discussion is also addressed in the Medicaid section of this rule at II.D.4.a.3.

Comment: One commenter expressed concern about the potential tax consequences associated with an EP's reassignment of the EHR incentive payment by an independent contractor to a larger organization. The commenter recommended that a 1099 independent contractor should consult with his/her tax advisor before agreeing to reassign incentive payments and to ensure that the election to reassign is made before payment is sent from CMS or the State Medicaid Agency.

Response: The commenter's

recommendation falls outside the scope of our authority. This is a matter for the 1099 independent contractor EP to consider.

Comment: Many national and state medical associations expressed concern regarding the proposed requirement that the EP must identify a Tax Identification Number (TIN) to which the EP's incentive payment should be made. They assert that this will not work for physicians who do not have a TIN, and are enrolled in Medicare or Medicaid through their Social Security Number (SSN). Therefore, the commenters recommend that CMS accept the SSN in lieu of the TIN, so that all eligible physicians are able to participate in the Medicare and Medicaid EHR incentive programs.

Response: We recognize that many physicians are enrolled in Medicare or Medicaid through their Social Security Number (SSN). Therefore, we are revising our proposal at $\S 495.10$ that an EP must submit, in a manner specified by CMS, the Taxpayer Identification Number (TIN) to which the EP's incentive payment should be made. In finalized §495.10(c), we provide that the TIN may be the EP's Social Security Number (SSN) to which the EP's incentive payment should be made. We note that if the physician is part of a group with more than one owner or organization that is incorporated, they would have a TIN for the corporation that is not the EP's SSN.

Comment: Some commenters recommended that the employer or entity to which an EP reassigns payment for covered services, should be deemed authorized to provide, on the EP's

behalf, any documentation necessary for the EP to qualify for EHR incentive payments.

Response: We believe that this should be resolved by the parties themselves. There is nothing in the statute that requires an EP's employer or other entity to which an EP reassigns payment to provide any necessary documentation for an EP to qualify for EHR incentive payments. Rather, the finalized regulatory provision at $\S 495.8$ provides that an EP must demonstrate that he or she satisfies each of the applicable objectives and associated measures under $\S 495.6$. If the parties wish to have the necessary documentation furnished by the employer or entity, they should resolve this pursuant to an employment or contractual agreement. We are finalizing our proposal because we believe that making a single, consolidated payment would be the least administratively burdensome for both CMS and EPs. In addition, we believe a single, consolidated payment would reduce the possibility of fraud and duplicate payments. Several of these issues related to reassignment of payment are also addressed in the Medicaid section. See II.D.3.e.

e. Payment Adjustment Effective in CY 2015 and Subsequent Years for EPs Who Are Not Meaningful Users of Certified EHR Technology

Section 1848(a)(7) of the Act, as amended by section 4101(b) of the HITECH Act, provides for payment adjustments effective for CY 2015 and subsequent years for EPs who are not meaningful EHR users during the relevant EHR reporting period for the year. In general, beginning in 2015, if an EP is not a meaningful EHR user for any EHR reporting period for the year, then the Medicare physician fee schedule amount for covered professional services furnished by the EP during the year (including the fee schedule amount for purposes of determining a payment based on the fee schedule amount) is adjusted to equal the "applicable percent" of the fee schedule amount (defined below) that would otherwise apply. The HITECH Act includes a significant hardship exception, discussed below, which, if applicable, could exempt certain EPs from this payment adjustment. The payment adjustments do not apply to hospitalbased EPs.

The term "applicable percent" means: "(I) for 2015, 99 percent (or, in the case of an EP who was subject to the application of the payment adjustment if the EP is not a successful electronic prescriber under section 1848(a)(5) for 2014, 98 percent); (II) for 2016, 98 percent; and (III) for 2017 and each subsequent year, 97 percent."

In addition, section 1848(a)(7)(iii) of the Act provides that if for 2018 and subsequent years the Secretary finds that the proportion of EPs who are meaningful EHR users is less than 75 percent, the applicable percent shall be decreased by 1 percentage point from the applicable percent in the preceding year, but in no case shall the applicable percent be less than 95 percent.

Significant Hardship Exceptionsection 1848(a)(7)(B) of the Act provides that the Secretary may, on a case-bycase basis, exempt an EP who is not a meaningful EHR user for the year from the application of the payment 
adjustment if the Secretary determines that compliance with the requirements for being a meaningful EHR user would result in a significant hardship, such as in the case of an EP who practices in a rural area without sufficient Internet access. The exemption is subject to annual renewal, but in no case may an EP be granted a hardship exemption for more than 5 years.

Comment: Some commenters believed that when an EP's performance leads to a negative financial impact under Medicare payment policy, it would be unfair and overly punitive for them to face a separate and potentially more significant financial impact-whether through a denial of funding and/or ARRA's penalties. Further, some commenters indicated that they interpreted these requirements to mean that Medicaid participants would or would not experience fee-schedule adjustments if they are not meaningful users by the end of 2014.

Response: We will reduce payments as specified under the statute. Under sections 4101(b) and (c) of the HITECH Act, we are required to pay EPs less than 100 percent of the fee schedule and to make downward adjustments to MAaffiliated EPs for their professional services if they are not meaningful users of certified EHR beginning in CY 2015. Under sections 4102(a), (a)(2), and (c) of the HITECH Act, we are authorized to pay eligible hospitals a reduced annual payment update, provide downward payment adjustment to CAHs for cost reporting periods, and provide downward payment adjustment to MAaffiliated hospitals respectively, if they are not meaningful users of certified EHR technology beginning in FY 2015. The Medicare fee schedule adjustments will impact any EP or subsection(d) hospital that is not a meaningful user by the end of 2014. The adjustments are not authorized under Medicaid, but the adjustments will still apply to Medicaid EPs who are also Medicare EPs and also to Medicaid acute care hospitals that are also subsection(d) hospitals. We are finalizing these provisions as proposed.

\section{Incentive Payments for Hospitals}

a. Definition of Eligible Hospital for Medicare

Section 1886(n) of the Act, as amended by section 4102(a)(1) of the HITECH Act, provides for incentive payments, beginning in FY 2011 (that is, October 1, 2010 through September 30, 2011) for eligible hospitals that are meaningful users of certified EHR technology during the EHR reporting period for the payment year. In the proposed rule, we proposed a new
$\S 495.104$ to implement this provision. As we noted in the proposed rule, section 1886(n)(6)(B) of the Act defines "eligible hospitals" for purposes of the incentive payments provision, as "subsection (d) hospitals," referring to the definition of that term in section 1886(d)(1)(B) of the Act. Section 1886(d)(1)(B) of the Act generally defines a "subsection (d) hospital" as a "hospital located in one of the fifty States or the District of Columbia." The term therefore does not include hospitals located in the territories or hospitals located in Puerto Rico. Section 1886(d)(9)(A) of the Act separately defines a "subsection (d) Puerto Rico hospital" as a hospital that is located in Puerto Rico and that "would be a subsection (d) hospital if it were located in one of the 50 states." Therefore, because section 4102(a)(1) of the HITECH Act does not refer to "subsection (d) Puerto Rico hospitals," we proposed that incentive payments for meaningful users of certified EHR technology would not available under this provision to hospitals located in Puerto Rico. The provision does apply to inpatient, acute care hospitals located in the State of Maryland. These hospitals are not currently paid under the IPPS in accordance with a special waiver provided by section 1814(b)(3) of the Act. Despite this waiver, the Maryland hospitals continue to meet the definition of a "subsection (d) hospital" because they are hospitals located in the 50 states. Therefore we proposed that incentive payments for meaningful users of certified EHR technology would be available under this provision to acute care hospitals located in the State of Maryland. The statutory definition of a subsection (d) hospital also does not apply to hospitals and hospital units excluded from the IPPS under section 1886(d)(1)(B) of the Act, such as psychiatric, rehabilitation, long term care, children's, and cancer hospitals. We also proposed that, for purposes of this provision, we would provide incentive payments to hospitals as they are distinguished by provider number in hospital cost reports. We proposed that incentive payments for eligible hospitals would be calculated based on the provider number used for cost reporting purposes, which is the CMS Certification Number (CCN) of the main provider (also referred to as OSCAR number). Payments to eligible hospitals are made to each provider of record. The criteria for being a meaningful EHR user, and the manner for demonstrating meaningful use, are discussed in section B.2. of this final rule.
Comment: We received numerous comments on our proposal to identify all individual hospitals eligible for incentive payments based on the provider number used for cost reporting purposes (the CCN of the main provider). These commenters, including national and regional hospital associations, hospital systems, and hospitals with multiple campuses, objected to the proposed policy on various grounds. Many of these commenters pointed out that there is no standard policy that defines the specific types of facilities to which a single CCN applies. As a result, a single CCN could encompass multiple hospitals within a hospital system in some cases, while in other cases multiple hospitals within a system could have separate CCNs. These commenters therefore maintained that our proposed policy would unjustifiably lead to disparate treatment of hospital systems based solely on whether the system had one or more provider numbers. Commenters also maintained that, because the Medicare and Medicaid payment incentives are calculated using a per-hospital base amount, plus a capped per-discharge amount per hospital, identifying individual hospitals solely by CCN would result in distributing payments in a manner that does not foster

widespread EHR adoption and use. The for this argument regarding limited EHR adoption and use is that multi-campus systems with a single CCN would receive only one base payment, and would be more likely to reach the discharge cap. Some commenters also argued that linking incentive payments only to a single CCN would not accurately reflect the pattern of costs required for deploying EHR systems across all sites in a hospital system. For example, even hospital sites that are part of the same system often require significant variations in their EHR systems, accommodating local policies and processes, as well as different legacy systems, physician preferences, clinical protocols, and other variables. Some commenters cited as a precedent our policy with regard to hospitals with one CCN, but multiple sites spanning more than one wage index region. CMS has instructed such hospitals to report wage data for each site separately on the cost report, and pays for discharges under the wage index that applies where the service is provided, that is, under a different wage index for each site.

These commenters recommended various approaches to recognizing and verifying the status of separate hospitals under one CCN number. Many of them 
recommended that we adopt a "multipronged approach that allows a "hospital" to be defined in ways that acknowledge the varied organizational structures of multi-hospital systems, including by a distinct CCN, a distinct emergency department, or a distinct hospital license." Commenters recommended that we indentify and verify the distinct hospitals within hospital systems either by revising the cost report or by developing an attestation process similar to the process employed under $\S 413.65$ of the regulations to verify provider-based status. Commenters also recommended that we either collect the data necessary for determining payment amounts (for example, discharge counts) directly from each hospital within a system with a single provider number, or develop a method of allocating discharges, bed days, and other relevant data among the hospital campuses represented in a hospital cost report under a single CCN.

Finally, a number of the commenters advocating a different approach contended that our proposed policy ran counter to the intent of the EHR incentive provision, which is to promote broader adoption of EHR systems. These commenters argued in various ways that recognizing each campus of a multi-campus hospital for separate payment was most consistent with the statute because it would provide a greater overall level of funding for EHR efforts, especially to hospital systems that have elected to enroll multiple campuses under a single Medicare provider agreement, and thus support diffusion of EHR systems more broadly. One of these commenters did, however, acknowledge that "in most circumstances the term 'subsection(d) hospital' under the Medicare Program includes all of a hospital system's inpatient facilities that operate under a single provider number," before going on to argue that CMS has both the authority and the obligation under the HITECH Act to diffuse EHR incentive payment more broadly by treating each facility under a hospital system as a separate hospital, regardless of whether any of the facilities share a single provider number.

Response: We appreciate the commenters' concerns, but we continue to believe that our proposal represents the best policy approach in determining what constitutes an "eligible hospital." In the absence of clear direction from the statute to the contrary, we believe that the most appropriate policy is to interpret the terms in subsection (d) "acute care hospital" and "children's hospital" in the light of existing Medicare and Medicaid program policies and precedents. It is quite true, as a number of the commenters noted, that hospital systems have considerable latitude (although not unlimited) in choosing whether to obtain one CCN for all their facilities, or to obtain separate CCNs for some or all of their facilities. However, once a hospital has sought and obtained a single CCN for two or more facilities, that hospital has chosen to represent itself to CMS as a single hospital, including for purposes of payment, cost reporting, and satisfying the conditions of participation. Such systems submit unified cost reports integrating data (including charges, discharges, bed days, and other relevant data) from every facility under the single CCN. For purposes of DSH and IME payments under the IPPS, both eligibility for payment and the applicable payment amounts are determined on the basis of this integrated data. Most significantly, the Medicare conditions of participation require that a system with a single $\mathrm{CCN}$ establish and maintain a single governing structure, medical staff, nursing staff, and record services. Section 482.2 states that a "hospital must have an organized medical staff that operates under by-laws approved by the governing body." Section 482.21(e) states that the governing body must ensure, among other matters, that "the hospital-wide quality assessment and performance improvement efforts address priorities for improved quality of care." In addition, $\S 482.24$ states that the hospital must have "a medical record service that has administrative for medical records.”. For these reasons, we believe that recognition of the decision made by each hospital or hospital to represent and organize itself as a single entity under one CCN, or as two or more distinct entities under separate CCNs is a strength, rather than a weakness, of our proposed policy. Each institution that has exercised available latitude to obtain one CCN for all their facilities not only represents itself as a single hospital, but also agrees to conduct itself in significant ways as a single hospital.

We also do not agree with those commenters who argue that our policy of applying different wage indexes to the campuses comprising a hospital system operating under a single CCN warrants our treating each campus as a separate eligible hospital for purposes of the EHR incentive payment program. Our policy for these few cases when a multi-campus hospital spans two or more wage index areas does not amount to recognizing that each campus is a separate hospital for payment purposes, but rather to accounting for the fact that, in these few cases, one hospital is located in two wage index areas. In these cases, it is appropriate to pay, and to account for wages, on the basis of where each discharge occurs rather than on the basis of where, for example, the main campus of a hospital may be located.

With regard to the disparate treatment argument advanced by a number of commenters, we acknowledge that, under our proposed policy, a single hospital system with two campuses will receive (all other things being equal) lower incentive payments than the combined incentive payments of twosingle-campus hospitals with the same number of discharges. However, an equivalent disparate treatment situation would arise under the policy advocated by these commenters. Under the policy of recognizing each campus of a multicampus system as a separate hospital, a single-campus hospital would received lower incentive payments than a multicampus hospital with the same number of discharges, despite the fact that both hospitals have a single CCN and are recognized for administrative and financial purposes, and for purposes of the conditions of participation, as a single hospital.

Example: Hospital A is a multicampus hospital with 30,000 discharges and a Medicare share of 50 percent. Hospital A's discharges are evenly split between its two campuses. Hospital B is a single campus hospital with 30,000 discharges and a Medicare share of 50 percent. During the first year of the transition, each campus of Hospital A would receive a separate incentive payment determined on the following manner: $(\$ 2,000,000$ base amount $+[(15,000-$ $1,149) \times \$ 200]$ discharge-related amount) $\times .5$ Medicare share $\times 1.0$ transition factor $=(\$ 2,000,000+$ $\$ 2,770,200) \times .5 \times 1.0=\$ 2,385,100$

Hospital A's total payment would therefore be $\$ 4,770,200$. In contrast, Hospital B would receive a single payment determined in the following manner:

$(\$ 2,000,000$ base amount $+[(23,000-$ $1,149) \times \$ 200$ ] discharge-related amount $\times .5$ Medicare share $\times 1.0$ transition factor $=(\$ 2,000,000+$ $\$ 4,370,200) \times .5 \times 1.0=\$ 3,185,100$

Hospital B would thus receive a payment that is $\$ 1,585,100$ smaller than Hospital A's total payment for the same number of discharges.

The change in policy recommended by these commenters will therefore replace one equity issue with another. We see no reason to privilege one of these arguments over the other, and 
therefore we believe that the decision on a final policy ought to turn on the other considerations that we discuss.

Finally, we cannot agree with the commenters that determining the appropriate policy on this question should turn on which alternative produces the greatest overall level of spending on EHR systems. Many decisions could result in lower potential payments to some or all potential meaningful users of EHR payments. Congress deliberately chose to limit incentive payments based on the statutory formula (using the current statutory and regulatory definition of "subsection (d) hospital"), and further limited the amount of incentive payments available to large hospitals by not increasing incentive payments above 23,000 discharges.

After consideration of the public comments received, we are finalizing our policy as proposed. For purposes of this provision, we will provide incentive payments to hospitals as they are distinguished by provider number in hospital cost reports. Incentive payments for eligible hospitals will be calculated based on the provider number used for cost reporting purposes, which is the CMS Certification Number (CCN) of the main provider (also referred to as OSCAR number). Payments to eligible hospitals will be made to each provider of record.

b. Incentive Payment Calculation for Eligible Hospitals: Initial Amount

Section 1886(n)(2) of the Act, as amended by 4102(a) of the HITECH Act, describes the methodology for determining the incentive payment amount for eligible hospitals that are meaningful users of certified EHR technology during the EHR reporting period for a payment year. In general, that section requires the incentive payment for each payment year to be calculated as the product of: (1) An initial amount; (2) the Medicare share; and (3) a transition factor applicable to that payment year.

As amended by section 4201(a) of the HITECH Act, section 1886(n)(2)(A)(i) of the Act defines the initial amount as the sum of a "base amount," as defined in section 1886(n)(2)(B) of the Act, and a "discharge related amount," as defined in section 1886(n)(2)(C) of the Act. The base amount is $\$ 2,000,000$, as defined in section 1886(n)(2)(B) of the Act. The term "discharge related amount" is defined in section 1886(n)(2)(C) of the Act as "the sum of the amount, estimated based upon total discharges for the eligible hospital (regardless of any source of payment) for the period, for each discharge up to the 23,000th discharge as follows:

(i) for the first through the 1,149th discharge, $\$ 0$.

(ii) for the 1,150th through the 23,000th discharge, $\$ 200$.

(iii) for any discharge greater than the 23,000th, \$0."

In addition to the base amount, the discharge related amount provides an additional \$200 for each hospital discharge during a payment year, beginning with a hospital's 1,150th discharge of the payment year, and ending with a hospital's 23,000th discharge of the payment year. No additional payment is made for discharges prior to the 1,150th discharge, or for those discharges subsequent to the 23,000th discharge. We proposed to implement the "initial amount" within the formula as that term is defined in the statute.

Comment: Several commenters requested that we identify the sources of the discharge data we plan to employ for purposes of determining the discharge related amount. These commenters also requested confirmation of their understanding that no type of discharge, regardless of source of payment, would be excluded from the discharge count for this purpose. Commenters specifically cited nursery discharges and discharges from non-PPS areas of a hospital as examples of discharges that should not be excluded under the statutory language, which they believe requires the inclusion of all patient discharges regardless of type of patient within the inpatient areas of the hospital.

Response: We cannot agree with the commenters that the statutory language includes all patient discharges within the inpatient areas of the hospital. Rather, the statutory language clearly restricts the discharges to be counted for purposes of determining the dischargerelated amount to discharges from the acute care portion of the hospital. As we discussed in the proposed rule, the term "discharge related amount" is defined in section 1886(n)(2)(C) of the Act as "the sum of the amount, estimated based upon total discharges for the eligible hospital (regardless of any source of payment) for the period, for each discharge up to the 23,000 th discharge as follows:

(i) for the first through the 1,149th discharge, $\$ 0$.

(ii) for the 1,150th through the 23,000th discharge, $\$ 200$.

(iii) for any discharge greater than the 23,000th, \$0."

The phrase "total discharges for the eligible hospital (regardless of any source of payment)" limits the count of discharges to the acute care inpatient discharges. This is because of the reference to "eligible hospital." "Eligible hospital" is defined in section 1886(n)(6)(B) of the Act for purposes of the incentive payments provision, as "a subsection (d) hospital," referring in turn to the definition of that term in section 1886(d)(1)(B) of the Act. Section 1886(d)(1)(B) of the Act generally defines a "subsection (d) hospital" as a "hospital located in one of the fifty States or the District of Columbia," excluding hospitals that are not paid under the IPPS in accordance with section 1886(d)(1)(B) of the Act, such as psychiatric, rehabilitation, long term care, children's, and cancer hospitals. However, 1886(d)(1)(B) also specifies that the "term 'subsection (d) hospital * * * does not include a psychiatric or rehabilitation unit of the hospital which is a distinct part of the hospital (as defined by the Secretary)." Therefore, the term "eligible hospital" for purposes of the incentive payments provision does not extend to the excluded units of the hospital. The term does, of course, include the inpatient portion of the hospital that receives payment for Medicare purposes under the inpatient PPS. The phrase "regardless of any source of payment," however, indicates that the count of "total discharges" for this purpose should include not only patients for whom Medicare is the source of payment, but also patients for whom payment is received from Medicaid or any other source of payment. Accordingly, in the revised cost report form that is currently pending and which will be finalized in time for the 2011 payment year, CMS Form 2552-10, Hospital and Hospital Health Care Complex Cost Report, we have included a cell for entry of "Total hospital discharges as defined in section 4102 of AARA," in the new Worksheet E-1, Part II, "Calculation of

Reimbursement for Settlement for HIT." This new cell is derived from line 14, from "Worksheet S-3, Part I column 15." In turn, this cell from Worksheet S-3, Part I, column 15 incorporate all discharges from the inpatient, acute care portion of the hospital, regardless of payment source. In this final rule, we have also revised the definition of "eligible hospital" in $\S 495.100$ of the regulations, as well as the specification of "initial amount" in $\S 495.104$ (c)(3) of the regulations, in order to clarify this point.

Section 1886(n)(2)(C) of the Act, as amended by section 4102(a) of the HITECH Act, specifies that a "12-month period selected by the Secretary" may be 
employed for purposes of determining the discharge related amount. While the statute specifies that the payment year is determined based on a Federal fiscal year (FY), section 1886(n)(2)(C) of the Act provides the Secretary with authority to determine the discharge related amount on the basis of discharge data from a relevant hospital cost reporting period, for use in determining the incentive payment during a FY. FYs begin on October 1 of each calendar year, and end on September 30 of the subsequent calendar year. Hospital cost reporting periods can begin with any month of a calendar year, and end on the last day of the 12th subsequent month. We proposed, for purposes of administrative simplicity and timeliness, for each eligible hospital during each incentive payment year, to use data on the hospital discharges from the hospital fiscal year that ends during the FY that is prior to the FY that serves as the payment year as the basis for making preliminary incentive payments. Similarly, we proposed that final payments would be determined at the time of settling the cost report for the hospital fiscal year that ends during the payment year, and settled on the basis of the hospital discharge data from that cost reporting period.

Example of proposal: FY 2011 begins on October 1, 2010 and ends on September 30, 2011. For an eligible hospital with a cost reporting period running from July 1, 2009 through June 30,2010 , we would employ the relevant data from the hospital's cost reporting period ending June 30, 2010 in order to determine the incentive payment for the hospital during FY 2011. This timeline would allow us to have the relevant data available for determining payments in a timely manner for the first and subsequent payment years. This timeline would also render it unnecessary to develop a cumbersome process to extract and employ discharge data across more than one hospital cost reporting period in order to determine the discharge related amount for a FYbased payment period. However, final payments would be based on hospital discharge data from the cost report ending June 30, 2011, and determined at the time of settlement for that cost reporting period.

Commenters raised several issues with regard to our proposals regarding the timing of the cost reports to be used for purposes of determining preliminary and final incentive payments. Each of these issues embraces the use of several data elements, including discharge counts, bed days, and other factors employed in the payment calculations. For purposes of simplicity, we will address these issues in general terms in this section. As we will note at several junctures below, this discussions of these issues, however, are applicable to the cost report data for other elements of the computation.

Comment: Several commenters called our attention to timing issues with regard to the cost reporting periods that we proposed to use for purposes of determining preliminary and final incentive payments. These commenters noted that, if we finalize our proposal to use data from the hospital fiscal year that ends during the FY prior to the FY that serves as the payment year as the basis for making preliminary incentive payments, hospitals with cost reporting periods on the October-to-September cycle would face a delay of two months or longer after potentially qualifying as a meaningful user before receiving a preliminary incentive payment.

Specifically, for hospitals on this cycle, the cost report that would be used for determining interim payments for the first payment year (the October 1, 2009 through September 30, 2010 cost report) would not be due until February 28, 2011, two months after the hospital may have been able to qualify as a meaningful user (January 1, 2011). For hospitals on the September-to-August cycle, the delay could be one month. The commenters pointed out that over one-fifth of subsection (d) hospitals have cost reporting periods beginning on September 1 or October 1. The commenters therefore recommended that we employ discharge and other data from a hospital's most recently filed cost report as the basis for determining the hospital's preliminary incentive payment once the hospital has qualified as a meaningful user.

Response: We agree with these commenters, and in this final rule we are therefore adopting the policy that we employ discharge and other data from a hospital's most recently filed 12-month (see discussion below) cost report as the basis for determining the hospital's preliminary incentive payment once the hospital has qualified as a meaningful user. However, the precise timing of payments, especially during the first payment year, may be affected by other factors such as the timeline for implementing the requisite systems to calculate and disburse the payments. We are adopting the policy recommended by the commenters in order to avoid any unnecessary delays in making interim payments due merely to the timing of cost reporting periods.

Example: FY 2011 begins on October 1, 2010 and ends on September 30, 2011. For an eligible hospital with a cost reporting period on the October-to-
September cycle, we would employ the relevant data from the hospital's most recently submitted cost reporting period in order to determine the incentive payment for the hospital during FY 2011. If the hospital qualifies for incentive payments on January 1, 2011, this would probably be the cost report for the period running from October 1, 2008 through September 30, 2009.

However, we would also employ the October 1, 2009 through September 30, 2010 cost report, if that cost report is submitted before the point when preliminary incentive payments can be calculated.

Comment: A number of commenters also raised concerns about our proposal to determine final incentive payments at the time of settling the cost report for the hospital fiscal year that ends during the payment year, and to be settled on the basis of the hospital discharge and other data from that cost reporting period. These commenters pointed out that the pending CMS Form 2552-10 will not be effective in time for all hospitals and CAHs to complete the new S-10 worksheet, Hospital Uncompensated Care and Indigent Care Data, reporting charity care for their cost reporting period ending during the payment year. The effective date of the new cost report will be for cost reporting periods beginning on or after May 1, 2010 (as opposed to February 1, 2010 date anticipated in the proposed rule). For purposes of our proposal for determining final incentive payments, including the Medicare share/charity calculation, the first cost reporting period for which the new cost report will be available is the period running from May 1, 2010 through April 30, 2011. This means that, for cost reporting periods ending in FY 2011 before April 30, hospitals will not be able to complete the new S-10 worksheet to report charity care charges. Therefore, these commenters recommended that we revise our proposed policy, so that final incentive payments will be determined at the time of settlement for the cost reporting period beginning in the payment year. In this way all hospitals, regardless of their cost reporting cycle, will have adequate time to submit the revised cost reports in time for determining final incentive payments.

Response: We agree with these commenters, and in this final rule we are therefore adopting the policy that we determine final incentive payments at the time of settling the 12-month (see discussion below) cost report for the hospital fiscal year that begins after the beginning of the payment year, and to be settled on the basis of the hospital 
discharge and other data from that cost reporting period.

Example: FY 2011 begins on October 1, 2010 and ends on September 30, 2011. For an eligible hospital with a cost reporting period running from July 1 through June 30, we would employ the relevant data from the hospital's cost reporting period ending June 30, 2009 in order to determine the preliminary incentive payment for the hospital during FY 2011 (or June 30, 2010, if that cost report was filed prior to the calculation). However, final payments would be based on hospital discharge data from the cost report beginning on July 1, 2011 and ending June 30, 2012, and determined at the time of settlement for that cost reporting period.

Comment: Several commenters requested that we explain how the occurrence of non-standard cost reporting periods will be taken into account in determining the appropriate cost reporting periods to employ for determining preliminary and final EHR incentive payments. Non-standard cost reporting periods run for periods shorter than the standard 12-month cost reporting periods (for example, 3 months, 6 months), and are typically employed to accommodate the circumstances of hospitals in several distinct situations, such as newly constructed hospitals, changes of ownership, and reorganization of a single multicampus hospital into multiple separate providers. In these cases, one non-standard cost reporting period may be employed before the hospital resumes (or begins) cost reporting on a 12-month cycle. One commenter recommended that we account for these situations by adopting three changes to our proposed regulations:

- For purposes of determining preliminary incentive payments, employ the most recently submitted 12month cost reporting period that ends in the year prior to the payment year, in order to account for those situations in which the most recent cost reporting period ending prior to the payment year is a non-standard period.

- For purposes of determining final incentive payments, employ the first 12month cost reporting period that begins after the start of the payment year, in order to account for those situations in which the cost reporting period ending during the payment year is a nonstandard period.

- Provide that a hospital may address the CMS regional office responsible for its payment area for determination of the appropriate cost reporting period to employ for calculating preliminary or final incentive payment in cases that are not anticipated by the rules adopted in the final regulation.

Response: We acknowledge that we failed to address the circumstances of non-standard cost reporting periods in the proposed rule, and we agree with the commenters that it is only appropriate to do so. Non-standard cost reporting periods are not likely to be truly representative of a hospital's experience, even if methods were to be adopted for extrapolating data over a normal 12-month cost reporting period. This is because these periods are often quite short (for example, 3 months), which makes it questionable to extrapolate the data over a full cost reporting period. In addition, these abbreviated periods often capture the experience of a hospital during a period of transition (for example, change of ownership), which often renders the data highly unrepresentative. We also agree with the logic of the policy revisions proposed by the commenter cited above, subject only to the necessity of adapting the

recommendations slightly to the revisions, as discussed above, we are also adopting to our proposals for identifying the cost reporting periods to be employed in determining preliminary and final EHR incentive payments.

After consideration of the public comments we receive with regard to the use of cost reporting periods for preliminary and final incentive payment determinations, we are adopting the following policies in this final rule.

- For purposes of determining preliminary incentive payments, we will employ discharge and other relevant data from a hospital's most recently submitted 12-month cost report once the hospital has qualified as a meaningful user.

- For purposes of determining final incentive payments, we will employ the first 12-month cost reporting period that begins after the start of the payment year, in order to settle payments on the basis of the hospital discharge and other data from that cost reporting period. In this final rule, we are revising section 495.104(c)(2) of the regulations accordingly. We are not adopting the recommendation to allow the CMS regional offices to make a determination about the appropriate cost reporting period in situations not anticipated by these rules because we believe that these two rules cover all possible situations. For example, even in complicated cases involving nonstandard cost reporting periods, the cost reporting period for a hospital adjusts to a standard 12-month cycle within a brief period. c. Incentive Payment Calculation for Eligible Hospitals: Medicare Share

As previously discussed, the initial amount must be multiplied by the eligible hospital's Medicare share and an applicable transition factor to determine the incentive payment to an eligible hospital for a payment year. As added by section 4102(a) of the HITECH Act, section 1886(n)(2)(D) of the Act defines the Medicare share for purposes of calculating incentive payments as a fraction based on estimated Medicare FFS and managed care inpatient bed days, divided by estimated total inpatient bed-days, modified by charges for charity care. This section specifies that the Medicare share fraction is determined for the incentive payment year "for an eligible hospital for a period selected by the Secretary." As in the case of the discharge data discussed above, this clause provides the Secretary with authority to determine the eligible hospital's Medicare share fraction on the basis of data from a relevant hospital cost reporting period, for use in determining the incentive payment during a FY. For purposes of administrative simplicity and timeliness equivalent to those discussed above with regard to discharge data, we proposed, for each eligible hospital during each payment year, to employ data on the hospital's Medicare fee-forservice and managed care inpatient bed days, total inpatient bed-days, and charges for charity care from the hospital FY that ends during the FY prior to the FY that serves as the payment year as the basis for preliminary payment. We also proposed that final payment would be made on the basis of the data from the hospital fiscal year that ends during the FY that serves as the payment year at the time of the settlement of the cost report for the latter period.

As a result of the changes we are making to these proposed policies in response to the comments discussed in the previous section, in this final rule we are adopting the following policies for employing data on the eligible hospital's Medicare fee-for-service and managed care inpatient bed days, total inpatient bed-days, and charges for charity care from the hospital in making preliminary and final EHR incentive payment determinations:

- For purposes of determining preliminary incentive payments, we will employ data on the hospital's Medicare fee-for-service and managed care inpatient bed days, total inpatient bed-days, and charges for charity care from a hospital's most recently submitted 12-month cost report once the 
hospital has qualified as a meaningful user

- For purposes of determining final incentive payments, we will employ the first 12-month cost reporting period that begins after the start of the payment year, in order to settle payments on the basis of the hospital's Medicare fee-forservice and managed care inpatient bed days, total inpatient bed-days, and charges for charity care data from that cost reporting period.

Section 1886(n)(2)(D) of the Act, as amended by section 4102 of the HITECH Act, defines the numerator and denominator of the Medicare share fraction for an eligible hospital in terms of estimated Medicare FFS and managed care inpatient bed-days, estimated total inpatient bed-days, and charges for charity care. Specifically, section 1886(n)(2)(D)(i) of the Act defines the numerator of the Medicare share fraction as the sum of-

- The estimated number of inpatientbed-days (as established by the Secretary) which are attributable to individuals with respect to whom payment may be made under part A; and

- The estimated number of inpatientbed-days (as so established) that are attributable to individuals who are enrolled with a MA organization under Part C.

We proposed to determine the numbers of Medicare Part A and Part C inpatient-bed-days using the same data sources and methods for counting those days that we employ in determining Medicare's share for purposes of making payments for direct graduate medical education costs, as provided under section 1886(h) of the Act and $\S 413.75$ of our regulations. Specifically, we proposed to derive "the estimated number of inpatient-bed-days * * * attributable to individuals with respect to whom payment may be made under part A" from lines 1, 6 through 9, 10, and 14 in column 4 on Worksheet S-3, Part I of CMS Form 2552-96, Hospital and Hospital Health Care Complex Cost Report. We stated that the data entered on these lines in the cost report include all patient days attributable to Medicare inpatients, excluding those in units not paid under the IPPS and excluding nursery days.

Comment: A number of commenters pointed out an apparent contradiction between the cost report sources from which we proposed to derive the "the estimated number of inpatient-bed-days * * * attributable to individuals with respect to whom payment may be made under part A" (lines 1, 6 through 9, 10, and 14 in column 4 on Worksheet S-3, Part I of CMS Form 2552-96,), and our statement that "the data entered on these lines in the cost report include all patient days attributable to Medicare inpatients, excluding those in units not paid under the IPPS and excluding nursery days." These commenters supported our proposal to employ the data from those lines of the cost report, on the grounds that these cost report lines "adequately capture the necessary data." However, as the commenters pointed out, the data on the identified lines do include patient days in units not paid under the inpatient PPS. These commenters also contended that the relevant statutory language ("inpatientbed-days * * * attributable to individuals with respect to whom payment may be made under part A"; emphasis supplied) would seem to include patient days in units not paid under the inpatient PPS.

Response: We agree with the commenters that our citation of the specific cost report sources from which we proposed to derive "the estimated number of inpatient-bed-days * * * attributable to individuals with respect to whom payment may be made under part A" was not consistent with our statement the data entered on these lines in the cost report include "all patient days attributable to Medicare inpatients, excluding those in units not paid under the IPPS and excluding nursery days." In this case, our error was in the specific cost report lines that we cited, rather than in our statement that the relevant statutory language ("inpatient-bed-days * * * attributable to individuals with respect to whom payment may be made under part $A$ ") includes "all patient days attributable to Medicare inpatients, excluding those in units not paid under the IPPS and excluding nursery days." As in the case which we discussed above with regard to counting "total discharges," the relevant statutory language directs that the numerator and denominator of the Medicare share fraction incorporate inpatient bed-day counts for the eligible hospital, and, as discussed in our section on total discharges, "eligible hospital" is defined with reference to section 1886(d)(1)(B) of the Act, which specifically excludes from the definition psychiatric or rehabilitation units that are a distinct part of the hospital. Specifically, the "Medicare share" is to be "specified * * * for an eligible hospital." The numerator of the Medicare share fraction is further defined as "the sum $(* * *$ with respect to the eligible hospital) of-

"(I) the estimated number of inpatientbed-days (as established by the Secretary) which are attributable to individuals with respect to whom payment may be made under part A; and

"(II) the estimated number of inpatient-bed-days (as so established) which are attributable to individuals who are enrolled with a Medicare Advantage organization under part C."

Finally, the denominator of the Medicare share fraction includes "the estimated total number of inpatient-beddays with respect to the eligible hospital." Therefore, the inpatient-bedday counts included in the Medicare share fraction for purposes of the incentive payments provision do not extend to inpatient-bed-days in excluded units of the hospital, but only to inpatient-bed-days in the acute care portion of the hospital that receives Medicare payment under the inpatient PPS. In this final rule, we are revising section 495.104(c)(4) of the regulations in order to clarify this point.

Since the publication of the proposed rule, we have adopted various changes to the Medicare cost report, including changes designed to accommodate the appropriate computation and final settlement of EHR incentive payments for qualifying hospitals. These changes are included in the pending cost report form, CMS Form 2552-10. In this revised form, the relevant Medicare inpatient days are entered in line 2 of the new Worksheet E-1, Part II, "Calculation of Reimbursement for Settlement for HIT." This new line is defined as the sum of lines 1 and 8 through 12, from Worksheet S-3, Part I, column 6 of CMS Form 2552-10. These lines include all patient days attributable to Medicare inpatients, excluding those in units not paid under the IPPS, and excluding nursery days.

Comment: Several commenters also contended that our proposed exclusion of nursery days from the determination of "inpatient-bed-days * * *

attributable to individuals with respect to whom payment may be made under part $A$ " is inappropriate. These commenters maintained that the statutory language is broad enough to include all inpatient days associated with Medicare eligible individuals without restriction based on the type of Part A patient.

Response: In excluding nursery days from the count of Medicare inpatient bed days, we are following the precedent of not counting such days for purposes of the direct medical education, indirect medical education, and disproportionate share adjustments under the Medicare IPPS. As in the case of the term "subsection (d)" hospital, we believe that, in the absence of clear direction from the statute to the contrary, the most appropriate policy is 
to interpret terms such as "inpatient bed-days" in the light of existing Medicare program policies and precedents. Under our policies for the direct medical education, indirect medical education and disproportionate share adjustments, a bed must be permanently maintained for lodging inpatients in order to be included in available inpatient bed and inpatient bed day counts. We exclude the days provided to newborns (except for those in intensive care units of the hospital) because healthy newborn infants are not provided with an acute level of hospital care. (This is not the case with newborns assigned to intensive care units, who are included in the counts for those units.) For these reasons, nursery days are explicitly excluded from:

- The counts of Medicare inpatient hospital days and total inpatient hospital days for purposes of direct graduate medical education payments under section 413.75(b) of the regulations, where the definition of Medicare patient load reads: "Inpatient days in any distinct part of the hospital are included and nursery days are excluded."

- The counts of bed days for purposes of the Medicare indirect graduate medical education adjustment under section 412.105(b): the "count of available bed days excludes bed days associated with * * * (5) Beds or bassinets in the healthy newborn nursery * * *."

- The count of beds for purposes of the Medicare DSH adjustment under section 412.106(a)(i) of the regulations: "The number of beds in a hospital is determined in accordance with $\S 412.105(\mathrm{~b}) . ”$

We note that, in addition to excluding nursery days from the numerator of the Medicare share fraction, these days are excluded for the same reasons from the count of total inpatient bed days in the denominator of the Medicare share fraction. We therefore do not believe that excluding these days would result in disadvantage to hospitals in determining their Medicare share fractions for purposes of calculating EHR incentive payments. (See our discussion of the cost report data employed to determine total inpatient bed days in the denominator of the Medicare share fraction, below.)

Comment: Other commenters maintained that swing bed days should also be included in the determination of "inpatient bed-days * * * attributable to individuals with respect to whom payment may be made under part A."

Response: Once again, as in the case of the term "subsection(d)" hospital, we believe that, in the absence of clear direction from the statute to the contrary, the most appropriate policy is to interpret terms such as "inpatient bed-days" in the light of existing Medicare program policies and precedents. We are therefore also following the precedent of Medicare payment adjustments in excluding certain swing bed days from the count of Medicare inpatient days. As in these cases, swing bed days are excluded when the swing bed is used to furnish SNF care, because only the days used for inpatient hospital care will be included in the count of "inpatient beddays * * * attributable to individuals with respect to whom payment may be made under part A." Otherwise, we would be including non-inpatient beddays in the count.

Comment: One commenter objected that, for purposes of the Medicare inpatient day count in the Medicare share, we appeared to be proposing to use only paid Medicare days. This commenter argued that all eligible Medicare days should be counted in order to reflect a hospital's true Medicare utilization. The commenter also maintained that the statute's reference to days "attributable to individuals with respect to whom payment may be made under part A" requires inclusion of all days when a beneficiary was eligible for Medicare, on the grounds that this language "does not require actual payment by Medicare.” The commenter further noted that the other factor in the numerator of the Medicare share fraction requires inclusion of all patient days associated with individuals enrolled in a Part C Medicare Advantage plan, and maintained that there "would be no rational basis for Congress to include all enrolled Part $\mathrm{C}$ days, quite clearly regardless of whether they are paid, but to limit part A days to those paid by Medicare."

Response: We assume that, by the term "unpaid" Medicare days, the commenter is referring to days provided to Medicare entitled beneficiaries for which the services are non-covered, such as the cases in which a beneficiary has exhausted coverage of inpatient hospital services, or in which the services are not covered under a national or local coverage determination. We do not agree with the commenter that these days ought to be included in the count of "inpatient-beddays * * * attributable to individuals with respect to whom payment may be made under part A." Indeed, we believe that the best reading of this statutory language suggests the opposite of what the commenter maintains: In cases of non-covered days, payment may not be made under Part A, and therefore these days should not be included in a count of days "attributable to individuals with respect to whom payment may be made under part $A$." We agree with the commenter that the language for the other factor in the numerator of the Medicare share fraction ("inpatient-beddays attributable * * * to individuals who are enrolled with a MA organization under Part C") is more inclusive. However, we must assume that the difference in the statutory language is meaningful. Therefore, we are finalizing our proposal not to include days provided to Medicare entitled beneficiaries for which the services are non-covered in the count of Medicare inpatient days. It is important to note that we do include such "nonpaid" days for purposes of other Medicare payment provisions, where it is appropriate to do so under the governing statutory provisions. For example, for purposes of the Medicare DSH adjustment the relevant statutory language requires inclusion of days associated with individuals who are "entitled" to benefits under Medicare Part A, rather than days for which "payment may be made under part A."

After consideration of these comments, we are finalizing our proposals with regard to the data to be used to determine the "inpatient beddays * * * attributable to individuals with respect to whom payment may be made under part A" in the numerator of the Medicare share fraction.

Accordingly, we will derive this information from Worksheet E-1, Part II, line 2 of the pending Medicare cost report, Form CMS-2552-10, which is defined as the sum of lines 1 and 8 through 12 in column 6, Worksheet S3 , Part I of the pending cost report. As we have just discussed, we are revising the cost report data sources from which we are deriving this information in order to be consistent with the statutory requirement. We are also revising $\S 495.104$ (c)(4)(ii)(A)(2) of the regulations to clarify this point.

Comment: One commenter inquired about the status of inpatient-bed-days attributable to individuals enrolled in the 1876 Medicare cost plan operating under "billing option 2," under which the section 1876 cost contractor pays hospitals for Part A benefits, and then claims reimbursement from CMS. The cost-contractor pays Part A benefits for its 36,000 enrolled Medicare beneficiaries to contracted hospitals in one State. The commenter maintained that a reasonable interpretation of the statutory language suggests that the inpatient bed days for these 
beneficiaries should be counted in the numerator of the Medicare share fraction. The commenter requested clarification concerning the inclusion of these days in the data sources we proposed to employ, or the development of an appropriate remedy in order to ensure that they are counted. Another commenter noted that Worksheet S-3, Part I, column 4, line 2 in the Medicare cost report, CMS 2552-96, has

historically been completed primarily by teaching hospitals, based on patient days reported on Provider Statistical and Reimbursement (PS\&R) Report Type 118. The commenter further stated that there have been many situations in which non-teaching hospitals reporting days on this cost report line have the days removed by the Medicare fiscal intermediary or Medicare administrative contractor (MAC), as PS\&R Report Type 118 contains no patient day data for non-teaching hospitals. The commenter recommended that we clarify our plans with regard to PS\&R Report Type 118 and allow the form to populate with accurate data for all hospitals submitting no-pay bills for Medicare beneficiaries who are enrolled in Medicare Advantage (MA) plans and who receive Medicare-covered hospital services. The commenter further noted that, at this time, CAHs and IPPS hospitals that do not receive the $\mathrm{DSH}$ adjustment are not required to submit no-pay bills for Medicare Advantage patients.

Response: We agree with the commenters that all these days should be counted in the numerator of the Medicare share fraction. With respect to MA plan enrollees, these patients are already included in the "estimated number of inpatient-bed-days attributable * * * to individuals who are enrolled with a MA organization under Part C." In order for the data on the inpatient days attributable to individuals enrolled in MA plans to be included on the Medicare cost report, the hospital must submit a "no-pay" bill to the Medicare contractor. We have issued instructions clarifying that hospitals must submit no-pay bills for inpatient days attributable to individuals enrolled in MA plans. Specifically, CR 5647, dated July 20, 2007, required all hospitals paid under the inpatient prospective payment system (IPPS), inpatient rehabilitation facility prospective payment system (IRF PPS), and long term care hospital prospective payment system (LTCH PPS) to submit informational only Medicare Advantage claims. Furthermore, CR 6821, dated May 5, 2010, provided that applicable IPPS, IRF
PPS and LTC hospitals will be given one final opportunity to comply with the requirement to submit FY 2007 informational only claims. In addition, these hospitals are required to attest in writing to their Medicare contractor that they have either submitted all of their Medicare Advantage claims for FY 2007 or that they have no Medicare Advantage claims for that fiscal year. After consideration of the comments, we are finalizing our proposals for determining the "inpatient bed-days * * * attributable to individuals with respect to whom payment may be made under part A" and the "estimated number of inpatient-bed-days attributable * * * to individuals who are enrolled with a MA organization under Part C." However, we are modifying the language of $\S 495.104$ (c)(4)(ii)(A)(1) regarding the counting of inpatient bed-days attributable to individuals with respect to whom payment may be under part $\mathrm{A}$ to clarify that this count includes days attributable to enrollees under section 1876 cost contracts where payments for Part A benefits are made by the section 1876 contractor. We intend to derive this information from Worksheet E-1, Part II, line 3 of the pending Medicare cost report, Form CMS-2552-10, which is derived from line 2 in column 6 , Worksheet S-3, Part I of the pending cost report. This data source on the revised Medicare cost report is the equivalent of the source we cited in the proposed rule.

Section 1886(n)(2)(D)(ii) of the Act defines the denominator of the Medicare share fraction as the product of-

- The estimated total number of inpatient-bed-days with respect to the eligible hospital during such period; and

- The estimated total amount of the eligible hospital's charges during such period, not including any charges that are attributable to charity care (as such term is used for purposes of hospital cost reporting under Title XVIII), divided by the estimated total amount of the hospital's charges during such period.

As in the case of Medicare Part A and Part C inpatient-bed days, for purposes of determining total inpatient-bed days in the denominator of the Medicare share fraction, we proposed to use the same data sources, and the same methods, that we employ in determining Medicare's share for purposes of making payments for direct graduate medical education costs. Specifically, we proposed to derive the relevant data from lines 1, 6 through 9, 10, and 14 in column 6 on Worksheet S-3, Part I of the Medicare cost report.
We noted that the data entered on these lines in the cost report include all patient days attributable to inpatients, excluding those in units not paid under the IPPS.

Comment: Several commenters noted, regarding our proposal concerning Medicare inpatient days in the denominator of the Medicare share fraction, an apparent contradiction between the cost report sources from which we proposed to derive "estimated total number of inpatient-bed-days with respect to the eligible hospital during such period" (lines 1, 6 through 9, 10, and 14 in column 6 on Worksheet S-3, Part I), and our statement that "the data entered on these lines in the cost report include all patient days attributable to inpatients, excluding those in units not paid under the IPPS ." These commenters supported our proposal to employ the data from those lines of the cost report, on the grounds that these cost report lines adequately capture the necessary data. However, as the commenters pointed out, the data on the identified lines do include patient days in units not paid under the inpatient PPS. And these commenters contended that the relevant statutory language ("the estimated total number of inpatient-beddays with respect to the eligible hospital during such period") would seem to include patient days in units excluded from the inpatient PPS.

Response: As in the case of the equivalent issue with regard to Medicare inpatient bed days, we agree with the commenters that our citation of the specific cost report sources from which we proposed to derive the "the estimated total number of inpatient-beddays with respect to the eligible hospital during such period" was not consistent with our statement that the data entered on these lines in the cost "include all patient days attributable to inpatients, excluding those in units not paid under the IPPS." And as in the case of Medicare inpatient-bed-days, our error was in the specific cost report lines that we cited, rather than in our statement that the relevant statutory language ("the estimated total number of inpatient-beddays with respect to the eligible hospital”) includes “all patient days attributable to inpatients, excluding those in units not paid under the IPPS.”. As we have discussed in connection with counting discharges and Medicare inpatient-bed-days, the relevant statutory language directs that the denominator of the Medicare share fraction incorporate inpatient bed-day counts for the eligible hospital.

Therefore, the inpatient-bed-day counts included in the Medicare share fraction for purposes of the incentive payments 
provision do not extend to inpatientbed-days in excluded units of the hospital, but only to inpatient-bed-days in the acute care portion of the hospital that receives payment for Medicare purposes under the inpatient PPS.

We are finalizing our proposal for determining the count of total inpatientbed days in the denominator of the Medicare share fraction as including all patient days attributable to inpatients, excluding those in units not paid under the IPPS. Accordingly, we will derive this information from Worksheet E-1, Part II, line 4 of the pending Medicare cost report, Form CMS-2552-10, which is defined as the sum of lines 1 and 8 through 12, in column 8, Worksheet S3 , Part I of the pending cost report. As we have just discussed, we are revising the cost report data sources from which we are deriving this information in order to be consistent with the statutory requirement. In this final rule, we are also revising $\S 495.104$ (c)(4)(ii)(B)(1) to clarify this point.

As we noted above, the denominator of the Medicare share fraction also includes the "estimated total amount of the eligible hospital's charges during such period, not including any charges that are attributable to charity care (as such term is used for purposes of hospital cost reporting under Title XVIII), divided by the estimated total amount of the hospital's charges during such period." We discuss the data sources and methods for calculating the charges and charity care portions of this formula in the next section.

d. Incentive Payment Calculation for Eligible Hospitals: Charity Care and Charges

In determining the denominator of the Medicare share fraction, we also must determine any charges that are attributable to charity care furnished by an eligible hospital or CAH. The exclusion of charges attributable to charity care has the effect of decreasing the denominator of the Medicare share fraction as the proportion of charity care (charity care charge ratio) provided by a hospital increases. This is because the ratio of estimated total hospital charges, not including charges attributable to charity care, to estimated total hospital charges during a period decreases, relatively speaking, as a hospital provides a greater proportion of charity care. The effect of a greater charity care factor on the denominator of the Medicare share fraction is therefore to decrease the denominator (as the total number of inpatient-bed days is multiplied by a relatively lower charity care charge ratio), as a hospital provides a greater proportion of charity care. A smaller denominator increases the Medicare share factor, providing for higher incentive payments, to a hospital that provides a greater proportion of charity care. Conversely, as a hospital provides a lower proportion of charity care, the ratio of estimated total hospital charges, not including charges attributable to charity care, to estimated total hospital charges during a period increases.

For the purposes of this final rule, we define charity care as part of uncompensated and indigent care described for Medicare cost reporting purposes in the Medicare cost report instructions at section 4012 of the

Provider Reimbursement Manual (PRM), Part 2; Worksheet S-10; Hospital Uncompensated and Indigent Care Data. Subsection (d) hospitals and CAHs are required to complete the Worksheet S-10.

As part of the Form CMS-2552-10 described above, the revised Worksheet S-10 instructions define uncompensated care as follows: “* * * charity care and bad debt which includes non-Medicare bad debt and non-reimbursable Medicare bad debt. Uncompensated care does not include courtesy allowances or discounts given to patients." These instructions further define charity care to include health services for which a hospital demonstrates that the patient is unable to pay. Charity care results from a hospital's policy to provide all or a portion of services free of charge to patients who meet certain financial criteria. For Medicare purposes, charity care is not reimbursable, and unpaid amounts associated with charity care are not considered as an allowable

Medicare bad debt. Therefore, we proposed to use the charity care charges that are reported on line 19 of the revised Worksheet $S-10$ in the computation of the Medicare share of the incentive payments. Line number 19 of the revised Worksheet S-10, as proposed, has changed to line number 20 based on the pending OMB approved final Form CMS-2552-10. Only the line number has changed as the instructions are the same for line 19 as proposed and for line 20 in the pending final OMB approved Worksheet S-10. Thus, the charity care charges used to calculate the final Medicare share is reported on line 20 of the pending final OMB approved Worksheet S-10.

Under section 1886(n)(2)(D) of the Act, if the Secretary determines that data are not available on charity care necessary to calculate the portion of the formula specified in clause (ii)(II) of section 1886(n)(2)(D) of the Act, the Secretary shall use data on uncompensated care and may adjust such data so as to be an appropriate proxy for charity care including a downward adjustment to eliminate bad debt data from uncompensated care data. In the absence of the data necessary for the Secretary to compute the amount described in clause (ii)(II) of section 1886(n)(2)(D) of the Act, the amount under such clause shall be deemed to be 1 .

We believe that the charity care charges reported on line 20 of the pending final OMB approved Worksheet S-10 represent the most accurate measure of charity care charges as part of the hospital's overall reporting of uncompensated and indigent care for Medicare purposes. Therefore, since eligible hospitals and CAHs are required to complete the Worksheet S-10, if a hospital has not properly reported any charity care charges on line 20, we may question the accuracy of the charges used for computing the final Medicare share of the incentive payments. With appropriate resources, we believe the charity care data can be obtained by the MAC. This data would be used to determine if the hospital's charity care criteria are appropriate, if a hospital should have reported charity care charges, and if the reported charges are proper. If we determine, as based on the determination of the MAC, that the hospital did not properly report charity care charges on line 20 of the pending final OMB approved Worksheet S-10, then we proposed to deem the portion of the denominator described in section 1886(n)(2)(D)(ii)(II) of the Act to be 1.

In the proposed rule, we specifically solicited public comments on the charity care financial criteria established by each hospital and reviewed by the MACs, the collection of charity care data on the Worksheet S-10, and whether proxies for charity care may be developed with other data available to us.

Comment: Some commenters requested that CMS clarify the definition of charity care. One commenter believed the CMS

incorrectly indicated that Medicare does not reimburse for charity care. The commenter believed this statement is inconsistent with section 312 of the Provider reimbursement Manual (PRM).

Response: Section 1886(n)(2)(D)(ii)(II) of the Act defines charity care charges to compute the Medicare share as such term is used for purposes of hospital cost reporting under Medicare. Thus, we are adopting our proposed definition of charity care as part of uncompensated and indigent care described for Medicare cost reporting purposes in the 
Medicare cost report instructions as described above.

In addition, we believe that our statement is correct in that Medicare does not pay for charity care in accordance with the regulations and manual instructions. Specifically, section 413.89(b)(1) of the Medicare regulations defines bad debts as amounts considered to be uncollectible from accounts and notes receivable that were created or acquired in providing services. "Accounts receivable" and "notes receivable" are designations for claims arising from the furnishing of services, and are collectible in money in the relatively near future. Section 413.89(b)(2) of the Medicare regulations defines charity allowances as reductions in charges made by the provider of services because of the indigence or medical indigence of the patient. Cost of free care (uncompensated services) furnished under a Hill-Burton obligation are considered as charity allowances. Furthermore, section $413.89(\mathrm{~g})$ states that charity allowances have no relationship to beneficiaries of the Medicare program and are not allowable costs. These charity allowances include the costs of uncompensated services furnished under a Hill-Burton obligation

Also, section 312 of the PRM states that, for Medicare bad debt purposes, a non-Medicaid beneficiary may be considered indigent or medically indigent and that once indigence is determined and the provider concludes that no improvements in the beneficiary's financial condition exist, the debt may be deemed uncollectible without applying the collection requirements of section 310 of the PRM. We believe that the instructions at section 312 of the PRM specify bad debt amounts that may be allowable under section 413.89 of the regulations and, thus, these instructions are not related to charity care amounts that are not allowable for Medicare.

After consideration of the public comments received, we are finalizing the definition of charity care these provisions as proposed.

Comment: We received some comments asking if CMS will adopt standards to determine if a hospital's charity care policy is sufficient to qualify for the inclusion of charges in the formula for EHR and whether that same policy would suffice to meet the criteria to determine the eligibility for Medicare bad debt.

Response: Currently for bad debt purposes, section 312 of the PRM requires the provider to perform asset/ income tests of patient resources for non-Medicaid beneficiaries. These tests will be used to determine if the beneficiary meets the provider's indigent policy to qualify an unpaid deductible and/or coinsurance amount as a Medicare bad debt. The provider is responsible for developing its indigent policy. Currently, the Medicare contractor will determine if the indigent policies are appropriate for determining allowable Medicare bad debt under section 312 of the PRM and $\S 413.89$ of the regulations. We believe that the Medicare contractor will continue to determine if the provider's indigent policy for bad debt purposes is appropriate and can determine if the same policy would be sufficient to use for charity care purposes.

Comment: We received many comments on the use of charity care charge data from line 19 of the revised worksheet S-10, as proposed.

Commenters urge CMS to calculate charity care costs by starting with the amount of charges a hospital has written off. Commenters noted that this modification would help streamline and unify charity care reporting across the Federal government (based on the way Internal Revenue Service (IRS)) requires charity care to be reported) ensure consistency of reporting, and avoid significantly increasing hospitals' administrative burden.

Response: As described above, we use charity care charges from line 20 of the pending final OMB approved worksheet S-10 that captures "total initial payment obligations of the patients who are given full or partial discounts, based on the hospital's charity care criteria (measured a full charge), for care delivered during the cost reporting period for the entire facility." Similar comments received on our proposed rule were also received on the Agency Information Collection Activities: Proposed Collection: Comment Request published in the July 2, 2009 Federal Register (74 FR 31738). CMS issued a revised package, Agency Information Collection Activities: Submission for OMB Review: Comment Request, in the April 30, 2010 Federal Register (75 FR 22810). The comment period for the submission for OMB review ended June 1, 2010. OMB will review the comments received and issue an approved Form CMS 2552 10. The OMB approved Form CMS-2552-10 will be effective for cost reporting periods beginning on or after May 1, 2010

Comment: Some commenters noted that the Hospital Uncompensated Care and Indigent Care Worksheet S-10 that CMS proposed to revise in the July 2 , 2009 Federal Register (74 FR 31738) would not be timely (based on the anticipated effective date for cost reporting periods beginning on or after February 1, 2010 as stated in the proposed rule), and therefore, hospitals with cost reporting periods beginning on November 1, 2009, December 1, 2009 or January 1, 2010 would not have the opportunity to report charity care data for the first year of the incentive payment. Commenters further highlighted their concern for available data necessary to be included in interim payments and for final payments for periods that end December 31, 2010. Commenters urged CMS to develop an interim mechanism for hospitals to report the necessary information so that no hospital receives a charity care adjustment of " 1 " merely because of its cost reporting cycle. Some commenters suggested that CMS use other charity care data. Some commenters suggested that CMS use the current version of the Medicare cost report, Form CMS-255296 , to determine interim incentive payments.

Response: To calculate the Medicare share, which includes the charges for charity care, we proposed in the proposed rule to employ data from the hospitals fiscal year that ends during the FY prior to the FY that serves as the payment year as the basis for preliminary payment. We further stated that final payment would be made on the basis of the data from the hospital fiscal year that ends during the FY that serves as the payment year. After consideration of the public comments received, we are revising the provision that for purposes of determining preliminary incentive payments, we will employ data on the hospital's/ CAH's Medicare fee-for-service and managed care inpatient bed days, total inpatient bed-days, and charges for charity care from a hospital's/CAH's most recently submitted 12-month cost report once the hospital has qualified as a meaningful user. For purposes of determining final incentive payments, we will employ the first 12-month cost reporting period that begins after the start of the payment year, in order to settle payments on the basis of the hospital's/CAH's Medicare fee-forservice and managed care inpatient bed days, total inpatient bed-days, and charges for charity care data from that cost reporting period.

In addition, as described in the proposed rule, hospitals have been required to fill out the worksheet $\mathrm{S}-10$ of the Form CMS 2552-96 since the BBRA of 1999 was enacted. We recognize that the charity care data from the 2552-96 worksheet S-10 may have some limitations because, in some cases, providers failed to complete the worksheet either partially or in its 
entirety. Furthermore, in the past CMS did not review the worksheet S-10 because the data had no Medicare payment implications. Thus, in the absence of availability of charity care data from the OMB approved Form CMS 2552-10, a hospital for the purposes of calculating the charity care charges in the interim may use the information from the 2552-96 worksheet S-10; line 22 until the revised worksheet is available. We believe that the Medicare contractor can make a determination if the charity care charges from the 255296 are appropriate, and if so, use such charges in determining the preliminary incentive payment amount for hospitals, as described above. Since CAHs were not required to fill out the 2552-96 worksheet S-10, charity care charges may not be available to determine preliminary incentive payments until the revised worksheet is available. However, using data from the first 12month cost reporting period that begins after the start of the payment year, as described above, hospitals and CAHs will calculate the final incentive payment amount with data from the pending Form CMS-2552-10 Medicare cost report that is effective for cost reporting periods beginning on or after May 1, 2010.

Comment: Several commenters pointed out that we had failed to identify the source of the data for "estimated total amount of the eligible hospital's charges" in the proposed rule.
Some of these commenters recommended that we employ Worksheet C, Column 8, line 103 for this purpose.

Response: We did neglect to identify the source of the data for "estimated total amount of the eligible hospital's charges" in the proposed rule. In the final rule, we are providing that, for this purpose, we will employ the data from Worksheet E-1, Part II, line 5 of the revised Medicare cost report, Form CMS-2552-10, which in turn derives this information from line 200 in column 8, Worksheet C, Part I of the pending cost report. We note that line 200 in column 8, Worksheet C, Part I of the revised cost report is the equivalent of 101, Column 8, Worksheet C of the current cost report. We are employing the equivalent of line 101, rather than the equivalent of line 103, as recommended by the commenters, because line 101 (current line 200) includes the charges for observation, and accordingly reflects the "total amount of the eligible hospital's charges" more truly than line 103, which excludes those charges.

e. Incentive Payment Calculation for Eligible Hospitals: Transition Factor

As we have previously discussed, the initial amount must be multiplied not only by the Medicare share fraction, but also by an applicable transition factor in order to determine the incentive payment to an eligible hospital for an incentive payment year. Section 1886(n)(2)(E)(i) of the Act designates that the applicable transition factor equals one (1) For the first payment year, three-fourths for the second payment year, one-half for the third payment year, one-fourth for the fourth payment year, and zero thereafter. However, section 1886(n)(2)(E)(ii) of the Act provides that if "the first payment year for an eligible hospital is after 2013, then the transition factor specified in this subparagraph for a payment year for such hospital is the same as the amount specified in clause (i) for such payment year for an eligible hospital for which the first payment year is 2013." Accordingly, if a hospital's first payment year is FY 2014, then the applicable transition factor equals threefourths $(3 / 4)$ for the first payment year (FY 2014), one-half (1/2) for the second payment year (FY 2015), one-fourth (1/4) for the third payment year (FY 2015), and zero thereafter. If a hospital's first payment year is FY 2015, then the applicable transition factor equals $(1 / 2)$ for the first payment year (FY 2015), (1/4) for the second payment year (FY 2016), and zero thereafter. As discussed in more detail below, under section 1886(n)(2)(E)(ii) of the Act, the transition factor for a hospital for which the first payment year is after 2015 equals zero for all years. In other words, 2015 is the last year for which eligible hospitals may begin participation in the Medicare EHR Incentive Program. 
Figure 1--Incentive Payment Calculation for Subsection D Hospitals

Incentive Amount $=$ [Initial Amount $] \times$ [Medicare Share $] \times$ [Transition Factor $]$

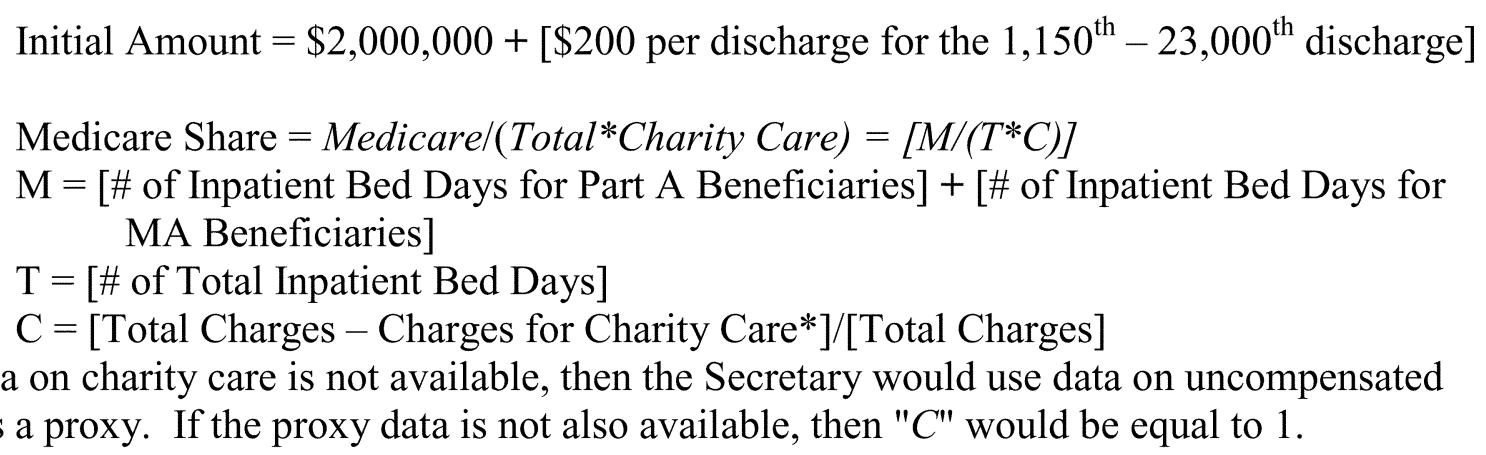

Table13: Transition Factor

\begin{tabular}{|c|c|}
\hline Consecutive Payment Year & Transition Factor \\
\hline 1 & 1 \\
\hline 2 & $3 / 4$ \\
\hline 3 & $1 / 2$ \\
\hline 4 & $1 / 4$ \\
\hline
\end{tabular}

f. Duration and Timing of Incentive Payments

Section 1886(n)(2)(E)(i) of the Act establishes that an eligible hospital that is a meaningful user of certified EHR technology could receive up to 4 years of financial incentive payments. The transition factor phases down the incentive payments over the 4-year period. Therefore, an eligible hospital that is a meaningful user of certified EHR technology during the relevant EHR reporting period, in payment year FY 2011, could receive incentive payments beginning with FY 2011 (transition factor equals 1), and for FY 2012 (transition factor equals 3/4), 2013 (transition factor equals $1 / 2$ ), and 2014 (transition factor equals $1 / 4$ ) if they continue to be a meaningful user of certified EHR technology during the relevant EHR reporting periods.

Section 1886(n)(2)(E)(ii) of the Act establishes the range of time during which a hospital may begin to receive incentive payments, and the applicable transition periods for hospitals that are permitted to begin receiving incentive payments after FY 2011. Specifically, that section provides that if the "first payment year for an eligible hospital is after 2015, the transition factor * * * for such hospital and for such year and subsequent year shall be 0 ." This clause in effect provides that no incentive payments will be available to a hospital that would begin to receive such payments after FY 2015. In other words, FY 2015 is the last FY in which a hospital can begin to receive incentive payments. Taken together, sections 1886(n)(2)(G)(i) and 1886(n)(2)(E)(ii) of the Act allow hospitals to begin receiving incentive payments during FYs 2011 through 2015. Section 1886(n)(2)(E)(ii) of the Act also establishes the transition periods and factors that will be in effect for hospitals that begin to receive transition payments during FY 2014 and 2015. As discussed previously, that section states that if "the first payment year for an eligible hospital is after 2013, then the transition factor specified in this subparagraph for a payment year for such hospital is the same as the amount specified in clause (i) for such payment year for an eligible hospital for which the first payment year is 2013." Section 1886(n)(2)(E)(ii) of the Act also establishes the transition periods that will be in effect for hospitals that begin to receive transition payments during FYs 2014 through 2015. That section states that if "the first payment year for an eligible hospital is after 2013, then the transition factor specified in this subparagraph for a payment year for such hospital is the same as the amount specified in clause (i) for such payment year for an eligible hospital for which the first payment year is 2013." By implication, this clause establishes that, for hospitals that begin to receive incentive payments in FYs 2012 and 2013, the transition periods are equivalent to those for hospitals that begin to receive such payments in FY 2011. An eligible hospital that is a meaningful user of certified EHR technology could receive incentive payments beginning with FY 2012 (transition factor equals 1), and for FY 2013 (transition factor equals $3 / 4$ ), FY 2014 (transition factor equals $1 / 2$ ), and FY 2015 (transition factor equals 1/4).

Similarly, an eligible hospital that is a meaningful EHR user could receive incentive payments beginning with FY 2013 (transition factor equals 1), and for FYs 2014 (transition factor equals $3 / 4$ ), 2015 (transition factor equals $1 / 2$ ), and 2016 (transition factor equals $1 / 4$ ).

However, this section also specifically provides that the transition factor is modified for those eligible hospitals that first become meaningful users of certified EHR technology beginning in 2014 or 2015. Such hospitals would receive payments as if they became meaningful EHR users beginning in 2013. In other words, if a hospital were to begin to demonstrate meaningful use of EHR certified technology in 2014, the transition factor used for that year (2014) would be $3 / 4$ instead of $1,1 / 2$ for the second year (2015), $1 / 4$ for the third year (2016), and zero thereafter. Similarly, if a hospital were to begin 
meaningful use of certified EHR technology in 2015, the transition factor used for that year would be $1 / 2$ instead of $1,1 / 4$ for the second year (2016), and zero thereafter.

Table 25 shows the possible years an eligible hospital could receive an incentive payment and the transition factor applicable to each year.

TABLE 14: Transition Factor for Medicare FFS Eligible Hospitals

\begin{tabular}{|c|r|r|r|r|r|}
\hline Fiscal Year & \multicolumn{5}{|c|}{ Fiscal Year that Eligible Hospital First Receives the } \\
& \multicolumn{5}{|c|}{ Incentive Payment } \\
\hline & $\mathbf{2 0 1 1}$ & $\mathbf{2 0 1 2}$ & $\mathbf{2 0 1 3}$ & $\mathbf{2 0 1 4}$ & $\mathbf{2 0 1 5}$ \\
\hline 2011 & 1.00 & ------ & ------ & ----- & ----- \\
\hline 2012 & 0.75 & 1.00 & ----- & ---- & ----- \\
\hline 2013 & 0.50 & 0.75 & 1.00 & ----- & ----- \\
\hline 2014 & 0.25 & 0.50 & 0.75 & 0.75 & ----- \\
\hline 2015 & ------ & 0.25 & 0.50 & 0.50 & 0.50 \\
\hline 2016 & ------ & ----- & 0.25 & 0.25 & 0.25 \\
\hline
\end{tabular}

Comment: Several commenters pointed out an apparent inconsistency in the regulation text that we proposed to implement the transition period and applicable transition factors for EHR incentive payments. Specifically, the commenters noted that proposed section 495.104(b)(5) states that hospitals "whose first payment year is FY 2015 may receive such payments for FY 2015 through 2017" (emphasis supplied), while proposed section 495.104(c)(5) states that the transition factors for hospitals "whose first payment year is FY 2015" are:

(A) $1 / 2$ for FY 2015; and

(B) $1 / 4$ for FY 2016. (Emphasis supplied.)

Response: These commenters are correct. Our proposed section 495.104(b)(5) contained a typographical error. In order to be consistent with the clear requirements of the statute, section 495.104(b)(5) should have stated that hospitals "whose first payment year is FY 2015 may receive such payments for FY 2015 through 2016.” In this final rule, we are revising section 495.104(b)(5) of the final regulations accordingly.

g. Incentive Payment Adjustment Effective in FY 2015 and Subsequent Years for Eligible Hospitals Who Are Not Meaningful EHR Users

In addition to providing for incentive payments for meaningful use of EHRs during a transition period, section 1886(b)(3)(B) of the Act, as amended by section 4102(b)(1) of the HITECH Act, provides for an adjustment to the market basket update to the IPPS payment rate for those eligible hospitals that are not meaningful EHR users for the EHR reporting period for a payment year, beginning in FY 2015. Specifically, section 1886(b)(3)(B) of the Act provides that, "for FY 2015 and each subsequent FY," an eligible hospital that is not "a meaningful EHR user * * * for an EHR reporting period" will receive a reduced update to the IPPS standardized amount. This reduction will apply to "three-quarters of the percentage increase otherwise applicable." For FY 2015 and each subsequent FY, the reduction to three-quarters of the applicable update for an eligible hospital that is not a meaningful EHR user will be " $33^{1 / 3}$ percent for FY 2015 , 66 $2 / 3$ percent for FY 2016, and 100 percent for FY 2017 and each subsequent FY." In other words, the Secretary is required to subject eligible hospitals who are not meaningful users to $1 / 4,1 / 2$, and $3 / 4$ reductions of their market basket updates in FY 2015, FY 2016, and FY 2017 and subsequent years respectively. Section 4102(b)(1)(B) of the HITECH Act also provides that such "reduction shall apply only with respect to the FY involved and the Secretary shall not take into account such reduction in computing the applicable percentage increase * * * for a subsequent FY.” This provision establishes a continuing incentive for hospitals to become meaningful EHR users, because a hospital that does become a meaningful EHR user in any year after the effective date of the update reduction will receive the same, fully updated standardized amount for that year, and subsequent years, as those hospitals that were already meaningful EHR users at the time when the update reduction went into effect (although hospitals would remain subject to a separate reduction for failure to report quality data under RHQDAPU). In order to conform with this new update reduction, section 4102(b)(1)(A) of the HITECH Act revises section 1886(b)(3)(B)(viii)(1) of the Act to provide that, beginning with FY 2015, the reduction to the IPPS applicable percentage increase for failure to submit data on quality measures to the Secretary shall be one-quarter of the applicable market basket update. In this way, even the combined reductions for EHR use and quality data reporting will not produce an update of less than zero for a hospital in a given FY as long as the hospital market basket remains a positive number.

In the proposed rule, we noted that specific proposals to implement these payment adjustments for subsection (d) hospitals that are not meaningful EHR users were not being made at that time, but would be subject to future rulemaking prior to the 2015 implementation date. We invited comments on these payment adjustments, and stated any comments received would be considered in developing future proposals to implement these provisions.

We received a few comments on this provision.

3. Incentive Payments for Critical Access Hospitals (CAHs)

Section 1814(1)(3)(A) of the Act, as amended by section 4102(a)(2) of the HITECH Act, also provides for incentive payments for CAHs that are meaningful users of certified EHR technology during an EHR reporting period for a cost reporting period beginning during a payment year after FY 2010 but before FY 2016. The criteria for being a meaningful EHR user, and the manner for demonstrating meaningful use, are discussed in section II.A.2. of this final rule. 


\section{a. Definition of CAHs for Medicare}

Section 1861(mm)(1) of the Act defines a $\mathrm{CAH}$ as a facility that has been certified as a critical access hospital under section 1820(c). CAHs are reimbursed for services furnished to Medicare beneficiaries under section 1814(l) of the Act for inpatient services and section $1834(\mathrm{~g})$ of the Act for outpatient services. Incentive payments for CAHs under section 1814(l)(3)(A) of the Act will be calculated based on the provider number used for cost reporting purposes, which is the CCN of the main provider. The process for making incentive payments to CAHs is discussed in section II.B.4.c. of this final rule.

Comment: We received many comments on the use of the CCN to identify CAHs. Most comments were similar to those received on the use of the CCN for determining incentive payments to eligible hospitals.

Response: We responded to the comments for eligible hospitals elsewhere in this final rule. Our responses to comments received on using the CCN to identify CAHs are the same as the responses for eligible hospital.

After consideration of the public comments received, we are finalizing our policy as proposed. For purposes of this provision, we will provide incentive payments to qualifying CAHs as they are distinguished by the provider number in the CAH's cost reports. Incentive payments for qualifying CAHs will be calculated based on the provider number used for cost reporting purposes, which is the CCN of the main provider (also referred to as OSCAR number). Payments to qualifying CAHs will be made to each provider of record.

\section{b. Current Medicare Payment of}

Reasonable Cost for CAHs

For Medicare purposes, CAHs are paid for most inpatient and outpatient services to Medicare beneficiaries on the basis of reasonable cost under section 1814(l) and section 1834(g) of the Act, respectively. Thus, CAHs are not subject to the IPPS and Hospital Outpatient Prospective Payment System (OPPS).

Section 1861(v)(1)(A) of the Act is the statutory basis for reasonable cost reimbursement in Medicare. Under the reasonable cost reimbursement methodology, payments to providers are based on the reasonable cost of furnishing Medicare-covered services to beneficiaries. Reasonable cost includes all necessary and proper costs in furnishing the services, subject to the principles of reasonable cost reimbursement relating to certain specific items of revenue and cost. Reasonable cost takes into account both direct and indirect costs of providers of services, including normal standby costs. The objective of the reasonable cost methodology is to ensure that the costs for individuals covered by the program are not borne by others not so covered, and the costs for individuals not so covered are not borne by the program. The reasonable costs of services and the items to be included are determined in accordance with the regulations at $42 \mathrm{CFR}$ part 413 , manual guidance, and other CMS instructions.

Currently, under section 1814(1)(1) of the Act and $\S 413.70$ (a) of the regulations, effective for cost reporting periods beginning on or after January 1 , 2004, payment for inpatient services of a CAH, other than services of a distinct part unit of a CAH, is 101 percent of the reasonable costs of the CAH in providing $\mathrm{CAH}$ services to its inpatients, as determined in accordance with section 1861(v)(1)(A) of the Act and with the applicable principles of cost reimbursement in Parts 413 and 415 of the regulations. However, payment for inpatient $\mathrm{CAH}$ services is not subject to the reasonable cost principles of the lesser of cost or charges, the reasonable compensation equivalent limits for physician services to providers, the ceilings on hospital operating costs, or the payment window provisions for preadmission services, specified in $\S 412.2(\mathrm{c})(5)$ and $\S 413.40(\mathrm{c})(2)$. Section $1834(\mathrm{~g})$ of the Act and $\S 413.70(\mathrm{~b})$ of the regulations describe the payment methodology for outpatient services furnished by a CAH.

Currently, reasonable cost reimbursement for CAHs includes payment for depreciation of depreciable assets used in providing covered services to beneficiaries, as described under Part 413 subpart G of our regulations and $\S 104$ of the Medicare Provider Reimbursement Manual (PRM). In general, the depreciation expense of an asset, representing a portion of the depreciable asset's costs which is allocable to a period of operation, is determined by distributing the acquisition costs of the depreciable asset, less any salvage costs, over the estimated useful life of the asset.

\section{c. Changes Made by the HITECH Act}

Sections 4102(a)(2) and 4102(b)(2) of the HITECH Act amended section 1814(l) of the Act, which governs payment for inpatient CAH services. The HITECH Act did not amend section $1834(\mathrm{~g})$ of the Act, which governs payment for outpatient CAH services.
Sections 4102(a)(2) and 4102(b)(2) of the HITECH Act amended section 1814(l) of the Act by adding new paragraphs (3), (4), and (5) as follows:

Section 1814(l)(3)(A) of the Act provides the following:

The following rules shall apply in determining payment and reasonable costs * * * for a critical access hospital that would be a meaningful EHR user (as would be determined under paragraph (3) of section 1886(n)) for an EHR reporting period for a cost reporting period beginning during a payment year if such critical access hospital was treated as an eligible hospital under such section:

(i) The Secretary shall compute reasonable costs by expensing such costs in a single payment year and not depreciating these costs over a period of years (and shall include as costs with respect to cost reporting periods beginning during a payment year costs from previous cost reporting periods to the extent they have not been fully depreciated as of the period involved).

(ii) There shall be substituted for the Medicare share that would otherwise be applied [to CAHs under section 1814(l)(1),] a percent (not to exceed 100 percent) equal to the sum of-

(I) The Medicare share (as would be specified under paragraph (2)(D) of section 1886(n)) for such critical access hospital if such critical access hospital was treated as an eligible hospital under such section; and

(II) 20 percentage points.

Section 1814(l)(3)(B) of the Act provides that the incentive payment for CAHs will be paid "through a prompt interim payment (subject to reconciliation) after submission and review of such information (as specified by the Secretary) necessary to make such payment." The provision also states that "[i]n no case may payment under this paragraph be made with respect to a cost reporting period beginning during a payment year after 2015 and in no case may a critical access hospital receive payment under this paragraph with respect to more than 4 consecutive payment years."

Section 1814(l)(3)(C) of the Act provides that the reasonable costs for which a CAH may receive an incentive payment are costs for the purchase of certified EHR technology to which purchase depreciation (excluding interest) would otherwise apply under section 1814(l)(1) of the Act.

Section 1814(l)(4)(A) of the Act provides for an adjustment, subject to the hardship exemption in section 1814(l)(4)(C) of the Act, to a CAH's reimbursement at 101 percent of its reasonable costs if the $\mathrm{CAH}$ has not met the meaningful EHR user definition for an EHR reporting period that begins in FY 2015 or a subsequent fiscal year. Section 1814(l)(4)(B) of the Act specifies that if a CAH is not a meaningful EHR 
user during the cost reporting period beginning in FY 2015, its reimbursement will be reduced from 101 percent of its reasonable costs to 100.66 percent. For FY 2016, the percentage of reimbursement for a $\mathrm{CAH}$ that is not a meaningful EHR user is reduced to 100.33 percent of its reasonable costs. For FY 2017 and each subsequent FY, the percentage of reimbursement is reduced to 100 percent of reasonable costs. Section 1814(l)(4)(C) of the Act states that, as provided for eligible subsection (d) hospitals, the Secretary may, on a caseby-case basis, exempt a CAH from this adjustment if the Secretary determines, subject to annual renewal, that requiring the CAH to be a meaningful EHR user during a cost reporting period beginning in FY 2015 or a subsequent fiscal year would result in a significant hardship, such as in the case of a CAH in a rural area without sufficient Internet access. However, in no case may a CAH be granted an exemption under this provision for more than 5 years.

Section 1814(1)(5) provides that there shall be no administrative or judicial review under sections 1869 or 1878 of the Act, or otherwise, of: (1) The methodology and standards for determining the amount of payment under section 1814(1)(3) of the Act and payment adjustments under section 1814(l)(4) of the Act; (2) the methodology and standards for determining a CAH to be a meaningful EHR user; (3) the methodology and standards for determining if the hardship exemption applies to a CAH; (4) the specification of EHR reporting periods; and (5) the identification of reasonable costs used to compute $\mathrm{CAH}$ incentive payments.

\section{d. Incentive Payment Calculation for CAHs}

Consistent with section 1814(l)(3)(A) of the Act, we proposed to amend $\S 413.70$ (a) to add a new paragraph (5) to provide for an incentive payment to a qualifying $\mathrm{CAH}$ for the reasonable costs incurred for the purchase of certified EHR technology in a cost reporting period beginning during a payment year after FY 2010 but before FY 2016. We proposed to include a cross-reference to $\$ 495.106$ which defines the terms associated with the $\mathrm{CAH}$ incentive payment, including the definition of a "qualifying CAH" that is eligible to receive the $\mathrm{CAH}$ incentive payment, and the methodology for determining the amount of that incentive payment. In addition, we proposed to amend $\S 413.70$ (a) to add a new paragraph (6) to provide for the adjustment of a CAH's reasonable costs of providing inpatient services starting in FY 2015 if the CAH is not a qualifying $\mathrm{CAH}$.

In computing the $\mathrm{CAH}$ incentive payment and applying the adjustments to a CAH's payment if the CAH is not a qualifying $\mathrm{CAH}$, we proposed to apply the definitions of certified EHR technology, EHR reporting period, meaningful EHR user and qualified EHR in $\S 495.4$ that are discussed elsewhere in this final rule.

In $\$ 495.106(\mathrm{a})$, we proposed to define a qualifying $\mathrm{CAH}$ as a CAH that would meet the meaningful EHR user

definition for eligible hospitals in $\S 495.4$, which is discussed in section II A.1. of this final rule if it were an eligible hospital. Also in $\$ 495.106$ (a), for the purposes of computing the $\mathrm{CAH}$ incentive payment, we proposed that the reasonable costs for the purchase of certified EHR technology mean the reasonable acquisition costs, excluding any depreciation and interest expenses associated with the acquisition, incurred for the purchase of depreciable assets as described at part 413 subpart $\mathrm{G}$, such as computers and associated hardware and software, necessary to administer certified EHR technology as defined in $\S 495.4$ of this final rule. We also proposed to define payment year for CAHs to mean a fiscal year beginning after FY 2010 but before FY 2016.

Under proposed § 495.106(b), we specified that a qualifying $\mathrm{CAH}$ must receive an incentive payment for its reasonable costs incurred for the purchase of certified EHR technology. The CAH incentive payment will be for a cost reporting period that begins during a payment year after FY 2010 but before FY 2016.

Consistent with section 1814(1)(3)(A) of the Act, we proposed under $\S 495.106$ (c) that the payment methodology for computing the incentive payment for a qualifying $\mathrm{CAH}$ for a cost reporting period during a payment year would be equal to the product of-(1) the reasonable costs incurred for the purchase of certified EHR technology in that cost reporting period and any similarly incurred costs from previous cost reporting periods to the extent they have not been fully depreciated as of the cost reporting period involved and (2) the CAH's Medicare share which equals the Medicare share as computed for eligible hospitals including the adjustment for charity care (described in sections II.A.2.b. and A.3. of this final rule) plus 20 percentage points. However, in no case will the resulting Medicare share for a CAH exceed 100 percent. This payment methodology will be used in place of payment at 101 percent of reasonable costs typically applied under section 1814(l)(1) of the Act and $\S 413.70(\mathrm{a})(1)$ of the regulations.

For example, a CAH first requests an incentive payment for its cost reporting period beginning on January 1, 2012 which is in FY 2012. The CAH incurred reasonable costs of $\$ 500,000$ for the purchase of certified EHR technology in its previous cost reporting period beginning on January 1, 2011. This CAH is a meaningful user of certified EHR technology during the relevant EHR reporting period and thus qualifies for an incentive payment for FY 2012. (For illustrative purposes this example assumes no salvage value of the assets acquired.) The CAH depreciated $\$ 100,000$ of the costs of these items in the cost reporting period beginning on January 1, 2011. As a result, the amount used to compute the incentive payment will be the remaining $\$ 400,000$ of undepreciated costs. The CAH's Medicare share is 90 percent (its Medicare share of 70 percent using the methodology described in section II.A.2.b. of this final rule plus 20 percentage points). Therefore, the CAH's incentive payment for FY 2012 is $\$ 360,000$ (\$400,000 times 90 percent). This CAH's first payment year is FY 2012, and it can receive incentive payments through 4 consecutive payment years which, in this example, would be FYs 2012 through 2015.

If, in the above example, the $\mathrm{CAH}$ also incurred reasonable costs of $\$ 300,000$ for the purchase of certified EHR technology in its cost reporting period beginning in FY 2012 that will not be depreciated, then the incentive payment for FY 2012 is $\$ 630,000(\$ 700,000$ $(\$ 400,000$ in FY 2011 plus $\$ 300,000$ in FY 2012) times 90 percent).

(The preceding examples are offered for illustrative purposes only and are not intended to encompass all possible computations of the $\mathrm{CAH}$ incentive payment.)

Under proposed § 495.106(d)(1), the amount of the incentive payment made to a qualifying CAH under this section represents the expensing and payment of the reasonable costs of certified EHR technology computed as described above in a single payment year and, as specified in $\$ 413.70$ (a)(5), such payment is made in lieu of any payment that would have been made under $\S 413.70(\mathrm{a})(1)$ for the reasonable costs of the purchase of certified EHR technology including depreciation and interest expenses associated with the acquisition. The Medicare contractor will review the CAH's current year and each subsequent year's cost report to 
ensure that the assets associated with the acquisition of certified EHR technology are expensed in a single period and that depreciation and interest expenses associated with the acquisition are not allowed.

Under proposed § 495.106(d)(2), the amount of the incentive payment made to a qualifying $\mathrm{CAH}$ under this section would be paid through a prompt interim payment for the applicable payment year after-(1) the CAH submits the necessary documentation, as specified by CMS or its Medicare contractor, to support the computation of the incentive payment amount; and (2) CMS or its Medicare contractor reviews such documentation and determines the interim amount of the incentive payment.

Under proposed § 495.106(d)(3), the interim incentive payment would be subject to a reconciliation process as specified by CMS and the final incentive payment as determined by CMS or its Medicare contractor would be considered payment in full for the reasonable costs incurred for the purchase of certified EHR technology in a payment year.

Under § 495.106(d)(4), we proposed that an incentive payment may be made with respect to a cost reporting period beginning during a payment year beginning with FY 2011 (October 1, 2010 through September 30, 2011) through FY 2015 (October 1, 2014 through September 30, 2015), but in no case may a CAH receive an incentive payment with respect to more than four consecutive payment years. Therefore, a $\mathrm{CAH}$, that is a meaningful EHR user, may begin receiving an incentive payment for its cost reporting period beginning in FY 2011 for the incurred reasonable costs for the purchase of certified EHR technology during that cost reporting period and in previous cost reporting periods to the extent that the item or items have not been fully depreciated. These incentive payments will continue for no more than 4 consecutive payment years and will not be made for a cost reporting period beginning during a payment year after 2015. As discussed above and in section II.B.4. of this final rule, the CAH must submit supporting documentation for its incurred costs of purchasing certified EHR technology to its Medicare contractor (Fiscal Intermediary (FI)/ MAC).

CAHs cannot receive an incentive payment for a cost reporting period that begins in a payment year after FY 2015. If the first payment year for a CAH is FY 2013 then the fourth consecutive payment year would be 2016 . However, the CAH cannot be paid an incentive payment for FYs 2016 and beyond. For FY 2016 and beyond, payment to CAHs for the purchase of additional EHR technology will be made under $\S 413.70(\mathrm{a})(1)$ in accordance with the reasonable cost principles, as described above, which would include the depreciation and interest cost associated with such purchase.

Comment: We received many comments requesting CMS to provide a list of those depreciable items that would be used to determine the $\mathrm{CAH}$ incentive payment under this provision. The commenters were concerned that certain expenses, such as staff training, associated with an EHR system may not be included in the CAH's incentive payment. We also received comments requesting a further explanation of what documentation will be required to support the reasonable costs incurred by the CAH.

Response: Section 1814(1)(3)(C) of the Act, as amended by the HITECH Act, provides that the costs for which a CAH may receive an incentive payment are reasonable costs for the purchase of certified EHR technology to which purchase depreciation (excluding interest) would otherwise apply under section 1814(1)(1) of the Act.

Furthermore, section 1814(l)(3)(A) of the Act, as amended by the HITECH Act, mandates that the Secretary shall compute reasonable costs for the purchase of certified EHR technology by expensing such costs in a single payment year and not depreciating these costs over a period of years (and shall include as costs with respect to cost reporting periods beginning during a payment year costs from previous cost reporting periods to the extent they have not been fully depreciated as of the period involved). As described in the proposed rule, for the purposes of computing the CAH incentive payment, we proposed that the reasonable costs for the purchase of certified EHR technology mean the reasonable acquisition costs, excluding any depreciation and interest expenses associated with the acquisition, incurred for the purchase of depreciable assets as described at part 413 subpart $\mathrm{G}$, such as computers and associated hardware and software, necessary to administer certified EHR technology as defined in $\S 495.4$ of this final rule.

CAHs will incur both depreciable and non-depreciable reasonable costs in a payment year that are associated with implementing and maintaining certified EHR technology. According to the statute, only the reasonable costs for the purchase of certified EHR technology to which purchase depreciation (excluding interest) would otherwise apply are to be included in the CAH incentive payment. Thus, CAHs will not have to depreciate these reasonable costs over the useful life of the EHR asset purchased as such costs will be expensed in a single payment year. Any non-depreciable reasonable costs incurred in that same single payment year that are associated with an EHR system may be paid for under the current Medicare reasonable cost payment system at 101 percent.

Currently, the CAH's Medicare contractor determines if an item purchased is a depreciable asset under Medicare principles or other accounting standards. The Medicare contractor also determines the CAH's reasonable cost for acquiring depreciable assets. For the purposes of computing the $\mathrm{CAH}$ incentive payment, we are not changing the Medicare contractor's current responsibilities described above. We, therefore, suggest that CAHs communicate with their Medicare contractors to determine the necessary documentation to support their reasonable costs incurred for the purchase of certified EHR technology and to determine if the items that they purchase are depreciable assets under Medicare principles or other accounting standards.

Comment: We received some comments requesting clarification of how the incentive payments will be computed if an eligible CAH converts to or from an eligible "subsection d" hospital.

Response: If during a payment year an eligible $\mathrm{CAH}$ is converted to or from a "subsection d" hospital, the CAH may receive an incentive payment as long as it incurred the reasonable costs of purchasing certified EHR technology in a payment year (or in a previous cost reporting period) when it was a $\mathrm{CAH}$ and as long as the affected providers meet the meaningful use criteria described elsewhere in this final rule. When a conversion takes place, the affected CAH and "subsection d" hospital are each required to file a Medicare cost report under section 413.24 of the regulations. For instance, if in month 6 of a cost reporting period that begins January 1, 2011 and ends December 31, 2011, a "subsection d" hospital converts to a CAH, the "subsection d" hospital will file a terminating 6-month cost report (January 1, 2011 to June 30, 2011). If the CAH retains the same year end of December 31, 2011, the CAH will file a 6-month cost report from July 1, 2011 to December 31, 2011. In this instance, the CAH's 6-month cost report would be used to determine if it incurred reasonable costs for the purchase of 
certified EHR technology that may qualify for a CAH incentive payment during that period. The "subsection d" hospital's 6 month terminating cost report would be used to determine the possible amount of any incentive payment for that eligible hospital.

After consideration of the public comments received, with the exception of a few minor, technical and conforming changes, we are finalizing the applicable provisions as proposed.

Comment: We received many comments regarding the use of data from the revised Medicare cost report (Form CMS-2552-10) described in the proposed rule to compute the Medicare share portion of the CAH incentive payment. Commenters were also concerned that certain cost report data may not be available at the time of computing a CAH's incentive payment.

Response: As discussed elsewhere in this final rule, we are addressing concerns with data from the revised cost report in a final collection that is currently in the Paperwork Reduction Act clearance process. In addition, we address the timing issues with the revised cost report data elsewhere in this final rule.

e. Reduction of Reasonable Cost Payment in FY 2015 and Subsequent Years for CAHs That Are Not Meaningful EHR Users

Section 4102(b)(2) of the HITECH Act amends section 1814(l) to include an adjustment to a CAH's reimbursement at 101 percent of its reasonable costs if the $\mathrm{CAH}$ has not met the meaningful EHR user definition for an EHR reporting period that begins in FY 2015, FY 2016, FY 2017, and each subsequent FY thereafter. Consistent with this provision, we proposed that under $\S 495.106(\mathrm{e})$ and $\S 413.70(\mathrm{a})(6)$, if a CAH has not demonstrated meaningful use of certified EHR technology for FY 2015, its reimbursement would be reduced from 101 percent of its reasonable costs to 100.66 percent. For FY 2016, its reimbursement would be reduced to 100.33 percent of its reasonable costs. For FY 2017 and each subsequent FY, its reimbursement would be reduced to 100 percent of reasonable costs.

However, as provided for eligible hospitals, a CAH may, on a case-by-case basis, be exempted from this adjustment if CMS or its Medicare contractor determines, on an annual basis, that requiring the $\mathrm{CAH}$ to be a meaningful EHR user would result in a significant hardship, such as in the case of a $\mathrm{CAH}$ in a rural area without sufficient Internet access. However, in no case may a CAH be granted an exemption under this provision for more than 5 years.

Comment: We received some comments requesting further clarification of how CMS will be determining whether a significant hardship exists to warrant an exemption.

Response: We received a few comments on this provision which is not effective until FY 2015. We will take these comments into account when we develop proposals for implementing this provision at a later date.

After consideration of the public comments received, we are finalizing sections 495.106(e) as proposed. We have renumbered proposed section 413.70(a)(6)(iv) as 413.70(a)(7), but are otherwise finalizing section $413.70(a)(6)$ as proposed.

Section 1814(l)(5) of the Act exempts the determinations made under paragraphs (1)(3) and (1)(4) from administrative and judicial review. Accordingly, under $\S 413.70(a)(6)(i v)$ and $\S 495.106(f)$, we proposed that there shall be no administrative or judicial review under sections 1869 or 1878 of the Act, or otherwise, of the following:

- The methodology and standards for determining the amount of payment under section 1814(1)(3) of the Act and payment adjustments under section 1814(l)(4) of the Act for CAHs, including selection of periods under section 1886(n)(2) of the Act for determining, and making estimates or using proxies of, inpatient-bed-days, hospital charges, charity charges, and the Medicare share under subparagraph (D) of section 1886(n)(2) of the Act;

- The methodology and standards for determining a CAH to be a meaningful EHR user under section 1886(n)(3) of the Act as would apply if the CAH was treated as an eligible hospital under section 1886(n) of the Act;

- The methodology and standards for determining if the hardship exemption under section 1814(1)(4)(C) of the Act applies to a $\mathrm{CAH}$;

- The specification of EHR reporting periods under section 1886(n)(6)(B) of the Act as applied under section 1814(l)(3) and (4) of the Act for CAHs; and

- The identification of reasonable costs used to compute the CAH incentive payment under section 1814(l)(3)(C) of the Act.

Comment: We received some comments requesting clarification of whether CAHs will be able to appeal their incentive payment amounts.

Response: We believe that the

limitation of administrative and judicial review does not apply to the amount of the $\mathrm{CAH}$ incentive payment. The $\mathrm{CAH}$ may appeal the statistical and financial amounts from the Medicare cost report used to determine the CAH incentive payment. The $\mathrm{CAH}$ would utilize the current provider appeal process pursuant to section 1878 of the Act.

Accordingly, after consideration of the public comments received, we are finalizing $§ 495.106(f)$ as proposed. We have renumbered proposed

$\S 413.70(a)(6)(i v)$ as $\S 413.70(a)(7)$, but are otherwise finalizing the provision as proposed.

4. Process for Making Incentive Payments Under the Medicare FFS Program

As previously discussed in section II.B.1. and 2. of this final rule and sections 1848(o)(1) and 1886(n)(1) of the Act, the statute provides for incentive payments to eligible professionals, eligible hospitals, and CAHS who are meaningful users of certified EHR technology as early as FY 2011 for qualifying eligible hospitals and CAHs and CY 2011 for qualifying EPs. The statute does not specify the process for making these payments to qualifying EPs and qualifying eligible hospitals and CAHs participating in the FFS Medicare incentive payment program, but instead leaves the payment process to the Secretary's discretion.

We proposed that FIs, carriers, and MACs, as appropriate, would be responsible for determining the incentive payment amounts for qualifying EPs and qualifying eligible hospitals and CAHs in accordance with the methodology set forth in section II.B.1.b. and B.2.b. of this final rule based on the previously discussed meaningful use criteria, disbursing the incentive payments to qualifying EPs and qualifying eligible hospitals and CAHs, and resolving any reconciliation issues.

\section{a. Incentive Payments to EPs}

We proposed that the carriers/MACs calculate incentive payment amounts for qualifying EPs, where incentive payments would be disbursed on a rolling basis, as soon as they ascertained that an EP demonstrated meaningful use for the applicable reporting period (that is, 90 days for the first year or a calendar year for subsequent years), and reached the threshold for maximum payment. In accordance with section 1848(1)(3)(B) of the Act, we proposed that if a qualifying $\mathrm{EP}$ is not eligible for the maximum incentive payment amount for the payment year and if the qualifying EP was also a qualifying MA EP, the qualifying MA organization with which the EP is affiliated would receive the incentive payment for the EP through 
the MA EHR incentive program. If the qualifying EP either does not also qualify as a MA EP or he or she qualifies as a MA EP but is not eligible for the maximum incentive payment for the payment year, we proposed that the carriers/MAC would calculate the amount of the qualifying EP's incentive payment and disburse the incentive payment to the qualifying EP in the year following the payment year. The proposed rule also outlined that incentive payments would not be issued to qualifying EPs if an incentive payment was already made under the Medicaid program for the relevant payment year, and as required by section $1848(\mathrm{~m})(2)$ of the Act as amended by section 4101(f) of the HITECH Act, qualifying EPs who received incentive payments from the Medicare EHR incentive payment program would not be eligible to receive an e-prescribing incentive payment. Additionally, we proposed that the incentive payments would be tracked at the qualifying EP's TIN level, and disbursed to the TIN that the qualifying EP indicated during the registration process; qualifying EPs who do not have individual TINs (that is, a qualifying EP who works solely in a group practice) would be paid at the group practice level's TIN. We proposed that qualifying EPs select one TIN for disbursement of their Medicare EHR incentive payment. Of course, after the payment is disbursed to their designated TIN, qualifying EPs may decide to allocate their incentive payment among the multiple practices in which they furnish covered professional services subject to applicable laws, regulations and rules, including, without limitation, those related to fraud, waste, and abuse.

To be clear, we note that financial relationships, including those arising from the reallocation/reassignment of incentive payments, between physicians and their employers/other entities may implicate certain fraud, waste, and abuse laws, regulations, and rules. Therefore, we proposed to include specific safeguards to limit the risk that the allocation/reassignment of incentive payments could raise under those and other applicable laws, regulations and rules. Section II.B.1.d. above finalizes our proposal at $\S 495.10$ (f) to permit EPs to reassign their incentive payments to their employer or to an entity with which they have a contractual arrangement, consistent with all rules governing reassignments including part 424, subpart F.

Comment: Several commenters expressed concern that the proposed rule contained limited information on how the incentive program for Medicare
EPs will be operationalized. They requested additional information on the expected timeframe and process for payments.

Response: The HITECH Act requires that EHR incentive program payments be separately tracked and monitored because these funds cannot be commingled with other Medicare funds. Therefore, to facilitate funds control, payments will be made through a single payment contractor rather than through the carriers/MACs as was originally proposed. Additionally, the Integrated Data Repository (IDR), rather than the carriers/MACs, will be accumulating the allowed charges for each qualified EP's NPI. Payments would be made on a rolling basis, as soon as we ascertain that an EP has successfully demonstrated meaningful use for the applicable reporting period (that is, 90 days for the first year or a calendar year for subsequent years) and the EP's allowed charges has reached the threshold that qualifies an EP for maximum incentive payment, for the relevant payment year. Once this determination has been made, the National Level Repository (NLR) will calculate the EP's incentive payment. The payment will then be made by the single payment contractor. We anticipate that it will take anywhere from 15 to 46 days from the time an EP successfully attests to being a meaningful user to the time an incentive payment is made, and that for FY 2011, incentive payments will be made to EPs who successfully demonstrate that they were meaningful EHR users for the EHR reporting period (that is, 90 days) as early as May 2011. As proposed, we will pay a qualifying EP a single consolidated incentive payment for a payment year, rather than make periodic installment payments. In order to accommodate different attestation dates throughout the first year for EPs, our payment cycle is on a monthly basis as previously described; however, qualifying EPs will receive one single payment per year. In other words, CMS will issue payments as soon as possible after a qualifying EP attested to meaningfully using a certified EHR system, hence the monthly payment cycle; however, an EP will only receive one incentive payment for each year he/she qualifies. For qualifying EPs whose allowed charges for the payment year do not reach the maximum thresholds, the single payment contractor will disburse an incentive payment in the following year.

Comment: One commenter recommended CMS make semi-annual incentive payments for the second and subsequent payment years to ensure physician practices have cash flow to deploy certified EHR systems and train employees how to use the systems.

Response: When the EHR reporting period is a full year, no EPs will have successfully demonstrated that they are meaningful users at the mid-year mark. Therefore, as previously described, qualifying Medicare EPs will receive a single payment per year, issued on a monthly payment cycle. We intend to finalize this provision as proposed; there will be a single successful attestation per year and a single payment following the attestation for qualifying EPs.

Comment: One commenter questioned whether the scopes of work for the MACs/Medicare Carriers would be revised to reflect the additional work that this program will entail.

Response: As previously discussed in the first comment and response, the IDR, rather than the MACs/Medicare Carriers, will accumulate the EPs allowed charges. The MAC/Carrier work related the Medicare EHR incentive program will be within their current scope of work and will be handled through the normal change request process.

Comment: One commenter believes an EP's program selection (Medicare or Medicaid) is tied to the TIN where the EP assigns incentive payments. The commenter recommended CMS permit additional changes in program selection if EPs change their TIN. The commenter believes allowing only one program change in the life of the program is too restricting given that patient mix might change due to a practice being purchased by another TIN or an EP becoming a part-time employee of another TIN.

Response: Section II.A.5.b. of this final rule outlines our policy decision around changing program selections.

After consideration of the public comments received, we are finalizing our policy as proposed. For purposes of this provision, payments will be made through a single payment contractor with the IDR accumulating the allowed charges for each qualified EP's NPI. Payments will be made on a rolling basis, as soon as we ascertain that an EP has successfully demonstrated meaningful use for the applicable reporting period (that is, 90 days for the first year or a calendar year for subsequent years), and reached the threshold for maximum payment then the NLR will calculate the incentive payment. We estimate it will take anywhere from 15 to 46 days from the time an EP successfully attests to being a meaningful user to the time an incentive payment is made. 
b. Incentive Payments to Eligible Hospitals

We proposed that the FIs/MACs would calculate incentive payments for qualifying eligible hospitals, and would disburse such payments on an interim basis once the hospital has demonstrated it is a meaningful EHR user for the EHR reporting period for the payment year. As discussed above in section B.2.b. of the final rule, the formula for calculating a qualifying eligible hospital's incentive payment requires the following data: (1) An initial amount; (2) the Medicare share; and (3) a transition factor applicable to that payment year. We proposed that FIs/MACs would use the prior-year cost report, Provider Statistical and Reimbursement (PS\&R) System data, and other estimates to calculate the interim incentive payment. As discussed in section II.B.2.c. of this final rule, beginning in 2010, cost reports will capture charity care data which will be used in calculating the Medicare share of the payment. We proposed that the MACs/FIs calculate a qualifying hospital's final incentive payment using data from the cost report for the hospital's fiscal year that ends during the FY prior to the FY that serves as the payment year. We also proposed that the FIs/MACs calculate the final incentive payment using actual cost report data report for the hospital's fiscal year that ends during the FY prior to the fiscal year that serves as the payment year, and would reconcile the incentive payment as necessary at settlement of the cost report.

Additionally, incentive payments for qualifying eligible hospitals would be calculated based on the provider number used for cost reporting purposes, which is the CCN of the main provider. Therefore, incentive payments for qualifying hospitals would be disbursed to the CCN rather than the TIN.

Comment: Several commenters expressed concern that the proposed rule contained limited information on how the incentive program for hospitals will be operationalized. They requested additional information on the expected timeframe and process for payments as well as requesting clarification that the incentive payments would be

distributed as a "lump sum payment." One commenter requested CMS disburse one lump sum payment at the start of each eligible year for those hospitals that meet all of the meaningful use requirements.

Response: Hospital EHR incentive payments will be calculated by the FIs/ MACs; however, to facilitate funds control, payments will be made through a single payment contractor. We will direct the payment contractor to issue to qualifying hospitals, that is those hospitals who successfully demonstrate that they are meaningful EHR users, a single initial payment for the year. We anticipate that payments will be made to qualifying Medicare hospitals beginning in May 2011. No payment will be made prior to an eligible Medicare hospital successfully demonstrating that it was a meaningful EHR user during the EHR period for the relevant payment year. For purposes of determining interim incentive

payments, we will employ data on the hospital's Medicare fee-for-service and managed care inpatient bed days, total inpatient bed-days, and charges for charity care from a hospital's most recently submitted 12-month cost report once the hospital has qualified as a meaningful user. For purposes of determining final incentive payments, we will employ the first 12-month cost reporting period that begins after the start of the payment year, in order to settle payments on the basis of the hospital's Medicare fee-for-service and managed care inpatient bed days, total inpatient bed-days, and charges for charity care data from that cost reporting period.

Comment: One commenter requested that CMS allow hospitals to make an interim attestation 90 days after the start of the second and subsequent payment years. They suggested the interim attestation would note that they are in compliance with the meaningful use rules and intend to remain in compliance. They requested that CMS instruct the contractor to issue interim EHR payments after receipt of such attestation. The commenter believes this would cut down on the time frame of 21 months between their first and second hospital interim payments.

Response: The reporting period requirements for a hospital's second and subsequent years are 365 days. Due to the year-long reporting period, we do not believe we can allow for an interim attestation that the provider is a meaningful EHR user. Under our definitions at $\S 495.4$, a provider is not a meaningful EHR user unless it has "for an EHR reporting period for a payment year," demonstrated meaningful use "in accordance with $\S 495.8$ by meeting the applicable objectives and associated measures under $\S 495.6$." Thus, we could not determine that the provider is a meaningful user at an interim point in time, and there would be no basis for providing the interim payment.

Comment: One commenter expressed confusion over the term "demonstration period" and questioned if a hospital had to complete the full demonstration period before payments would be made.

Response: We assume the commenter means EHR “reporting period” when using the phrase, "demonstration period.” A hospital must demonstrate that it met the requirements for meaningful use for the full EHR reporting period for the relevant payment year before we will direct the payment contractor to issue an incentive payment to the hospital for the payment year. A hospital therefore must complete the full EHR reporting period before demonstrating that it was a meaningful EHR user and before any payments would be made.

Comment: Several commenters recommended that CMS' payment process for eligible hospitals be consistent with its payment process for EPs, and that hospital's initial incentive payment thus be distributed no later than two months after the hospital successfully demonstrates meaningful use. The same commenters requested CMS specify that the final incentive payment be issued no later than two months after the hospital submits its cost report from the FY that ends during the payment year.

Response: We anticipate that for FY 2011, interim incentive payments will be made to eligible hospitals that successfully demonstrate that they were meaningful EHR users for the EHR reporting period for FY 2011 (that is, 90 days) as early as May 2011. The exact timing of when a qualifying eligible hospital receives its interim incentive payment will depend on when the hospital successfully demonstrates that it was a meaningful EHR user; the sooner a hospital successfully demonstrate that is was a meaningful EHR user during the EHR reporting period for the payment year, the sooner it will receive its interim incentive payment. For a Medicare hospital's second and subsequent participation years, after a hospital successfully demonstrates that it was a meaningful EHR user during the EHR reporting period (that is, the federal fiscal year) for the payment year, the hospital will receive the interim incentive payment in the following year; the initial incentive payments will be made on a monthly payment cycle beginning shortly after the hospital is determined to be a meaningful user. To the commenters' point of requesting that we be consistent with the approach to paying EPs, there seems to be confusion around what was proposed as to the timing and distribution of the EP's incentive payment. The proposal for the EP's incentive payment was that EP's 
accumulated allowed charges would be based on claims submitted not later than two months after the end of the payment year. The incentive payment for a qualifying EP's second and subsequent payment years was always to be disbursed in the year following the payment year. We did not propose paying an EP within two months of being deemed a meaningful user.

Comment: Several commenters questioned how CMS would treat a hospital that qualified for an incentive payment one year, but did not qualify the next or subsequent years; what is the impact on the stream of incentive payments to the hospital?

Response: An eligible hospital's first payment year is the first year they successfully demonstrate that they were a meaningful EHR user for the EHR reporting period for the payment year. Section 1886(n)(2)(G) of the Act defines the second through fifth payment years for a hospital as each successive year immediately following the first payment year for such hospital. An eligible hospital's second payment year, then, is the year following its first payment year, regardless of whether the eligible hospital qualifies for an incentive payment in the year following its first payment year. Similarly, an eligible hospital's third, fourth, and fifth payment year are the third, fourth, and fifth years, respectively, following the hospital's first payment year, even if the hospital does not receive an incentive payment for one or more of those years.

Comment: Several commenters requested that CMS clarify that EHR incentive payments for which a hospital qualifies or receives under the EHR incentive program (whether directly or pursuant to an assignment, reassignment or other transfer) shall not affect or be taken into account in the calculation or other payments made to the eligible hospital under Medicare, Medicaid, or any other state or federal healthcare program, such as disproportionate share payments, graduate medical education and indirect medical education payments, and payments for un-compensated care payments.

Response: EHR incentive payments will have no bearing on the hospital's Medicare disproportionate share, indirect medical education or direct graduate medical education payments. This discussion is also addressed in the Medicaid section at II.D.4.b.

After consideration of the public comments received, we are finalizing our policy as proposed. For purposes of this provision, Hospital incentive payments will be calculated by the FIs/ MACs; however, to facilitate funds control, payments will be made through a single payment contractor. We will direct the payment contractor to issue to qualifying hospitals a single initial payment per year, and expect initial payment may begin as early as May 2011, for those who demonstrate they are meaningful EHR users at the earliest date possible. We estimate it will take anywhere from 15 to 46 days from the time a hospital successfully attests to being a meaningful user to the time an incentive payment is made.

\section{c. Incentive Payments to CAHs}

In the proposed rule, CMS proposed that because CAHs are paid on a cost reimbursement basis once a CAH incurs actual EHR costs, it could submit supporting documentation to the FI/ MAC for review. The FIs/MACs would determine an incentive payment amount, as discussed in section II.A.3 of the proposed rule by substituting for the Medicare share amount that would otherwise be applied under the formula used for computing payments for eligible hospitals, a percent (not to exceed 100 percent) equal to the sum of-(1) the Medicare share for such $\mathrm{CAH}$, and (2) 20 percentage points.

As discussed in the proposed rule, the FIs/MACs would reconcile the cost report and ensure the EHR expenses are adjusted on the cost report to avoid duplicate payments. Incentive payments for qualifying CAHs would be calculated based on the provider number used for cost reporting purposes, which is the CCN number of the main provider. Therefore, incentive payments for qualifying CAHs would be based on the CCN rather than the TIN.

Comment: Several commenters expressed concern that the proposed rule contained limited information on how the incentive program would be operationalized for CAHs. They requested additional information on the expected timeframe and process for payments to CAHs.

Response: To facilitate funds control, payments will be made through a single payment contractor. In order to receive a HITECH incentive payment, a CAH will have to attest that it is a meaningful user, and submit documentation to its FI/MAC to support the costs incurred for its HIT system. Once the FI/MAC reviews the documentation and the allowable amount is determined, we will direct the payment contractor to release to the $\mathrm{CAH}$ a single incentive payment in the next HITECH payment cycle. Payment cycles will begin in May 2011.

Comment: Several commenters requested more information on the timing of the distribution of payments to
CAHs once the necessary

documentation has been submitted and that recommended CMS be consistent with its proposal on incentive payments for EPs and specify that the CAH's initial incentive payment will be distributed no later than two months after it submits the necessary documentation. The same commenters requested that CMS specify that the final incentive payment be issued no later than two months after the $\mathrm{CAH}$ submits its cost report.

Response: CAHs will receive a single initial incentive payment per year with the initial payments beginning in May 2011. Once the FIs/MACs review the documentation and the allowable amount is determined, we will direct the payment contractor to release a single incentive payment in the next incentive payment cycle to qualifying CAHs. We anticipate the initial payments will generally be made within two months of the determination of the allowable amount. The final payment will be calculated on the cost report, and the process to settle the cost report will not be modified for these incentive payments. It will continue to follow the normal final settlement process. For the CAHs' second and subsequent participation years, CAHs will also receive a single initial incentive payment per year and a final incentive payment as described above. With respect to the commenters' request that we be consistent with the proposed approach to paying EPs, there seems to be confusion around what was proposed as to the timing and distribution of incentive payments to EPs. The proposal for EP incentive payments was that an EP's accumulated allowed charges would be based on claims submitted not later than two months after the end of the payment year. The incentive payment for a qualifying EP's second and subsequent payment years was always to be disbursed in the year following the payment year. We did not propose to make incentive payments to an EP within two months of the EP being deemed a meaningful user.

Comment: Several commenters questioned what is considered "necessary documentation" for CAHs to submit in order to receive Medicare $\mathrm{CAH}$ incentive payments. The same commenters requested CMS propose and obtain comments on "necessary documentation" and finalize a rule before FY 2011.

Response: The documentation submitted should include information reflecting what was purchased, and support the costs incurred. Such documentation may include invoices, receipts, or other comparable materials. 
Comment: One commenter recommended CMS (not the MACs/FIs) should make all determinations regarding CAHs.

Response: The documentation review process for Medicare CAH incentive payments is similar to processes currently performed by FIs/MACs. Also, the data needed to calculate the Medicare Share is on the cost reports, which are submitted to the FIs/MACs. Accordingly, we believe it would be most appropriate for the payment determinations be made by the FIs/ MACs, and not by CMS.

After consideration of the public comments received, we are finalizing our policy as proposed. For purposes of this provision, CAH payments will be calculated by the FIs/MACs; however, as discussed above, to facilitate funds control, payments will be made through a single payment contractor. Once the FIs/MACs review the documentation and the allowable amount is determined, we will direct the payment contractor to release to the CAH a single incentive payment in the next HITECH payment cycle. Payment cycles will begin in May 2011.

\section{d. Payment Accounting Under Medicare}

We will conduct selected compliance reviews of EPs, eligible hospitals, and qualified CAHs who register for the incentive programs and of recipients of incentive payments for the meaningful use of certified EHR technology. The reviews will validate provider eligibility through their meaningful use attestations including verification of meaningful use and would also review components of the payment formulas.

We will identify and recoup overpayments made under the incentive payment programs that result from incorrect or fraudulent attestations, quality measures, cost data, patient data, or any other submission required to establish eligibility or to qualify for a payment. The overpayment will be recouped by CMS or its agents from the $\mathrm{EP}$, eligible hospital, MA organization, $\mathrm{CAH}$, other entities to whom the right to payment has been assigned/reassigned, or, in the case of Medicaid, from the State Medicaid agencies. Medicare FFS EPs and eligible hospitals will need to maintain evidence of qualification to receive incentive payments for 10 years after the date they register for the incentive program.

\section{Preclusion of Administrative and Judicial Review}

We did not discuss preclusion of administrative and judicial review in our proposed rule. We are now including a discussion, in order to make the public aware of the preclusion. Also, the sections of this final rule discussing payments to Medicare Advantage (MA) organizations and CAHs both include a description of the preclusion, as well as accompanying regulation text.

Therefore, while we believe statutory provisions on preclusion of review are self-implementing, below, we include a discussion of the preclusion of review that applies to EPs and eligible hospitals. We have also added regulation text to maintain consistency with the CAH and MA organization provisions.

For EPs, section 1848(o)(3)(C) of the Act prohibits administrative or judicial review under section 1869, section 1878, or otherwise, of all of the following:

- The methodology and standards for determining EP incentive payment amounts.

- The methodology and standards for determining the payment adjustments that apply to EPs beginning with 2015.

- The methodology and standards for determining whether an EP is a meaningful EHR user, including: (1) The selection of clinical quality measures; and (2) the means of demonstrating meaningful EHR use.

- The methodology and standards for determining the hardship exception to the payment adjustments.

- The methodology and standards for determining whether an EP is hospitalbased.

- The specification of the EHR reporting period, as well as whether payment will be made only once, in a single consolidated payment, or in periodic installments.

For eligible hospitals, section 1886(n)(4)(A) of the Act similarly prohibits administrative or judicial review under section 1869, section 1878, or otherwise, of the following:

- The methodology and standards for determining the incentive payment amounts made to eligible hospitals, including: (1) The estimates or proxies for determining discharges, inpatientbed-days, hospital charges, charity charges, and Medicare share; and (2) the period used to determine such estimate or proxy.

- The methodology and standards for determining the payment adjustments that apply to eligible hospitals beginning with FY 2015.

- The methodology and standards for determining whether an eligible hospital is a meaningful EHR user, including: (1) The selection of clinical quality measures; and (2) the means of demonstrating meaningful EHR use.
- The methodology and standards for determining the hardship exception to the payment adjustments.

- The specification of the EHR reporting period, as well as whether payment will be made only once, in a single consolidated payment, or in periodic installments.

We note that the above listing may summarize or abbreviate portions of the statute. For precise language on the preclusion of judicial review, readers should always refer to the statute.

\section{Medicare Advantage (MA) Organization Incentive Payments}

\section{Definitions}

\section{a. Qualifying MA Organization}

Section 1853(1)(1) of the Act, as added by section 4101(c) of the HITECH Act, provides for incentive payments to qualifying MA organizations for certain of their affiliated EPs who are meaningful users of certified EHR technology during the relevant EHR reporting period for a payment year. Section 1853(1)(5) of the Act defines the term "qualifying MA organization" as an MA organization that is organized as a health maintenance organization (HMO) as defined in section 2791(b)(3) of the PHS Act. Section 2791(b)(3) of the PHS Act in turn defines a health maintenance organization as a federally qualified $\mathrm{HMO}$, an organization recognized as an HMO under State law, or a similar organization regulated for solvency under State law in the same manner and to the same extent as an HMO. Since there are few federally qualified HMOs, we expect MA organizations to primarily qualify for incentive payments as State-licensed HMOs, or as organizations regulated for solvency under State law in the same manner and to the same extent as HMOs.

In $§ 495.200$ we proposed to define "qualifying MA organization." Specifically, in § 495.202(a)(2), we proposed to deem MA organizations offering MA HMO plans that are not federally-qualified HMOs to meet the definition of HMO in section 2791(b)(3) of the PHS Act, as HMOs recognized under State law, or as entities subject to State solvency rules in the same manner as HMOs. We believe this is reasonable because under the MA application process, State regulators are required to certify that MA organizations operating in their State are authorized to offer the type of MA plan they proposed to offer, and meet solvency standards that are adequate for these purposes. For each MA organization offering MA HMO plans, the State has thus recognized that the organization is able to assume risk 
as an HMO. Therefore, we have determined that absent evidence to the contrary, an MA organization offering HMO plans is recognized by the State as a health maintenance organization, or that it is subject to State solvency standards in the same manner and to the same extent as an HMO and therefore provides sufficient assurance that the section 2791(b)(3) of the PHS Act definition is met.

In $\S 495.202(\mathrm{a})(3)$, for MA organizations that offer other coordinated care MA plans (Preferred Provider Organization (PPO) plans, Provider Sponsored Organization (PSO) plans, and Regional Preferred Provider Organization (RPPO) plans) and for other MA organizations offering other MA plan types (private fee-for-service (PFFS) plans, Medical Savings Account (MSA) plans), we proposed that the sponsoring MA organization would be required to attest that the MA organization is recognized under State law as an HMO, or that it is a similar organization regulated under State law for solvency in the same manner and to the same extent as an HMO before we would make a determination that the MA organization is a qualifying MA organization for purposes of incentive payments.

Although we did not receive any comments on these provisions and are finalizing them as proposed, there is one exception. In order to bring 422.202(a) into conformance with the change we are making to $422.202(\mathrm{~b})(1)$, we are changing the date by which MAOs are required to identify themselves to us from the bidding deadline in June 2010 (for plan year 2011) to the bidding deadline in June 2011 (for plan year 2012).

\section{b. Qualifying MA Eligible Professional} (EP)

A qualifying MA organization may receive an incentive payment only for those EPs described under section 1853(1)(2) of the Act, as added by section 4101(c) of the HITECH Act. Section 1853(l)(2) of the Act provides that MA EPs must be "eligible professionals" as defined under section 1848(o) of the Act as added by section 4101(a) of the HITECH Act, and must either-

- Be employed by the qualifying MA organization; or

- Be employed by, or be a partner of, an entity that through contract with the qualifying MA organization furnishes at least 80 percent of the entity's Medicare patient care services to enrollees of the qualifying MA organization.

Further, the EP must furnish at least 80 percent of his or her professional services covered under Title XVIII (Medicare) to enrollees of the qualifying MA organization and must furnish, on average, at least 20 hours per week of patient care services.

As discussed in section II.A.1. of this final rule, an EP is defined as a physician (under section 1861(r) of the Act).

We said we interpreted "employed by" to mean that the EP is considered an employee of a qualifying MA organization or qualifying entity under the usual common law rules applicable in determining the employer-employee relationship under section 3121(d)(2) of the Internal Revenue Code of 1986.

We said we interpreted "to be a partner of" to mean that the qualifying MA EP has an ownership stake in the entity. Under this interpretation, a professional that contracts with an entity, but who has no ownership stake in the entity, would not be considered a qualifying MA EP.

We said we interpreted "furnishing at least 80 percent" of the entity's "patient care services" to enrollees of the organization to mean at least 80 percent of the qualifying entity's total Medicare revenue in a year (that is, total revenue from Medicare FFS as well as from all MA organizations) must be from a single qualifying MA organization.

We proposed to interpret the requirement that a qualifying MA EP furnish at least 80 percent of their professional services covered under Title XVIII to enrollees of the organization to mean that at least 80 percent of the professional's total Medicare revenue in a year (that is, total revenue from Medicare FFS as well as from all MA organizations) must be from a single qualifying $\mathrm{MA}$ organization. We said we believed that in establishing the rule that qualifying MA EPs need to furnish at least 80 percent of their Title XVIII covered services "to enrollees of the organization," the statute limits payment related to any specific qualifying MA EP to a single qualifying MA organization. Thus, if a qualifying MA EP provided an average of 20 hours per week of patient care services to two distinct qualifying MA organizations, we said we would pay the qualifying MA organization for the MA EP only if such a qualifying EP provided at least 80 percent of his or her professional services covered under Title XVIII to enrollees of that organization.

For purposes of determining whether a qualifying MA EP furnishes, on average, at least 20 hours per week of patient care services, we interpreted the requirement to include both Medicare and non-Medicare patient care services.
Moreover, we proposed that the relevant time period for determining whether an MA EP furnishes at least 20 hours per week of patient care services should be the EHR reporting period. (We discuss the definition of EHR reporting period in section II.A.1.e. of this final rule.) Therefore, we said that over the EHR reporting period, the qualifying MA EP must provide on average 20 hours per week of patient care services. Finally, we interpreted "patient care services" to mean services that would be considered "covered professional services" under sections 1848(o)(5)(A) and (k)(3) of the Act. That is, health care services for which payment would be made under, or for which payment would be based on, the fee schedule established under Medicare Part B if they were furnished by an eligible professional to a Medicare beneficiary.

We considered various methods of determining when at least 20 hour per week, on average, of patient care services would be considered to be provided by MA EPs. We considered methods such as defining a dollar or service threshold, or the number of hours of direct patient care services actually provided. After due consideration we proposed to require qualifying MA organizations to attest to the fact that MA EPs for whom they are requesting EHR incentive payments have provided, on average, 20 hours of patient care services during the EHR reporting period.

Comment: A few commenters referenced the Report to Congress required by section $4101(d)$ of the HITECH Act. The commenters suggested ways in which we could combine original FFS Medicare claims-payment data and MA services provided by EPs in order to arrive at a single, combined EHR payment. One commenter asked whether payments to a provider from a Medicare Advantage plan can contribute to the volume of Allowed Charges for the purpose of calculating maximum Meaningful Use rewards, saying that he believed that they should. Another commenter said that a substantial percentage of senior citizens receive their care from EPs providing services by way of Medicare Advantage plans. The commenter continued that current proposed rules provide incentive payment only to EPs in whose practices 80 percent or more of total services are to Medicare Advantage patients. The commenter concluded that this would exclude many EPs treating our most vulnerable citizens from the opportunity to meaningfully adopt EHRs in their practices and that the 80 percent [MA] practice requirement should be eliminated. Other commenters argued 
that the regulation was unclear regarding an exclusion of covered professional services of an EP not employed by an MAO when determining their participation or level of payment because those services are provided to MA beneficiaries. The commenter believed that the Secretary should provide a mechanism, whereby EPs can supplement their record to the appropriate carrier/MAC with their MA charges.

Response: We do not have statutory authority to combine payments across the FFS and MA EHR incentive payment programs. The statutory provision at section 1853(l)(3)(B) of the Act, as added by section 4101 of the HITECH, entitled "Avoiding Duplication of Payments," specifically prohibits us from making payments to EPs for both FFS and MA services. Additionally, had Congress wanted CMS to combine FFS and MA charges it could have included a provision similar to the provision in section 1886(n)(2)(D)(i) of the Act, as added by section 4102(a) of the HITECH Act, where FFS and MA inpatient-beddays are added together to derive the numerator of the Medicare share fraction. We do not have the authority to eliminate the requirement that an EP provide 80 percent of Medicare services to enrollees of an MA organization, as that requirement is set forth in section 1853(l)(2)(A)(i)(II) of the Act, as added by the HITECH Act, which is clear in requiring that an MA EP provide " 80 percent of * * * professional services * * * covered under this title to enrollees of the [MA] organization."

Comment: One commenter recommended that CMS retain its proposal regarding how the 80 percent and the 20 hours per week criteria will be met by MA EPs. Another commenter said that many EPs in Puerto Rico would not qualify for incentives under this test. The commenter said that the single MA organization requirement of 80 percent revenue and 20 hours per week for MA EPs would not be met due to the competition and market changes from year to year. The commenter suggested eliminating the single MA organization requirement. Instead, the commenter said we should change the standards to consider all enrollees of all MA organizations to which an EP furnishes services. The commenter continued by saying that if the requirements are not modified to accept multiple MA organizations, the commenter anticipated several unintended consequences in the Puerto Rico market. First, the commenter said, it would be impossible for providers to meet the single MA organization requirement of 80 percent revenue and
20 hours per week, and therefore, the standard would create disinterest in adopting EHRs in their practice. Second, the commenter said, the single MA organization requirement standard would stymie competition. An unanticipated consequence of the requirement would be providers dropping out of MA plans to consolidate revenue in order to meet the standard from a single MA organization. Third, the commenter concluded, patients would have fewer options to select among MA plans, and to a lesser degree, MA enrollees might be forced to discontinue care with long time MA providers in light of the providers' determination to consolidate revenue under a single MA organization.

Response: As noted above, the 80 percent of Medicare revenue standard is set forth in the statute, and may not be changed by regulation. The 20 hour per week rule is also statutory and based on section 1853(1)(2)(B) of the Act, as added by the HITECH Act. We note, however, that it is not the case that all 20 hours of patient care services per week be provided by an EP to MA enrollees of a single MA organization. Rather, the 20 hours of patient care services to enrollees of a single MA organization can include both Medicare and non-Medicare services and patients.

Comment: One commenter asked CMS to continue to work with Congress to develop an equitable mechanism by which to provide incentives to physicians that provide health care services through participation with more than one MAO.

Response: As previously mentioned in the preamble to this final rule, the statute clearly limits payment related to any specific MA EP to a single qualifying MA organization. Potential changes in the statute are outside the scope of this rulemaking.

After consideration of the public comments received, we are implementing the foregoing provisions as proposed.

As discussed in section II.B. of this final rule relating to Medicare FFS EPs, a qualifying MA EP is also defined as a physician under section 1861(r) of the Act. Section 1853(l)(1) of the Act, as added by section 4101(c) of the HITECH Act, provides that the provisions of sections 1848(o) and 1848(a)(7) of the Act, as amended and added by sections 4101(a) and (b) of the HITECH Act, respectively, which establish the incentive payments for EPs under Medicare FFS, apply to a qualifying MA organization's qualifying MA EPs “in a similar manner" as they apply to EPs under Medicare FFS. As discussed above in section II.A.6. of this final rule, section 1848(o)(1)(C)(i) of the Act, as added by section 4101(a) of the HITECH Act, states that hospital-based EPs are not eligible for incentive payments. Therefore, we proposed that, similar to the Medicare FFS incentive program, MA incentive payments would also not be available for hospital-based EPs. We note that the hospital where a hospitalbased EP provides his or her Medicare covered services would be potentially entitled to an incentive payment either through the Medicare FFS incentive program, or through the MA-affiliated hospital EHR incentive program. Therefore, we proposed that for such a hospital-based MA EP, a qualifying MA organization would be no more entitled to an MA EP incentive payment under the MA EHR incentive program than a similarly situated EP would be entitled to an incentive payment under the Medicare FFS EHR incentive program.

Comment: We received one comment related to hospital-based MA EPs, and specifically to our proposal in the proposed rule that "similar to the Medicare FFS incentive program, MA incentive payments would also not be available for hospital-based EPs.” The commenter noted, however, that unlike the proposed regulatory definition of "Qualifying Eligible Professional (EP)" under the Medicare FFS incentive program, the proposed regulatory definition of "Qualifying MA EP" under the MA EHR incentive program did not expressly exclude hospital-based EPs. The commenter went on to say that if hospital-based MA EPs are excluded from the MA EHR incentive program (for example, because they provide $90 \%$ or more of their covered services in the CY preceding the payment year in an outpatient hospital setting), unless there is an exception for MA EPs who are hospital-based in qualifying MAAffiliated Eligible Hospitals that would not qualify for an incentive payment under the MA Affiliated hospital EHR incentive program payment criteria, Qualifying MA Organizations with MA EPs who are hospital-based in such qualifying MA-Affiliated Hospitals would not qualify for an incentive, with regard to those MA EPs, under any HITECH Act Medicare incentive program. The commenter concluded that this outcome would not be consistent with the objective of the HITECH Act to promote widespread adoption of HIT through the payment of monetary incentives for meaningful use of EHRs. The commenter recommended that if hospital-based MA EPs are excluded from the MA EHR incentive program, then we should include an exception for MA EPs who are hospital- 
based in Qualifying MA-Affiliated Eligible Hospitals that would not qualify for an incentive payment (or would only qualify for a very minimal incentive payment) under the MA-Affiliated hospital EHR incentive program payment criteria.

Response: We thank the commenter for pointing out our oversight in not including the hospital-based physician exclusion in the proposed regulation text related to the MA EP EHR incentive program. We will include in regulation text the fact that an MA EP is not a "hospital-based EP," as that term is defined in $\S 495.4$ of this final rule. As to a possible exception for hospitalbased EPs who are practicing in MAaffiliated hospitals that do not qualify for incentive payments (or that qualify for very minimal incentive payments), we cannot provide such an exception. MA-affiliated eligible hospitals will receive EHR incentive payments based on the same statutory formula used to make EHR incentive payments to other "subsection (d)" hospitals-see section II.C.3. of this final rule, below. There is no statutory authority nor is there a valid reason to treat MA EPs, in this respect, any differently that other EPs that are hospital-based.

After consideration of the public comment received, we are modifying the regulation text related to the definition of MA EP by the additional of an item 5) to the definition of "Qualifying MA EP" at $§ 495.200$ to add a specific hospital-based MA EP exclusion.

As discussed in the proposed rule, an MA EP must either be employed by the qualifying MA organization, or be employed by, or be a partner of, an entity that through contract with the qualifying MA organization furnishes at least 80 percent of the entity's Medicare patient care services to enrollees of the qualifying MA organization. With respect to the later criteria, we did not propose to define the term "entity," but instead recognized that there exist a range of entities with which MA organizations contract for patient care services, including physician groups, Independent Practice Associations (IPAs), Exclusive Provider Organizations (EPOs), Physician Hospital Organizations (PHOs), and Preferred Provider Organizations (PPOs).

Moreover, we recognized that an EP may contract with more than one such entity, and that these entities often contract with a number of MA organizations and other health care insurers. An EP also may directly contract with more than one MA organization. In general, we said, it is only when an EP is employed by a single qualifying MA organization, or is employed by or in partnership with an entity that contracts with a single qualifying MA organization, that an EP can satisfy the criteria to be an MA EP.

We said that the qualifying MA organization must attest to the fact that each MA EP is a meaningful user of certified EHR technology in accordance with $\S 495.4$. If all of these conditions are met, such an individual is identified as an MA EP. We proposed to define the term "MA eligible professional (EP)" at $\S 495.200$ as an EP who satisfies all of these conditions.

Finally, we discussed section 4101(d) of the HITECH Act which directed the Secretary to study and report on "nearly exclusive" physicians that primarily treat MA enrollees and that would not otherwise qualify for incentive payments under current law. We explained that this rule does not address such individuals, as it is limited to codifying in regulation existing statutory language as discussed herein.

We did not receive any comments on these provisions and are finalizing them as proposed.

\section{c. Qualifying MA-Affiliated Eligible} Hospital

We proposed to define "qualifying MA-affiliated eligible hospital" in $\S 495.200$. A qualifying MA organization may receive an incentive payment only for a qualifying MA-affiliated eligible hospital described under section 1853(m)(2) of the Act, as added by section 4102(c) of the HITECH Act, that is a meaningful user of certified EHR technology as defined in $\S 495.4$. Section 1853(m)(2) of the Act provides that such MA-affiliated eligible hospitals are "eligible hospitals" as defined under section 1886(n)(6) of the Act and must be under common corporate governance with a qualifying MA organization that serves individuals enrolled under MA plans offered by such organization where more than twothirds of the Medicare hospitals discharges (or bed-days) are Medicare individuals enrolled under MA plans offered by such organization. As discussed in section II.A.1. of this final rule, section 1886(n)(6) of the Act defines an "eligible hospital" as a subsection (d) hospital (as defined under section 1886(d)(1)(B) of the Act). In $\S 495.200$, we also proposed to define "under common corporate governance", as a qualifying MA organization and a qualifying MA-affiliated eligible hospital that have a common parent corporation, where one is a subsidiary of the other, or where the organization and the hospital have a common board of directors.

Section 1853(m)(3)(B)(i) of the Act, as added by section 4101(c) of the HITECH Act, provides that if for a payment year at least one-third (33 percent) of an MA eligible hospital's discharges (or beddays) of Medicare patients are covered under Part A (rather than under Part C), the hospital may only receive an incentive payment under section 1886(n) of the Act-the Medicare FFS incentive program.

In $§ 495.200$ we proposed to define "inpatient-bed-days" in the same manner as that term is defined for purposes of implementing section 4201(a) of the HITECH Act in the preamble of this final rule. The term will be used in the same way in computing incentive payments due qualifying MA organizations under the qualifying MA-affiliated eligible hospital incentive payment program.

We note that, as discussed in section II.B.2.b. of this final rule, under section 1886(n)(2)(D)(i)(II) of the Act, the portion of the Medicare FFS hospital incentive payment comprising the discharge related amount, or Medicare share, is based in part on the estimated number of inpatient-bed-days attributable to individuals enrolled in MA plans under Part C. This means that hospitals that treat individuals enrolled in MA plans will receive a Medicare FFS hospital incentive payment partially based on the number of MAenrollee bed-days. To the extent a hospital does not meet the 33 percent threshold requiring payment through the FFS Medicare EHR hospital incentive program, incentive payments can be made to a qualifying MA organization under common corporate governance to the extent other requirements of the MA EHR hospital incentive program are met. (See section II.C. 3 of this final rule for the computation of incentive payments to qualifying MA organizations.)

Therefore, we proposed to make EHR incentive payments to qualifying MAaffiliated eligible hospitals under the FFS EHR incentive program. Finally, we said that to the extent such data necessary to estimate the inpatient-beddays-related incentive payment amount are not already available to us through the normal submission of hospital cost reports; we proposed to require that qualifying MA organizations seeking reimbursement for qualifying MAaffiliated eligible hospitals submit similar data.

We did not receive any comments on these provisions and are finalizing them as proposed. 
2. Identification of Qualifying MA Organizations, MA EPs, and MAAffiliated Eligible Hospitals

In $\S 495.202$ we proposed to require an MA organization that intended to ask for reimbursement under the MA EHR incentive payment program to so indicate as part of submissions of their initial bid under section 1854(a)(1)(A) of the Act, and to attest, in some cases, that they meet the requirements of a qualifying MA organization. For MA organizations offering an MA HMO plan type, we proposed to deem such organizations to meet the definition of HMO in 42 U.S.C. $300-\operatorname{gg}(\mathrm{b})(3)$, (that is, section 2791(b)(3) of the PHS Act). As noted previously, for MA organizations offering plan types other than HMOs, we proposed to require an attestation by the organization that the MA organization is recognized under State law as an HMO, or that it is a similar organization regulated under State law for solvency in the same manner and to the same extent as an HMO before we would make a determination that the MA organization is a qualifying MA organization for purposes of incentive payments. We proposed to require this beginning with bids due in June 2010 (for plan year 2011) for MA organizations seeking reimbursement for MA EPs and MA-affiliated eligible hospitals.

We also proposed requiring qualifying MA organizations, as part of their initial bids starting with plan year 2011, to make a preliminary identification of potentially qualifying MA EPs and potentially qualifying MA-affiliated eligible hospitals for which the organizations would seek EHR incentive payments.

In developing the preliminary and final lists of potentially qualifying MA EPs, qualifying MA organizations, we said that qualifying MA organizations must exclude hospital-based MA EPs. We proposed that qualifying MA organizations identify hospital-based MA EPs using the same criteria outlined in section II.A.6 of this final rule for identifying hospital-based EPs in the Medicare FFS EHR incentive program.

Along with both the preliminary and final lists of potentially qualifying MA EPs and MA-affiliated hospitals, we said that qualifying MA organizations would be required to submit an attestation that these professionals and hospitals meet the criteria to be considered eligible. For example, for hospitals, the qualifying MA organization would need to attest that they are under common corporate governance with the qualifying MA organization and for EPs, the qualifying MA organization would need to attest that the list does not include any

hospital-based EPs.

We proposed requiring qualifying MA organizations to provide final identification of potentially qualifying MA EPs by the end of the MA EP payment year (December 31), and final identification of potentially qualifying MA-affiliated eligible hospitals by the end of the MA-affiliated hospital payment year (the FFY ending on September 30), for which MA EHR incentive payments were sought. We also proposed requiring qualifying MA organizations to report the name, practice address, and other identifying information, like NPI, for all physicians that meet the requirements of a qualifying MA EP for which the qualifying MA organization would be requesting payment under the MA EHR incentive payment program.

We said that once a qualifying MA organization identifies potential EPs, we are required to ensure that such EPs did not receive the maximum EHR incentive payment for the relevant payment year under the Medicare FFS program under section 1848(o)(1)(A) of the Act, as added by section 4101(a) of the HITECH Act, before releasing an incentive payment to a qualifying MA organization related to such EP. (See section 1853(l)(3)(B)(i) of the Act, as added by section 4101(c) of the HITECH Act). Therefore, in order to allow us time to determine whether an MA EP received the maximum EHR incentive payment under the Medicare FFS program, we proposed not to make incentive payments to qualifying MA organizations for the MA EPs for a payment year until after the final computation of EP incentive payments for that year under the Medicare FFS program. Additionally, we proposed to require qualifying MA organizations to ensure that all MA EPs are enumerated through the NPI system, in order to detect and prevent duplicate payment for EPs under both the FFS and MA EHR incentive payment programs.

Comment: Two commenters contended that requiring MA organizations to provide even a preliminary list of MA EPs by June 2010 (for payment year 2011) would be unrealistic and burdensome, especially when publication of a Final Rule seems unlikely before May 2010 at the earliest. For 2011, any preliminary list will be inaccurate, despite good faith efforts and reasonable due diligence. Moreover, CMS has not stated any justifiable purpose for requiring such a preliminary list.

Response: We agree with the commenters that it would be unnecessarily burdensome and unrealistic to require MA organizations to provide preliminary lists as early as June of 2010 of potential MA EPs for incentive payment year 2011. We will change the timing of this requirement in $\S 495.202$ (b)(1) to say that as part of initial bids for plan year $2012 \mathrm{MA}$ organizations will be required to submit preliminary lists in June of 2011 (when bids are due for 2012) of potential MA EPs for incentive payment year 2011. Thus, we will delay the requirement for a full year. The purpose of such preliminary lists is to identify potential MA EPs that have, for instance, registered as FFS Medicare or Medicaid EPs on the National Level Repository. The intent of getting these lists before payment is due, or before a final determination of eligibility can be made, is to help qualifying MA organizations know of any potential conflicts in time to "cure" them before final payment determinations are made.

Comment: One commenter objected to CMS' proposal that MA organizations be required to submit final lists of MA EPs and MA hospitals by the last day of the payment year, including the attestations of meaningful use and accurate payment calculation. The commenter argued that this timing would not allow sufficient time to ensure that data are complete and accurate, especially considering that MA organizations bear the additional burden of having to develop and support internal administrative systems to determine eligibility and to calculate payment (we will calculate FFS EP payments based on claims submitted). The commenter recommended that we extend the deadlines to produce both preliminary and final lists of MA EPs and hospitals. The commenter suggested that MA organizations be given until 90 to 120 days after the close of the payment year to identify and list eligible EPs and hospitals (for example, after 31

December 2011 for plan year 2011).

Response: We agree with the commenter that additional time should be permitted and we are therefore adding a due date in $\S 495.202(b)(3)$ for final identification of potentially qualifying MA EPs and MA-affiliated eligible hospitals of 60 days after the close of the payment year. We believe 60 days is reasonable, since it is the same as the time in which FFS EPs have to submit claims for consideration under the Medicare FFS EHR incentive payment program.

After consideration of the public comments received, we are modifying the regulation text related to the timing of both preliminary and final identification of MA EPs and MAaffiliated eligible hospitals. Preliminary 
identification of MA EPs and MAaffiliated hospitals for payment year 2011 will need to occur by the bidding deadline in June 2011, and final identification will need to occur within 60 days of the close of the payment year. Accordingly, we are respectively modifying the regulation text at $\S 495.202(\mathrm{~b})(1)$ and $\S 495.202(\mathrm{~b})(3)$. We are also modifying the regulation text at $\S 495.204$ (b)(2) to be consistent with the change to $\S 495.202(\mathrm{~b})(3)$, since final identification in $\S 495.202(\mathrm{~b})(3)$ should occur at the same time as final revenue reporting under $\S 495.204(b)(2)$, so calculations of payments due under the MA EP incentive payment program can be finalized. We are also modifying the regulation text at $\S 495.210$ (b) and (c) to be consistent with the changes to \$495.204(b)(2) and § 495.202(b)(3), since the deadline for attestations of meaningful use should be consistent with deadlines for revenue reporting for MA EPs, and final identification of MA EPs and MA-affiliated hospitals. Finally, as noted (above) in our discussion of the definition of qualifying MA

organizations, we are modifying the date in $\S 495.202(a)(1)$ by which MAOs are required to identify themselves to us from the bidding deadline in June 2010 (for plan year 2011) to the bidding deadline in June 2011 (for plan year 2012).

We also proposed to require all qualifying MA organizations to selfreport and identify themselves, regardless of whether they have qualifying MA EPs or MA-affiliated eligible hospitals for whom or which the organization plans to claim incentive payments at the time the initial bid is due (the first Monday of June, see section 1854(a)(1)(A) of the Act) beginning in 2014 for bids related to plan year 2015. We proposed to require this reporting by all qualifying MA organizations in years beginning with 2014 in anticipation of the statutory requirement in sections $1853(\mathrm{l})(4)$ and $1853(\mathrm{~m})(4)$ of the Act, to negatively adjust our capitation payments to qualifying MA organizations for MA EPs and MA-affiliated eligible hospitals that are not meaningful users of certified EHR technology for years beginning with 2015.

We did not receive any comments on these provisions and are finalizing them as proposed.

3. Computation of Incentives to Qualifying MA Organizations for MA EPs and Hospitals

In $\S 495.204$, we proposed a methodology under which payments to qualifying MA organizations for qualifying MA EPs will be computed.
Section 1853(l)(3)(A) of the Act provides that in applying section 1848(o), instead of the additional payment amount specified under section 1848(o)(1)(A) of the Act, the Secretary may substitute an amount determined by the Secretary, to the extent feasible and practical, to be similar to the estimated amount in the aggregate that would be payable under, or would be based on, the Medicare physician fee schedule under Part B instead of Part C. Section II.B.1. of this final rule discusses these provisions.

Section 1853(m)(3)(A) of the Act provides that, in providing an incentive payment to qualifying MA organizations for MA-affiliated hospitals, we substitute for the amount specified under section 1886(n)(2) of the Act-the incentive payment amount under Medicare FFS for qualifying eligible hospitals-an amount determined by the Secretary to be similar to the estimated amount in the aggregate that would be payable if payment for services furnished by such hospitals was payable under Part A instead of Part C. (For more detailed information see section II.B.2. of this final rule.)

Sections 1848(o)(1)(D)(i) and 1886(n)(2)(F) of the Act permit us to make incentive payments for a year in installments, although we proposed to make a single lump sum payment with respect to MA EPs. With respect to MA EP incentive payments, we said we read the term "aggregate" to mean the aggregate installment payments made by us under the FFS EHR incentive program to a qualifying EP over the course of the relevant payment year.

The duplicate payment provisions in section 1853(l)(3)(B)(i)(II) of the Act direct us to make payment for EPs "only under" the MA EHR incentive program "and not under" the Medicare FFS EHR incentive program to the extent any EP earned "less than [the] maximum incentive payment for the same period" under the Medicare FFS EHR incentive program. We noted in the proposed rule that section 1853(l)(1) of the Act provides that section 1848(o) of the Act applies in a "similar," but not the same, manner to qualifying MA organizations as it applies to EPs under Part B. The Medicare FFS incentive payment program under section 1848(o) does not include payment for professional services provided to MA enrollees, but rather only for services paid under Part B. In a similar manner we proposed to limit payment to an MA organization to only payment for their EPs' services to MA enrollees of plans offered by the MA organization. We said we did not believe it would be appropriate to provide an incentive payment to an MA organization for services provided to individuals covered under Part B. Therefore, we proposed, that in calculating qualifying MA EP incentive payments, we would only consider covered professional services provided to enrollees of MA plans offered by qualifying MA organizations and would not include in the calculation any services reimbursed by Medicare FFS.

Comment: Many commenters asked if MA plan beneficiaries and services would be counted in the calculation of FFS EHR incentives and, if so, if it would require separate submissions to each MA plan in the local market.

Response: As we explained in the preamble of the proposed rule, we cannot make MA EP incentive payments for Part B services covered and paid for on a fee-for-service basis under the original Medicare program. We also cannot make MA EP incentive payments to entities other than qualifying MAOs. In short, the Medicare Advantage services provided by EPs that are not qualifying MA EPs-defined in statute and in this rule at $\S 495.200$-are not reimbursable under the EHR incentive payment program.

Comment: Two commenters contended that the proposed Medicare Advantage incentive computation was inconsistent. They said that sections II.C.3. through 5. of this final rule discuss compensation, but the preamble says that the Secretary may substitute a different amount. This discrepancy should be clarified.

Response: We disagree. The statute says that we can substitute an amount "that is similar to the estimated amount that would be payable or based on the fee schedule." It does not say that we can substitute a different amount.

After consideration of the public comments received, we are

implementing these provisions as proposed.

We also said that under the Medicare FFS EHR incentive program, an EP's incentive payment could not exceed the annual limits specified under section 1848(o)(1)(B)(i) of the Act. We proposed that similar payment limits apply to qualifying MA organizations for their qualifying MA EPs. Specifically, section 1848(o)(1)(B) of the Act provides that the incentive payment for an EP for a given year shall not exceed the following amounts:

- For the EP's first payment year, $\$ 15,000$ (or, if the first payment year is 2011 or $2012, \$ 18,000)$.

- For the EP's second payment year, $\$ 12,000$.

- For the EP's third payment year, $\$ 8,000$.

- For the EP's fourth payment year, $\$ 4,000$. 
- For the EP's fifth payment year, $\$ 2,000$.

- For any succeeding year, $\$ 0$.

Note that, similar to the Medicare FFS EHR incentive program, there will be no incentive payments made with respect to a year after 2016. We proposed similar restrictions related to qualifying MA organizations. So, the maximum cumulative incentive payment over 5 years to a qualifying MA organization for each of its qualifying MA EPs that meaningfully use certified EHRs beginning on or before 2012 would be $\$ 44,000$ per qualifying MA EP. For qualifying MA organizations first reporting the meaningful use of certified EHRs by qualifying MA EPs after 2014, there is no incentive payment amount available. Subject to an exception discussed below, for MA organizations first reporting the meaningful use of certified EHRs by qualifying MA EPs in 2013 or 2014, the maximum potential incentive payment per qualifying $\mathrm{EP}$ is, respectively, $\$ 39,000$ over 4 years, and $\$ 24,000$ over 3 years.

We did not receive any comments on these provisions and are finalizing them as proposed.

We proposed to make MA EP incentive payments to qualifying MA organizations on the same payment cycle for all employed/partnering qualifying EPs of the organization. In other words, all MA EPs of a specific qualifying MA organization will be in the same payment year with respect to the amount of the incentive payment per qualifying EP that we will make. So, for instance, if a qualifying MA organization is in its second payment year in 2013 and it hires a new EP for which the qualifying MA organization had not previously received an EHR incentive payment, we will nevertheless make a second year incentive payment (up to $\$ 12,000$ in 2013) with respect to such an MA EP-assuming all other conditions are met. Thus, the limits on MA EP incentive payments discussed above are applied to the qualifying MA organization's entire MA EP population in any specific payment year relative to that MA organization, regardless of the length of employment/partnership of/ between that specific MA EP and that specific qualifying MA organization.

Under section 1848(o)(1)(B)(iv) of the Act, the annual incentive payment limit for EPs who predominantly furnish Part $B$ services in a geographic health professional shortage area (HPSA) is increased by 10 percent. While we do not anticipate that MA EPs would generally practice in a HPSA area, to the extent that an MA EP practices in an area where he or she would be entitled to the 10 percent increase, that amount would apply to MA EPs as well.

We did not receive any comments on these provisions and are finalizing them as proposed.

We explored various ways of computing the EP-level incentive payments due qualifying MA organizations whose qualifying MA EPs meaningfully use certified EHR technology. One option that we considered was using MA plan bidding and payment data to estimate average annual MA revenue for qualifying MA EPs with respect to a qualifying MA organization. However, we did not pursue this option because the approach results in an average revenue amount across all potentially qualifying MA EPs with respect to a qualifying MA organization and, therefore, would include revenue amounts that exceed the annual per-professional ceiling on incentive payments under FFS for all EPs. We said we believed such a result is contrary to the legal requirement that qualifying MA organizations are to receive incentive payments only for qualifying MA EPs that actually provide at least 20 hours per week of patient care services. Under this method there would be also no way to know if the EP provided 80 percent of his/her professional Medicare services to enrollees of the organization.

We also considered a reporting system for which qualifying MA organizations would be required to report eligibleprofessional-specific information along with MA patient encounters for nonhospital-based office visits. Specifically, we examined requiring qualifying MA organizations reporting qualifying MA EP encounters with MA plan enrollees based on the five levels of office visit codes recognized by Medicare FFS.

We said we believed that such a process would be administratively burdensome and difficult to operationalize. Therefore, we proposed an alternative approach, but sought input from interested parties as to which of the approaches, or perhaps others, would best address the statutory requirement to compensate qualifying MA organizations for qualifying MA EPs the amount that would be payable if payment for services furnished by such professionals were made under Part B instead of Part C.

Therefore, in $\S 495.204(b)(1)$ through (3) we proposed an approach in which the revenue received by the qualifying MA EP for services provided to enrollees of the qualifying MA

organization would serve as a proxy for the amount that would have been paid if the services were payable under Part
B. Under our proposed approach, the qualifying MA organization would report to us the aggregate annual amount of revenue received by each qualifying MA EP for MA plan enrollees of the MA organization. We said we would calculate the incentive payment amount due the qualifying MA organization for each qualifying MA EP as an amount equal to 75 percent of the reported annual MA revenue of the qualifying MA EP, up to the maximum amounts specified under section 1848(o)(1)(B) of the Act.

For qualifying MA EPs who were compensated on a salaried basis, we proposed in § 495.204(b)(4) requiring the qualifying MA organization to develop a methodology for estimating the portion of the qualifying MA EP's salary attributable to providing services that would otherwise be covered as professional services under Part B of Medicare to MA plan enrollees of the MA organization. The methodology, which would require review and approval by us, could be based on the relative share of patient care hours spent with MA enrollees of the organization or another reasonable method. So, for instance, if a qualifying MA EP spends 30 percent of his or her time providing covered Part B physician office services to MA plan enrollees, then the qualifying MA organization would report 30 percent of the qualifying MA EP's salary as annual revenue, which would be used to compute the amount of the MA incentive payment due to the qualifying MA organization for the qualifying MA EP. Thus, if the qualifying MA EP had a base salary of $\$ 150,000,30$ percent would be $\$ 45,000-$ which is well over the threshold of $\$ 24,000$ needed by the MA organization to qualify for a maximum incentive payment of up to $\$ 18,000$ (70 percent of $\$ 24,000$ ) for such a qualifying MA EP in any year. We also proposed to require that salaries be prorated to ensure that the amount reported reflects the salary paid for the applicable year, where necessary.

We also said that salaried physicians' compensation typically does not include an allowance for administrative practice costs. Given that Part B allowed amounts do include practice expense costs, we proposed allowing qualifying MA organizations to identify, where appropriate, an additional amount related to overhead that would be added to the qualifying MA EP's estimated Part $B$ compensation. To the extent Medicare FFS compensation to physicians includes an amount for office space rental, office staffing, and equipment, we believe that qualifying MA organizations should also be permitted 
to include an amount for overhead related to such costs not directly experienced by salaried qualifying MA EPs. In §495.204(b)(4)(ii), we proposed requiring qualifying MA organizations to develop a methodology for estimating the additional amount related to overhead attributable to providing services that would otherwise be covered under Part B of Medicare. We said the methodology would require review and approval by us.

For qualifying MA EPs who are not salaried (that is, who are paid on a capitated or fee-for-service basis), we proposed in $\S 495.204(\mathrm{~b})(5)$ to require qualifying MA organizations to obtain attestations from such EPs and to submit to us information from the attestations as to the amount of compensation received by the EPs for MA plan enrollees of the MA organization. We are proposing such attestations because many EPs are not paid directly by MA organizations, but rather by intermediary contracting entities, such as physician groups, and as a result the qualifying MA organization may not otherwise know how much compensation is received by each qualifying MA EP. In reporting compensation, we are proposing that the EPs include only those amounts for professional services that would otherwise be payable under Part B and for which payment would be made under, or would be based on, the Medicare physician fee schedule.

Comment: One commenter recommended that final CMS regulations retain the exact requirements outlined in $\S \S 495.204(\mathrm{~b})(4)$ and (5). Two commenters said that CMS should allow flexibility in methods MA organizations propose for computing incentive payments so long as the organization's approach is reasonable, straightforward, and fairly equates to the Medicare feefor-service approach without imposing undue burdens on MA organization systems or compromising EP privacy. The proposed rule describes how incentive payment amounts will be calculated for eligible hospitals and EPs. The proposed rule presents options for a MA payment methodology, but expressly solicits comments from MA organizations about how such a methodology could be designed to fairly approximate the FFS payment calculation. The commenters included recommendations about how MA organizations could be reimbursed and what methodology would be a reasonable proxy for the Part B-based payment applied to FFS physicians, based on the amount of individual physician care provided to MA members. The commenters said that MA EPs who are employed by their organizations are independent physician group practices that contract exclusively with their organizations to meet the health needs of their members, including MA enrollees. Their organizations do not pay the salaries of MA EPs who provide patient care services to their members and patients. They said that CMS has proposed that the organization that directly pays the EP salaries would perform a calculation and attest to the MA organization about the amount of payment. They said that while this would mitigate some of the confidentiality concerns related to sharing salary information with the health plans, salary information would still be potentially exposed to CMS. They said that another disadvantage of using actual salary as a basis for calculating the incentive payment is that this approach potentially introduces unacceptable variability into the estimation of proxy amounts for Medicare services. For example, two MA EPs, whose salaries vary significantly but provide the same Medicare services in a reporting period, would have different proxy amounts. Further, they said, if such EPs were billing under Part B, the amount of Medicare services each billed would be the same, regardless of whether their incomes were the same. These commenters went on to propose an alternative method of computing a proxy Part B amount. They said that as a first step, the MA organization would calculate the percentage of clinic time each physician spends caring for MA members. This MA Practice percentage could be derived by either: (1) Capturing the total scheduled appointment time for MA members for each MA EP and dividing that amount by the total scheduled time for that MA EP (for all appointments); or (2) capturing the number of MA member visits/ procedures for each MA EP and dividing that amount by the total number of visits/procedures for that MA EP (for all members). The organization would then calculate the average practice cost by specialty for all specialties identified in the annual American Medical Group Association's (“AMGA") salary survey. The commenters explained that AMGA survey provides the median compensation per physician in most specialties as well as the noncompensation related clinic costs per physician (staffing, supplies, overhead, etc.) in most specialties. Adding specialty specific compensation data (for groups $>100$ physicians) to the combined average non-compensation related clinic costs for that specialty (for all sized groups) would provide a surrogate amount for each specialty's total operating costs. This would produce the Average Operating Costs by Specialty. Multiplying each MA EP's MA Practice percentage and the Average Operating Costs by Specialty for that MA EP's practice specialty would produce a surrogate Medicare Part B amount. For each MA EP, the MA organization would be paid an incentive equal to 75 percent of the surrogate Medicare billing amount for that physician, such incentive not to exceed the maximum incentive for each payment year of the program (for example, $\$ 18,000$ if the first year of participation is 2011).

Response: While we appreciate the thought and effort that went into this proposed alternative method of calculating MA EP incentive payments, we are reluctant to adopt it for the simple reason that where salaries, practice costs, or actual MA EP compensation can be known, we believe it is a better read of statutory requirements to work from that actual compensation and cost data than it would be to allow estimation of both. In many respects the proposed alternative method is similar to the method discussed and disposed of in the proposed rule related to estimating physician compensation based on MA bidding and payment data. Although the commenters' alternative version factors in actual practice time, we believe using AMGA salary survey data would be inferior to using actual physician compensation practice cost information. To the extent actual salary information is unknown or unavailable to the MA organization, we believe it could be provided to us in a manner that would protect the privacy of individual MA EPs and physician groups. Furthermore, the proposal also estimates "noncompensation related clinic costs" based on AMGA data, which is, again, inappropriate, when actual overhead costs might be quite different in a specific MA organization. However, based on the commenters concerns regarding provider privacy and the need to develop a consistent and verifiable method of computing the amount payable to qualifying MA organizations for MA EPs we are modifying the regulation text at $\S 495.204(\mathrm{~b})(5)$ to say that qualifying MA organizations "may" obtain attestations from qualifying MA EPs and "may" submit such information to us-rather than "must." And, we add a new subparagraph (6) that allows the physician group or other payer to 
provide EP reimbursement information directly to us. We also provide assurances that we will use the EP reimbursement data for no other purpose than to compute the MA EP incentive payment due the qualifying MA organization.

Comment: One commenter said that in the proposed rule the methodology for estimating the portion of the qualifying MA EP's salary attributable to providing services that would otherwise be covered as professional services under Part B of Medicare to MA plan enrollees of the MA organization would require review and approval by CMS; and that such methodology "could be based on the relative share of patient care hours spent with MA enrollees of the organization or another reasonable method." However, the commenter opined, the proposed rule offers no details about how the review and approval process would be conducted, including dates and timelines for the process. Thus, the commenter recommended that CMS permit flexibility in allowing MA organizations to develop methodologies that will be reasonable in light of organization structure and systems, it is important to provide some guidance about how CMS will review and approve such proposals. CMS should permit, the commenter said, any reasonable payment methodology method that is fair, relatively easy to administer, subject to audit and that provides a reliable approximation of Medicare Part B billing. In addition, the commenter concluded, CMS should provide a simple process for submission and approval of MA payment methodologies.

Response: In the proposed rule at $\S 495.204(\mathrm{~b})(4)$ we offered flexibility related to the "methodology for estimating the portion of each qualifying MA EP's salary attributable to providing services that would otherwise be covered as professional services under Part B," said that the methodology had to be "approved by CMS," and that the amount could include an "additional amount related to overhead." Based on this comment we are adding a new clause (iii) that says that such methodological proposals must be submitted to CMS by June of the payment year, must be auditable by an independent third-party, and that CMS will review and approve or disapprove such proposals in a timely manner.

Comment: One commenter wanted to know what percentage of the incentive payments will go to eligible professionals under Medicare Advantage.
Response: No known percentage of incentive payment will go to eligible professionals under Medicare Advantage, since MA EP payments are made solely to qualifying MA organizations.

In the proposed rule we said that in applying the instruction in section $1853(\mathrm{~m})(3)(\mathrm{A})$ of the Act to substitute for the amount specified under section 1886(n)(2) of the Act an amount similar to the estimated amount in the aggregate that would be payable if payment for the hospitals' services were made under Part A instead of Part C, we read the term "aggregate" to mean the aggregate installment payments made by us if EHR incentive payments were made under Part A instead of Part C.

Incentive payments to eligible hospitals under the Medicare FFS EHR incentive program are comprised of three components: (1) An initial amount composed of a base incentive payment of $\$ 2,000,000$ and a second incentive payment amount of $\$ 200$ per discharge for discharges 1,150-23,000 during a 12-month period selected by the Secretary; (2) the Medicare share; and (3) a transition factor. As discussed in the preamble related to $\S 495.104$ (c), for purposes of calculating incentive payments to eligible hospitals under the Medicare FFS EHR incentive program, we are proposing that the 12-month period be based on the FFY. For the purpose of calculating incentive payments for qualifying MA-affiliated eligible hospitals, we similarly are proposing that the 12-month period be based on the FFY.

Section II.B. of this final rule discusses our methodology for calculating the incentive payment for qualifying eligible hospitals under the Medicare FFS EHR program. As set forth in $\S 495.204$ (c)(2), we proposed to use the FFS EHR hospital incentive program for purposes of calculating and making the incentive payment for qualifying MA-affiliated hospitals. To the extent data are not available to reimburse MAaffiliated hospitals through the FFS hospital incentive program, we proposed to require submission of such data to us and adopt the same definition of "inpatient-bed-days" and other terms under the Medicare FFS EHR hospital incentive program specified in $§ 495.104$ of this final rule. In such a case we proposed in $\S 495.204$ (c)(1) to make payment for such MA-affiliated eligible hospitals to the qualifying MA organization.

The formula for calculating the hospital incentive payment under the Medicare FFS hospital incentive program is an initial amount of the sum of the base amount of $\$ 2,000,000$ per hospital plus an additional $\$ 200$ per discharge for discharges 1,150 through 23,000 for that hospital in that payment year. This initial amount is then multiplied by a transition factor and then again by the Medicare share. These last two numbers are fractions and will tend to reduce the initial amount computed in the first step.

Similar to the Medicare FFS EHR hospital incentive program, we proposed to use inpatient-bed-day data, discharges, and other components of the FFS calculation for each qualifying MAaffiliated eligible hospital from the hospital-specific fiscal year that ends during the FFY prior to the FFY that serves as the payment year. To the extent such data are not already available to us through the normal submission of hospital cost reporting data; we proposed requiring qualifying MA organizations seeking reimbursement for their qualifying MAaffiliated eligible hospitals to submit similar data.

We said we can only pay for qualifying MA-affiliated eligible hospitals under common corporate governance based on inpatient-bed-days computed on a fiscal year basis where less than one third of the inpatient-beddays of Medicare patients are covered under Medicare FFS-Part A. However, it does not appear that reimbursement only under the MA EHR incentive program is required for qualifying MAaffiliated eligible hospitals that are under common corporate governance. Rather, section 1853(m)(3)(B), of the Act only prohibits payment under the MA EHR incentive program when Medicare hospital inpatient-bed-days covered under Part A exceed 33 percent of all Medicare inpatient-bed-days. Although eligibility under the MA EHR hospital incentive program is not available to qualifying MA organizations for any specific hospital when FFS inpatientbed-days exceed 33 percent of the Medicare total, a qualifying MA organization could be reimbursed through the Medicare FFS EHR hospital incentive payment program for qualifying hospitals under common corporate governance even for hospitals with very low ratios of FFS to MA inpatient-bed days.

Given that the hospital incentive payment methodology and payment amount will be identical under the Medicare FFS EHR incentive program and the MA EHR incentive program, and given that there is no statutory prohibition on reimbursing a qualifying MA-affiliated eligible hospital through the Medicare FFS EHR incentive program, for purposes of administrative efficiency, and pursuant to our authority 
under section $1857(\mathrm{e})$ of the Act to add new "appropriate" contract terms (incorporated for Part D by section 1860D-12(b)(3)(D) of the Act), we proposed requiring that qualifying MA organizations receive incentive payments for qualifying MA-affiliated eligible hospitals through their affiliated hospitals under the Medicare FFS EHR incentive program if they are eligible for such payments, rather than through the MA EHR incentive program. We believe this is the most efficient way in which to administer the MA EHR hospital incentive program in light of the expected low volume of MA-affiliated eligible hospitals (approximately 50 hospitals), and in light of preliminary data which indicates that MA-affiliated eligible hospitals already submit Medicare cost reporting data to us from which we can compute hospital incentive payments due. To the extent sufficient data do not exist to make such payments under the Medicare FFS EHR incentive program, qualifying MA organizations will be required to submit additional data to us.

We did not receive any comments on these provisions and are finalizing them as proposed.

To the extent payments are made to qualifying MA organizations for qualifying MA EPs or qualifying MAaffiliated eligible hospitals, we proposed to conduct selected compliance reviews to ensure that EPs and eligible hospitals for which such organizations received incentive payments were actually meaningful users of certified EHR technology, in accordance with our existing authority in section 1857 (d) of the Act and 42 CFR 422.504 of the regulations related to protections against fraud. The reviews would include validation of meaningful user attestations, the status of the organization as a qualifying MA organization, and verification of both meaningful use and data used to calculate incentive payments. We proposed requiring MA organizations to maintain evidence of compliance with all aspects of the MA EHR incentive payment program for 10 years after the date payment is made with respect to a given payment year. Payments that result from incorrect or fraudulent attestations, cost data, or any other submission required to establish eligibility or to qualify for a payment, will be recouped by CMS from the MA organization.

We did not receive any comments on these provisions and are finalizing them as proposed.

Finally, as we indicated above in section II.C.2. of this final rule, we are modifying the regulation text at
$\S 495.204(\mathrm{~b})(2)$ to be consistent with the change to $\S 495.202(\mathrm{~b})(3)$, since final identification in $\S 495.202(\mathrm{~b})(3)$ should occur at the same time as final revenue reporting under $\S 495.204(\mathrm{~b})(2)$, in order to ensure that calculations of payments due under the MA EP incentive payment program can be finalized.

\section{Timeframe for Payment}

For payments to qualifying MA EPs, in $\S 495.206$ we proposed the timeframe for payment to be after the Medicare FFS program computes incentive payments due under the Medicare FFS EHR incentive program-so the first possible incentive payments would be made sometime in early 2012 . We proposed that payments for qualifying MA-affiliated eligible hospitals under common corporate governance occur in the same manner and in the same time frame as payments made under the Medicare FFS EHR incentive program to "subsection (d)" hospitals as discussed in section II.B.2.d. of this final rule.

We proposed to define "payment year" with respect to qualifying MA EPs in $\S 495.200$. Section 1853(1)(3)(C) of the Act directs us to establish the same first payment year for all EPs with respect to any specific qualifying MA organization. Consistent with the statute, we proposed to pay a qualifying MA organization on the same schedule for all of its qualifying MA EPs. In other words, the first year during which the qualifying MA organization receives an incentive payment for its qualifying EPs will be considered the first payment year for all of its qualifying EPs. Accordingly, for purposes of determining the applicable incentive payment limits, the second, third, fourth, and fifth years during which the qualifying MA organization receives an incentive payment for its qualifying EPs will be considered the second, third, fourth, and fifth payments years for each of its qualifying EPs, regardless of whether the MA organization claimed an incentive payment for a particular EP for a prior payment year. Such a consistent payment cycle relative to qualifying MA organizations and qualifying MA EPs obviates the need to track payment years and payment adjustment years based on prior payments or adjustments with respect to any individual qualifying MA EP. Rather, for purposes of payment years and payment adjustment years, any EP employed by or partnering with any specific MA organization will be on the same cycle with respect to that organization.

We said that similar to the Medicare FFS EHR incentive program, payment to qualifying MA organizations for qualifying MA EPs and payment for qualifying MA-affiliated eligible hospitals is available only for a finite number of years. As previously discussed in the section on the calculation of MA incentive payments, above, a qualifying MA organization can receive an incentive payment of up to $\$ 18,000$ for each of its qualifying MA EPs for its first payment year if its first payment year is 2011 or 2012 , or up to $\$ 15,000$, if its first payment year is 2013 , or up to $\$ 12,000$, if its first payment year is 2014. Note that, similar to the Medicare FFS EHR incentive program, there would be no incentive payments made with respect to a year after 2016 .

We proposed to define "payment year" with respect to qualifying MAaffiliated eligible hospitals in $\S 495.200$. For incentive payments for qualifying MA-affiliated eligible hospitals, the first year for which an MA organization may claim payment is FY 2011. Similar to the Medicare FFS EHR hospital incentive program, we proposed to use the hospital inpatient bed-days data from the hospital FY that ends during the FFY prior to the FY that serves as the payment year. For qualifying MAaffiliated eligible hospitals, we proposed to compute hospital EHR incentive payments due in the same manner as they are being computed in the Medicare FFS hospital incentive payment program. For qualifying MAaffiliated eligible hospitals for which the first payment year is 2011 through 2013, up to 3 additional years of incentive payments are available. For qualifying MA-affiliated eligible hospitals for which the first payment year is after 2015, no EHR payment incentive can be made for that year or any subsequent year. Finally, for qualifying MAaffiliated eligible hospitals for which the first payment year is 2014 or 2015, only 2 (or 1) additional year(s) of hospital incentive payments will be available.

Unlike the fixed schedule for application of limitation on incentive payments for MA EPs discussed previously in this section of the final rule in which all employed/partnering MA EPs will be paid on the same schedule (first payment year, second payment year, etc.) with respect to any specific qualifying MA organization, we proposed to make payments to MA organizations for MA-affiliated eligible hospitals on a hospital specific basis. In other words, if a qualifying MA organization has some MA-affiliated eligible hospitals with a first payment year of FY 2011, it may have other MAaffiliated eligible hospitals with a first payment year of FYs 2012 through 2015.

Comment: Two commenters said that payments to MA organizations will be 
delayed every year by an unspecified amount of time. The commenters said that it was understood that CMS is charged by statute to avoid making duplicate payments, however MA organizations should be paid without unspecified delay. A suggested alternative by the commenters was to permit MA organizations to attest that their MA EPs will not seek any payment under the Medicare FFS Incentive Program. Alternatively, the commenters suggested, CMS could use an installment payment system (permitted under statute as stated) for MA

organizations. The commenters said that this would permit partial payment until the resolution of the duplicate payment issue and would avoid long delays in paying MA incentives.

Response: We do not agree that MA organization EHR incentive payments are subject to "unspecified delay." Rather, since MA organizations will be paid for MA EPs only if such EPs were not paid the maximum incentive payment under the FFS EHR incentive payment program, and since final claims data will not be available until two months after the close of the payment year-see § 495.102(a)(2)CMS will not be able to compute MA EP payments until the FFS EHR incentive payment program has completed its calculations. This will occur in the early spring of the year after the close of a payment year. Moreover, MA-affiliated eligible hospitals will receive EHR incentive payments on the same schedule as other "subpart (d)" hospitals. Finally, note that MA EPs are free to leave qualifying MA organizations at any time, and since EPs are also free to register for eligibility under FFS Medicare or Medicaid EHR incentive payments, an attestation by a qualifying MA organization would have little merit. For these reasons we cannot accept the suggestion that qualifying MA organizations receive interim or partial mid-year payments for MA EPs.

After consideration of the public comment received, we are implementing these provisions as proposed.

\section{Avoiding Duplicate Payment}

We proposed duplicate payment avoidance provisions in $\S 495.208$. Section 1853(1)(3)(B) of the Act, as added by the HITECH Act, is entitled "Avoiding Duplication of Payments." Subclause (I) of clause (i) of this paragraph of the Act states that to the extent an MA EP is entitled to the maximum incentive payment under section 1848(o)(1)(A) of the Act, the Medicare FFS EHR incentive payment program, such incentive payment will only be made under the Medicare FFS EHR incentive program. Therefore, before payments can be made to qualifying MA organizations for MA EPs, we must first determine if a maximum incentive payment under the Medicare FFS program has been previously earned by potential MA EPs. Under the Medicare FFS incentive payment program, incentive payment calculations will not be completed for the first payment year, 2011, until the early part of 2012. Therefore, we said we would not be able to make payments to qualifying MA organizations for MA EPs until claims submissions counted for Medicare FFS incentive payments for CY 2011 have been closed, and payment calculations for participating EP under the Medicare FFS EHR incentive program have been completed. This will occur in the early part of CY 2012. In the MA EHR incentive payment program we proposed to follow the FFS EHR incentive payment program schedule-first computing Medicare FFS incentive payments for EPs and then computing and paying MA EP incentive payments, where appropriate-in all subsequent payment years.

We went on to explain that subclause (II) of section 1853(1)(3)(B)(i) of the Act further states that to the extent an MA EP is entitled to less than the maximum incentive payment under the Medicare FFS EHR incentive program, that payment is to be made solely under the MA provision. In other words, we will need to withhold Medicare FFS incentive payments from EPs of less than the maximum to the extent such professionals are also identified as MA EPs under section 1853(l)(2) of the Act. Again, we would need to await the computation of payments due EPs under the Medicare FFS EHR incentive program before we can determine whether the EP is entitled to less than the maximum payment amount under the Medicare FFS EHR program, in which case any incentive payment for the EP will only be made to the qualifying MA organization under the MA EHR program, and not to the EP under the Medicare FFS EHR program.

We also said that section 1853(m)(3)(B) of the Act states that incentive payments for qualifying MAaffiliated eligible hospitals are to be made under either the Medicare FFS hospital incentive payment program, or under the MA hospital incentive payment program. If more than 33 percent of discharges or bed-days of all Medicare patients for a year are covered under Part A, then payment for that year is to only be made under section 1886(n) of the Act-the Medicare FFS
EHR incentive program-and no payment is to be made under the MA hospital incentive payment program. Otherwise, to the extent less than 33 percent of bed days of all Medicare patients for an incentive payment year are covered under Part A, then payment for that incentive payment year may be made under the MA EHR incentive payment program.

Unlike the process we proposed to follow related to qualifying EPs (where we will wait for the Medicare FFS incentive payment program to compute eligible physician incentive payments due under that program before determining the amount due under the MA EHR incentive program), we would not need to rely on Medicare FFS EHR incentive payment program calculations before determining eligibility for MAaffiliated hospital incentive payments. We said we would reimburse all hospitals, including MA-affiliated eligible hospitals, under the Medicare FFS hospital incentive program. We believe that by doing so, we will prevent duplicate payments being made for the same hospitals by Medicare FFS and the MA incentive payment programs. To the extent that qualifying MA organizations are to receive incentive payments through the MA program rather than through their hospitals under the Medicare FFS EHR incentive program due to a lack of sufficient data to make payments under the FFS program, we would identify and reimburse only appropriate qualifying MA organizations for qualifying MAaffiliated eligible hospitals. Such reimbursement will be in a manner similar to the manner in which the Medicare FFS EHR incentive program will reimburse eligible hospitals due an incentive payment under the Medicare FFS EHR incentive program.

Finally, we said that in order to avoid duplicate payments and in accordance with section 1853(m)(3)(B)(ii)(II) of the Act, we will not make MA EHR hospital incentive payments to qualifying MA organizations for MA-affiliated eligible hospitals other than through the Medicare FFS EHR hospital incentive payment program without first ensuring that no such payments under the Medicare FFS EHR hospital incentive payments were made.

We did not receive any comments on these provisions and are finalizing them as proposed.

\section{Meaningful User Attestation}

We proposed meaningful user attestation requirements in $\S 495.210$. For each MA EP and MA-affiliated hospital for which a qualified MA organization seeks an incentive 
payment, the organization must attest, in a form and manner specified by us, that its MA EPs and MA-affiliated eligible hospitals are meaningful EHR users, as required by sections 1853(1)(6) and $1853(\mathrm{~m})(1)$ of the Act. We further proposed to adopt the definitions of meaningful user under the Medicare FFS program related to EPs and eligible hospitals in $\S 495.4$. We are requiring qualifying MA organizations to attest each payment year whether each of its MA EPs and MA-affiliated eligible hospitals for which it is seeking an incentive payment was a meaningful EHR user for the EHR reporting period for a payment year. A qualifying MA organization must make this attestation for each payment year for which it is seeking an incentive payment for MA EPs and MA-affiliated eligible hospitals. We believe attestations should occur toward the end of a year with respect to that year, since qualifying MA organizations will need to attest to, based on our proposed rule, meaningful use for the appropriate duration and during the appropriate period related to MA EPs and MA-affiliated eligible hospitals before claiming incentive payments for them.

In the proposed rule we said that unlike the Medicare FFS EHR incentive program, where we will require the reporting of clinical quality measuressee $\$ 495.8$-we will not require qualifying MA organizations to submit clinical quality measures per section 1848(o)(2)(B) of the Act, with respect to EPs, and section 1886(n)(3)(B) of the Act, with respect to eligible hospitals. Consistent with sections

1848(o)(2)(B)(iii) and 1886(n)(3)(B)(iii) of the Act, we note that qualifying MA organizations sponsoring coordinated care MA plans are already required to submit Healthcare Effectiveness Data and Information Set (HEDIS), Health Outcomes Survey (HOS), and Consumer Assessment of Healthcare Providers and Systems (CAHPS) measures per $\S 422.152$ and $\S 422.516$. Coordinated care MA plans include HMO, PPO and RPPO (Regional PPO) plans. Beginning with CY 2010, PFFS and MSA plans will also be required to begin collecting and submitting administrative HEDIS measures.

We believe that all qualifying MA organizations will be organizations offering MA coordinated care plans, and therefore; those MA organizations from which we routinely receive complete HEDIS dataset reporting. Pursuant to sections 1848(o)(2)(B)(iii) and 1886(n)(3)(B)(iii) of the Act, for clinical quality measures which overlap between the existing MA quality reporting program and under the EHR incentive program, we proposed to allow qualifying MA organizations to continue reporting under the existing MA quality reporting program. For those HITECH clinical quality measures that do not overlap and that are appropriate for the MA program, we are considering requiring that qualifying $\mathrm{MA}$ organizations that receive an incentive payment report those measures to CMS. This would ensure that clinical quality measure reporting under HITECH is consistent between the FFS program and MA. An alternative approach would be to require that qualifying $\mathrm{MA}$ organizations that receive an incentive payment report all of the HITECH clinical quality measures under section II.A.2 of this final rule that are appropriate for the MA program directly to CMS, while also reporting those HEDIS, HOS, and CAHPS measures under the existing MA quality program. This may result in duplicative reporting under the HITECH program and current MA quality reporting, but may provide us with more direct access to quality data under the HITECH program. We invite public comment on these approaches, including alternative methods to consistently treat MAaffiliated providers and FFS providers under the HITECH Medicare incentive program.

Comment: The meaningful use criteria make reference to checking eligibility electronically and submitting claims electronically for 80 percent of patients seen. This would not be possible for us because, for most of our visits, there is no insurance company with which to check, and there is no eligibility to submit claims to. We are a capitated system and for most of the patient visits, the concept of checking eligibility and submitting claims in not relevant.

Response: This comment points out the difficulty in adopting FFS Medicare meaningful use measures for qualifying MA organizations, MA-affiliated hospitals and MA EPs. For purposes of determining meaningful use in a Medicare Advantage environment, we agree that submitting claims

electronically is not a useful standard in a capitated environment where virtually all patients are members of the same insurance plan.

Comment: One commenter said that given the sensitivity of the data, and the RHQDAPU program specifications, the commenter believes CMS should never request that hospitals submit patientlevel data to CMS, but that the data submitted should always be at the aggregated, summary level. The commenter encouraged us to state specifically that this is its intention in FY 2012 and all future years of EHR incentive program reporting. Some other commenters said that their health care delivery systems were based on an integrated care delivery model, where coordination of care is supported through program-wide EHR

implementation that enables a patient's medical record to be shared among the members of the patient's care team. The commenters said they believed patientcentric electronic medical record models that integrate clinical information across providers align with goals of ONC's Strategic Plan and reform efforts that seek to enable more patientcentric integration of care. The commenters said that during any given reporting period under the EHR incentive payment program, patients may receive health care services from various providers (for example, the primary care physician, one or more specialists, nurse practitioners, etc.). The commenters said they had adopted program-wide policies and procedures for using their EHR system to promote coordinated delivery of care. Thus, the commenters said they intended to use their EHR system to support the functionality and care delivery criteria of meaningful use for all providers across their organizations. Within their organizations, they said, a single provider is never solely responsible for all the information in a given patient's electronic medical record. In fact, they said, many providers may access the patient's electronic record to view or add information, order tests or medications, review results, etc. They said the shared record makes it extremely difficult to reliably track all the meaningful use criteria to each EP in their organizations without adding additional administrative functionality to their systems that would do nothing to improve patient care. It would be inappropriate and not the intent of the EHR incentive payment program, they said they believed, to add unnecessary redundancy in care delivery (that is, providers re-entering correct

demographic information to get "credit" for that measure). They said they intended to participate in the EHR incentive payment program under provisions for Medicare Advantage organizations. They went on to say that since the proposed rule states, "the qualifying MA organization must attest to the fact that each MA EP is a meaningful user of certified EHR technology * * *, " they believed such attestation can be based on measuring criteria at a MA organizational level. While they acknowledged that meeting basic eligibility criteria is appropriate on an individual provider level (that is, 
the MA EP must meet the same definition for EP under FFS, satisfy minimum hours per week delivering patient care services, not be hospitalbased, etc.), they said they should be able to meet meaningful use criteria as a MA organization on behalf of all of their individual EPs, so long as they are able to demonstrate that their EHR system itself meets the criteria and its use is pervasive and consistent throughout their healthcare delivery sites. They recommended that where a patient's electronic medical record is shared among a team of providers within a MA organization, the meaningful use criteria be measured on an organizational versus an individual provider level. As an alternative they proposed that for any provider who treats a given patient, if the criterion is met in that patient's electronic record, all EPs who are members of the patient's care delivery team would receive "credit" for meeting that measure.

Response: We agree with the commenters in large part. We believe that continued reporting by qualifying MA organizations under the HEDIS program is the most appropriate way to protect personally identifiable patient information. We also believe that in integrated care delivery systems, it does not make sense to require specific individuals to enter specific data in order to obtain meaningful user statusespecially in a Medicare Advantage environment where we will require only continued HEDIS reporting as a demonstration of meaningful use. Finally, we believe that reporting of clinical quality measures at the MA organization level is the most effective and appropriate means of attaining the ultimate goal of EHR adoptionimproved patient outcomes and reduced healthcare costs.

Comment: Some commenters said that the proposed rule states that, "unlike the Medicare FFS EHR Incentive Program, where we will require the reporting of clinical quality measures * * * we will not require qualifying MA organizations to submit clinical quality measures * * * with respect to EPs * * * and with respect to eligible hospitals * * *. $[W]$ e note that qualifying MA organizations sponsoring coordinated care plans are already required to submit Healthcare Effectiveness Data and Information Set ("HEDIS"), Health Outcomes Survey ("HOS"), and Consumer Assessment of Healthcare Providers and Systems ("CAHPS") measures." The proposed rule suggests allowing MA organizations to continue reporting these measures, but also considers requiring that $\mathrm{MA}$ organizations report those HITECH clinical quality measures that do not overlap with these currently reported measures "and are appropriate for the MA program.” We believe this current reporting is both appropriate and sufficient to measure the clinical quality of MA programs and should be deemed to satisfy the clinical quality reporting requirements under the EHR incentive payment program. HEDIS, HOS and CAHPS reporting are well-established and subject to audit. The measures are specifically chosen to capture quality within MA organizations, in particular to measure the clinical quality of the team approach we use to deliver care. While we support consistency across the EHR incentive payment program, we are concerned that requiring $\mathrm{MA}$ organizations to create new mechanisms for this additional reporting would be unduly burdensome, especially if these additional measures would have to be reported at the individual provider or patient level. Another commenter said that their considerable experience with developing responses for new measures demonstrated how resource and labor intensive clinical quality measurement can be. For example, the commenter continued, during a recent effort to automate ten TJC (The Joint

Commission) measures, we identified 87 data elements, only 37 of which are captured as discrete data. Of the remaining 50 measures, some are captured using discrete data in different places in the EHR, and some are captured using free text (for example, clinical trials and other irregular exclusion criteria) and will require the creation of new documentation tools. We estimate it will take one to two years of work for these ten measures to be fully automated, despite our relatively sophisticated use of data warehousing tools and our high level of automation in the data management process. The burden is especially heavy when measurement elements are ill-defined. Under meaningful use clinical quality reporting, over 120 measures have been proposed. Of these, 94 would be measures not currently calculated or reported on a routine basis. We anticipate a considerable increase in workload to create and maintain these measures. Adding new and duplicatepossibly less reliable-measures and reporting systems will be costly, timeconsuming and may not have an incrementally significant impact on improving patient care. While we are not opposed to new metrics (those without similar known specifications), such measures should be field tested prior to becoming requirements; in particular, subject to rigorous testing of the electronic specifications. Such measures should also be supported by robust clinical evidence to show they will impact clinical outcomes. MA organizations should be deemed to have satisfied all clinical quality reporting required in the EHR incentive payment program by meeting their current reporting requirements. If additional measures are required, we recommend staged adoption, beginning with those measures that MA organizations already report or can report in the near future. We recommend eliminating measures that have little or no evidence to link them to improved outcomes. Overall, we strongly recommend that CMS significantly reduce the overall number of clinical quality measures that would be required for meaningful use.

Response: We agree with the commenters and believe that HEDIS, HOS and CAHPS are the appropriate means of reporting measures for both MA EPs and MA-affiliated hospitals. Where appropriate we will consider adding elements to these already existing quality reporting programs. We will consider adding HEDIS elements over time, as experience and clinical data warrant.

Comment: One commenter said one of the five priorities specified by CMS is to improve care coordination. However, the siloed nature of the incentive payments, lack of a robust set of care coordination measures, and the narrow definition of eligible professionals do not fully support this priority. The commenter also said that the current structure of the proposed incentive program, as required by statute, maintains the current siloed structure of Medicare and Medicaid payments. The selected functionality and quality measures in large part do the same. However, this siloed structure does not support or encourage integrated coordinated care across providers and settings. As greater attention is paid to improving care coordination and the quality of care through integrated care models (for example, accountable care organizations, patient-centered medical homes), greater attention should be given to selecting measures that focus on patient-centered episodes of care. Furthermore, consideration should be given to refining the incentive payment structure to foster integration and accountability among and across providers and settings.

Response: We believe that HEDIS reporting and other existing quality reporting programs (that is, HOS and CAHPS) go a long way toward assuring that coordination and integration of care will continue to occur in the Medicare Advantage environment. One of the 
purposes of EHR adoption is to facilitate the coordination of care in health care environments where care coordination is not currently perceived to occur. We are asking providers to pick a program through which they are most likely to be eligible for EHR incentive payments. For MA organizations that treat Medicare, Medicaid and dually-eligible patients, EHR incentive payments will be made only under one program (Medicare or Medicaid) with respect to any specific EP. However care coordination should occur regardless of health insurance or EHR incentive payer. After consideration of the public comments received we are not changing our proposed policy to allow qualifying MA organizations to establish meaningful use through attestation and to demonstrate meaningful use through continued HEDIS reporting.

Finally, we proposed requiring qualifying MA organizations to submit attestations to us related to meaningful use by MA-affiliated hospitals within 30 days of the close of the FFY-which is the payment year for MA-affiliated hospitals-by October 30. We also proposed requiring qualifying MA organization to submit attestations to us related to meaningful use by MA EPs within 30 days of the close of the MA EP payment year-which is a CY-by January 30. In this final rule we are modifying the regulation text at $\S 495.210$ (b) and (c) to be consistent with the changes to $\S 495.204(b)(2)$ and $\S 495.202(\mathrm{~b})(3)$, since the deadline for attestations of meaningful use should be consistent with deadlines for revenue reporting for MA EPs, and final identification of MA EPs and MAaffiliated hospitals. We are extending the timeframe for reporting meaningful use to 60 days after the close of the payment year.

\section{Posting Information on the CMS Web} Site

In the proposed rule we said that sections 1853(l)(7) and 1853(m)(5) of the Act require us to post information on an Internet Web site related to the receipt of incentive payments under the MA EHR incentive program. We said posted information would include the names, business addresses, and business phone numbers of each qualifying MA organization receiving an incentive payment under this section for qualifying MA EPs and hospitals. A list of the names of each qualifying MA EP and qualifying MA-affiliated eligible hospital for which an incentive payment has been made would also be posted. Since this requirement is applicable to other Medicare EPs and eligible hospitals, we have included this requirement in $\S 495.108$.

We did not receive any comments on these provisions and are finalizing them as proposed.

\section{Limitation on Review}

In the proposed rule we said that section 1853(l)(8) of the Act states that there shall be no administrative or judicial review under section 1869 of the Act, section 1878 of the Act, or otherwise of the methodology and standards for determining payment amounts and payment adjustments under the MA EHR EP incentive program. We said this includes provisions related to duplication of payment avoidance and rules developed related to the fixed schedule for application of limitation on incentive payments for all qualifying MA EPs related to a specific qualifying MA organization. This also includes the methodology and standards developed for determining qualifying MA EPs and the methodology and standards for determining a meaningful EHR user, including the means of demonstrating meaningful use and the selection of measures. We proposed to codify these requirements in $\S 495.212$ (b).

Section 1853(m)(6) of the Act, as added by the HITECH Act, states that there shall be no administrative or judicial review under section 1869, section 1878, or otherwise of the methodology and standards for determining payment amounts and payment adjustments under the MA EHR hospital incentive program. This includes provisions related to duplication of payment. This also includes the methodology and standards developed for determining qualifying MA hospitals and the methodology and standards for determining a meaningful EHR user, including the means of demonstrating meaningful use and the selection of measures. We proposed to codify these requirements in $\S 495.212(\mathrm{c})$.

We did not receive any comments on these provisions and are finalizing them as proposed.

\section{Conforming Changes}

In the proposed rule we said that sections 4101(e) and 4201(d)(2) and (3) of the HITECH Act provide conforming amendments to Part C of the Social Security Act. Therefore, we proposed the following conforming changes to the regulations text:

- Revising $\$ 422.304$ by adding a new paragraph (f) to account for the amendment to section 1853(a)(1)(A) of the Act referencing the additional EHR incentive payments that may be made to qualifying MA organizations in the section of the statute that provides for monthly capitation payments to MA organizations. (This addition would also act as a cross-reference to MA EHR incentive payment rules in subpart $\mathrm{C}$ of part 495 of this chapter.)

- Revising $\$ 422.306(\mathrm{~b})(2)$ by adding a new paragraph (iv) to address the amendments to section 1853(c)(1)(D)(i) of the Act which exclude the EHR incentive payments made to EPs and hospitals under the Medicare FFS program from the computation of FFS costs in a year for the purpose of computing MA monthly capitation amounts.

- Revising $\S 422.308$ by adding a new paragraph (a)(1) to address the amendments to section 1853(c)(1)(D)(1) and (c)(6)(A) of the Act regarding the exclusion of FFS Medicare EHR incentive payments and adjustments from the calculation of the national per capita growth percentage.

- Revising $\$ 422.322$ by adding a new paragraph (a)(3) to account for the amendments to section 1853(c)(6)(A) and (f) of the Act specifying that the source of EHR incentive payments to qualifying MA organizations are from the Federal Hospital Insurance Trust Fund or the Supplementary Medical Insurance Trust Fund.

- Revising $\$ 422.322(\mathrm{~b})$ by adding a reference to $\$ 495.204$ to address the amendment to section 1851(i)(1) of the Act that indicates that EHR incentive payments are instead of incentive payments that would otherwise be payable under original Medicare.

We did not receive any comments on these provisions and are finalizing them as proposed.

10. Payment Adjustment and Future Rulemaking

In the proposed rule we said that in future rulemaking we will develop standards related to payment adjustments to qualifying MA organizations related to MA EPs and MA-affiliated eligible hospitals that are not meaningful users of certified EHR technology. We solicited comment on how we can most effectively and efficiently apply payment adjustments to qualifying MA organizations whose MA eligible EPs and hospitals have not successfully meaningfully used certified EHR technology.

The statutory requirement related to imposition of payment adjustments with respect to MA EPs is set forth in section 1853(l) of the Act. Specifically, section 1853(l)(4) of the Act requires that instead of applying the payment adjustment in section 1848(a)(7) of the Act, we apply the payment adjustment 
to the Medicare physician expenditure proportion. This is our estimate of the proportion of the expenditures under Parts A and B paid to the qualifying MA organization in the form of capitation payments under section 1853 of the Act that are not attributable to the EHR incentive payment program, that are attributable to expenditures for physician services. In the case of a qualifying MA organization that attests that not all MA EPs of the organization are meaningful EHR users with respect to years beginning with 2015, we are directed to apply the payment adjustment on the proportion of the capitation payment with respect to all such EPs of the organization that are not meaningful users for such year. The adjustment amount is 1 percent for 2015, 2 percent in 2016, and 3 percent in 2017 and subsequent years.

Comment: Two commenters said that the EHR Incentive Program (the Medicare component) is limited to providers who bill for Part B covered services under traditional FFS Medicare or for MA organizations that provide equivalent services to MA beneficiaries. In addition to incentive payments, the program will impose penalties on providers who do not adopt technology and meet criteria for meaningful use of electronic health records; those penalties will be in the form of percentage reductions in Medicare reimbursements, beginning in 2016 . Medicare section 1876 (of the Act) cost contract programs by statute are not eligible for the EHR Incentive Program. The proposed rule does not expressly state whether physicians paid under a cost plan will be required to meet meaningful use criteria to avoid the payment adjustments that will take effect after 2015. CMS should clearly state that those providers who are not eligible to participate in the EHR Incentive Program will not be subject to reductions in payment for not achieving meaningful use, for instance any providers reimbursed under Medicare cost contract arrangements.

Response: While it is true that current statute applies payment adjustments beginning in 2015 only to FFS and MA providers, it is also true that cost plan providers might provide either FFS or MA services to which adjustments would apply. So, while it is true that cost plan payments are unaffected, a blanket statement that cost plan providers are unaffected is not possible.

The statutory requirement related to imposition of payment adjustments with respect to $\mathrm{MA}$-affiliated eligible hospitals is provided in section 1853(m) of the Act. Specifically, section 1853(m)(4) of the Act requires us to apply the adjustment to the hospital expenditure proportion, which is our estimate of the proportion of the expenditures under Parts A and B paid to the qualifying MA organization in the form of capitation payments under section 1853 of the Act that are not attributable to the EHR incentive payment program, that are attributable to expenditures for inpatient hospital services. In the case of a qualifying MA organization that attests that not all MAaffiliated eligible hospitals of the organization are meaningful EHR users with respect to years beginning with 2015, we are directed to apply the payment adjustment on the proportion of all such MA-affiliated eligible hospitals of the organization that are not meaningful users for such year. The adjustment amount is of three-fourths of the market basket increase related to a hospital by a $33^{1 / 3}$ percent reduction in 2015 , by a $66^{2} / 3$ percent reduction in 2016 , and by a 100 percent reduction in 2017 and all subsequent years.

Effectively, the reduction is of all but 25 percent of the market basket increase for a specific hospital in years after 2016.

We received no additional comments.

\section{Medicaid Incentives}

\section{Overview of Health Information} Technology in Medicaid

Under the HITECH Act, State Medicaid programs, at their option, may receive Federal financial participation (FFP) for expenditures for incentive payments to certain Medicaid providers to adopt, implement, upgrade, and meaningfully use certified EHR technology. Additionally, FFP is available to States for reasonable administrative expenses related to administration of those incentive payments as long as the State meets certain conditions. Section 1903(a)(3)(F)(i) of the Act, as amended by section 4201 of the HITECH Act, establishes 100 percent FFP to States for providing incentive payments to eligible Medicaid providers (described in section 1903(t)(2) of the Act) to adopt, implement, upgrade, and meaningfully use certified EHR technology. The incentive payments are not direct reimbursement for the purchase and acquisition of such technology, but rather are intended to serve as incentives for EPs and eligible hospitals to adopt and meaningfully use certified EHR technology.

Section 1903(a)(3)(F)(ii) of the Act, as amended by section 4201 of the HITECH Act, also establishes 90 percent FFP to States for administrative expenses related to carrying out the substantive requirements associated with the incentive payments.

Finally, as required by section 1903(t)(10) of the Act, CMS will be reporting to Congress on the status, progress, and oversight of the overall EHR incentive program. These reports will discuss steps taken to avoid duplicate Medicare and Medicaid incentive payments to EPs, the extent to which Medicaid EPs and hospitals have adopted certified EHR technology as a result of the incentive payments, and any improvements in health outcomes, clinical quality, or efficiency resulting from the adoption of such technology.

Comment: A commenter requested additional discussion in the final rule of the many challenges that exist to adopting electronic health record technology experienced by the Medicaid Transformation Grantees.

Response: The primary challenges faced by the Medicaid Transformation Grantees involved assisting providers to adopt the EHRs and to successfully integrate utilization of the EHRs into their practice workflow. Workflow redesign is unique to each practice based upon practice size, clinical specialty area, practice operation (for example, medical home teams or specialty care) and the providers' hardware and software. In addition, Grantees reported that providers value the EHRs only in so far as the patient data in the EHR is timely and complete. Therefore lagging data feeds or gaps in data from certain sources, such as labs or Part D claims for dual eligibles, were observed to discourage providers from investing their time and effort into learning how to use the EHRs. Many Grantees noted that early negative experiences with workflow or with timely and accurate access to relevant data discouraged providers from using the system. They reported needing to dedicate significant time and resources to provider outreach, technical assistance and training. Some Grantees focused on identifying or developing the right EHR product only to conclude afterwards that their focus needed to be equally, if not more, on supporting their providers' use of the EHR, including fostering health information exchange through interface development. In summary, the Medicaid Transformation Grantees affirmed that the barriers faced by Medicaid providers to EHR adoption and use were not unique to Medicaid. There were several challenges to HIT/ EHR implementation that were specific to Medicaid programs that may be useful for States in light of HITECH. These include, integration of HIT into the State Medicaid Management Information System (MMIS); churning 
of Medicaid patients on/off Medicaid eligibility; issues of consent with patients with diminished capacity, children and their parents and caregivers, and foster children/wards of the State; costs associated with transaction fees for pharmacy hubs on a statewide scale; and how to calculate return on investment and quality outcomes as a result of HIT programs that are running concurrent with other quality initiatives with the same goals, such as the medical home model, disease management/care coordination and provider pay-for-performance.

While this information is valuable in terms of understanding and addressing the challenges to EHR adoption, we continue to believe that the benefits of meaningful use of EHRs far outweigh the implementation challenges.

\section{General Medicaid Provisions}

In $\S 495.320$ and $\S 495.322$ we provide the general rule that States, at their option, may receive: (1) 90 percent FFP for State expenditures related to the administration of an EHR incentive program for certain Medicaid providers that are adopting, implementing, or upgrading and meaningfully using certified EHR technology; and (2) 100 percent FFP for State expenditures for those incentive payments.

We did not receive any comments and we are finalizing these provisions as proposed.

3. Identification of Qualifying Medicaid EPs and Eligible Hospitals

\section{a. Overview}

As specified in section 1903(t)(2) of the Act, only certain Medicaid providers will be eligible for incentive payments. This section discusses some of these eligibility requirements, including requirements relating to patient volume, whether a provider is hospital-based, and whether an EP is practicing predominantly in a federally-qualified health center (FQHC) or a rural health clinic (RHC). Regulations relating to these requirements may be found at $\S 495.304$ through $\S 495.306$.

\section{b. Program Participation}

As specified under section 1903(t)(2)(A) of the Act, Medicaid participating providers who wish to receive a Medicaid incentive payment must meet the definition of a "Medicaid EP." This definition (1903(t)(3)(B) of the Act) lists five types of Medicaid professionals: Physicians, dentists, certified nurse-midwives, nurse practitioners, and physician assistants practicing in an FQHC or RHC that is so led by a physician assistant.
Additionally, to qualify for incentives, most Medicaid EPs cannot be "hospitalbased." We will use the same definition of "hospital-based" as used in the Medicare EHR incentive program, as sections 1848(o)(1)(C) and 1903(t)(3)(D) of the Act use almost identical definitions of the term. We refer readers to section II.A. for a definition of "hospital-based," and for a thorough discussion of our methodology.

The only exception to this rule is that Medicaid EPs practicing predominantly in an FQHC or RHC are not subject to the hospital-based exclusion.

Medicaid EPs must also meet the other criteria for Medicaid incentive payment eligibility, such as the patient volume thresholds or practicing predominantly in an FQHC or RHC, as described in this subpart. Since the statute at 1903(t)(2)(A)(iii) of the Act does not define "practices predominantly," we specify that an EP practices predominantly at an FQHC or an RHC when the clinical location for over 50 percent of his or her total patient encounters over a period of 6 months occurs at an FQHC or RHC.

Acute care and children's hospitals are listed in section 1903(t)(2) of the Act as the only two types of institutional providers potentially eligible for Medicaid incentive payments. These terms are specific to the Medicaid EHR incentive program and are not currently defined in the Medicaid regulations. Consequently, we define these terms in $\S 495.302$.

As specified under section 1903(t)(2)(B) of the Act, to qualify for incentive payments acute care hospitals also must meet patient volume

threshold requirements, as specified in $\S 495.306$. Children's hospitals do not have patient volume requirements for Medicaid incentive program

participation.

Comment: Commenters expressed confusion about the restrictions on physician assistants' (PAs)

participation. Numerous commenters suggested that PAs should be eligible without conditions, particularly the condition that they are practicing in an FQHC or RHC that is "so led by a physician assistant" and/or CMS should exercise flexibility in defining "so led," in order to capture the highest number of PAs. We received specific comments on how to define "so led" to provide the greatest flexibility to PAs. Suggestions included allowing clinics under a larger FQHC to be led by a PA, but not necessarily the entire FQHC. Also, commenters asked that we consider "led" to mean the dominant clinical provider, which is the case for PAs in many RHCs.
Response: As stated in the statute at 1903(t)(3)(B)(v), regarding the program eligibility for PAs, PAs are eligible when they are a "physician assistant insofar as the assistant is practicing in a rural health clinic that is led by a physician assistant or is practicing in a Federally qualified health center that is so led." These conditions on PAs' eligibility apply whether the PA is qualifying because they meet Medicaid patient volume requirements or if they are qualifying because they practice predominantly in an FQHC or RHC. Since this language requiring that a $\mathrm{PA}$ must be leading the FQHC or RHC is derived from statute, we have no flexibility to change or remove it.

However, we agree that we have the authority to interpret what it means for a PA to lead an FQHC or RHC, and we believe a PA would be leading an FQHC or RHC under any of the following circumstances:

(1) When a PA is the primary provider in a clinic (for example, when there is a part-time physician and full-time PA, we would consider the PA as the primary provider);

(2) When a PA is a clinical or medical director at a clinical site of practice; or

(3) When a PA is an owner of an RHC.

We agree that FQHCs and RHCs that have PAs in these leadership roles can be considered "PA-led." Furthermore, since RHCs can be practitioner owned (FQHCs cannot), we will allow ownership to be considered "PA-led."

With the exception of this clarification of PA-led, we are adopting this language as proposed. We have not changed our regulatory language, as we consider this clarification to be an interpretation of our regulations as to what it means to be a PA to be leading an FQHC or RHC.

Comment: We received questions about eligibility related to FQHC lookalikes, tribal clinics, and other similar facilities.

Response: As previously mentioned, in accordance with section 1903(t)(2)(B), the only two facilities eligible for incentives are acute care and children's hospitals. However, EPs at facilities such as FQHCs, RHCs, and tribal clinics may be eligible for participation when they practice predominantly at an FQHC or RHC or meet the other patient volume requirements. The statute defines FQHCs at 1905(l)(2)(B) and defines RHCs at 1905(l)(1) by essentially incorporating the definition in 1861(aa).

Comment: Numerous commenters opposed the proposed definition for "hospital-based."

Response: This is a consideration for Medicare and Medicaid and is addressed in II.A. 
After consideration of the public comments received, we are making changes under II.A.

\section{(1) Acute Care Hospitals}

For purposes of Medicaid incentive payments, we proposed to define an "acute care hospital" as a health care facility where the average length of patient stay is 25 days or fewer and with a CCN that has the last four digits in the series 0001 through 0879 (that is, shortterm general hospitals and the 11 cancer hospitals in the United States).

We excluded from this proposed definition a category of long-term care hospitals, which are defined for Medicare purposes in regulations at § 412.23(e). Specifically § 412.23(e)(2)(i) states that the hospital must have an average Medicare inpatient length of stay of greater than 25 days (which includes all covered and non-covered days of stay of Medicare patients).

Comment: We received numerous comments recommending that CAHs be included in the definition of acute care hospitals for purposes of the Medicaid EHR incentive payment program. Commenters pointed out that the CAHs would qualify on all criteria except for the requirement to have a CCN in the range 0001-0879. CAHs have CCNs in the range 1300-1399. Moreover, many commenters pointed out that, because of their rural location and distance from other hospitals to which they frequently transfer patients, the CAHs would benefit from having electronic records that could be shared with the subsequent provider of care to the patient. Commenters also asked what reimbursement methodology CMS would use if it decided to include CAHs in the Medicaid incentive payment program.

Response: We agree with the commenters that CAHs conform to our definitional criteria for acute care hospital except for the CCN range. Moreover, we recognize the positive impact on quality that may ensue from the CAH's being able to electronically communicate with the hospitals to which it transfers patients. Therefore, in the final rule, we are amending the definition of acute care hospital for purposes of the Medicaid EHR incentive payment program as "those hospitals with an average patient length of stay of 25 days or fewer, and with a CCN that falls in the range 0001-0879 or 13001399." This definition will now encompass general short-term hospitals, cancer hospitals, and critical access hospitals that meet the Medicaid patient volume criteria. Since we are including CAHs under the category of "acute care hospital," we are not developing a separate Medicaid incentive payment calculation for CAHs. States will pay the incentive payment to qualifying CAHs using the acute care methodology described at section 495.310(g). In summary, CAHs will be eligible for the Medicaid hospital incentive insofar as they meet the requirements under an acute care hospital described here. While the statute issued specific calculation requirements for CAHs under Medicare, there is no special Medicaid calculation. Like other acute care hospitals, some CAHs may be eligible for Medicare and Medicaid incentives.

We will reflect this definitional change in the final regulation at section 495.302 .

Comment: Further guidance was requested on the determination of average length of stay. Commenters questioned whether the average length of stay should be calculated relative to the fiscal year prior to the payment year or relative to the calendar year prior to the payment year. Commenters also questioned whether outliers in terms of extremely long length of stay could be left out of the calculation, and if so, could CMS provide detail on this and any similar exclusions; for example, other exclusions with respect to observation stays.

Response: After consideration of these comments, we believe the best policy is to allow the States to decide whether they will use a fiscal year or calendar year for calculating length of stay, as the State will be in the best position to determine what documentation exists in order to support any length of stay calculation. With respect to outliers, we point readers to the State Operations Manual, page 303, Revision 57, dated January 29, 2010 and we note that these long (and short) stay outliers are included in average length of stay calculations for other purposes, such as reporting statistics to States, Medicare, and other payers. We do not find a basis for excluding outliers from the average length of stay for purposes of the incentive payment. In fact, since acute care hospitals have CCNs in either the 0001-0879 or the 1300-1399 range, and length of stay is one of the definitional criteria for CCNs in these ranges, all of the acute care hospitals are very likely to meet length of stay criteria.

Observation stays are considered to be outpatient services and, therefore, cannot be included in average length of stay calculations. This is consistent with the treatment of observation days under Medicare.

In summary we are making no revisions to the regulation as a result of this comment.

\section{(2) Children's Hospitals}

For purposes of the Medicaid EHR incentive program, in the proposed rule, we proposed one definition to include only separately certified children's hospitals, with CCNs in the 3300-3399 series in the definition of eligible “children's hospital.” By defining "children's hospital" in this way, we: (1) Prevented general acute care hospitals, which could not themselves qualify for the incentive because they did not meet the 10 percent Medicaid patient volume, from using the fact that they have a pediatric wing as justification for requesting a Medicaid incentive payment; (2) excluded many of the facilities that are perceived by the public as children's hospitals, but do not meet the Medicare standards as either freestanding or hospital-withinhospital children's hospitals; and (3) excluded some pediatric specialty hospitals which have CCNs as psychiatric or rehabilitation hospitals.

An alternative definition of a “children's hospital” was also proposed to include those hospitals with Medicare provider numbers in the following series:

- 0001 through 0879-Short-term (General and Specialty) Hospitals.

- 3025 through 3099-Rehabilitation Hospitals (Excluded from Prospective Payment Systems).

- 3300 through 3399-Children's Hospitals (Excluded from Prospective Payment Systems).

- 4000 through 4499-Psychiatric Hospitals (Excluded from Prospective Payment Systems).

This definition, for the purposes of the Medicaid HIT incentive payments, applied only to those freestanding hospitals within the above mentioned series that exclusively furnish services to individuals under age 21 .

This broader definition still: (1) Prevented acute care hospitals that cannot independently qualify for the incentive because they do not meet the 10 percent Medicaid patient volume from using the fact that they have a pediatric wing as justification for requesting an HIT incentive payment; (2) allowed for participation in the incentive program by the greatest number of children's hospitals, including rehabilitative and psychiatric specialty hospitals; and (3) aligned with Federal efforts aimed at improving healthcare quality for all children, including those with physical and mental diseases/disabilities.

Comment: CMS received several comments on this issue. Specifically, the commenters stated that the proposed rule limited the definition of children's 
hospitals to those that provide care to individuals under the age of 21 ; the commenters stated that children's hospitals actually may provide care to older individuals who have conditions such as congenital cardiac problems, sickle cell disease and cystic fibrosis.

Response: We agree with the commenters that children's hospitals do on occasion treat patients who are over the age of 21 , especially if the patient is on a continued course of treatment for a condition that began in childhood, such as those conditions mentioned. Accordingly, in the proposed rule published on January 13, 2010 at section 495.302, we defined a children's hospital for purposes of the HIT incentive payment program as a hospital that is separately certified as a children's hospital, with a CCN in the 3300-3399 series and predominantly treats individuals under the age of 21. We used the term "predominantly" to recognize that not all patients of the children's hospital are in fact under age 21.

This definition addresses the commenters' concerns and we are not revising it in the final rule. The commenter's may have been responding to the alternate definition in which we requested comments. While that alternate definition mentioned specialty hospitals that exclusively treat individuals under the age of 21 , we are not adopting that definition in this final rule, as noted in the response to the comment below.

Comment: CMS also received a few comments that supported our proposed definition of children's hospital as those that are separately certified and predominantly treating individuals under 21 years of age. The commenters urged us to adopt this definition rather than the alternate definition discussed in the proposed rule and on which we requested comments.

Response: We agree with the commenters and are adopting the definition that we originally proposed at section 495.302. See the response to the comment below.

Comment: CMS received one comment that recommended use of the alternative definition as providing more opportunity for hospital participation.

Response: We considered the merits of both definitions and we have decided to maintain the definition originally proposed in section 495.302 as representing the clearest definition of a children's hospital. As previously stated, we only intend to include children's hospitals with CCNs within a specific range; this will not include pediatric wings of larger hospitals.
In summary, after considering the comments, we are adopting the definition of children's hospital as originally proposed.

c. Medicaid Professionals Program Eligibility

For Medicaid EPs, the general rule (subject to the two exceptions listed below) is that the EP must have at least 30 percent patient volume attributable to those who are receiving Medicaid. Section 1903(t)(2)(A)(i) of the Act provides authority to the Secretary to establish the methodology by which such patient volume will be estimated; our proposed methodologies which follow, are based on this discretion. To establish such patient volume, we proposed that the EP have a minimum of 30 percent of all patient encounters attributable to Medicaid over any continuous, representative 90-day period within the most recent calendar year prior to reporting. There are two statutory exceptions to the general 30 percent rule discussed previously. The first exception is that a pediatrician may have at least 20 percent patient volume attributable to those who are receiving medical assistance under the Medicaid program, as estimated in accordance with a methodology established by the Secretary (section 1903(t)(2)(A)(ii) of the Act). Again, the method we proposed to use was that the pediatrician have a minimum 20 percent of all patient encounters attributable to Medicaid over any continuous, representative 90 -day period within the most recent calendar year prior to reporting.

The second exception is that Medicaid EPs practicing predominantly in an FQHC or RHC must have a minimum of 30 percent patient volume attributable to "needy individuals."

Again, the method we would use is that 30 percent of all patient encounters be attributable to needy individuals over any continuous 90-day period within the most recent calendar year prior to reporting.

Section 1903(t)(3)(F) of the Act defines needy individuals as individuals meeting any of the following three criteria: (1) They are receiving medical assistance from Medicaid or the Children's Health Insurance Program (CHIP); (2) they are furnished uncompensated care by the provider; or (3) they are furnished services at either no cost or reduced cost based on a sliding scale determined by the individual's ability to pay.

Comment: Many commenters requested that CMS consider groups outside of the statute eligible for incentive payments. These facilities and practitioners included: Community mental health centers and other behavioral health providers (including psychiatric clinics); nursing homes, nursing facilities, and skilled nursing facilities; long-term care providers (community and institutional), including home health care providers; pharmacists and pharmacies; social workers; blood centers; provider based departments; professional societies; Medicaid-participating health plans; speech-language pathologists and audiologists; FQHCs, RHCs, tribal providers, and other community clinics; health aides; and podiatrists. The commenters included numerous testimonials, research, and statements to note that these providers are critical partners in improving the quality and coordination of care for the Medicaid population. Some of the commenters acknowledged that this is a statutory issue but assert that exclusion of such providers impacts Medicaid's ability to improve the quality and efficiency of care. Furthermore, some of these commenters based several additional comments upon presumed eligibility. For example, some commenters said that social workers could not afford EHRs and should not be required to participate.

Another group of comments came from health care professionals that sought eligibility for incentives by virtue of early adoption of EHRs but who do not participate in either Medicaid or Medicare. They suggested a third incentive option available for providers that either do not participate with Medicaid/Medicare or would not reach the threshold of patient visits to receive Medicaid incentive payments.

Response: We note that the

commenters are correct to recognize that this is a statutory issue. The definition of a "Medicaid EP," at 1903(t)(3)(B) of the Act, lists five types of professionals that are eligible for Medicaid incentive payments: physicians, dentists, certified nurse-midwives, nurse practitioners, and physician assistants practicing in an FQHC that is led by a physician assistant or RHC that is so led.

Additionally, the statute at 1903(t)(2)(B) designates acute care hospitals and children's hospitals as the only two types of facilities eligible for the Medicaid incentives. These providers must also meet all other program requirements, including Medicaid patient volume thresholds.

Since the commenters recommend including providers that are not among those explicitly mentioned in the statute, these providers cannot be eligible for the incentive payments.

Additionally, professionals who do not participate in either Medicaid or 
Medicare are also not eligible for incentives due to the statutory requirements associated with each program. Specifically, the Medicaid incentives program requires providers to meet Medicaid patient volume thresholds or practice predominantly in an FQHC or RHC, where they must serve needy individuals (as defined at section 495.10). Additionally, the hospital calculations for Medicare and Medicaid are based, in part, on Medicare or Medicaid inpatient beddays. For Medicare EPs, the incentive is based on the associated Medicare claims. Hence, these professionals cannot meet the statutory requirements for eligibility.

After consideration of these comments, we are maintaining the list of providers eligible for the Medicaid incentive payment program as originally proposed and as identified by statute.

It is worth noting that while the facilities recommended for inclusion by the commenters will not be considered eligible to participate in these incentives, some of the EPs at these facilities may be eligible. One example is that a psychiatrist (physician) or NP is likely to treat individuals at a behavioral health facility. Per our rules at section 495.10 , the EP must identify a TIN to which the incentive payment should be made. We believe that, in accordance with 1903(t)(6)(A) of the Act, an EP could reassign payment to a TIN associated with his or her employer or the facility in which she or he works. This facility could be one of those recommended for inclusion by the commenters. Any reassignment of payment must be voluntary and we believe the decision as to whether an EP does reassign incentive payments to a specific TIN is an issue which EPs and these other parties should resolve. Any reassignment of payment must be consistent with applicable laws, rules, and regulations, including, without limitation, those related to fraud, waste and abuse.

We have provided clarifying language at section 495.10 (f) to further clarify the reassignment of incentive payments by EPs to specific TINs.

\section{d. Calculating Patient Volume Requirements}

As required by section 1903(t)(2) of the Act and discussed in the previous section, all EPs and the vast majority of hospitals will need to meet certain patient volume thresholds in order to be eligible for incentive payments. (The only exception to this rule is for children's hospitals, which have no patient volume threshold requirement).
In addition, where patient volume is a criterion, most providers will be evaluated according to their "Medicaid" patient volume, while some professionals (those practicing predominantly in an FQHC or RHC) will be evaluated according to their "needy individual" patient volume.

We define "patient volume" in $\S 495.302$ to be a minimum participation threshold for each individual Medicaid provider (with the exception of children's hospitals). In the proposed rule, we proposed methodologies for estimating the patient volume thresholds and listed them by entity type.

Further, we proposed that States could submit alternative approaches to the established timeframe for estimating patient volume, through their State Medicaid HIT Plans (SMHP) and we would make a determination of whether it was an acceptable alternative.

In determining the "needy individual" patient volume threshold that applies to EPs practicing predominantly in FQHCs or RHCs, section 1902(t)(2) of the Act authorizes the Secretary to require the downward adjustment to the uncompensated care figure to eliminate bad debt data. We interpret bad debt to be consistent with the Medicare definition, as specified at $\$ 413.89(b)(1)$. In order to remain as consistent as possible between the Medicare and Medicaid EHR incentive programs, States will be required to downward adjust the uncompensated care figure. Under Medicare, bad debts are amounts considered to be uncollectible from accounts and notes receivable that were created or acquired in providing services. "Accounts receivable" and "notes receivable" are designations for claims arising from the furnishing of services, and are collectible in money in the relatively near future. Providers should be required to use cost reports (for FQHCs and clinics this would be the Medicare 222-92 cost report, or the most recent version of the 222), or other auditable records to identify bad debts. All information under attestation is subject to audit. Our proposed regulations on calculating the needy individual patient volume can be found at $\S 495.302$ and $\S 495.306$.

Further, in establishing the Medicaid patient volume thresholds for EPs and acute care hospitals, section 1902(t)(2) of the Act requires that individuals enrolled in a Medicaid managed care plan be included. We interpret this to mean that individuals enrolled in MCOs, prepaid inpatient health plans (PIHPs), or prepaid ambulatory health plans (PAHPs), under 42 CFR Part 438 be included in the calculation.
Therefore, in determining patient volume, providers and States should be aware that individuals enrolled in such plans will be included in the patient volume calculation. Acute care hospitals have to meet the 10 percent Medicaid volume threshold.

Comment: Commenters recommended that CMS provide flexibility in the specific volume thresholds required for program participation (for example, 30 percent for most EPs, 20 percent for pediatricians) and apply a lower percentage or a minimum number of encounters. Some commenters referenced research stating that practices with a 30 percent patient volume may not be financially viable.

Response: The patient volume thresholds of 30 percent and 20 percent are required by statute and cannot be changed in the rulemaking process.

After consideration of the public comments received, we are not making any changes to these statutory requirements.

Comment: Commenters suggested that CMS define "encounter" and take a menu approach to patient volume to allow States several options, based on their data sources. Some commenters provided specific suggestions for patient volume "menu" items. Some

commenters further noted that there were inconsistencies in how we applied "encounter" data. Finally, one commenter noted that we should consider how "encounter" data is applied to EPs that bill services through another provider (for example, PAs that bill through MDs). Other commenters asked for a clarification of how "encounters" would apply to the duallyeligible Medicare/Medicaid beneficiaries. Additionally, several commenters provided specific suggestions for alternative methods making an approximate determination of providers' patient volume by [not using patient volume] and extending the look-back period to two years.

Response: We agree with the approach of offering at least some options to States regarding patient volume. This approach allows States to audit their programs using the data sources available to them, while also including the largest number of providers that may treat Medicaid patients. We believe our new approach will correct the inconsistencies in how we applied "encounter." Furthermore, our new definition of encounter will capture the dually-eligible beneficiaries, as well as individuals who are in a Title XIX-funded 1115 demonstration project. Specifically, the statute at 1903(t)(2) states that Medicaid patient volume will be "attributable to individuals who are 
receiving medical assistance under [Title XIX]," and also states that the patient volume calculation for those practicing predominantly in an FQHC or RHC will be "attributable to needy individuals." Needy individual is defined at $1903(\mathrm{t})(3)(\mathrm{F})$ as "an individual-(i) who is receiving assistance under Title XIX; (ii) who is receiving assistance under Title XXI; (iii) who is furnished uncompensated care by the provider; or (iv) for whom charges are reduced by the provider on a sliding scale basis based on the individual's ability to pay." We believe our final rule definition of "encounter" captures care to all of these individuals.

Additionally, consistent with the statute, we expect providers and States to make estimation in accordance with the methodologies we established here. This estimation would need to be made with reasonable effort, using verifiable data sources by the provider and the State.

Finally, we do not agree with any of the suggestions from commenters that involve using a benchmark number of Medicaid patients or other suggestions that involve a deviation from the statutory language. The statute is clear that Medicaid patient volume must be considered and explicitly specified percentages of caseload mix compositions attributable to either Medicaid and/or "needy" individuals that must be achieved for participation in the incentive program. We also do not agree with allowing the provider to consider a period longer than a year prior to registering because that is not a current, accurate portrayal of the provider's participation in Medicaid.

After consideration of the public comments received, we are revising the patient volume approach to the following two options. The State may choose one of the two options listed below (or both options), or a Stateproposed alternative, if approved by CMS. The State's strategy must be submitted for review and approval through the SMHP, in accordance with all requirements at section 495.332 .

A Medicaid provider may demonstrate patient volume by:

(1) Having patient encounters within the 90-day period by using the same methodology we proposed in the proposed rule.

This first option preserves the methodology we proposed in the proposed rule, however we clarify "encounter" below. For the Medicaid patient volume, the methodology for estimating patient volume would require calculation of a threshold (represented below) using as the numerator the individual hospital's or EP's total number of Medicaid patient encounters in any representative continuous 90-day period in the preceding calendar year and the denominator is all patient encounters for the same individual professional or hospital over the same 90-day period. We are not prescribing standards for what is a "representative" period, but we intend to apply a plain meaning test. In other words, if a reasonable person would not consider the selected period to be representative (for example, because the selected period included a short-term temporary Medicaid outreach program), then it would not support a threshold calculation.

[Total (Medicaid) patient encounters in any representative continuous 90-day period in the preceding calendar year/ Total patient encounters in that same 90-day period] * 100

For the needy individual patient volume, the methodology for estimating patient volume would require the same calculation, but with the numerator equal to the EP's total number of needy individual patient encounters in any representative 90-day period in the preceding calendar year.

[Total (Needy Individual) patient encounters in any representative continuous 90-day period in the preceding calendar year/Total patient encounters in that same 90-day period] * 100

Table 15, below, demonstrates the above-referenced patient volume thresholds. (This same Table appeared in the proposed rule, with a few minor clarifications included in this Table).

\section{TABLE 15: Qualifying Patient Volume Threshold for Medicaid EHR Incentive Program}

\begin{tabular}{|c|c|c|}
\hline Entity & $\begin{array}{l}\text { Minimum 90-day Medicaid } \\
\text { Patient Volume Threshold }\end{array}$ & \multirow{7}{*}{$\begin{array}{l}\text { Or the Medicaid EP } \\
\text { practices predominantly } \\
\text { in an FQHC or RHC - } \\
30 \% \text { "needy individual" } \\
\text { patient volume threshold }\end{array}$} \\
\hline Physicians & $30 \%$ & \\
\hline Pediatricians & $20 \%$ & \\
\hline Dentists & $30 \%$ & \\
\hline Certified nurse midwives & $30 \%$ & \\
\hline $\begin{array}{l}\text { Physician Assistants when } \\
\text { practicing at an FQHC/RHC } \\
\text { led by a physician assistant }\end{array}$ & $30 \%$ & \\
\hline Nurse Practitioner & $30 \%$ & \\
\hline Acute care hospital & $10 \%$ & N/A \\
\hline Children's hospital & N/A & N/A \\
\hline
\end{tabular}

(2) Having a Medicaid enrollee on the panel assigned to the EP (for example, managed care or medical homes) within that representative 90-day period.

With more than 70 percent of Medicaid and CHIP enrollees receiving care in a managed care delivery system, and additional enrollees in medical homes, we determined that it was necessary to look for flexibility in how we applied these requirements. Under this option, we wanted to capture the EP's panel assignments, as well as any additional unduplicated Medicaid encounters. In other words, we do not intend for the EP to count an assigned patient who was also an encounter more than once.

The methodology for estimating the Medicaid patient volume threshold (represented above) would use as the numerator the individual hospital's or EP's total number of Medicaid patients assigned through a Medicaid managed 
care panel, medical or health home program panel, or similar provider structure with capitation and/or case assignment, plus all other Medicaid encounters for that EP. The assignment must be current within the 90-day period and we will consider as a proxy for this an encounter with any patient on the panel within the previous calendar year prior to the representative 90-day period when the patient was on the panel. Note that, as stated above, while the EP may add in encounters with other, non-panel Medicaid patients to the numerator, these encounters must be patients who are not assigned to a panel and would be encounters that occurred during the representative 90day period. The denominator is all patients assigned to the EP or hospital for the same 90-day period, also with whom the provider had at least one encounter in the prior calendar year as a proxy, as well as any other unduplicated Medicaid encounters during the representative 90-day period.

$\{$ [Total (Medicaid) patients assigned to the provider in any representative continuous 90-day period in the preceding calendar year, with at least one encounter taking place during the calendar year preceding the start of the 90-day period] + [Unduplicated (Medicaid) encounters in the same 90day period]/[Total patients assigned to the provider in that same 90-day period, with at least one encounter taking place during the calendar year preceding the start of the 90-day period] + [All unduplicated encounters in that same 90-day period] $\}$ * 100

For the needy individual patient volume for EPs enrolled in managed care and medical homes, the threshold (represented below) would be calculated in the same manner, but with the numerator equal to the EP's total number of needy individuals assigned to the patient panel in any representative 90-day period in the preceding calendar year with at least one encounter within that year.

\{[Total (Needy Individual) patients assigned to the provider in any representative continuous 90-day period in the preceding calendar year, with at least one encounter taking place during the year preceding the 90-day period] + [Unduplicated (Needy Individual) encounters in the same 90-day period]/ [Total patients assigned to the provider in that same 90-day period, with at least one encounter taking place during the year preceding the 90-day period] + [All unduplicated encounters in that same 90-day period] $\}$ * 100
Table 15 demonstrates the abovereferenced patient volume thresholds per provider type.

In order to resolve any inconsistencies with the definitions of "encounter," for purposes of EP patient volume, we have allowed the following to be considered Medicaid encounters:

(1) Services rendered on any one day to an individual where Medicaid or a Medicaid demonstration project under section 1115 of the Act paid for part or all of the service; or

(2) Services rendered on any one day to an individual for where Medicaid or a Medicaid demonstration project under section 1115 of the Act paid all or part of their premiums, co-payments, and/or cost-sharing.

For purposes of calculating hospital patient volume, we have allowed the following to be considered Medicaid encounters:

(1) Services rendered to an individual per inpatient discharges where

Medicaid or a Medicaid demonstration project under section 1115 paid for part or all of the service;

(2) Services rendered to an individual per inpatient discharge where Medicaid or a Medicaid demonstration project under section 1115 of the Act paid all or part of their premiums, co-payments, and/or cost-sharing;

(3) Services rendered to an individual in an emergency department on any one day where Medicaid or a Medicaid demonstration project under section 1115 of the Act either paid for part or all of the service; or

(4) Services rendered to an individual in an emergency department on any one day where Medicaid or a Medicaid demonstration project under section 1115 of the Act paid all or part of their premiums, co-payments, and/or costsharing.

We wanted to adequately reflect what an encounter looked like for a hospital and apply these concepts consistently across the numerous areas of this final rule. We used inpatient discharges and emergency department services for the hospitals because this is consistent with how we will make hospital-based determinations for EPs and how we collect meaningful use information for hospitals. We decided that services rendered on one day would be an encounter. An emergency department must be part of the hospital under the qualifying CCN.

For purposes of calculating needy individuals patient volume, we have allowed the following to be considered needy patient encounters:

(1) Services rendered on any one day to an individual where Medicaid or CHIP or a Medicaid or CHIP demonstration project under section 1115 of the Act paid for part or all of the service;

(2) Services rendered on any one day to an individual where Medicaid or CHIP or a Medicaid or CHIP demonstration project under section 1115 of the Act paid all or part of their premiums, co-payments, and/or costsharing; or

(3) Services rendered to an individual on any one day on a sliding scale or that were uncompensated.

We understand that multiple providers may submit an encounter for the same individual. For example, it may be common for a PA or NP to provide care to a patient, then a physician to also see that patient. It is acceptable in circumstances like this to include the same encounter for multiple providers when it is within the scope of practice.

We considered whether Medicaid providers or States should pick from the two options provided above. Since States are responsible for auditing the program and must have reliable sources of data, we agree with commenters that it must be States that make a determination as to whether either option will be permitted (or both).

In the proposed rule, we also proposed that if States had an alternative approach for the timeframe in accounting for the methodology, they would be allowed to submit it in the SMHP for review and approval. For the final rule, we are modifying this option. As stakeholders' understanding of the program matures and new technologies become available, there may be new solutions that we did not consider here, but would be a better option for one or several States. To that end, in this final rule we are providing flexibility to consider States' alternative methodologies for measuring not just the timeframe that is used in establishing patient volume, but all of the elements included in the patient volume calculation (except the thresholds established by statute). Therefore, we have revised our final regulations to allow States to offer alternatives regarding the methodology used to establish patient volume, and for the Secretary to adopt these options, so that they may be used by other States as well. An alternative would need a verifiable data source. A State also would need to provide us with an analysis to demonstrate that the methodology being proposed by the State did not result, in the aggregate, in fewer providers becoming eligible than under the two options presented in this final rule. Finally, if a State is reviewed and approved for an alternative 
methodology, we will post this alternative methodology on the CMS internet Web site, and allow other States to adopt the methodology as well, thereby ensuring that the alternative is a methodology that is "established by the Secretary." While we believe that States will not submit alternative methodologies until after the first year of the program, allowing for such alternatives will permit the patient volume calculation to evolve along with State and provider experience of the program.

We believe that these solutions will help address issues for providers practicing across State lines, who may have their Medicaid patient volume derived from more than one State. We encourage States to build partnerships, particularly through data sharing agreements. Medicaid providers must still annually re-attest to meeting the patient volume thresholds.

After consideration of the comments, we are revising $\S 495.302$, 495.306, and $\S 495.332$ regarding patient volume, patient encounters and the associated revisions to the SMHP requirements.

Comment: Commenters asked CMS to include all individuals receiving services through section 1115

demonstrations as eligible encounters.

Response: Although the commenter did not elaborate, we believe the commenter is referring to section 1115 demonstrations under the authority of section 1115(a)(2) of the Act. Our final regulations allow two alternate methods for States to estimate Medicaid patient volume. Under both methods, however, the State must review whether a Medicaid "patient encounter" occurred. Our regulations, at 495.306(e) state that a Medicaid encounter will exist where Medicaid (or a Medicaid demonstration project approved under section 1115) paid for part or all of the service; or where Medicaid (or a Medicaid demonstration project approved under section 1115) paid all or part of the individual's premiums, co-payments and/or cost-sharing. Because our methodology is based upon Medicaid payment for an encounter, and because we believe it will be difficult or impossible for EPs and eligible hospitals to distinguish between payment that is due to patients receiving medical assistance under Title XIX and payment that is due to expansion populations (who are not receiving Title XIX medical assistance), we will allow providers to include in the patient volume calculation individuals who are part of expansion populations under section 1115(a)(2) of the Act. The statute confers broad authority on the Secretary to establish the methodology that is used to estimate the patient volume percentage. Thus, although individuals in section 1115(a)(2) demonstrations are not receiving Title XIX medical assistance, we use our broad authority to allow a methodology that considers these individuals in the estimate that is used. (Limited to Medicaid patient volume determinations, the same reasoning would not apply to CHIP demonstrations or to State-only programs, because no Title XIX funding is received for these projects. However, in calculating Needy Individual patient volume, it is permissible to consider Medicaid or CHIP demonstration projects approved under section 1115.) Our above discussion noting what will be considered a patient encounter includes encounters which were paid for with Title XIX funds under a section 1115 Medicaid demonstration.

Comment: Several commenters asked that CMS allow CHIP patients to be considered in the Medicaid patient volume requirements, particularly for pediatricians.

Response: The requirement that the methodology for estimating Medicaid patient volume is based on Medicaid and not CHIP is related to the statutory language at section 1903(t)(2)(A)(i)-(ii)). Such language requires that the Secretary establish a methodology that can be used to estimate "Medicaid" patient volume for those individuals receiving medical assistance under Title XIX. However, the statute at 1903(t)(2)(A)(iii) allows for an EP practicing predominantly in an FQHC or RHC to consider CHIP patients under the needy individual patient volume requirements.

After consideration of these public comments, we are making no further revisions to this section of the rule.

Comment: Many commenters urged CMS to allow practice- or clinic-level patient volume data to apply to practitioners as a proxy to establish patient volume. This would apply for both Medicaid and needy individual patient volume calculations. The commenters stated that many clinics and group practices do not necessarily track the pay or data per EP and it would be very disruptive to their current practice to begin collecting data like this.

Response: We agree with commenters and acknowledge that it is not our intent to disrupt the practice with new additional burdens, but rather to leverage efficiencies. We will allow clinics and group practices to use the practice or clinic Medicaid patient volume (or needy individual patient volume, insofar as it applies) and apply it to all EPs in their practice under three conditions: (1) The clinic or group practice's patient volume is appropriate as a patient volume methodology calculation for the EP (for example, if an EP only sees Medicare, commercial, or self-pay patients, this is not an appropriate calculation); (2) there is an auditable data source to support the clinic's patient volume determination; and (3) so long as the practice and EPs decide to use one methodology in each year (in other words, clinics could not have some of the EPs using their individual patient volume for patients seen at the clinic, while others use the clinic-level data). The clinic or practice must use the entire practice's patient volume and not limit it in any way. EPs may attest to patient volume under the individual calculation or the group/ clinic proxy in any participation year. Furthermore, if the EP works in both the clinic and outside the clinic (or with and outside a group practice), then the clinic/practice level determination includes only those encounters associated with the clinic/practice.

We have revised our regulations to make clear that when patient volume is calculated on a group-practice/clinic level, the above rules will apply.

Comment: Similar to the last comment, we received comments requesting clarification on how the patient volume requirements will apply in States with seamless eligibility determinations and payments for their program. For example, some States have streamlined their programs so that the potential beneficiary is applying for any public health care program for which they might be eligible (for example, Medicaid, CHIP, State-only) in one application. Often these States have one enrollment card as well. In other words, it is likely that both the beneficiary and the health care provider might have no indication as to whether the beneficiary is receiving assistance under Title XIX, Title XXI, or State-only funds. This becomes a problem when attempting to determine if the provider meets the patient volume requirements.

Response: If there is a combined program like the one in the example, this does not mean that all the encounters are being paid for with Title XIX funds (or the individual's premium or cost-sharing is funded through Title XIX), which is how we explained we would determine Medicaid patient encounters. We do not believe it would be reasonable to allow an encounter that is paid for with Title XXI or State-only funds to be considered a "Medicaid encounter." Thus, States with combined programs (for example, Medicaid/CHIP expansion programs), may indeed have difficulty determining who is eligible 
for participation in this incentive program.

Considering these States have made enormous strides to reduce the confusion and burden associated with eligibility and payment for these programs, and also to reduce the stigma sometimes associated with Medicaid, we want to support the work they have done.

After consideration of the public comments received, we believe that the best course of action is to work with these States on a case-by-case basis through providing guidance as they develop the SMHP. We believe that each State will have different data and information available to them. The States should make sure that the health IT coordinators are working closely with the Medicaid (and CHIP, as it pertains to this program) policy staff on all aspects of the program. The goal will be to find a solution that leverages the State's existing and/or future data sources, as well as looking for flexible alternatives, while still honoring Congress' intent for the patient volume requirements, as established in the statute.

Comment: Some commenters pointed out that not all Medicaid providers use an EHR or submit electronic claims, making it tedious to capture a numerator and denominator for patient volume until the providers have adopted an EHR. Additionally, some commenters expressed concern about how providers would determine the denominator for patient volume and how States would audit the resulting percentage.

Response: While the commenters may be correct about the assertion that not all providers use an EHR or submit electronic claims, we do not believe it will prevent EPs and eligible hospitals from participating. These providers are businesses and there is an expectation that they are tracking their receivables from all entities (including Medicaid) associated with specific patients. In other words, we do not see a connection between electronic claims and current EHR use and calculation of the patient volume. Furthermore, when EHRs are used with practice management systems, we believe that in most cases, this data should be derived from the electronic systems.

When States consider their audit strategies, they should leverage existing data sources to the extent possible, but also consider future data sources. Part of the Medicaid Information Technology Architecture (MITA) principles associated with the SMHP development includes consideration of the "as is" world, as well as the "to be" world.
While States may not have the systems in place today for a complete picture, we expect a longer-term strategy leveraging better data systems.

After consideration of the public comments received, we are not making any change on the basis of this comment. We provided additional flexibility in the patient volume requirements, which may help providers more easily calculate their patient volume and provide for flexibility when States begin to audit providers.

Comment: Commenters requested clarification on how to determine eligibility for the five types of Medicaid EPs. Commenters also noted that there was a potential difference between Medicare and Medicaid for the definition of "physician." Finally, other commenters were confused if, as a specialty practitioner, they qualified as one of the EP types.

Response: We agree with the commenters that there is a distinction between the Medicare and Medicaid definitions of physician. The Medicare statute at section 1848(o)(5)(C) defines an eligible professional as including all the professionals listed in section 1861(r) of the Act (which, generally stated, includes podiatrists, chiropractors and optometrists), the Medicaid statute does not incorporate all of 1861(r). Rather, the Medicaid statute defines what are physician services for purposes of qualifying as medical assistance under section 1905(a)(5)(A) of the Act, and states that physician services constitutes services furnished by a physician as defined in section 1861(r)(1) (which includes only doctors of medicine or osteopathy legally authorized to practice medicine and surgery by their State). In addition, section 1905(e) permits States the option to consider optometrist services as physician services. In this case, the State plan must specifically provide that the term “physicians' services" includes services of the type which an optometrist is legally authorized to perform.

Thus, in keeping with the statute, a physician would be limited to doctors of medicine or osteopathy legally authorized to practice in their State, and, in cases where States have specifically adopted the option of 1905(e) in their State plans,

optometrists.

In addition, States would need to refer to their own scope of practice rules to determine whether an individual qualifies as providing dental, nurse practitioner, physician assistant, or certified nurse midwife services. Also, States and EPs would need to refer to
CMS regulations. These regulations, at 42 CFR 440.60 require that practitioners be licensed and that they are within the scope of practice defined under State law (see also 1905(a)(6)). 42 CFR 440.100(b), defines a dentist as an individual licensed to practice dentistry or dental surgery in his or her State. 42 CFR 440.165 defines a nurse midwife as a registered professional nurse who meets the following requirements: (1) Is currently licensed to practice in the State as a registered professional nurse; (2) is legally authorized under State law or regulations to practice as a nursemidwife, (3) has completed a program of study and clinical experience for nursemidwives as specified in the State, unless the State does not specify such a program. (4) In the case where the State has not specified a particular program of study and clinical experience, the regulation provides alternative means for demonstrating this training. See also section 1905(a)(17), defining certified nurse midwife with reference to section 1861 (g). 42 CFR 440.166 contains a definition of what qualifies as nurse practitioner services and requires a nurse practitioner to be a registered professional nurse who meets the State's advanced educational and clinical practice requirements, if any, beyond the 2 to 4 years of basic nursing education required of all registered nurse. States will have a Medicaid State Plan (and often State regulations) that designates how each provider is eligible to participate in the Medicaid program by practice type. All of these practitioners must meet all other eligibility requirements (including Medicaid patient volume) in order to participate.

Regarding the confusion by some specialty providers (for example, advanced practice nurses, pediatricians, physician sub-specialties, etc.), so long as an EP qualifies as a practitioner within the State's scope of practice rules for each of the five EP types, they are eligible for this program. In other words, since pediatricians are physicians, they must meet the physician scope of practice rules and then they may be eligible for an incentive when they meet all other requirements. Advanced practice nurses who meet their State's criteria for qualifying as a nurse practitioner would qualify as nurse practitioners. We believe most States would recognize APNs as NPs within their scope of practice rules. Eligible provider types must be specified in a State's SMHP.

After consideration of the public comments received, we are revising the definition of these EPs under section 
495.304 to clarify additional scope of practice requirements.

Comment: Commenters requested clarification on how full- or part-time status impacts an EP's eligibility for incentives.

Response: Full or part-time status does not affect patient volume calculations or whether an EP's practice is predominantly in an FQHC or RHC. There is no mention of requisite number of hours in the statute or this final rule as a pre-condition for eligibility.

After consideration of the public comments received, we are not making any revisions to this section of the final rule.

e. Entities Promoting the Adoption of Certified EHR Technology

We define "promoting the adoption of certified EHR technology" in $§ 495.302$. Under section 1903(t)(6)(A)(i), incentive payments must generally be made directly to the EP. Section

1903(t)(6)(A)(ii) of the Act provides an exception to permit payment of incentive payments to "entities promoting the adoption of certified EHR technology," as designated by the State, if participation in the payment arrangement is voluntary for the EP involved. Additionally, the entity must not retain more than 5 percent of the payment for costs unrelated to certified EHR technology (and support services including maintenance and training) that is for, or is necessary for, the operation of the technology. While the Act authorizes States to designate these entities, the Secretary nevertheless retains authority to define what it means to be "promoting the adoption of certified EHR technology," as specified in section 1903(t)(6)(A)(ii) of the Act. Section 1102 of the Act authorizes the Secretary to "make and publish such rules and regulations, not inconsistent with this Act, as may be necessary to the efficient administration of the functions with which he or she is charged under this Act." Since one of our functions is to approve Title XIX plans under sections 1902(b) and 1116 of the Act, and States would need to submit plans as to how they would spend section 4201 of the HITECH Act funds, we have the authority to determine whether a State's plan for allowing EPs to assign their Medicaid incentive payments to these entities is in compliance with our interpretation of the Act.

We define "promoting" certified EHR adoption to mean the enabling and oversight of the business, operational and legal issues involved in the adoption and implementation of EHR and/or exchange and use of electronic health information between participating providers, in a secure manner, including maintaining the physical and organizational relationship integral to the adoption of certified EHR technology by EPs. Under 1903(t)(6)(A)(ii) of the Act and as proposed in $\S 495.332$, States must establish verification procedures that enable Medicaid EPs to voluntarily assign payments to entities promoting EHR technology. States must guarantee that the assignment is voluntary and that the entity does not retain more than 5 percent of those assigned Medicaid incentive payments for costs unrelated to certified EHR technology. We proposed requiring States to publish and make available to all Medicaid EPs the procedures they developed for assigning incentive payments to the third party entities before payments can be assigned. Such publication must also include information about the State's verification mechanism. The State's method must assure compliance with the requirement that no more than 5 percent of the Medicaid EP's annual incentive payment is retained by the entity for costs not related to certified EHR technology.

Although section 1903(t)(6)(A)(ii) of the Act allows assignment of payment to entities promoting the adoption of EHR technology, we wish to clarify that such assignment would not remove the responsibility of the Medicaid EP to individually demonstrate meaningful use of the EHR technology (as discussed in greater detail below). Therefore, entities promoting the adoption would not receive the assigned payments unless the Medicaid EP meets all eligibility criteria. Our definition for promoting the adoption of certified EHR technology is in $\S 495.302$.

Comment: A commenter recommended that CMS require that entities designated by States that promote the adoption of EHR technology must use qualified EHR technology and be able to capture, query and/or exchange data from beyond a practice or closed system in order to foster interoperability, and to promote competition among EHR vendors with vendor-neutral and provider-neutral solutions. The commenter

recommended that entities that promote the adoption of certified EHR technology be certified to an electronic hub that permits the exchange of electronic structured data on a providerneutral basis.

Commenters also requested that the Regional Extension Centers funded by ONC be permissible as entities designated by the State to be eligible to receive EPs assigned incentive payments.
Response: States will have the discretion to identify entities that promote the adoption of certified EHR technology in accordance with our definition in regulation. We do not agree that the definition of "promotion of the adoption of EHR technology" requires the designated entity itself to utilize certified EHR technology. A variety of entities might offer services that meet the language included in this final rule defining promoting EHR adoption. We wish to point out that there is also a discussion of reassignment of payments in Section II.B.1.d. of this rule.

After consideration of the comments, we are adopting the language as written with the additional clarification that we encourage States to consider how they will verify on an ongoing basis that the entities that they designate are in fact promoting EHR adoption, per the requirements. Their responsibility to audit this element might be a factor in identifying which entities they wish to designate, in terms of tangible EHR promotion activities.

We agree that our definition of “promoting EHR adoption” does not preclude the ONC-funded Regional Extension Centers from being designated by States for this role.

4. Computation of Amount Payable to Qualifying Medicaid EPs and Eligible Hospitals

The statute, at sections 1903(t)(1), $(\mathrm{t})(4)$, and $(\mathrm{t})(5)$ of the Act, creates different payment formulas for Medicaid EPs versus hospitals. The payment methodology for Medicaid hospitals shares many aspects of the methodology used for Medicare hospitals.

a. Payment Methodology for EPs

\section{(1) General Overview}

Pursuant to section 1903(t)(1)(A) of the Act, payment for EPs equals 85 percent of "net average allowable costs." While the Secretary is directed to determine "average allowable costs" based upon studies of the average costs of both purchasing and using EHR technology, the net average allowable costs that set payment are capped by statute. As discussed in more detail further on, generally stated, these caps equal $\$ 25,000$ in the first year, and $\$ 10,000$ for each of 5 subsequent years (there is an exception for pediatricians with under 30 percent Medicaid patient volume, whose caps are two-thirds of these amounts). Thus, the maximum incentive payment an EP could receive from Medicaid equals 85 percent of $\$ 75,000$, or $\$ 63,750$, over a period of 6 years. EPs must begin receiving 
incentive payments no later than $\mathrm{CY}$ 2016.

\section{(2) Average Allowable Costs}

Section 1903(t)(4)(C) of the Act gives the Secretary the authority to determine average allowable costs. Specifically, the Secretary is directed to study the average costs associated with the purchase, initial implementation, and upgrade of certified EHR technology, including support services, and integral related training. The Secretary also is directed to study the average costs of operating, maintaining, and using certified EHR technology. The statute permits the Secretary to use studies submitted by the States.

We conducted a literature review of recent studies on EHR technology to determine the average allowable cost of implementing and using such technology. We reviewed the results from four recent, comprehensive studies.

In conducting a review of the data, we determined that the studies demonstrate a cross-sectional view of small and large practices and community health centers. There was adequate data to support a depiction of costs across multiple provider types.

To summarize, we determined that the average costs of EHRs vary greatly because of the size and type of provider practices, the differences in available features of systems, and the additional costs associated with licensing, support, training, and maintenance. However, based on the information reviewed, we determined that the average costs for initial EHR systems currently can range from $\$ 25,000$ to $\$ 54,000$ in the implementation year, per professional. Since the average costs of EHR technology in the first year can be as much as $\$ 54,000$ and no less than $\$ 25,000$, and since we believe the costs of such technology will be increasing, we set the average allowable cost at $\$ 54,000$. We established this average allowable cost at the high end of the range since the data we reviewed is based on certification criteria that may not be appropriate moving forward. Specifically, since the ONC is establishing new certification criteria for EHR technology, we believe the average cost of certified EHR technology incorporating the new criteria will be higher than the current costs of EHR technology. It is our assumption that making improvements to incorporate the new certification standards into current EHR technology will be costly. Thus, we believe that establishing the average allowable cost at $\$ 54,000$ is reasonable.
Additionally, our analysis determined that the range for subsequent incentive payment year costs for most providers will fall into a large range, based on a number of factors. On one end of the range, costs related to maintenance could be as low as $\$ 3,000$ to $\$ 9,000$ per provider, where other studies state that maintenance will be as high as $\$ 18,000$ to $\$ 20,610$ per provider. Given the requirements in the ONC interim final rule for the adoption of an initial set of standards, implementation specifications, and certification criteria for EHRs and the health measures data discussed in this final rule that CMS and the States will need to collect from professionals, we believe that the costs for maintaining certified EHR technology will also be on the higher end of the range at $\$ 20,610$.

\section{(3) Net Average Allowable Costs.}

As originally required by section 1903(t)(3)(E) of the Act, in order to determine "net" average allowable costs, average allowable costs for each provider must be adjusted in order to subtract any payment that is made to Medicaid EPs and is directly attributable to payment for certified EHR technology or support services of such technology. The only exception to this requirement is that payments from State or local governments do not reduce the average allowable costs. The resulting figure is the "net" average allowable cost, that is, average allowable cost minus payments from other sources (other than State or local governments). The statute indicates that EPs may receive 85 percent of a maximum net average allowable cost in the first year of $\$ 25,000$ and a maximum net average allowable cost of $\$ 10,000$ in subsequent years. This would mean that, as required by the statute, the net average allowable costs are capped at these amounts.

Since we set the average allowable cost at $\$ 54,000$ in the first year, EPs could receive as much as $\$ 29,000$ in funding from sources (other than from State or local governments) as contributions to the certified EHR technology and the incentive payment would still be based on 85 percent of the maximum net average allowable cost of $\$ 25,000$ (or $\$ 21,250$ ). This is appropriate since $\$ 54,000$ (the average allowable cost) minus $\$ 29,000$ (contributing sources of funding from other than State or local governments) equals $\$ 25,000$. Since $\$ 25,000$ is equal to the level of the maximum net average allowable cost or capped amount discussed above, providers could receive 85 percent of $\$ 25,000$ or $\$ 21,250$ in year one as a Medicaid incentive payment.
The same logic would hold true for subsequent years. Specifically, if in the following years an eligible professional received as much as $\$ 10,610$ in contributing funds from sources other than State or local governments, the maximum incentive payment of $\$ 8,500$ would be unaffected in such subsequent years. This result is due to the fact that the average allowable costs of $\$ 20,610$ for maintaining EHR technology minus the $\$ 10,610$ received would still equal $\$ 10,000$, the maximum net average allowable costs permitted under the statute.

In reviewing whether a reduction in the net average allowable cost was warranted based on other contributions to EHR technology, we considered the situation of EPs who may have been provided with the actual certified EHR technology, as well as training, support services, and other services that would promote the implementation and meaningful use of such technology. In some cases, we do not believe the contribution would reduce average allowable costs at all. For example, if an FQHC or RHC has provided technology to its staff EPs to use, we do not believe that such technology provision would be considered a "payment" from another source that would reduce average allowable costs. Moreover, we believe the situations in which an EP has been provided with the actual technology, support service, or training from another source are extremely limited in light of the statutory prohibitions on "kickbacks" at Section 1128B(b) of the Act.

Comment: Several commenters are concerned that States are required to develop a method to determine the payment amount for each provider. Commenters believed that incentive payments should be based on the maximum amount and that individual calculations are cumbersome and a difficult process for both States and eligible professionals.

Response: We would like to clarify the requirements in the statute and the process by which incentive payments will be established. Specifically, the Secretary is directed to study the average costs associated with the purchase, initial implementation, and upgrade of certified EHR technology, including support services, and integral related training. The Secretary is also directed to study the average costs of operating, maintaining, and using certified EHR technology. The statute permits the Secretary to use studies submitted by the States. CMS conducted a literature review of recent studies on EHR technology to determine the average allowable cost of implementing 
and using such technology. CMS reviewed the results from four recent, comprehensive studies and determined that these costs are $\$ 54,000$ per professional. We recognize that this cost is variable and since the ONC is establishing certification criteria for EHR technology, we believe this cost is reasonable since we expect that current EHR technology will need to be upgraded in order to meet the new certification criteria.

Next, in accordance with the statute, in order to determine the net average allowable costs for each provider, average allowable costs for each provider must be adjusted in order to subtract any payment that is made to Medicaid eligible professionals and is directly attributable to payment for certified EHR technology or support services of such technology. The only exception to this requirement, as discussed above, is that payments from State, or local governments do not reduce the average allowable costs. The resulting figure is the net average allowable costs. The statute further indicates that Medicaid eligible professionals can receive up to 85 percent of a maximum of the net average allowable cost. In year one the maximum net average allowable cost is $\$ 25,000$ and in subsequent years is $\$ 10,000$. Additionally, the statute indicates that Medicaid eligible professionals are responsible for the remaining 15 percent of the net average allowable cost (1903(t)(6)(B)). We believe the commenters are concerned with the 85 percent of net average allowable cost maximum incentive payment amount and the responsibility of the Medicaid professional for the remaining 15 percent of the net average allowable cost.

Since the statute is clear that to get to the net average allowable cost, payments made to the EP that are directly attributable to the payment for certified EHR technology or support services for such technology for each provider have to be subtracted from the average allowable cost, this must be an individual provider calculation. We do not believe we have discretion to change this netting process directed by the Congress. We have provided an example calculation so that in using the average allowable cost established by the Secretary of $\$ 54,000$ professionals could receive as much as $\$ 29,000$ in payments from outside sources and still receive 85 percent of the maximum capped net average allowable cost of $\$ 25,000$. We have also required that States must have a process in place and a methodology for verifying that payment incentives are not paid at amounts higher than 85 percent of the net average allowable cost and a process in place and a methodology for verifying that professionals pay 15 percent of the net average allowable cost of the certified EHR technology.

States may wish to establish a process whereby individuals attest to having completed their forms correctly and risk the circumstance of audit in the event the State has reason to believe individuals did not complete the forms appropriately. States could develop a process for providers to attest to having received no other sources of funding from other than State and local governments as payment that is directly attributable to the cost of the technology. States could select a random sample of providers to audit after the incentive payment has been paid. Additionally, States could determine that certain types of providers should be selected for a more extensive review since it may be true that this particular provider group was most likely to have received payment for certified EHR technology from sources other than State, or local governments. This process could eliminate some of the burden.

Comment: Commenters also asked that we provide some examples of the costs that must be subtracted to get to the net average allowable cost and therefore the incentive payment amount. Commenters do not want to be penalized because they did not have a fair chance at understanding the rule before participating in the program. Commenters further argued that reducing incentive payments due to other non-State/local resources could immobilize innovation and temper research activities.

Response: When States begin to think through the payments that are not considered acceptable and that must be subtracted from the average allowable cost to get to the net average allowable costs and consequently, the incentive payment, we believe that States should consider the situation in which professionals may have been provided with the certified EHR technology through, for example, an employer/ employee relationship. We do not believe in this case that there could be any payments directly attributable to the professional for the certified EHR technology; therefore, there are no payments that must be subtracted. This situation would apply in the case of clinics like FQHCs/RHCs or IHS facilities. Additionally, States should consider that any in-kind contributions such as EHR technology or free software provided by vendors are not cash payments and therefore are also not costs that must be subtracted. Further, in the case of grants like the HRSA Capital Improvement Program grants that are used to finance many projects within an organization; for example, research projects, infrastructure,

construction or repair and renovation of health centers, health care services, etc., we do not believe these grants are directly attributable as payments for the certified technology but rather are payments for several projects of the organization. Again, we do not believe that these costs are directly attributable to payment costs for the certified technology and therefore must be subtracted. These are just some examples but the clarifying point is that any costs that are subtracted from the average allowable cost to get to the net average allowable cost have to be cash payment that is "directly attributable to the professional for the certified EHR technology." Aside from specific costs related to computer hardware, software, staff training, and/or upgrades of the technology, we believe there are limited situations that exist in which cash payment has been made that is directly attributable to the professional solely for the purpose of certified EHR technology.

In any case, we are requiring that States submit to CMS for review and approval a description of their process and methodology for verifying payment incentives in State Medicaid HIT plans. CMS has the flexibility to approve State Medicaid HIT plans that require provider attestation initially with subsequent auditing of either a random sample, or a sample of payment incentive recipients most likely to have received funding from other sources.

We also would like to provide clarifying information concerning the responsibility of the professional for 15 percent of the net average allowable cost. Section 1903(t)(6)(B) of the Act dictates that EPs are responsible for payment of the remaining 15 percent of the net average allowable cost and States are responsible for ensuring that the Secretary pays no more than 85 percent of the net average allowable cost as incentive payments. In ensuring EPs' responsibility for the remaining 15 percent, we believe States may consider funding that the EP receives from other sources as essentially meeting the EPs responsibility. For example, as stated earlier, States should consider the previous examples of employer/ employee relationship, certain grants, and in-kind contributions. Specifically, if a professional is an employee at an FQHC/RHC or IHS facility, since the employer has provided the technology to the employee it is assumed that the employer has contributed the 15 percent 
to the net average allowable cost on behalf of the employee. Additionally, in the case of in-kind contributions, the professional's 15 percent responsibility to the net average allowable cost is of no consequence since the entity has assumed that responsibility for the professional. It should be noted that in the case of a vendor supplying the 15 percent on behalf of the EP because the technology, training, support services, etc. was either in-kind contributions or free, conflict of interest safeguards apply and the parties should be mindful of the requirement to comply with applicable fraud, waste, and abuse laws, rules, and regulations.

In those cases in which the professional himself must satisfy the responsibility for the 15 percent net average allowable costs, we believe in determining the calculation, States should consider costs related to the providers' efforts to address workflow redesign and training to facilitate meaningful use of EHRs as contributing to the providers' 15 percent share.

Considering the costs of training, preparing for, and installing or upgrading EHR technology, we believe the vast majority of EPs will spend, or receive funding from other sources in the amount of 15 percent of the maximum net average allowable cost (or $\$ 3,750$ in the first year and $\$ 1,500$ in subsequent years). We also believe that for providers' first payment for having adopted, implemented or upgraded certified EHR technology, States should take into consideration providers' verifiable contributions up through the date of attestation. For example, if a provider adopted EHR technology for \$100 in January 2010 and then paid for the upgrade to the newly certified version for an additional \$100 in December of 2010, the sum of both investments; that is, $\$ 200$, should be applicable to their 15 percent of the net average allowable cost.

In summary, in response to these comments, we are clarifying in the final rule that State Medicaid HIT plans must explain the process and methodology States will put in place to ensure that Medicaid eligible professionals comply with this responsibility (see section 495.332). Additionally, we have clarified the rules at section 495.310 that providers are responsible for 15 percent of the net average allowable costs of the certified EHR technology.

The following chart is useful in depicting the effect of this calculation.

\section{Payments: NAACcalculation}

\section{Average allowable costs (AAC) minus payments from other sources:}

\section{- State and local sources not considered \\ $=$ Net average allowable costs (NAAC)}

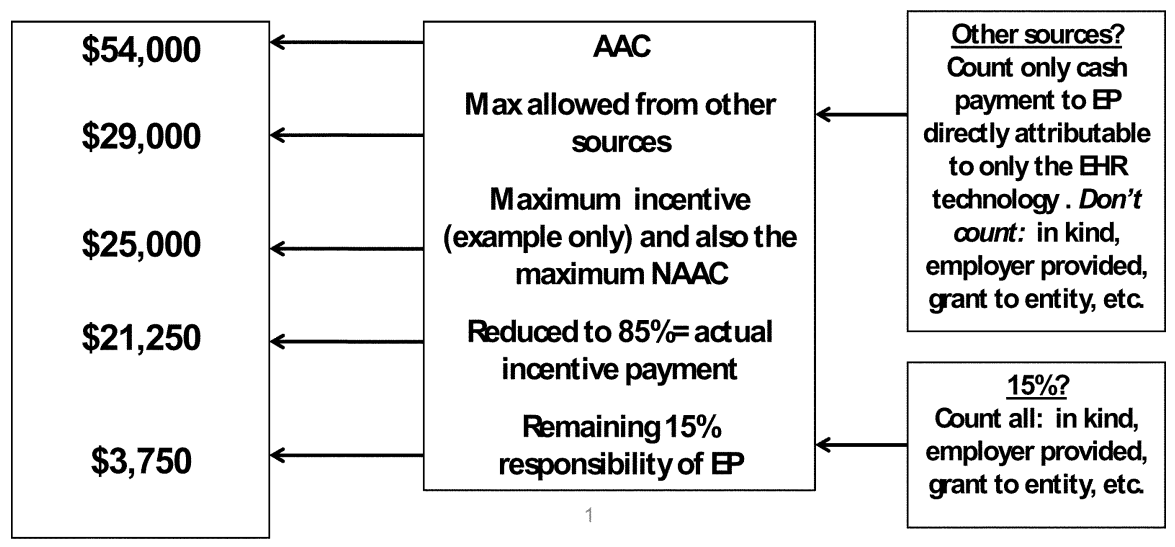

Comment: Several commenters have raised questions about the cost of the certified EHR technology for hospitals. Specifically, commenters believed that $\$ 54,000$ is identified as the initial costs for providers with 20 percent per year thereafter for ongoing costs; and \$5 million for initial costs for hospitals with 20 percent per year thereafter for ongoing costs. The commenters believed that the $\$ 54,000$ assumption for providers may be accurate; however, the \$5 million assumption for hospitals could be off by a factor of 4 or 5 . Other commenters believed that even the $\$ 54,000$ assumption seriously underestimates the total cost of ownership for EHR systems and their ongoing expenses and argued that this assumption does not account for the training and labor costs associated with implementation of an EHR system, nor does it account for the lost revenues resulting from the decreases in productivity during the initial implementation phase. One commenter questioned whether the $\$ 54,000$ average allowable cost for certified EHR technology takes into account leasing of an ASP (applicable service provider web based) model as an allowable cost.

Response: As explained above, we conducted a literature review of recent studies on EHR technology and determined that these costs are $\$ 54,000$ per professional. We are not establishing an average allowable cost for hospitals. The reference to the costs of EHRs for hospitals was only to make the point that the costs of EHRs vary greatly because of the size and type of provider practices, differences in available features of systems, and the additional costs associated with licensing, support, training and maintenance. Additionally, there is no reason to establish the average allowable costs of EHR technology for hospitals since the hospital incentive payments are based on a formula that is defined in the statute and that does not rely on the 
average allowable cost. In terms of the $\$ 54,000$ average allowable cost figure, we indicated that we believe this is a reasonable figure but recognize that there are many variables to determining the average allowable cost of certified EHR technology because of practice size, the differences in available features of systems, and the additional costs associated with licensing, support, training and maintenance. The $\$ 54,000$ average allowable cost figure does take into account web based models since the Secretary is tasked to study the average costs associated with the purchase, initial implementation, and upgrade of certified EHR technology, including support services, and integral related training.

We are making no additional revisions to this section of the final rule as a result of this comment.

Comment: One commenter requested that CMS make clear that any funding an FQHC receives because the Medicaid eligible professional voluntarily chooses to reassign his/her incentive payment or any funds the center may have received through HRSA Capital Improvement Funds cannot be the basis for a State reducing its per visit payment to FQHCs required under Section 1902(bb).

Response: We agree with the commenter with respect to the incentive payments authorized under section 1903(t); however, we are not addressing the HRSA Capital Improvement funds, as this funding is outside the scope of this rulemaking. Since FQHCs are not eligible providers, incentive payments will not be made to FQHCs. It is true, however, that an eligible professional could choose to reassign his/her incentive payment to the FQHC. Any reassignment of payments must be consistent with applicable laws, rules, and regulations, including, without limitation, those related to fraud, waste, and abuse. Incentive payments are payments designed to promote the adoption and meaningful use of certified EHR technology and are not payments for medical assistance provided in the FQHC. We do not have the authority under this program to provide that these funds be the basis for the State to reduce its per visit payment to the FQHC.

After consideration of this comment, we are making no further additions to this section of the final rule.

(4) Payments for Medicaid Eligible Professionals

One important difference we proposed between the payments to Medicaid EPs and hospitals is that States would disburse the payments to EPs in alignment with the calendar year, whereas hospitals will receive payments in alignment with the fiscal year, as described in section II.D.4.b. of this final rule. There are two primary reasons for this. The first is to align Medicaid incentive payment disbursements with that of the Medicare program, in order to support consistency between the two programs, as well as among the States. We will undertake national outreach activities to encourage provider EHR adoption and to align the annual payment periods.

As previously discussed in this final rule, based on the 85 percent threshold applied to the net average allowable costs, we proposed that most Medicaid EPs may receive up to a maximum incentive payment of $\$ 21,250$ in the first payment year.
In subsequent years of payment, Medicaid EPs' incentive payments will be limited to 85 percent of the $\$ 10,000$ cap on net average allowable cost, or up to a maximum of $\$ 8,500$ annually for most Medicaid EPs.

Since pediatricians are qualified to participate in the Medicaid EHR incentive program as physicians, and therefore classified as Medicaid EPs, they may qualify to receive the full incentive (that is, the 85 percent threshold applied to the net average allowable cost) if the pediatrician is not hospital-based and can demonstrate that they meet the minimum 30 percent Medicaid patient volume requirements discussed in this subpart.

Pediatricians who are not hospitalbased, and have a minimum of 20 percent of their patient encounters paid by Medicaid are also encouraged to participate in the Medicaid EHR incentive program. The maximum payment amount for these pediatricians, who meet the 20 percent Medicaid patient volume, but fall short of the 30 percent patient volume, is reduced to two-thirds of the net average allowable cost, subject to the 85 percent threshold. The reduction accounts for the reduced patient volume, but the intent is to offer an incentive to attract pediatricians to participate. This means pediatricians with a minimum 20 percent patient volume may qualify for up to a maximum of $\$ 14,167$ in the first incentive payment year and to up a maximum of $\$ 5,667$ in the 5 subsequent incentive payment years, or no more than $\$ 42,500$ over the maximum 6 year period.

TABLE 16: Maximum Incentive Payment Amount for Medicaid Professionals

\begin{tabular}{|c|c|c|}
\hline $\begin{array}{c}\text { Cap on Net Average Allowable Costs, } \\
\text { per the HITECH Act }\end{array}$ & \begin{tabular}{c|}
85 percent \\
Allowed for \\
Eligible \\
Professionals
\end{tabular} & \begin{tabular}{|c|} 
Maximum \\
Cumulative \\
Incentive over 6-year \\
Period
\end{tabular} \\
\hline$\$ 25,000$ in Year 1 for most professionals & $\$ 21,250$ & \\
\hline$\$ 10,000$ in Years 2-6 for most professionals & $\$ 8,500$ & $\$ 63,750$ \\
\hline $\begin{array}{l}\$ 16,667 \text { in Year } 1 \text { for pediatricians with a } \\
\text { minimum } 20 \text { percent patient volume, but less than } \\
30 \text { percent patient volume, Medicaid patients }\end{array}$ & $\$ 14,167$ & \\
\hline $\begin{array}{l}\$ 6,667 \text { in Years } 2-6 \text { for pediatricians with a } \\
\text { minimum } 20 \text { percent patient volume, but less than } \\
30 \text { percent patient volume, Medicaid patients }\end{array}$ & $\$ 5,667$ & $\$ 42,500$ \\
\hline
\end{tabular}


All State Medicaid EHR incentive program calculations, payments, and limits under this section are subject to our review.

Comment: Commenters suggested that CMS apply the health professional shortage area (HPSA) bonus offered under Medicare to Medicaid providers.

Response: There is no statutory authority for HPSA bonuses in the Medicaid incentive program. However, it is worth noting that in comparing the maximum participation period for EPs in Medicare and Medicaid, EPs can earn higher total incentive payments under Medicaid, even when compared to the Medicare payments with the HPSA bonus.

We are not making any changes to this rule as a result of this comment.

Comment: Commenters requested clarification on how the Medicare payment adjustments apply to Medicaid providers. Commenters suggested that if these apply to Medicaid providers, it could be a reason not to participate. One commenter asked about a provider who began in the Medicare incentive program and then switched to Medicaid, but then stopped meaningfully using the certified EHR.

Response: The Medicaid program does not have the payment adjustments that apply, beginning in 2015, in the Medicare program. However, all Medicare providers will have a payment reduction in 2015 if they are not demonstrating meaningful use, regardless of whether they participate in the Medicare or Medicaid EHR incentive program. Whether an EP, hospital or CAH is a meaningful user of certified EHR technology will continue to be determined on a year-by-year basis. A provider who stops meaningfully using certified EHR cannot receive an incentive payment. This is discussed in greater detail in II.A.

We are not making any changes to this rule as a result of this comment.

(5) Basis for Medicaid EHR Incentive Program First Payment Year and Subsequent Payment Years

(i) Medicaid EP Who Begins

Adopting, Implementing or Upgrading
Certified EHR Technology in the First Year

A Medicaid EP who begins by adopting, implementing, or upgrading certified EHR technology in the first year will be eligible for the incentive payments not in excess of the maximum amount. Under section 1903(t)(4) of the Act he or she is eligible to receive up to the maximum first year Medicaid incentive payments discussed in the previous sections, plus additional incentive payments for up to 5 years for demonstrating meaningful use of certified EHR technology. In other words, these providers may participate in the Medicaid EHR incentive program for up to 6 years.

Table 17 demonstrates the payment scenarios available to a Medicaid EP who begins in their first year by adopting, implementing, or upgrading certified EHR technology, and receives all six years of payments consecutively. As can be seen from the table, the EP can begin receiving payments as late as 2016, and still receive up to the maximum payments under the program.

\section{TABLE 17: Payment Scenarios For Medicaid EPs Who Begin Adoption in the First Year}

\begin{tabular}{|c|c|c|c|c|c|c|}
\hline \multirow[b]{2}{*}{ Calendar Year } & \multicolumn{6}{|c|}{ Medicaid EPs who begin adoption in } \\
\hline & 2011 & 2012 & 2013 & 2014 & 2015 & 2016 \\
\hline 2011 & $\$ 21,250$ & --- & ------- & -------. & -------- & \\
\hline 2012 & $\$ 8,500$ & $\$ 21,250$ & ------------ & ------------ & ------------ & \\
\hline 2013 & $\$ 8,500$ & $\$ 8,500$ & $\$ 21,250$ & ------ & ------- & \\
\hline 2014 & $\$ 8,500$ & $\$ 8,500$ & $\$ 8,500$ & $\$ 21,250$ & --------. & --- \\
\hline 2015 & $\$ 8,500$ & $\$ 8,500$ & $\$ 8,500$ & $\$ 8,500$ & $\$ 21,250$ & \\
\hline 2016 & $\$ 8,500$ & $\$ 8,500$ & $\$ 8,500$ & $\$ 8,500$ & $\$ 8,500$ & $\$ 21,250$ \\
\hline 2017 & ------------ & $\$ 8,500$ & $\$ 8,500$ & $\$ 8,500$ & $\$ 8,500$ & $\$ 8,500$ \\
\hline 2018 & ------------- & -------------- & $\$ 8,500$ & $\$ 8,500$ & $\$ 8,500$ & $\$ 8,500$ \\
\hline 2019 & ------------- & ------------- & ------- & $\$ 8,500$ & $\$ 8,500$ & $\$ 8,500$ \\
\hline 2020 & ------------- & -------------- & ------------- & ------------- & $\$ 8,500$ & $\$ 8,500$ \\
\hline 2021 & 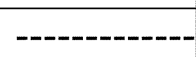 & - & 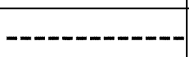 & 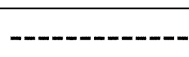 & ------------ & $\$ 8,500$ \\
\hline TOTAL & $\$ 63,750$ & $\$ 63,750$ & $\$ 63,750$ & $\$ 63,750$ & $\$ 63,750$ & $\$ 63,750$ \\
\hline
\end{tabular}

(ii) Medicaid EP who has Already Adopted, Implemented or Upgraded Certified EHR Technology and Meaningfully Uses EHR Technology

For a Medicaid EP who has already adopted, implemented, or upgraded certified EHR technology and can meaningfully use this technology in the first incentive payment year, we proposed that the Medicaid EP be permitted to receive the same maximum payments, for the same period of time, as the Medicaid EP who merely adopted, implemented or upgraded certified EHR technology in the first year. Section 1903(t)(6)(C)(ii) of the Act states that for a Medicaid EP or hospital who has completed "adopting, implementing, or upgrading” certified EHR technology "prior to the first year of payment * * * clause (i)(I) shall not apply and clause (i)(II) [discussing the demonstration of meaningful use] shall apply to each year of payment to the Medicaid provider under this subsection, including the first year of payment." We believe this provision supports an interpretation that a
Medicaid EP who has already adopted certified EHR technology, would still receive a "first year" of payment under section 1903(t)(4) of the Act, and like all other first years of payment, this payment could not exceed $\$ 21,250$. Then, under section 1903(t)(4)(A)(ii) and (iii) of the Act, such Medicaid EPs could receive an additional 5 years of payment for subsequent years of payment, with payments not exceeding $\$ 8,500$ in each of these 5 subsequent years. This approach allows early adopters of certified EHR to begin 
meaningfully using technology, without being at a competitive disadvantage, and without losing incentive payments for the previous costs associated with adopting, implementing, or upgrading certified EHR technology.
Thus, the maximum incentive payments for Medicaid EPs demonstrating that they are meaningful users in the first payment year, would be identical to the maximum payments available to those demonstrating adoption, implementation, or upgrading certified EHR technology in the first year, as depicted in Table 18.

\section{TABLE 18: Maximum Incentive Payments for Medicaid EPs Who Are Meaningful Users in the First Payment Year}

\begin{tabular}{|c|c|c|c|c|c|c|}
\hline \multirow[b]{2}{*}{ Calendar Year } & \multicolumn{6}{|c|}{ Medicaid EPs who begin meaningful use of certified EHR technology in-- } \\
\hline & 2011 & 2012 & 2013 & 2014 & 2015 & 2016 \\
\hline 2011 & $\$ 21,250$ & -------- & & & & \\
\hline 2012 & $\$ 8,500$ & $\$ 21,250$ & & -- &.-- & \\
\hline 2013 & $\$ 8,500$ & $\$ 8,500$ & $\$ 21,250$ & $----\cdot-$ & --- & \\
\hline 2014 & $\$ 8,500$ & $\$ 8,500$ & $\$ 8,500$ & $\$ 21,250$ & -1 & \\
\hline 2015 & $\$ 8,500$ & $\$ 8,500$ & $\$ 8,500$ & $\$ 8,500$ & $\$ 21,250$ & 敒 \\
\hline 2016 & $\$ 8,500$ & $\$ 8,500$ & $\$ 8,500$ & $\$ 8,500$ & $\$ 8,500$ & $\$ 21,250$ \\
\hline 2017 & |------------ & $\$ 8,500$ & $\$ 8,500$ & $\$ 8,500$ & $\$ 8,500$ & $\$ 8,500$ \\
\hline 2018 & ------------- & & $\$ 8,500$ & $\$ 8,500$ & $\$ 8,500$ & $\$ 8,500$ \\
\hline 2019 & ------------- & |----------- & ----- & $\$ 8,500$ & $\$ 8,500$ & $\$ 8,500$ \\
\hline 2020 & -------------- & -------------- & ------ & -------- & $\$ 8,500$ & $\$ 8,500$ \\
\hline 2021 & --- & - & ------ & & & $\$ 8,500$ \\
\hline TOTAL & $\$ 63,750$ & $\$ 63,750$ & $\$ 63,750$ & $\$ 63,750$ & $\$ 63,750$ & $\$ 63,750$ \\
\hline
\end{tabular}

We also requested comment on an alternative approach that would limit the incentive payment for Medicaid EPs who have already adopted,

implemented, or upgraded certified EHR technology to 5 years of payment, at a maximum payment of $\$ 8,500$ per year. We refer readers to our proposed rule (75 FR 1937) for a discussion of this approach.

Medicaid EPs are not required to participate on a consecutive annual basis, however, the last year an EP may begin receiving payments is 2016, and the last year the EP can receive payments is 2021. See our discussion on consecutive versus non-consecutive payments in section II.A. of this final rule. We wish to point out to readers that this is one area where the Medicare and Medicaid incentive payment programs differ. That is, Medicare EPs do not have the same flexibility afforded to Medicaid EPs, who are permitted to participate in a non-consecutive annual basis, or to skip years, in other words, without the omitted years necessarily reducing the total number of years for which they may receive payment. The tables in this section demonstrate how a Medicaid EP would maximize the aggregate incentive under different scenarios, considering that a Medicaid
EP may initiate participation in 2011 through 2016. Additionally, these tables do not include the alternative Medicaid maximum incentive payment for pediatricians discussed in the previous section, which is two-thirds of the total amount listed in Tables 27 through 30 . Finally, these tables do not represent EPs whose incentive payments may be reduced because net average allowable costs may actually be lower than $\$ 25,000$ in the first year, or $\$ 10,000$ in subsequent years, due to payments from other, non-State/local sources.

Comment: Some commenters rejected the alternative scenario (including 5 years of payment instead of 6), as it would effectively result in a penalty for early adopters, and reward those who delayed adoption.

Response: We agree that early adopters should not be penalized.

Further, we agree that Medicaid EPs that have adopted EHR technology before the first year should have an opportunity for the same maximum incentive payments as EPs that are meaningful users in the first year. Accordingly, the alternative scenario we presented in Table 30 of the proposed rule will not be used for incentive payments.

As we are adopting our proposed policy as final, we are not making any changes to the regulations as a result of this comment.

b. Payment Methodology for Eligible Hospitals

Statutory parameters placed on Medicaid incentive payments to hospitals are largely based on the methodology applied to Medicare incentive payments. The specifications described in this section are limits to which States must adhere when developing aggregate EHR hospital incentive amounts for Medicaid-eligible hospitals. States will calculate hospitals' aggregate EHR hospital incentive amounts on the FFY to align with hospitals participating in the Medicare EHR incentive program.

States may pay children's hospitals and acute care hospitals up to 100 percent of an aggregate EHR hospital incentive amount provided over a minimum of a 3-year period and a maximum of a 6-year period. Section 1905(t)(5)(D) requires that no payments can be made to hospitals after 2016 unless the provider have been paid a payment in the previous year; thus, while Medicaid EPs are afforded flexibility to receive six years of payments on a non-consecutive, annual basis, hospitals receiving a Medicaid 
incentive payment must receive payments on a consecutive, annual basis after the year 2016. Prior to 2016, Medicaid incentive payments to hospitals can be made on a nonconsecutive, annual basis. The maximum incentive amounts for these providers are statutorily defined by a formula at section 1903(t)(5)(B) of the Act. The statute requires that Medicaid refer, with some adjustments, to the calculation for the Medicare hospital incentive payment described at sections 1886(n)(2)(A), 1886(n)(2)(C), and 1886(n)(2)(D) of the Act, to determine the aggregate EHR amount allowable for individual hospitals. The aggregate EHR hospital incentive amount is calculated using an overall EHR amount multiplied by the Medicaid share.

States are responsible for using auditable data sources to calculate Medicaid aggregate EHR hospital incentive amounts, as well as determining Medicaid incentive payments to those providers. Auditable data sources include-

- Providers' Medicare cost reports;

- State-specific Medicaid cost reports;

- Payment and utilization information from the State's MMIS (or other automated claims processing systems or information retrieval systems); and

- Hospital financial statements and hospital accounting records.

All State Medicaid EHR incentive program calculations, payments, and limits under this section are subject to our review.

For purposes of the Medicaid EHR hospital incentive program, the overall EHR amount is equal to the sum over 4 years of (I)(a) the base amount (defined by statute as $\$ 2,000,000$ ); plus (b) the discharge related amount defined as $\$ 200$ for the 1,150th through the 23,000 th discharge for the first year (for subsequent years, States must assume discharges increase by the provider's average annual rate of growth for the most recent 3 years for which data are available per year): multiplied by (II) the transition factor for each year equals 1 in year $1,3 / 4$ in year $2,1 / 2$ in year 3 , and $1 / 4$ in year 4 .

The statute specifies that the payment year is determined based on a Federal fiscal year. Section 1886(n)(2)(C) of the Act provides the Secretary with authority to determine the discharge related amount on the basis of discharge data from a relevant hospital cost reporting period, for use in determining the incentive payment during a Federal fiscal year. Federal fiscal years begin on October 1 of each calendar year, and end on September 30 of the subsequent calendar year. Hospital cost reporting periods can begin with any month of a calendar year, and end on the last day of the 12th subsequent month in the next calendar year. For purposes of administrative simplicity and timeliness, we require that States use data on the hospital discharges from the hospital fiscal year that ends during the Federal fiscal year prior to the fiscal year that serves as the first payment year.

The discharge-related amount is $\$ 200$ per discharge for discharges 1,150 through 23,000. To determine the discharge-related amount for the 3 subsequent years that are included in determining the overall EHR amount, States should assume discharges for an individual hospital have increased by the average annual growth rate for an individual hospital over the most recent 3 years of available data from an auditable data source. Note that if a hospital's average annual rate of growth is negative over the 3 year period, it should be applied as such.

The overall hospital EHR amount requires that a transition factor be applied to each year. This transition factor equals 1 for year $1, \frac{3}{4}$ for year 2 , $\frac{1}{2}$ for year 3 , and $\frac{1}{4}$ for year 4 , as provided for in sections 1886(n)(2)(A) and 1886(n)(2)(E) of the Act, and as incorporated through section 1902(t)(5)(B) of the Act. We note that although, for purposes of the Medicare incentives, section 1886(n)(2)(E)(ii) of the Act requires a transition factor of 0 , if the first payment year is after 2013, we do not believe this rule would apply in the context of the Medicaid incentive payments. Nothing in section 1903(t) of the Act specifically cross references this 0 transition factor, and, notably, section 1903(t) of the Act allows Medicaid incentive payments to begin as late as 2016.

The "Medicaid Share," against which the overall EHR amount is multiplied, is essentially the percentage of a hospital's inpatient, non-charity care days that are attributable to Medicaid inpatients.

More specifically, the Medicaid share is a fraction expressed as-

- Estimated Medicaid inpatient-beddays plus estimated Medicaid managed care inpatient-bed-days;

Divided by;

- Estimated total inpatient-bed days multiplied by ((estimated total charges minus charity care charges) divided by estimated total charges).

As indicated in the above formula, the Medicaid share includes both Medicaid inpatient-bed-days and Medicaid managed care inpatient-bed-days. This is in keeping with section 1903(t)(5)(C) of the Act, which provides that in computing inpatient-bed-days, the
Secretary shall take into account inpatient-bed-days that are paid for individuals enrolled in a Medicaid managed care plan under sections 1903(m) or 1932 of the Act. We interpreted these managed care individuals to be individuals enrolled in an managed care organization (MCO), prepaid inpatient health plan (PIHP), or prepaid ambulatory health plan (PAHP) under 42 CFR part 438.

Some Medicaid managed care entities (that is, MCOs, PIHPs, and PAHPs with risk contracts) provide substitute services (or, "in-lieu-of services") in more cost effective or efficient settings than the State plan services in the managed care contract. For example, in a hospital inpatient setting, these services could be in a different unit, such as a sub-acute wing or skilled nursing wing, so long as States and contracting entities are in compliance with the actuarial soundness rules in $\S 438.6$ (c), provision of substitute services is allowed. Although we understand that these substitute service days may be used to achieve efficiency and cost effectiveness, we do not believe such substitute service days should count as "inpatient-bed-days" in the hospital EHR incentive payment calculation. The statute requires us to calculate the Medicaid share "in the same manner" as the Medicare share under section 1886(n)(2)(D) of the Act and such substitute service days would not be considered "in the same manner." Thus, we proposed that for purposes of the Medicaid formula, we would count only those days that would count as inpatient-bed-days for Medicare purposes under section 1886(n)(2)(D) of the Act.

In addition, because the formula for calculating the Medicaid share requires a determination of charity care charges, States should use the revised Medicare 2552-10, Worksheet S-10 or another auditable data source to determine the charity care portion of the formula. In the absence of sufficient charity care data to complete the calculation, section 1886(n)(2)(D) of the Act, requires the use of uncompensated care data to derive an appropriate estimate of charity care, including a downward adjustment for bad debts. We interpreted bad debt to be consistent with the Medicare definition of bad debt as promulgated at $\S 413.89(b)(1)$.

Finally, per section 1886(n)(2)(D) of the Act, to the extent there is simply not sufficient data that would allow the State to estimate the inpatient bed-days attributable to Medicaid managed care patients, the statute directs that such figure is deemed to equal 0 . Likewise, if there is simply not sufficient data for 
the State to estimate the percentage of inpatient bed days that are not charity care (that is, [estimated total chargescharity care charges]/estimated total charges), the statute directs that such figure is deemed to equal 1.

Unlike Medicaid EPs, who must waive rights to duplicative Medicare incentive payments, hospitals may receive incentive payments from both Medicare and Medicaid, contingent on successful demonstration of meaningful use and other requirements under both programs.

The last year that a hospital may begin receiving Medicaid incentive payments is FY 2016. States must make payments over a minimum of 3 years and a maximum of 6 years.

Additionally, in any given payment year, no annual Medicaid incentive payment to a hospital may exceed 50 percent of the hospital's aggregate incentive payment. Likewise, over a 2year period, no Medicaid payment to a hospital may exceed 90 percent of the aggregate incentive.

Table 19 demonstrates several scenarios for Medicaid hospitals. However, there are other scenarios not included here. For example, this table assumes that a hospital would participate on a consecutive annual basis until the incentive is exhausted. The purpose of Table 19 is to illustrate the general timeline for Medicaid hospital incentives.

\section{TABLE 19: Hospital Incentives}

\begin{tabular}{|c|c|c|c|c|c|c|c|c|}
\hline \multicolumn{9}{|c|}{$\begin{array}{l}\text { States will monitor compliance of hospitals coming onto the program with different } \\
\text { requirements depending on the year. Incentive determination will also be based on Y1 } \\
\text { versus subsequent years. This chart is an example, noting that hospitals may collect the } \\
\text { incentive over 3-6 years. }\end{array}$} \\
\hline \multirow{12}{*}{ 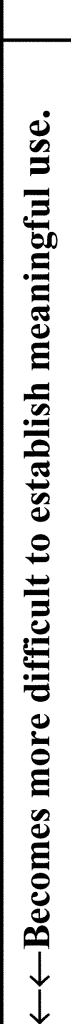 } & $\mathbf{C Y}$ & \multicolumn{7}{|c|}{ Demonstration of Compliance } \\
\hline & 2011 & Y1 & \multicolumn{6}{|c|}{$\begin{array}{l}\text { Y1 participants must demonstrate that they engaged in effort } \\
\text { to adopt, implement, or upgrade to certified EHR technology } \\
\text { However, if users already adopted, they may proceed to Y2 } \\
\text { requirements in Y1. }\end{array}$} \\
\hline & 2012 & Y2 & Y1 & \multicolumn{5}{|c|}{$\begin{array}{l}\text { Y1, same as above. Y2 must become a meaningful } \\
\text { EHR user. We expect to issue definition of } \\
\text { meaningful use on a biannual basis beginning in } \\
2011 .\end{array}$} \\
\hline & 2013 & Y3 & Y2 & Y1 & \multicolumn{4}{|c|}{ Y1, same as above. Y2-3 will be the same. } \\
\hline & 2014 & Y4 & Y3 & Y2 & Y1 & \multicolumn{3}{|c|}{$\begin{array}{l}\text { Y1, same as above. Y2-4, same as } \\
\text { above. }\end{array}$} \\
\hline & 2015 & Y5 & Y4 & Y3 & $\mathrm{Y} 2$ & Y1 & $\begin{array}{l}\mathrm{Y} 1 \\
\text { sam }\end{array}$ & $\begin{array}{l}\text { as above. Y2-5, } \\
\text { above. }\end{array}$ \\
\hline & 2016 & Y6 & Y5 & Y4 & Y3 & $\mathrm{Y} 2$ & Y1 & \multirow{6}{*}{$\begin{array}{l}\text { Y1, same as above } \\
\text { Y2-6, same as } \\
\text { above. }\end{array}$} \\
\hline & 2017 & & Y6 & Y5 & Y4 & Y3 & $\mathrm{Y} 2$ & \\
\hline & 2018 & & & Y6 & Y5 & Y4 & Y3 & \\
\hline & 2019 & & & & Y6 & Y5 & Y4 & \\
\hline & 2020 & & & & & Y6 & Y5 & \\
\hline & 2021 & & & & & & Y6 & \\
\hline
\end{tabular}

Comment: Many commenters recommended that CMS instruct States to provide hospitals the maximum incentive payments possible in their first two payment years. Commenters provided many examples of how CMS should instruct States to make payments. For instance, commenters suggested that CMS require States to pay 50 percent of hospitals' aggregate incentive payment in the first year and another 40 percent in the second yearas a limited source of capital for adoption, implementation, and upgrades. Many commenters stated that it is critical that EHR incentive payments be made in a timely manner and not delayed or affected by State budgetary problems or changes.

Response: After consideration of the public comments received, we are finalizing these provisions as originally proposed, with one clarification to ensure the statutory requirement that eligible hospitals, after 2016, may not receive an incentive payment, unless a payment was received in the prior year. The statute is imposing maximums on what the State is authorized to pay eligible hospitals. At section 1903(t)(5)(A) the statute requires that a State can make no more than 50 percent of the hospital's aggregate incentive payment in any one year. Likewise, over a 2-year period, the State cannot pay more than 90 percent of the aggregate incentive. Finally, under 1903(t)(5)(D) no more than six years of payment may be made, and payment may not be paid 
for any year beginning after 2016, unless the hospital was provided an incentive payment for the preceding year.

However, these are limits on State payments, not required minimums. We believe that States should work with their provider communities to determine the best timeframes for implementing their EHR programs and making payments to providers.

Comment: Some commenters indicated that incentive payments should not be included in any calculation of total Medicaid payments for the purpose of determining Medicaid shortfalls, disproportionate share payments, upper payment limits, or any general Medicaid program service.

Response: According to the statute, Medicaid HIT incentive payments are made to encourage the adoption and use of certified EHR technology defined by the statute, as well as support services including maintenance and training that is for, or is necessary for the adoption and operation of, such technology. Payments to providers under this rule are not being made for the provision of services or the cost of the provision of services to Medicaid beneficiaries or the uninsured. Therefore, we are clarifying that EHR incentive payments made to providers in accordance with the statute and final regulation are not subject to the same limits as payments for items and services provided to Medicaid beneficiaries and the uninsured including Medicaid upper payment limits and disproportionate share hospital limits. This comment is also addressed in the Medicare section at II.B.4.b.

Comment: One commenter noted a technical error in the proposed rule at 495.310 (g) (2) Medicaid Share. The commenter questioned whether (2)(iii) meant to qualify (2)(ii) or (2)(i), noting that the latter would result in dual eligibles being removed from Medicaid days (the numerator) and would not conform to the Act which would require that they be removed from the denominator.

Response: We agree that the regulation includes a technical error, and we read the statute as requiring that dually eligible individuals be excluded from the denominator. Section 1903(t)(5)(C) states that the Medicaid share should be calculated using a numerator that does not include individuals "described in section 1886(n)(2)(D)(i).” Individuals described in that section are individuals for whom payment may be made under Medicare Part A as well as individuals enrolled with a Medicare Advantage Organization under Part C. Thus, dually eligible individuals are excluded from the numerator in determining the

Medicaid share.

We are therefore revising section $495.310(\mathrm{~g})(2)(\mathrm{iii})$ to ensure that it refers to clause (i), rather than clause (ii), of $\S 495.310$ (g)(2).

Comment: One commenter highlighted a technical error in the proposed rule at $\S 495.310$ (g)(1)(i)(B) when he requested clarification for that section which reads: "The discharge related amount for a 12-month period selected by the State but with the Federal fiscal year before the hospital's fiscal year that serves as the payment year." He interpreted the language to mean that if the payment year begins in 2011, the Federal fiscal year would be 2010; and the discharge related amount would be for 2009 .

Response: Section 495.310(g)(1)(i)(B) is improperly worded in the proposed rule and should read, "The discharge related amount for a 12-month period selected by the State, but ending in the Federal fiscal year before the hospital's fiscal year that serves as the first payment year." For example: FY 2011 begins on October 1, 2010 and ends on September 30, 2011. For an eligible hospital with a cost reporting period running from July 1, 2010 through June 30,2011 , the State would employ the relevant data from the hospital's cost reporting period ending June 30, 2010 in order to determine the EHR incentive payment amount for the hospital.

We are revising this language in the final rule at section $495.310(\mathrm{~g})(1)(\mathrm{i})(\mathrm{B})$ to be clear.

Comment: Some commenters indicated that CMS should specify an alternative source of charity care data that States may use so that Medicare and Medicaid incentive payments can be determined appropriately. Others commented that while CMS has proposed the Medicare cost report, Medicaid cost report data, MMIS data, hospital financial statements, and accounting records to determine Medicaid EHR incentives, there is no absence of State-level usable data to implement this definition.

Response: We agree that there are a number of data sources available at the State and hospital levels that would allow States to accurately capture charity care data for the purposes of calculating hospital EHR amounts. However, we have no vehicle for identifying which of these tools exist in individual States or across the country. Medicare cost reports, Medicaid cost report data, MMIS data, hospital financial statements, and accounting records are all items that we feel confident are accessible to all States and providers. Additionally, we believe that
States and their provider communities are better versed at determining the tools that will be most beneficial for their individual programs. As such, we included the standard items listed as auditable data sources, but did not prohibit the use of other appropriate auditable data sources. States must describe their auditable data sources in their SMHP and submit to CMS for review and approval.

After consideration of this comment, we are making no further additions to this section of the final rule.

Comment: One commenter asked whether the criteria for determining Medicaid eligible days and Medicaid managed care days in the Medicaid share portion of the hospital incentive payment calculation is the same criteria for determining Medicare DSH payments.

Response: The criteria for determining Medicaid eligible days and Medicaid managed care days for Medicare DSH and Medicaid managed care days for EHR incentive payments are not the same. Medicare DSH includes unpaid days, while the EHR incentive payment calculation requires the inclusion of only paid inpatient-bed days.

After consideration of this comment, we are making no further additions to this section of the final rule.

Comment: One commenter asked for clarification of the term "estimated" Medicaid inpatient bed days.

Response: We are unclear about the commenter's question. Specifically, the statute permits the use of "estimated" days in the Medicaid share portion of the EHR hospital incentive payment calculation. Therefore, we refer the reader to the hospital calculation at section 1903(t)(5) and section 495.310 of this rule.

After consideration of this comment, we are making no further additions to this section of the final rule.

Comment: One commenter requested that for purposes of accurately calculating and auditing the Medicaid Share, CMS should eliminate data provisions at 2080.18 of the State Medicaid Manual.

Response: We disagree. The provisions at 2080.18 of the State Medicaid Manual do not adversely impact the calculation or auditing of the Medicaid Share.

We have not made any changes to the regulation related to this comment.

Comment: On commenter requested that we include as an auditable data sources, data acquired through authorized trading partners, such as clearing houses, eligibility systems maintained by CMS, state Medicaid programs, and/or their agents. 
Response: We agree that there are a number of data sources available that would allow States to accurately data for the purposes of calculating the Medicaid Share. However, we have no vehicle for identifying which of these tools exist in individual States or across the country. Medicare cost reports, Medicaid cost report data, MMIS data, hospital financial statements, and accounting records are all items that we feel confident are accessible to all States and providers. Additionally, we believe that States and their provider communities are better versed at determining the tools that will be most beneficial for their individual programs. As such, we included the standard items listed as auditable data sources, but did not prohibit the use of other appropriate auditable data sources.

After consideration of this comment, we are making no further additions to this section of the final rule.

Comment: One commenter asked whether the Medicaid payment is based on an annually-calculated Medicaid Share, or is the Medicaid Share established in the base year only and to be applied to the duration of payments.

Response: For purposes of calculating the Medicaid hospital incentive, the Medicaid Share is established in the base year.

After consideration of this comment, we are making no further additions to this section of the final rule.

c. Alternative and Optional Early State Implementation to Make Incentive Payments for Adopting, Implementing, or Upgrading Certified EHR Technology

Unlike Medicare, Medicaid has no statutory implementation date for making EHR incentive payments. In our proposed rule we discussed the fact that some States might be prepared to implement their programs and make EHR incentive payments to Medicaid providers in 2010 for adopting, implementing, or upgrading certified EHR technology. We proposed to allow States to initiate implementation of these payments to Medicaid EPs and hospitals after the effective date of the final rule if they could successfully demonstrate to CMS that they are ready to make timely and accurate payments through the SMHP. States would include an additional attestation for providers assuring that they are not accepting payment in any other State.

We also proposed that to be approved for early implementation, a State would be required to have an electronic system for provider registration capable of collecting the relevant information (this information is identified in section II.A.5.c of this final rule, where we describe the data collection requirements).

Participating States would be responsible for transmitting the required data to CMS so that CMS could ensure that no duplicate payments were made to providers. We proposed to use the single provider election repository described in section II.A.5.c. of this final rule to assure no duplicative payments were made between States.

We did not propose that States would be able to make early payments to meaningful users. Rather, our proposal was intended to offer Medicaid providers an early opportunity for capital so that they would be more likely to have the certified EHR technology required to demonstrate meaningful use in successive periods. We stated that since hospitals may qualify under both programs, we hoped that they would use the early capital to qualify as meaningful users under the Medicare program in the first year.

Comment: We received comments suggesting that our proposal on early State implementation creates unreasonable pressure on States, particularly given the status and timeline of the ONC rule on certification criteria.

Response: We agree with commenters. We proposed this option in order for States with very mature programs to proceed with early incentive payments for adoption, implementation, and upgrading certified EHR technology. However, in considering the complexity associated with States establishing an electronic registration system (which would only be temporary), as well as the fact that very few providers (if any) will have certified EHR technology early enough for this option, we believe that this may not be an efficient, costeffective option for many States.

Consequently, as a result of these comments, we are removing this option. States will not be permitted to make payments until January 2011.

Additionally, we wish to reiterate that States must have a SMHP approved by CMS before making any payments to EPs and eligible hospitals.

\section{d. Process for Making and Receiving} Medicaid Incentive Payments

The process for making payments involves coordination between Medicare and State Medicaid agencies to avoid duplication of payments, prevent fraud and abuse, and create program efficiencies to encourage adoption. While we have responsibility regarding payments to Medicare EPs and eligible hospitals, State Medicaid agencies (or their contractors) are fully responsible for administering and disbursing the incentive payments to Medicaid eligible providers.

We proposed to require that EPs make a selection between receiving incentive payments through either the Medicare or Medicaid EHR incentive programs. Medicaid EPs who practice in multiple States would be required to choose only one State from which to receive Medicaid incentive payments in each payment year. (We note that readers should also refer to section section II.A of this final rule for additional information regarding the EHR reporting period and the single provider election repository).

As we noted in the proposed rule, the statute anticipates coordination between the Medicare and Medicaid EHR incentive programs to ensure no duplicate payments are made to EPs (see 1903(t) and 1848(o)(1)(D)(iii).

Additionally, section 1848(o)(1)(B) of the Act requires that Medicare incentive payments for eligible professionals begin no earlier than 2011. While the Medicaid provisions have no statutory start date, before States may begin implementing the Medicaid EHR incentives, CMS, and ONC need to provide further direction to States in the form of rulemaking and other policy guidance. To that end, Medicaid will not begin to provide 100 percent FFP for incentive payments any earlier than January 1, 2011. This also gives CMS, ONC, and States an opportunity to coordinate between Medicare and Medicaid, which will simplify administrative complexity in the EHR incentive program and facilitate provider adoption.

Under this final rule Medicaid EPs, as discussed in section II.D.5 and II.A.5.C, will enroll in the program through the single provider election repository. Once an EP selects the Medicaid EHR incentive program, States must have a system for reporting and tracking necessary information to qualify an EP for an incentive payment. In addition, as detailed in $\S 495.316$ States are required to submit to CMS data on the number, type and practice location(s) of providers who qualified for an incentive payment on the basis of having adopted, implemented, or upgraded certified EHR technology or who qualified for an incentive payment on the basis of having meaningfully used such technology as well as aggregate deidentified data on meaningful use. States' systems and processes must receive prior approval, concurrent with the requirements described in section II.D.8 of this final rule for review and approval of the SMHP.

The specific timeframes for EPs and eligible hospitals to report and submit 
the required information in order to demonstrate they have adopted, implemented, or upgraded certified EHR technology, as well as meaningful use of such EHR technology are discussed in section II.A.1.e. of this final rule. As discussed in that section, for the first payment year based on meaningful use, the reporting period for eligible hospitals and EPs will be a continuous 90-day period that both starts and ends within the payment year. As long as the period spans the 90-day continuous period and ends within the payment year (fiscal year for hospitals, calendar year for EPs), the reporting period can begin at any time during such payment year. States also are expected to process payments on a rolling basis. We will issue further guidance regarding the timing expectations needed for State systems to coordinate with CMS and make timely payments

Comment: Several commenters were concerned that Medicaid EPs and eligible hospitals that qualify for incentive payments in their first year by adopting, implementing or upgrading certified EHR technology are not afforded the same flexibility as Medicare EPs and eligible hospitals in their second payment year. The commenters wrote that they would be required to demonstrate meaningful use for the full year, rather than 90 days in their second payment year, (even though it will be their first year demonstrating meaningful use). The commenters recommended that Medicaid EPs and eligible hospitals be subject to a 90-day reporting period in their second payment year when it is the first year they are demonstrating meaningful use.

Response: We agree with the commenters and as discussed in section II.A., we clarify that there is no EHR reporting period for adopting, implementing, or upgrading certified EHR technology for Medicaid provider's first payment year. In order to offer parity with Medicare providers who must achieve meaningful use in the first year over a 90-day period and over 12 months in subsequent years, the same policy will apply to Medicaid providers. In other words, Medicaid providers in their second participation year (or in their first payment year if they are qualifying based on meaningful use) shall demonstrate meaningful use over a 90-day reporting period and over 12months for their third and subsequent years.

\section{e. Avoiding Duplicate Payment}

In our proposed rule, we discussed the statutory requirement at section 1903(t)(7) of the Act that the Medicare and Medicaid programs coordinate payments to avoid duplication, and that CMS and the States coordinate payments through a data matching process, utilizing NPIs to the extent practicable. We also discussed section 1903(t)(2) of the Act, which states that Medicaid EPs must waive rights to Medicare incentive payments under sections 1848(o) and 1853(l) of the Act; hospitals, however, may qualify for incentives under both programs. We also proposed requirements under the review and approval of SMHPs in part 495 subpart D for States to verify that providers meet these requirements.

In section II.A of this final rule, we discuss the final requirements we are adopting in order to avoid duplicate payments in the Medicare and Medicaid incentive programs. We also respond to comments in that section (see section II.A.5.c. of this final rule). As discussed in that section of the final rule, to ensure against duplicate incentive payments, we believe three conditions are required: (1) Knowing which EHR incentive program a provider has selected, (2) uniquely identifying each provider participating in each incentive program; and (3) ensuring that each State has access to the information on which EPs or hospitals intend to receive incentive payments from another State, or from the Medicare program.

To achieve all three of these conditions, we will collect this data in a single provider election repository. Next, in administering each State

Medicaid EHR incentive program, States will cross-check for potential duplicative payments through the data available to them through the single provider election repository, which is based on the NPIs. We believe that this coordinates with our requirements that a State must have an approved SMHP that will include a mechanism for crosschecking this information prior to payment.

f. Flexibility for EPs To Alternate Between Medicare and Medicaid EHR Incentive Programs One Time

We refer readers to section II.A.5.b of this final rule, which discusses rules that would allow Medicare and Medicaid EPs to make one EHR incentive program election change prior to the 2015 payment year, and not to permit any switching after the 2014 payment year. Under such a proposal, even if an EP initially received incentive payments under the Medicare program, such an EP could still switch to the Medicaid program one time prior to 2015 (assuming the professional meets all eligibility criteria for the Medicaid incentives program). Similarly, an EP who initially selected the Medicaid EHR incentive program could switch to the Medicare program one time prior to 2015. (In other words, the last payment year an EP could switch would be the 2014 payment year.)

Comments received on these policies are addressed in section II.A.5.b. of this final rule.

\section{g. One State Selection}

In the proposed rule, we proposed that EPs and hospitals with multi-State Medicaid practice locations annually pick only one State from which to receive incentive payments. In other words, a provider would not be able to receive incentive payments from more than one State in the same year.

Medicaid EPs and hospitals could annually change the State they select when they re-attest to program requirements.

We considered the possible impact of this proposed approach with respect to patient volume calculations on Medicaid EPs and hospitals in border State areas, stating that because the Medicaid incentive payment for EPs will remain the same-regardless of whether they receive payment from one State or from multiple States-we did not think the administrative complexity associated with dividing and administering payments between or among more than one State could be justified. We recommended, however, that States consider border State providers when developing their policies on patient volume and the attestation methodology. We afforded additional flexibility in the patient volume at proposed $\S 495.306$ to account for unique circumstances and data collection.

Comment: Providers inquired whether it is permissible for an EP who practices in more than one State to aggregate patient encounters in order to achieve the 30 percent Medicaid patient volume criteria.

Response: First, it is not clear that aggregating patient volume across States will be an issue once EPs actually begin tallying up patient volume. Patient volume is calculated as a percentage, and not an absolute number. Thus, it does not appear that, but for aggregating patient volume across multiple States, an EP would not be able to qualify for incentive payments in any State. For example, if an EP has 10 percent patient volume in one State (10 of 100 encounters are Medicaid) and 20 percent patient volume in a second State (20 of 100 encounters are Medicaid), this does not add up to 30 percent patient volume (but, rather, results in a 15 percent patient volume 
as a result of dividing 30 by 200). To restate, we do not believe that an $\mathrm{EP}$ will need to sum patient encounters across multiple States in order to reach the 30 percent patient volume-as in order to reach this patient volume threshold, the EP would likely meet the 30 percent in at least one State. Indeed, it appears that the only benefit of aggregating patient volume across States would be to permit an EP who has more than a 30 percent patient volume in one State to receive incentive payments from another State in which s/he does not meet the 30 percent threshold.

Nevertheless, we recommend that States consider the circumstances of border State providers when developing their policies and attestation methodologies. To afford States maximum flexibility to develop such policies, we will not be prescriptive about whether a State may allow a Medicaid EP to aggregate his/her patients across practice sites, if the State has a way to verify the patient volume attestation when necessary. States will propose their policies and attestation methodologies to CMS for approval in their State Medicaid HIT plans.

We are making no additional revisions to this section of the rule as a result of this comment.

\section{Single Provider Election Repository and State Data Collection}

We refer readers to section II.A.5.c of this final rule for a discussion of the single provider election repository and the comments received on this policy. As discussed in that section, the repository will collect a minimum amount of information on all EPs and hospitals to prevent duplicative payments and coordinate technical assistance.

6. Collection of Information Related to the Eligible Professional's National Provider Identifier and the Tax Identification Number

In our proposed rule, we proposed that EPs in multiple group practices or multiple types of practice locations would be required to select one TIN for Medicaid EHR payment disbursement. In other words, such EPs would not be permitted to require a State to divide payments among different practices or practice locations based upon group TINs. We explained that requiring EPs to use only one TIN would reduce administrative complexity, as it would ensure that States are not put in the position of dividing payments in any way an EP requests (such as by patient encounters or amount contributed to EHR technology). We also stated that requiring reimbursement to be made to one TIN would reduce opportunities for fraud or abuse, as States would be able to cross-check EP and TIN combinations more easily to verify EP attestations.

We also stated that although the State would not divide payments among the various TINs of an individual EP, Medicaid EPs could, themselves, decide to divide payment. These EPs could independently distribute funds among their respective group practices or practice locations after the initial disbursement from the State to their designated TIN.

Comment: We received comments suggesting that EPs should be allowed to proportion their payments and give multiple TINs

Response: For these reasons advanced in the proposed rule, we believe that permitting an EP to divide the incentive payment among multiple TINs would introduce an unnecessary level of administrative complexity into this temporary program. It also could increase the opportunities for fraud and abuse as it would be more administratively cumbersome for States to track multiple payments (to ensure correct payments) and to track and verify multiple eligibility-related EP attestations. Once a payment is disbursed from the State, nothing precludes the EP from further disbursing the incentive payment, subject to the applicable fraud, waste, and abuse laws, regulations, and rules.

After consideration of the public comments received, we are finalizing these provisions as proposed.

\section{Activities Required To Receive} Incentive Payments

\section{a. General Overview}

As we discussed in our proposed rule, to qualify to receive a first year Medicaid incentive payment, section 1903(t)(6)(C)(i) of the Act indicates that EPs and eligible hospitals must demonstrate that they are "engaged in efforts to adopt, implement, or upgrade certified EHR technology." For providers who meet this standard in their first year of participation in the Medicaid incentive program, in subsequent years of participation, they must then demonstrate "meaningful use of certified EHR technology through a means that is approved by the State and acceptable to the Secretary," and that may be based upon the methods employed under the Medicare incentive payments to physicians and hospitals, per sections 1848(o) or 1886(n) of the Act. b. Definitions Related to Certified EHR Technology and Adopting, Implementing or Upgrading Such Technology

\section{(1) Certified EHR Technology}

As noted previously, in order to receive a Medicaid incentive payment the EHR technology must be "certified." Section 1903(t)(3) of the Act defines "certified EHR technology" as "a qualified electronic health record (as defined in section 3000(13) of the Public Health Service Act) that is certified pursuant to section 3001(c)(5) of such Act as meeting standards adopted under section 3004 of such Act that are applicable to the type of record involved (as determined by the Secretary), such as an ambulatory electronic health record for office-based physicians or an inpatient hospital electronic health record for hospitals)." In section II.A of this final rule, for both Medicare and Medicaid, we discussed incorporating ONC's definition of certified EHR technology.

\section{(2) Adopting, Implementing or Upgrading}

Unlike the Medicare incentive programs, the Medicaid program allows eligible providers to receive an incentive payment even before they have begun to meaningfully use certified EHR technology. These providers may receive a first year of payment if they are engaged in efforts to "adopt, implement, or upgrade" certified EHR technology. In proposed § 495.302, we define adopting, implementing or upgrading certified EHR technology as the process by which providers have installed and commenced utilization of certified EHR technology capable of meeting meaningful use requirements; or expanded the available functionality and commenced utilization of certified EHR technology capable of meeting meaningful use requirements at the practice site, including staffing, maintenance, and training.

For the purposes of demonstrating that providers adopted, implemented, or upgraded certified EHR technology, we proposed that Medicaid EPs and hospitals would have to attest to having adopted, (that is, acquired and installed) or commenced utilization of (that is, implemented) certified EHR technology; or expanded (that is, upgraded) the available functionality of certified EHR technology and commenced utilization at their practice site. We proposed that States would be responsible for ensuring that processes are in place to verify that providers have actually adopted, implemented or upgraded certified EHR technology, patient volume, as well as 
other requirements in this section, including verifying that attestations are consistent with methodologies to combat fraud and abuse (see proposed $\S 495.366$ through 370, Financial Oversight, Program Integrity, and Provider Appeals). We proposed that the State's SMHP would detail these processes.

The CMS Medicaid Transformation Grants demonstrated the many challenges that exist to adopting EHR technology. EHR system availability is not the same as EHR system utilization. It is for that reason that we proposed to include staff training and efforts to redesign provider workflow under the definition of implementing certified EHR technology. We explained that success is not simply defined by the acquisition and installation of new or upgraded certified EHR technology, but more importantly by providers demonstrating progress towards the integration of EHRs into their routine health care practices to improve patient safety, care, and outcomes.

In establishing criteria for the "adoption" portion of the "adopt, implement, or upgrade" requirement, we proposed that there be evidence that a provider demonstrated actual installation prior to the incentive, rather than "efforts" to install. We stated that this evidence would serve to differentiate between activities that may not result in installation (for example, researching EHRs or interviewing EHR vendors) and actual purchase/ acquisition or installation. As Medicaid incentive payments are intended to stimulate meaningful use of EHR technology, we stated our belief that the payments need to result in tangible adoption, implementation, or upgrading of certified EHR technology. We stated that States would be responsible for verifying this evidence of EHR adoption.

In establishing criteria for the "implementation" portion of "adopt, implement or upgrade" requirement, we proposed that "implementation" mean that the provider has installed certified EHR technology and has started using the certified EHR technology in his or her clinical practice. Implementation activities would include staff training in the certified EHR technology, the data entry of their patients' demographic and administrative data into the EHR, or establishing data exchange agreements and relationships between the provider's certified EHR technology and other providers, such as laboratories, pharmacies, or HIEs.

In establishing the criteria for the "upgrade" portion of "adopt, implement or upgrade" requirement, we proposed "upgrade" to mean the expansion of the functionality of the certified EHR technology, such as the addition of clinical decision support, e-prescribing functionality, CPOE or other enhancements that facilitate the meaningful use of certified EHR technology. We proposed that States describe in their SMHPs the process that would be in place for ensuring that providers have actually adopted, upgraded or implemented certified EHR technology. We encourage States to consider the submission of a vendor contract from providers to ensure the existence of EHR technology.

Comment: Several commenters recommended that CMS clarify if "upgrade" does or does not apply to an already certified EHR. They recommended that CMS confirm that an upgrade is intended to enable a provider to expand existing functionality of an EHR so that it meets the new certification criteria.

Response: To clarify this question, an example of upgrading that would qualify for the EHR incentive payment would be upgrading from an existing EHR to a newer version that is certified per the EHR certification criteria promulgated by ONC related to meaningful use. Upgrading may also mean expanding the functionality of an EHR in order to render it certifiable per the ONC EHR certification criteria.

We are making no additional revisions to this section of the final rule as a result of this comment.

Comment: Commenters wrote that given that adopt/implement/upgrade (AIU) involves significant practice workflow redesign and that the States' overarching goal is to increase the level of provider participation, the commenters recommended that CMS require only AIU for participation Year 1 and Year 2. They further recommended that CMS allow AIU compliance to be further defined as the provider developing, submitting, and following a customized plan for the necessary workflow changes with timelines (whose development can be assisted by the Regional Extension Centers); the provider would have to meet their timelines for each year in Stage 1 to qualify for the incentive payment; and the AIU plan timelines would have to be structured so submission of HIT and clinical quality measures would begin in Stage 2 .

Response: The statute at section 1903(t)(6)(C) permits Medicaid providers to receive the EHR incentives for adopting, implementing or upgrading to certified EHR technology in their first participation year. A provider's first participation year may be any year between 2011 through 2016 .
In their State Medicaid HIT Plans, States will propose to CMS how they will audit and oversee Medicaid providers' adoption, implementation or upgrading to certified EHR technology. States should propose further details to CMS about how they will verify that providers have met this requirement.

After consideration of the comments received, we do not believe that just the development and submission of an implementation plan for EHR adoption is a significant enough commitment to warrant the AIU incentive payment. There is nothing binding, nor is there any financial contribution towards such a plan.

We are making no additional revisions to this section of the final rule as a result of this comment.

Comment: Many commenters suggested that they believe the goal of this incentive is to help defray some of the costs of adopting, implementing, and upgrading to certified EHR technology. As such, the commenters believe "proof" of AIU should not require completion of AIU but demonstrated commitment to AIU. For example, a proof of purchase, a schedule for training and implementation, and periodic reporting from practices on progress on the schedule could suffice. The commenters requested that States have flexibility to define what is sufficient to trigger payment.

Response: States should provide details to CMS on how they will audit and oversee Medicaid providers' adoption, implementation or upgrading to certified EHR technology in their SMHP. States' SMHP should include further details about how they will verify that providers have met this requirement. However, while States may propose how they will determine what AIU activities are sufficient for the EHR incentive payment; CMS must approve their proposals via the SMHP. The definitions included in this final regulation by CMS for adopt, implement or upgrade do imply completion of at least one of the three tasks. A proof of purchase or signed contract would likely be an acceptable indicator of EHR adoption per the States. Implementation is on-going, therefore working actively with Regional Health IT Extension Centers on implementation, completion of specific benchmarks or other activities towards implementation would be acceptable.

We are making no additional revisions to this section of the final rule as a result of this comment.

Comment: A commenter recommended that State Medicaid agencies provide eligible hospitals with 
the maximum incentive payments for their first two payment years as a limited source of capital for AIU.

Response: The Medicaid hospital calculation was part of the HITECH statute and not defined by CMS. Eligible Medicaid hospitals can receive their first year's payment for AIU and not meaningful use, but must meet the meaningful use requirement in their second and subsequent participation years.

We are making no additional revisions to this section of the final rule as a result of this comment.

Comment: A commenter recommended that a Medicaid provider be permitted to qualify for their first year Medicaid EHR incentive even if they have not actually installed certified EHR technology but have spent or are committed to spend an amount equal to at least the lesser of $\$ 50,000$ or 5 percent of the Medicaid EHR incentive amount.

Response: In consideration of the comments, we are clarifying that the final definition of adopt, implement or upgrade is inclusive of providers' acquisition, such as a purchase, of a certified EHR. Providers will be responsible for providing documentation which substantiates AIU as required by the State Medicaid Agency.

We are revising the definition of adopt, implement, and upgrade as a result of these comments, see section 495.302.

\section{c. Other General Terminology}

In our proposed rule, we proposed definitions for "EHR reporting period" and "payment period," stating that these definitions relate to the requirements for Medicaid EPs participating in the Medicaid EHR incentive program. As discussed previously, the reporting period is significant for EPs and eligible hospitals because it will define the period during which the provider must demonstrate meaningful use of certified EHR technology. The reporting period also is significant for States, because States will refer to such reporting periods in assuring us that providers are eligible to participate in the Medicaid EHR incentive program. (Requirements relating to the components that must be included in the SMHP were specified in proposed § 495.332). In the proposed rule, we specified that States would need to refer to the providers' reports of the activities that establish their efforts to adopt, implement, or upgrade certified EHR technology. Similarly, once meaningful use of EHR technology is required, States would need to refer to providers' reports on meaningful use, including reporting of clinical quality measures (see section II.A. of this final rule for requirements for clinical quality measures), in accordance with the appropriate EHR reporting period. States could not appropriately make incentive payments in the absence of such reporting.

We proposed that States would be required to validate to us that the Medicaid EPs and hospitals meet all of the eligibility criteria to qualify for Medicaid incentive payments, including the applicable patient volume

thresholds, hospital-based requirements, and all other requirements. States would develop their own administration, payment and audit processes, and as described in $\S 495.332$, we would require that States include in their SMHPs how they would obtain Medicaid EPs' and hospitals' attestations of eligibility to qualify for the Medicaid incentive payments. We proposed that permissible means for ensuring patient volume and all of the requirements described in this section would include survey, attestation, or the creation of special codes on claims, subject to our prior approval.

Section 1903(t)(6)(C)(ii) of the Act also indicates that in the case of an early adopter, that is, a Medicaid EP or eligible hospital that has already adopted certified EHR technology, such provider would receive payment in the first year and all subsequent years of the incentive program by demonstrating meaningful use.

In our proposed rule, we discussed our expectation that the bar for demonstrating meaningful use of certified EHR technology will rise in years to come. In this final rule, meaningful use and its evolving criteria are discussed in section II.A. In order to receive Medicaid incentive payments, providers will be required to demonstrate (and States will be required to track and validate) meaningful use, as described in section II.A.2. of this final rule. In section II.D.8 of this final rule, we also discuss our policies regarding States' ability to require additional objectives in the demonstration of "meaningful use," or otherwise add to the Federal definition of meaningful use. We also discuss the requirement that States receive prior approval of any such additions.

As we discussed in the proposed rule, we believe that States should carefully consider how to build upon their existing EHR activities and

infrastructure without deterring eligible Medicaid providers from participating by compelling them to use a particular system. We encourage States that were awarded Federal HIT/EHR grants, such as the Medicaid Transformation Grants, to the extent practicable, to connect the tools and infrastructure developed under their Federal grant funds with providers' efforts to adopt, implement, and upgrade certified EHR technology and to become meaningful users of certified EHR technology. We will be evaluating States' HIT Planning Advanced Planning Documents (PAPDs) and SMHPs with this objective in mind, as described section II.D.8 of this final rule.

As we discussed in the proposed rule, States' system requirements for monitoring meaningful use must include the capacity to determine the appropriate stage of meaningful use and the appropriate incentive payment amount, depending upon the providers' payment year. In other words, regardless of the calendar year, a provider's first year as a participant in the Medicaid EHR incentive program is when that provider must demonstrate either adoption, implementation, upgrading or meaningful use of certified EHR

technology. States' systems must be able to track a provider's year of entry into the Medicaid EHR incentive program to determine the correct eligibility criteria and generate the appropriate Medicaid incentive payments.

Once States are giving providers the Medicaid EHR incentive payments for being meaningful users of EHRs, and in 2012 begin receiving clinical quality measures data from those providers, we proposed that States would be required to share any such reported data with CMS in an aggregated, de-identified manner, on an annual basis. The timetable and format for sharing the clinical quality measurement data would be provided to States in future policy guidance issued by CMS. States' failure to submit these required reports to us could result in discontinued funding or disallowances. See the discussion below regarding the SMHP and the State reporting requirements. We would use the States' reports, including data on meaningful use and clinical quality measures, in order for the Secretary to fulfill her responsibilities to Congress under section 1903(t)(10) of the Act. This provision requires that the Secretary report to Congress on the improvement of health outcomes, clinical quality, or efficiency as a result of implementing this program. For hospitals eligible for both the Medicare and Medicaid EHR incentive programs, we proposed that we would use the meaningful use measures hospitals report to us to make quality data on Medicaid eligible hospitals available to States.

Comment: Commenters requested clarification on the reporting period for 
adopting, implementing, and upgrading, and whether this period is similar to the 90-day period for demonstrating meaningful use in the first year.

Response: As discussed earlier, we are clarifying that there is a no reporting period for AIU for the providers' first participation year. However, there is a 90-day reporting period for the first participation year in which Medicaid providers qualify by demonstrating meaningful use. The rationale is that we understand that not all AIU activities require 90 days, such as EHR acquisition. States will determine how they plan to implement this requirement.

As a result of this comment and a similar comment above, we are revising section 495.4 to indicate that there is no EHR reporting period for adopting, implementing, or upgrading in Medicaid providers' first participation year, if they qualify based on AIU, and there is a 90-day reporting period for both the first year that a Medicaid provider demonstrates MU (regardless of whether they demonstrated AIU in their first participation year or are qualifying based on MU in their first participation year).

Comment: Several commenters requested that CMS clarify the process that will assure Medicaid access to Medicare meaningful use data, at a minimum for (1) hospitals who receive both Medicaid and Medicare payments and (2) eligible providers that may switch once between the Medicaid and Medicare incentive programs. Commenters requested that CMS provide States with Medicare quality reporting/data in a timely fashion (for example, within 30 days of receipt of such information). Alternatively, commenters suggested that the providers could be required to report separately to both Medicare and Medicaid.

Response: We are finalizing our policy as proposed. We believe that it would represent an undue burden on hospitals eligible for both EHR incentive payments to report their data to both CMS and the States. We will issue further guidance about how States will be able to access the meaningful use data submitted to CMS by hospitals eligible for both Medicare and Medicaid EHR incentive payments in order for the State to meet its audit and oversight requirements. It is not clear to CMS why a State would require access from CMS to an eligible professional's meaningful use data if they were a Medicare EHR Incentive Program participant in the prior year. States can only base a Medicaid provider's EHR incentive payment, as it pertains to meaningful use, on the current participation year's EHR reporting period.

We are making no additional revisions to our regulations as a result of this comment.

Other than the changes explained above, we are finalizing the remainder of our proposed policies as they were proposed.

\section{d. Quality Measures}

We refer readers to section II.A.3 of this final rule-for a discussion of the clinical quality measure reporting required for demonstrating meaningful use of certified EHR technology. As discussed previously, we intend to update our definition of meaningful use biennially, and we expect that our updated, Stage 2 definition would include additional Medicaid clinical quality measures to be reported from EHRs. We intend to work with the quality measurement community to develop these Stage 2 quality measures (see section II.B.1.d. of this final rule).

Comment: Several commenters believe that the current clinical measures do not reflect key clinical services and issues for the Medicaid population, including behavioral health, dental, long-term care, and care coordination (particularly across physical and behavioral health care).

The commenters recommend that CMS work with the Medicaid Medical Directors and ONC and consider the development and inclusion of clinical and non-clinical quality measures that are more representative of the Medicaid population. Alternatively they wrote that CMS and ONC should have a "placeholder" to accommodate data and interoperability for these measures. Commenters wrote that the areas with gaps are behavioral health, dental care, long-term care, special needs populations and care coordination, particularly across physical and behavioral health. The commenters recommended that new clinical quality measures be added as "placeholders" for care provided by non-eligible, but critical Medicaid providers, such as Community Mental Health Centers, Home Health, and Renal Dialysis Centers.

Many commenters noted that with regard to pediatric clinical quality measures, they recommend that firstyear measures focus on immunizations, diabetes, asthma, autism, and lead screening. They also recommend measures to introduce in 2012 and beyond to include smoking, obesity, disease- or condition-specific measures, and measures aimed at reducing disparities. They further recommended measures to introduce in 2013 and beyond include the development of clinical quality measures on psychology, child abuse, developmental delays, and efficiency measures.

Response: We agree that these measures (listed directly above) have clinical relevance for providers. However we are aligning with the Medicare Stage 1 meaningful use provisions regarding publication and opportunity for public comment on quality measures before they are finalized. We are not including additional meaningful use objectives and measures that were not discussed in the proposed rule.

Comment: Several commenters believed that the quality measures proposed in the interim rule do not match the quality measures that HRSA currently requires FQHCs to report. The commenters would like to work with CMS and HRSA to move forward and harmonize the quality measures by 2013 but requested that until quality measures are harmonized across the federal government system, FQHCs and the EPs who qualify and assign their Medicaid incentive payments to the FQHC should be allowed to report on the current HRSA measures.

Response: Meaningful use applies to each individual EP. Therefore the HRSA quality measures, which are facilitybased, not necessarily NQF-endorsed, or reportable from EHRs are not an acceptable alternative for EPs who practice at an FQHC. Furthermore, as explained in section II.A. of this final rule, we are not including in the final rule quality measures that were not included in the proposed rule. To ensure uniformity across both programs, we have adopted this same policy for Medicaid. We believe it is important to offer Medicaid providers and stakeholders the same opportunity for public comment on quality measures.

We agree with the goal of harmonizing quality measure reporting across Federal programs and will engage with stakeholders and experts to address this priority as part of the development of the Stage 2 definition of meaningful use.

We are finalizing these provisions as proposed and we will continue to work to identify, and develop electronic specifications for additional clinical quality measures that address current gaps, such as long-term care, behavioral health, pediatrics and oral health for Stage 2 of meaningful use. In particular, we recognize the lack of endorsed oral health clinical quality measures, with identified and tested electronic specifications. This poses a challenge for dentists, who are eligible professionals for the Medicaid EHR 
incentives, to demonstrate meaningful use, other than with the general, profession-neutral measures.

While an eligible professional can report "zero" for the denominator of any measure for which s/he does not have any relevant patients, we will work to include in Stage 2 of meaningful use, clinical quality measures that would provide useful data to CMS and States on oral health care as reported by EHRs.

In addition, in order to minimize provider burden, and to maximize measure reporting efforts and resources, we seek to align the quality measures for the Stage 2 definition of meaningful use with other quality measures development and reporting related to health care reform and other CMS quality measures programs, as appropriate and feasible. Stage 1 of meaningful use is limited to objectives and measures that are already in existence, not those still under development. Measures will be included that have operational relevance to the care provided to Medicaid and CHIP beneficiaries by eligible professionals and hospitals defined in the HITECH Act.

8. Overview of Conditions for States To Receive Federal Financial Participation (FFP) for Incentive Payments and Implementation Funding

Section 1903(a)(3)(F) of the Act provides that States are eligible for 100 percent FFP for direct payment expenditures to certain Medicaid EPs and eligible hospitals to encourage the adoption and use of certified EHR technology. States are also eligible for 90 percent FFP for reasonable administrative expenses, contingent on State compliance with the following requirements: (1) Using the funds to administer Medicaid incentive payments for certified EHR technology, including tracking of meaningful use by Medicaid EPs and eligible hospitals; (2) conducting oversight of the Medicaid EHR incentive program, including routine tracking of meaningful use attestations and reporting mechanisms; and (3) pursuing initiatives to encourage the adoption of certified EHR technology for the promotion of health care quality and the exchange of health care information. (See 1903(t)(9) of the Act.)

This section of the final rule discusses the requirements for States to request FFP from CMS for the Medicaid EHR incentive program. Additionally, this section is closely connected to the requirements outlined in Financial Oversight, Program Integrity and Providers Appeals for purposes of oversight and accountability.
In proposed § 495.302, we defined terms used in the Medicaid subpart of the regulations governing State requests for FFP. Although some of these terms have been defined in other portions of our regulations, for ease of reference, and in order to define the terms in this specific context, we proposed to separately include definitions in part 495.

We proposed to include in our regulations the requirements that in order to qualify to receive FFP for administering the incentive program, States must develop a SMHP, an HIT Planning APD (PAPD), and an HIT Implementation APD (IAPD). These documents lay out the process used by States to implement and oversee the EHR incentive program, and will help States to construct an HIT roadmap to develop the systems necessary to support eligible providers in their adoption and meaningful use of certified EHR technology. The development of a SMHP (see also $\S 495.332$ ) provides States with the opportunity to analyze and plan for how EHR technology, over time, can be used to enhance quality and health care outcomes, while reducing overall health care costs. The uses of EHR technology can be integrated with existing State resources to achieve these goals.

We provided guidance in a State Medicaid Director's (SMD) letter on September 1, 2009, on this process and the State efforts necessary to receive the 90 percent FFP for planning-related expenditures. As stated in that letter, and as further required through this rulemaking, our review process ensures that States are complying with requirements of the HITECH Act, and that they demonstrate to the "satisfaction of the Secretary" that they are using the funds in the manner anticipated by the law. For example, because of our oversight

responsibilities, simply proposing activities would not ensure the 90 percent FFP. As explained in the letter, and as further reflected in this rulemaking, we must review and prior approve all elements of the State's SMHP, and APD documents, and work with States to determine the appropriate level and type of FFP.

States are required to submit these advance planning documents in order for us to approve receipt of the 90 percent Federal match. Specifically, prior approval is required for the HIT PAPD (see also § 495.336). The deliverable resulting from the HIT PAPD is the SMHP. The SMHP must be reviewed and approved before it is included in an IAPD (see also $\S 495.338$ ). The IAPD also must be prior approved. Until approval is granted States cannot draw down funds.

For purposes of the Medicaid EHR incentive program, we believe there are two high-level phases in the process of planning and implementing the incentive program, as well as the promoting the adoption of EHR. Phase I includes initial planning, including an assessment of the State EHR environmental landscape, and development of the SMHP. As explained in our September 1, 2009 letter, the vehicle for informing us of Phase I activities is the HIT PAPD, and indeed, over 40 States have already submitted their PAPDs and have received funding to begin Phase I activities. Phase II then involves further development and full implementation of the SMHP. Consequently, the HIT IAPD is the vehicle for reporting of Phase II activities. As discussed in the SMD letter, and as further reflected in this final rule, States need to receive prior approval of their planning documents. In fact, we have already worked closely with the majority of States in developing their HIT PAPDs, prior to them initiating their EHR planning activities, and we expect this close coordination to continue between the States and CMS.

Also, as proposed, in this final rule we will require States to obtain prior written approval of funding, planning documents, proposed budgets, project schedules, and certain implementation activities that a State may wish to pursue in support of the Medicaid EHR incentive program to encourage the adoption and use of certified EHR technology in line with the 90 percent FFP available to States. To minimize the burden on States, we designed the prior approval conditions, and the prior approval process, to mirror what is presently used in support of acquiring automated data processing equipment and services in conjunction with development and operation of State MMIS (the State's automated mechanized claims processing and information retrieval system approved by CMS).

As proposed, this final rule (at 495.348) will require State Medicaid programs to comply with current procurement standards. Specifically, at 495.348 we have included language that accords with the procurement requirements in 45 CFR part 95 subpart $\mathrm{F}$ and incorporates many of the procurement standards previously contained in 42 CFR part 74 . Inclusion of these procurement requirements maintains the long-standing procurement standards and policies for State information technology contracts. 
Under these standards the State must ensure that when procuring HIT equipment and/or services, there is maximum practical open and free competition, and that any procured materials or services are obtained in a cost-effective manner. The regulations also make clear that the State, as the grantee, is responsible for meeting its contractual responsibilities under any of its procurements, and will not have recourse to the Federal government to settle or satisfy its contractual and administrative issues. Further, States must have written standards of conduct regarding the performance of its employees that are engaged in the award and administration of the HIT equipment/services contracts (including conflict of interest rules contained in 495.348(c)). States must have written procurement procedures that accord with 495.348(e) and a system for administering contracts in accordance with 495.348(f). Procurement contracts must meet the additional requirements contained in 495.348(g) as well as describe the conditions under which the contract may be terminated for default or because of circumstances beyond the control of the contractor (see 495.348(h)). Procurement contracts must include provisions allowing State and Federal access to the materials and staff of the contractor, in accordance with 495.348(i).

As was proposed, our final regulations at 495.346 also will require the State agency to allow the Department access to all records and systems operated by the State in support of the program. Final regulations at 495.352 impose reporting requirements on States to submit to the Department, on a quarterly basis, a progress report documenting specific implementation and oversight activities performed during the quarter. Regulations at 495.354 through 495.360 contain rules for charging equipment, nondiscrimination requirements, requirements for cost allocation plans, and requirements for ownership rights in software. Our rules would require termination of FFP in the case of States failing to provide access to information relating to any of the requirements we have included in this subpart. We believe the procurement and other rules discussed above are authorized under section 1902(a)(4) of the Act, as well as under section 1903(t)(9) of the Act requiring a State to conduct adequate oversight of its program, and use its funds to administer the incentive payments. In addition, any reporting and other requirements will assist us in submitting the reports that are required under section 1903(t)(10) of the Act, which requires us to monitor and report on the progress of implementation of the EHR provisions.

As proposed, State Medicaid agencies will be required to attest, as required by section 1903(t)(6)(A)(i) of the Act, that States make Medicaid incentive payments to a Medicaid EP or eligible hospital directly (or to an employer or facility to which such Medicaid EP or eligible hospital has assigned their Medicaid incentive payments) without any deduction or rebate. States must also attest that payments to an entity promoting the adoption of certified EHR technology, as designated by the State, will only be made if participation in such a payment arrangement is voluntary for the Medicaid EP involved, and if such entity does not retain more than 5 percent of such assigned Medicaid incentive payments for costs not related to such technology. (See 495.332 of our final rules). States are required to attest that the entire incentive payment has been forwarded to the eligible Medicaid provider, and that no Medicaid eligible professional or hospital is required to return any portion of the incentive payment to the State Medicaid agency. States must establish a process to ensure that any existing fiscal relationships with eligible professionals or hospitals to disburse the Medicaid incentive payments through Medicaid managed care plans does not result in payments that exceed 105 percent of the capitation rate, in order to comply with the Medicaid managed care incentive payment rules at $\S 438.6$ (c)(5)(iii) and a methodology for verifying such information.

Additionally, we are requiring that termination of funding approved under this proposed Part 495 subpart D or disallowance of FFP may result if the State fails to meet the requirements and undertakings of the approved PAPD, SMHP, and IAPD, or fails to provide access to the required information

Since section 4201 of the HITECH Act amends section 1903(a)(3) of the Act to provide for 90 percent FFP for costs associated with certain administrative activities performed by a State, we have allowed for claiming of such reasonable costs incurred on or after February 18, 2009, prior to publication of the final rule. Specifically, a State that can show that initial planning stages of moving the State in the direction of meaningful use of certified EHR technology through such activities as training efforts, staff support, or contracting with a vendor may potentially receive retroactive FFP back to the date in which these efforts began, with CMS approval, but not before February 18, 2009.
Comment: Several commenters expressed concerns about the timing of planning and implementation and request flexibility in this area.

Commenters indicated that there will be a need for ongoing planning while rules and guidelines are being promulgated. Commenters indicated that they envision a phased approach to implementation, and request that CMS permit simultaneous expenditure of both planning and implementation funds.

Response: We proposed specific requirements for States to request FFP from CMS for the Medicaid EHR incentive program modeled on the process States use to request FFP from CMS for Medicaid Management Information Systems technology projects. CMS proposed to utilize information and documentation that will result from the process described in this section to evaluate approaches proposed by States, track and monitor progress of implementation, and perform the statutory program and financial oversight required for this new program.

In establishing the requirements we believe States will have flexibility to request FFP for planning and implementation activities to implement the provisions of the EHR incentive program in a manner that is similar to and consistent with current approaches to receive enhanced FFP for MMIS systems under the Medicaid program. This will enable States to modify or adapt as changes occur during the planning and implementation phases envisioned under this proposed rule. Further, we believe that the information required is consistent with section 1903(t)(9) of the Act that States must demonstrate to the satisfaction of the Secretary that the State is conducting adequate oversight.

We agree with the need for flexibility in planning for the Medicaid incentive program, and the conduct of

implementation activities to ensure the program is successful in the long-term. We have added additional clarifying information in the sections regarding the HIT PAPD, HIT IAPD, As-needed HIT PAPD update and as-needed HIT IAPD update, Annual HIT IAPD requirements, and SMHP requirements. These clarifications are consistent with guidance issued in our State Medicaid Director's letter on September 1, 2009, which indicated that CMS anticipates a phased approach to planning and implementation activities.

Finally, for the final rule we are making numerous changes in order to be more specific and provide additional clarity regarding certain terms and 
requirements. These revisions are reflected here; however, regulations text is not updated since the concepts of these terms remain the same.

Clarifications are as follows:

We have further defined the terms "service oriented architecture (SOA)", or "service component based architecture" to indicate that they are a means of organizing and developing information technology capabilities as collaborating services that interact with each other based on open standards. We are defining this term in the context of health IT projects authorized under the Act to ensure that different systems and programming languages provide a basis for interoperability among and between applications that may reside on different platforms through a communication protocol to achieve health information exchange required under the Act. CMS anticipates that States will describe proposed HIT projects in the context of SOA principles, and intends to evaluate plans for health information exchange, and interoperable health IT based on these commonly used information technology principles.

We have also further defined the term "State self-assessment (SS-A)," a component of MITA, as a process that a State will use to review its Medicaid information technology strategic goals and objectives, measure its current baseline business processes and capabilities against defined MITA business capabilities, and develop targeted future capabilities to transform the Medicaid enterprise to be consistent with the MITA principles of interoperability and exchange of health information. Although we are including a definition of State self assessment in this final rule, we are deleting the requirement that a State provide the MITA SS-A, as we believe the as-is assessment supercedes the need for a separate MITA SS-A. However, we believe it is important to keep a definition of SS-A, because there is an inter-connection between activities accomplished under the Medicaid EHR Incentive Program and States' MMIS enhancements. For example, data exchanges between various State systems that comprise the Medicaid enterprise of the State might also support the State's administration of the EHR Incentive Program.

We are further defining MITA, because we expect that States will describe proposed health IT projects as well as their "as is" landscapes using MITA concepts and principles. We intend to evaluate States' proposed strategies and plans for development of Medicaid health information exchange and interoperable health IT using these
MITA principles, as applicable. These strategies and plans must be included in the State Medicaid Health Information Technology Plan (SMHP), a term discussed below. We have previously published a document entitled "MITA Framework 2.0" on the CMS Web site at http://www.cms.hhs.gov/

MedicaidInfoTechArch. The MITA

Framework 2.0 was developed by CMS in collaboration with State Medicaid agencies and information technology vendors to facilitate the adoption of information technology principles and practices that will lead to increased deployment of state-of-the-art technologies and improved management of the Medicaid program. States presently are utilizing MITA and the SS-A for Medicaid IT projects approved by CMS, and application of these principles for activities required under this proposed rule will not add additional burden to State efforts to adopt HIT as envisioned under the Section 1903(a)(3)(F) of the Act.

The MITA principles and tools foster integrated business processes and IT transformation for all States. It achieves this in part by demonstrating that planned enhancements to Medicaid systems, including MMIS, support State and Medicaid strategic goals and how intra-state systems other than the MMIS have been considered in developing the solutions. We believe that as States and providers implement EHRs, it will be necessary and essential to plan technology upgrades that will facilitate health information exchange with Medicaid providers receiving incentive funding.

We are further clarifying that we are defining the Medicaid Management Information System (MMIS) as it relates to specific requirements for Medicaid claims processing and information retrieval contained in current regulations at 42 CFR part 433 , subpart C. We proposed a definition of the term MMIS because it is the common term that CMS, State Medicaid agencies, and industry use to refer to the Mechanized Claims Processing and Information Retrieval Systems specified in section 1903(a)(3) of the Social Security Act. MMIS means the system of software and hardware used to process Medicaid claims from providers of medical care and services for the medical care and services furnished to recipients under the medical assistance program and to retrieve and produce service utilization and management information required by the Medicaid single State agency and Federal Government for program administration and audit purposes. The objectives of the MMIS include claims processing and retrieval of utilization and management information necessary for program administration and audit and must coordinate with other mechanized systems and subsystems that perform other functions, such as eligibility determination. The MMIS is also compatible with the claims processing and information retrieval systems used in the administration of the Medicare program.

We believe that States will utilize their MMIS extensively in administering the provisions of this proposed rule, including but not limited to payment and tracking of Medicaid incentive payments, access to data and information necessary to establish the vision for Medicaid health IT, and achieving interoperability and health information exchange envisioned in the Act.

In the proposed regulation at $\S 495.332$ we proposed a definition of the term State Medicaid Health Information Technology Plan (SMHP) as an integral part of planning and implementation of the EHR incentive program. The SMHP is a comprehensive document that describes the State's current and future health IT activities in support of the Medicaid EHR incentive program. We further clarify that we require that the SMHP will be developed by the State Medicaid agency, after consulting with other stakeholders across the State. The SMHP will be reviewed and approved by CMS prior to any activities described in the SMHP being funded and implemented. We anticipate State agencies will engage a wide range of stakeholders within and outside of State and Federal government to develop a vision of how the Medicaid EHR incentive program will operate in concert with the larger health system and statewide efforts. The SMHP is required to participate in the Medicaid incentive program because we believe that States must develop a strategic vision and plan that includes clear targets and measurable outcomes to be consistent with the intent of section 1903(a)(3)(F) of the Act to encourage the adoption and meaningful use of certified EHR technology.

The SMHP is intended to serve as the vision for developing the desired future state for the Medicaid IT environment that furthers the goals of health information exchange and meaningful use envisioned under the Act. The SMHP should be coordinated and integrated with the Statewide plan for health IT developed under section 3013 of the Public Health Service Act, which is developed by the designated statewide entity. To ensure that the SMHP is coordinated and integrated 
with the Statewide plan, we will develop criteria and processes for the evaluation of the SMHP consistent with ONC's review of the Statewide plans. The SMHP must contain: (a) A current health IT landscape assessment; (b) a vision of the State's HIT future landscape, and (c) the specific actions necessary to implement the incentive payments program, including a health IT roadmap to achieve those actions. This deliverable will be the "plan" to determine how the incentive payments will be administered; however, it is not the implementation of such plan. The SMHP must include all of the elements listed in 495.332; however, we realize that States may not have all of the answers initially. States will not be permitted to make incentive payments to providers unless they have a comprehensive EHR incentive payment program established. However, if States are not completely clear, for example, about their "to be" world at the time of the submission of their SMHP, States can present the components that are finalized and revise the SMHP to further discuss their "to be" world at a later time. Additionally, as stated previously in this final rule, we have revised the rule to include a requirement that the SMHP must describe the process in place and the methodology for verifying that eligible professionals meet their responsibility for 15 percent of the net average allowable cost for certified EHR technology and that the SMHP include information about how States will validate the patient volume consistent with the menu of options listed in $\S 495.306$.

For this final rule, we are also explaining our understanding that the elements of the SMHP, as listed in $\S 495.332$, may be separated into four categories, as follows:

(1) Assessment and Planning. This category of SMHP elements addresses requirements in the Act relating to increasing the use of health IT, including EHR, ensuring interoperability, and meaningful use of certified EHRs. As proposed, States will perform comprehensive assessments of the current health IT landscape environment in the State, including the inventory of existing health IT in the State, including "as is" and "to be" landscape assessments. Also, as proposed, States will develop a 5-year strategic plan, and a description of how the State Medicaid HIT plan will be planned, designed, developed and implemented, including how it will be implemented, and a description of how intrastate systems, including the MMIS, and other claims systems, have been considered in developing a health IT solution. The SMHP will include a description of data-sharing components of proposed health IT solutions, including security provisions, and description of how the State will support integration of clinical and administrative data.

(2) Ensuring improvements in health outcomes, clinical quality, and efficiency. This category of SMHP elements will address requirements in the Act relating to improving healthcare quality and lowering costs. As proposed, States will include components that describe a process for ensuring improvements in health outcomes, clinical quality, or efficiency resulting from the adoption of certified EHR technology by recipients of Medicaid EHR incentive payments and a methodology for verifying such information. As proposed, we are requiring a description of how the State will address, in the long-term, the needs of underserved and vulnerable populations such as children, individuals with chronic conditions, Title IV-E foster care children, individuals in long-term care settings and the aged, blind, and disabled. We proposed that in order to obtain approval for their SMHP and implementation funding, a State would have to detail how their EHR Incentive Program addressed the concepts of selfdirection including budget development and expenditure tracking for persons with disabilities. After additional consideration, CMS decided that these concepts are not directly applicable to electronic health records or meaningful use, per se, and while important, are more associated with other e-Health tools, such as personal health records. Furthermore, the provider types to whom this is most directly relevant, such as home, institutional and community-based providers and facilities, are not eligible for EHR incentives so including planning for this issue was not perceived as rising to the level of a requirement. It is anticipated that Stage 2 of meaningful use will include greater levels of patient engagement, including via personal health records. However, we think it is premature to require that States fully address this issue in their SMHPs order to initiate their EHR Incentive Programs for Stage 1.

As proposed, we will also require a description of the process in place for ensuring that any certified EHR technology used as the basis for incentive payments to Medicaid providers is compatible with State or Federal administrative management systems, including the MMIS, or other automated claims processing system or information retrieval system, and a methodology for verifying such information.

(3) Interoperability and Health Information Exchange. This category of SMHP elements will address requirements in the Act relating to ensuring interoperability and increasing health information exchange. We proposed a series of elements that explain how the State will adopt national data standards for health and data exchange and open standards for technical solutions as they become available. These elements of the SMHP also are included in our final rule.

(4) Administration and Oversight. This category of SMHP elements address the requirements in the Act relating to implementation and financial oversight of the program. For provider eligibility, we proposed that States provide a description of the process they will use for ensuring that each EP and eligible hospital meets provider enrollment eligibility criteria upon enrollment and re-enrollment to the Medicaid EHR payment incentive program, and the process for ensuring patient volume consistent with the criteria in $\S 495.304$ and $\S 495.306$, and for ensuring that each Medicaid EP is not hospital-based and that there is a methodology in place used to verify such information. We are finalizing most of these requirements, as proposed. However, in response to comments suggesting that CMS define the term "encounter" and take a menu approach to patient volume to allow States several options, based on their data sources, CMS has included changes to the SMHP requirements for the patient volume requirement in § 495.302, § 495.306, and § 495.332. These changes are discussed under the patient volume section of this final rule. We note that States that wish to offer an alternative for estimating patient volume would be required to involve key stakeholders in the determination of such alternative. We also proposed, and are finalizing, specific elements in the SMHP relating to monitoring and validation of information, including a method of ensuring all information from provider attestations is captured, stored, and verified, and any information added to the CMS Single Provider Repository is all true and accurate. We also proposed, and are finalizing, that States include a list of the specific actions planned to implement the EHR incentive program, including a description and organizational charts for workgroups within State government and external partners. As proposed, States will need to describe the process they have in place to ensure that no 
amounts higher than 100 percent of FFP will be claimed for reimbursement of expenditures for State payments to Medicaid eligible providers for the certified EHR incentive payment program, and a methodology for verifying such information is available and the process to ensure that no amounts higher than 90 percent of FFP will be claimed for CMS-approved administrative expenses in administering the certified EHR technology incentive payment program, including a methodology for verifying such information. As proposed, States will need to include mechanisms for making timely and accurate payments and a requirement that providers attest that they are not receiving a payment in any other State under the Medicaid EHR incentive program. This category also includes elements relating to financial management and auditing necessary to ensure the proper and efficient management and oversight of the program and FFP.

Finally, we proposed that the States may propose in the SMHP alternatives to measuring patient volume or achieving meaningful use. The rules for proposing alternatives are discussed elsewhere in this final rule.

We are further clarifying the definition of Health Information Technology Planning Advance Planning Document (HIT PAPD) (and any necessary update documents) to mean a plan of action that requests FFP and approval to initiate and accomplish planning activities necessary for a State agency to determine the need for and plan the acquisition of HIT equipment and services, and to acquire information necessary to prepare a HIT

Implementation Advanced Planning Document (HIT IAPD), described below, or common procurement instruments, such as requests for proposals, or requests for qualifications and quotations, necessary to implement the SMHP. CMS is including a definition of the HIT PAPD so that States may submit proposed resources and planning activities, which are described in further detail in our State Medicaid Director's letter on September 1, 2009, to receive the 90 percent FFP match for initial planning activities related to the Medicaid EHR incentive payment program. In order to qualify for the 90 percent FFP administrative match, section 1903(t)(9) of the Act requires a State to demonstrate, to the satisfaction of the Secretary, compliance with three specific criteria:

(A) The State uses the funds for purposes of administering the incentive payments, including the tracking of meaningful use of certified EHR technology by Medicaid providers;

(B) The State conducts adequate oversight of the incentive program, including routine tracking of meaningful use attestations and reporting mechanisms; and

(C) The State pursues initiatives to encourage adoption of certified EHR technology to promote health care quality and the exchange of health care information under Medicaid, subject to applicable laws and regulations governing such exchange, while ensuring privacy and security of data provided to its data exchange partners.

We are further clarifying the definition of Health Information Technology Implementation Advance Planning Document (HIT IAPD) (and any necessary update documents) to mean a plan of action that requests approval of FFP to acquire necessary resources to implement and administer the activities and objectives of the State's proposed SMHP, once the SMHP is approved by CMS, including the allocation or acquisition of human resources, services and equipment. To qualify to receive FFP for administering the incentive program, States must develop an HIT PAPD, SMHP, and an HIT IAPD. These documents would lay out the process States will use to implement and oversee the EHR incentive program, and would help States to construct and maintain a health IT roadmap to develop the systems necessary to support providers in their adoption and meaningful use of certified EHR technology.

With respect to FFP under the Medicaid incentive program, we are clarifying that the incentive payments to providers are matched at 100 percent FFP as described above, and therefore there is no non-Federal share for these payments. However, there is a nonFederal share necessary for the administration of the payment incentives. That is, CMS is reimbursing States at 90 percent FFP for reasonable expenses related to the administration of the payment incentives. States must fund the 10 percent non-Federal share of Medicaid health information technology (health IT) administrative payments consistent with existing rules and regulations regarding funding of the non-Federal share. We review non-

Federal share funding sources to ensure compliance with existing statute and regulations. Consistent with current practice, we will review non-Federal share funding sources on an individual basis using information provided by the State and gathered by CMS staff. Existing rules permit States to provide the non-Federal share of administrative claims through various sources, including appropriations, intergovernmental transfers, certified public expenditures, bona fide donations, and permissible health care related taxes. CMS' regional financial management staff will review funding sources and will review the Medicaid Budget and Expenditure System to ensure that all claims for reimbursement are appropriate. Additionally, States are required to submit SMHPs outlining their process for making payments and ensuring that all claims for reimbursement are appropriate to CMS for review and approval.

At $\S 495.324$ we proposed to review and prior approve all elements of the State's APD documents and SMHP described in this rule to ensure that all of the intended objectives of the program are addressed. We are finalizing this proposal. States are required to submit these APD documents and the SMHP in order for us to approve FFP. Specifically, prior approval is required for the HIT PAPD (see also § 495.336). The deliverable resulting from the HIT PAPD is the SMHP. The SMHP will be reviewed and approved before it is included in an HIT Implementation APD (HIT IAPD) (see also $§ 495.338$ ). The HIT IAPD also must be prior approved. After a HIT PAPD is approved for planning activities, and these planning activities are complete, we anticipate that in certain cases, States may decide to submit the SMHP and HIT IAPD together in one submission for CMS review and approval. In all cases, until approval is granted, States cannot draw down Federal funds. We envision that the prior approval process described at $\S 495.324$ will permit States to work closely with CMS in developing the HIT PAPD prior to initiating EHR planning activities and prior to submission of the initial HIT PAPD.

We are defining "as needed" and "annual" updates to the HIT PAPD and HIT IAPD at $\S 495.340$ and $\S 495.342$. In consultation with States and other key stakeholders, CMS has determined that planning and implementing the Medicaid EHR incentive payment program will be a complex process that will result in a need for "as needed" and "annual" updates to the original scope of work. Therefore, we proposed that the APD process would allow States to update their APD documents when they anticipate changes in the amount of FFP, duration of the project, or scope of work or activities under the APD. We are finalizing this proposal, as it allows States flexibility to add additional tasks and milestones as the project evolves, as determined since the date the APD was 
initially approved or since the most recently updated and approved APD. We initially proposed that we envision two phases in the process of planning and implementing the incentive program, as well as the promotion of adoption and meaningful use of EHR. We are further clarifying that based on submission of HIT PAPDs in response to guidance provided in our State Medicaid Director's letter of September 1, 2009, initial planning timelines are ranging from 6 months to 18 months to develop the SMHP. CMS envisions that States will begin to administer the EHR incentive program on January 1, 2011, once the SMHP and IAPD are approved. As proposed, we will issue additional written guidance, similar to our earlier SMD letter, concerning timelines for implementation of the EHR incentive program as States develop the SMHP.

We require the HIT IAPD as the vehicle for informing us of Phase II activities. We anticipate that States will also have ongoing planning needs as implementation activities, once approved under the IAPD, are under way. We further envision that the IAPD "annual" or "as needed" updates may also include requests for approval of FFP for other Phase II that are necessary to continue planning and development for the ongoing implementation phases of the program. In section 495.388, we proposed to require that States submit information in the IAPD regarding an estimate of prospective cost allocation (OMB Circular A-87, Cost Principles for State, Local, and Indian Tribal

Governments) to the various State and Federal funding sources and the proposed procedures for distributing costs including a detailed payment list file to include NPI, name, and type of provider for which the State will provide incentive payments. For the final rule, we are continuing to require the estimate of prospective cost distribution and the procedures for distributing costs; however, we are eliminating the requirement that States have to submit NPI, name and provider type as part of the estimates for cost distribution since we realize that in continuing to require this information States will not be able to submit approvable IAPDs to CMS because States will not have this information at the time of submittal; hence, States will not be successful in implementing this program.

We wish to further clarify that in proposing termination of funding if the State fails to meet the requirements and undertakings of the approved HIT PAPD, SMHP, and HIT IAPD, or fails to provide access to the required information, this requirement is necessary to ensure the proper and efficient use of FFP and is consistent with present authority under the Act and existing regulations that are promulgated by CMS, including at 45 CFR Part 95, Subpart F.

Comment: One commenter questioned whether the EHR incentive payments will be required to be processed through the Medicaid Management Information System (MMIS).

Response: Payments under the Medicaid EHR incentive program are authorized under Title XIX of the Social Security Act as part of the Medicaid program. We require that States have an automated claims processing and information and retrieval system, known as MMIS to manage health care provider payments for health care services, and provide information for program management, administration, and auditing. As such, we believe that most States will choose to process, monitor, and report Medicaid incentive payments to eligible professionals and hospitals participating in the Medicaid EHR incentive program using the MMIS. States may propose alternative methods to process, monitor, and report Medicaid incentive payments in their SMHP. Any proposed method to process, monitor, and report Medicaid incentive payments, including utilization of the State's MMIS, must be approved by CMS. Through guidance issued in a State Medicaid Directors Letter and via case by case analysis of APDs, CMS will collaborate with States to approve system development and enhancement expenditures under the most appropriate funding source, HITECH or MMIS.

Comment: One commenter provided comments on $\S 495.348(d)$, Procurement standards; Competition, and

$\S 495.360$ (a). The commenter agrees that procurement transactions are conducted to provide, to the maximum extent practicable, open and free competition and recommends that procurement transactions require that bidders bid specifically for the EHR portion of any project (to ensure that the discrete costs are clearly identified), (2) no certified EHR technology may be excluded from bidding, and (3) all projects must be both EHR-neutral and provider-neutral. They further comment that CMS could consider having either a cap or percentage limits on the amount of administrative costs or consulting fees to ensure that the bulk of the award is used for the hard costs of the project: equipment, connectivity, and training.

Response: The requirement in $\S 495.348(d)$ is limited to States and other grantees of Federal funds authorized under Title XIX of the Social Security Act and does not apply to procurement standards for vendors bidding on EHR technology for eligible providers. However, CMS will encourage States to include adoption of interoperable solutions that align with the MITA principles that address IT architectural and platform neutrality.

We are making no additional revisions to this section of the rule as a result of this comment.

Comment: One commenter recommended that CMS reconsider the general rule set forth in $\S 495.360$ that "the State or local government must include a clause in all procurement instruments that provides that the State or local government will have all ownership rights in software or modifications thereof and associated documentation designed, developed or installed with FFP under this Subpart." The commenter states that it is typical for the vendor to own the underlying software, and State or local governments are provided a license to use the software, and this is contrary to the proposed general rule.

Response: We disagree with the recommendation to exclude a clause in all State procurement instruments that provides that the State or local government will have all ownership rights in software developed or modified using Federal funding. This is a long-standing principal for use of FFP associated with the development of information technology solutions that may be licensed for use by other State or Federal government agencies to benefit the Medicaid program, at no additional cost for the license. CMS clarifies that costs of the license agreements for proprietary software may be reimbursable under the provisions of 1903(a)(3)(F)(ii) of the Act that provides for 90 percent FFP for costs associated with certain administrative activities performed by a State. However, costs associated with developing or modifying software may not be funded with Federal funds unless the State has ownership rights to that software. This provision does not apply to eligible providers or hospitals purchasing software for which Federal funding has been provided by States through the Medicaid EHR incentive program. Proposed costs may be submitted for review and consideration for approval by CMS as part of the HIT PAPD and HIT IAPD requirements described in this proposed rule under $\S 495.336$ and $\S 495.338$.

We are making no additional revisions to this section of the rule as a result of this comment. 
Comment: One commenter indicated that the process for State Medicaid plans seems to be lengthy, with no timeframes specified for initial submission from the State to the Department, nor is there a timeline for the approval process from CMS back to the State. There is also no timeline for the implementation of the health IT programs after a State receives approval. The commenter also notes that with the burden for administration on the States, there may not be adequate time to get all of the activities completed to have infrastructure and processes in place to accept data or attestations from the Eligible Providers and Eligible Hospitals.

Response: We provided specific guidance on timelines and process prior to the initial planning period regarding State planning activities and administrative expenses for provider incentive payments in our State Medicaid Director's letter on September 1, 2009. We also indicated in our letter that CMS will work with States to determine when each State is ready to begin making payments. We have provided additional rationale about the process for submitting documents and required content in the final rule. In the near future, CMS will issue more guidance on specific implementation activities and timelines, prior to States submission of their SMHP and IAPD.

We are making no additional

revisions to this section of the rule as a result of this comment.

Comment: One commenter requested that CMS require that States pass through the matching funds to providers.

Response: The regulation at section 495.366 requires that States have a process in place to assure that Medicaid EHR incentive payments are made without reduction or rebate, have been paid directly to an eligible provider or to an employer, a facility, or an eligible third party entity to which the Medicaid eligible provider has assigned payments. This language is consistent with the statutory language at 1903(t)(6). We will require that this process be established in the SMHP.

We are making no additional revisions to this section of the rule as a result of this comment.

Comment: One commenter requested that CMS clarify that use of certified public expenditures (CPE) or intergovernmental transfers in the context of the Medicaid EHR incentive payments would be inappropriate, since these payments do not have a nonfederal share. If CMS does permit use of CPEs in the Medicaid EHR incentive program context, CMS must require that
States pass through the matching funds to providers.

Response: We believe the commenter is not clear. As explained above incentive payments to providers are matched at 100 percent; thus, there is no non-Federal share for these payments. However, there is a non-Federal share necessary for the administration of the payment incentives. CMS is reimbursing States at 90 percent for reasonable expenses related to the administration of the payment incentives and States must fund the 10 percent non-Federal share of Medicaid health information technology administrative payments consistent with existing rules and regulations regarding funding of the non-Federal share. Please see our above discussion of this issue for further detail.

We are making no additional revisions to this section of the rule as a result of this comment.

Comment: One commenter questioned why Medicaid is allowed to determine its own requirements and the impact this may have on other stakeholders.

Response: We are clarifying that we have provided specific guidance for State planning activities that must be addressed in order to qualify to receive FFP for administering the incentive program. We provided guidance in a State Medicaid Director's letter published on September 1, 2009, on this process. CMS intends to require submission of documentation that will enable the agency to evaluate whether the activities for which FFP was, or may be approved for, are being completed according to Federal requirements, including any terms and conditions of FFP approval. States must develop a HIT PAPD, a SMHP, and a HIT IAPD. These documents would describe the processes and resources States will use to implement and oversee the EHR incentive program, and would help States to construct an health IT roadmap to develop the systems necessary to support providers in their adoption and meaningful use of certified EHR technology. The development of a SMHP (see also § 495.332) also provides States with the opportunity to analyze and plan for how EHR technology, over time, can be used to enhance quality and health care outcomes and reduce overall health care costs. Our review process ensures that States are complying with requirements in the Act, and that they demonstrate to the "satisfaction of the Secretary" that they are using the funds in the manner anticipated by the law. For example, because CMS is responsible for overseeing States in their administration of the Medicaid program, as well as ensuring the overall financial integrity of the program, States cannot simply propose activities in order to secure the 90 percent FFP. We propose to review and prior approve all elements of the State's SMHP, and APD documents described in this rule to ensure that all of the intended objectives of the program are addressed. One of the key components of the SMHP is stakeholder collaboration and coordination to ensure that an integrated strategy is developed addressing stakeholder needs.

We are making no additional revisions to this section of the rule as a result of this comment.

Comment: One commenter recommended that all the source materials needed to create the quality measure registry, is submitted to the MITA Information Architecture Review Board (IARB) for approval as a MITA standard and all the source materials be added to the MITA artifact repository. Doing this will prevent duplicative efforts and associated expense both by CMS and the participating States.

Response: We agree with the commenter. We support the concept that States should apply MITA principles to any IT development work performed for the EHR incentive program, where applicable. If a State chooses to integrate a clinical data warehouse into its MMIS system, all recommended steps, and required approvals, for MMIS development, including application of MITA guidelines, should apply. The goal of MITA is not to focus on creating new standards so much as utilizing data standards developed by other national organizations, such as those responsible for implementation of HITECH and also defining information requirements for new business processes. If a State is going to develop its own clinical data repository to store Medicaid providers' submitted clinical quality measures data (one of the MU objectives), then use of the MITA Governance boards would be a recommended approach. States whose SMHPs successfully apply MITA to their EHR incentive program systems are encouraged to store approved artifacts in the Clemson University MITA repository so that other States may benefit: http://mita.clemson.edu.

We are making no additional revisions to this section of the rule as a result of this comment.

Comment: One commenter, as a large pediatric provider with five physicians and four nurses in a relatively rural area, is concerned that States have not yet sent, or had approved by CMS, the State's Medicaid requirements. 
Response: States are in the process of developing their SMHPs. States could not be approved to start offering incentives prior to a final rule becoming effective.

We are making no additional revisions to this section of the rule as a result of this comment.

Comment: Some commenters asked for clarification on how managed care entities would be involved in this program besides potentially being used to disburse incentive payments, as mentioned in the proposed rule. Examples included things like monitoring providers in the health plans to ensure compliance. The commenters suggested that any work done by the managed care entity should be reflected in the capitation rate.

Response: Service agreements between States and their managed care contractors are not governed by this regulation, but must be in compliance with 42 CFR part 438 . We agree there are many opportunities to leverage the efficiencies of the managed care entities activities and role with the larger goals and State responsibilities for administering the payments. We suggest that activities like distributing informational materials about the incentive program and health IT to health plan providers and enrollees would fall under most current contracts and would be considered part of the cost of doing business, which may be reflected in the administrative portion of the capitation rate.

If more significant activities are expected, such as monitoring and reporting information on the providers, health plans may exceed the normal costs of doing business and what would be adequately reflected in the administrative portion of the capitation rate. An alternative option would be for the State and managed care organization to have contractual requirements and deliverables separate from the capitation rate, including the administrative component. In the latter scenario, it would be acceptable to develop a contract amendment specifying the terms.

We are making no additional revisions to this section of the rule as a result of this comment.

Comment: A commenter asked whether or not a State would need to file a State Plan Amendment that incorporates the SMHP into their State Plan, or if the SMHP can stand alone. The commenter further asked that if the SMHP can stand alone, then would the state need to file a State Plan Amendment that references the SMHP in their plan.
Response: CMS clarifies that the State does not need to file a State Plan Amendment or reference the SMHP in their State Plan. As part of the Advance Planning Document process, the SMHP is a deliverable that is submitted to CMS for review and approval prior to expending funds for the incentive program implementation activities.

We are making no additional revisions to this section of the rule as a result of this comment.

\section{Financial Oversight, Program} Integrity and Provider Appeals

Pursuant to section 1903(t)(9) of the Act, which requires States to conduct adequate oversight of the incentive program, and in order to ensure that ARRA funds are expended wisely and in a manner that impedes waste, fraud or abuse of Federal taxpayer money, at $\S 495.366$, we proposed requirements for States' financial oversight and monitoring of expenditures.

Additionally, we proposed at $\$ 495.368$ to provide State requirements for combating fraud and abuse.

Specifically, States would be responsible for estimating the expenditures for the Medicaid EHR incentive program on the State's quarterly budget estimate reports. These reports are used as the basis for Medicaid quarterly grant awards that would be advanced to the State for the Medicaid EHR incentive program. The State submits this Form electronically to CMS via the Medicaid and State CHIP Budget and Expenditure System (MBES/ CBES). States must assure that requests for reimbursement of FFP comply with all sections of this new part and that the amounts reported on the Form CMS-64 and its attachments represent actual expenditures for which all supporting documentation, in readily reviewable form, has been compiled and which is available at the time the claim for reimbursement of provider payment incentives and administration funding is filed.

We would assure that State expenditures claimed for Federal matching under the Medicaid program are programmatically reasonable, allowable, and allocable in accordance with existing Federal laws, regulations, and policy guidance. States would be responsible for establishing policies, computer systems, edits to process Medicaid EHR incentive payments; and for conducting analyses of providers' patterns of practice (data-mining) and taking other reasonable steps to ensure that no duplicate or otherwise improper EHR incentive payments have been made. States will be responsible for ensuring that provider information, including but not limited to, attestations, survey, and any information added to CMS' single provider election repository indicates that any falsification of documentation or concealment of material facts may be prosecuted under Federal and State laws. States would be responsible for recovering and returning to CMS FFP for any HIT incentive payments that are discovered to be improper. State Agencies must have information processing systems, which may include an MMIS - the automated mechanized claims processing and information retrieval system, to process Medicaid EHR incentive payments. MMIS systems can also help to manage information for program administration and audit purposes.

States must assure that any requests for reimbursement of the 90 percent Federal match for administration of the program are being requested only because the State has used the funds for purposes related to administering payments to qualified Medicaid providers for certified EHR technology, including for tracking of meaningful use of such technology, is conducting adequate oversight of the program including routine tracking of meaningful use attestations and reporting mechanisms; and is pursuing initiatives to encourage the adoption of certified EHR technology to promote health care quality and the exchange of health care information because of such technology. Any initiatives for health information exchange must be consistent with Federal laws and regulations governing the exchange.

We would monitor State Agency compliance through systems performance reviews, on-site reviews, and audits of the APD process. Additionally, we would monitor provider demonstration of meaningful use.

As a result of the authority extended to the Secretary under section 1902(a)(4) of the Act requiring the effective and efficient administration of the State plan, as well as section 1903(t)(9) of the Act, requiring that a State demonstrate to the satisfaction of the Secretary that it is conducting adequate oversight of the program, we also are requiring States to establish § 495.370, Provider Appeals. This section specifies that Medicaid providers who believe that they have been denied an incentive payment or have received an incorrect payment amount under this part because of incorrect determinations of eligibility, including, but not limited to, measuring patient volume;

demonstrating meaningful use of, or the efforts to adopt, implement, or upgrade 
to, certified EHR technology; whether the professional is hospital-based; whether the professional is practicing predominantly in an FQHC or RHC; whether the hospital qualifies as an acute care or children's hospital; or whether the provider is already participating in the Medicare incentive program and therefore ineligible duplicate Medicaid incentive program payments can appeal the decision using current Federal processes established at $\S 447.253(\mathrm{e})$.

Comment: One individual commented on potential fraud and abuse opportunities if large amounts of medical data can be mined, as a result of electronic health records.

Response: First, it is important to note that as part of demonstrating meaningful use providers will be submitting only aggregated, not individually identifiable data, to States. Second, we wish to clarify that providers will be required to comply with the Health Insurance Portability and Accountability Act of 1996 (HIPAA) to the extent that they are covered entities. States must provide CMS with details about how their implementation of the EHR incentive program will address Federal and State privacy laws and how all data will be secured in the SMHP.

Additionally, the act of preventing fraud should be paramount in implementing this program. In accordance with Section 1903(t)(9) of the Social Security Act, States must demonstrate to the satisfaction of the Secretary that they are conducting adequate oversight of this program and that they are complying with Federal requirements to: (a) Ensure the qualifications of providers who request Medicaid EHR incentive payments, (b) detect improper payments and (c) refer suspected cases of fraud and abuse to the Medicaid fraud control unit. In conducting required oversight responsibilities, States can receive 90 percent matching funds for allowable expenditures. States are required to assure CMS through the State's Medicaid HIT plan that they have processes in place to prevent against fraud and abuse. CMS will review and approve each State's Medicaid HIT plan.

We are making no additional revisions to this section of the rule as a result of this comment.

Comment: One commenter noted that use of electronic health records may provide claims adjudication auditors with documentation to verify that items or services provided are reasonable and necessary, supporting an upfront clean claims process and the opportunity to conduct pre- and post-pay audits without the need to request documentation in retrospect. Another commenter wanted an assurance that CMS will perform audits of a random sample of attestation surveys and that any providers that are found to be making false claims would be penalized and listed in a public report posted on CMS' Web site.

Response: We thank the commenter for the comments, but point out that meaningful use currently would not include using EHRs to provide electronic documentation in support of claims adjudication. We do, however, want to address the issue of pre- and post-audits. While one commenter is concerned with the process for adjudicating claims, the other commenter is concerned that there are other areas of this program that will necessitate pre- and post-pay audits. For Medicaid, States are required to provide information to CMS in the State Medicaid HIT plan outlining the processes and methodologies they will use to ensure that payments are being made to the right person, at the right time, for the right reason. Specifically, in year one in order to receive an incentive payment, providers will be attesting to, among other things, whether they are using a certified EHR, demonstrating meaningful use, demonstrating adopting, implementing or upgrading certified EHR technology, etc. States will be required to "look behind" provider attestations. We believe that this will require audits both pre- and post-pay. CMS believes a combination of approaches is in order which should result in accurate payments. CMS wishes to point out that States must provide assurances to CMS that they are conducting adequate oversight in order to receive the 90 percent FFP for administration of the incentive payments. Additionally, it should be noted that this program is consistent with other programs under Title XIX. States must properly administer the program or risk FFP. All costs claimed under the program are subject to review or audit. Furthermore, CMS' approval of the State Medicaid HIT plan does not relieve the State of its responsibility to comply with changes in Federal laws and regulations and to ensure that claims for Federal funding are consistent with all applicable requirements. We should point out that for Medicaid there is no statutory requirement to post individual provider's name and/or incentive payment program information to the CMS Web site.

We are making no additional revisions to this section of the rule as a result of this comment.
Comment: One commenter is concerned about the circumstances under which Medicaid is required to recoup incentive payments from providers. Specifically, the commenter requests clarification on the scenario in which a provider receives a payment for demonstrating adoption,

implementation, or upgrading EHR technology in year one, demonstrating meaningful use in years two and three, but receives no payment in year four because the provider could not demonstrate meaningful use. The commenter is concerned that Medicaid will be responsible for recouping payments made in years one, two, and three.

Response: First, it should be noted that it is possible for a provider to be able to demonstrate meaningful use in one year, but not others. Thus, the failure of the provider to demonstrate meaningful use in year four would not necessarily mean that the provider failed to demonstrate meaningful use in prior years, although it could possibly alert the State to more closely review a specific provider's prior year attestations or demonstrations of meaningful use. For hospitals demonstrating meaningful use in both the Medicare and Medicaid incentive payment programs, CMS will issue further guidance about how States will be able to access the meaningful use data submitted to CMS in order for the State to meet its audit and oversight requirements. States will be required to outline in the SMHP the process for "looking behind" provider attestations and the demonstration of meaningful use including any record retention requirements.

In accordance with section 1903(t)(9) of the Social Security Act and $\S 495.332$ (c) and (e) of the regulations as well as $\S 495.368$, States are required to include in their State's Medicaid HIT plan processes for detecting improper payments and for combating fraud and abuse. This would mean that States will be responsible for conducting audits of providers and ensuring that any requests for reimbursement for $\mathrm{FFP}$ meet all requirements of this subpart. When States conduct audits and determine that improper payments have been made, States are responsible for recovering and returning to CMS FFP for any incentive payments that are discovered to be improper.

We are making no additional revisions to this section of the rule as a result of this comment.

Comment: Another commenter is concerned with a similar issue. That is, the commenter requested that CMS identify and develop "safe harbor" 
processes and methods for

administering the incentive program that would assure States that if these processes/methods are used, States would not be at risk if the processes/ methods are less successful than anticipated. An example would include a process for auditing the adoption, implementation, and upgrading process. If an audit approach was agreed to but ended up being less than effective when applied, the State should not be responsible for re-auditing providers for previous years, nor would it be denied participation in the incentive program and lose the FFP. Another commenter is similarly concerned that this is a new program and they requested that CMS explicitly recognize the States' ability to revise and redirect the program without penalty from CMS.

Response: Our focus is on ensuring that EHR incentive payments are made to the eligible provider, and are for the correct amount in the appropriate payment year (or payment cycle). CMS will ensure that State expenditures claimed for Federal matching under the Medicaid program are programmatically reasonable, allowable, and allocable in accordance with existing Federal laws, regulations, and policy guidance.

States can receive FFP if they are conducting adequate oversight and States must provide their plans for financial oversight and the processes and methodologies they will use to verify provider information to CMS for review and approval as part of its State's Medicaid HIT plan. We believe States may want to consider multiple ways in which to audit their providers; for example, to ensure that a provider is not excluded from the program, the State should review on a prepay basis the Office of the Inspector General's List of Excluded Individuals and Entities to determine if providers are excluded. Additionally, States may wish to consider attestation in year one for demonstrating adopting, implementing, or upgrading or meaningfully using certified EHR technology. States will have to "look behind" these attestations and we assume this will be done on a post-pay basis. One size does not fit all and we believe several audit options should be used by States to ensure "adequate oversight." However, if it is determined that the State's audit methodologies are proving to be less than effective we will require that the State update its State Medicaid HIT plan and present more effective audit strategies that will work to accomplish conducting adequate oversight of the program. States must ensure due diligence in conducting adequate oversight and all requirements of this subpart must be met or FFP could be at risk.

We are making no additional revisions to this section of the rule as a result of this comment.

Comment: One commenter requested information regarding the appeals process.

Response: For Medicaid, CMS has specified the appeals process for a Medicaid provider receiving electronic health record incentive payments in $\S 495.370$. Specifically, the State must have a process in place consistent with the requirements established at $\S 447.253(\mathrm{e})$ to allow for providers to appeal incentive payments, incentive payment amounts, provider eligibility determinations, and the demonstration of adopting, implementing or upgrading and meaningful use of certified EHR technology. CMS is requiring that the State Medicaid HIT plan describe the process in place for provider appeals. We believe the States, not the Federal government, are in the best position to determine the administrative process that would best meet their needs and we believe States are in a position to design an effective appeal procedure; thus, we are providing for a great deal of State flexibility. Within the parameters of the regulation, States are free to establish reasonable criteria for appeals, to limit the issues on appeal that may be appropriate, or to adopt other procedures to prevent frivolous appeals. However, State appeal processes should be consistent with the requirement in $\S 447.253(\mathrm{e})$ for prompt administrative review. (States define what would constitute a prompt review, and we have not specified a time period for conducting or concluding a provider appeal.) This requirement is in keeping with providing States flexibility while retaining for providers an opportunity to avail themselves of an exception process when they believe an exception is warranted. Additionally, § 447.253(e) provides that the Medicaid agency must allow providers an opportunity to submit additional evidence. Our regulations at $\S 495.370$ also require that the appeals processes established by the States comply with the State's own administrative procedure laws and that the State provide any additional appeal rights that would otherwise be available under the procedures established by the State.

We are making no additional revisions to this section of the rule as a result of this comment.

\section{Information Collection Requirements}

Under the Paperwork Reduction Act of 1995, CMS is required to provide 60- day notice in the Federal Register and solicit public comment before a collection of information requirement is submitted to the Office of Management and Budget (OMB) for review and approval. In order to fairly evaluate whether an information collection should be approved by OMB, section 3506(c)(2)(A) of the Paperwork Reduction Act of 1995 requires that CMS solicit comment on the following issues:

- The need for the information collection and its usefulness in carrying out the proper functions of our agency.

- The accuracy of our estimate of the information collection burden.

- The quality, utility, and clarity of the information to be collected.

- Recommendations to minimize the information collection burden on the affected public, including automated collection techniques.

The following is a discussion of the requirements we believe are subject to PRA and collection of information requirements as a result of this final rule. This analysis finalizes our projections which were proposed in the January 13, 2010 Federal Register (75 FR 1844 through 2011). The projected numbers of EPs and eligible hospitals, MA organizations, MA EPs and MAaffiliated hospitals are based on the numbers used in the impact analysis assumptions as well as in Table 32 in section IV of this final rule.

\section{A. ICRs Regarding Demonstration of Meaningful Use Criteria (\$ 495.8)}

Section 495.8(a)(1) of the proposed rule contained requirements for EPs, in CY 2011, to attest, through a secure mechanism, to meeting meaningful use criteria. As described in the proposed rule (75 FR 1949), we divided meaningful use objectives/measures into Sets A and B. We estimated that the total burden for an EP to attest to $\S 495.8(\mathrm{a})(1)(\mathrm{i})$ and (ii) for Set A meaningful use objectives/measures and ambulatory quality measures would be one hour. For all 442,600 non-hospitalbased Medicare and Medicaid EPs (323,500 Medicare EPs, 80,900 dual Medicare/Medicaid EPs, and 38,200 Medicaid-eligible-only EPs), the burden therefore equaled 442,600 hours. We estimated that the associated cost burden was $\$ 79.33$ for an EP to attest to $\S 495.8(a)(1)(i)$ and (ii) for Set A meaningful use objectives/measures and ambulatory quality measures, and the total associated annual cost burden for all EPs to attest was $\$ 35,111,458$. We invited comments on the estimated percentages and the numbers of (registered) EPs that will attest to the above including Set A meaningful use 
objectives/measures in CY 2011, but did not receive any on this issue.

In the proposed rule, we also estimated that it would take 8 hours for an EP to attest to meeting the Set B meaningful use objectives/measures. We estimated that the total annual burden for all 442,600 non-hospital-based EPs to attest to Set B meaningful use objectives and measures was 3,540,800 hours. We estimated the associated cost burden for an EP to attest was $\$ 634.64$ and the total cost burden for all nonhospital-based EPs to attest was $\$ 280,891,664$. We solicited comments on the estimated percentages and the numbers of (registered) EPs that will attest to Set B objectives and measures in CY 2011, but did not receive any on this issue.

Although, as we proposed, we continue to have an attestation requirement in $\S 495.8(\mathrm{a})(1)$, we are revising the burden estimates for two reasons. First, as described elsewhere in this final rule, the definition of hospitalbased EP has changed, resulting in about 73,000 outpatient hospital EPs becoming potentially eligible to participate in the EHR incentive program. Therefore, we are increasing the number of EPs in our burden estimates. We estimate that in CY 2011, there will be 521,600 non-hospitalbased Medicare and Medicaid EPs (382,000 Medicare EPs, 95,500 dual Medicare/Medicaid EPs, and 44,100 Medicaid-eligible-only EPs) participating in the EHR incentive program. Second, in response to public comments, we have made significant changes in $\S 495.6$ meaningful use objectives and measures for EPs, eligible hospitals and CAHs, which has changed the burden estimates.

In section II.A.2.d. of this final rule, Stage 1 Criteria for Meaningful Use in this final rule, we have re-categorized meaningful use objectives/measures as core criteria and menu criteria. Unless an exception applies, § 495.6(a) requires that an EP must meet all 15 Stage 1 meaningful use core criteria under $\S 495.6(d)$ and 5 out of 10 meaningful use menu criteria under $\S 495.6(\mathrm{e})$. The burden associated with the requirements in $\S 495.8$ and $\S 495.6$ is the time and effort required to attest to the required elements.

To comply with $\S 495.8(a)(1)$, we estimate that it would take an EP 8 hours 52 minutes to prepare and attest that during the EHR reporting period, the EP used certified technology, specify the technology, and satisfied all 15 mandatory Stage 1 meaningful use core criteria. We estimate that it would take an EP an additional 0.5 hours to select and attest to the clinical quality measures, in the format and manner specified by CMS. We estimate the total burden associated with this requirement for an EP is 9 hours 22 minute (8 hours 52 minutes +0.5 hours) and the total burden for all the EPs to attest to these requirements is $4,855,827$ hours (521,600 EPs $\times 9$ hours 22 minutes). We estimate the associated cost burden for an EP to attest to these requirements is $\$ 743.08$ (9 hours 22 minutes $\times \$ 79.33$ (mean hourly rate for physicians based on the May 2008 Bureau of Labor Statistics)), and the total cost burden for all EPs to attest to these requirements is $\$ 387,592,672(4,855,827$ hours $\times$ \$79.33).

We recognize that some Stage 1 meaningful use menu set measures are easier to accomplish than others. We cannot predict which of the measures in the menu set an EP will select.

Therefore, our burden estimates are based on two scenarios to illustrate how different scenarios would impact the burden incurred. Our "least

burdensome" or "low" scenario of meaningful use demonstration assumes that an EP defers the five most burdensome objectives/measures while our "most burdensome" or "high" scenario of meaningful use demonstration assumes that an EP defers the five least burdensome meaningful use menu set measures. We recognize that in reality, nothing is absolute, and we have no basis for estimating the "all low" or "all high" scenario and have therefore created estimates for both. To compensate for the uncertainties of selection of meaningful use criteria by an EP, we use the averages of the "high" and "low" scenario estimates in Table 33. Section 495.6(a) requires that an EP must meet five out of 10 Stage 1 meaningful use menu set measures (unless exceptions apply). The burden involved is the time and effort to select and attest to the meaningful use menu set measures. In the "low" scenario, we estimate that an EP may defer the five most burdensome meaningful use measures. We estimate it will take an EP 42 minutes to comply with the remaining five Stage 1 meaningful use menu set measures. We estimate the total burden for all 521,600 EPs to comply with the meaningful use menu set criteria is 365,120 hours (521,600 EPs $\times 42$ minutes). In the high scenario, we estimate that an EP may defer the five least burdensome meaningful use criteria. We estimate that it will take an EP 2 hours 40 minutes to comply with the remaining five Stage 1 meaning use menu measures. We estimate that the total burden for all 521,600 EPs to comply with the meaningful use menu set criteria is 1,390,586 hours (521,600 EPs $\times 2$ hours 40 minutes). Based on the two scenarios, the average burden for an EP to comply with meaningful use menu set criteria is 1 hour 41 minutes ( 42 minutes +2 hours 40 minutes)/2). Based on the two scenarios, the average burden for all EPs to comply with meaningful use menu set criteria is 877,853 hours $((365,120$ hours + $1,390,586$ hours)/2). We estimate the cost burden for an EP to comply with the "low" scenario Stage 1 meaningful use menu criteria is $\$ 55.53$ (42 minutes $\times \$ 79.33$ (mean hourly rate for physicians based on the May 2008 Bureau of Labor Statistics)), and the total cost burden for all 521,600 EPs to comply is $\$ 28,964,970$ (521,600 EPs $\times$ \$55.53). We estimate that the cost burden for an EP to comply with the "high" scenario Stage 1 meaningful use menu criteria is $\$ 211.49$ (2 hours 40 minutes $\times \$ 79.33$ ), and the total cost burden for all EPs is $\$ 110,315.156$ (521,600 EPs $\times \$ 211.49)$. The average cost burden estimate for an EP to comply with the meaningful use menu set criteria is $\$ 133.51$ ( $(\$ 55.53+$ $\$ 211.49) / 2$ ). The average cost burden estimate for all 521,600 EPs to comply with meaningful use menu set criteria is $\$ 69,640,063((\$ 28,964,970+$ $\$ 110,315.156) / 2)$.

In the proposed rule, we expected that there would be steady growth in the number of participating EPs. We estimated that in 2012, there would be 447,400 non-hospital-based Medicare, and Medicaid EPs $(326,900$ Medicare EPs, 81,700 dual Medicare/Medicaid EPs and 38,800 Medicaid-eligible-only EPs) qualified to receive EHR incentive payment. We estimated that the burden for meeting § 495.8(a)(2), which required attestation for most meaningful use measures, and electronic reporting of clinical quality measures in CY 2012, would be 0.5 hours for an EP to attest to the Set A objectives and measures and 8 hours to gather information and attest to the Meaningful Use Set B objectives/measures. For burden estimate purposes, we estimated that all 447,400 non-hospital-based Medicare, and Medicaid EPs might attest. We estimated that the total annual attestation burden for all EPs was 223,700 hours for the Set A objectives/ measures and 3,579,200 hours for Set B objectives/measures. We estimated that the associated cost burden was \$39.67 for the Set A meaningful use objectives/ measures and $\$ 634.64$ for the Set B meaningful use objectives/measures. The total cost burden for all EPs was $\$ 17,746,121$ for Set A and $\$ 283,937,936$ 
for Set B. We invited comments on the estimated percentages and the numbers of registered EPs that would attest to EHR technology used and Meaningful Use Set A and Set B objectives/measures in CY 2012, but we did not receive any comments on this issue.

We expect steady growth in EPs in CY 2012. In the final rule, based on legislation altering the definition of "hospital-based," we are increasing our estimates of participating EPs, and estimate that in CY 2012, there will be about 527,254 non-hospital-based Medicare and Medicaid EPs (385,954 Medicare EPs, 96,500 dual Medicare/ Medicaid EPs and 44,800 Medicaideligible-only EPs) who are qualified to receive EHR incentive payments. The Stage 1 meaningful use criteria (core and menu sets) are the same for CY 2011 and CY 2012. We estimate that it would take 8 hours 52 minutes for an EP to attest that during the EHR reporting period, the EP used certified technology, specify the technology, and satisfied all 15 mandatory Stage 1 meaningful use core criteria. We estimate the total burden associated with this requirement for all EPs is -4,675,161 hours $(527,254$ EPs $\times 8$ hours 52 minutes). The associated cost burden for an EP to comply with this requirement is $\$ 703.42$ (8 hours 52 minute $\times \$ 79.33$ ) and the associated cost burden for all EPs is $\$ 370,880.589(44,675,161$ hours $\times$ $\$ 79.33$ (mean hourly rate of physicians based on the May 2008 Bureau of Labor Statistics)).

The Stage 1 meaningful use objectives and measures are the same for CY 2011 and CY 2012. Therefore, in CY 2012, the burden associated with attesting to Stage 1 meaningful use core and menu criteria for an EP is the same as CY 2011. Again, we cannot predict which of the measures in the menu set will be selected by an EP. Therefore, as explained above, we use a "low" and "high" scenario to estimate burden. For the "low" scenario, we estimate it will take an EP 42 minutes to attest to five Stage 1 meaningful use menu-set measures. The total burden for all $527,254 \mathrm{EPs}$, therefore, would be estimated at 369,078 hours $(527,254$ EPs $\times 42$ minutes). Under the "high" scenario, we estimate it will take 2 hours 40 minutes for an EP to attest to five Stage 1 meaningful use menu-set criteria. The total burden for all 527,254 EPs, therefore, is estimated to be $1,405,659$ hours $(527,254$ EPs $\times 2$ hours 40 minutes). Based on the two scenarios, the average burden hours for an EP to attest to meaningful use menu set measures is 1 hour 41 minutes ((42 minutes +2 hours 40 minutes)/2), and the total average burden for all EPs is
887,369 hours $((369,078$ hours + $1,405,659$ hours)/2). Under the "low scenario," we estimate that the cost burden for an EP is $\$ 55.53$ (42 minutes $\times \$ 79.33$ (mean hourly rate for physicians based on the May 2008 Bureau of Labor Statistics)), and the total cost burden for all 527,254 EPs to comply with is $\$ 29,278,942(527,254$ EPs $\times \$ 55.53)$. For the "high scenario," we estimate that the cost burden is $\$ 211.49$ (2 hours 40 minutes $\times \$ 79.33$ ), and the total cost burden for all EPs is $\$ 111,510,942(527,254$ EPs $\times \$ 211.49)$. The average cost burden is $\$ 133.51$

$((\$ 55.53+\$ 211.49) / 2)$. The average cost burden for all $527,254 \mathrm{EPs}$ is $\$ 70,394,942((\$ 29,278,942+$ 111,510,942)/2).

Section 495.8(a)(2)(iii) requires that for CY 2012, EPs must report electronically to CMS, or, in the case of Medicaid EPs, the States, clinical quality information in the form and manner specified by CMS. We have limited the required measures only to those that can be automatically calculated by a certified EHR, and to those for which we have electronic specifications currently available and we are able to post as final by the date of display of this final rule. The burden associated with this requirement is the time and efforts to report the required clinical quality measures. We estimate the burden for an EP to comply with this requirement is 0.5 hours and the total burden for all EPs to comply with this requirement is 263,627 hours $(527,254$ EPs $\times 0.5$ hours). We believed that an EP may assign a medical secretary to submit the specific clinical quality measures to CMS or the States. We estimate the cost burden for an EP to comply with this requirement is $\$ 7.40$ (0.5 hours $\times \$ 14.81$ (mean hourly rate of medical secretaries based on the May 2008 Bureau of Labor Statistics)) and the cost burden for all EPs to comply with this requirement is $\$ 3,904,316(263,627$ hours $\times \$ 14.81)$.

To estimate capital costs for EPs, we assume a certified EHR system will cost roughly $\$ 54,000$. If 521,600 EPs adopt these EHRs, total capital costs prior to incentives would be roughly $\$ 23.9$ billion. We also estimate that in 2011, $\$ 0.2$ billion of Medicare incentive payments and $\$ 0.2$ billion of Medicaid incentive payments would be provided to EPs under a low scenario, and $\$ 0.6$ billion Medicare incentive payments and $\$ 0.9$ billion of Medicaid incentive payments would be provided to EPs under a high scenario to help offset those costs. Therefore, we estimate that total net capital costs for EPs in 2011 would be $\$ 23.5$ billion ( $\$ 23.9$ billion $-\$ 0.2$ billion $-\$ 0.2$ billion) under a low scenario and $\$ 22.4$ billion ( $\$ 23.9$ billion $-\$ 0.6$ billion $-\$ 0.9$ billion). These capital costs would decrease over the course of the EHR incentive programs as additional incentives are provided. Therefore, in 2012, the total net capital costs for EPs would be $\$ 22.1$ billion ( $\$ 23.5$ billion $-\$ 1.0$ billion of Medicare incentives $-\$ 0.4$ billion of Medicaid incentives) under the low scenario and 419.0 billion ( $\$ 22.4$ billion $-\$ 2.3$ billion $-\$ 1.1$ billion) under the high scenario.

As with EPs, for eligible hospitals and CAHs, we proposed, at section 495.8(b) of the proposed rule, that hospitals demonstrate they are meaningful EHR users through an attestation mechanism. As with EPs, we divided meaningful use criteria into Sets A and B. We estimated that it would take an eligible hospital or CAH 0.5 hours to attest to the requirements in $\S 495.8(\mathrm{~b})(1)(\mathrm{i})$ and (ii) including the Set A meaningful use objectives/measures, .0.5 hours to select and attest to the hospital quality measures, and 7 hours to comply with gathering the information, attesting and reporting Set B objectives/measures. Therefore, the estimated the total burden for all 5,011 Medicare and Medicaid eligible hospitals and CAHs (3,620 acute care hospitals, 1,302 critical access hospitals, 78 Medicaid children's hospitals, and 11 Medicaid cancer hospitals) equaled 5,011 hours. For Set B objectives and measures, we estimated the total burden at 35,077 hours.

We believed that an eligible hospital or CAH might assign an attorney to attest on their behalf. We estimated the cost burden for an eligible hospital or $\mathrm{CAH}$ to attest to the Set $\mathrm{A}$ and hospital quality requirements was $\$ 59.98$ and the total estimated annual cost burden for all eligible hospitals and CAHs to attest was $\$ 300,560$. For Set B objectives/ measures, we estimated a per-hospital cost burden of $\$ 419.86$, and a total cost burden of $\$ 2,103,918$, not including capital costs. We solicited public comments on the estimated percentages and the numbers of (registered) eligible hospitals and CAHs that would attest in FY 2011, but we did not receive any comments on this issue. We also invited comments on the type of personnel or staff that would mostly likely attest on behalf of eligible hospitals and CAHs, but we did not receive any comments on this issue.

For the final rule, as proposed, $\S 495.8(\mathrm{~b})$ will require demonstration of meaningful use through an attestation mechanism. However, as with EPs, we have revised the burden estimates due to the changes in meaningful use 
objectives and measures, in response to comments. Unless an exception applies, $\S 495.6(\mathrm{~b})$ requires that an eligible hospital or CAH must meet all 14 Stage 1 meaningful use core criteria under $\S 495.6$ (f) and five out of 10 meaningful use menu criteria under $§ 495.6(\mathrm{~g})$. The burden associated with the requirements in $\S 495.8$ and $\S 495.6$ is the time and effort required to attest to the required elements.

To comply with § 495.8(b)(1), we estimate that it would take an eligible hospital or CAH 8 hours 42 minutes to prepare and attest that during the EHR reporting period, the hospital or CAH used certified technology, specify the technology, and satisfied all 14 mandatory Stage 1 meaningful use core criteria. We estimate that it will take an eligible hospital or CAH an extra 0.5 hours to select and attest to the hospital quality measure, in the format and manner specified by CMS. We estimate the total burden associated with this requirement for an eligible hospital or CAH is 9 hours 12 minutes ( 8 hours 42 minutes +0.5 hours) and the total burden all eligible hospitals and CAHs to attest to these requirements is 46,101 hours ( 9 hours 12 minutes $\times 5,011$ hospitals). We believe an eligible hospital or CAH may use an attorney to attest on their behalf. We estimate the associated cost burden for an eligible hospital or CAH to attest to these requirements is $\$ 551.82$ (9 hours 12 minutes $\times \$ 59.98$ (mean hourly rate for attorneys based on the May 2008 Bureau of Labor Statistics)) and the total cost burden for all eligible hospitals and CAHs to attest to these requirements is $\$ 2,765,150$ ( $\$ 551.82 \times 5,011$ hospitals and CAHs)).

We recognize that some Stage 1 meaningful use menu criteria are easier to accomplish than others. Therefore, as with the EPs, our burden estimates are based on a "low" and "high" scenario. Unless an exception applies, § 495.6(b) requires that an eligible hospital or $\mathrm{CAH}$ must meet five out of 10 Stage 1 meaningful use menu criteria. The burden involved is the time and effort to select and attest to the meaningful use menu-set measures. Under the "low" scenario, we estimate it will take an eligible hospital or CAH 42 minutes to attest to five Stage 1 meaningful use menu-set measures, resulting in a total burden for all 5,011 eligible hospitals and CAHs of 3,508 hours $(5,011$ hospitals $\times 42$ minutes). Under the high scenario, we estimate it will take an eligible hospital or CAH 3 hours 30 minutes to attest to five Stage 1 meaningful use menu-set measures, resulting in a total burden for all 5,011 eligible hospitals and CAHs of 17,539 hours $(5,011$ hospitals $\times 3$ hours 30 minutes). Based on the two scenarios, the average burden is 2 hours 6 minutes (42 minutes +3 hours 30 minutes)/2), and the average burden for all eligible hospitals and CAHs is 10,523 hours $(3,508$ hours $+17,539$ hours $) / 2)$.

We believe an eligible hospital or CAH may use an attorney to attest on their behalf. For menu-set meaningful use criteria, low scenario, we estimate the associated cost burden for an eligible hospital or CAH is $\$ 41.99$ (42 minutes $\times \$ 59.98$ (mean hourly rate for attorneys based on the May 2008 Bureau of Labor Statistics)) and the total cost burden for all eligible hospitals and CAHs is $\$ 210,392(\$ 41.99 \times 5,011$ hospitals and CAHs). For menu-set meaningful use criteria, high scenario, we estimate the associated cost burden for an eligible hospital or $\mathrm{CAH}$ is $\$ 209.93$ (3 hours 30 minutes $\times \$ 59.98$ ) and the total cost burden for all eligible hospitals and CAHs is $\$ 1,051,959$ (\$209.93 × 5,011 hospitals and CAHs). Based on the two scenarios, the average cost burden for an eligible hospital or $\mathrm{CAH}$ to attest to meaningful use menu set criteria is $\$ 125.96$ ( $\$ 41.99+$ $\$ 209.93) / 2$ ). The average burden for all eligible hospitals and CAHs to attest to meaningful use menu set criteria is $\$ 631,176$ ( $\$ 210,392+\$ 1,051,959) / 2)$.

As with EPs, our proposed regulations (at $\S 495.8(b)(2)$ ) required that for FY 2012 and subsequent years, eligible hospitals and CAHs demonstrate meeting most meaningful use criteria through attestation, and electronically report hospital quality measures. As with EPs, we divided meaningful use objectives and measures into Sets $A$ and B. For Set A, we estimated that it would take an eligible hospital or CAH 0.5 hours to attest to the requirements in $\S 495.8(b)(2)$. For Set B, we estimated it would take an eligible hospital or $\mathrm{CAH}$ 7 hours to gather information and attest. Assuming that 5,011 hospitals might attest, we estimated that the total annual attestation burden for all eligible hospitals and CAHs was 2,506 hours (Set A) and 35,077 hours (Set B). We estimated the total annual cost burden for all eligible hospitals and CAHs to attest was $\$ 150,310$ (Set A) and $\$ 2,103,918$ (Set B). We invited public comments on the estimated percentages and the numbers of registered EPs that would attest to EHR technology used in CY 2012, but we did not receive any comments on this issue.

In the final rule, we also require that for FY 2012, eligible hospitals and CAHs demonstrate meeting meaningful use criteria through attestation, except for clinical quality measures, which must be electronically reported to CMS or the States. We do not expect growth in the number of eligible hospitals or CAHs. The meaningful use criteria (core and menu sets) are the same for FY 2011 and FY 2012. To comply with $\S 495.8(\mathrm{~b})(1)$, we estimate that it would take an eligible hospital or CAH 8 hours 41 minutes to prepare and attest that during the EHR reporting period, the eligible hospital or CAH used certified technology, specify the technology, and satisfied all 14 mandatory Stage 1 meaningful use core criteria. We estimate the total burden associated with this requirement for all eligible hospitals and CAHs to attest to these requirements is 43,596 hours ( 8 hours 42 minutes $\times 5,011$ hospitals). We believe an eligible hospital or CAH may use an attorney to attest on their behalf. We estimate the associated cost burden for an eligible hospital or CAH to attest to these requirements is $\$ 521.83$ (8 hours 42 minutes $\times \$ 59.98$ (mean hourly rate for attorneys based on the May 2008 Bureau of Labor Statistics)) and the total cost burden for all eligible hospitals and CAHs to attest to these requirements is $\$ 2,614,870$ ( $\$ 521.83 \times 5,011$ hospitals and CAHs).

We recognize that some Stage 1 meaningful use menu criteria are easier to accomplish than others. We cannot predict which of the measures in the menu criteria will be selected by an eligible hospital or CAH. Therefore, as with EPs, our burden estimates are based on a "low" and "high" scenario. Unless an exception applies, § 495.6(b) requires that an eligible hospital or $\mathrm{CAH}$ must meet five out of 10 Stage 1 meaningful use menu criteria. The burden involved is the time and effort to select and attest to the meaningful use menu criteria. Under the "low" scenario, we estimate it will take an eligible hospital or CAH 42 minutes to attest to five Stage 1 meaningful use menu-set measures, resulting in a total burden of 3,508 hours $(5,011$ hospitals $\times 42$ minutes). Under the high scenario, we estimate it will take an eligible hospital or CAH 3 hours 30 minutes to attest to five Stage 1 meaningful use menu-set measures, resulting in a total burden of 17,539 hours (5,011 hospitals $\times 3$ hours 30 minutes). Based on the two scenarios, the average burden for an eligible hospital or CAH to attest to meaningful use menu set criteria is 2 hours 6 minutes ( 42 minutes +3 hours 30 minutes)/2), and the average burden hours for all eligible hospitals and CAHs is 10,523 hours $((3,508$ hours $+17,539$ hours) $/ 2$ )

We believe an eligible hospital or CAH may use an attorney to attest on their behalf. For menu-set meaningful use criteria, low scenario, we estimate 
the associated cost burden for an eligible hospital or CAH is \$41.99 (42 minutes $\times \$ 59.98$ ) and the total cost burden for all eligible hospitals and CAHs is $\$ 210,392(\$ 41.99 \times 5,011$ hospitals and CAHs). For menu-set meaningful use criteria, high scenario, we estimate the associated cost burden for an eligible hospital or $\mathrm{CAH}$ is $\$ 209.93$ (3 hours 30 minutes $\times \$ 59.98$ ) and the total cost burden for all eligible hospitals and CAHs is $\$ 1,051,959$ (\$209.93 × 5,011 hospitals and CAHs). Based on the two scenarios, the average cost burden for an eligible hospital or $\mathrm{CAH}$ to attest to meaningful use menu set criteria is $\$ 125.96$ ( $\$ 41.99+$ $\$ 209.93) / 2$ ). The average burden for all eligible hospitals and CAHs to attest to meaningful use menu set criteria is $\$ 631,175((\$ 210,392+\$ 1,051,959) / 2)$.

Section 495.8(b)(2)(iii) requires that for FY 2012, eligible hospitals or CAHs must report electronically to CMS, or, in the case of Medicaid hospitals, the States, clinical quality information in the format and manner specified by CMS. Given that we limit the required measures only to those that can be automatically calculated by a certified EHR and to those for which we have electronic specifications currently available that we are able to post as final by date of display of this final rule. The burden associated with this requirement is the time and effort to report the required hospital quality measures. We estimate the burden for an eligible hospital or CAH to comply with this requirement is 0.5 hours and the total burden for all eligible hospitals or CAHs to comply with this requirement is 2,506 hours $(5,011$ hospitals and CAHs $\times 0.5$ hours). We believe that an eligible hospital or CAH may assign a medical secretary to submit the specific hospital clinical quality measures to CMS or the States. We estimated the cost burden for an eligible hospital or CAH to comply with this requirement is $\$ 7.40$ (0.5 hours $\times \$ 14.81$ (mean hourly rate of medical secretary based on May 2008 Bureau of Labor Statistics)) and the cost burden for all eligible hospitals or CAHs to comply with this requirement is $\$ 37,107(2,506$ hours $\times \$ 14.81)$.

To estimate capital costs for eligible hospitals and CAHs, consistent with the sources cited in section V.G.4 of this final rule, we assume that achieving meaningful use will require roughly a \$5 million capital investment for the average hospital. If 5,011 hospitals adopt these EHRs, total capital costs prior to incentives would be roughly $\$ 25.1$ billion. We also estimate that in 2011, $\$ 0.2$ billion of Medicare incentive payments and $\$ 0.4$ billion of Medicaid incentive payments would be provided to eligible hospitals and CAHs under the low scenario, and $\$ 0.5$ billion of Medicare incentive payments and $\$ 0.8$ billion of Medicaid incentive payments would be provided to eligible hospitals and CAHs under the high scenario to help offset those costs. Therefore, we estimate that total net capital costs for hospitals in 2011 would be $\$ 24.5$ billion ( $\$ 25.1$ billion $-\$ 0.2$ billion $-\$ 0.4$ billion) under the low scenario and $\$ 23.8$ billion ( $\$ 25.1$ billion $-\$ 0.5$ billion $-\$ 0.8$ billion) under the high scenario. These capital costs would decrease over the course of the EHR incentive programs as additional incentives are provided. Therefore, in 2012, the total net capital costs for hospitals would be $\$ 23.5$ billion ( $\$ 24.5$ billion $-\$ 0.9$ billion of Medicare incentives $-\$ 0.1$ billion of Medicaid incentives) under the low scenario, and $\$ 21.4$ billion ( $\$ 23.8$ billion $-\$ 2.1$ billion of Medicare incentives $-\$ 0.3$ billion of Medicaid incentives) under the high scenario.

Comment: Some commenters believed that CMS grossly underestimated the cost and hour burden for EPs, eligible hospitals and CAHs to comply with meaningful use Set A and Set B measures. Some commenters stated that we should take into consideration all the time required to prepare all attestation of meaningful use measures, including the manual counting of numerators and denominators in our burden estimates.

Response: Prior to and after the publication of the proposed rule, we have worked with ONC to ensure that our meaningful use objectives/measures are well aligned with certified EHR technology. In the final rule, we only require meaningful use measures that can be achieved by the functionality and capability of certified EHR technology. Furthermore, based on comments, we have explained in section II.A.2.d. of this final rule that we are including a substantial amount of flexibility in the final rule to lower the burden for EPs, eligible hospitals and CAHs in meeting the attestation and demonstration of meaningful use criteria. Some examples of such flexibility are the categorization of Stage 1 meaningful use core and menu (optional) criteria, reducing the number of meaningful use objectives/ measures for 2011 and 2012, limiting the denominators, in certain cases, only to patients whose records are maintained using certified EHR technology, and lowering thresholds for many of the meaningful use measures. We believe these changes reduce burden without compromising the intent of the Congress, and the ability of EHR technology to begin to improve health care quality, efficiency, and outcomes. We have considered the comments and we have made some revisions on our previous burden estimates. While this requirement is subject to PRA, we have no way of accurately quantifying the burden. We will continue to monitor the burden associated with the implementation of EHR technology as our experience continues to grow and as EHR technology continues to evolve.

Comment: CMS received numerous comments regarding the burden (economic and other) of reporting on the large number of measures and the overall quality reporting burden this will add to EPs and other healthcare providers. Others suggested reporting on significantly smaller set of measures.

Response: As we have explained in section II.A.3.(d) of this final rule, we have reduced the reporting burden by decreasing the number of required clinical quality measures and limiting measures to those that can be automatically calculated by a certified EHR. We believe that the proposed burden estimate, which was estimated to be an additional 0.5 hours in 2011 and 2012, is reasonable and we are finalizing it.

Table 20 below lists the objectives and associated measures in which we estimate the burden to fulfill "core set," "menu set", and clinical quality measures requirements. Estimates of total capital costs at the bottom of Table 20 are derived from the estimates used in the "Industry Costs" section in Section V.G.4. of this final rule. BILLING CODE 4120-01-P 


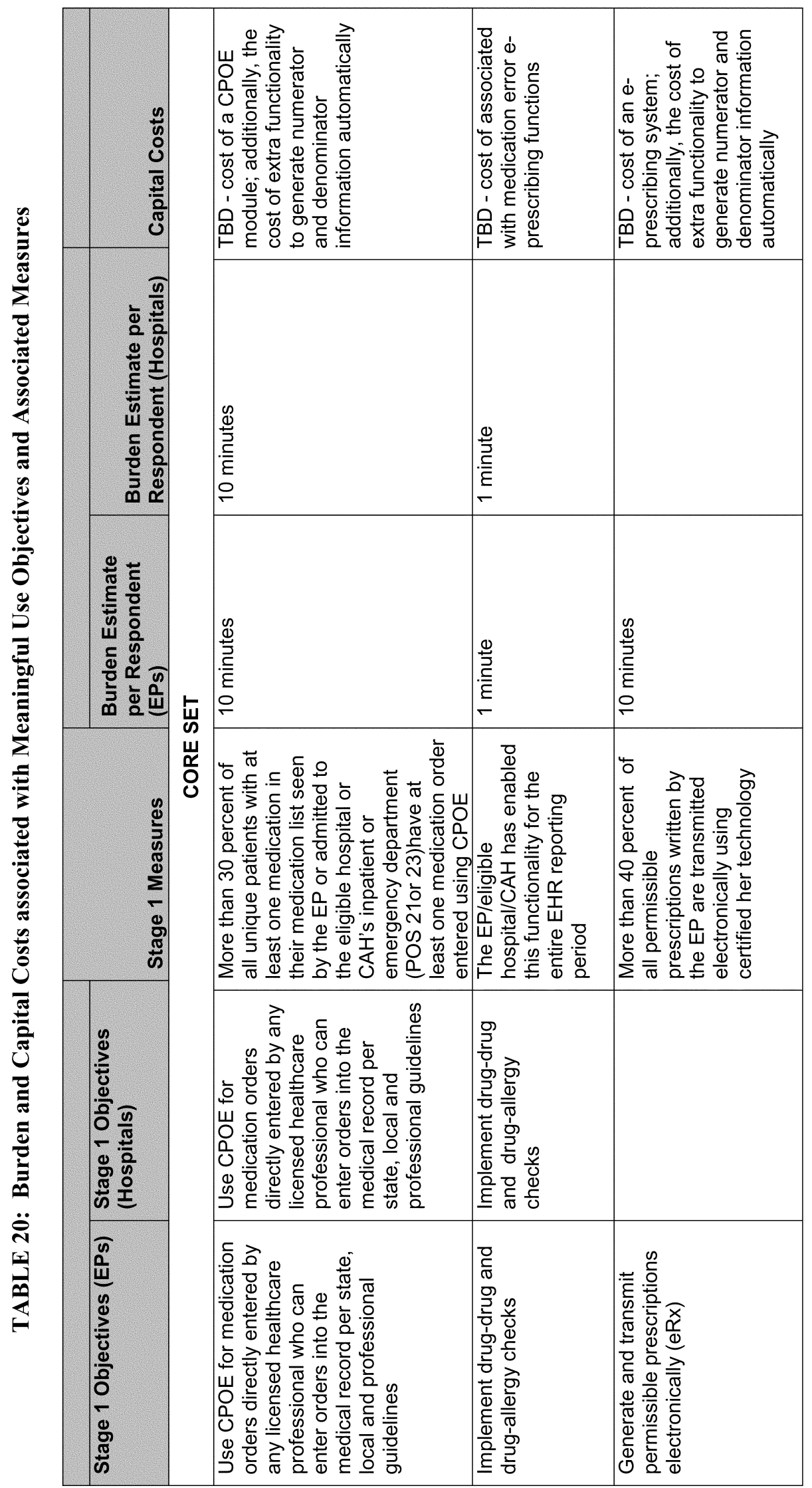




\begin{tabular}{|c|c|c|}
\hline 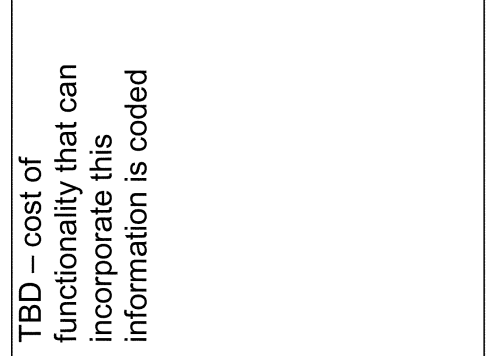 & 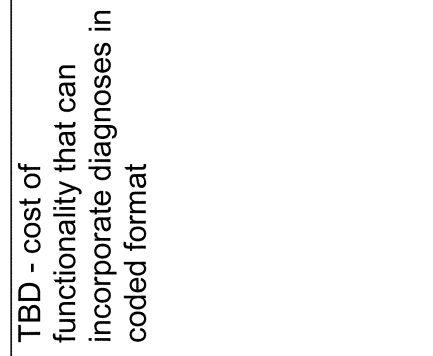 & 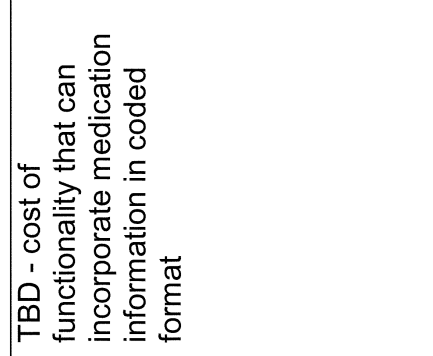 \\
\hline 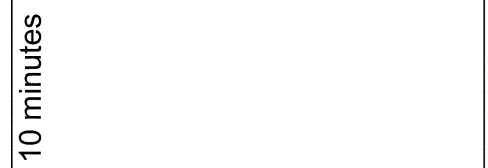 & 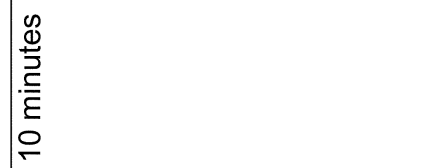 & 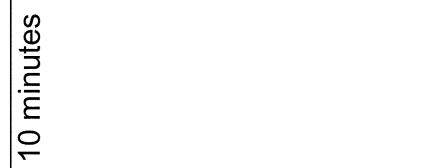 \\
\hline 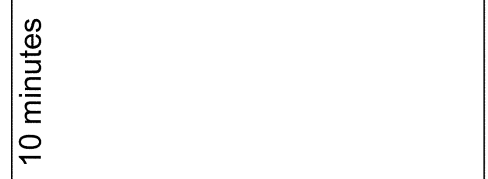 & 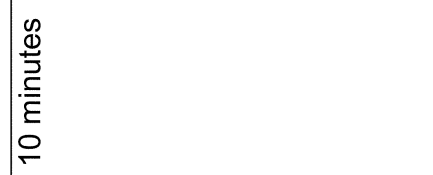 & 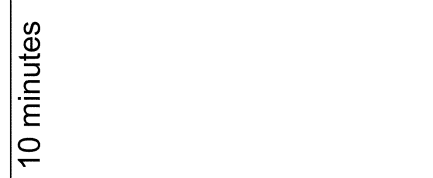 \\
\hline 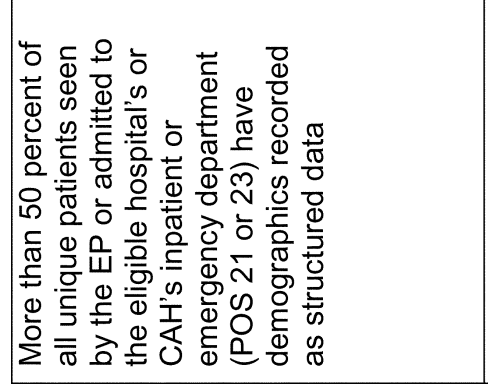 & 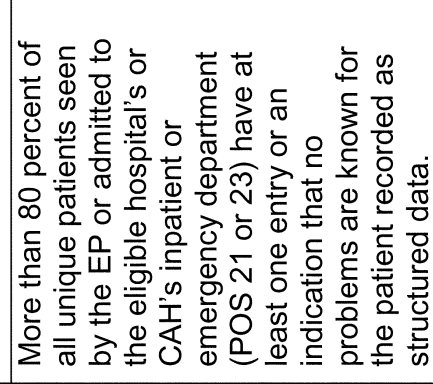 & 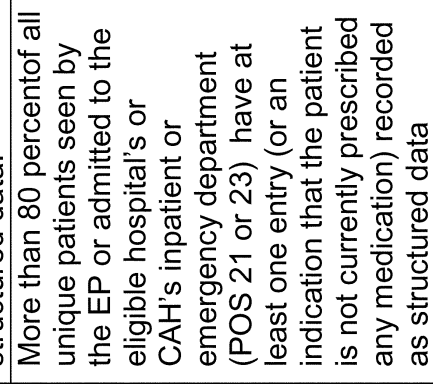 \\
\hline 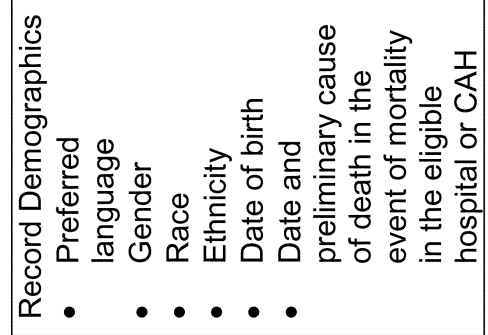 & 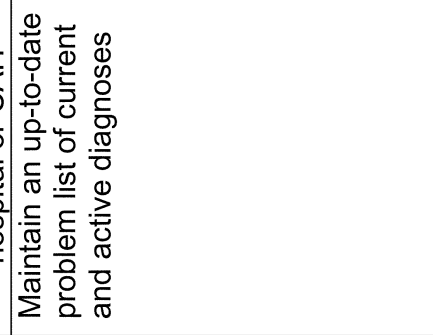 & 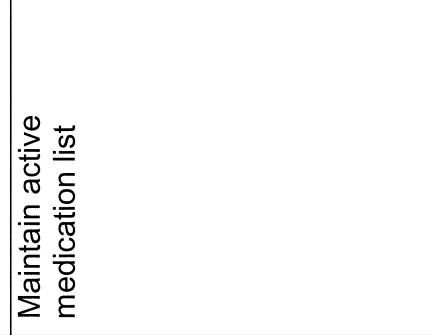 \\
\hline 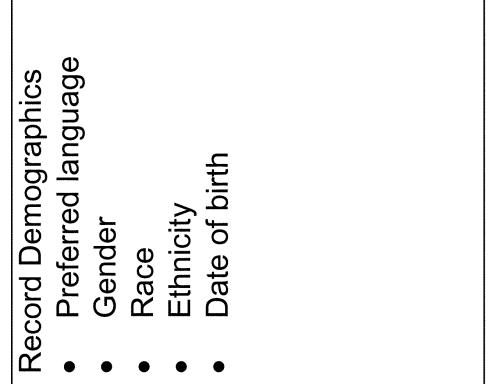 & 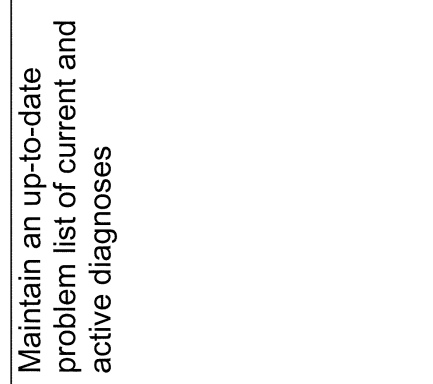 & 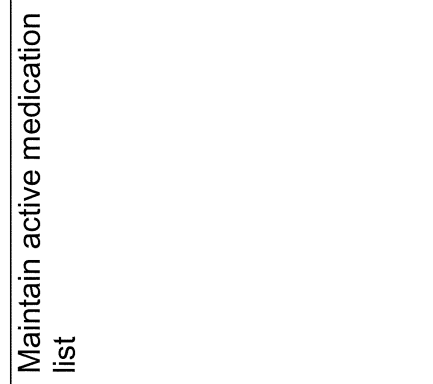 \\
\hline
\end{tabular}




\begin{tabular}{|c|c|c|c|}
\hline 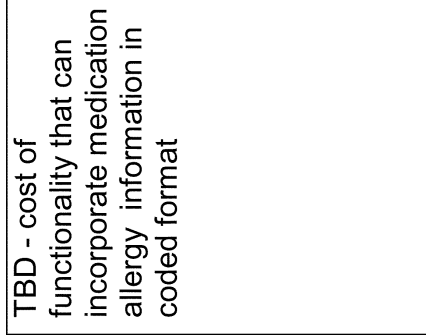 & 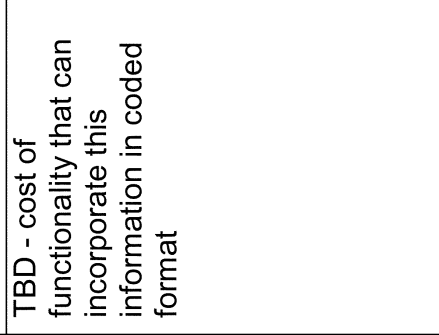 & 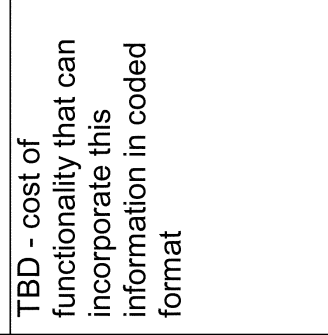 & 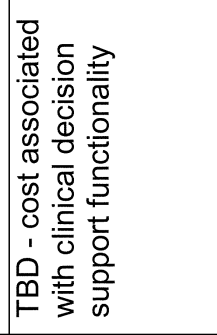 \\
\hline 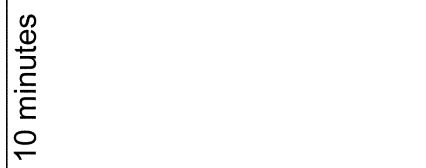 & 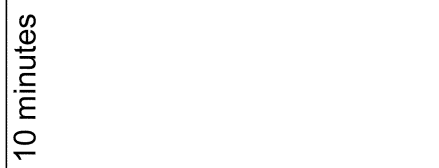 & 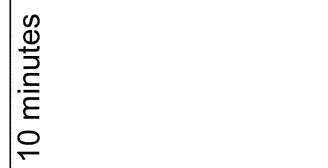 & 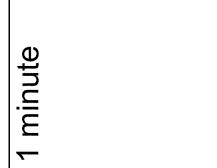 \\
\hline 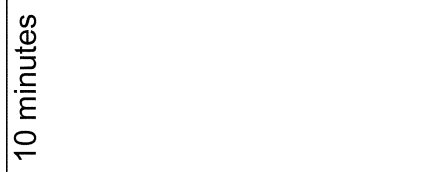 & 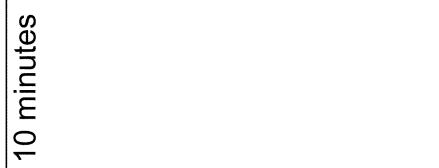 & 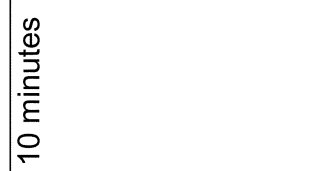 & 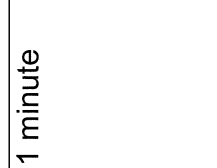 \\
\hline 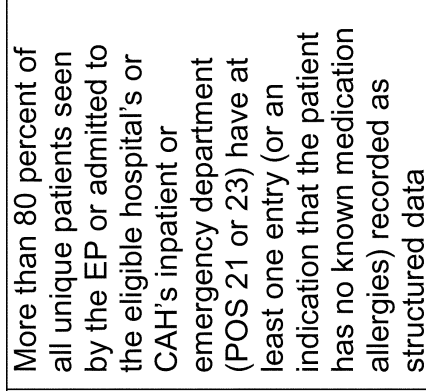 & 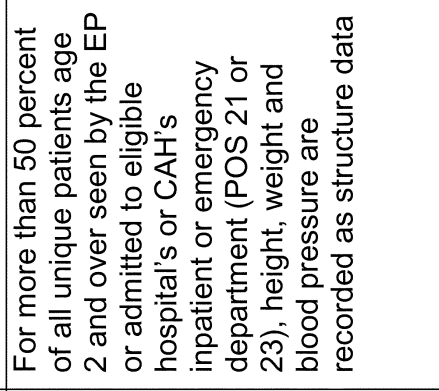 & 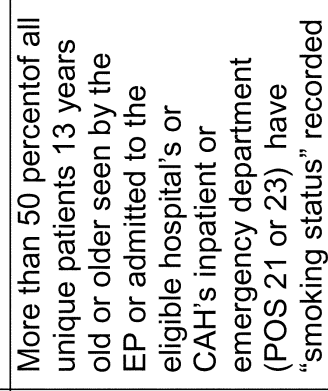 & 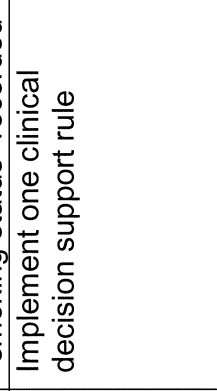 \\
\hline 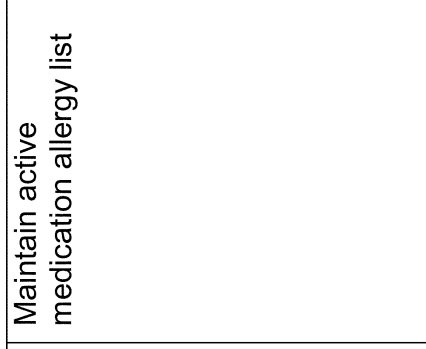 & 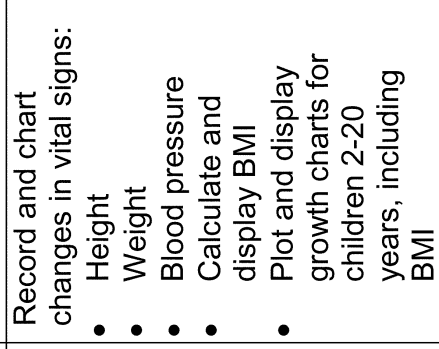 & 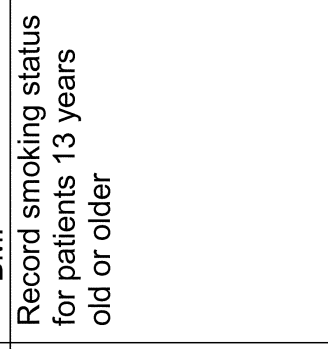 & 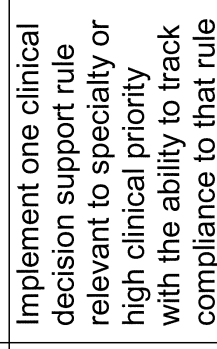 \\
\hline 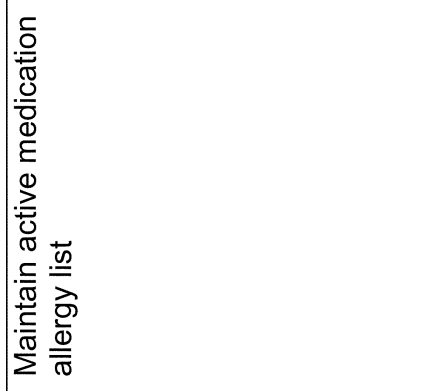 & 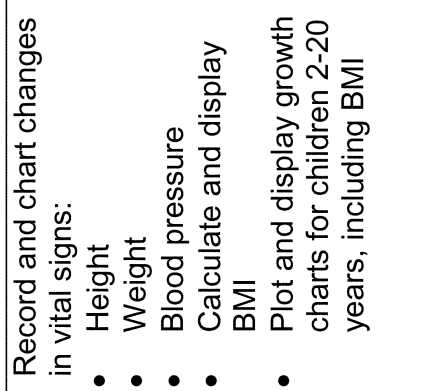 & 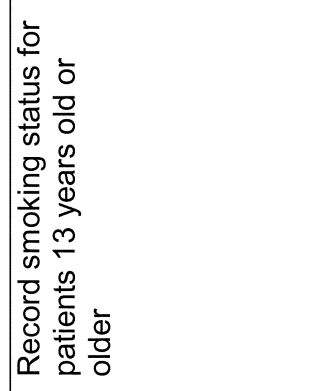 & 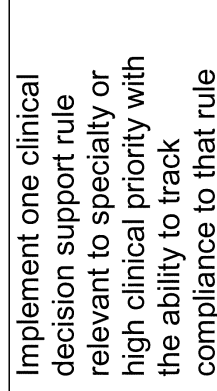 \\
\hline
\end{tabular}




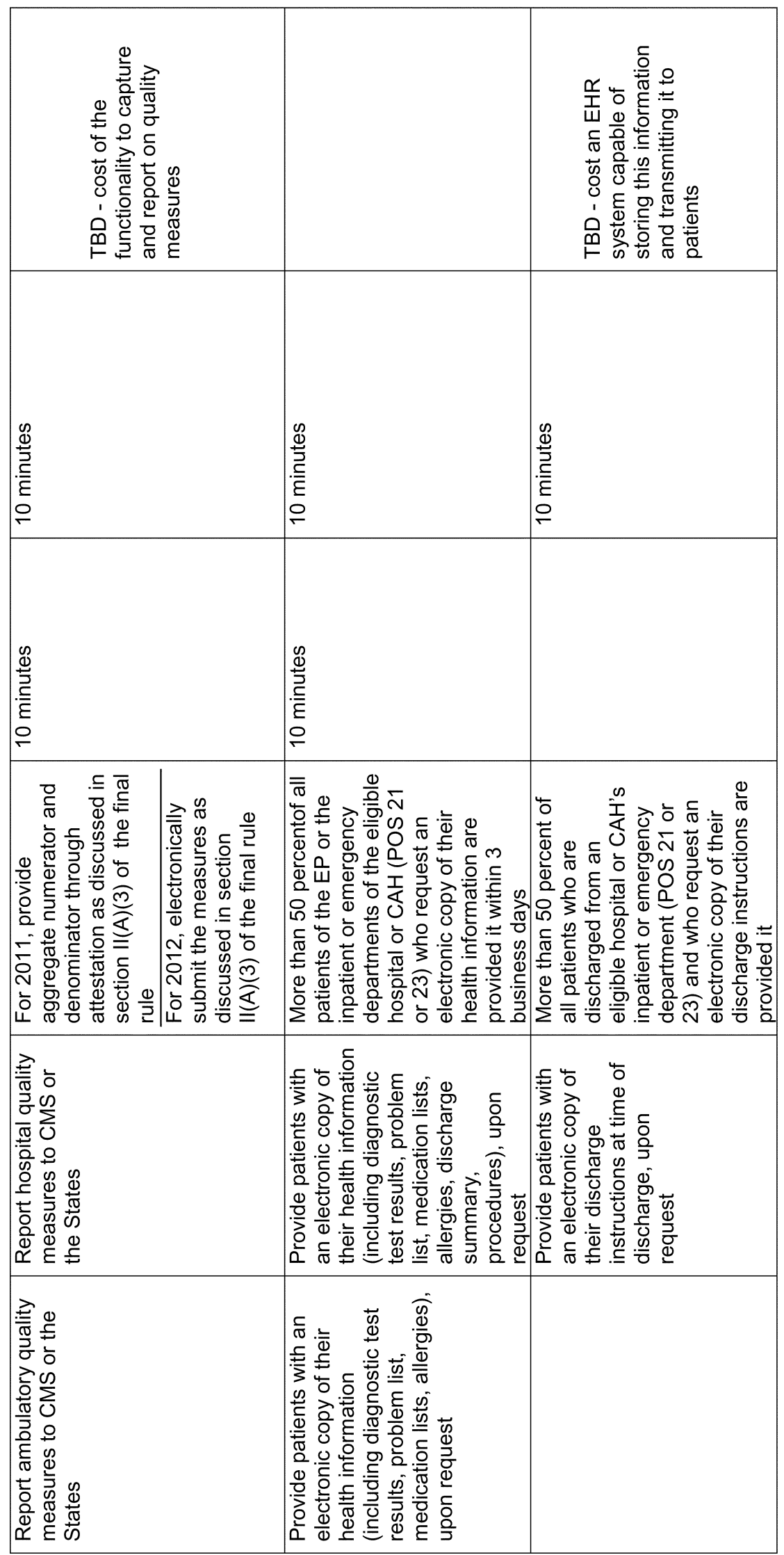




\begin{tabular}{|c|c|c|c|c|}
\hline 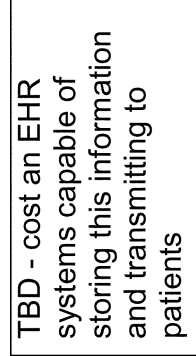 & 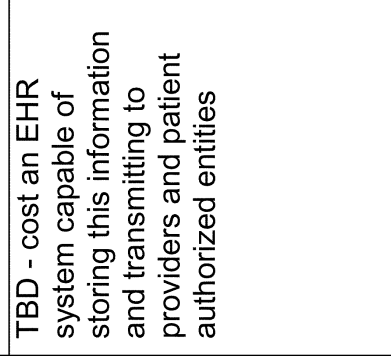 & 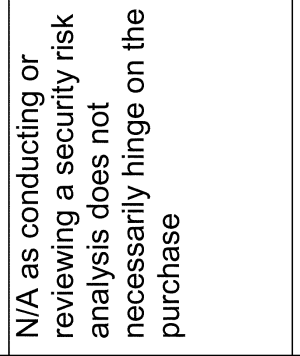 & & \multirow{6}{*}{ 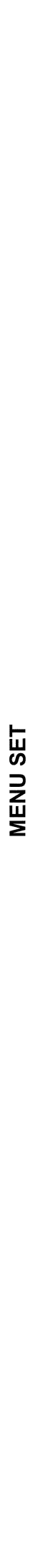 } \\
\hline & 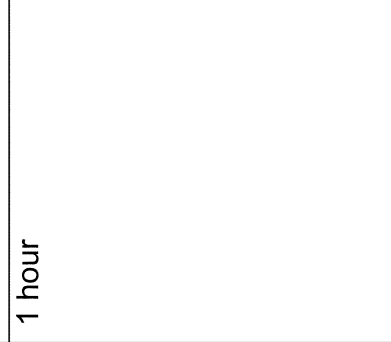 & 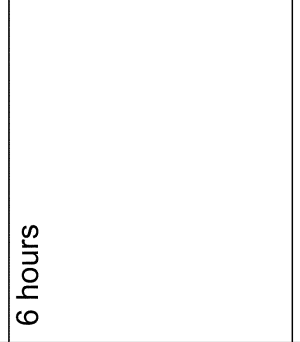 & 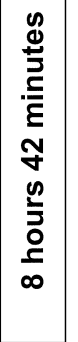 & \\
\hline 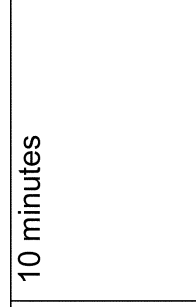 & 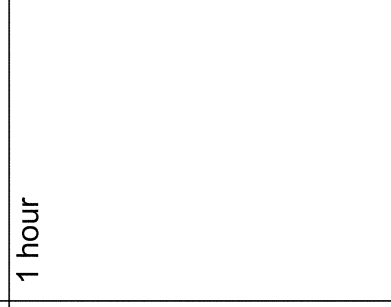 & $\begin{array}{l}\frac{\infty}{3} \\
\frac{0}{0} \\
\frac{0}{0}\end{array}$ & 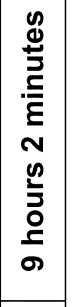 & \\
\hline \multirow[t]{2}{*}{ 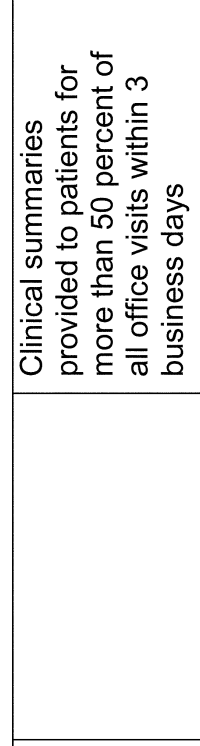 } & 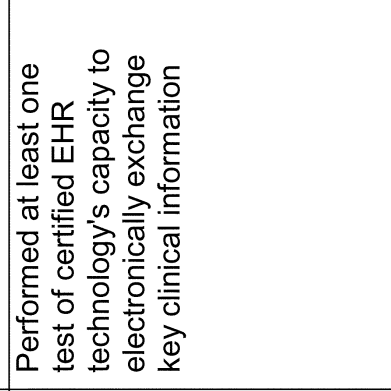 & 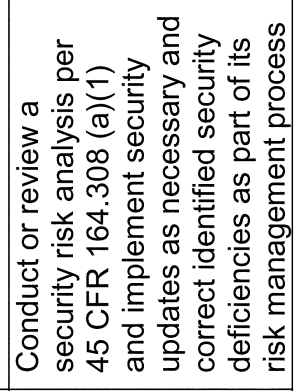 & \multirow{3}{*}{ 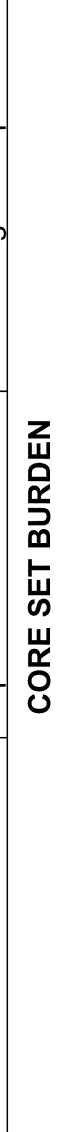 } & \\
\hline & 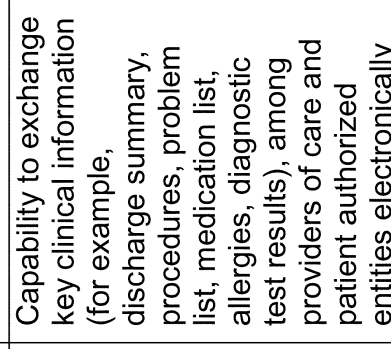 & 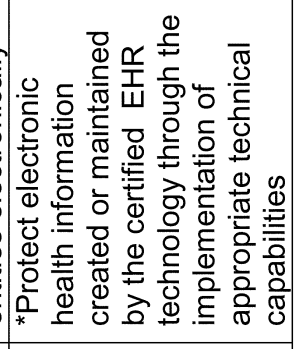 & & \\
\hline 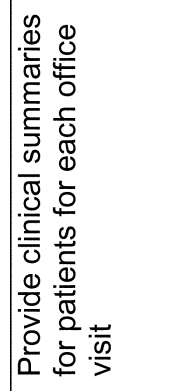 & 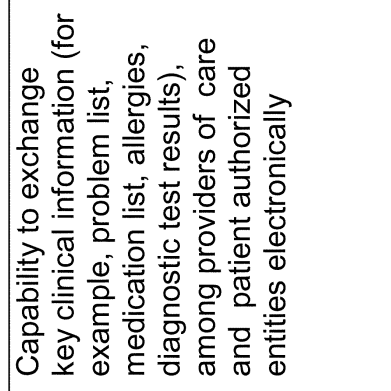 & 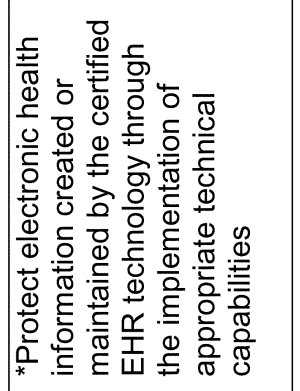 & & \\
\hline
\end{tabular}




\begin{tabular}{|c|c|c|}
\hline 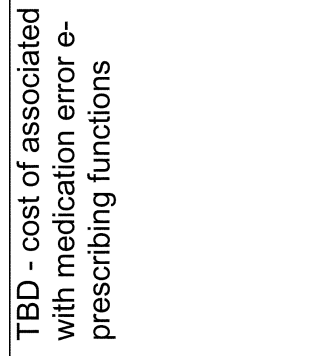 & & 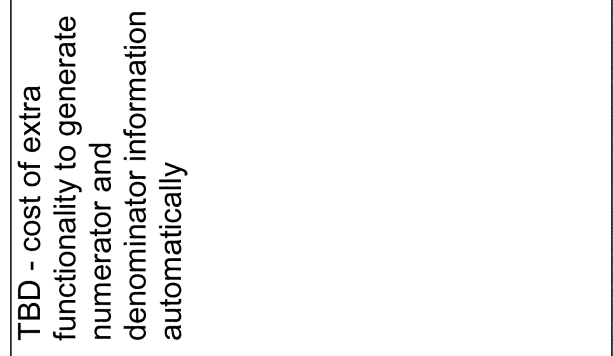 \\
\hline 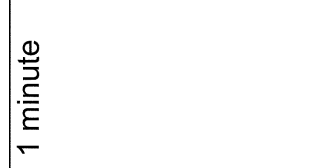 & 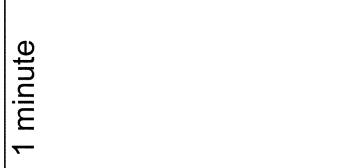 & 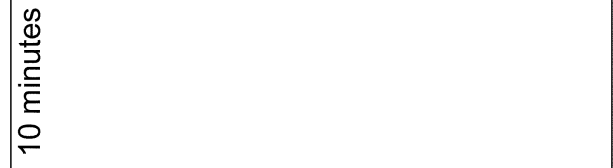 \\
\hline 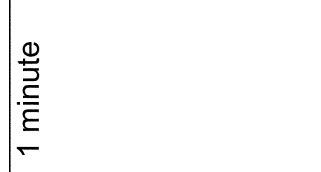 & & 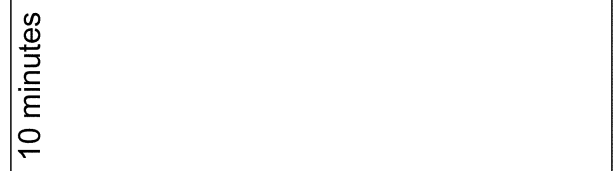 \\
\hline 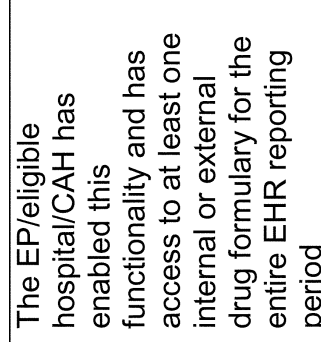 & 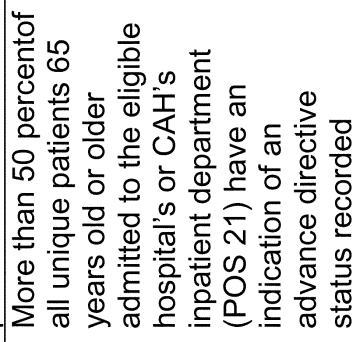 & 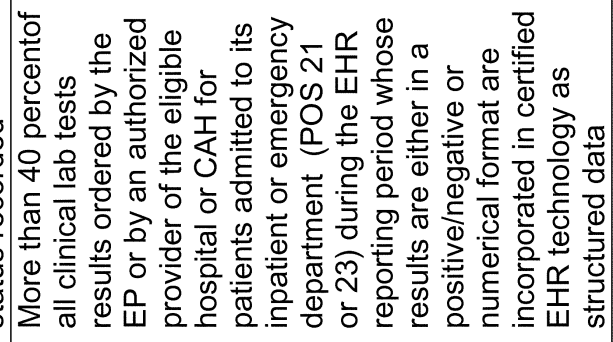 \\
\hline 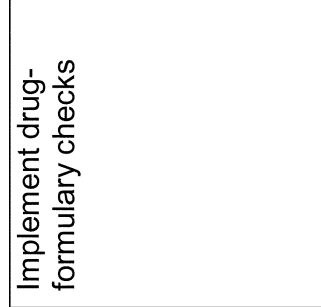 & 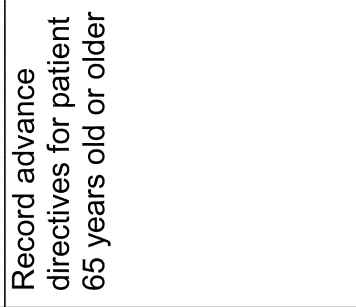 & 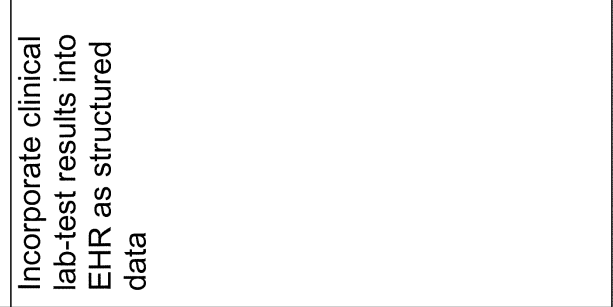 \\
\hline 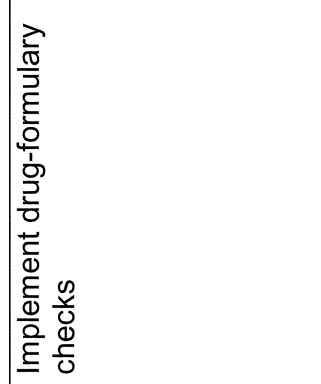 & & 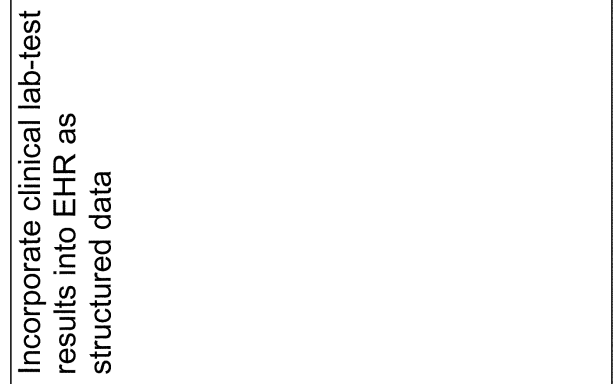 \\
\hline
\end{tabular}




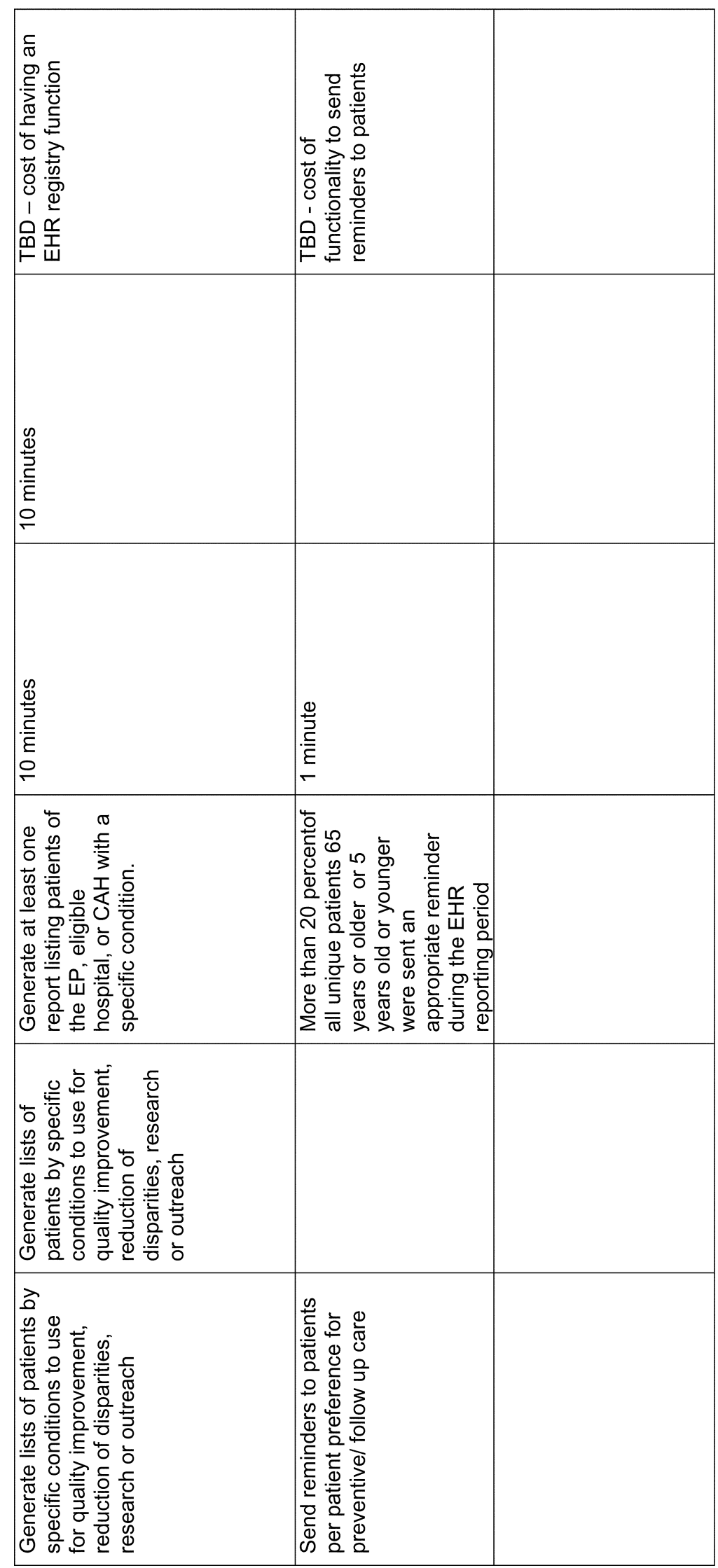




\begin{tabular}{|c|c|c|}
\hline 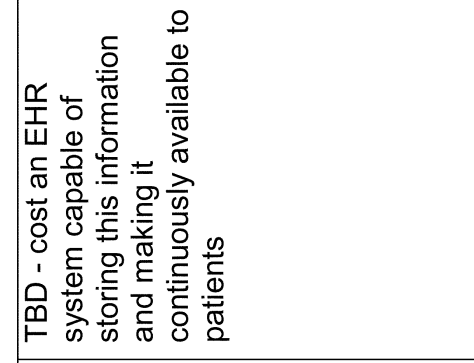 & & 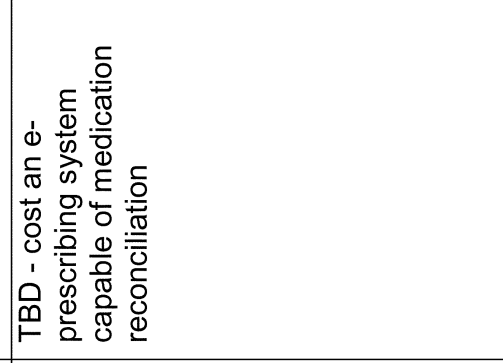 \\
\hline & 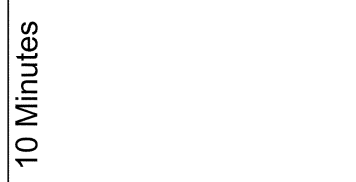 & 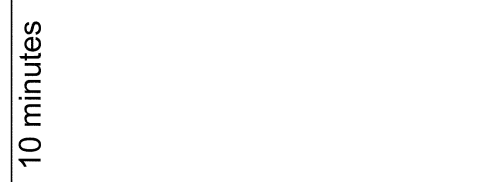 \\
\hline 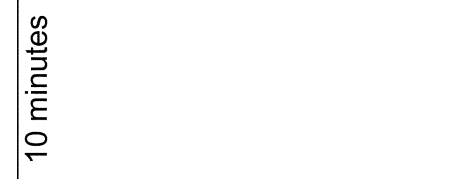 & 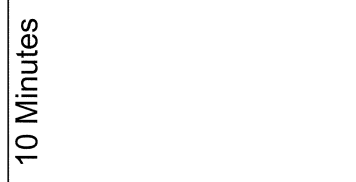 & 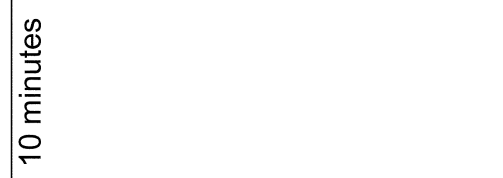 \\
\hline \multirow[t]{2}{*}{ 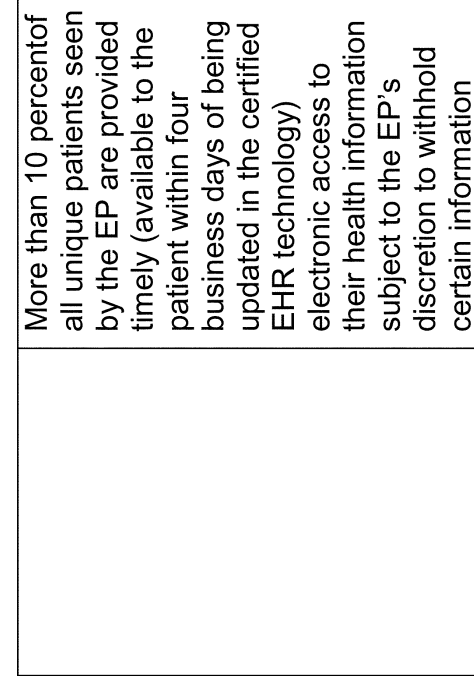 } & 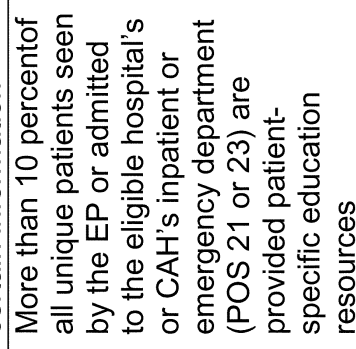 & 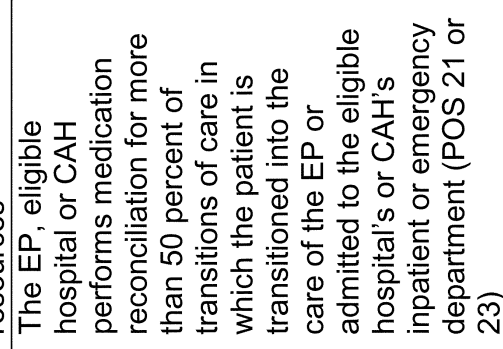 \\
\hline & 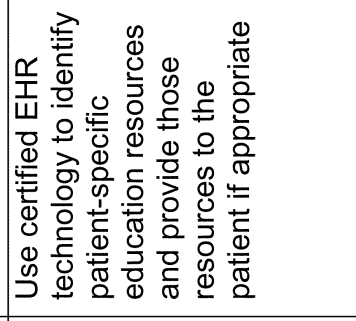 & 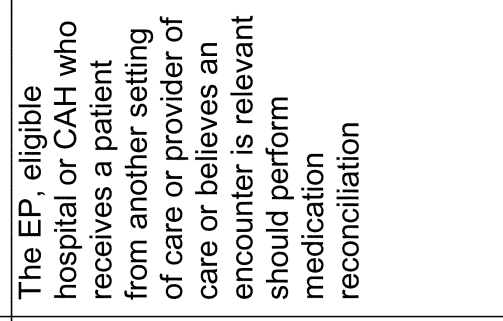 \\
\hline 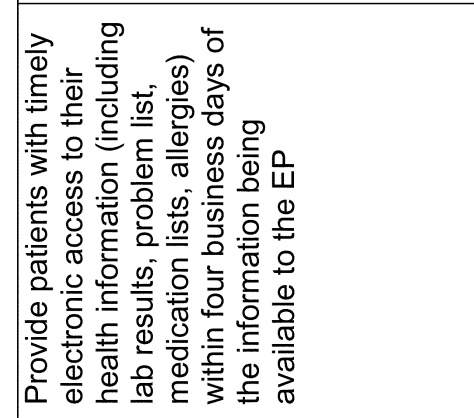 & 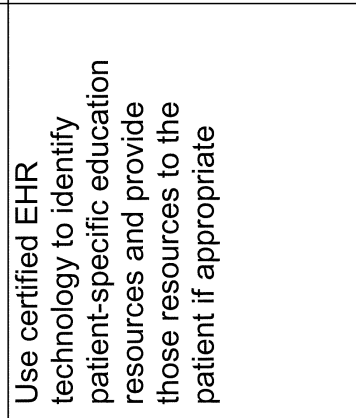 & 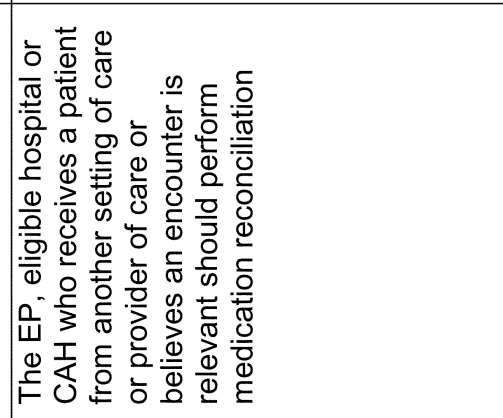 \\
\hline
\end{tabular}




\begin{tabular}{|c|c|}
\hline 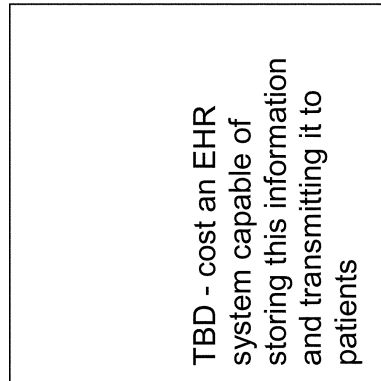 & 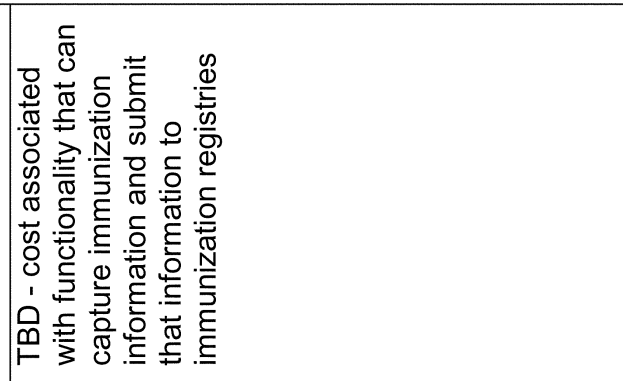 \\
\hline 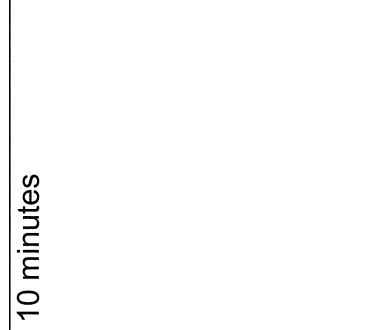 & 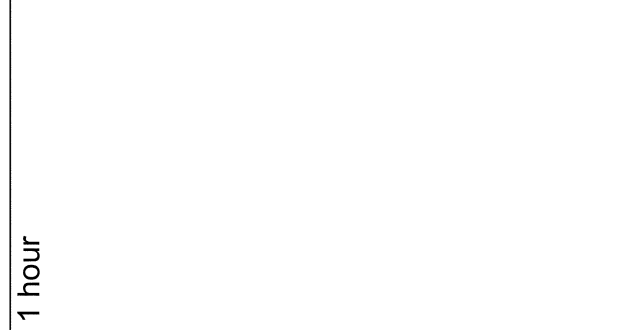 \\
\hline 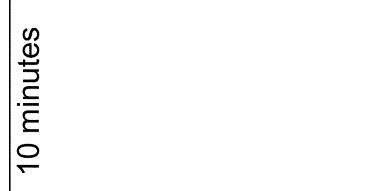 & $\begin{array}{l}\text { 호 } \\
\text { 음 }\end{array}$ \\
\hline 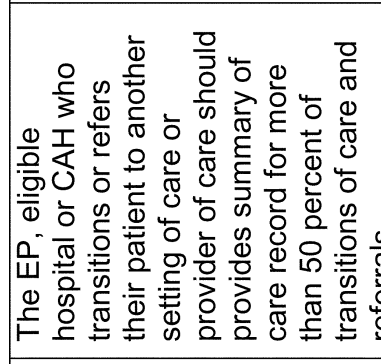 & 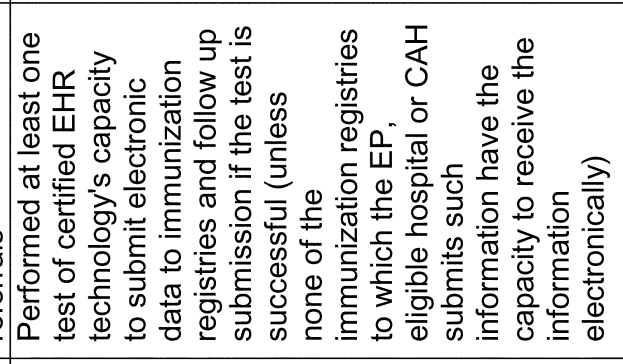 \\
\hline 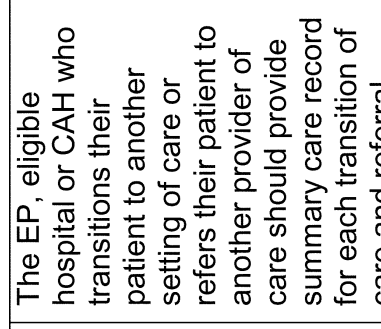 & 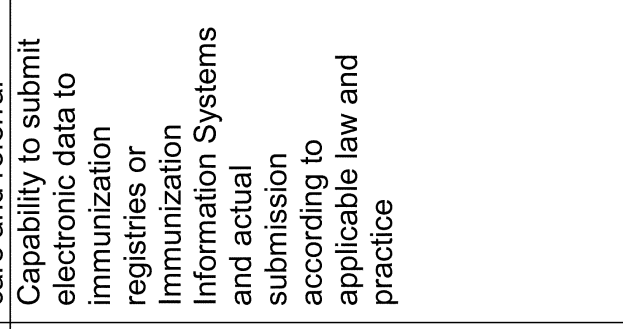 \\
\hline 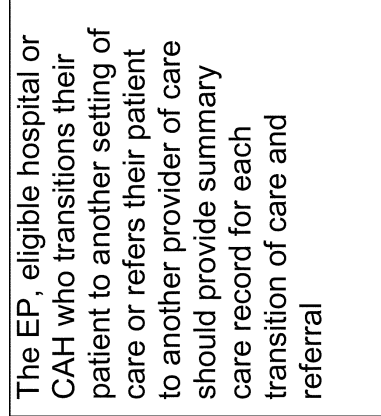 & 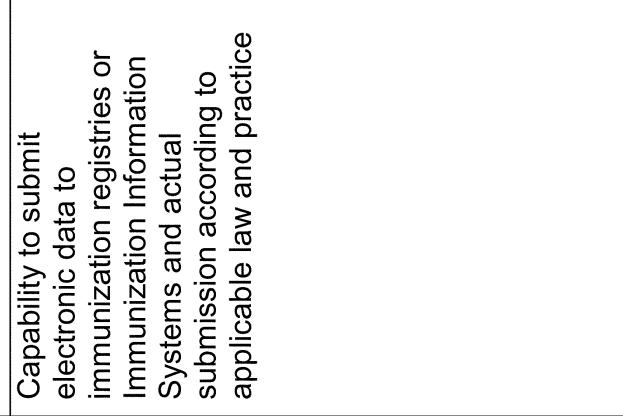 \\
\hline
\end{tabular}




\begin{tabular}{|c|c|c|}
\hline 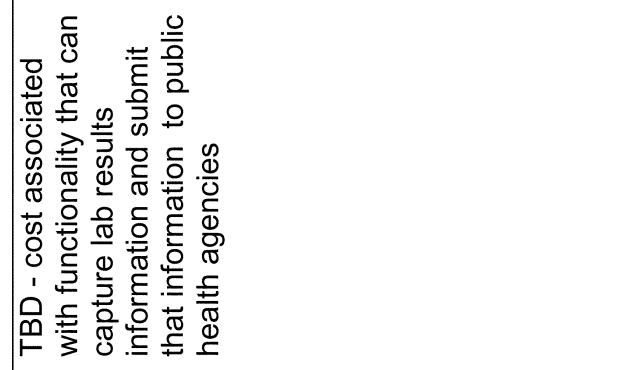 & 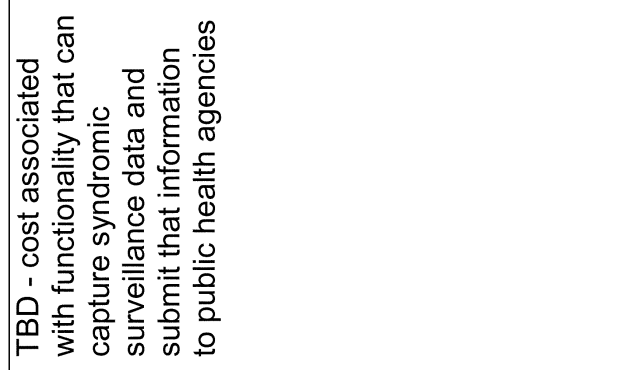 & 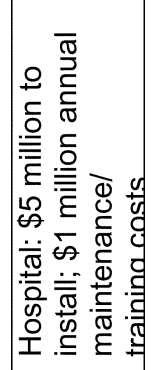 \\
\hline 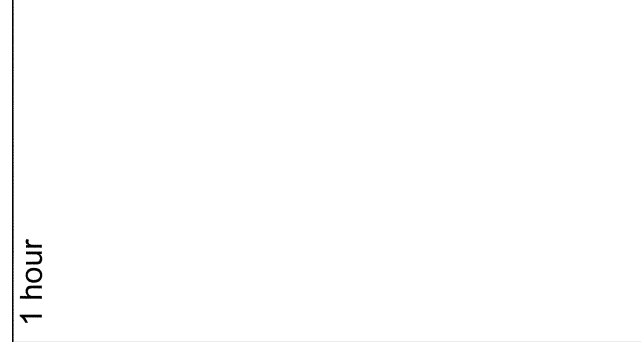 & 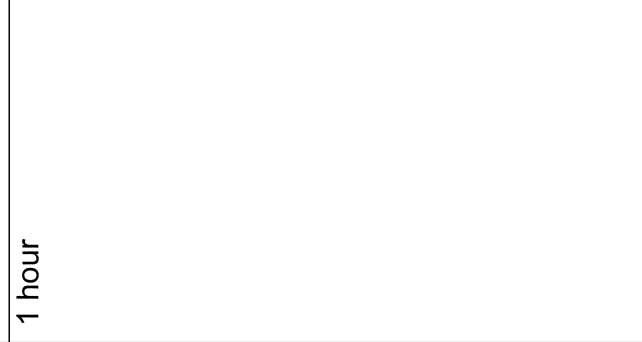 & 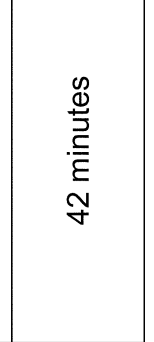 \\
\hline & 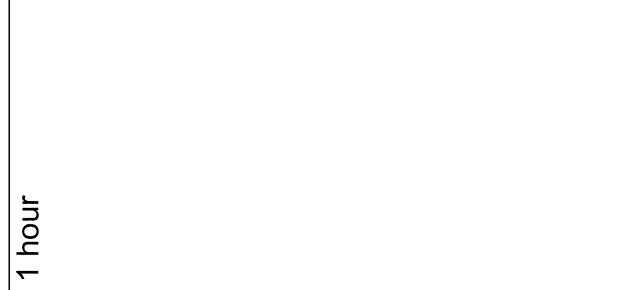 & 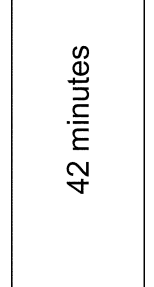 \\
\hline 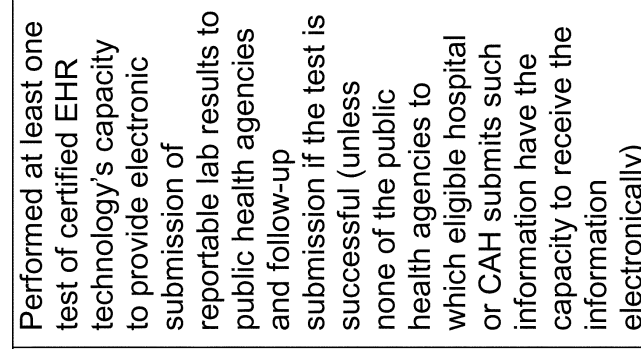 & 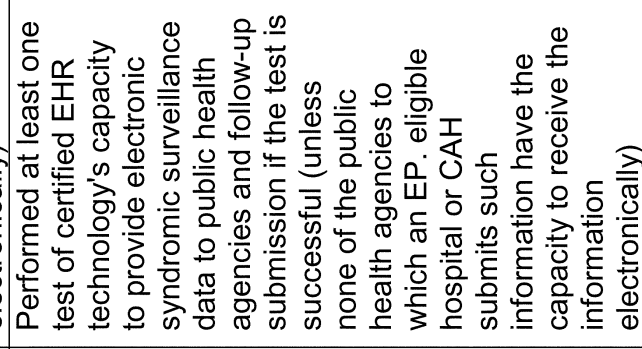 & \multirow{3}{*}{ 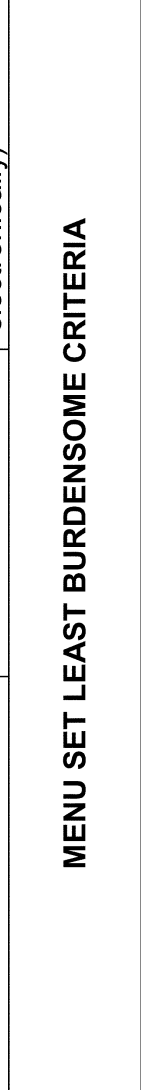 } \\
\hline 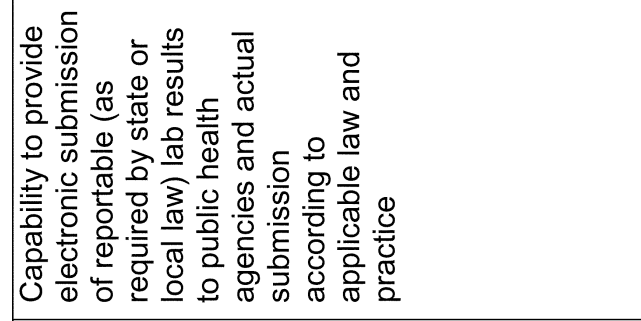 & 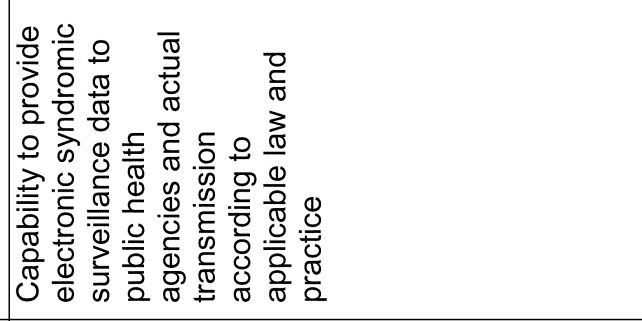 & \\
\hline & 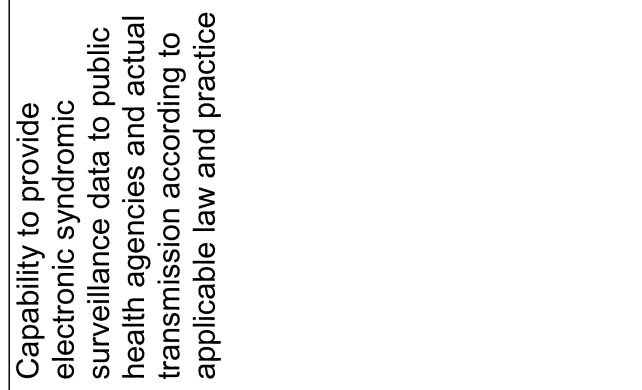 & \\
\hline
\end{tabular}




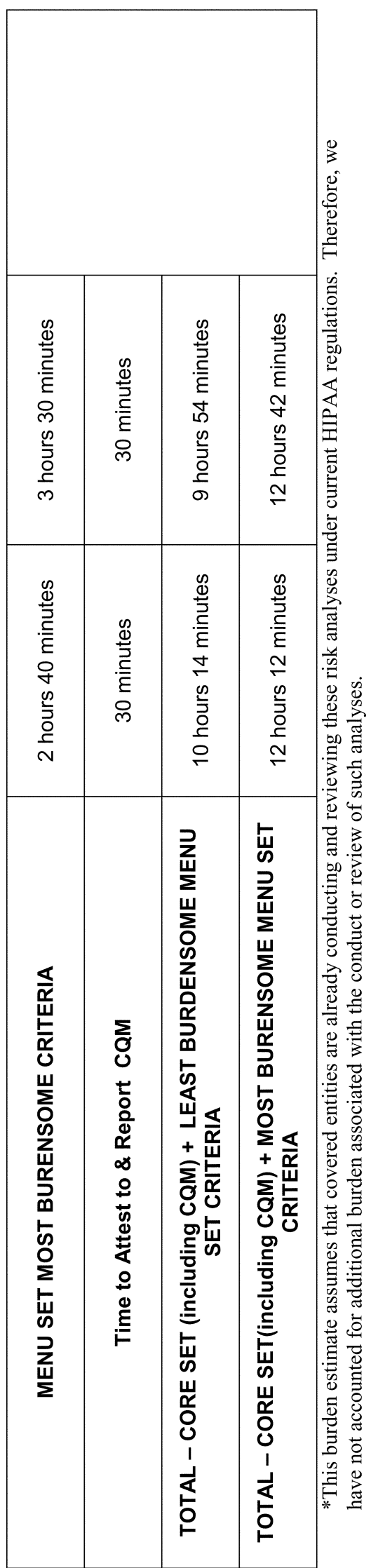


B. ICRs Regarding Participation

Requirements for EPs, Eligible

Hospitals, and CAHs (§ 495.10)

Since the EHR incentive payment program is new, we do not have enough information to estimate the information collection requirements burden beyond the first payment year for an EP, eligible hospital, or $\mathrm{CAH}$ for this provision. Furthermore, the EPs, eligible hospitals, and CAHs can enroll any time during the first 5 years; therefore, it is difficult to predict with certainty the burden beyond the first payment year as the burden depends on the number of participants. Therefore, we provide a best estimate of what we believe the burden associated with this provision might be.

For the proposed rule, $\S 495.10$ (a) through (c), we estimated that all 442,600 non-hospital-based Medicare, and Medicaid EPs would register in 2011 to receive an EHR incentive payment, and that it would take no more than 0.5 hours to complete the registration, resulting in a total estimated annual registration burden for all EPs of 221,300 hours $(442,600$ EPs $\times$ 0.5 hours). As we could not predict whether an EP or a medical secretary (on the EP's behalf) would register, we did one high-end and one low-end burden estimate. The cost burden for an $\mathrm{EP}$ who chose to register in the EHR incentive payment program himself or herself was $\$ 39.67$ (0.5 hours $\times \$ 79.33$ (mean hourly rate for physicians based on the May 2008 Bureau of Labor Statistics)), with a total estimated annual cost burden for all EPs of $\$ 17,555,729(221,300$ hours $\times \$ 79.33)$. Similarly, the cost burden for an EP who chose to use a medical secretary to register on their behalf was $\$ 7.41(0.5$ hours $\times \$ 14.81$ ), with a total estimated annual cost burden for all EPs of $\$ 3,277,453$ (221,300 hours $\times \$ 14.81)$. We used the average of the two estimates in the tally in Table 34 of the proposed rule. We invited comments on whether we should use the higher cost burden estimate $(\$ 17,555,729)$ or the lower cost burden estimate $(\$ 3,277,453)$, but we did not receive any comments on this issue. We invited public comments on the estimated percentages or the numbers of EPs that will register in CY 2011 and subsequent years, but we did not receive any comments on this issue.

We are finalizing both the lower cost estimate using the medical secretary as the personnel registering for the EP and the high cost estimate of the EP registering him or herself. Due to the revised estimates of non-hospital-based EPs eligible for the EHR incentive program, we are revising our burden estimates to reflect this change. In the final rule, we estimate that 521,600 nonhospital-based Medicare, and Medicaid EPs may register in CY 2011 to receive an EHR incentive payment. We believe that an EP may use a medical secretary to register on his/her behalf (low burden) or the EP may register him or herself (high burden). We estimate that it would take no more than 0.5 hours to complete the registration. The low cost burden for a medical secretary to register an EP is $\$ 7.41$ (0.5 hours $x$ $\$ 14.81$ (mean hourly rate of medical secretaries based on the May 2008 Bureau of Labor statistics)). The total estimated annual registration burden hours for the low cost estimate is 260,800 (521,600 EPs $\times 0.5$ hours) in the first payment year. The total estimated low cost burden for all EPs to register in CY 2011 is $\$ 3,862,448(260,800$ hours $\times$ $\$ 14.81)$. The high cost burden for an EP to register him or herself is $\$ 39.67$ (0.5 hours $\times \$ 79.33$ (mean hourly rate for physicians based on the May 2008 Bureau of Labor Statistics)). In the first payment year, the total estimated annual registration burden hours for the high cost estimate is 260,800 (521,600 EPs $\times 0.5$ hours). The total estimated high cost burden for all EPs to register in CY 2011 is $\$ 20,689,264(260,800$ hours $\times \$ 79.33$ ). We only use the average of the two estimates in the tally in Table 34.

Section 495.10(d) proposed that if there were subsequent changes in the initial registration information, the EP was responsible for providing us with updated changes in the manner specified by us. Based on our experience with provider enrollment, we estimated that about 11 percent of the Medicare and Medicaid EPs might need to update their registration information during a 1-year period. We estimated that 49,214 EPs (11 percent) might only have one occasion that required updating of information in a given year. For each occasion, we estimated that it would take no more than 0.5 hours to notify us of the changes. With that, we estimated that the annual total burden hours for 49,214 EPs to update changes were 24,607.

However, we could not predict if the EP would update the registration information himself or herself or assign a medical secretary to do it. Therefore, we did two burden estimates for an EP and his/her medical secretary. The cost burden for an EP who chose to update the registration information himself or herself was \$39.67. The total estimated annual cost burden for all 49,214 EPs to update registration information themselves was $\$ 1,952,073$. Similarly, the cost burden for the EP who chose to use a medical secretary to update registration information on his/her behalf was $\$ 7.41$. The total estimated annual cost burden for 49,214 EPs who chose to use medical secretaries to update registration information on their behalf was $\$ 364,429$. We used the average of the two estimates in the tally in Table 34. We invited comments on whether we should use the higher cost burden estimate $(\$ 1,952,073)$ or the lower cost burden estimate $(\$ 364,429)$ but we did not receive any comments on this issue. We also invited public comments on the estimated percentages and the numbers of EPs that will need to submit subsequent registration changes to us over the course of the EHR incentive payment program but we did not receive any comments on this issue.

We are finalizing both the lower cost estimate using the medical secretary as the personnel to update registration information for the EP and the high cost estimate of the EP updating their registration information. Due to the revised estimates of non-hospital-based EPs eligible for the EHR incentive program pursuant to legislative inclusion of EPs who practice in outpatient hospital setting, we are revising our burden estimate for this requirement to reflect this change. In the final rule, we estimate that about 11 percent of the Medicare and Medicaid EPs may need to update their registration information during a 1-year period. We estimate that 57,998 EPs $(527,254$ (revised estimated number of EPs for CY 2012) $\times 11$ percent) may only have one occasion that requires them to update their information in a given year. For each occasion, we estimate that it will take no more than 0.5 hours to notify us of the changes. With that, we estimate that the annual total burden hours for 57,998 EPs to update registration changes are 28,999 . The lower cost burden estimate for a medical secretary to update an EP's registration is $\$ 7.41$ ( $\$ 14.81$ (mean hourly rate for medical secretary based on the May 2008 Bureau of Labor Statistics) $\times 0.5$ hours). The total lower cost burden for all EPs to update registration information is $\$ 429,475$ (28,999 hours $\times$ \$14.81). The high cost burden for an EP to update their own registration information is $\$ 39.67$ ( 0.5 hours $\times$ $\$ 79.33$ (mean hourly rate for physicians based on May 2008 Bureau of Labor Statistics)). The total estimated annual high cost burden to update registration information is $\$ 2,300,491(28,999$ hours $\times \$ 79.33$ ). We only use the average of the two estimates in the tally in Table 34 . 
In $\S 495.10$ (a) and (b), we estimate that in FY 2011, there are 5,011 Medicare and Medicaid eligible hospitals, and CAHs that may be qualified to receive EHR incentive payment. Since we cannot predict how many eligible hospitals, and CAHs will participate in the EHR incentive payment program, we estimate that all 5,011 hospitals may register for the incentive program for burden estimate purposes. We estimate that it would take no more than 0.5 hours for an eligible hospital or $\mathrm{CAH}$ to register. We estimate the total annual burden hours for registration will be 2,506 $(5,011$ hospitals $\times 0.5$ hours). Once the decision to participate in the incentive program is made, we believe eligible hospitals or CAHs may assign a medical secretary to submit the registration information. The cost burden for an eligible hospital or CAH to register is $\$ 7.41$ (0.5 hours $\times \$ 14.81$ (mean hourly rate for medical secretaries based on the May 2008 Bureau of Labor Statistics)). We estimate that the total annual cost burden for eligible hospitals and CAHs to register is $\$ 37,106(5,011$ hospitals $\times$ 0.5 hours $\times \$ 14.81$ ) (mean hourly rate for medical secretaries based on the May 2008 Bureau of Labor Statistics)). We invited public comments on the estimated percentages or the number of eligible hospitals and CAHs that will register for the EHR incentive payment program in 2011 and subsequent years but we did not receive any comments on this issue. We are finalizing the burden estimates as proposed.

In $\S 495.10$ (d), we proposed that if there were subsequent changes in the initial registration information, the eligible hospital or $\mathrm{CAH}$ was responsible for providing us with updated information in the manner specified by us. Based on our experience with provider enrollment, we estimated that about 8 percent of the Medicare and Medicaid eligible hospitals and CAHs $(5,011$ hospitals and CAHs $\times 8$ percent $=401$ hospitals $)$ might need to update their registration information during a 1-year period. We estimated that eligible hospitals in this 8 percent pool might only have 1 occasion that required updating of registration information in a given year. For each occasion, we estimated that it would take no more than 0.5 hours to notify us of the changes. With that, we estimated that the total annual burden hours for eligible hospitals and CAHs to update CMS of registration changes were 201 (401 hospitals and CAHs $\times 0.5$ hours). We believe that eligible hospitals or CAHs might assign a medical secretary to update the registration information. We estimated the total annual cost burden for eligible hospitals and CAHs to update CMS of registration changes is $\$ 2,969$ (401 hospitals and CAHs $\times 0.5$ hours $\times$ $\$ 14.81$ ) (mean hourly rate for medical secretaries based on the May 2008 Bureau of Labor Statistics)). We invited public comments on the estimated percentages and the numbers of eligible hospitals and CAHs that will submit subsequent registration changes to us over the course of the EHR incentive payment program but we did not receive any comments on this issue. We are finalizing the estimated burden for hospitals and CAHs that will be making subsequent registration changes for $\mathrm{FY}$ 2012 as proposed.

In $\S 495.10$ (e)(1), we proposed that for participation in the EHR incentive payment programs, prior to the first payment year, an EP must notify us in a specified manner as to whether he or she elects to participate in the Medicare or Medicaid EHR incentive program. We estimated that in 2011, there would be about 80,900 dual Medicare/Medicaid EPs who might make the initial Medicare and Medicaid program selection. The standard full amount of Medicaid incentive payments that an EP could receive is larger than the standard full amount for the Medicare EP incentive payments. Therefore, for burden estimate purposes, we believed that all of the 80,900 dual Medicare/ Medicaid EPs might make the Medicaid program selection. We estimated that it would take no more than 0.5 hours to submit the initial Medicare or Medicaid selection notification to us. We could not predict if the EP would submit the notification to CMS himself or herself or assign a secretary to do it. Therefore, we did one high end estimate and one low end burden estimate for an EP and a medical secretary respectively. The total estimated burden hours for all the dual Medicare/Medicaid EPs to notify CMS of program selection were 40,450 in the first payment year. The cost burden for these EPs who notify CMS of Medicare or Medicaid program selection himself or herself was $\$ 39.67$. The total estimated annual cost burden for all dual Medicare/Medicaid EPs to notify CMS of program selection themselves was $\$ 3,208,899$. Similarly, the cost burden for an EP who chose to use a medical secretary to notify CMS of program selection was $\$ 7.41$. The total estimated annual cost burden for all dual Medicare/Medicaid EPs who use medical secretaries to notify CMS of program selection was $\$ 599,065$. We used the average of the two estimates in the tally in Table 34 . We invited comments on whether we should use the higher cost burden estimate $(\$ 3,208,899)$ or the lower cost burden estimate $(\$ 599,065)$, but we did not receive any comments on this issue. We also invited public comments on the estimated percentages and the number of dual Medicare/Medicaid EPs that would submit initial Medicare or Medicaid program selection in 2011, 2012, 2013, or 2014 but we did not receive any comments.

In the final rule, we are finalizing both the low burden cost estimate using a medical secretary for dual-Medicare/ Medicaid EPs to notify CMS of program selection and the high burden cost estimate of an EP who may do this him or herself. We have revised the total number of dual-Medicare/Medicaid EPs and the associated burden estimates pursuant to the legislative inclusion of EPs, who practice in outpatient hospital, in the incentive program. We estimate that in CY 2011, there will be 95,500 dual Medicare/Medicaid EPs who may use a medical secretary to notify CMS of the initial Medicare and Medicaid program selection. We estimate that it would take no more than 0.5 hours to submit the initial Medicare or Medicaid selection notification to us. The estimated burden for all the dualMedicare/Medicaid EPs to comply with this requirement is 47,750 hours $(95,500$ EPs $\times 0.5$ hours). The associated low cost burden for a dual-Medicare/ Medicaid EP is $\$ 7.41$ (0.5 hours $\times$ $\$ 14.81$ (mean hourly rate for medical secretaries based on May 2008 Bureau of Labor Statistics) and the total low cost burden for all the dual-Medicare/ Medicaid EPs is $\$ 707,178(47,750$ hours $\times \$ 14.81)$. The associated high cost burden for a dual-Medicare/Medicaid $\mathrm{EP}$ is $\$ 39.67$ (0.5 hours $\times \$ 79.33$ (mean hourly rate for physicians based on the May 2008 Bureau of Labor Statistics)) and the total high cost burden estimate for all dual-Medicare/Medicaid EPs is $\$ 3,788,008(47,750$ hours $\times \$ 79.33)$. We only use the average of the two estimates in the tally in Table 34 .

In $\S 495.10(\mathrm{e})(2)$ we proposed that EPs might switch from Medicare to Medicaid EHR incentive program or vice versa one time, and only for payment year 2014 or earlier. The burden associated with this requirement was the time required for the EP to make the Medicare/Medicaid program selection. Since we had no knowledge of how many EPs will make the subsequent changes in program selection, we assumed that all 81,700 (estimated number of dual-Medicare/ Medicaid EPs for CY 2012) dual Medicare/Medicaid EPs might make subsequent program selection changes 
for burden estimate purposes. We estimated that it would take no more than 0.5 hours to submit the Medicare/ Medicaid selection change to us. We could not predict if the EP would submit the change to CMS himself or herself or assign a secretary to do it. Therefore, we did one high end burden estimate for an EP and one low end estimate for a medical secretary. We used the average of the two estimates in the tally in Table 34. The total estimated burden hours for all dual-Medicare/ Medicaid EPs to notify CMS of program changes were 40,850 in a given year. The higher cost burden for the EP who chose to notify CMS of Medicare/ Medicaid program change him or herself was $\$ 39.67$. The total estimated annual cost burden for all dual Medicare/ Medicaid EPs to notify CMS of program changes themselves was $\$ 3,240,630$. Similarly, the lower cost burden for an EP who chose to use a medical secretary to notify CMS of program changes was $\$ 7.41$. The total estimated annual cost burden for all dual-Medicare/Medicaid EPs who use medical secretaries to notify CMS of program changes was $\$ 604,989$. We invited comments on whether we should use the higher cost burden estimate $(\$ 3,240,630)$ or the lower cost burden estimate $(\$ 604,989)$ but we did not received any comments on this issue. We also invited comments on the estimated percentages and the number of dual-Medicare/Medicaid EPs that would submit initial Medicare or Medicaid program changes in 2012, 2013, or 2014 but we did not receive any comments on this issue.

We are finalizing both the lower cost burden for EPs for may assign medical secretaries as the personnel to submit Medicare/Medicaid program selection changes to CMS and the high cost burden for EPs who may do this him or herself. We revised our burden estimates and the number of dualMedicare/Medicaid EPs, pursuant to legislative inclusion of EPs who practice at outpatient hospital setting in the incentive program. For CY 2012, we estimate that there will be 96,500 dualMedicare/Medicaid EPs. The notification will take 0.5 hours and the total burden for all dual-Medicare/ Medicaid EPs will be 48,250 hours (96,500 EPs $\times 0.5$ hours). The lower cost burden for each EP is $\$ 7.41$ (0.5 hours $\times \$ 14.81$ (mean hourly rate for medical secretaries based on the May 2008

Bureau of Labor Statistics) and the total lower cost burden for all the dualMedicare/Medicaid EPs will be $\$ 714,583(48,250$ hours $\times \$ 14.81)$. The high cost burden for each EP is $\$ 39.67$ (0.5 hours $\times \$ 79.33$ (mean hourly rate for physicians based on the May 2008 Bureau of Labor Statistics)) and the total high cost burden for all dual-Medicare/ Medicaid EPs is $\$ 3,827,673(48,250$ hours $\times \$ 79.33$ ). We only use the average of the two estimates in the tally in Table 34.

\section{ICRs Regarding Identification of} Qualifying MA Organizations, MA-EPS and MA-Affiliated Eligible Hospitals (\$ 495.202)

Section 495.202(a)(1) states that beginning with bids due in June 2011 (for plan year 2012), MA organizations seeking reimbursement for qualifying MA EPs and qualifying MA-affiliated eligible hospitals under the MA EHR incentive program are required to identify themselves to CMS in a form an manner specified by CMS, as part of submissions of initial bids under section 1854(a)(1)(A) of the Act. There is no burden associated with this requirement for qualifying MA organizations offering MA HMO plans, since they are deemed to meet the definition of HMO in 42 U.S.C. 300gg-91(b)(3) of the PHS Act in accordance with $\S 495.202(a)(2)$.

However, per $\S 495.202(\mathrm{a})(3)$, for MA organizations offering types of MA plans other than HMOs, the burden is the amount of time it will take them to attest to the fact that they meet the definition of HMO in 42 U.S.C. 300gg91(b)(3). We believe the burden associated with this requirement for MA organizations not offering HMO type plans would be approximately 1 hour per MA organization. We do not believe that there are any MA organizations that are not offering MA HMO type plans that will request reimbursement for qualifying MA EPs or MA-affiliated eligible hospitals under the MA EHR incentive payment program. Although the timeframe goes beyond the effective date of the proposed information collection period ( 3 years from the effective date of the final rule), we do not believe there are any MA organizations with potentially qualifying MA EPs or potentially qualifying MA-affiliated eligible hospitals that will need to report to us beginning in 2014 (for plan year 2015) per $\S 495.202(\mathrm{a})(4)$. Therefore, we believe there will be no burden associated with identification of qualifying MA organizations per $\S 495.202(\mathrm{a})(1)$ through (4).

Section 495.202(b)(1) and (2) require a qualifying MA organization, as part of its initial bid starting with its bid for plan year 2012, to make preliminary identification of potentially qualifying MA EPs and potentially qualifying MAaffiliated eligible hospitals for which the organization is seeking incentive payments for the current plan year (2011). The burden associated with this requirement would be the time required for a MA organization to identify their MA-affiliated hospitals to CMS. In the proposed rule, we explained that when MAOs identify amounts of compensation per $\S 422.204(\mathrm{~b})(2)$ and (5) they will also be identifying MA EPs per this requirement, and therefore there is will be no additional burden related to this requirement with respect to MA EPs. There are approximately 29 MAaffiliated eligible hospitals and approximately $12 \mathrm{MA}$ organizations, or an average of 2.42 eligible hospitals for each MA organization. In the proposed rule, we estimated that the total burden hours for all MA organizations to identify their affiliated hospitals to CMS would be 3 hours. We believe a MA organization may use a billing clerk to identify the eligible hospital to us. The total cost burden for all MA organizations to identify their eligible hospitals to us would be $\$ 46.32$.

Sections 495.202(b)(1) and (2), state that a MA organization, as part of its initial bid starting with plan year 2012, must make a preliminary identification of potentially qualifying MA EPs and potentially qualifying MA-affiliated eligible hospitals for which the organization is seeking incentive payments for the current plan year. A qualifying MA organization must provide the following information on their MA-affiliated EPs and eligible hospitals: (A) name of the EP or eligible hospital; (B) address of the EP's practice or eligible hospital's location; and (C) NPI. We believe that it is within the customary business practices of an MA organization to keep the information in (A), (B), and (C) on file. The burden associated with this requirement would be the time required to provide this information to CMS along with an attestation that the MA EPs or MAaffiliated eligible hospitals meet the eligibility criteria. In the proposed rule, we estimated that it would take 0.5 hours for a MA organization to comply with this attestation requirement. We estimated that the total burden for all MA organizations to attest would be 6 hours. We believe that MA organizations may use an attorney to attest on their behalf. In the proposed rule, we estimated that the cost burden for a MA organization to attest is $\$ 29.99$ and the total estimated cost burden for all MA organizations to attest would be $\$ 359.88$.

Section 495.202(b)(4) states that all qualifying MA organizations, as part of their initial bids in June 2015 for plan year 2016, must identify potentially qualifying MA EPs and potentially 
qualifying MA-affiliated eligible hospitals. An attestation that each professional or hospital either meets or does not meet the eligibility criteria must be included as part of the identification submission. We cannot estimate the collection burden for this requirement as the timeframe goes beyond the scope of the effective date of the proposed information collection period (3 years from the effective date of the final rule).

\section{ICRs Regarding Incentive Payments} to Qualifying MA Organizations for MAEPs and Hospitals (\$ 495.204)

Section 495.204(b)(2) requires a qualifying MA organization to report to CMS within 60 days of the close of the calendar year, the aggregate annual amount of revenue attributable to providing services that would otherwise be covered as professional services under Part $B$ received by each qualifying MA EP for enrollees in MA plans of the MA organization in the payment year. Since the tracking of salaries or compensation for MA EPs constitutes usual and customary business practices, the only burden associated with this requirement would be the time required to submit the aggregated annual amount of revenue received by each qualifying MA EP for enrollees in MA plans of the MA organization. In the proposed rule, we estimated that there were $12 \mathrm{MA}$ organizations and 28,000 MA EPs. We believe that it will take a MA organization 40 hours annually to report the required aggregate revenue data for all its salaried MA EPs, given that all the data are readily available. The total estimated annual burden hours for all MA organizations to comply with this requirement would be 480 . We believe MA organizations may involve a billing clerk to report the required data to CMS. We estimated that the cost burden for a MA organization to report was $\$ 617.6$ (40 hours $\times \$ 15.44$ (mean hourly rate of billing clerk based on the May 2008 Bureau of Labor Statistics)) and we estimated the total annual cost burden for all MA organizations to comply with this requirement would be $\$ 7,411$.

Section 495.204(b)(4) states that for qualifying MA EPs who are compensated on a salaried basis, CMS requires the qualifying MA organization to develop a methodology for estimating the portion of each qualifying MA EP's salary attributable to providing services that would otherwise be covered under Part B to MA plan enrollees of the MA organization. The methodology: (i) Must be approved by CMS; (ii) may include an additional amount related to overhead, where appropriate, estimated to account for the MA-enrollee related
Part B practice costs of the salaried qualifying MA EP; and (iii) methodological proposals must be submitted to CMS by June of the payment year and must be auditable by an independent third party. CMS will review and approve or disapprove such proposals in a timely manner. In the proposed rule, we estimated that it might take a MA organization one and a half hour to develop the methodology. We estimated that there are about two MA organizations that may have the need to develop the methodology. We estimated the total burden hours for the two MA organizations to develop the methodology would be 3 hours. We believed that a MA organization may use an accountant to develop the methodology. We estimated the cost burden for a MA organization was $\$ 47.48$ (1.5 hours $\times \$ 31.65$ (mean hourly rate for accountants based on the May 2008 Bureau of Labor Statistics)), and the total cost burden for the two MA organizations to develop the methodology would be $\$ 94.95$ (47.48 × 2 MA organizations).

Section 495.204(b)(5) states that for qualifying MA EPs who are not salaried, qualifying MA organizations may obtain and submit to CMS, attestations from such qualifying MA EPs as to the amount of compensation received by such EPs for MA plan enrollees of the MA organization. We estimate that about 10 percent of the MA EPs were not salaried and that was an average of 233 non-salaried EPs in each MA organization. Further, we estimate that it might take 0.25 hour to electronically obtain and compile each attestation into a document for transmission to CMS. We estimate the total burden hours for a MA organization would be 58.3 , and the total estimated burden hours for all MA organizations would be 699.6 (58.3 hours $\times 12$ MA organizations). We believe an MA organization may involve a billing clerk to compile and submit the compensation information from such attestations. We estimate the cost burden for a MA organizations to comply with this requirement would be $\$ 900.15$ (58.3 hours $\times \$ 15.44$ (mean salary of a billing clerk based on the May 2008 Bureau of Labor Statistics)). We estimate the total annual cost burden for all MA organizations to comply with this requirement would be $\$ 10,801.82$ (\$900.15 × $12 \mathrm{MA}$

organizations).

Section 495.204(b)(6) states that for qualifying MA EPs who are not salaried, qualified MA organizations may also have qualifying MA EPs send MA organization compensation information directly to CMS. We estimated the burden associated with this requirement is the time it would take the MA EP to send the information directly to CMS. However, we believe that the nonsalaried MA EPS are employed by a third-party physician group which will be responsible for sending the required information to CMS. Again, we estimate that about 10 percent of the MA EPs are not salaried and that there is an average of 233 non-salaried EPs in each of the third-party physician groups. Further, we estimate that it might take 0.25 hour to electronically obtain and compile the information into a document for transmission to CMS. We estimate the total burden hours for a third-party physician group will be 58.3, and the total estimated burden hours for all third-party physician groups will be 699.6 (58.3 hours $\times 12$ third-party physician group). We believe a thirdparty physician group may involve a billing clerk to compile and submit the compensation information. We estimate the cost burden for a third-party physician group to comply with this requirement will be $\$ 900.15$ (58.3 hours $\times \$ 15.44$ (mean salary of a billing clerk based on the May 2008 Bureau of Labor Statistics)). We estimate the total annual cost burden for all third-party physician groups to comply with this requirement will be $\$ 10,801.82$ ( $\$ 900.15 \times 12$ thirdparty physician groups). Note that this is the same burden we estimate with respect to $\$ 422.204(\mathrm{~b})(5)$. Further, an MAO will either submit non-salary information directly to CMS, or it will have someone else do it on behalf of the MA EPs with respect to that MAO. We believe the burden related to

$\S 422.204(\mathrm{~b})(6)$ is counted in the burden we already projected with respect to $\S 422.204(\mathrm{~b})(5)$. We do not believe any MAO will submit under both $\S 422.204(\mathrm{~b})(5)$ and (6).

\section{E. ICRs Regarding Meaningful User Attestation ( $\$ 495.210$ )}

Section 495.210(b) requires qualifying MA organizations to attest within 60 days after the close of a calendar year whether each qualifying MA EP is a meaningful EHR user. We anticipate that the adopted EHR technology will capture the data for determination whether each qualifying MA EP is a meaningful EHR user. We estimate the burden associated with this requirement would be the time necessary to attest to the required information. We estimated that there were approximately $12 \mathrm{MA}$ organizations and approximately 28,000 MA EPs, or an average of approximately 2,333 MA EPs affiliated with each qualifying MA organization. We believe that it would take a MA organization about 40 hours annually to attest whether each qualifying MA EP is a 
meaningful user, given that all the data are captured in the certified EHR technology and that meaningful use will be demonstrated through the continued reporting of HEDIS data. We estimate the total estimated annual burden hours for all MA organizations to comply with this requirement will be 480 . We believe MA organizations might involve an attorney to attest on their behalf. We estimate the cost burden for a MA organization to attest will be $\$ 2,399$ (40 hours $\times \$ 59.98$ (mean hourly rate of attorney based on the May 2008 Bureau of Labor Statistics)). We estimate the total annual cost burden for all MA organizations to comply with attestation for MA EPs will be $\$ 28,790$.

Section 495.204(c)(2) states that to the extent data are available, qualifying MA organizations must receive hospital incentive payments through their affiliated hospitals under the Medicare FFS EHR hospital incentive program, rather than through the MA EHR hospital incentive program. Under $\S 495.210$ (c), we proposed that qualifying MA organizations be required to attest within 60 days after the close of a calendar year whether each qualifying MA-affiliated eligible hospital is a meaningful EHR user. While the EHR incentive payments for Medicare FFS and MA-affiliated hospitals are treated the same as all Medicare-certified MA affiliated hospitals they will demonstrate clinical quality measures through the continued reporting of HEDIS data. This means that $§ 495.210$ (c) generally applies to a MA-affiliated hospital that is not Medicare certified, and such a type of hospitals does not exist currently. We do not expect there to be any MAaffiliated hospitals that will not be covered under the Medicare FFS EHR hospital incentive program because section 1852(a)(1)(A) of the Act requires MA organizations to provide Part A inpatient services solely through providers that meet applicable requirements of the Medicare program. We have already addressed the attestation burden on hospitals, including MA-affiliated hospitals under $\S 495.10(\mathrm{~b})(2)(\mathrm{i})(\mathrm{ii})$ and through our existing PRA package related to HEDIS reporting by MA organizations-OMB control number 0938-NEW.

\section{F. ICRs Regarding Establishing Patient Volume (\$ 495.306)}

This section of the final rule contains patient volume requirements, and requires EPs and certain hospitals to attest to meeting such requirement using representative periods in order to qualify for a Medicaid EHR incentive. The minimum patient volume requirements are as follows: 30 percent Medicaid patient volume for most EPs, 20 percent Medicaid patient volume for pediatricians, 30 percent needy individual patient volume for EPs practicing predominantly in an FQHC or RHC, and 10 percent Medicaid patient volume for acute-care hospitals. The burden associated with the requirements in this section is the time and effort necessary to submit the information to CMS. In the proposed rule, in each instance, we estimated it would take no longer than 0.5 hours to submit the necessary information to CMS. We estimated that 119,000 entities would submit the required information to meet 30 percent (or 20 percent pediatrician) requirements for most EPs. We estimated the total annual burden to be 59,500 hours, with total labor cost amounting to $\$ 4,720,135$ (assuming that physicians (rather than staff assistants) establish patient volume (\$79.33 mean hourly rate for physicians based on May 2008 Bureau of Labor Statistics).

For hospitals to attest to patient

volume, we estimated that 3,631 entities would submit required information, and estimated a total burden of $1,815.50$ hours $(3,631$ entities $\times .5$ hours $)$. The total labor cost associated with this requirement is $\$ 25,617$. This cost burden was based on a secretary reporting patient volume on behalf of the acute care hospital at $\$ 14.11$ (mean hourly rate for secretaries based on May 2008 Bureau of Labor Statistics).

We received no comments on this section; however, since we have revised our definition of hospital-based EP, the burden is revised to account for the additional number of Medicaid EPs that could now be eligible to receive Medicaid incentive payments. We currently estimate that there are an additional Medicare/Medicaid 75,700 EPs that could be eligible for an incentive payment because of the new definition of hospital-based EP. We believe there are 553,200 Medicare EPs of which 86 percent are non-hospital based or 477,500 . We believe 20 percent or 95,500 will meet patient volume requirements, and therefore, potentially qualify for Medicaid EHR incentive payments. Additionally, there are 44,100 Medicaid-only EPs (nurse practitioners, certified nurse-midwives, dentists, and physician assistants) that we believe will meet patient volume. Specifically, we believe that 139,600 EPs $(95,500+44,100)$ could be reporting patient volume information. Thus, the updated annual burden associated with the requirements in $\S 495.306$ at 0.5 hours for EPs is 69,800.

The total labor cost associated with the requirement is $(69,800 \times 79.33)$
$\$ 5,537,234$. The total labor cost associated with each requirement is $\$ 5,537,234$.

For hospitals reporting patient volume, we have updated the burden to account for the additional CAHs that meet the definition of acute care hospital. Specifically, there are 3,620 acute care hospitals, 11 cancer hospitals, and 1,302 CAHs that must report 10 percent Medicaid patient volume, or 4,933 entities. The updated annual burden associated with the requirement, at 0.5 hours is $2,466.5$ $(4,933 \times .05)$. The total labor cost is $\$ 34,803.30$.

\section{G. ICRs Regarding Process for Payments (\$ 495.312)}

Section 495.312(b) states that in order to receive a Medicaid EHR incentive payment, a provider must report all necessary data (including data required by subpart A of the regulations, such as meaningful use data) within the EHR reporting period. We believe the information collections associated with this requirement are discussed in the relevant sections discussing each particular requirement that would necessitate data reporting (for example, the burden for demonstrating meaningful use is discussed in the information collection section on meaningful use). Therefore, we have not calculated a separate information collection burden for this requirement.

H. ICRs Regarding Activities Required

To Receive an Incentive Payment

(\$ 495.314)

Section 495.314(a)(1) states that in the first payment year, to receive an incentive payment, the Medicaid EP or eligible hospital must meet one of the following criteria. The Medicaid EP or eligible hospital must demonstrate that during the EHR reporting period for a payment year, it has adopted,

implemented, or upgraded certified EHR technology, as defined in $\S 495.302$; or, the Medicaid EP or eligible hospital must demonstrate that during the EHR reporting period for a payment year it is a meaningful user of certified EHR technology as defined in $\S 495.4$.

The burden associated with the requirements in proposed

$\S 495.314(\mathrm{a})(1)$ is the time and effort necessary for a Medicaid EP or eligible hospital to demonstrate that it meets one of the criteria in $\S 495.314(\mathrm{a})(1)(\mathrm{i})$ through (ii). We believe we already accounted for this burden in the earlier discussion of the burden associated with $\S 495.8$

Section 495.314(a)(2) states that a provider may notify the State of its nonbinding intention to participate in 
the incentives program prior to having fulfilled all of the eligibility criteria. This requirement constitutes a thirdparty disclosure. The burden associated with this requirement is the time and effort necessary for a provider to send notification to the State. We estimated that this burden will be the same burden associated with $\S 495.10$ since the information necessary to notify the State of the providers non-binding intention to participate in the program could be the same information as submitted by those providers that have committed to participating in the program, that is, the National Provider Identifier, the tax identification number, etc.

Section 495.314(b)(1) states that in the second, third, fourth, fifth, and sixth payment years, to receive an incentive payment, the Medicaid EP or eligible hospital must demonstrate that during the EHR reporting period for the applicable payment year, it is a meaningful user of certified EHR technology, as defined in $\S$ 495.4. The burden associated with this requirement is the time and effort necessary for a Medicaid EP or eligible hospital to demonstrate that it is a meaningful user of certified EHR technology. We discussed the burden associated with this requirement in our discussion of the burden associated with $\S 495.6$ and 495.8 .

We did not receive any comments on the information collection burdens we estimated for the proposed rule.

I. ICRs Regarding State Monitoring and Reporting Regarding Activities Required To Receive an Incentive Payment (\$ 495.316)

Section 495.316(a) requires States to be responsible for tracking and verifying the activities necessary for a Medicaid EP or eligible hospital to receive an incentive payment for each payment year, as described in $\S 495.314$. Burden is calculated for each State's process for the administration of the Medicaid incentive payments, including tracking of attestations and oversight, and the process for approving, processing, and making timely payments.

For the proposed rule, we estimated that it would take 5 hours per State to accomplish this. The estimated annual burden for States associated with the aforementioned submission requirements is 280 hours (56 StatesTerritories $\times 5.0$ hours/State-Territory). The cost burden was estimated based on an employee contracting with the State Agency. The burden associated with $\S 495.316$ is already in the OMB approval process. We announced the information collection in a Federal
Register notice that published on September 11, 2009 (74 FR 467330). Comment: Some commenters asked CMS to clarify if States are responsible for collecting the MU measure data or if providers will report data directly to CMS. If the collection and reporting of MU data are States' responsibility, this would create tremendous burden on States. The commenters also asked CMS to clarify if States are responsible for validating attestations by eligible providers.

Response: For EPs and some hospitals, States are responsible for collecting the MU measure data; for hospitals that are eligible for both the Medicare and Medicaid incentives, hospitals that meet the Medicare MU objectives are deemed to have met MU for Medicaid; thus, since hospitals are required to report $\mathrm{MU}$ data to CMS for the Medicare EHR incentives program, these hospitals do not, in addition, have to report MU data to States. States are required to submit a State Medicaid HIT plan to CMS for review and approval outlining their methodology for collecting MU measure data and other required information outlined in this final rule. States are also responsible for validating attestations by providers. We do not believe collecting data or validating attestations is a tremendous burden on States as noted by our estimates. States can receive 90 percent FFP for administering the incentive payments to providers and for conducting adequate monitoring and oversight. In addition, it should be noted that States voluntarily participate in the Medicaid EHR incentive program.

\section{J. ICRs Regarding State Responsibilities} for Receiving FFP (\$ 495.318)

Section 495.318 states that in order to be provided FFP under section 1903(a)(3)(F) of the Act, a State must demonstrate to the satisfaction of the Department, that the State is conducting the activities listed at $\S 495.318$ (a) through (c). This burden is the same as that listed above in the burden discussion for $\S 495.316$.

\section{K. ICRs Regarding Prior Approval Conditions (\$ 495.324)}

Section 495.324(a) requires a State to obtain prior written approval from the Department as specified in paragraph (b) of this section, when the State plans to initiate planning and implementation activities in support of Medicaid provider incentive payments encouraging the adoption and use of certified EHR technology with proposed Federal financial participation (FFP). Specifically, § 495.324(b) states that to receive 90 percent match, each State must receive prior approval for all of the requirements listed in $\S 495.324(\mathrm{~b})(1)$ through (3).

Section 495.324(c) requires a State to obtain prior written approval from the Department of its justification for a sole source acquisition, when it plans to acquire non-competitively from a nongovernmental source HIT equipment or services, with proposed FFP under subpart D of Part 495 in the regulations, if the total State and Federal acquisition cost is more than $\$ 100,000$. Burden must be calculated for State Medicaid Agencies to submit the planning and implementation documents and the SMHP to CMS. This burden is the same as that listed above in the burden discussion for $\S 495.316$.

L. ICRs Regarding Termination of Federal Financial Participation (FFP) for Failure To Provide Access to Information (\$ 495.330)

Section 495.330(a) states that the Department can terminate FFP at any time if the Medicaid agency fails to provide State and Federal representatives with full access to records relating to HIT planning and implementation efforts, and the systems used to interoperate with electronic HIT, including on-site inspection. Section 495.330(b) states that the Department may request such access at any time to determine whether the conditions in this subpart are being met. The burden associated with the requirements in this section is the time and effort necessary to make the information available to the Department upon request so it can monitor compliance. The Department estimated that it will make 1 request per State/ Territory per year for information and that it will take each State 5 hours to compile and furnish the information. For States to collect and submit the information required, we estimated it would take 5 hours per State. The estimated annual burden for States associated with the aforementioned submission requirements is 280 hours (56 States-Territories $\times 5.0$ hours/StateTerritory).

The annual cost burden for a State employee to provide the above information is $\$ 9,904$ (280 hours $\times$ $\$ 35.37$ (mean hourly rate for a management analyst based on the May 2008 Bureau of Labor Statistics)). We believe that a secretary may compile State information and provide the information to the Department. In that case the annual cost burden for the secretary to provide this information is $\$ 3,951$ (280 hours $\times \$ 14.11$ (mean hourly rate for secretaries based on the May 2008 Bureau of Labor Statistics)). 
M. ICRs Regarding State Medicaid Agency and Medicaid EP and Hospital Activities (\$ 495.332 Through \$ 495.344)

The burden associated with this section is the time and effort associated with completing the single provider election repository and each State's process for the administration of the Medicaid incentive payments, including tracking of attestations and oversight; the submission of the State Medicaid HIT Plan and the additional planning and implementation documents; enrollment or reenrollment of providers, and collection and submission of the data for providers to demonstrate that they have adopted, implemented, or upgraded certified EHR technology or that they are meaningful users of such technology. We believe much of the burden associated with these requirements has already been accounted for in our discussion of the burden for $\S 495.316$.

\section{N. ICRs Regarding Access to Systems and Records (\$ 495.346)}

Section 495.346 states that the State agency must allow the Department access to all records and systems operated by the State in support of this program, including cost records associated with approved administrative funding and incentive payments to Medicaid providers. State records related to contractors employed for the purpose of assisting with implementation or oversight activities or providing assistance, at such intervals as are deemed necessary by the Department to determine whether the conditions for approval are being met and to determine the efficiency, economy, and effectiveness of the program.

The Department believes that the burden associated with maintaining the records is exempt under 5 CFR $1320.3(b)(2)$ as this burden is part of a usual and customary business practice; the time, effort, and financial resources necessary to comply with a collection of information that would be incurred by persons in the normal course of their activities (for example, in compiling and maintaining business records) will be excluded from the "burden" if the agency demonstrates that the reporting, recordkeeping, or disclosure activities needed to comply are usual and customary.

However, there is burden associated with making the information available to the Department upon request. This burden is described in the burden discussion for $\S 495.330$.

\section{O. ICRs Regarding Procurement Standards (\$ 495.348)}

Section 495.348(c) states that a grantee must maintain written standards of conduct governing the performance of its employees engaged in the award and administration of contracts. Although most States may already have these written standards of conduct, we have estimated the burden associated with this requirement as the time and effort necessary for a grantee to develop and maintain written standards of conduct. We estimate it will take each of the 56 grantees 0.5 hours to develop and maintain standards of conduct. The total estimated annual burden is 28 hours (56 grantees $\times 0.5$ hours). The annual cost burden for a grantee to develop and maintain standards of conduct is $\$ 990$ (28 hours $\times \$ 35.37$ (mean hourly rate for a management analyst based on the May 2008 Bureau of Labor Statistics)).

Section 495.348(e) requires that all grantees establish written procurement procedures. At a minimum, the standards must provide for the information listed in $\S 495.348(e)(1)$ through (13). The burden associated with this requirement is the time and effort necessary for a grantee to develop and maintain written procurement procedures. Although most States probably have these procedures already, we estimate that it will take each of the 56 grantees 0.5 hours to develop and maintain written procurement procedures. The total estimated annual burden is 28 hours (56 grantees $\times 0.5$ hours). The annual cost burden for a grantee to develop and maintain written procurement procedures is $\$ 990$ (28 hours $\times \$ 35.37$ (mean hourly rate for a management analyst based on the May 2008 Bureau of Labor Statistics)).

Section 495.348(f) imposes recordkeeping requirements. This section states that a system for contract administration must be maintained to ensure contractor performance with the terms, conditions and specifications of the contract and to ensure adequate and timely follow up on all purchases. The burden associated with this requirement is the time and effort necessary to develop and maintain a system for contract administration. We estimate that it will take each of the 56 grantees 5 hours to develop and maintain a system for contract administration. The total estimated annual burden is 280 hours ( 56 grantees $\times 5$ hours). The annual cost burden for a grantee to develop and maintain a system for contract administration is $\$ 9,904$ (280 hours $\times \$ 35.37$ (mean hourly rate for a management analyst based on the May 2008 Bureau of Labor Statistics)).
P. ICRs Regarding State Medicaid Agency Attestations (\$ 495.350)

Section 495.350 requires States to provide assurances to the Department that amounts received with respect to sums expended that are attributable to payments to a Medicaid provider for the adoption of EHR are paid directly to such provider, or to an employer or facility to which such provider has assigned payments, without any deduction or rebate. The burden associated with this requirement is the time and effort necessary for a State to verify that the sums expended are attributable to payments to a Medicaid provider for the adoption of EHR are paid directly to such provider, or to an employer or facility to which such provider has assigned payments, without any deduction or rebate. Additionally, there is burden associated with submitting an attestation to the Department to that effect. The estimated burden associated with these requirements is 0.5 hours to verify the information and 0.5 hours to submit the attestation to the Department, for a total of 1 hour. The estimated annual burden for States associated with the aforementioned submission requirements is 56 hours (56 StatesTerritories $\times 1$ hour State-Territory). The annual cost burden for a State employee to provide the above information is $\$ 1,981$ (56 hours $\times \$ 35.37$ (mean hourly rate for a management analyst based on the May 2008 Bureau of Labor Statistics)). We believe that that a secretary may compile State information and provide the information to the Department. In that case the annual cost burden for the secretary to provide this information is $\$ 790$ (56 hours $\times \$ 14.11$ (mean hourly rate for secretaries based on the May 2008 Bureau of Labor Statistics)).

\section{Q. ICRs Regarding Reporting Requirements (\$ 495.352)}

Section 495.352 requires each State to submit to the Department on a quarterly basis a progress report documenting specific implementation and oversight activities performed during the quarter, including progress in implementing the State's approved Medicaid HIT plan. The burden associated with this requirement is the time and effort necessary for a State to draft and submit quarterly progress reports to the Department. For States to collect and submit the information required, we estimate it will take 5 hours per State. The estimated annual burden for States associated with the aforementioned submission requirements is 280 hours 
(56 States-Territories $\times 5$ hours/StateTerritory).

The annual cost burden for a State employee to provide the above information is $\$ 9,904$ (280 hours $x$ $\$ 35.37$ (mean hourly rate for a management analyst based on the May 2008 Bureau of Labor Statistics)). We believe that a secretary may compile State information and provide the information to the Department. In that case the annual cost burden for the secretary to provide this information is $\$ 3,951$ (280 hours $\times \$ 14.11$ (mean hourly rate for secretaries based on the May 2008 Bureau of Labor Statistics)).

R. ICRs Regarding Retroactive Approval of FFP With an Effective Date of February 18, 2009 (\$ 495.362)

Section 495.362 states that for administrative activities performed by a State, without obtaining prior approval, which are in support of planning for incentive payments to providers, a State may request consideration of FFP by recorded request in a HIT planning advance planning document or implementation advance planning document update. While this requirement is subject to the PRA, we believe the burden is already covered in the discussion of proposed $\S 495.332$ through § 495.344 .

\section{S. ICRs Regarding Financial Oversight and Monitoring Expenditures (\$ 495.366)}

Section 495.366(a)(2) requires a State to have a process in place to report actual expenditures for the Medicaid EHR incentive program using the Medicaid Budget Expenditure System. Since States already have to report Medicaid expenditures to the Medicaid Budget and Expenditure System, there is no need for States to develop and implement a reporting process.

However, States will need to estimate and report the expenditures related to the provider incentive payments and the cost of the administration of the incentive payments. The estimated annual burden for States associated with the aforementioned requirements is 280 hours (56 States-Territories $\times 5$ hours State-Territory).

The annual cost burden for a State employee to provide the above information is $\$ 9,904$ (280 hours $\times$ \$35.37 (mean hourly rate for a management analyst based on the May 2008 Bureau of Labor Statistics)). We believe that a secretary may compile State information and provide the information to the Department. In that case the annual cost burden for the secretary to provide this information is $\$ 3,951$ (280 hours $\times \$ 14.11$ (mean hourly rate for secretaries based on the May 2008 Bureau of Labor Statistics)).

Section 495.366(a)(3) requires a State to have an automated payment and information retrieval mechanized system, (Medicaid Management Information System) to make EHR payment incentives, to ensure Medicaid provider eligibility, to ensure the accuracy of payment incentives, and to identify potential improper payments. Since States already have an automated payment and information retrieval system, there is no need to estimate this burden.

Section 495.366(b) lists the information collection requirements associated with provider eligibility as a basis for making payment. States must, subject to $\S 495.332$, collect and verify information on Medicaid providers. This burden is the same as that listed above in the discussion of $\S 495.316$.

Section § 495.366(c)(1) states that subject to $\S 495.332$, the State must annually collect and verify information regarding the efforts to adopt, implement, or upgrade certified EHR technology and the meaningful use of said technology before making any payments to providers. This burden has already been discussed in our burden explanation for $\$ 495.8$.

Section 495.366(d)(1) states that subject to paragraph $\S 495.332$, the State must assure that State expenditures are claimed in accordance with, including but not limited to, applicable Federal laws, regulations and policy guidance. Section 495.366(d)(2) specifies that subject to $\S 495.332$, the State must have a process in place to assure that expenditures for administering the Medicaid EHR incentive payment program will not be claimed at amounts higher than 90 percent of the cost of such administration. Section 495.366(d)(3) states that subject to $\S 495.332$, the State must have a process in place to assure that expenditures for payment of Medicaid EHR incentive payments will not be claimed at amounts higher than 100 percent of the cost of such payments to Medicaid providers. This burden is the same as that listed above in the discussion of $\S 495.316$.

Section 495.366(e) discusses the information collection requirements associated with improper Medicaid electronic health record payment incentives. The burden associated with the requirements listed in proposed $\S 495.366(\mathrm{e})(1)$ through (7) is the time and effort necessary to develop processes to provide the necessary assurances discussed in this section. This burden is the same as that listed above in the discussion of $\S 495.316$.

T. ICRs Regarding Appeals Process for a Medicaid Provider Receiving Electronic Health Record Incentive Payments (\$ 495.370)

Section 495.370(a) requires states to have a process in place consistent with the requirements established in $\S 447.253(\mathrm{e})$ for a provider or entity to appeal incentive payments, incentive payment amounts, provider eligibility determinations, and the demonstration of adopting, implementing, or upgrading and meaningful use of certified EHR technology. This burden is the same as that listed above in the discussion of $\S 495.316$.

We continue to believe that these numbers are subject to a substantial amount of uncertainty and actual experience may be significantly different. The range of possible experience is greater than under most other rules for the following reason; specifically, this rule provides the option for States to participate in the Medicaid certified electronic health record technology incentive payment program. To the extent that States participate more or less than assumed here (that is, the number of States, EPs and hospitals) the burden associated may be greater than or less than estimated.

\section{U. General Comments Regarding the Information Collection Requirements}

Comment: Some commenters recommended that EPs and eligible hospitals should start tracking time and resources estimates on their overall cost for complying with all the required data collection to achieve meaningful use during the reporting period. They believed the information is beneficial for CMS in developing and assessing future meaningful use objectives and measures.

Response: We welcome provider input on the required resources to comply with the meaningful use requirements. We believe the information would help us to fine-tune burden estimates for future rulemaking for subsequent stages of meaningful use demonstration.

BILLING CODE 4120-01-P 


\begin{tabular}{|c|c|c|c|c|c|c|c|c|c|c|}
\hline 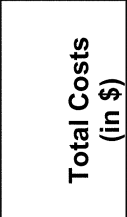 & 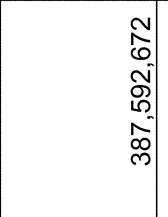 & $\begin{array}{l}0 \\
5 \\
50 \\
5 \\
0 \\
0 \\
0 \\
-5 \\
\bar{N}\end{array}$ & 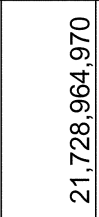 & 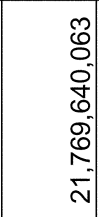 & $\begin{array}{l}0 \\
0 \\
0 \\
0 \\
0 \\
0 \\
0 \\
0 \\
\infty\end{array}$ & $\begin{array}{l}\bar{\sigma} \\
0 \\
0 \\
\overline{0} \\
\overline{5} \\
\vdots 0 \\
\dot{7}\end{array}$ & 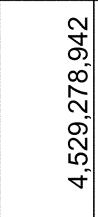 & 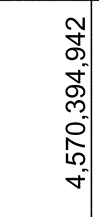 & $\begin{array}{l}0 \\
0 \\
0 \\
\delta \\
0 \\
m\end{array}$ & 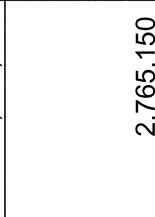 \\
\hline 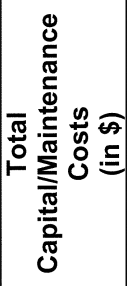 & 0 & $\begin{array}{l}8 \\
8 \\
8 \\
8 \\
8 \\
8 \\
- \\
-\end{array}$ & 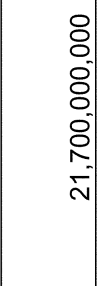 & $\begin{array}{l}8 \\
8 \\
8 \\
8 \\
8 \\
\stackrel{-}{-} \\
\dot{N}\end{array}$ & 0 & $\begin{array}{l}8 \\
8 \\
8 \\
8 \\
0 \\
0 \\
\dot{7}\end{array}$ & 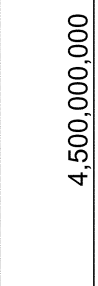 & \begin{tabular}{l}
8 \\
0 \\
0 \\
0 \\
0 \\
0 \\
+ \\
\hdashline
\end{tabular} & 0 & 0 \\
\hline 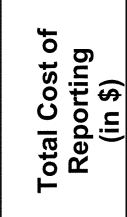 & 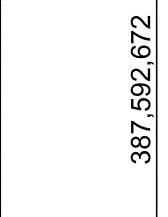 & $\begin{array}{l}0 \\
0 \\
5 \\
\frac{5}{5} \\
0 \\
0 \\
\frac{1}{7}\end{array}$ & $\begin{array}{l}0 \\
0 \\
0 \\
0 \\
0 \\
\infty \\
\sim\end{array}$ & $\begin{array}{l}0 \\
0 \\
0 \\
\vdots \\
0 \\
0\end{array}$ & $\begin{array}{l}0 \\
0 \\
0 \\
0 \\
0 \\
0 \\
0 \\
0 \\
\text { m. }\end{array}$ & $\begin{array}{l}\bar{\sigma} \\
\delta \\
0 \\
\vdots \\
\check{c} \\
\check{c}\end{array}$ & 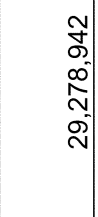 & $\begin{array}{l}\text { Tे } \\
5 \\
+ \\
0 \\
0 \\
0\end{array}$ & $\begin{array}{l}0 \\
\stackrel{m}{ } \\
\dot{0} \\
0 \\
\text { m. }\end{array}$ & 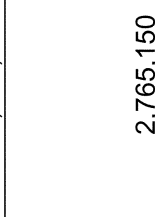 \\
\hline 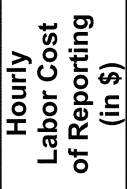 & $\begin{array}{l}\stackrel{0}{2} \\
\stackrel{\rho}{2}\end{array}$ & $\begin{array}{c}\stackrel{m}{0} \\
\stackrel{\rho}{2}\end{array}$ & $\begin{array}{l}\overparen{e} \\
\stackrel{\rho}{R}\end{array}$ & 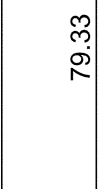 & 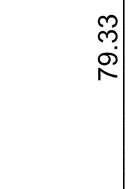 & 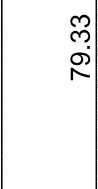 & $\begin{array}{l}\stackrel{m}{2} \\
\stackrel{2}{R}\end{array}$ & $\begin{array}{l}\text { } \\
\stackrel{0}{2} \\
\stackrel{2}{2}\end{array}$ & $\begin{array}{l}\bar{\infty} \\
\dot{v}\end{array}$ & $\begin{array}{l}\infty \\
\stackrel{2}{\circ} \\
\stackrel{8}{\circ}\end{array}$ \\
\hline 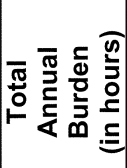 & $\begin{array}{l}\text { N } \\
\infty \\
\infty \\
\infty \\
\infty \\
\forall\end{array}$ & $\begin{array}{l}0 \\
0 \\
0 \\
0 \\
0 \\
0 \\
-\end{array}$ & $\begin{array}{l}\text { Nิ} \\
\stackrel{0}{0} \\
0\end{array}$ & $\begin{array}{l}\text { م⿱ } \\
\infty \\
\hat{N} \\
\infty\end{array}$ & $\begin{array}{l}\overline{0} \\
0 \\
5 \\
0 \\
0 \\
0\end{array}$ & $\begin{array}{l}0 \\
0 \\
0 \\
0 \\
0 \\
0 \\
-\end{array}$ & $\begin{array}{l}\infty \\
0 \\
0 \\
0 \\
0\end{array}$ & \begin{tabular}{l}
0 \\
0 \\
0 \\
\hdashline \\
$\infty$ \\
$\infty$
\end{tabular} & 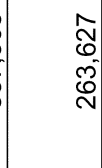 & $\frac{5}{0}$ \\
\hline 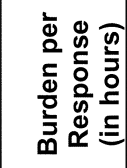 & $\begin{array}{l}\hat{\omega} \\
\tilde{\omega} \\
\sigma\end{array}$ & 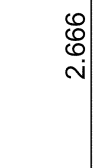 & $\begin{array}{l}8 \\
? \\
0\end{array}$ & $\begin{array}{l}0 \\
0 \\
0 \\
\stackrel{-}{-}\end{array}$ & $\begin{array}{l}\hat{0} \\
\infty \\
\infty \\
\infty\end{array}$ & 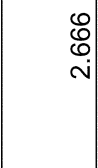 & $\begin{array}{l}8 \\
0 \\
0\end{array}$ & \begin{tabular}{l}
0 \\
0 \\
0 \\
\hdashline
\end{tabular} & $\begin{array}{l}8 \\
0 \\
0 \\
0\end{array}$ & $\begin{array}{l}\text { ¿े } \\
\text { নे }\end{array}$ \\
\hline 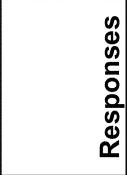 & 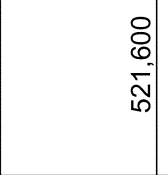 & $\begin{array}{l}8 \\
0 \\
- \\
-1\end{array}$ & $\begin{array}{l}8 \\
0 \\
-1 \\
-1\end{array}$ & 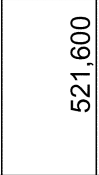 & 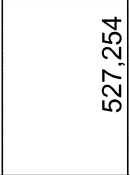 & 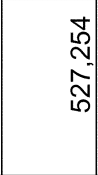 & 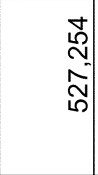 & 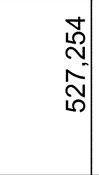 & 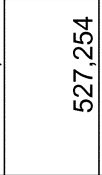 & $\bar{c}$ \\
\hline 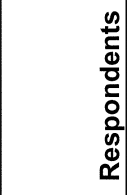 & 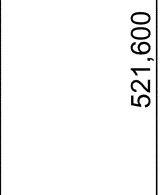 & 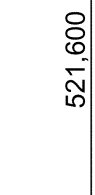 & 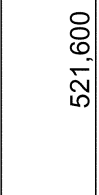 & 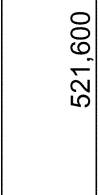 & 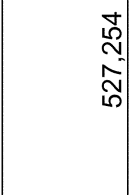 & 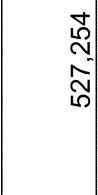 & 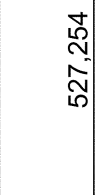 & 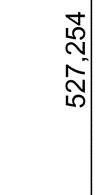 & 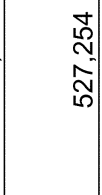 & $\bar{\delta}$ \\
\hline 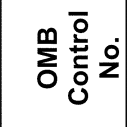 & 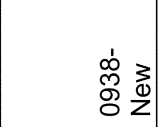 & 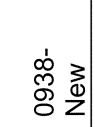 & 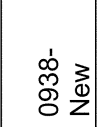 & 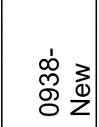 & 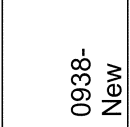 & 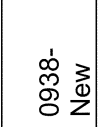 & $\begin{array}{l}\infty \\
\tilde{O} \\
\delta \\
O\end{array}$ & 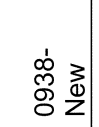 & 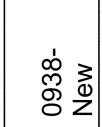 & 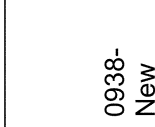 \\
\hline $\begin{array}{l}\mathscr{D} \\
\mathscr{8} \\
\mathscr{8}\end{array}$ & 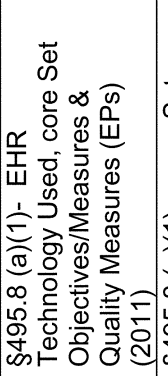 & 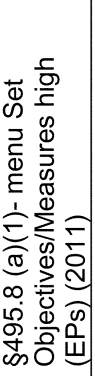 & 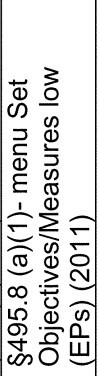 & 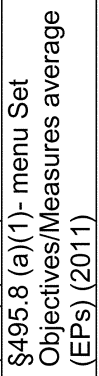 & 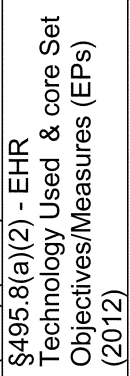 & 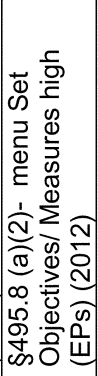 & 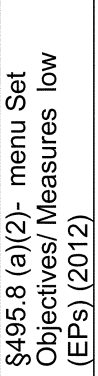 & 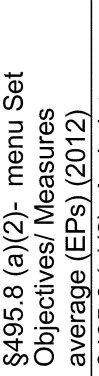 & 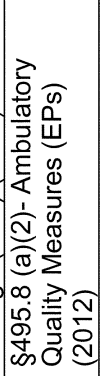 & 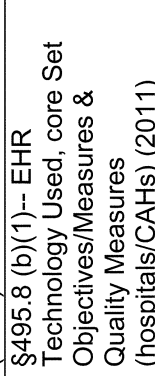 \\
\hline
\end{tabular}




\begin{tabular}{|c|c|c|c|c|c|c|c|c|c|c|c|c|c|c|}
\hline 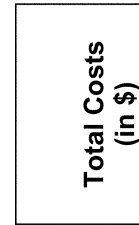 & $\begin{array}{l}\text { N. } \\
0 \\
0 \\
0 \\
0 \\
0 \\
0 \\
\vdots \\
0\end{array}$ & 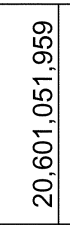 & 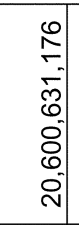 & 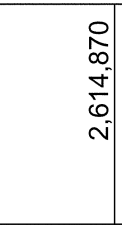 & 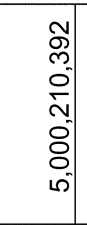 & 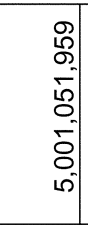 & $\begin{array}{l}0 \\
\vdots \\
-0 \\
0 \\
0 \\
0 \\
6 \\
\text { i. }\end{array}$ & ) & $\mid$ & $\left|\begin{array}{l}0 \\
0 \\
0 \\
0 \\
0 \\
0 \\
0 \\
0\end{array}\right|$ & 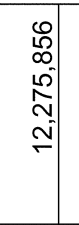 & 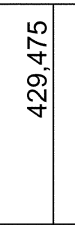 & & $\begin{array}{c}0 \\
0 \\
o \\
0 \\
0 \\
m \\
- \\
-1\end{array}$ \\
\hline 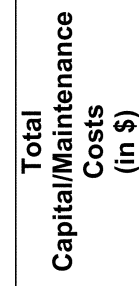 & $\begin{array}{l}8 \\
0 \\
0 \\
0 \\
0 \\
0 \\
\text { N. }\end{array}$ & $\begin{array}{l}\text { o. } \\
0 \\
0 \\
0 \\
0 \\
0 \\
\text { N. }\end{array}$ & $\begin{array}{l}8 \\
0 \\
0 \\
0 \\
0 \\
0 \\
\text { ¿. }\end{array}$ & 0 & $\begin{array}{l}8 \\
8 \\
8 \\
8 \\
8 \\
8 \\
0 \\
1\end{array}$ & $\begin{array}{l}8 \\
\vdots \\
0 \\
0 \\
0 \\
0 \\
\iota^{-}\end{array}$ & $\begin{array}{l}8 \\
8 \\
8 \\
0 \\
8 \\
\vdots \\
1\end{array}$ & 0 & 0 & 0 & o & 0 & 0 & 0 \\
\hline 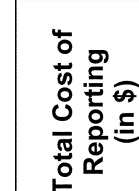 & $\begin{array}{l}\tilde{N} \\
0 \\
0 \\
\stackrel{0}{N}\end{array}$ & 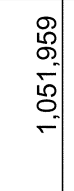 & $\begin{array}{l}0 \\
0 \\
\overline{8} \\
0\end{array}$ & $\begin{array}{l}0 \\
0 \\
0 \\
+ \\
0 \\
i \\
\text { in }\end{array}$ & $\begin{array}{l}\mathbb{N} \\
0 \\
0 \\
\stackrel{N}{N}\end{array}$ & 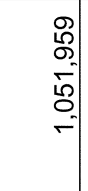 & $\begin{array}{l}0 \\
0 \\
\overline{0} \\
0\end{array}$ & $\begin{array}{l}0 \\
0 \\
i \\
\bar{m}\end{array}$ & 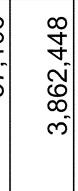 & 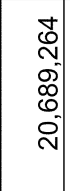 & 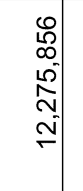 & 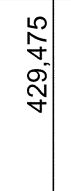 & 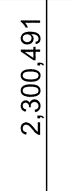 & $\begin{array}{l}0 \\
0 \\
0 \\
0 \\
0 \\
0 \\
-1\end{array}$ \\
\hline 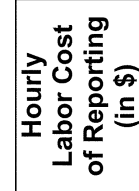 & $\begin{array}{l}0 \\
0 \\
0 \\
0 \\
0\end{array}$ & $\begin{array}{l}0 \\
\text { o. } \\
0 \\
0\end{array}$ & $\begin{array}{l}0 \\
0 \\
0 \\
0 \\
b\end{array}$ & \begin{tabular}{l} 
o \\
$\stackrel{\circ}{0}$ \\
\hdashline \\
\hdashline
\end{tabular} & 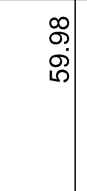 & $\begin{array}{l}0 \\
\stackrel{\infty}{0} \\
0 \\
0\end{array}$ & \begin{tabular}{l}
0 \\
0 \\
0 \\
\hdashline
\end{tabular} & $\mid \begin{array}{c}\overline{0} \\
\dot{\sim}\end{array}$ & $\mid \begin{array}{l}\bar{\infty} \\
\dot{f} \\
\dot{f}\end{array}$ & $\begin{array}{l}\stackrel{m}{0} \\
\stackrel{\rho}{R}\end{array}$ & f & $\mid \begin{array}{c}\bar{o} \\
\dot{f}+\end{array}$ & & $\hat{f}$ \\
\hline 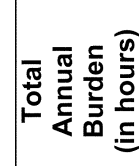 & 思 & 惫 & 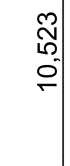 & 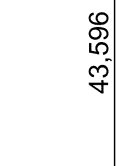 & | & 盢 & 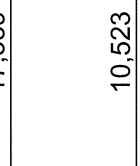 & 离 & $\begin{array}{l}0 \\
\infty \\
0 \\
0 \\
\sim\end{array}$ & $\begin{array}{l}8 \\
0 \\
\infty \\
0 \\
ه \\
\sim\end{array}$ & 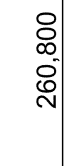 & 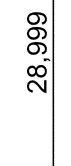 & & $\begin{array}{l}\mathscr{8} \\
\stackrel{\leftrightarrow}{\circ} \\
\infty \\
\sim\end{array}$ \\
\hline 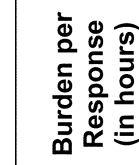 & 옹 & 总 & $\stackrel{8}{\stackrel{\circ}{N}}$ & 﨎 & : & 总 & $\stackrel{\circ}{\stackrel{\circ}{\sim}}$ & $\begin{array}{ll}0 & 0 \\
0 \\
0 \\
0\end{array}$ & o & 资 & | & 资 & $\begin{array}{ll}0 & 0 \\
0 \\
0 \\
0\end{array}$ & $\begin{array}{l}\text { : } \\
\text { d. }\end{array}$ \\
\hline 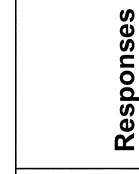 & $c_{i}^{\bar{c}}$ & $\begin{array}{c}\bar{c} \\
i_{0}\end{array}$ & $\begin{array}{c}\bar{c} \\
\vdots \\
\omega^{\circ}\end{array}$ & $\begin{array}{l}\bar{c} \\
\overline{1} \\
\omega^{2}\end{array}$ & $c_{i}^{\bar{c}}$ & 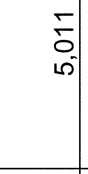 & $\begin{array}{l}\bar{c} \\
\overline{0} \\
\omega\end{array}$ & $\begin{array}{c}\bar{c} \\
\vdots \\
i s\end{array}$ & $\mid \begin{array}{c}0 \\
0 \\
i \\
i\end{array}$ & 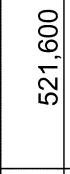 & : & 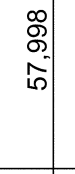 & 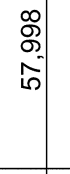 & 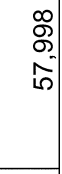 \\
\hline 总 & co & $\begin{array}{c}\bar{c} \\
\bar{c} \\
i^{\circ}\end{array}$ & $\begin{array}{c}\bar{c} \\
\vdots \\
i\end{array}$ & $\begin{array}{c}\bar{c} \\
\vdots \\
\omega^{\circ}\end{array}$ & $\begin{array}{c}\bar{c} \\
\omega^{\circ}\end{array}$ & $\begin{array}{c}\bar{c} \\
\omega^{\circ}\end{array}$ & $\begin{array}{c}\bar{c} \\
\dot{0} \\
\omega^{\circ}\end{array}$ & 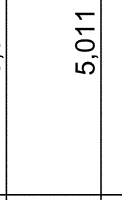 & $\mid \begin{array}{c}0 \\
0 \\
\overleftarrow{0} \\
0\end{array}$ & $\left|\begin{array}{l}0 \\
0 \\
0 \\
i \\
i\end{array}\right|$ & 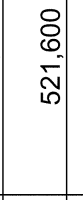 & 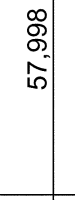 & & $\begin{array}{l}\infty \\
\stackrel{\infty}{8} \\
i \\
i\end{array}$ \\
\hline 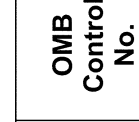 & 离 $\begin{array}{c}3 \\
0\end{array}$ & 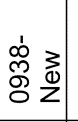 & 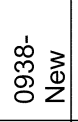 & 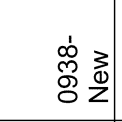 & $\begin{array}{l}\infty \\
0 \\
0 \\
0 \\
0 \\
z\end{array}$ & $\begin{array}{l}\infty \\
0 \\
0 \\
0 \\
0 \\
z\end{array}$ & 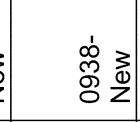 & 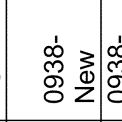 & 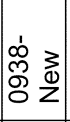 & 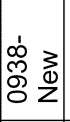 & & & $\begin{array}{l}0 \\
0 \\
0 \\
0 \\
3\end{array}$ & \\
\hline & $=$ & 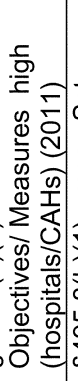 & 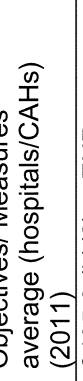 & 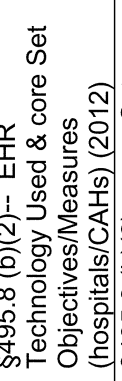 & 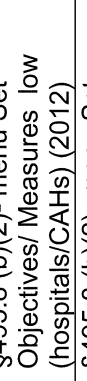 & 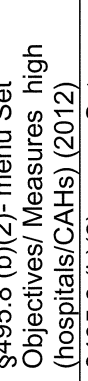 & 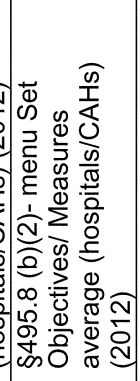 & 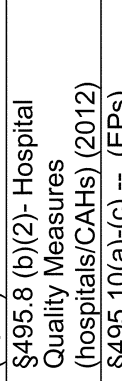 & 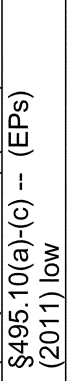 & 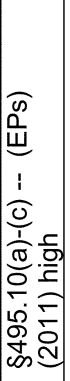 & 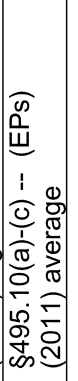 & 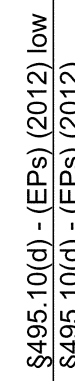 & 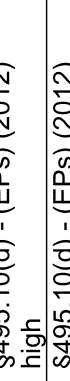 & 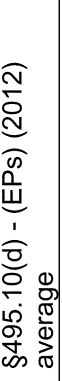 \\
\hline
\end{tabular}




\begin{tabular}{|c|c|c|c|c|c|c|c|c|c|c|c|c|c|c|c|c|c|c|c|}
\hline 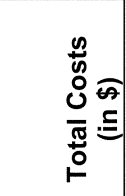 & 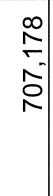 & $\begin{array}{l}\infty \\
0 \\
\infty \\
\infty \\
0 \\
\omega \\
-1\end{array}$ & 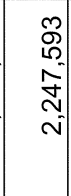 & 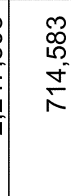 & 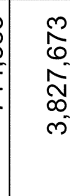 & 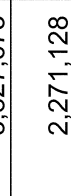 & $\begin{array}{c}0 \\
0 \\
\hat{m} \\
\end{array}$ & 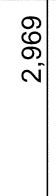 & 迥 & $\stackrel{0}{q}$ & : & $\mathscr{q}$ & 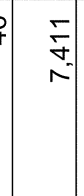 & ๘ & $\begin{array}{c}\mathbb{N} \\
0 \\
0 \\
0\end{array}$ & $\begin{array}{l}\text { @ } \\
\stackrel{1}{0} \\
\stackrel{0}{0}\end{array}$ & 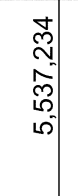 & 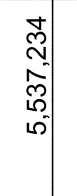 & 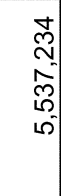 \\
\hline 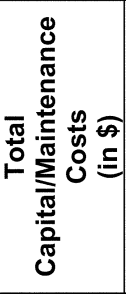 & 0 & 0 & 0 & 0 & 0 & 0 & 0 & 0 & 0 & 0 & 0 & 0 & 0 & 0 & 0 & 0 & 0 & & $c$ \\
\hline 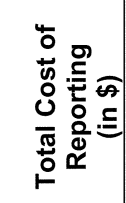 & 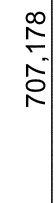 & $\begin{array}{l}\infty \\
0 \\
\infty \\
0 \\
0 \\
\rho^{2}\end{array}$ & 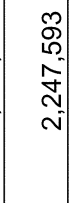 & 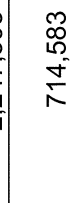 & 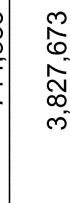 & 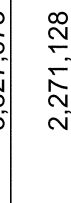 & 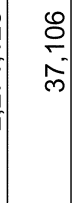 & 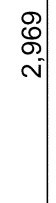 & $\begin{array}{l}\text { ठ } \\
\text { (1) }\end{array}$ & $\varphi$ & : & $\stackrel{\varphi}{q}$ & זٓ⿱宀 & เి & $\begin{array}{l}\mathrm{N} \\
\mathrm{D} \\
0 \\
0 \\
0\end{array}$ & $\begin{array}{l}\text { Q } \\
\stackrel{2}{0} \\
\stackrel{\infty}{N}\end{array}$ & 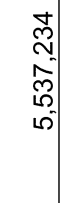 & 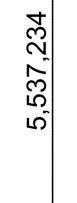 & 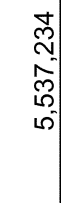 \\
\hline 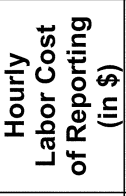 & $\begin{array}{l}\bar{o} \\
\dot{f}\end{array}$ & $\begin{array}{l}\text { ल. } \\
\stackrel{2}{R}\end{array}$ & $\hat{f}$ & $\begin{array}{l}\bar{\infty} \\
\stackrel{+}{\sim}\end{array}$ & 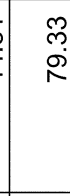 & ร & $\begin{array}{l}\overline{0} \\
\dot{f}\end{array}$ & $\begin{array}{l}\overline{0} \\
\dot{f}\end{array}$ & \begin{tabular}{l} 
o. \\
$\stackrel{0}{0}$ \\
\hdashline
\end{tabular} & 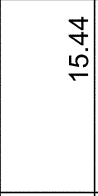 & \begin{tabular}{l}
$\infty$ \\
$\circ$ \\
$\stackrel{0}{0}$ \\
\hdashline
\end{tabular} & $\begin{array}{c}7 \\
5 \\
6\end{array}$ & 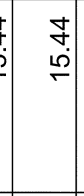 & $\begin{array}{l}\text { @ִ } \\
\dot{m}\end{array}$ & $\begin{array}{l}7 \\
\dot{7} \\
i\end{array}$ & 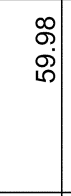 & 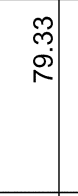 & $\begin{array}{l}\stackrel{m}{2} \\
\stackrel{i}{N}\end{array}$ & o. \\
\hline 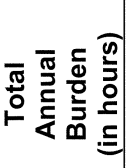 & $\begin{array}{l}0 \\
\stackrel{\circ}{2} \\
\hat{f}\end{array}$ & 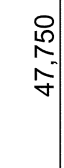 & 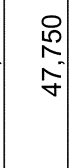 & 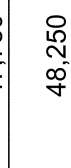 & $\begin{array}{l}\text { 오 } \\
\text { N } \\
\text { o } \\
\text { † }\end{array}$ & 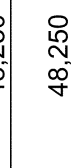 & $\begin{array}{l}0 \\
0 \\
i \\
\end{array}$ & ర్ & 0 & $m$ & 0 & $m$ & $\begin{array}{l}\stackrel{\circ}{+} \\
\stackrel{+}{*}\end{array}$ & $m$ & ঃ & 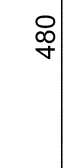 & $\begin{array}{l}8 \\
8 \\
0 \\
0\end{array}$ & 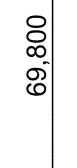 & $\begin{array}{l}8 \\
0 \\
0 \\
0 \\
0\end{array}$ \\
\hline 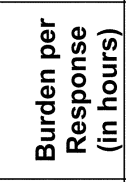 & $\begin{array}{l}8 \\
\text { ధొ } \\
0\end{array}$ & $\begin{array}{l}8 \\
\text { ధొ } \\
0\end{array}$ & $\begin{array}{l}\text { D. } \\
\text { م. } \\
0\end{array}$ & $\begin{array}{l}8 \\
\text { ధํ. } \\
0\end{array}$ & $\begin{array}{l}8 \\
0 \\
0 \\
0\end{array}$ & $\begin{array}{l}8 \\
\text { in } \\
0\end{array}$ & $\begin{array}{l}\text { Dे } \\
\text { ధ. } \\
0\end{array}$ & $\begin{array}{l}\text { : } \\
\text { ధొ. } \\
0\end{array}$ & $\begin{array}{l}8 \\
0 \\
0 \\
0\end{array}$ & 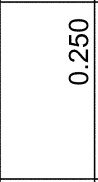 & $\begin{array}{l}8 \\
\text { ధొ } \\
0\end{array}$ & $\begin{array}{l}\text { مै } \\
\text { on }\end{array}$ & 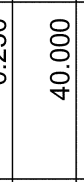 & 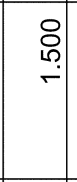 & 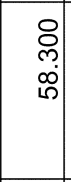 & 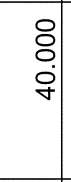 & 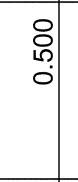 & 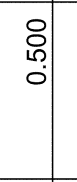 & | \\
\hline 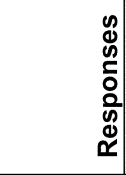 & $\begin{array}{l}8 \\
0 \\
\circ \\
\circ \\
\circ\end{array}$ & 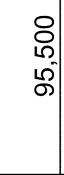 & 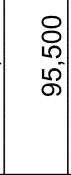 & $\begin{array}{l}8 \\
\circ \\
\mathscr{0} \\
8\end{array}$ & $\begin{array}{l}8 \\
10 \\
8 \\
8\end{array}$ & $\begin{array}{l}8 \\
6 \\
0 \\
8\end{array}$ & $\begin{array}{l}\bar{\tau} \\
\bar{\delta} \\
\end{array}$ & 휘 & 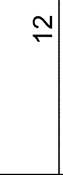 & 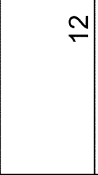 & $\cong$ & $\simeq$ & 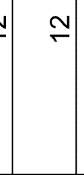 & $N$ & 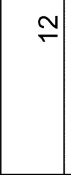 & 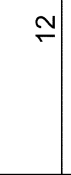 & $\begin{array}{l}8 \\
0 \\
0 \\
m\end{array}$ & $\begin{array}{l}8 \\
0 \\
0 \\
0 \\
\end{array}$ & $\begin{array}{l}\text {. } \\
\text { के } \\
\text { m. }\end{array}$ \\
\hline 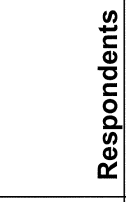 & $\begin{array}{l}8 \\
0 \\
0 \\
0 \\
0\end{array}$ & $\begin{array}{l}8 \\
0 \\
0 \\
0 \\
0\end{array}$ & $\begin{array}{l}8 \\
10 \\
5 \\
\circ\end{array}$ & $\begin{array}{l}8 \\
8 \\
8 \\
8\end{array}$ & $\begin{array}{l}8 \\
1 \\
8 \\
8\end{array}$ & $\begin{array}{l}8 \\
0 \\
8 \\
8\end{array}$ & $\begin{array}{c}\bar{\sigma} \\
\bar{\sigma} \\
\sigma^{\circ}\end{array}$ & ᄒे & $\cong$ & $\cong$ & $\cong$ & $\cong$ & $\cong$ & $N$ & 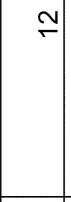 & $\cong$ & \begin{tabular}{l}
8 \\
0 \\
0 \\
\hdashline \\
-1
\end{tabular} & \begin{tabular}{l}
0 \\
0 \\
0 \\
0 \\
\hdashline
\end{tabular} & 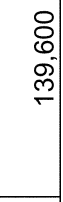 \\
\hline 罗竞 & & & & 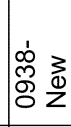 & & & oj & & & 8 & (๘) & 82 & 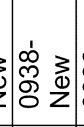 & z & $z \check{z}$ & B. & ס & 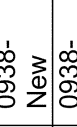 & 禹 \\
\hline 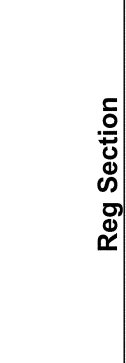 & 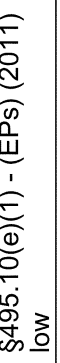 & 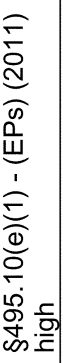 & 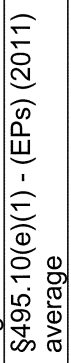 & 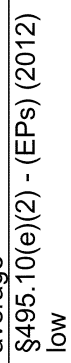 & 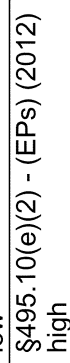 & 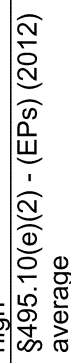 & 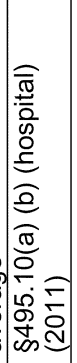 & 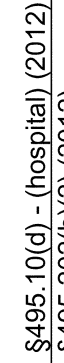 & 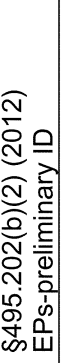 & 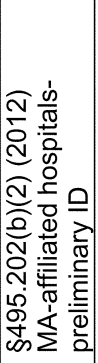 & 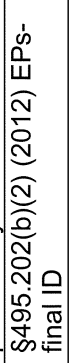 & 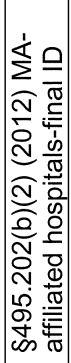 & 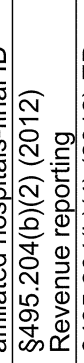 & 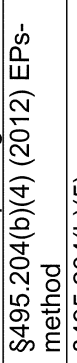 & 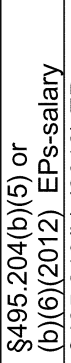 & 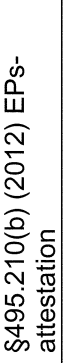 & 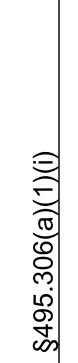 & 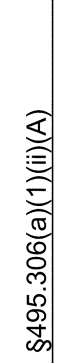 & 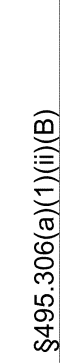 \\
\hline
\end{tabular}




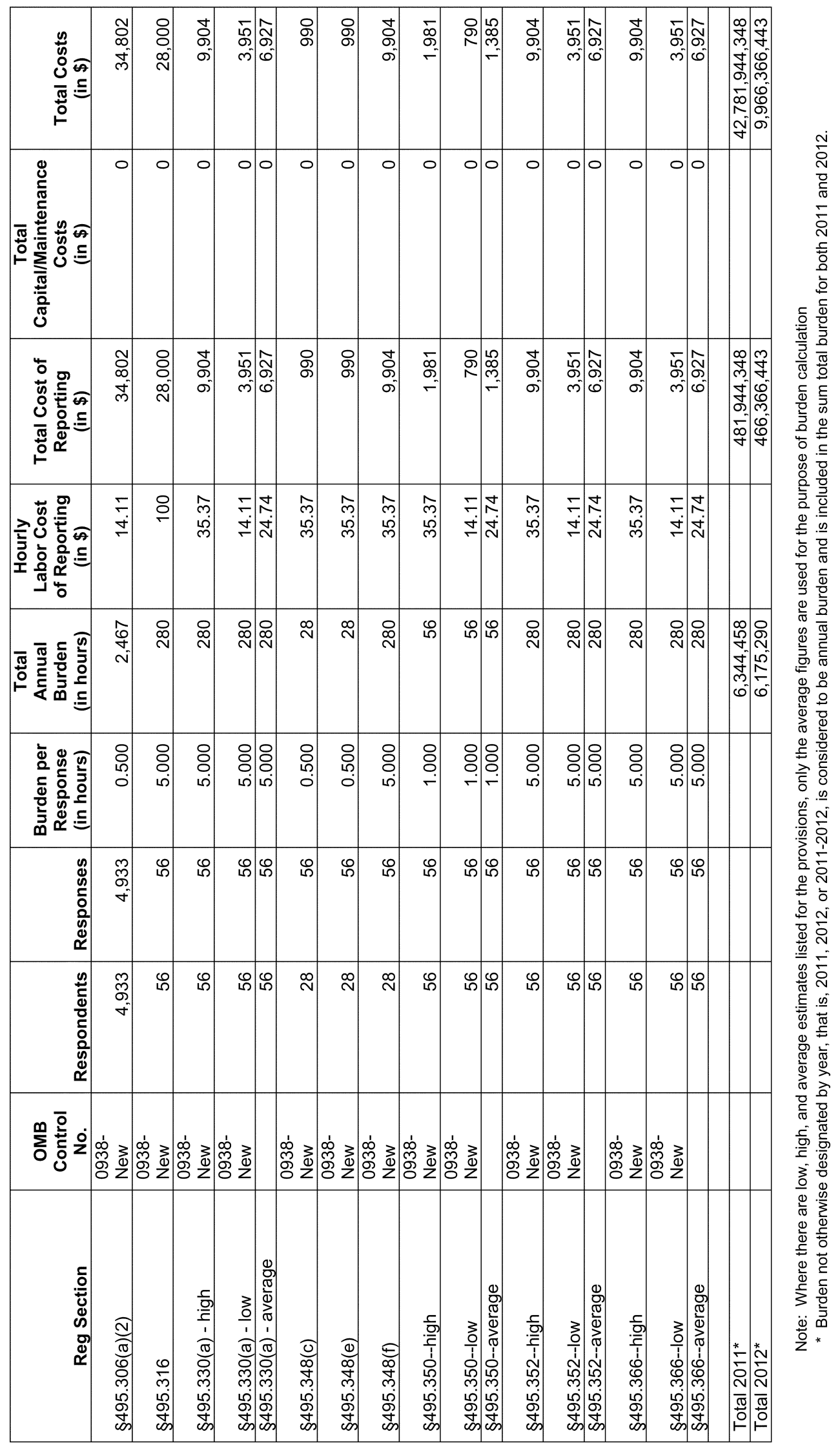


We will accept comments on the aforementioned information collection requirements for 60 days from the date of display for this final rule. At the conclusion of the 60-day comment period, we will publish an additional notice announcing the submission of the information collection request associated with this final rule for OMB approval. At that time, the public will have an additional 30 days to submit public comments to OMB for consideration.

To obtain copies of the supporting statement associated with the information collection requirements contained herein, access CMS' Web site at http://www.cms.hhs.gov/

PaperworkReductionActof1995, or Email your request, including your address, phone number, OMB number, and CMS document identifier, to Paperwork@cms.hhs.gov, or call the Reports Clearance Office on (410) 7861326.

In commenting on the information collection requirements, please reference the information collection request identifier (CMS-10336). To be assured consideration, comments and recommendations must be submitted in one of the following ways by September 13, 2010:

1. Electronically. You may submit your comments electronically to http:// www.regulations.gov. Follow the instructions for "Comment or Submission" or "More Search Options" to find the information collection document(s) accepting comments.

2. By regular mail. You may mail written comments to the following address: CMS, Office of Strategic Operations and Regulatory Affairs, Division of Regulations Development, Attention: Document Identifier/OMB Control Number, Room C4-26-05, 7500 Security Boulevard, Baltimore, Maryland 21244-1850.

\section{Regulatory Impact Analysis}

\section{A. Overall Impact}

We have examined the final impacts of this rule as required by Executive Order 12866, the Regulatory Flexibility Act, section 1102(b) of the Social Security Act regarding rural hospital impacts, the Unfunded Mandates Reform Act, Executive Order 13132 on Federalism, and the Congressional Review Act.

Executive Order 12866 directs agencies to assess all costs and benefits of available regulatory alternatives and, if regulation is necessary, to select regulatory approaches that maximize net benefits (including potential economic, environmental, public health and safety effects, distributive impacts, and equity). A regulatory impact analysis (RIA) must be prepared for rules with economically significant effects (\$100 million or more in any 1 year). This final rule is anticipated to have an annual effect on the economy of $\$ 100$ million or more, making it an economically significant rule under the Executive Order and a major rule under the Congressional Review Act.

Accordingly, we have prepared a RIA that to the best of our ability presents the costs and benefits of the final rule.

This final rule is one of three coordinated rulemakings undertaken to implement the goals and objectives of the HITECH Act related to the adoption and meaningful use of certified EHR technology. The other two are HHS's interim final rule establishing certification criteria, standards, and implementation specifications for certification of EHR systems, and HHS' final rule on EHR certification programs. Each rule assessed the direct economic effects of its provisions. This final rule on Medicare and Medicaid EHR Incentive Programs addresses the impacts related to the actions taken by EPs or eligible hospitals, or CAHs to demonstrate meaningful use of certified EHR technology, including purchasing or developing in-house certified EHR technology or EHR technology modules.

A number of factors will affect the adoption of EHR systems and

demonstration of meaningful use. Many of these are addressed in this final analysis, but also the final provisions of the other rules. Readers should understand that these forecasts are also subject to substantial uncertainty since demonstration of meaningful use will depend not only on the standards and requirements for FYs 2011 and 2012 for eligible hospitals and CYs 2011 and 2012 for EPs, but on future rulemakings issued by the HHS.

The HITECH Act provides Medicare and Medicaid incentive payments for the meaningful use of certified EHR technology. Additionally, the Medicaid program also provides incentives for the adoption, implementation, and upgrade of certified EHR technology. Payment adjustments are incorporated into the Medicare program for providers unable to demonstrate meaningful use. The absolute and relative strength of these is unclear. For example, a provider with relatively small Medicare billings will be less disadvantaged by payment adjustments than one with relatively large Medicare billings. Another uncertainty arises because there are likely to be "bandwagon" effects as the number of providers using EHRs rises, thereby inducing more participation in the incentives program, as well as greater adoption by entities (for example, clinical laboratories) that are not eligible for incentives or subject to penalties, but do business with EHR adopters. It is impossible to predict exactly if and when such effects may take hold.

One legislative uncertainty arises because under current law, physicians are scheduled for payment reductions under the sustainable growth rate (SGR) formula for determining Medicare payments. Under the current law, physician payments were reduced by 23 percent beginning December 1, 2010, and are scheduled for further reductions in CY 2011. Such reductions could cause major changes in physician behavior, enrollee care, and other Medicare provider payments, but the specific nature of these changes is exceptionally uncertain. Under a current law scenario, the EHR

incentives or payment adjustments would exert only a minor influence on physician behavior relative to these very large payment reductions. However, the Congress has legislatively avoided physician payment reductions in each of the past 7 years. Behavioral changes resulting from these scheduled Medicare physician payment reductions are not included in our estimate and

likewise we do not assume any

additional behavioral changes from EHR incentive payments for Medicare physicians.

All of these factors taken together make it impossible to predict with precision the timing or rates of adoption and ultimately meaningful use.

Therefore, we show two scenarios, which illustrate how different scenarios would impact overall costs. Our "high" scenario of meaningful use

demonstration assumes that roughly a decade from now, nearly 100 percent of hospitals and 70 percent of EPs will be "meaningful users." This estimate is based on the substantial economic incentives created by the combined direct and indirect factors affecting providers. We appreciate that in the real world nothing is ever 100 percent, and can even identify factors that would certainly lead providers to forego implementing an EHR. For example, a physician nearing retirement with a low Medicare caseload might well decide to accept the relatively low adverse consequences of declining to demonstrate meaningful use of certified EHR technology. Alternatively, EPs, eligible hospitals and CAHs may choose not to adopt and meaningfully use EHRs if the total costs of purchasing certified EHRs and the total costs of complying with this rule are higher than the value 
of the total EHR incentive payments (and adjustments, if applicable). However, we have no reliable basis for estimating the rate of such "holdouts." To emphasize the uncertainties involved, we have also created a "low" scenario estimate for the demonstration of meaningful use each year, which assumes less robust adoption and meaningful use. Our "low" scenario of meaningful use demonstration assumes that roughly a decade from now, nearly 95.6 percent of hospitals and 36 percent of EPs will be "meaningful users."

Both the high and low scenario estimates are based on current law, which includes a scheduled physician payment cut of 23 percent on December 1,2010 . Such a reduction could cause major changes in physician behavior, enrollee care, and other Medicare provider payments, but the specific nature of these changes is exceptionally uncertain. In our estimates, we did not assume changes in physician behavior as a result of these payment cuts, as this reflects the standard practice used in forecasts of government spending (including effects on the private sector) by the Boards of Trustees for the Hospital Insurance and Supplementary Medical Insurance Trust Funds, and the Office of the Actuary in HHS.

Since this RIA was published in the proposed rule, legislation has been enacted that increases the number of EPs that may be eligible to receive an incentive payment by changing the determination of hospital-based. A complete discussion of the issue, including comments and responses are available in section 2 of this rule stated The determination of whether an EP is hospital-based will be based upon whether substantially all of the EP's services are furnished in places of service classified under place of service codes 21 (Inpatient Hospital) or 23 (Emergency Room, Hospital). Previously under the old definition, CMS estimated that 27 percent of EPs would meet the definition of hospital-based, however, now, under this final definition of hospital-based EPs, about 14 percent of Medicare EPs would be considered hospital-based and thus not eligible to receive any incentive payments.

There are many estimates of current EHR adoption and usage rates. There is one EHR function-e-prescribing-for which adoption and usage rates for both physicians and hospitals may exceed 50 percent. However, high estimates are misleading because they focus on particular elements, not on comprehensive systems that provide a full range of functions, similar in scope to those established in ONC's final rule that adopts standards, implementation specifications, and certification criteria for the technical requirements and capabilities that EHR systems will need to meet in order to be certified. Based on several peer-reviewed studies, only a small proportion of physicians and hospitals have invested in EHR technology that encompasses such a broad range of functions. For example, a study entitled "Electronic Health Records in Ambulatory Care-A National Survey of Physicians" (Catherine DesRoches et al., New England Journal of Medicine, July 3, 2008), found that in 2007 only "four percent of physicians reported having an extensive, fully functional electronicrecords system, and 13 percent reported having a basic system." (Additional results from the same survey can be found at the Department's Health IT Adoption Initiative Web site at http:// healthit.hhs.gov/portal/server.pt?open= $512 \&$ mode $=2 \&$ cached $=$ true $\& o b j I D=$ 1152.) Another study entitled "Use of Electronic Health Records in U.S. Hospitals" (Ashish Jha et al., New England Journal of Medicine, April 16, 2009) found that in 2007 "only 1.5 percent of U.S. hospitals have a comprehensive electronic-records system * * * and an additional 7.6 percent have a basic system." Computerized order entry (CPOE) for drugs was fully implemented in only 17 percent of hospitals.

Most physicians and hospitals have not yet invested in the hardware, software, testing and training to implement advanced EHRs for a number of reasons-lack of standards, lack of interoperability, limited physician acceptance, fear of maintenance costs, and lack of capital. Perhaps most importantly, adoption of EHR technology necessitates major changes in business processes and practices throughout a provider's office or facility. Business process reengineering on such a scale is not undertaken lightly. However, the availability of the HITECH Act incentives, grants for technical support, more consistent use of standards and specified certification criteria, and other factors addressed in this RIA are likely to increase the adoption of EHR technology very substantially over the next 10 yearsperhaps approaching complete adoption for physicians, hospitals, and many other types of providers, despite, as those providers have commented, not being included in this final rule.

Overall, we expect spending under the EHR incentive program for transfer payments to Medicare and Medicaid providers over 10 years to be $\$ 9.7$ billion under the low scenario, and $\$ 27.4$ billion under the high scenario (these estimates include net payment adjustments for Medicare providers who do not achieve meaningful use in 2015 and beyond in the amount of $\$ 3.9$ billion under the high scenario and $\$ 8.1$ billion under the low scenario). We have also estimated "per entity" costs for EPs, eligible hospitals, and CAHs. We estimate also that adopting entities will achieve dollar savings at least equal to their total costs, and that there will be additional benefits to society. We remain persuaded after consideration of the public comments that implementation costs will be significant for each participating entity because providers who would like to qualify as meaningful users of EHRs will need to purchase certified EHR technology. We further acknowledge that certified EHRs may differ in many important respects from the types of EHRs noted in these comments and the functionalities they contain may differ. However, we still anticipate that the short-term costs to demonstrate meaningful use of certified EHR technology will be outweighed by the long-term benefits, including practice efficiencies and improvements in medical outcomes. Thus it remains that although both cost and benefit estimates are highly uncertain, the RIA that we have prepared to the best of our ability presents the costs and benefits of the final rulemaking.

\section{B. Regulatory Flexibility Analysis}

The Regulatory Flexibility Act requires agencies to prepare an Initial Regulatory Flexibility Analysis to describe and analyze the impact of the final rule on small entities unless the Secretary can certify that the regulation will not have a significant impact on a substantial number of small entities. In the healthcare sector, Small Business Administration size standards define a small entity as one with between $\$ 7$ million and \$34 million in annual revenues. For the purposes of the Regulatory Flexibility Act, essentially all non-profit organizations are considered small entities, regardless of size. Individuals and States are not included in the definition of a small entity. Since the vast majority of Medicare providers (well over 90 percent) are small entities within the Regulatory Flexibility Act's definitions, it is the normal practice of HHS simply to assume that all affected providers are "small" under the Regulatory Flexibility Act. In this case, most EPs, eligible hospitals, and CAHs are either nonprofit or meet the SBA's size standard for small business. We also believe that the effects of the incentives program on many and probably most of these affected entities will be economically 
significant. Accordingly, this RIA section, in conjunction with the remainder of the preamble, constitutes the required Initial Regulatory Flexibility Analysis. We believe that the adoption and meaningful use of EHRs will have an impact on virtually every EP and eligible hospital, as well as CAHs and some EPs and hospitals affiliated with MA organizations. While the program is voluntary, in the first 5 years it carries substantial positive incentives that will make it attractive to virtually all eligible entities.

Furthermore, entities that do not demonstrate meaningful use of EHR technology will be subject to significant Medicare payment reductions after the fifth year. The anticipation of these Medicare payment adjustments will also motivate EPs, eligible hospitals, and CAHs to adopt and meaningfully use certified EHR technology.

For some EPs, CAHs and eligible hospitals the EHR technology that they have in place before the HITECH requirements will be able to be upgraded to meet the criteria for certified EHR technology as defined for this program. These costs may be minimal, involving no more than a software upgrade. "Home-grown" EHR systems that might exist may also require an upgrade to meet the HITECH certification requirements.

We believe that most EPs using EHR systems will require significant changes to achieve certification and that EPs, CAHs and eligible hospitals will have to make process changes to achieve meaningful use. Further, given what we know about the current low levels of EHR adoption we believe that the majority of EPs will need to purchase certified EHR technology, implement this new technology, and train their staff on its use. The costs for implementation and complying with the criteria of meaningful use could lead to higher operational expenses. However, we believe that the combination of payment incentives and long-term overall gains in efficiency will compensate for the initial expenditures.

\section{Number of Small Entities}

In total, we estimate that there are approximately 624,000 healthcare organizations (EPs, eligible hospitals, or CAHs that will be affected by the incentive program. These include hospitals and physician practices as well as doctors of medicine or osteopathy, dental surgery or dental medicine, podiatric medicine, optometry or a chiropractor. Additionally, eligible nonphysicians (such as certified nurse-midwives, etc.) will be eligible to receive the Medicaid incentive payments.

Of the 624,000 healthcare organizations we estimate will be affected by the incentive program, we estimate that 94.71 percent will be EPs, 0.8 percent will be hospitals, and 4.47 percent will be MAO physicians or hospitals. We further estimate that EPs will spend approximately $\$ 54,000$ to purchase and implement a certified EHR and \$10,000 annually for ongoing maintenance according to the CBO. In that paper, Evidence on the Costs and Benefits of Health Information Technology, May 2008, in attempting to estimate the total cost of implementing health IT systems in office-based medical practices, recognized the complicating factors of EHR types, available features and differences in characteristics of the practices that are adopting them. The CBO estimated a cost range of $\$ 25,000$ to $\$ 45,000$ per physician. For all eligible hospitals, the range is from $\$ 1$ million to $\$ 100$ million. Though reports vary widely, we anticipate that the average would be $\$ 5$ million to achieve meaningful use. We estimate \$1 million for maintenance, upgrades, and training each year. See the Costs of EHR adoption in section a under Background and Assumptions portion of this analysis for a discussion regarding the costs of adoption and variation by size and details on our estimates for the number of entities that are eligible for the incentive within each eligibility type category.

Comment: One commenter suggested that the Regulatory Flexibility Act analysis did not include an assessment of the cost to implement the rule at state and local health departments. State and local health departments do operate clinics and provide care to the public. Some state and local health departments would be considered small businesses under the Regulatory Flexibility Act and an assessment of the implementation costs for these entities would allow us to work together to identify possible funding sources and cost savings strategies.

Response: Under Medicaid, clinics such as rural health clinics or FQHCs are not eligible providers that can receive incentive payments. However, EPs within these clinics can receive incentive payments if they meet all other eligibility requirements. The Federal costs and payments associated with EHR implementation for EPs are captured on in Tables 32 and 33.

\section{Alternatives Considered}

This final rule implements new provisions of the Act for providing incentives for EPs, eligible hospitals, and CAHs that adopt and demonstrate meaningful use of certified EHR technology. HHS has no discretion to change the incentive payments or Medicare payment reductions specified in the statute for providers that adopt or fail to adopt EHR and achieve meaningful use of EHR technology. The only substantial alternatives within the discretion of the Department revolve around how best to meet the requirements of the HITECH Act through the definition of meaningful use for FY 2011 and beyond. Requirements that are too stringent could have the adverse effect of preventing many EPs, eligible hospitals, and CAHs from achieving meaningful use and thus preventing them from receiving an incentive payment. Our meaningful use requirements for 2011 are designed to encourage more widespread adoption of certified EHR technology and allow more EPs, eligible hospitals, and CAHs to qualify for incentives while they are also adjusting their practice patterns and training staff to operate the EHR technology in preparation for more stringent meaningful use requirements over time. We recognize that there may be incremental costs that result from requiring additional functionality over the base level defined in the HITECH

Act. We note that with regard to reporting of clinical quality measures for purposes of demonstrating meaningful use, we initially considered requiring EPs, eligible hospitals, and CAHs to report quality measures electronically in the initial year of the program; however, ultimately we determined that many providers would not be able to comply with a requirement to report all quality measures at the beginning of the program. The alternative approach, consistent with the requirements of this final rule, is to require reporting of quality measures in phases. In 2011, there will be a requirement to report clinical quality measures through attestation with a numerator, denominator, and exclusions. Electronic clinical quality measure reporting will begin in FY 2012 for hospitals and CY 2012 for EPs. We expect that additional clinical quality measure reporting will be added in later years.

Under Medicaid, we considered numerous alternatives regarding how to demonstrate eligibility for the incentive payments as well as adoption and meaningful use of the certified EHR technology. These alternatives, including the time period for demonstrating adequate patient volume, and the requirements and methods for demonstrating meaningful use are 
discussed in section II.D. of this final rule.

\section{Conclusion}

As discussed later in this analysis, we believe that there are many positive effects of adopting EHR on health care providers, quite apart from the incentive payments to be provided under this rule. While economically significant, we do not believe that the net effect on individual providers will be negative over time except in very rare cases. (The statute provides for hardship exemption in such cases.) Accordingly, we believe that the object of the Regulatory Flexibility Act to minimize burden on small entities are met by this rule as final.

Comment: Commenters cited the variation in the costs of EHR adoption across EP settings. For example, smaller practices believe their costs of EHR adoption to be higher per physician than larger counterparts. They believe they cannot realize the staff reductions and related cost savings from EHR adoption due to greater crossfunctionality for their staff.

Response: We acknowledge the different experiences EPs have with EHR adoption and implementation. Two additional studies relating to the costs of adoption among small practices (Miller et al. (2005) "The Value Of Electronic Health Records In Solo Or Small Group Practices" Health Affairs 24(5): 11271137, and Zaroukian and Sierra (2006) "Benefiting from Ambulatory EHR Implementation: Solidarity, Six Sigma, and Willingness to Strive" The Journal of Healthcare Information Management 20(1): 53-60) estimate the cost per physician to be $\$ 44,000$ per year with roughly $\$ 8,500$ to $\$ 13,000$ in ongoing maintenance. However, even among these studies there was still variation in experience. The per provider design of meaningful use incentive payments and orientation of other government health IT grant programs is to facilitate adoption and positive return on investment across health care settings. Thus we continue to hold that our cost estimates are reasonable estimations of provider experience while acknowledging that variations in experiences will be inevitable.

\section{Small Rural Hospitals}

Section 1102(b) of the Act requires us to prepare a RIA if a rule would have a significant impact on the operations of a substantial number of small rural hospitals. This analysis must conform to the provisions of section 604 of the Regulatory Flexibility Act. For purposes of section 1102(b) of the Act, we define a small rural hospital as a hospital that is located outside of a metropolitan statistical area and has fewer than 100 beds. This final rule would affect the operations of a substantial number of small rural hospitals because they are required to adopt certified EHR technology by 2015, or face adjusted Medicare payments. As stated above, we have determined that this final rule would create a significant impact on a substantial number of small entities, and have prepared a Regulatory Flexibility Analysis as required by the Regulatory Flexibility Act and, for small rural hospitals, section 1102(b) of the Act. Furthermore, any impacts that would arise from the implementation of certified EHR technology in a rural eligible hospital would be positive, with respect to the streamlining of care and the ease of sharing information with other EPs to avoid delays, duplication, or errors.

Comment: Several commenters have disagreed with our assessment, noting that the unique circumstances of small rural hospitals will not lead to efficiency and lower costs as it might with urban hospitals, but would lead to increased costs related to loss of productivity among the staff for implementing and learning an EHR system, and in later years, Medicare payment adjustments because of the lack of broadband access in these areas among other reasons.

Response: Although we agree that small rural hospitals will have challenges inherent in their location, size and staffing complexity, we also acknowledge that smaller, more rural hospitals could experience added burden in achieving meaningful use. Supplemental funding to Regional Extension Centers to assist CAHs will work to lessen disparity between urban and rural hospitals. We also believe that the presence of incentive payments, market demands and rewards for data exchange, and future cost savings resulting from meaningful use will increase hospital adoption and meaningful use of EHRs.

\section{Unfunded Mandates Reform Act}

Section 202 of the Unfunded Mandates Reform Act of 1995 (UMRA) requires that agencies assess anticipated costs and benefits before issuing any rule whose mandates would require spending in any 1 year $\$ 100$ million in 1995 dollars, updated annually for inflation. In 2010, that threshold is approximately \$135 million. UMRA does not address the total cost of a rule. Rather, it focuses on certain categories of cost, mainly those "Federal mandate" costs resulting from-(1) imposing enforceable duties on State, local, or tribal governments, or on the private sector, or (2) increasing the stringency of conditions in, or decreasing the funding of, State, local, or tribal governments under entitlement programs.

This rule imposes no substantial mandates on States. This program is voluntary for States and States offer the incentives at their option. The State role in the incentive program is essentially to administer the Medicaid incentive program. While this entails certain procedural responsibilities, these do not involve substantial State expense. In general, each State Medicaid Agency that participates in the incentive program will be required to invest in systems and technology to complyStates will have to identify and educate providers, evaluate their attestations and pay the incentive. However, the Federal government will fund 90 percent of the State's related administrative costs, providing controls on the total State outlay.

The investments needed to meet the meaningful use standards and obtain incentive funding are voluntary, and hence not "mandates"within the meaning of the statute. However, the potential reductions in Medicare reimbursement after FY 2015 are effectively mandates. We note that we have no discretion as to those potential payment reductions. Private sector EPs that voluntarily choose not to participate in the program may anticipate potential costs in the aggregate that may exceed \$135 million; however, because EPs may choose for various reasons not to participate in the program, we do not have firm data for the percentage of participation within the private sector.

This RIA, taken together with the remainder of the preamble, constitutes the analysis required by UMRA.

\section{E. Federalism}

Executive Order 13132 establishes certain requirements that an agency must meet when it promulgates a final rule that imposes substantial direct requirement costs on State and local governments, preempts State law, or otherwise has Federalism implications. This final rule would not have a substantial direct effect on State or local governments, preempt State law, or otherwise have a Federalism implication. Importantly, State Medicaid agencies are receiving 100 percent match from the Federal government for incentives paid and a 90 percent match for expenses associated with administering the program. As previously stated, we believe that State administrative costs are minimal. We note that this final rule does add a new 
business requirement for States, because of the systems that will need to be implemented to track and report on provider attestations, applications, and payments. States will also expend funds on the systems that must be built to conduct the tracking and reporting activities. States will interface with the NLR since registration of providers will be stored in the NLR. For tracking and making payments, we believe that most States will use their current MMIS system to make payments. States must inform us of their plans for payments, systems, etc, via the SMHP, PAPD and IAPD; additionally, States will indicate the costs associated with these activities in their PAPD and IAPD. CMS is providing 90 percent FFP to States for building the interface and/or for updates to the MMIS related to EHR incentive payment administration. We believe the Federal share of the 90 percent match will protect the States from burdensome financial outlays and, as noted above, States offer the Medicaid EHR incentive program at their option.

\section{F. Anticipated Effects}

The objective of the remainder of this RIA is to summarize the costs and benefits of the HITECH incentive program for the Medicare FFS, Medicaid, and Medicare Advantage (MA) programs. We also provide assumptions and a narrative addressing the potential costs to the industry for implementation of this technology.

\section{G. HITECH Impact Analysis}

\section{Need for Regulation}

This final rule would implement the provisions of the American Recovery and Reinvestment Act of 2009 (ARRA) (Pub. L. 111-5) that provide incentive payments to EPs, eligible hospitals, and CAHs participating in Medicare and Medicaid programs that adopt and meaningfully use certified EHR technology. The final rule specifies the initial criteria that an EP, eligible hospital, or CAH must meet in order to qualify for the incentive payment; calculation of the incentive payment amounts; payment adjustments under Medicare for covered professional services and inpatient hospital services provided by EPs, and eligible hospitals failing to meaningfully use certified EHR technology; and other program participation requirements. As noted earlier in this RIA, changes both in legislation and policy based on comments from the public have been taken into account for the preparation of this final impact analysis.

\section{Alternatives Considered}

As previously discussed in the alternatives section of the regulatory flexibility analysis, HHS has no discretion to change the incentive payments or payment reductions specified in the statute for providers that adopt or fail to adopt EHR and demonstrate meaningful use of certified EHR technology. However, we have discretion around how best to meet the HITECH Act requirements for meaningful use for FY 2011 and beyond, which we have exercised in this final rule. Additionally, we have used our discretion to appropriately time the registration, attestation and payment requirements to allow EPs and eligible organizations as much time as possible in coordination with the anticipated certification of EHR technology to obtain and meaningfully use certified EHRs. We recognize that there may be additional costs that result from various discretionary policy choices such as requiring additional functionality over the base level defined in the HITECH Act, however, those costs cannot be estimated and are not captured in this analysis.

\section{Background and Assumptions}

The principal costs of this final rule are the additional expenditures that will be undertaken by eligible entities in order to obtain the Medicare and Medicaid incentive payments to adopt, implement or upgrade and/or demonstrate meaningful use of certified EHR technology, and to avoid the Medicare payment adjustments that will ensue if they fail to do so. The estimates for the provisions affecting Medicare and Medicaid EPs, eligible hospitals, and CAHs are somewhat uncertain for several reasons: (1) The program is voluntary although payment adjustments will be imposed on Medicare providers who are unable to demonstrate meaningful use starting in 2015; (2) the criteria for the demonstration of meaningful use of certified EHR technology has been finalized for stage one but will change over time; (3) the HHS certification process although defined, has yet to be implemented;, and, (4) the impact of the financial incentives and payment adjustments on the rate of adoption of certified EHR technology by EPs, eligible hospitals, and CAHs is difficult to predict. The net costs and savings shown for this program represent two possible scenarios and actual impacts could differ substantially.

As written in the preamble, this final rule describes the incentive payments for EPs, eligible hospitals, and CAHs for adopting and demonstrating meaningful use of certified EHR technology. This impact analysis addresses the costs and benefits to the Medicare and Medicaid programs, as well as general implementation costs for eligible hospitals, CAHs and EPs.

Detailed information about the incentive program, the specific payment amounts and how those payments will be paid, is provided in section II of this final rule. Based on input from a number of internal and external sources, including the Government

Accountability Office (GAO) and CBO, we calculated the numbers of EPs and eligible hospitals, including CAHs under Medicare, Medicaid, and MA and used them throughout the analysis.

- About 553,200 Medicare FFS EPs in 2011 (some of which will also be Medicaid EPs).

- About 14 percent of the total EPs are hospital-based Medicare EPs, and are not eligible for the program. This leaves approximately 477,500 nonhospital-based Medicare EPs in 2011.

- Twenty percent of the nonhospitalbased Medicare EPs (approximately 95,500 Medicare EPs in 2011) are also eligible for Medicaid (meet the 30 percent Medicaid patient volume criteria), but can only be paid under one program. We assume that any EP in this situation will choose to receive the Medicaid incentive payment, because it is larger.

- About 44,100 non-Medicare eligible EPs (such as dentists, pediatricians, and eligible non-physicians such as certified nurse-midwives, nurse practitioners and physicians assistants) will be eligible to receive the Medicaid incentive payments.

- 5,011 eligible hospitals comprised of the following:

$++3,620$ acute care hospitals.

$++1,302$ CAHs

++ 78 children's hospitals (Medicaid only).

++ 11 cancer hospitals (Medicaid only).

- All eligible hospitals, except for children's and cancer hospitals, may qualify and apply for both Medicare and Medicaid incentive payments.

- 12 MA Organizations (about 28,000 EPs, and 29 hospitals) would be eligible for incentive payments.

- Payments can begin as early as third quarter FY 2011.

\section{Industry Costs and Adoption Rates}

To estimate the impact on healthcare providers we used information from four studies cited previously. Based on these studies, we continue to estimate for EPs, the average adopt/implement/ 
upgrade cost is $\$ 54,000$ per physician FTE, while annual maintenance costs average $\$ 10,000$ per physician FTE.

For all eligible hospitals, the range is from $\$ 1$ million to $\$ 100$ million. Although reports vary widely, we anticipate that the average would be $\$ 5$ million to achieve meaningful use, because providers who would like to qualify as meaningful users of EHRs will need to purchase certified EHRs. We further acknowledge that "certified EHRs" may differ in many important respects from the EHRs currently in use and may differ in the functionalities they contain. We estimate $\$ 1$ million for maintenance, upgrades, and training each year. Industry costs are important, in part, because EHR adoption rates will be a function of these industry costs and the extent to which the costs of "certified EHRs" are higher than the total value of EHR incentive payments available to EPs and eligible hospitals (as well as adjustments, in the case of the Medicare EHR incentive program) and any perceived benefits including societal benefits. Because of the uncertainties surrounding industry cost estimates, we have made various assumptions about adoption rates in the following analysis in order to estimate the budgetary impact on the Medicare and Medicaid programs.

For an eligible Medicaid EP, the first year incentive can be based on adoption, implementation, and upgrade costs. Previously, we noted that section 1903(t)(4)(C) of the Act gives the Secretary the authority to determine average allowable costs for certified EHR technology. The Secretary studied average costs associated with the purchase, initial implementation, and upgrade of certified EHR technology, including support services and initial training.

Sections 1903(t)(1)(A) and 1903(t)(4) of the Act specify that EPs may not receive incentive payments in excess of 85 percent of the net average allowable costs of certified EHR technology, with such net average allowable costs capped at $\$ 25,000$ in the first year (and $\$ 10,000$ in each of the subsequent years).

\section{a. Costs of EHR Adoption for EPs}

Previously, we described four studies used to estimate costs of implementation including the purchase and installation of hardware and software, training, as well as productivity losses associated with implementation and training. Each of these studies was conducted several years ago, and did not control for type of EHR, functionality, physician practice type or size. Furthermore, EHRs were not being built against any particular consensus standard, nor was the concept of "meaningful use" a factor. Thus, the cost of implementing and maintaining certified EHR technology which meets the requirements established in this regulation might exceed the estimates from these studies.

One average estimate of the cost per physician for implementation is around $\$ 35,000$. A similar study of community health centers estimated costs to average $\$ 54,000$ per physician FTE. In this study, the authors explained that implementation costs varied between entities for hardware, software, installation, and training. After implementation, there were ongoing operating costs estimated at $\$ 21,000$ per year for a practice of four physicians. The CBO paper, Evidence on the Costs and Benefits of Health Information Technology, May 2008, in attempting to estimate the total cost of implementing health IT systems in office-based medical practices, recognized the complicating factors of EHR types, available features and differences in characteristics of the practices that are adopting them. The CBO estimated a cost range of $\$ 25,000$ to $\$ 45,000$ per physician. In the CBO study, operating costs added $\$ 3,000$ to $\$ 9,000$ per physician per year. Finally, a 2005 paper from AHRQ stated that the average purchase and implementation cost of an EHR could be \$32,606 per FTE physician. Maintenance costs were an additional $\$ 1,500$ per physician, per month, or $\$ 18,000$ per year. Smaller practices had the highest implementation costs per physician at $\$ 37,204$. Based on the studies cited, eligible providers will be eligible to receive the maximum incentive permitted under the statute, because the implementation and maintenance costs we have estimated exceed the caps for net average allowable costs set in the statute.

In calculating the impact of the EHR incentive program for Medicaid EPs, we assumed that approximately 20 percent of the EPs eligible for the Medicare incentive payment program are also eligible for Medicaid EHR incentive payments (about 95,500 in 2011). Since the Medicaid incentive payments are higher than those for Medicare and EPs can only receive payments from one program, we assume the dually eligible EPs will receive their payments through the Medicaid program. It is also important to note that just as there is overall variation in state Medicaid programs, we anticipate there will be variation in the design and timing of state Medicaid EHR incentive programs. New data on the pace of state planning for meaningful use was used to adjust
Medicaid adoption scenarios. Thus, how and when providers apply for meaningful use through Medicaid will likely differ by state. Medicaid also offers incentive payments for dentists, certified nurse-midwives, nurse practitioners and certain physicians' assistants. While minimal, we have incorporated the sum of these groups in Table 51. We have estimated a range of Medicaid EPs that will be meaningful users each calendar year. The last line represents the range of predicted meaningful EHR users each calendar year. The Medicaid penetration rate for EPs is consistent with the analysis that was used for the Medicare EPs, but without the behavioral limitations imposed by the Medicare current statute SGR payment reductions. We assumed a modest behavioral response by

Medicaid EPs to the Medicaid incentive payments resulting in an increase over baseline participation.

b. Costs of EHR Adoption for Eligible Hospitals

The American Hospital Association (AHA) conducts annual surveys that among other measures, track hospital spending. We have updated these data to reflect the latest figures from the 2008 AHA Survey. Costs at these levels of adoption were significantly higher in 2008 than 2007. This may better reflect the costs of implementing additional functionalities. We have also updated the number of discharges using the most recent cost report data available. The range in yearly information technology spending among hospitals is largefrom $\$ 36,000$ to over $\$ 32$ million based on 2007 and 2008 AHA data. EHR system costs specifically were reported by experts to run as high as $\$ 20$ million to $\$ 100$ million; HHS discussions with experts led to cost ranges for adoption that varied by hospital size and level of EHR system sophistication. Research to date has shown that adoption of comprehensive EHR systems is limited. In the aforementioned AHA study, 1.5 percent of these organizations had comprehensive systems, which were defined as hospital-wide clinical documentation of cases, test results, prescription and test ordering, plus support for decision-making that included treatment guidelines. Some 10.9 percent have a basic system that does not include physician and nursing notes, and can only be used in one area of the hospital. Applying a similar standard to the 2008 AHA data results in roughly 3-4 percent of hospitals having comprehensive systems and 12 to 13 percent having basic systems. According to hospital CEOs, the main barrier to adoption is the cost of the 
systems, and the lack of capital. Hospitals have been concerned that they will not be able to recoup their investment, and they are already operating on the smallest of margins. Because uptake of advanced systems is low, it is difficult to get a solid average estimate for implementation and maintenance costs that can be applied across the industry. In addition, we recognize that there are additional industry costs associated with adoption and implementation of EHR technology that are not captured in our estimates that eligible entities will incur. Because the impact of those activities, such as reduced staff productivity related to learning how to use the EHR technology, the need to add additional staff to work with HIT issues, administrative costs related to reporting, and the like are unknown at this time and difficult to quantify.

5. Medicare Incentive Program Costs a. Medicare Eligible Professionals (EPs)

In the proposed rule, CMS said that an EP would be a hospital-based EP and therefore ineligible to receive a Medicare or Medicaid EHR incentive payment if more than 90 percent of their services are provided in the following place of service (POS) codes for HIPAA standard transactions: 21-Inpatient Hospital, 22-Outpatient Hospital, 23Emergency Room.

However, as previously noted here and discussed elsewhere in this final rule, Congress amended the law to include only POS codes 21 (inpatient) and 23 (emergency room), excluding 22 (outpatient hospital), thereby permitting some hospital-based EPs to qualify for the incentive payment. Accordingly we have updated our tables to reflect the increased number of EPs that may now qualify for the incentive payments, and those revisions to the numbers flow throughout these updated tables.

To determine the estimated costs of the Medicare incentives for EPs we first needed to determine the EPs with Medicare claims. Then, we calculated that about 14 percent of those EPs are hospital-based, based on the definition final in $\$ 495.4$, and therefore, do not qualify for incentive payments. This percentage of EPs were subtracted from the total number of EPs who have claims with Medicare. These numbers were tabulated from Medicare claims data.

We have also estimated that about 20 percent of EPs that are not hospitalbased will qualify for Medicaid incentive payments and will choose that program because the payments are higher. Of the remaining EPs, we have estimated the percentage which will be meaningful users each calendar year. As discussed previously our estimates for the number of EPs that will successfully demonstrate meaningful use of certified EHR technology is uncertain, so we established high and low scenarios to account for high and low rates of demonstration of meaningful use.

The percentage of Medicare EPs who will satisfy the criteria for demonstrating meaningful use of certified EHR technology and will qualify for incentive payments is a key, but a highly uncertain factor. Our Medicare EHR adoption assumptions for EPs are also affected by the current situation with Medicare physician fee schedule payment rates. As noted previously, under current law (that is, the SGR system formulas), physician payments will be reduced by 21.3 percent beginning June 1, 2010, and are scheduled to be further reduced beginning in CY 2011. Such reductions would almost certainly cause major changes in physician behavior, enrollee care, and other Medicare provider payments, but the specific nature of these changes is exceptionally uncertain. Under a current law scenario, the EHR incentives or Medicare payment adjustments would exert only a minor influence on physician behavior relative to these very large payment reductions. Behavioral changes resulting from these scheduled payment reductions are not included in our estimate and likewise do not assume any additional behavioral changes from EHR incentive payments. Accordingly, the estimated number of non-hospital based Medicare EPs, (including those additional EPs who may now qualify under the revised definition), who will demonstrate meaningful use of certified EHR technology over the period CYs 2011 through 2019 is as shown in Table 22.

\section{TABLE 22: Medicare EPs Demonstrating Meaningful Use of Certified EHR Technology, High and Low Scenario}

\begin{tabular}{|c|c|c|c|c|c|c|c|c|c|}
\hline & \multicolumn{9}{|c|}{ Calendar Year } \\
\hline & 2011 & 2012 & 2013 & 2014 & 2015 & 2016 & 2017 & 2018 & 2019 \\
\hline $\begin{array}{l}\text { EPs who have claims with } \\
\text { Medicare (thousands) }\end{array}$ & 553.2 & 558.9 & 564.6 & 570.3 & 576.0 & 581.7 & 587.5 & 593.3 & 599.0 \\
\hline $\begin{array}{l}\text { Non-Hospital Based EPs } \\
\text { (thousands) }\end{array}$ & 477.5 & 482.4 & 487.3 & 492.2 & 497.1 & 502.1 & 507.1 & 512.0 & 517.0 \\
\hline $\begin{array}{l}\text { EPs that are both Medicare and } \\
\text { Medicaid EPs (thousands) }\end{array}$ & 95.5 & 96.5 & 97.5 & 98.4 & 99.4 & 100.4 & 101.4 & 102.4 & 103.4 \\
\hline \multicolumn{10}{|l|}{ Low Scenario: } \\
\hline $\begin{array}{l}\text { Percent of EPs who are } \\
\text { Meaningful Users }\end{array}$ & 10 & 13 & 15 & 18 & 21 & 24 & 28 & 32 & 36 \\
\hline Meaningful Users (thousands) & 39.9 & 48.7 & 58.8 & 70.2 & 83.1 & 97.3 & 112.9 & 129.9 & 148.1 \\
\hline \multicolumn{10}{|l|}{ High Scenario: } \\
\hline $\begin{array}{l}\text { Percent of EPs who are } \\
\text { Meaningful Users }\end{array}$ & 36 & 40 & 44 & 49 & 53 & 58 & 62 & 66 & 70 \\
\hline Meaningful Users (thousands) & 136.8 & 154.7 & 173.3 & 192.6 & 212.2 & 231.9 & 251.3 & 270.4 & 288.8 \\
\hline
\end{tabular}


Under the HITECH Act, EPs can receive up to 5 years of Medicare incentive payments for the demonstration of meaningful use of certified EHR technology. These payments are the lesser of 75 percent of the physician's allowed charges for the year or a specified maximum amount, which declines from a possible $\$ 18,000$ incentive payment for the first payment year (2011 or 2012) to a $\$ 2,000$ incentive payment for the fifth payment year. EPs in HPSAs receive incentives that are 10 percent higher than the maximum amounts. Hospital-based EPs are not eligible for the Medicare EP incentive payments. EPs may choose to receive incentive payments from either Medicare or Medicaid, (with some limitations on switching programs) but not from both.

The standard full amount of Medicaid incentive payments that an EP could receive is larger than the standard full amount for the Medicare EP incentive payments: of $\$ 63,750$ versus $\$ 44,000$ for Medicare. Medicare incentive payments can first be paid to EPs in CY 2011; and 2012 is the last year that an EP can start to receive incentives and obtain the full 5 years of payments. EPs who first qualify in CY 2013 would be limited to an incentive of $\$ 15,000$ for the first year, and may be eligible to receive 4 years of incentive payments. EPs who first qualify in CY 2014 would be limited to an incentive of $\$ 12,000$ for the first year and may be eligible to receive 3 years of incentive payments. For the Medicare program, incentives are not payable after CY 2016, and EPs who first demonstrate meaningful use in CY 2015 or later are not eligible for EHR incentive payments.

Medicare payment adjustments will apply in CY 2015 and later to EPs who cannot demonstrate meaningful use of certified EHR technology, regardless of whether they received an EHR incentive payment or not. Specifically, the Medicare Physician Fee Schedule payments for an EP who cannot demonstrate meaningful use of certified EHR technology would be reduced by 1 percentage point in CY 2015, two percentage points in CY 2016, and 3 percentage points in CY 2017, and between 3 and 5 percentage points starting in CY 2018. The HITECH Act gives the Secretary the authority, beginning in CY 2018, to increase these reductions by 1 percentage point each year, but not more than 5 percentage points overall, if the Secretary finds the proportion of EPs who are meaningful EHR users is less than 75 percent.

Each year a transfer will be made between the general fund of the Treasury and the Part B account of the Supplemental Medical Insurance (SMI) trust fund to offset the incentives paid or payment adjustments made during the year. In this way, the Part B beneficiary premium will not be affected by the EP payment incentives.

We estimate that there are $12 \mathrm{MA}$ organizations that might be eligible to participate in the EHR incentive program. Those plans have about 28,000 EPs.

Our estimates of the incentive payment costs and payment adjustment savings reflect our assumptions about the proportion of EPs who will demonstrate meaningful use of certified EHR technology. These assumptions were developed based on a review of recent studies and discussions with subject matter experts. We project that a growing proportion of EPs will adopt certified EHR technology that meets the standards even in the absence of the legislated incentives. This number could be higher or lower depending on the final meaningful use definition adopted, physicians' access to capital and implementation expertise, the success of the other HITECH programs in reaching physicians, and other factors.

Specifically, our assumptions are based on literature estimating current rates of physician EHR adoption and rates of diffusion of EHRs and similar technologies. There are a number of studies that have attempted to measure the rate of adoption of electronic medical records (EMR) among physicians prior to the enactment of the HITECH Act (see, for example, Funky and Taylor (2005) The State and Pattern of Health Information Technology Adoption. RAND Monograph MG-409. Santa Monica: The RAND Corporation; Ford, E.W., Menachemi, N., Peterson, L.T., Huerta, T.R. (2009) "Resistance is Futile: But it is Slowing the Pace of EHR Adoption Nonetheless" Journal of the American Informatics Association 16(3): 274-281). We started with the estimated rate of EHR adoption from the study with the most rigorous definition, but note that the meaningful criteria are not equivalent to a fully functional system as defined in this study. (DesRoches, CM, Campbell, EG, Rao, SR et al. (2008) "Electronic Health Records in

Ambulatory Care-A National Survey of Physicians" New England Journal of Medicine 359(1): 50-60). For the low scenario, we then inflated that number (4 percent) to a 2011 baseline using the numbers of physicians reporting in that survey that they had EHR

implementation underway. We assumed that the same proportion of them would be implementing fully-functional EHRs as in the baseline (30 percent of those with basic systems.) We then trended this number forward using the trajectory mapped out by Ford et al. using the data from the period prior to FY 2004 since the slower rate of adoption during the FY 2005 through 2007 period was thought to be caused by policy uncertainty which this regulation should resolve.

Given the revisions to the meaningful use criteria in this final rule and the nationwide implementation of the Regional Extension Center Program, the likelihood of reaching the high scenario has increased. However, actual adoption trends could be significantly different from these assumptions, given the elements of uncertainty we describe throughout this analysis.

Net costs for the low scenario of the Medicare EP portion of the HITECH Act are shown in Table 23. 
TABLE 23: Estimated Costs (+) and Savings (-) for Medicare EPs Demonstrating Meaningful Use of Certified EHR Technology, Low Scenario (in billions)

\begin{tabular}{|c|c|c|c|c|}
\hline Fiscal Year & $\begin{array}{c}\text { Incentive } \\
\text { Payments }\end{array}$ & $\begin{array}{c}\text { Payment } \\
\text { Adjustment } \\
\text { Receipts }\end{array}$ & $\begin{array}{c}\text { Benefit } \\
\text { Payments }\end{array}$ & Net Total \\
\hline 2009 & - & - & - & - \\
\hline 2010 & - & - & - & - \\
\hline 2011 & $\$ 0.2$ & - & - & $\$ 0.2$ \\
\hline 2012 & $\$ 1.0$ & - & - & $\$ 1.0$ \\
\hline 2013 & $\$ 0.9$ & - & - & $\$ 0.9$ \\
\hline 2014 & $\$ 0.6$ & - & - & $\$ 0.6$ \\
\hline 2015 & $\$ 0.5$ & $-\$ 0.6$ & - & $-\$ 0.1$ \\
\hline 2016 & $\$ 0.3$ & $-\$ 1.0$ & - & $-\$ 0.6$ \\
\hline 2017 & $\$ 0.1$ & $-\$ 1.4$ & - & $-\$ 1.3$ \\
\hline 2018 & - & $-\$ 1.6$ & - & $-\$ 1.6$ \\
\hline 2019 & - & $-\$ 1.6$ & - & $-\$ 1.6$ \\
\hline Total, $2009-2014$ & $\$ 2.6$ & - & - & $\$ 2.6$ \\
\hline Total, $2009-2019$ & $\$ 3.6$ & $-\$ 6.1$ & - & $-\$ 2.5$ \\
\hline
\end{tabular}

The estimated net costs for the high scenario of the Medicare EP portion of the HITECH Act are shown in Table 24.

TABLE 24: Estimated Costs (+) and Savings (-) for Medicare EPs Demonstrating Meaningful Use of Certified EHR Technology, High Scenario (in billions)

\begin{tabular}{|c|c|c|c|c|}
\hline Fiscal Year & $\begin{array}{c}\text { Incentive } \\
\text { Payments }\end{array}$ & $\begin{array}{c}\text { Payment } \\
\text { Adjustment } \\
\text { Receipts }\end{array}$ & $\begin{array}{c}\text { Benefit } \\
\text { Payments }\end{array}$ & Net Total \\
\hline 2009 & - & - & - & - \\
\hline 2010 & - & - & - & - \\
\hline 2011 & $\$ 0.6$ & - & - & $\$ 0.6$ \\
\hline 2012 & $\$ 2.3$ & - & - & $\$ 2.3$ \\
\hline 2013 & $\$ 2.0$ & - & - & $\$ 2.0$ \\
\hline 2014 & $\$ 1.3$ & - & - & $\$ 1.3$ \\
\hline 2015 & $\$ 1.1$ & $-\$ 0.4$ & - & $\$ 0.7$ \\
\hline 2016 & $\$ 0.7$ & $-\$ 0.6$ & - & $\$ 0.1$ \\
\hline 2017 & $\$ 0.3$ & $-\$ 0.8$ & - & $-\$ 0.5$ \\
\hline 2018 & - & $-\$ 0.8$ & - & $-\$ 0.8$ \\
\hline 2019 & - & $-\$ 0.8$ & - & $-\$ 0.8$ \\
\hline Total, 2009-2014 & $\mathbf{\$ 6 . 2}$ & - & - & $\$ 6.2$ \\
\hline Total, 2009-2019 & $\mathbf{\$ 8 . 3}$ & $-\mathbf{\$ 3 . 4}$ & - & $\mathbf{\$ 5 . 0}$ \\
\hline
\end{tabular}


b. Medicare Eligible Hospitals and CAHs

In brief, the estimates of hospital adoption were developed by calculating projected incentive payments (which are driven by discharges), comparing them to projected costs of attaining meaningful use, and then making assumptions about how rapidly hospitals would adopt given the fraction of their costs that were covered. In addition, our estimates have been updated to reflect that the additional challenges likely to be experienced in the adoption of EHRs among CAHs will be partially ameliorated by supplements to Regional Extension Center funding to assist CAHs with EHR adoption.

Specifically, the first step in preparing estimates of Medicare program costs for eligible hospitals was to determine the amount of Medicare incentive payments that each hospital in the country could potentially receive under the statutory formula, based on its admission numbers (total patients and Medicare patients). The total incentive payments potentially payable over a 4-year period vary significantly by hospitals' inpatient caseloads, ranging from a low of about $\$ 11,000$ to a high of $\$ 12.9$ million, with the median being $\$ 3.8$ million. The potential Medicare incentive payments for each eligible hospital were compared with the hospital's expected cost of purchasing and operating certified EHR technology. Costs of adoption for each hospital were estimated using data from the 2008 AHA annual survey and IT supplement. Estimated costs varied by size of hospital and by the likely status of EHR adoption in that class of hospitals. Hospitals were grouped first by size (CAHs, non-CAH hospitals under 400 beds, and hospitals with 400 or more beds) because EHR adoption costs do vary by size: namely, larger hospitals with more diverse service offerings and powerful physician staffs generally implement more customized systems than smaller hospitals that might purchase off-the-shelf products. We then calculated the proportion of hospitals within each class that were at one of three levels of EHR adoption: (1) Hospitals which had already implemented relatively advanced systems that included CPOE systems for medications; (2) hospitals which had implemented more basic systems through which lab results could be shared, but not CPOE for medications; and (3) hospitals starting from a base level either neither CPOE or lab reporting. The CPOE for medication standard was chosen because expert input indicated that the CPOE standard in the final meaningful use definition will be the hardest one for hospitals to meet. Table 25 provides these proportions.

TABLE 25: Hospital IT Capabilities By Hospital Size

\begin{tabular}{|c|c|c|c|c|c|c|c|c|}
\hline \multirow[b]{3}{*}{ Hospital Size } & \multicolumn{8}{|c|}{ Levels of Adoption } \\
\hline & \multicolumn{2}{|c|}{ Any CPOE Meds } & \multicolumn{2}{|c|}{ Lab Results } & \multicolumn{2}{|c|}{ Neither } & \multicolumn{2}{|c|}{ Total } \\
\hline & $\begin{array}{c}\text { Number } \\
\text { of } \\
\text { Hospitals }\end{array}$ & Percentage & $\begin{array}{c}\text { Number } \\
\text { of } \\
\text { Hospitals }\end{array}$ & Percentage & $\begin{array}{c}\text { Number } \\
\text { of } \\
\text { Hospitals }\end{array}$ & Percentage & $\begin{array}{c}\text { Number } \\
\text { of } \\
\text { Hospitals }\end{array}$ & Percentage \\
\hline CAHs & 176 & $19 \%$ & 440 & $48 \%$ & 293 & $32 \%$ & 909 & $23 \%$ \\
\hline Small/Medium & 817 & $31 \%$ & 1,352 & $51 \%$ & 462 & $18 \%$ & 2,631 & $67 \%$ \\
\hline
\end{tabular}

We then calculated the costs of moving from these stages to meaningful use for each class of hospital, assuming that even for hospitals with CPOE systems they would incur additional costs of at least 10 percent of their IT budgets. These costs were based on cross-sectional data from the AHA survey and thus do not likely represent the true costs of implementing systems. We have updated these data to reflect the latest figures from the 2008 AHA Survey. Costs at these levels of adoption were significantly higher in 2008 than 2007. This may better reflect the costs of implementing additional functionalities. We have also updated the number of discharges using the most recent cost report data available. Under the HITECH Act, an eligible hospital can receive up to 4 years of Medicare incentive payments for the demonstration of meaningful use of certified EHR technology. These payments reflect the ratio of Medicare inpatient days to total inpatient days and are adjusted by transition factors of $100,75,50$, and 25 percent for the first through fourth implementation years respectively. [Medicare incentive payments can first be paid to hospitals in FY 2011, and FY 2013 is the last year that a hospital can start to receive incentives and obtain the full 4-year transition rates.] Eligible hospitals that first qualify in FY 2014 or FY 2015 will only receive the transition portions that apply to eligible hospitals who implement their EHR in FY 2013 (for example, 75 percent in FY 2014 and 50 percent in FY 2015). Eligible hospitals first demonstrating meaningful use in FY 2016 or later are not eligible for incentive payments. Medicare payment adjustments will be applied beginning in FY 2015 to eligible hospitals that cannot demonstrate meaningful use of certified EHR technology. Special rules apply to CAHs.

We estimate that there are $12 \mathrm{MAOs}$ that might be eligible to participate in the incentive program. Those plans have 29 eligible hospitals. The costs for the MA program have been included in the overall Medicare estimates.

Again to illustrate the uncertainty, we are providing two scenarios for our estimates. Our high scenario estimated net costs for section 4102 of the HITECH Act are shown in Table 26: Estimated costs $(+)$ and savings $(-)$ for eligible hospitals adopting certified EHRs. This provision is estimated to increase Medicare hospital expenditures by a net total of $\$ 10.1$ billion during FYs -2011 through 2019. 
TABLE 26: Estimated Costs (+) and Savings ( $($ ) for Medicare Eligible Hospitals Demonstrating Meaningful Use of Certified EHR Technology, High Scenario (in billions)

\begin{tabular}{|c|c|c|c|c|}
\hline Fiscal Year & $\begin{array}{c}\text { Incentive } \\
\text { Payments }\end{array}$ & $\begin{array}{c}\text { Payment } \\
\text { Adjustment } \\
\text { Receipts }\end{array}$ & $\begin{array}{c}\text { Benefit } \\
\text { Payments }\end{array}$ & Net Total \\
\hline 2009 & - & - & - & - \\
\hline 2010 & - & - & - & - \\
\hline 2011 & $\$ 0.5$ & - & $\left(^{1}\right)$ & $\$ 0.5$ \\
\hline 2012 & $\$ 2.1$ & - & $\left({ }^{1}\right)$ & $\$ 2.1$ \\
\hline 2013 & $\$ 2.2$ & - & $\left(^{1}\right)$ & $\$ 2.2$ \\
\hline 2014 & $\$ 1.9$ & - & $\left(^{1}\right)$ & $\$ 1.9$ \\
\hline 2015 & $\$ 2.1$ & $-\$ 0.3$ & $\left(^{1}\right)$ & $\$ 1.8$ \\
\hline 2016 & $\$ 1.3$ & $-\$ 0.1$ & $\left(^{1}\right)$ & $\$ 1.2$ \\
\hline 2017 & $\$ 0.5$ & $-\$ 0.1$ & $\left(^{1}\right)$ & $\$ 0.5$ \\
\hline 2018 & - & $\left({ }^{1}\right)$ & $\left(^{1}\right)$ & $\left({ }^{1}\right)$ \\
\hline 2019 & - & - & $\left(^{1}\right)$ & $(1)$ \\
\hline Total, 2009-2014 & $\$ 6.7$ & - & $-\$ 0.1$ & $\$ 6.7$ \\
\hline Total, 2009-2019 & $\$ 10.7$ & $-\$ 0.5$ & $-\$ 0.2$ & $\$ 10.1$ \\
\hline
\end{tabular}

${ }^{1}$ Savings of less than $\$ 50$ million.

TABLE 27: Estimated Costs (+) and Savings (-) for Medicare Eligible Hospitals Demonstrating Meaningful Use of Certified EHR Technology, Low Scenario (in billions)

\begin{tabular}{|c|c|c|c|c|}
\hline Fiscal Year & $\begin{array}{c}\text { Incentive } \\
\text { Payments }\end{array}$ & $\begin{array}{c}\text { Payment } \\
\text { Adjustment } \\
\text { Receipts }\end{array}$ & $\begin{array}{c}\text { Benefit } \\
\text { Payments }\end{array}$ & Net Total \\
\hline 2009 & - & - & - & - \\
\hline 2010 & - & - & - & - \\
\hline 2011 & $\$ 0.2$ & - & $\left(^{1}\right)$ & $\$ 0.2$ \\
\hline 2012 & $\$ 0.9$ & - & $\left(^{1}\right)$ & $\$ 0.9$ \\
\hline 2013 & $\$ 1.1$ & - & $\left(^{1}\right)$ & $\$ 1.1$ \\
\hline 2014 & $\$ 1.2$ & - & $\left(^{1}\right)$ & $\$ 1.2$ \\
\hline 2015 & $\$ 1.4$ & $-\$ 0.9$ & $\left(^{1}\right)$ & $\$ 0.5$ \\
\hline 2016 & $\$ 1.2$ & $-\$ 0.6$ & $\left(^{1}\right)$ & $\$ 0.6$ \\
\hline 2017 & $\$ 0.6$ & $-\$ 0.3$ & $\left(^{1}\right)$ & $\$ 0.3$ \\
\hline 2018 & - & $-\$ 0.2$ & $\left({ }^{1}\right)$ & $-\$ 0.2$ \\
\hline 2019 & - & $-\$ 0.1$ & $(1)$ & $-\$ 0.1$ \\
\hline Total, 2009-2014 & $\$ 3.5$ & - & $-\$ 0.1$ & $\$ 3.5$ \\
\hline Total, 2009-2019 & $\$ 6.7$ & $-\$ 2.0$ & $-\$ 0.2$ & $\$ 4.6$ \\
\hline
\end{tabular}

\footnotetext{
${ }^{1}$ Savings of less than $\$ 50$ million.
} 
Based on the comparison of Medicare incentive payments and implementation/operating costs for each eligible hospital, (described above), we made the assumptions shown in Table 28 , related to the prevalence of certified EHR technology for FY 2011 through
2018. As indicated, eligible hospitals that could cover the full cost of an EHR system through Medicare incentive payments were assumed to implement them relatively rapidly, and vice-versa. In other words, eligible hospitals will have an incentive to purchase and implement an EHR system if they perceive that a large portion of the costs will be covered by the incentive payments. Table 28 shows the high scenario estimates:

TABLE 28: Assumed Proportion of Eligible Hospitals with Certified EHR Technology, by Percentage of System Cost Covered by Medicare Incentive Payments, High Scenario

\begin{tabular}{|c|c|c|c|c|c|}
\hline \multirow{2}{*}{$\begin{array}{c}\text { Fiscal } \\
\text { Year }\end{array}$} & \multicolumn{5}{|c|}{$\begin{array}{c}\text { Incentive Payments as Percentage of EHR } \\
\text { Technology Cost }\end{array}$} \\
\cline { 2 - 6 } & $\mathbf{1 0 0 + \%}$ & $\mathbf{7 5 - 1 0 0 \%}$ & $\mathbf{5 0 - 7 5 \%}$ & $\mathbf{2 5 - 5 0 \%}$ & $\mathbf{0 - 2 5 \%}$ \\
\hline 2011 & 0.8 & 0.5 & 0.3 & 0.2 & 0.1 \\
\hline 2012 & 0.95 & 0.65 & 0.5 & 0.35 & 0.2 \\
\hline 2013 & 1.0 & 0.8 & 0.7 & 0.6 & 0.4 \\
\hline 2014 & 1.0 & 0.95 & 0.85 & 0.75 & 0.6 \\
\hline 2015 & 1.0 & 1.0 & 0.95 & 0.9 & 0.8 \\
\hline 2016 & 1.0 & 1.0 & 1.0 & 0.95 & 0.9 \\
\hline 2017 & 1.0 & 1.0 & 1.0 & 1.0 & 0.95 \\
\hline 2018 & 1.0 & 1.0 & 1.0 & 1.0 & 1.0 \\
\hline
\end{tabular}

For instance, under the high scenario 50 percent of eligible hospitals whose incentive payments would cover between 75 percent and 100 percent of the cost of a certified EHR system were assumed to have a certified system in FY 2011. In FY 2012, 65 percent of those hospitals were assumed to have a certified EHR system. All such hospitals were assumed to have a certified EHR system in FY 2015 and thereafter.

High rates of EHR adoption are anticipated prior to FY 2015 due to the large payment adjustments that will be imposed on eligible hospitals that are unable to demonstrate meaningful use beginning in FY 2015. Specifically, the Medicare "market basket" payment updates would be reduced (on a noncumulative basis) by one-fourth, one-half, and three-fourths for FYs 2015, 2016, and 2017 and later, respectively, for eligible hospitals that were not meaningful users of certified EHR technology. However, we heard from industry experts that issues surrounding the capacity of vendors and expert consultants to support implementation, issues of access to capital, and competing priorities in responding to payer demand will limit the number of hospitals that can adopt advanced systems in the short-term. Therefore, we cannot be certain of the adoption rate for hospitals due to these factors and others previously outlined in this preamble, and so we provide two scenarios which are examples of what we believe are possible low rates and high rates of adoption.

Table 29 shows the low scenario estimates.

TABLE 29: Assumed Proportion of Eligible Hospitals with Certified EHR Technology, by Percentage of System Cost Covered by Medicare Incentive Payments, Low Scenario

\begin{tabular}{|c|c|c|c|c|c|}
\hline \multirow{2}{*}{$\begin{array}{c}\text { Fiscal } \\
\text { Year }\end{array}$} & \multicolumn{5}{|c|}{$\begin{array}{c}\text { Incentive Payments as Percentage of EHR } \\
\text { Technology Cost }\end{array}$} \\
\cline { 2 - 6 } & $\mathbf{1 0 0 + \%}$ & $\mathbf{7 5 - 1 0 0 \%}$ & $\mathbf{5 0 - 7 5 \%}$ & $\mathbf{2 5 - 5 0 \%}$ & $\mathbf{0 - 2 5 \%}$ \\
\hline 2011 & 0.6 & 0.35 & 0.2 & 0.1 & 0.05 \\
\hline 2012 & 0.65 & 0.4 & 0.25 & 0.15 & 0.1 \\
\hline 2013 & 0.75 & 0.55 & 0.4 & 0.25 & 0.15 \\
\hline 2014 & 0.9 & 0.75 & 0.55 & 0.4 & 0.3 \\
\hline 2015 & 1.0 & 0.9 & 0.75 & 0.6 & 0.5 \\
\hline 2016 & 1.0 & 1.0 & 0.9 & 0.85 & 0.75 \\
\hline 2017 & 1.0 & 1.0 & 0.95 & 0.9 & 0.85 \\
\hline 2018 & 1.0 & 1.0 & 1.0 & 0.95 & 0.9 \\
\hline 2019 & 1.0 & 1.0 & 1.0 & 1.0 & 1.0 \\
\hline
\end{tabular}


For large, organized facilities such as hospitals, we believe that the revenue losses caused by these payment adjustments would be a substantial incentive to adopt certified EHR technology, even in instances where the Medicare incentive payments would cover only a portion of the costs of purchasing, installing, populating, and operating the EHR system. Based on the assumptions about incentive payments as percentages of EHR technology costs in Table 29, we estimated that the great majority of eligible hospitals would qualify for at least a portion of the Medicare incentive payments that they could potentially receive, and only a modest number would incur penalties. Nearly all eligible hospitals are projected to have implemented certified
EHR technology by FY 2019. Table 30 shows our high scenario estimated percentages of the total potential incentive payments associated with eligible hospitals that could demonstrate meaningful use of EHR systems. Also shown are the estimated percentages of potential incentives that would actually be paid each year.

TABLE 30: Estimated Percentage of Medicare Incentives Which Could be Paid for Meaningful Use of Certified EHR Technology Associated with Eligible Hospitals and Estimated Percentage Payable in Year, High Scenario

\begin{tabular}{|c|c|c|}
\hline $\begin{array}{c}\text { Fiscal } \\
\text { Year }\end{array}$ & $\begin{array}{c}\text { Percent Associated } \\
\text { with Eligible Hospitals }\end{array}$ & $\begin{array}{c}\text { Percent Payable } \\
\text { in Year }\end{array}$ \\
\hline 2011 & $38.4 \%$ & $38.4 \%$ \\
\hline 2012 & $53.5 \%$ & $53.5 \%$ \\
\hline 2013 & $70.2 \%$ & $70.2 \%$ \\
\hline 2014 & $82.6 \%$ & $82.6 \%$ \\
\hline 2015 & $92.6 \%$ & $54.2 \%$ \\
\hline 2016 & $96.9 \%$ & $43.4 \%$ \\
\hline 2017 & $99.0 \%$ & - \\
\hline 2018 & $100.0 \%$ & - \\
\hline
\end{tabular}

For instance in FY 2012 under the high scenario, 53.5 percent of the total amount of incentive payments which could be payable in that year would be for eligible hospitals who have demonstrated meaningful use of certified EHR technology and therefore will be paid. In FY 2015 under the high scenario, 92.6 percent of the total amount of incentive payments which could be payable will be for hospitals who have certified EHR systems, but some of those eligible hospitals would have already received 4 years of incentive payments, and therefore 54.2 percent of all possible incentive payments actually paid in that year.

Table 31 shows the low scenario estimates.

\section{TABLE 31: Estimated Percentage of Medicare Incentives Which could be paid for the Meaningful Use of Certified EHR Technology Associated with Eligible Hospitals and Estimated Percentage Payable in Year, Low Scenario}

\begin{tabular}{|c|c|c|}
\hline $\begin{array}{c}\text { Fiscal } \\
\text { Year }\end{array}$ & $\begin{array}{c}\text { Percent Associated with } \\
\text { Eligible Hospitals }\end{array}$ & $\begin{array}{c}\text { Percent Payable in } \\
\text { Year }\end{array}$ \\
\hline 2011 & $16.8 \%$ & $16.8 \%$ \\
\hline 2012 & $21.8 \%$ & $21.8 \%$ \\
\hline 2013 & $32.1 \%$ & $32.1 \%$ \\
\hline 2014 & $47.6 \%$ & $47.6 \%$ \\
\hline 2015 & $66.4 \%$ & $49.6 \%$ \\
\hline 2016 & $85.9 \%$ & $64.1 \%$ \\
\hline 2017 & $91.4 \%$ & - \\
\hline 2018 & $95.6 \%$ & - \\
\hline
\end{tabular}

The estimated payments to eligible hospitals were calculated based on the hospitals' qualifying status and individual incentive amounts under the statutory formula. Similarly, the estimated penalties for nonqualifying hospitals were based on the market basket reductions and Medicare revenues. The estimated savings in Medicare eligible hospital benefit expenditures resulting from the use of hospital certified EHR systems are 
discussed under "general

considerations" at the end of this section. We assumed no future growth in the total number of hospitals in the U.S. because growth in acute care hospitals has been minimal in recent years.

Comment: The AHA surveyed 795 hospitals in January 2010 asking whether their EHR systems could meet each of the meaningful use objectives now and in coming years: 45 percent reported they could meet all Stage 1 objectives by 2015 meaning that the remainder might be subject to penalties.

Response: Their survey was based on our proposed definition of meaningful use. The definition of meaningful use in this final rule offers more flexibility and lower thresholds which we believe will make it easier for eligible hospitals to qualify for incentives. However we do acknowledge that the meaningful use criteria described in this final rule may still challenge hospitals to use their IT in ways that improve patient care and outcomes. We also acknowledge that smaller, more rural hospitals could experience added burden in achieving meaningful use related to timing and costs of implementation. Supplemental funding to Regional Extension Centers to assist CAHs will work to lessen disparity between urban and rural hospitals. We also believe that the presence of incentive payments, market demands and rewards for data exchange, and future cost savings resulting from meaningful use will increase hospital adoption and meaningful use of EHRs

\section{c. Critical Access Hospitals (CAHs)}

We estimate that there are 1,302 CAHs eligible to receive EHR incentive payments. Given the financial assistance available under HITECH for Regional
Extension Centers, whose priorities include assisting CAHs in EHR adoption, we estimate that the 19 percent of CAHs with relatively advanced EHR systems will achieve meaningful use before 2016. We also estimate that most of the remaining CAHs that have already adopted some kind of EHR system (48 percent of CAHs) will also achieve meaningful use by 2016 . Our estimates regarding the incentives that will be paid to CAHs are incorporated into the overall Medicare and Medicaid program costs.

We note that in response to comments this final rule amends the definition of acute care hospital for purposes of the Medicaid EHR incentive payment program to generally include critical access hospitals that meet the Medicaid patient volume criteria. Thus, the change in the definition has required that we update our tables to reflect the increased number of hospitals that now may qualify for the Medicaid EHR incentive payment program under this new definition. The numbers and percentages from the revised tables are reflected throughout this final impact analysis. Additionally, EHR adoption rates have been adjusted now that CAHs will be eligible for both Medicare and Medicaid EHR incentive payments.

\section{Medicaid Incentive Program Costs}

Under section 4201 of the HITECH Act, States can voluntarily participate in the Medicaid incentive payment program and we have based our Medicaid incentive program costs on all States participating. Eligible hospitals and EPs can qualify for a Medicaid incentive payment for adopting, implementing, or upgrading in their first participation year, or for meaningful use, and up to an additional 5 years of incentive payments for demonstrating meaningful use of certified EHR technology. Under Medicaid, EPs include physicians (including pediatricians), dentists, certified nursemidwives, nurse practitioners, and certain physician assistants. Initial incentive payments are available through 2016, and incentive payments cannot be made after 2021. The Medicaid hospital incentives are similar to those specified in section 4102 of the HITECH Act for Medicare, except that they must be paid out over at least 3 years and are spread out over a maximum of 6 years, are based on the ratio of Medicaid inpatient days to total days, and are not phased down as quickly as the Medicare payments based on the first year of payment. Medicaid hospitals can begin incentive payments through 2016, and incentive payments cannot be made after 2021. There are also additional hospitals, such as children's and cancer hospitals that are only eligible for Medicaid incentives.

EPs may qualify for Medicaid incentive payments if at least 30 percent of their patient volume is from Medicaid. (Separate rules apply for pediatricians.) As mentioned above, the Medicaid maximum incentive payments are larger than the corresponding Medicare payments. Various maximums are specified for eligible hospital and EP incentive payments. There are no Medicaid penalties for non-adoption of EHR systems or for failing to demonstrate meaningful use. The Federal costs for Medicaid incentive payments to providers who can demonstrate meaningful use of EHR technology were estimated similarly to the estimates for Medicare eligible hospital and EP. Table 32 shows our high estimates for the net Medicaid costs for eligible hospitals and EP. 
TABLE 32: Estimated Federal Costs (+) and Savings (-) under Medicaid, High Scenario (in Sbillions)

\begin{tabular}{|c|c|c|c|c|}
\hline \multirow[b]{2}{*}{ Fiscal year } & \multicolumn{2}{|c|}{ Incentive payments } & \multirow[b]{2}{*}{$\begin{array}{c}\text { Benefit } \\
\text { payments }\end{array}$} & \multirow[b]{2}{*}{ Net total } \\
\hline & Hospitals & $\begin{array}{c}\text { Eligible } \\
\text { professionals }\end{array}$ & & \\
\hline 2009 & - & - & - & - \\
\hline 2010 & - & - & - & - \\
\hline 2011 & 0.8 & 0.9 & $(1)$ & 1.7 \\
\hline 2012 & 0.3 & 1.1 & $(1)$ & 1.4 \\
\hline 2013 & 0.9 & 1.0 & $(1)$ & 1.9 \\
\hline 2014 & 0.7 & 0.9 & $(1)$ & 1.6 \\
\hline 2015 & 0.6 & 1.1 & $(1)$ & 1.7 \\
\hline 2016 & 0.5 & 1.1 & $(1)$ & 1.7 \\
\hline 2017 & 0.4 & 0.9 & $(1)$ & 1.3 \\
\hline 2018 & 0.2 & 0.6 & $(1)$ & 0.7 \\
\hline 2019 & 0.0 & 0.3 & $(1)$ & 0.3 \\
\hline Total, 2009-14 & 2.5 & 4.0 & 0.0 & 6.5 \\
\hline Total, 2009-19 & 4.3 & 8.0 & -0.1 & 12.2 \\
\hline
\end{tabular}

${ }^{1}$ Less than $\$ 50$ million impact

Table 33 shows the low estimates for Medicaid costs and savings.

TABLE 33: Estimated Federal Costs (+) and Savings (-) under Medicaid, Low Scenario (in Sbillions)

\begin{tabular}{|c|c|c|c|c|}
\hline & \multicolumn{2}{|c|}{ Incentive Payments } & \multirow{2}{*}{$\begin{array}{c}\text { Benefit } \\
\text { Payments }\end{array}$} & \\
\cline { 2 - 3 } Fiscal Year & Hospitals & $\begin{array}{c}\text { Eligible } \\
\text { Professionals }\end{array}$ & & Net Total \\
\hline 2009 & - & - & - & - \\
\hline 2010 & - & - & - & - \\
\hline 2011 & 0.4 & 0.2 & $\left(^{1}\right)$ & 0.6 \\
\hline 2012 & 0.1 & 0.4 & $\left(^{1}\right)$ & 0.5 \\
\hline 2013 & 0.4 & 0.4 & $\left(^{1}\right)$ & 0.8 \\
\hline 2014 & 0.4 & 0.4 & $\left({ }^{1}\right)$ & 0.8 \\
\hline 2015 & 0.5 & 0.5 & $\left({ }^{1}\right)$ & 1.0 \\
\hline 2016 & 0.7 & 0.6 & $\left({ }^{1}\right)$ & 1.3 \\
\hline 2017 & 0.8 & 0.5 & $\left(^{1}\right)$ & 1.3 \\
\hline 2018 & 0.4 & 0.4 & $\left(^{1}\right)$ & 0.9 \\
\hline 2019 & 0.1 & 0.3 & $\left(^{1}\right)$ & 0.4 \\
\hline Total, 2009-14 & 1.3 & 1.4 & 0.0 & 2.7 \\
\hline Total, 2009-19 & 3.8 & 3.8 & 0.0 & 7.6 \\
\hline
\end{tabular}

${ }^{1}$ Less than $\$ 50$ million impact.

a. Medicaid EPs

To determine the Medicaid EP incentive payments, we first determined the number of qualifying EPs. As indicated above, we assumed that 20 percent of the non-hospital-based Medicare EPs would meet the requirements for Medicaid incentive payments (30 percent of patient volume from Medicaid). All of these EPs were assumed to choose the Medicaid incentive payments, as they are larger. 
In addition, the total number of Medicaid EPs was adjusted to include EPs who qualify for the Medicaid incentive payments but not for the Medicare incentive payments, such as most pediatricians, dentists, certified nurse-midwives, nurse practitioners and physicians assistants. As noted previously there is much uncertainty about the rates of demonstration of meaningful use that will be achieved. Therefore, as we estimated for the
Medicare EPs, we are providing high and low scenario estimates for Medicaid EPs.

Our high scenario estimates are listed in the Table 34.

\section{TABLE 34: Assumed Number of Nonhospital Based Medicaid EPs Who Will Be Meaningful Users of Certified EHR Technology, High Scenario (All population figures are in thousands)}

\begin{tabular}{|c|c|c|c|c|c|c|c|c|c|}
\hline & $\underline{2011}$ & $\underline{2012}$ & $\underline{2013}$ & $\underline{2014}$ & 2015 & 2016 & $\underline{2017}$ & $\underline{2018}$ & $\underline{2019}$ \\
\hline EPs who have claims with Medicare & 553.2 & 558.9 & 564.6 & 570.3 & 576.0 & 581.7 & 587.5 & 593.3 & 599.0 \\
\hline Non-Hospital based EPs & 477.5 & 482.4 & 487.3 & 492.2 & 497.1 & 502.1 & 507.1 & 512.0 & 517.0 \\
\hline $\begin{array}{l}\text { EPs who meet the Medicaid patient Volume } \\
\text { Threshold }\end{array}$ & 95.5 & 96.5 & 97.5 & 98.4 & 99.4 & 100.4 & 101.4 & 102.4 & 103.4 \\
\hline Medicaid Only EPs ${ }^{1}$ & 44.1 & 44.8 & 45.5 & 46.3 & 47.1 & 47.8 & 48.6 & 49.3 & 50.1 \\
\hline Total Medicaid & 139.6 & 141.3 & 143.0 & 144.7 & 146.5 & 148.2 & 150.0 & 151.7 & 153.5 \\
\hline $\begin{array}{l}\text { Number of EPs receiving incentive payment } \\
\text { during year }\end{array}$ & 66.0 & 93.7 & 109.6 & 119.0 & 125.4 & 131.7 & 65.7 & 38.0 & 22.1 \\
\hline $\begin{array}{l}\text { Percent of EPs who have ever received } \\
\text { incentive payment }\end{array}$ & $47.3 \%$ & $66.3 \%$ & $76.6 \%$ & $82.2 \%$ & $85.6 \%$ & $88.8 \%$ & $91.9 \%$ & $94.7 \%$ & $95.9 \%$ \\
\hline $\begin{array}{l}\text { Number of EPs who have ever received } \\
\text { incentive payment }\end{array}$ & 66.0 & 93.7 & 109.6 & 119.0 & 125.4 & 131.7 & 137.7 & 143.6 & 147.2 \\
\hline
\end{tabular}

${ }^{11}$ Includes non hospital-based eligible pediatricians, dentists, certified nurse-midwives, nurse practitioners and physicians assistants. These numbers were not based on tabulated Medicaid data. Rather, a different methodology was used to estimate the EP counts for each group.

It should be noted that since the Medicaid EHR incentive payment program provides that a Medicaid EP can receive an incentive payment in their first year because he or she has demonstrated a meaningful use or because he or she has adopted, implemented, or upgraded certified EHR 35 shows our low scenario estimates. technology, these participation rates include not only meaningful users but eligible providers implementing certified EHR technology as well. Table

\section{TABLE 35: Assumed Number of Nonhospital Based Medicaid EPs Who Will Be Meaningful Users of Certified EHR Technology, Low Scenario (All population figures are in thousands)}

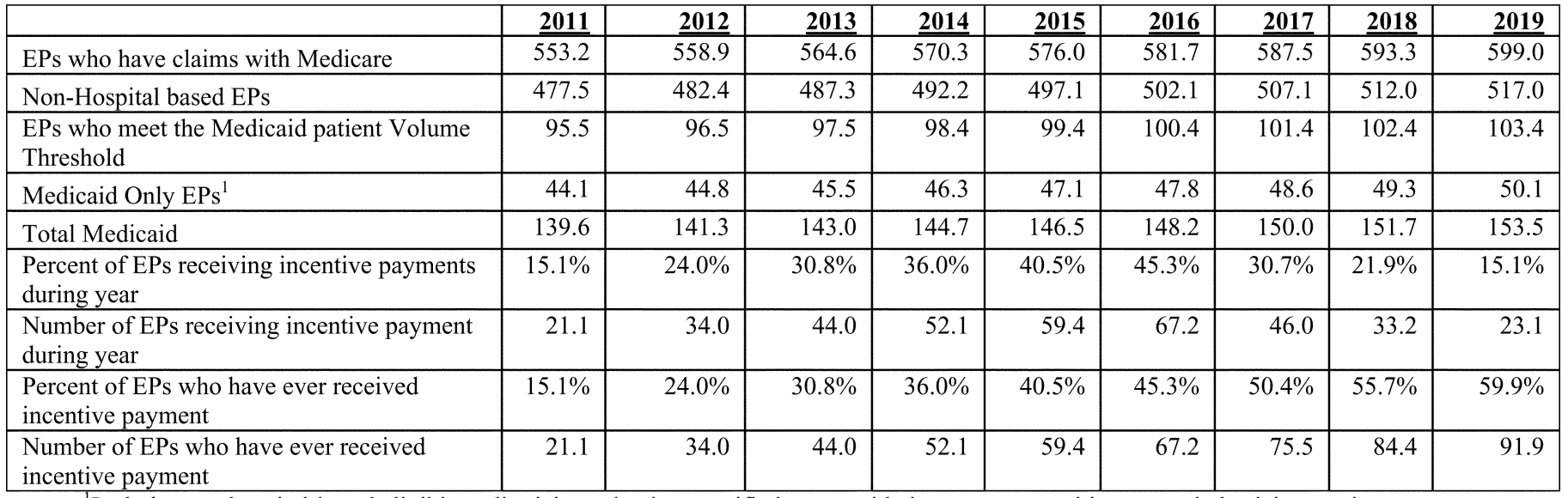

${ }^{1}$ Includes non hospital-based eligible pediatricians, dentists, certified nurse-midwives, nurse practitioners, and physicians assistants. These numbers were not based on tabulated Medicaid data. Rather, a different methodology was used to estimate the EP counts for each group. 


\section{b. Medicaid Hospitals}

Medicaid incentive payments to most acute-care hospitals were estimated using the same adoption assumptions and methodology as described previously for Medicare eligible hospitals and shown in Table 36. Because hospitals' Medicare and Medicaid patient loads differ, we separately calculated the range of percentage of total potential incentives that could be associated with qualifying hospitals, year by year, and the corresponding actual percentages payable each year. Acute care hospitals and children's hospitals can spread aggregate Medicaid incentive payments over no less than 3 years, but no more than 6 years of payments, and acute care hospitals may qualify to receive both the Medicare and Medicaid incentive payments.

As stated previously, the estimated eligible hospital incentive payments were calculated based on the hospitals' qualifying status and individual incentive amounts payable under the statutory formula. The estimated savings in Medicaid benefit expenditures resulting from the use of certified EHR technology are discussed under "general considerations." We estimated the Medicaid incentives payable to children's hospitals as an add-on to the base estimate, using data on the number of children's hospitals compared to nonchildren's hospitals.

\section{TABLE 36: Estimated Percentage of Potential Medicaid Incentives Associated with Eligible Hospitals and Estimated Percentage Payable Each Year, High Scenario}

\begin{tabular}{|c|c|c|}
\hline $\begin{array}{c}\text { Fiscal } \\
\text { Year }\end{array}$ & $\begin{array}{c}\text { Percent Associated } \\
\text { with Eligible Hospitals }\end{array}$ & $\begin{array}{c}\text { Percent Payable } \\
\text { in Year }\end{array}$ \\
\hline 2011 & $39.1 \%$ & $39.1 \%$ \\
\hline 2012 & $54.4 \%$ & $54.4 \%$ \\
\hline 2013 & $70.9 \%$ & $70.9 \%$ \\
\hline 2014 & $83.1 \%$ & $44.0 \%$ \\
\hline 2015 & $92.9 \%$ & $38.5 \%$ \\
\hline 2016 & $97.1 \%$ & $26.2 \%$ \\
\hline 2017 & $99.0 \%$ & $14.0 \%$ \\
\hline 2018 & $100.0 \%$ & $4.2 \%$ \\
\hline 2019 & $100.0 \%$ & $0.0 \%$ \\
\hline
\end{tabular}

Table 37 shows our low scenario estimates.

\section{TABLE 37: Estimated Percentage of Potential Medicaid Incentives Associated with Eligible Hospitals and Estimated Percentage Payable Each Year, Low Scenario}

\begin{tabular}{|c|c|c|}
\hline $\begin{array}{c}\text { Fiscal } \\
\text { Year }\end{array}$ & $\begin{array}{c}\text { Percent Associated with } \\
\text { Eligible Hospitals }\end{array}$ & $\begin{array}{c}\text { Percent Payable } \\
\text { in Year }\end{array}$ \\
\hline 2011 & $18.3 \%$ & $18.3 \%$ \\
\hline 2012 & $23.3 \%$ & $23.3 \%$ \\
\hline 2013 & $33.7 \%$ & $33.7 \%$ \\
\hline 2014 & $49.2 \%$ & $30.9 \%$ \\
\hline 2015 & $67.8 \%$ & $44.5 \%$ \\
\hline 2016 & $86.5 \%$ & $52.8 \%$ \\
\hline 2017 & $91.8 \%$ & $37.3 \%$ \\
\hline 2018 & $95.9 \%$ & $18.7 \%$ \\
\hline 2019 & $100.0 \%$ & $0.0 \%$ \\
\hline
\end{tabular}

7. Benefits for All EPs and All Eligible Hospitals

In this final rule we have not quantified the overall benefits to the industry, nor to eligible hospitals, or EPs in the Medicare, Medicaid, or MA programs. We believe that the first 5 years of the incentive program will be dedicated to implementation activities, from installation of the technology to training to operational and behavioral changes. Information on the costs and benefits of adopting systems specifically meeting the requirements in this rule does not yet exist-and information on costs and benefits overall is limited (Goldzweig et al. 2009 "Costs and Benefits of Health Information 
Technology: New Trends from the Literature" Health Affairs.)

Nonetheless, we believe there are benefits that can be obtained by eligible hospitals and EPs, including: reductions in medical record-keeping costs, reductions in repeat tests, decreases in length of stay, and reduced errors. Furthermore, there is limited but growing evidence to support the costsaving benefits anticipated from wider adoption of EHRs. For example, at one hospital emergency room in Delaware, the ability to download and create a file with a patient's medical history saved the ER \$545 per use, mostly on reduced waiting times. A pilot study of ambulatory practices found a positive ROI within 16 months and annual savings thereafter (Greiger et al. 2007, A Pilot Study to Document the Return on Investment for Implementing an Ambulatory Electronic Health Record at an Academic Medical Center http:// www.journalacs.org/article/S10727515\%2807\% 2900390-0/abstractarticle-footnote-1s.) Some vendors have estimated that EHRs could result in cost savings of between $\$ 100$ and $\$ 200$ per patient per year. As adoption increases, there will be more opportunities to capture and report on cost savings and benefits. A number of relevant studies are required in the HITECH Act for this specific purpose, and the results will be made public, as they are available.

\section{Benefits to Society}

According to the recent CBO study "Evidence on the Costs and Benefits of Health Information Technology" http:// www.cbo.gov/ftpdocs/91xx/doc9168/0520-HealthIT.pdf when used effectively, EHRs can enable providers to deliver health care more efficiently. For example, the study states that EHRs can reduce the duplication of diagnostic tests, prompt providers to prescribe cost-effective generic medications, remind patients about preventive care reduce unnecessary office visits and assist in managing complex care. Further, the report claims that there is a potential to gain both internal and external savings from widespread adoption of health IT, noting that internal savings would likely be in the reductions in the cost of providing care, and that external savings could accrue to the health insurance plan or even the patient, such as the ability to exchange information more efficiently. The benefits resulting specifically from this final regulation are even harder to quantify because they represent, in many cases, adding functionality to existing systems and reaping the network externalities created by larger numbers of providers participating in information exchange.

Since the CBO study, additional research has emerged documenting the association of EHRs with improved outcomes among diabetics (Hunt, JS et al. (2009) "The impact of a physiciandirected health information technology system on diabetes outcomes in primary care: a pre- and post-implementation study" Informatics in Primary Care 17(3):165-74; Pollard, C et al. (2009) "Electronic patient registries improve diabetes care and clinical outcomes in rural community health centers" Journal of Rural Health 25(1):77-84) and trauma patients (Deckelbaum, D. et al. (2009)

"Electronic medical records and mortality in trauma patients "The Journal of Trauma: Injury, Infection, and Critical Care 67(3): 634-636), enhanced efficiencies in ambulatory care settings (Chen, C et al. (2009) "The Kaiser Permanente Electronic Health Record: Transforming and Streamlining Modalities Of Care."Health Affairs 28(2):323-333), and improved outcomes and lower costs in hospitals

(Amarasingham, R. et al. (2009)

"Clinical information technologies and inpatient outcomes: a multiple hospital study" Archives of Internal Medicine 169(2):108-14).

\section{General Considerations}

The estimates for the HITECH Act provisions were based on the economic assumptions underlying the President's 2011 Budget. Under the statute, Medicare incentive payments for certified EHR technology are excluded from the determination of MA capitation benchmarks. As noted previously, there is considerable uncertainty about the rate at which eligible hospitals, CAHs and EPs will adopt EHRs and other HIT. Nonetheless, we believe that the Medicare incentive payments and the prospect of significant payment penalties for not demonstrating meaningful use will result in the great majority of hospitals implementing certified EHR technology in the early years of the Medicare EHR incentive program. We expect that a steadily growing proportion of practices will implement certified EHR technology over the next 10 years, even in the absence of the Medicare incentives. Actual future Medicare and Medicaid costs for eligible hospital and EP incentives will depend in part on the standards developed and applied for assessing meaningful use of certified EHR technology. We expect to administer the requirements in such a way as to encourage adoption of certified EHR technology and facilitate qualification for incentive payments, and expect to adopt progressively demanding standards at each stage year. Certified EHR technology has the potential to help reduce medical costs through efficiency improvements, such as prompter treatments, avoidance of duplicate or otherwise unnecessary services, and reduced administrative costs (once systems are in place), with most of these savings being realized by the providers rather than by Medicare or Medicaid. To the extent that this technology will have a net positive effect on efficiency, then more rapid adoption of such EHR systems would achieve these efficiencies sooner than would otherwise occur, without the EHR incentives.

The CBO has estimated a modest level of such savings attributable to EHRs, with much of the amount associated with reductions in adverse drug-to-drug interactions. We expect a negligible impact on benefit payments to hospitals and EPs from Medicare and Medicaid as a result of the implementation of EHR technology.

In the process of preparing the estimates for this rule, we consulted with and/or relied on internal CMS sources, as well as the following sources:

- Congressional Budget Office (staff and publications).

- American Medical Association (staff and unpublished data).

- American Hospital Association.

- Actuarial Research Corporation.

- RAND Health studies on:

++ "The State and Pattern of Health Information Technology Adoption" (Fonkych \& Taylor, 2005);

++ "Extrapolating Evidence of Health

Information Technology Savings and

Costs" (Girosi, Meili, \& Scoville,

2005); and

++ "The Diffusion and Value of Healthcare Information Technology" (Bower, 2005).

- Kaiser Permanente (staff and publications).

- Miscellaneous other sources (Health Affairs, American Enterprise Institute, news articles and perspectives).

As noted at the beginning of this analysis, it is difficult to predict the actual impacts of the HITECH Act with much certainty at this time. We believe the assumptions and methods described herein are reasonable for estimating the financial impact of the provisions on the Medicare and Medicaid programs, but acknowledge the wide range of possible outcomes.

All financial analysis is calculated over a 10-year planning horizon, because though the incentive payments for Medicare EPs, CAHs and eligible 
hospitals will only be paid for 5 years, the Medicaid incentives will cease in CY 2021. Starting in CY 2015, Medicare payment adjustments will begin.

\section{Summary}

The total cost to the Medicare and Medicaid programs is estimated to be
$\$ 9.7$ billion in transfers under the low scenario, and $\$ 27.4$ billion under the high scenario, over a 10-year timeframe. The main reasons for the changes from the proposed rule are revised definitions of hospital-based eligible professional and Medicaid acute care hospitals, and updated data on discharges and costs of adoption among hospitals. We do not estimate total costs to the provider industry, but rather provide a possible per EP and per eligible hospital outlay for implementation and maintenance operations.

TABLE 51: Estimated EHR Incentive Payments and Benefits Impacts on the Medicare and Medicaid Programs of the HITECH EHR Incentive Program. (Fiscal Year) - (in billions) Low Scenario

\begin{tabular}{|c|c|c|c|c|c|}
\hline \multirow{2}{*}{$\begin{array}{c}\text { Fiscal } \\
\text { Year }\end{array}$} & \multicolumn{2}{|c|}{ Medicare Eligible } & \multicolumn{2}{c|}{ Medicaid Eligible } & \multirow{2}{*}{ Total } \\
\cline { 2 - 5 } & Hospitals & Professionals & Hospitals & Professionals & \\
\hline 2011 & $\$ 0.2$ & $\$ 0.2$ & $\$ 0.4$ & $\$ 0.2$ & $\$ 1.0$ \\
\hline 2012 & $\$ 0.9$ & $\$ 1.0$ & $\$ 0.1$ & $\$ 0.4$ & $\$ 2.4$ \\
\hline 2013 & $\$ 1.1$ & $\$ 0.9$ & $\$ 0.4$ & $\$ 0.4$ & $\$ 2.8$ \\
\hline 2014 & $\$ 1.2$ & $\$ 0.6$ & $\$ 0.4$ & $\$ 0.4$ & $\$ 2.6$ \\
\hline 2015 & $\$ 0.5$ & $-\$ 0.1$ & $\$ 0.5$ & $\$ 0.5$ & $\$ 1.4$ \\
\hline 2016 & $\$ 0.6$ & $-\$ 0.6$ & $\$ 0.7$ & $\$ 0.6$ & $\$ 1.3$ \\
\hline 2017 & $\$ 0.3$ & $-\$ 1.3$ & $\$ 0.8$ & $\$ 0.5$ & $\$ 0.3$ \\
\hline 2018 & $-\$ 0.2$ & $-\$ 1.6$ & $\$ 0.4$ & $\$ 0.4$ & $-\$ 1.0$ \\
\hline 2019 & $-\$ 0.1$ & $-\$ 1.6$ & $\$ 0.1$ & $\$ 0.3$ & $-\$ 1.3$ \\
\hline TOTAL & $\$ 4.6$ & $\mathbf{- \$ 2 . 5}$ & $\$ 3.8$ & $\$ 3.8$ & $\$ 9.7$ \\
\hline
\end{tabular}

Table 39 shows the total costs from 2011 through 2019 for the high scenario after which the payment adjustments will be invoked.

\section{Table 39: Estimated EHR Incentive Payments and Benefits Impacts on the Medicare and Medicaid Programs of the HITECH EHR Incentive Program. (Fiscal Year) - (in billions) High Scenario}

\begin{tabular}{|c|c|c|c|c|c|}
\hline \multirow[b]{2}{*}{ Fiscal Year } & \multicolumn{2}{|c|}{ Medicare Eligible } & \multicolumn{2}{|c|}{ Medicaid Eligible } & \multirow[b]{2}{*}{ Total } \\
\hline & Hospitals & Professionals & Hospitals & Professionals & \\
\hline 2011 & $\$ 0.5$ & $\$ 0.6$ & $\$ 0.8$ & $\$ 0.9$ & $\$ 2.8$ \\
\hline 2012 & $\$ 2.1$ & $\$ 2.3$ & $\$ 0.3$ & $\$ 1.1$ & $\$ 5.8$ \\
\hline 2013 & $\$ 2.2$ & $\$ 2.0$ & $\$ 0.9$ & $\$ 1.0$ & $\$ 6.1$ \\
\hline 2014 & $\$ 1.9$ & $\$ 1.3$ & $\$ 0.7$ & $\$ 0.9$ & $\$ 4.8$ \\
\hline 2015 & $\$ 1.8$ & $\$ 0.7$ & $\$ 0.6$ & $\$ 1.1$ & $\$ 4.2$ \\
\hline 2016 & $\$ 1.2$ & $\$ 0.1$ & $\$ 0.5$ & $\$ 1.1$ & $\$ 2.9$ \\
\hline 2017 & $\$ 0.5$ & $-\$ 0.5$ & $\$ 0.4$ & $\$ 0.9$ & $\$ 1.3$ \\
\hline 2018 & - & $-\$ 0.8$ & $\$ 0.2$ & $\$ 0.6$ & 0.0 \\
\hline 2019 & - & $-\$ 0.8$ & - & $\$ 0.3$ & $-\$ 0.5$ \\
\hline TOTAL & $\$ 10.1$ & $\$ 5.0$ & $\$ 4.3$ & $\$ 8.0$ & $\$ 27.4$ \\
\hline
\end{tabular}

11. Explanation of Benefits and Savings Calculations

In our analysis, we assume that benefits to the program would accrue in the form of savings to Medicare, through the Medicare EP payment adjustments. Expected qualitative benefits, such as improved quality of care, better health outcomes, and the like, are still unable to be quantified at this time.

\section{H. Accounting Statement}

Whenever a rule is considered a significant rule under Executive Order 12866, we are required to develop an Accounting Statement indicating the 
classification of the expenditures associated with the provisions of this final rule. Monetary annualized benefits and nonbudgetary costs are presented as discounted flows using 3 percent and 7 percent factors. Additional expenditures that will be undertaken by eligible entities in order to obtain the Medicare and Medicaid incentive payments to adopt and demonstrate meaningful use of certified EHR technology, and to avoid the Medicare payment adjustments that will ensue if they fail to do so are noted by a placeholder in the accounting statement. We are not able to explicitly define the universe of those additional costs, nor specify what the high or low range might be to implement EHR technology in this final rule.
Expected qualitative benefits include improved quality of care, better health outcomes, reduced errors and the like. Private industry costs would include the impact of EHR activities such as temporary reduced staff productivity related to learning how to use the EHR, the need for additional staff to work with HIT issues, and administrative costs related to reporting.

\section{TABLE 40: Accounting Statement: Classification of Estimated Expenditures CYs 2010 through 2019}

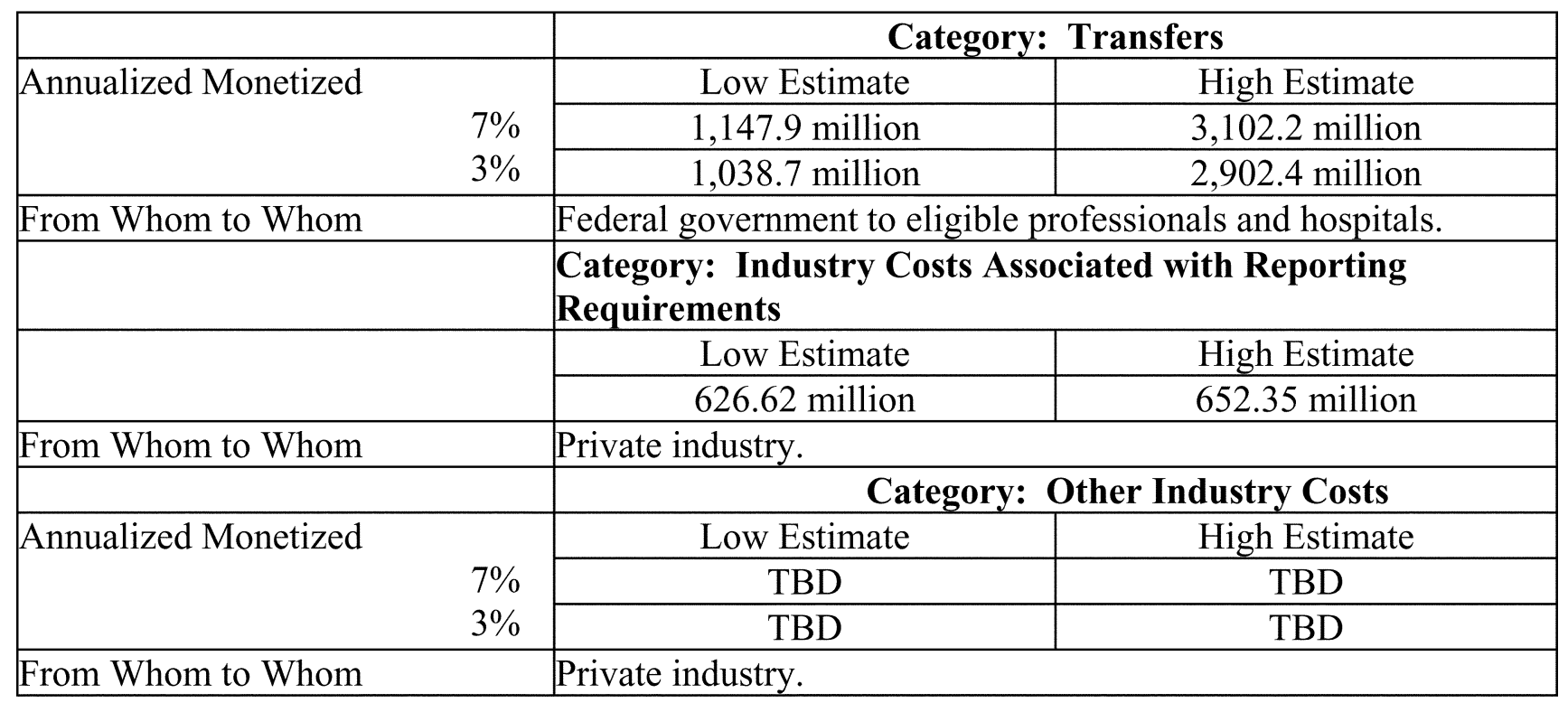

In accordance with the provisions of Executive Order 12866, this final rule was reviewed by the Office of Management and Budget.

\section{List of Subjects}

\section{CFR Part 412}

Administrative practice and procedure, Health facilities, Medicare, Puerto Rico, Reporting and recordkeeping requirements.

\section{CFR Part 413}

Health facilities, Kidney diseases, Medicare, Reporting and recordkeeping requirements.

\section{CFR Part 422}

Administrative practice and procedure, Health facilities, Health maintenance organizations (HMO), Medicare, Penalties, Privacy, Reporting and recordkeeping requirements.

\section{CFR Part 495}

Administrative practice and procedure, Electronic health records, Health facilities, Health professions, Health maintenance organizations
(HMO), Medicaid, Medicare, Penalties, Privacy, Reporting and recordkeeping requirements.

- For the reasons set forth in the preamble, the Centers for Medicare \& Medicare Services amends 42 CFR Chapter IV as follows:

PART 412-PROSPECTIVE PAYMENT SYSTEMS FOR INPATIENT HOSPITAL SERVICES

- 1. The authority citation for part 412 continues to read as follows:

Authority: Secs. 1102 and 1871 of the Social Security Act (42 U.S.C. 1302 and 1395hh).

\section{Subpart D-Basic Methodology for Determining Prospective Payment Federal Rates for Inpatient Operating Costs}

2. Section 412.64 is amended as follows:

- A. Revising paragraph (d)(2)(i)(B). - B. Adding new paragraphs (d)(2)(i)(C) and $(\mathrm{d})(3)$.

The revision and additions read as follows:
\$412.64 Federal rates for inpatient operating costs for Federal fiscal year 2005 and subsequent fiscal years.

(d) $* * *$

(2) ***

(i) ***

(B) For fiscal year 2007 through 2014, by 2 percentage points.

(C) For fiscal year 2015 and subsequent fiscal years, by one-fourth.

(3) Beginning in fiscal year 2015, in the case of a "subsection (d) hospital," as defined under section 1886(d)(1)(B) of the Act, that is not a meaningful electronic health record (EHR) user as defined in part 495 of this chapter, three-fourths of the applicable percentage change specified in paragraph (d)(1) of this section is reduced-

(i) For fiscal year 2015 , by $33^{1 / 3}$ percent;

(ii) For fiscal year 2016, by $66^{2} / 3$ percent; and

(iii) For fiscal year 2017 and subsequent fiscal years, by 100 percent. 


\section{PART 413-PRINCIPLES OF REASONABLE COST REIMBURSEMENT; PAYMENT FOR END-STAGE RENAL DISEASE SERVICES; OPTIONAL PROSPECTIVELY DETERMINED PAYMENT RATES FOR SKILLED NURSING FACILITIES}

3. The authority citation for part 413 continues to read as follows:

Authority: Secs. 1102, 1812(d), 1814(b), 1815, 1833(a), (i), and (n), 1861(v), 1871, 1881,1883 , and 1886 of the Social Security Act (42 U.S.C. 1302, 1395d(d), 1395f(b), 1395g, 1395l(a), (i), and (n), 1395x(v), 1395hh, 1395rr, 1395tt, and 1395ww); and sec. 124 of Public Law 106-133 (113 Stat. 1501A-332).

\section{Subpart E-Payments to Providers}

- 4. Section 413.70 is amended as follows:

- A. Revising paragraph (a)(1).

- B. Adding new paragraphs (a)(5),

(a)(6) and (a)(7).

The revision and additions read as follows:

\section{\$413.70 Payment for services of a CAH}

(a) Payment for inpatient services furnished by a CAH (other than services of distinct part units). (1) Effective for cost reporting periods beginning on or after January 1, 2004, payment for inpatient services of a CAH, other than services of a distinct part unit of the $\mathrm{CAH}$ and other than the items included in the incentive payment described in paragraph (a)(5) of this section and subject to the adjustments described in paragraph (a)(6) of this section, is 101 percent of the reasonable costs of the $\mathrm{CAH}$ in providing CAH services to its inpatients, as determined in accordance with section 1861(v)(1)(A) of the Act and the applicable principles of cost reimbursement in this part and in part 415 of this chapter, except that the following payment principles are excluded when determining payment for $\mathrm{CAH}$ inpatient services:

(i) Lesser of cost or charges;

(ii) Ceilings on hospital operating costs;

(iii) Reasonable compensation equivalent (RCE) limits for physician services to providers; and

(iv) The payment window provisions for preadmission services, specified in $\S 412.2(\mathrm{c})(5)$ of this subchapter and $\S 413.40(\mathrm{c})(2)$ of this part.

(5) A qualifying CAH receives an incentive payment for the reasonable costs of purchasing certified EHR technology in a cost reporting period during a payment year as determined under $§ 495.106$ of this chapter in lieu of payment for such reasonable costs under paragraph (a)(1) of this section.

(6)(i) For cost reporting periods beginning in or after FY 2015, if a CAH is not a qualifying $\mathrm{CAH}$, as defined in $\S 495.106$ (a) of this chapter, then notwithstanding the percentage applicable in paragraph (a)(1) of this section, the reasonable costs of the $\mathrm{CAH}$ in providing $\mathrm{CAH}$ services to its inpatients are adjusted, by the following applicable percentage:

(A) For cost reporting periods beginning in FY 2015, 100.66 percent.

(B) For cost reporting periods beginning in FY 2016, 100.33 percent.

(C) For cost reporting periods beginning in FY 2017 and each subsequent fiscal year, 100 percent.

(ii) A CAH may, on a case-by case basis, be exempt from the application of the adjustments made under this paragraph, if CMS or its Medicare contractors determine, on an annual basis, that requiring the $\mathrm{CAH}$ to become a qualifying CAH under $\S 495.106$ of this chapter would result in a significant hardship, such as in the case of a $\mathrm{CAH}$ in a rural area without sufficient Internet access.

(iii) In no case may a CAH be granted an exemption under this paragraph (a)(6) for more than 5 years.

(7) There is no administrative or judicial review under section s1869 and 1878 of the Actor otherwise of the following:

(i) The methodology and standards for determining the amount of payment under paragraph (a)(5) of this section, including the calculation of reasonable costs under $\S 495.106$ (c) of this chapter.

(ii) The methodology and standards for determining the amount of payment adjustments made under paragraph (a)(6).

(iii) The methodology and standards for determining a CAH to be a qualifying CAH under $\S 495.106$ of this chapter.

(iv) The methodology and standards for determining if the hardship exemption applies to a CAH under paragraph (a)(6)(ii) of this section.

(v) The specification of the cost reporting periods, payment years, or fiscal years as applied under this paragraph.

\section{PART 422-MEDICARE ADVANTAGE PROGRAM}

5. The authority citation for part 422 continues to read as follows:

Authority: Secs. 1102 and 1871 of the Social Security Act (42 U.S.C. 1302 and 1395hh).
Subpart G-Payments to Medicare Advantage Organizations

- 6. Section 422.304 is amended by adding a new paragraph (f) to read as follows:

\section{$\$ 422.304$ Monthly payments. \\ * * * * *}

(f) Separate payment for meaningful use of certified EHRs. In the case of qualifying MA organizations, as defined in $\S 495.200$ of this chapter, entitled to MA EHR incentive payments per $\S 495.220$ of this chapter, such payments are made in accordance with sections 1853(l) and (m) of the Act and subpart $\mathrm{C}$ of Part 495 of this chapter.

- 7. Section 422.306 is amended as follows:

- A. Removing "and" from the end of paragraph (b)(2)(ii).

- B. Removing the period at the end of paragraph (b)(2)(iii) and adding "; and" in its place.

- C. Adding a new paragraph (b)(2)(iv).

The addition reads as follows:

\section{$\$ 422.306$ Annual MA capitation rates.}

(b) ***

(2) ***

(iv) Adjusted to exclude costs attributable to payments under sections 1848(o) and 1886(n) of the Act of Medicare FFS incentive payments for meaningful use of electronic health records.

- 8. Section 422.308 is amended as

follows:

- A. Redesignating paragraph (a) as paragraph (a)(1).

- B. Adding a new paragraph (a)(2).

The addition reads as follows:

\$422.308 Adjustments to capitation rates, benchmarks, bids, and payments.

(a) ***

(2) The amount calculated in paragraph (a)(1) of this section must exclude expenditures attributable to sections 1848(a)(7) and (o) and sections 1886(b)(3)(B)(ix) and (n) of the Act.

- 9. Section 422.322 is amended as

follows:

- A. Adding paragraph (a)(3).

- B. Revising paragraph (b).

The addition and revision read as follows:

\$422.322 Source of payment and effect of MA plan election on payment.

(a) * * *

(3) Payments under subpart C of part 495 of this chapter for meaningful use of certified EHR technology are made 
from the Federal Hospital Insurance Trust Fund or the Supplementary Medical Insurance Trust Fund. In applying section $1848(0)$ of the Act under sections 1853(l) and 1886(n)(2)of the Act under section $1853(\mathrm{~m})$ of the Act, CMS determines the amount to the extent feasible and practical to be similar to the estimated amount in the aggregate that would be payable for services furnished by professionals and hospitals under Parts B and A, respectively, under title XVIII of the Act.

(b) Payments to the MA organization. Subject to $\S 412.105(\mathrm{~g}), \S 413.86(\mathrm{~d})$, and $\S 495.204$ of this chapter and $\S \S 422.109$, 422.316, and 422.320, CMS' payments under a contract with an MA organization (described in $\S 422.304$ ) with respect to an individual electing an MA plan offered by the organization are instead of the amounts which (in the absence of the contract) would otherwise be payable under original Medicare for items and services furnished to the individual.

\section{SUBCHAPTER G-STANDARDS AND CERTIFICATIONS}

10. A new part 495 is added to read as follows:

\section{PART 495-STANDARDS FOR THE ELECTRONIC HEALTH RECORD TECHNOLOGY INCENTIVE PROGRAM}

\section{Subpart A-General Provisions}

Sec.

495.2 Basis and purpose.

495.4 Definitions.

495.6 Meaningful use objectives measures for EPs, eligible hospitals, and CAHs.

495.8 Demonstration of meaningful use criteria.

495.10 Participation requirements for EPs, eligible hospitals, and CAHs.

Subpart B-Requirements Specific to the Medicare Program

495.100 Definitions.

495.102 Incentive payments to EPs

495.104 Incentive payments to eligible hospitals.

495.106 Incentive payments to CAHs. 495.108 Posting of required information.

495.110 Preclusion on administrative and judicial review.

Subpart C-Requirements Specific to Medicare Advantage (MA) Organizations

495.200 Definitions.

495.202 Identification of qualifying MA organizations, MA-EPs, and MAaffiliated eligible hospitals.

495.204 Incentive payments to qualifying MA organizations for MA-EPs and MAaffiliated eligible hospitals.

495.206 Timeframe for payment to qualifying MA organizations.

495.208 Avoiding duplicate payment.

495.210 Meaningful EHR user attestation.
495.212 Limitation on review.

\section{Subpart D-Requirements Specific to the Medicaid Program}

495.300 Basis and purpose.

495.302 Definitions.

495.304 Medicaid provider scope and eligibility.

495.306 Establishing patient volume.

495.308 Net average allowable costs as the basis for determining the incentive payment.

495.310 Medicaid provider incentive payments.

495.312 Process for payments.

495.314 Activities required to receive an incentive payment.

495.316 State monitoring and reporting regarding activities required to receive an incentive payment.

495.318 State responsibilities for receiving FFP.

495.320 FFP for payments to Medicaid providers.

495.322 FFP for reasonable administrative expenses.

495.324 Prior approval conditions.

495.326 Disallowance of FFP.

495.328 Request for reconsideration of adverse determination.

495.330 Termination of FFP for failure to provide access to information.

495.332 State Medicaid health information technology (HIT) plan requirements.

495.334 Reserved.

495.336 Health information technology planning advance planning document requirements (HIT PAPD).

495.338 Health information technology implementation advance planning document requirements (HIT IAPD)

495.340 As-needed HIT PAPD update and as-needed HIT IAPD update requirements.

495.342 Annual HIT IAPD requirements.

495.344 Approval of the State Medicaid HIT plan, the HIT PAPD and update, the HIT IAPD and update, and the annual HIT IAPD.

495.346 Access to systems and records.

495.348 Procurement standards.

495.350 State Medicaid agency attestations.

495.352 Reporting requirements.

495.354 Rules for charging equipment.

495.356 Nondiscrimination requirements.

495.358 Cost allocation plans.

495.360 Software and ownership rights.

495.362 Retroactive approval of FFP with an effective date of February 18, 2009.

495.364 Review and assessment of administrative activities and expenses of Medicaid provider health information technology adoption and operation.

495.366 Financial oversight and monitoring of expenditures.

495.368 Combating fraud and abuse.

495.370 Appeals process for a Medicaid provider receiving electronic health record incentive payments.

Authority: Secs. 1102 and 1871 of the Social Security Act (42 U.S.C. 1302 and 1395hh).

\section{Subpart A-General Provisions}

\$495.2 Basis and purpose.

This part implements the following:

(a) Section 1848(o) of the Act by

establishing payment incentives under Medicare Part B for eligible professionals who adopt and meaningfully use certified electronic health record (EHR) technology.

(b) Section 1853(1) of the Act to provide incentive payments to Medicare Advantage organizations for certain affiliated professionals who meaningfully use certified EHR technology and meet certain other requirements.

(c) Section 1886(n) of the Act by establishing incentives payments for the meaningful use of certified EHR technology by subsection (d) hospitals, as defined under section 1886(d)(1)(B) of the Act, participating in the Medicare FFS program.

(d) Section 1814(l) of the Act to provide an incentive payment to critical access hospitals that meaningfully use certified EHR technology based on the hospitals' reasonable costs.

(e) Section 1853(m) of the Act to provide incentive payments to MA organizations for certain affiliated hospitals that meaningfully use certified EHR technology.

(f) Sections 1903(a)(3)(F) and 1903(t) of the Act to provide 100 percent Federal financial participation (FFP) to States for incentive payments to certain eligible providers participating in the Medicaid program to purchase, implement, and operate (including support services and training for staff) certified EHR technology and 90 percent FFP for State administrative expenses related to such incentive payments.

(g) Sections 1848(a)(7), 1853(l)(4), 1886(b)(3)(B)(ix)(I), and 1853(m)(4) of the Act, providing for payment reductions for inpatient services furnished on or after October 1, 2014 to Medicare beneficiaries by hospitals that are not meaningful users of certified EHR technology, and for covered professional services furnished on or after January 1, 2015 to Medicare beneficiaries by certain professionals who are not meaningful users of certified EHR technology.

\section{\$495.4 Definitions.}

In this part, unless otherwise indicated-

Certified electronic health record technology has the same definition as this term is defined at 45 CFR 170.102.

Critical access hospital (CAH) means a facility that has been certified as a critical access hospital under section 1820(e) of the Act and for which 
Medicare payment is made under section 1814(l) of the Act for inpatient services and under section 1834(g) of the Act for outpatient services.

EHR reporting period means either of the following:

(1) For an eligible professional (EP)-

(i) For the first payment year, any continuous 90-day period within a calendar year;

(ii)(A) Except as specified in paragraph (1)(ii)(B) of this definition, for the second, third, fourth, fifth, or sixth payment year, the calendar year.

(B) For Medicaid providers who are demonstrating they are meaningful EHR users for the first time in their second payment year, the EHR reporting period during such second payment year is any continuous 90-day period within the calendar year.

(2) For an eligible hospital or a $\mathrm{CAH}-$

(i) For the first payment year, any continuous 90-day period within a federal fiscal year; and

(ii)(A) Except as specified in paragraph (2)(ii)(B) of this definition, for the second, third, fourth, fifth, or sixth payment year, the Federal fiscal year.

(B) For Medicaid providers who are demonstrating they are meaningful EHR users for the first time in their second payment year, the EHR reporting period during such second payment year is any continuous 90-day period within the Federal fiscal year.

Eligible hospital means an eligible hospital as defined under $\S 495.100$ or Medicaid eligible hospital under subpart D of this part.

Eligible professional (EP) means an eligible professional as defined under $\S 495.100$ or a Medicaid eligible professional under subpart D of this part.

Hospital-based EP is an EP (as defined under this section) who furnishes 90 percent or more of his or her covered professional services in a hospital setting in the year preceding the payment year. For Medicare, this will be calculated based on the Federal FY prior to the payment year. For Medicaid, it is at the State's discretion if the data is gathered on the Federal FY or CY prior to the payment year. A setting is considered a hospital setting if it is a site of service that would be identified by the codes used in the HIPAA standard transactions as an inpatient hospital, or emergency room setting.

Meaningful EHR user means:

(1) Subject to paragraph (3) of this definition, an EP, eligible hospital or $\mathrm{CAH}$ that, for an EHR reporting period for a payment year, demonstrates in accordance with $\S 495.8$ meaningful use of certified EHR technology by meeting the applicable objectives and associated measures under § 495.6; and

(2)(i) Except as specified in paragraph (2)(ii) of this definition, a Medicaid EP or Medicaid eligible hospital, that meets the requirements of paragraph (1) of this definition and any additional criteria for meaningful use imposed by the State and approved by CMS under $\S 495.316$ and $\S 495.332$.

(ii) An eligible hospital or $\mathrm{CAH}$ is deemed to be a meaningful EHR user for purposes of receiving an incentive payment under subpart D of this Part, if the hospital participates in both the Medicare and Medicaid EHR incentive programs, and the hospital meets the requirements of paragraph (1) of this definition.

(3) To be considered a meaningful EHR user, at least 50 percent of an EP's patient encounters during the EHR reporting period during the payment year must occur at a practice/location or practices/locations equipped with certified EHR technology.

Payment year means:

(1) For an EP, a calendar year beginning with CY 2011; and

(2) For a CAH or an eligible hospital, a Federal fiscal year beginning with FY 2011.

Qualified EHR has the same definition as this term is defined at 45 CFR 170.102 .

First, second, third, fourth, fifth, or sixth payment years mean as follows:

(1) The first payment year is: with respect to an EP, the first calendar year for which the EP receives an incentive payment under this part; and with respect to an eligible hospital or $\mathrm{CAH}$, the first FY for which the hospital receives an incentive payment under this part.

(2) The second, third, fourth, fifth, or sixth payment year is:

(i) With respect to a Medicare EP, the second, third, fourth or fifth successive CY immediately following the first payment year; and with respect to a Medicare eligible hospital or CAH, the second, third, or fourth successive Federal FY immediately following the first payment year. (Note: Medicare EPs are not eligible for a sixth payment year and Medicare eligible hospitals are not eligible for a fifth or sixth payment year.)

(ii)(A) With respect to a Medicaid EP, the second, third, fourth, fifth, or sixth $\mathrm{CY}$ for which the EP receives an incentive payment under subpart D, regardless of whether the year immediately follows the prior payment year; and

(B) With respect to a Medicaid eligible hospital, for years prior to FY 2017, the second, third, fourth, fifth, or sixth
Federal FY for which the hospital receives an incentive payment under subpart D of this part, regardless of whether the year immediately follows the prior payment year. Beginning with FY 2017, payments to Medicaid eligible hospitals must be consecutive, and the hospital is not eligible for an incentive payment under subpart D of this part unless it received such incentive payment for the prior fiscal year.

\$495.6 Meaningful use objectives and measures for EPs, eligible hospitals, and CAHs.

(a) Stage 1 criteria for EPs-(1) General rule regarding Stage 1 criteria for meaningful use for EPs. Except as specified in paragraphs (a)(2) and (a)(3) of this section, EPs must meet all objectives and associated measures of the Stage 1 criteria specified in paragraph (d) of this section and five objectives of the EP's choice from paragraph (e) of this section to meet the definition of a meaningful EHR user.

(2) Exclusion for non-applicable objectives. (i) An EP may exclude a particular objective contained in paragraphs (d) or (e) of this section, if the EP meets all of the following requirements:

(A) Must ensure that the objective in paragraph (d) or (e) of this section includes an option for the EP to attest that the objective is not applicable.

(B) Meets the criteria in the applicable objective that would permit the attestation.

(C) Attests.

(ii) An exclusion will reduce (by the number of exclusions applicable) the number of objectives that would otherwise apply. For example, an EP that has an exclusion from one of the objectives in paragraph (e) of this section must meet four (and not five) objectives of the EP's choice from such paragraph to meet the definition of a meaningful EHR user.

(3) Exception for Medicaid EPs who adopt, implement or upgrade in their first payment year. For Medicaid EPs who adopt, implement, or upgrade certified EHR technology in their first payment year, the meaningful use objectives and associated measures of the Stage 1 criteria specified in paragraphs (d) and (e) apply beginning with the second payment year, and do not apply to the first payment year.

(b) Stage 1 criteria for eligible hospitals and CAHs-(1) General rule regarding Stage 1 criteria for meaningful use for eligible hospitals or CAHs. Except as specified in paragraphs (b)(2) and (b)(3) of this section, eligible hospitals and CAHs must meet all objectives and associated measures of 
the Stage 1 criteria specified in paragraph (f) of this section and five objectives of the eligible hospital's or CAH's choice from paragraph $(\mathrm{g})$ of this section to meet the definition of a meaningful EHR user.

(2) Exclusions for nonapplicable objectives. (i) An eligible hospital or CAH may exclude a particular objective that includes an option for exclusion contained in paragraphs (f) or (g) of this section, if the hospital meets all of the following requirements:

(A) The hospital meets the criteria in the applicable objective that would permit an exclusion.

(B) The hospital so attests.

(ii) An exclusion will reduce (by the number of exclusions received) the number of objectives that would otherwise apply. For example, an eligible hospital that is excluded from one of the objectives in paragraph (g) of this section must meet four (and not five) objectives of the hospital's choice from such paragraph to meet the definition of a meaningful EHR user.

(3) Exception for Medicaid eligible hospitals that adopt, implement or upgrade in their first payment year. For Medicaid eligible hospitals that adopt, implement, or upgrade certified EHR technology in their first payment year, the meaningful use objectives and associated measures of the Stage 1 criteria specified in paragraphs (f) and (g) of this section apply beginning with the second payment year, and do not apply to the first payment year.

(c) Many of the objective and associated measures in paragraphs (d) through $(\mathrm{g})$ of this section rely on measures that count unique patients or actions.

(1) If a measure (or associated objective) in paragraphs (d) through (g) of this section references paragraph (c) of this section, then the measure may be calculated by reviewing only the actions for patients whose records are maintained using certified EHR technology. A patient's record is maintained using certified EHR technology if sufficient data was entered in the certified EHR technology to allow the record to be saved, and not rejected due to incomplete data.

(2) If the objective and associated measure does not reference this paragraph (c) of this section, then the measure must be calculated by reviewing all patient records, not just those maintained using certified EHR technology.

(d) Stage 1 core criteria for EPs. An EP must satisfy the following objectives and associated measures, except those objectives and associated measures for which an EP qualifies for an exclusion under paragraph (a)(2) of this section specified in this paragraph:

(1)(i) Objective. Use computerized provider order entry (CPOE) for medication orders directly entered by any licensed healthcare professional who can enter orders into the medical record per state, local and professional guidelines.

(ii) Measure. Subject to paragraph (c) of this section, more than 30 percent of all unique patients with at least one medication in their medication list seen by the EP have at least one medication order entered using CPOE.

(iii) Exclusion in accordance with paragraph (a)(2) of this section Any EP who writes fewer than 100 prescriptions during the EHR reporting period.

(2)(i) Objective. Implement drug-drug and drug-allergy interaction checks.

(ii) Measure. The EP has enabled this functionality for the entire EHR reporting period.

(3)(i) Objective. Maintain an up-todate problem list of current and active diagnoses.

(ii) Measure. More than 80 percent of all unique patients seen by the EP have at least one entry or an indication that no problems are known for the patient recorded as structured data.

(4)(i) Objective. Generate and transmit permissible prescriptions electronically (eRx).

(ii) Measure. Subject to paragraph (c) of this section, more than 40 percent of all permissible prescriptions written by the EP are transmitted electronically using certified EHR technology.

(iii) Exclusion in accordance with paragraph (a)(2) of this section Any EP who writes fewer than 100 prescriptions during the EHR reporting period.

(5)(i) Objective. Maintain active medication list.

(ii) Measure. More than 80 percent of all unique patients seen by the EP have at least one entry (or an indication that the patient is not currently prescribed any medication) recorded as structured data.

(6)(i) Objective. Maintain active medication allergy list.

(ii) Measure. More than 80 percent of all unique patients seen by the EP have at least one entry (or an indication that the patient has no known medication allergies) recorded as structured data.

(7)(i) Objective. Record all of the following demographics:

(A) Preferred language.

(B) Gender.

(C) Race.

(D) Ethnicity.

(E) Date of birth.

(ii) Measure. More than 50 percent of all unique patients seen by the EP have demographics recorded as structured data. (8)(i) Objective. Record and chart changes in the following vital signs: (A) Height.

(B) Weight.

(C) Blood pressure.

(D) Calculate and display body mass index (BMI).

(E) Plot and display growth charts for children 2-20 years, including BMI.

(ii) Measure. Subject to paragraph (c) of this section, more than 50 percent of all unique patients age 2 and over seen by the EP, height, weight and blood pressure are recorded as structured data.

(iii) Exclusion in accordance with paragraph (a)(2) of this section. Any EP who either see no patients 2 years or older, or who believes that all three vital signs of height, weight, and blood pressure of their patients have no relevance to their scope of practice.

(9)(i) Objective. Record smoking status for patients 13 years old or older.

(ii) Measure. Subject to paragraph (c) of this section, more than 50 percent of all unique patients 13 years old or older seen by the EP have smoking status recorded as structured data.

(iii) Exclusion in accordance with paragraph (a)(2) of this section. Any EP who sees no patients 13 years or older.

(10)(i) Objective. Report ambulatory clinical quality measures to CMS or, in the case of Medicaid EPs, the States.

(ii) Measure. Subject to paragraph (c) of this section, successfully report to CMS (or, in the case of Medicaid EPs, the States) ambulatory clinical quality measures selected by CMS in the manner specified by CMS (or in the case of Medicaid EPs, the States).

(11)(i) Objective. Implement one clinical decision support rules relevant to specialty or high clinical priority along with the ability to track compliance with that rule.

(ii) Measure. Implement one clinical decision support rule.

(12)(i) Objective. Provide patients with an electronic copy of their health information (including diagnostics test results, problem list, medication lists, medication allergies) upon request.

(ii) Measure. Subject to paragraph (c) of this section, more than 50 percent of all patients who request an electronic copy of their health information are provided it within 3 business days.

(iii) Exclusion in accordance with paragraph (a)(2) of this section. Any EP that has no requests from patients or their agents for an electronic copy of patient health information during the EHR reporting period.

(13)(i) Objective. Provide clinical summaries for patients for each office visit.

(ii) Measure. Subject to paragraph (c) of this section, clinical summaries 
provided to patients for more than 50 percent of all office visits within 3 business days.

(iii) Exclusion in accordance with paragraph (a)(2) of this section. Any EP who has no office visits during the EHR reporting period.

(14)(i) Objective. Capability to exchange key clinical information (for example, problem list, medication list, allergies, and diagnostic test results), among providers of care and patient authorized entities electronically.

(ii) Measure. Performed at least one test of certified EHR technology's capacity to electronically exchange key clinical information.

(15)(i) Objective. Protect electronic health information created or maintained by the certified EHR technology through the implementation of appropriate technical capabilities.

(ii) Measure. Conduct or review a security risk analysis in accordance with the requirements under 45 CFR 164.308(a)(1) and implement security updates as necessary and correct identified security deficiencies as part of its risk management process.

(e) Stage 1 menu set criteria for EPs. An EP must meet five of the following objectives and associated measures, one of which must be either paragraph (e)(9) or (e)(10) of this section, except that the required number of objectives and associated measures is reduced by an EP's paragraph (a)(2) of this section exclusions specified in this paragraph:

(1)(i) Objective. Implement drugformulary checks.

(ii) Measure. The EP has enabled this functionality and has access to at least one internal or external formulary for the entire EHR reporting period.

(2)(i) Objective. Incorporate clinical lab-test results into EHR as structured data.

(ii) Measure. Subject to paragraph (c) of this section, more than 40 percent of all clinical lab tests results ordered by the EP during the EHR reporting period whose results are either in a positive/ negative or numerical format are incorporated in certified EHR technology as structured data.

(iii) Exclusion in accordance with paragraph (a)(2) of this section. An EP who orders no lab tests whose results are either in a positive/negative or numeric format during the EHR reporting period.

(3)(i) Objective. Generate lists of patients by specific conditions to use for quality improvement, reduction of disparities, research, or outreach.

(ii) Measure. Subject to paragraph (c) of this section, generate at least one report listing patients of the EP with a specific condition. (4)(i) Objective. Send reminders to patients per patient preference for preventive/follow-up care.

(ii) Measure. Subject to paragraph (c) of this section, more than 20 percent of all patients 65 years or older or 5 years old or younger were sent an appropriate reminder during the EHR reporting period.

(iii) Exclusion in accordance with paragraph (a)(2) of this section. An EP who has no patients 65 years old or older or 5 years old or younger with records maintained using certified EHR technology.

(5)(i) Objective. Provide patients with timely electronic access to their health information (including lab results, problem list, medication lists, and allergies) within 4 business days of the information being available to the EP.

(ii) Measure. At least 10 percent of all unique patients seen by the EP are provided timely (available to the patient within four business days of being updated in the certified EHR technology) electronic access to their health information subject to the EP's discretion to withhold certain information.

(iii) Exclusion in accordance with paragraph (a)(2) of this section. Any EP that neither orders nor creates any of the information listed at 45 CFR 170.304(g) during the EHR reporting period.

(6)(i) Objective. Use certified EHR technology to identify patient-specific education resources and provide those resources to the patient if appropriate.

(ii) Measure. More than 10 percent of all unique patients seen by the EP are provided patient-specific education resources.

(7)(i) Objective. The EP who receives a patient from another setting of care or provider of care or believes an encounter is relevant should perform medication reconciliation.

(ii) Measure. Subject to paragraph (c) of this section, the EP performs medication reconciliation for more than 50 percent of transitions of care in which the patient is transitioned into the care of the EP.

(iii) Exclusion in accordance with paragraph (a)(2) of this section. An EP who was not the recipient of any transitions of care during the EHR reporting period.

(8)(i) Objective. The EP who transitions their patient to another setting of care or provider of care or refers their patient to another provider of care should provide summary care record for each transition of care or referral.

(ii) Measure. Subject to paragraph (c) of this section, the EP who transitions or refers their patient to another setting of care or provider of care provides a summary of care record for more than 50 percent of transitions of care and referrals.

(iii) Exclusion in accordance with paragraph (a)(2) of this section. An EP who neither transfers a patient to another setting nor refers a patient to another provider during the EHR reporting period.

(9)(i) Objective. Capability to submit electronic data to immunization registries or immunization information systems and actual submission according to applicable law and practice.

(ii) Measure. Performed at least one test of certified EHR technology's capacity to submit electronic data to immunization registries and follow up submission if the test is successful (unless none of the immunization registries to which the EP submits such information has the capacity to receive the information electronically).

(iii) Exclusion in accordance with paragraph $(a)(2)$ of this section. An EP who administers no immunizations during the EHR reporting period or where no immunization registry has the capacity to receive the information electronically.

(10)(i) Objective. Capability to submit electronic syndromic surveillance data to public health agencies and actual submission according to applicable law and practice.

(ii) Measure. Performed at least one test of certified EHR technology's capacity to provide electronic syndromic surveillance data to public health agencies and follow-up submission if the test is successful (unless none of the public health agencies to which an EP submits such information has the capacity to receive the information electronically).

(iii) Exclusion in accordance with paragraph (a)(2) of this section. An EP who does not collect any reportable syndromic information on their patients during the EHR reporting period or does not submit such information to any public health agency that has the capacity to receive the information electronically.

(f) Stage 1 core criteria for eligible hospitals or CAHs. An eligible hospital or CAH must meet the following objectives and associated measures except those objectives and associated measures for which an eligible hospital or CAH qualifies for a paragraph (b)(2) of this section exclusion specified in this paragraph:

(1)(i) Objective. Use CPOE for medication orders directly entered by any licensed healthcare professional who can enter orders into the medical 
record per State, local, and professional guidelines.

(ii) Measure. Subject to paragraph (c) of this section, more than 30 percent of all unique patients with at least one medication in their medication list admitted to the eligible hospital's or CAH's inpatient or emergency department (POS 21 or 23) have at least one medication order entered using CPOE.

(2)(i) Objective. Implement drug-drug and drug-allergy interaction checks.

(ii) Measure. The eligible hospital or $\mathrm{CAH}$ has enabled this functionality for the entire EHR reporting period.

(3)(i) Objective. Maintain an up-todate problem list of current and active diagnoses.

(ii) Measure. More than 80 percent of all unique patients admitted to the eligible hospital's or CAH's inpatient or emergency department (POS 21 or 23) have at least one entry or an indication that no problems are known for the patient recorded as structured data.

(4)(i) Objective. Maintain active medication list.

(ii) Measure. More than 80 percent of all unique patients admitted to the eligible hospital's or CAH's inpatient or emergency department (POS 21 or 23) have at least one entry (or an indication that the patient is not currently prescribed any medication) recorded as structured data.

(5)(i) Objective. Maintain active medication allergy list.

(ii) Measure. More than 80 percent of all unique patients admitted to the eligible hospital's or CAH's inpatient or emergency department (POS 21 or 23) have at least one entry (or an indication that the patient has no known medication allergies) recorded as structured data.

(6)(i) Objective. Record all of the

following demographics;

(A) Preferred language.

(B) Gender.

(C) Race.

(D) Ethnicity.

(E) Date of birth.

(F) Date and preliminary cause of death in the event of mortality in the eligible hospital or CAH.

(ii) Measure. More than 50 percent of all unique patients admitted to the eligible hospital's or CAH's inpatient or emergency department (POS 21 or 23) have demographics recorded as structured data.

(7)(i) Objective. Record and chart changes in the following vital signs:

(A) Height.

(B) Weight.

(C) Blood pressure.

(D) Calculate and display body mass index (BMI).
(E) Plot and display growth charts for children 2-20 years, including BMI.

(ii) Measure. Subject to paragraph (c) of this section, for more than 50 percent of all unique patients age 2 and over admitted to the eligible hospital's or CAH's inpatient or emergency department (POS 21 or 23), height, weight, and blood pressure are recorded as structured data.

(8)(i) Objective. Record smoking for patients 13 years old or older.

(ii) Measure. Subject to paragraph (c) of this section, more than 50 percent of all unique patients 13 years old or older or admitted to the eligible hospital's inpatient or emergency department (POS 21 or 23) have smoking status recorded as structured data.

(iii) Exclusion in accordance with paragraph (b)(2) of this section. Any eligible hospital or CAH that admits no patients 13 years or older to their inpatient or emergency department (POS 21 or 23).

(9)(i) Objective. Report hospital clinical quality measures to CMS or, in the case of Medicaid eligible hospitals, the States.

(ii) Measure. Subject to paragraph (c) of this section, successfully report to CMS (or, in the case of Medicaid eligible hospitals or CAHs, the States) hospital clinical quality measures selected by CMS in the manner specified by CMS (or, in the case of Medicaid eligible hospitals or CAHs, the States).

(10)(i) Objective. Implement one clinical decision support rule related to a high priority hospital condition along with the ability to track compliance with that rule.

(ii) Measure. Implement one clinical decision support rule.

(11)(i) Objective. Provide patients with an electronic copy of their health information (including diagnostic test results, problem list, medication lists, medication allergies, discharge

summary, procedures), upon request.

(ii) Measure. Subject to paragraph (c) of this section, more than 50 percent of all patients of the inpatient or emergency departments of the eligible hospital or CAH (POS 21 or 23) who request an electronic copy of their health information are provided it within 3 business days.

(iii) Exclusion in accordance with paragraph (b)(2) of this section. Any eligible hospital or CAH that has no requests from patients or their agents for an electronic copy of patient health information during the EHR reporting period.

(12)(i) Objective. Provide patients with an electronic copy of their discharge instructions at time of discharge, upon request. (ii) Measure. Subject to paragraph (c) of this section, more than 50 percent of all patients who are discharged from an eligible hospital or CAH's inpatient or emergency department (POS 21 or 23) and who request an electronic copy of their discharge instructions are provided it.

(iii) Exclusion in accordance with paragraph (b)(2) of this section. Any eligible hospital or CAH that has no requests from patients or their agents for an electronic copy of the discharge instructions during the EHR reporting period.

(13)(i) Objective. Capability to exchange key clinical information (for example, problem list, medication list, medication allergies, and diagnostic test results), among providers of care and patient authorized entities electronically.

(ii) Measure. Performed at least one test of certified EHR technology's capacity to electronically exchange key clinical information.

(14)(i) Objective. Protect electronic health information created or maintained by the certified EHR technology through the implementation of appropriate technical capabilities.

(ii) Measure. Conduct or review a security risk analysis in accordance with the requirements under 45 CFR 164.308(a)(1) and implement security updates as necessary and correct identified security deficiencies as part of its risk management process.

(g) Stage 1 menu set criteria for eligible hospitals or CAHs. Eligible hospitals or CAHs must meet five of the following objectives and associated measures, one which must be specified in paragraph $(\mathrm{g})(8),(\mathrm{g})(9)$, or $(\mathrm{g})(10)$ of this section, except that the required number of objectives and associated measures is reduced by a hospital's paragraph (b)(2) of this section exclusions specified in this paragraph:

(1)(i) Objective. Implement drugformulary checks.

(ii) Measure. The eligible hospital or $\mathrm{CAH}$ has enabled this functionality and has access to at least one internal or external formulary for the entire EHR reporting period.

(2)(i) Objective. Record advance directives for patient 65 years old or older.

(ii) Measure. Subject to paragraph (c) of this section, more than 50 percent of all unique patients 65 years old or older admitted to the eligible hospital's or CAH's inpatient (POS 21) have an indication of an advance directive status recorded as structured data.

(iii) Exclusion in accordance with paragraph (b)(2) of this section. An eligible hospital or $\mathrm{CAH}$ that admits no 
patients age 65 years old or older during the EHR reporting period.

(3)(i) Objective. Incorporate clinical lab-test results into EHR as structured data.

(ii) Measure. Subject to paragraph (c) of this section, more than 40 percent of all clinical lab test results ordered by an authorized provider of the eligible hospital or $\mathrm{CAH}$ for patients admitted to its inpatient or emergency department (POS 21 and 23) during the EHR reporting period whose results are either in a positive/negative or numerical format are incorporated in certified EHR technology as structured data.

(4)(i) Objective. Generate lists of patients by specific conditions to use for quality improvement, reduction of disparities, research, or outreach.

(ii) Measure. Subject to paragraph (c) of this section, generate at least one report listing patients of the eligible hospital or CAH with a specific condition.

(5)(i) Objective. Use certified EHR technology to identify patient-specific education resources and provide those resources to the patient if appropriate.

(ii) Measure. More than 10 percent of all unique patients admitted to the eligible hospital's or CAH's inpatient or emergency department (POS 21 or 23) are provided patient-specific education resources.

(6)(i) Objective. The eligible hospital or CAH who receives a patient from another setting of care or provider of care or believes an encounter is relevant should perform medication reconciliation.

(ii) Measure. Subject to paragraph (c) of this section, the eligible hospital or $\mathrm{CAH}$ performs medication reconciliation for more than 50 percent of transitions of care in which the patient is admitted to the eligible hospital's or CAH's inpatient or emergency department (POS 21 or 23).

(7)(i) Objective. The eligible hospital or CAH that transitions their patient to another setting of care or provider of care or refers their patient to another provider of care should provide summary care record for each transition of care or referral.

(ii) Measure. Subject to paragraph (c) of this section, the eligible hospital or $\mathrm{CAH}$ that transitions or refers their patient to another setting of care or provider of care provides a summary of care record for more than 50 percent of transitions of care and referrals.

(8)(i) Objective. Capability to submit electronic data to immunization registries or immunization information systems and actual submission according to applicable law and practice. (ii) Measure. Performed at least one test of certified EHR technology's capacity to submit electronic data to immunization registries and follow up submission if the test is successful (unless none of the immunization registries to which the eligible hospital or CAH submits such information has the capacity to receive the information electronically).

(iii) Exclusion in accordance with paragraph (b)(2) of this section. An eligible hospital or CAH that administers no immunizations during the EHR reporting period or where no immunization registry has the capacity to receive the information electronically.

(9)(i) Objective. Capability to submit electronic data on reportable (as required by State or local law) lab results to public health agencies and actual submission according to applicable law and practice.

(ii) Measure. Performed at least one test of certified EHR technology's capacity to provide electronic submission of reportable lab results to public health agencies and follow-up submission if the test is successful (unless none of the public health agencies to which an eligible hospital or CAH submits such information has the capacity to receive the information electronically).

(iii) Exclusion in accordance with paragraph (b)(2) of this section. No public health agency to which the eligible hospital or CAH submits such information has the capacity to receive the information electronically.

(10)(i) Objective. Capability to submit electronic syndromic surveillance data to public health agencies and actual submission according to applicable law and practice.

(ii) Measure. Performed at least one test of certified EHR technology's capacity to provide electronic syndromic surveillance data to public health agencies and follow-up submission if the test is successful (unless none of the public health agencies to which an eligible hospital or CAH submits information has the capacity to receive the information electronically).

(iii) Exclusion in accordance with paragraph (a)(2) of this section. No public health agency to which the eligible hospital or CAH submits information has the capacity to receive the information electronically.

(h) Stage 2 criteria for EPs. Beginning when final regulations for Stage 2 are effective, an EP must satisfy the following objectives and associated measures: (1)(i) Objective. Use computerized provider order entry (CPOE) for medication orders directly entered by any licensed healthcare professional who can enter orders into the medical record per state, local and professional guidelines.

(ii) Measure. More than 60 percent of all unique patients with at least one medication in their medication list seen by the EP have at least one medication order entered using CPOE.

(iii) Exclusion. Any EP who writes fewer than 100 prescriptions during the EHR reporting period.

(2) [Reserved].

(i) Stage 2 criteria for eligible hospitals or CAHs. Beginning when final regulations for Stage 2 are effective, an eligible hospital or CAH must satisfy the following objectives and associated measures:

(1)(i) Objective. Use computerized provider order entry (CPOE) for medication orders directly entered by any licensed healthcare professional who can enter orders into the medical record per state, local and professional guidelines.

(ii) Measure. More than 60 percent of all unique patients with at least one medication in their medication list admitted to the eligible hospital's or CAH's inpatient or emergency department (POS 21 or 23) have at least one medication order entered using CPOE.

(2) [Reserved].

\section{\$495.8 Demonstration of meaningful use criteria.}

(a) Demonstration by EPs. An EP must demonstrate that he or she satisfies each of the applicable objectives and associated measures under $\S 495.6$ of this subpart as follows:

(1) For CY 2011-(i) Attestation. Attest, through a secure mechanism, in a manner specified by CMS (or for a Medicaid EP, in a manner specified by the State), that during the EHR reporting period, the EP-

(A) Used certified EHR technology, and specify the technology used;

(B) Satisfied the required objectives and associated measures under $\S 495.6(\mathrm{~d})$ and $\S 495.6(\mathrm{e})$ of this subpart;

(C) Must specify the EHR reporting period and provide the result of each applicable measure for all patients seen during the EHR reporting period for which a selected measure is applicable;

(ii) Additional requirements for Medicaid EPs. For Medicaid EPs, if, in accordance with $\S 495.316$ and $\S 495.332$, CMS has approved a State's revised definition for meaningful use, in addition to meeting paragraphs (a)(1)(i) through (ii) of this section, the EP must 
also demonstrate meeting the State revised definition using the method approved by CMS; and

(iii) Exception for Medicaid EPs. If a Medicaid EP has adopted, implemented or upgraded certified EHR technology in the first payment year, the EP need not demonstrate meaningful use until the second payment year, as described in $\S 495.6$ and $\S 495.8$ of this subpart.

(2) For CY 2012 and subsequent years-

(i) Attestation. Attest, through a secure mechanism, in a manner specified by CMS (or for a Medicaid EP, in a manner specified by the State) that during the EHR reporting period, the $\mathrm{EP}-$

(A) Used certified EHR technology and specify the technology used.

(B) Satisfied the required objectives and associated measures under $\S 495.6(\mathrm{~d})$ and $\S 495.6(\mathrm{e})$, except $\S$ 495.6(d)(10) "Report ambulatory clinical quality measures to CMS or, in the case of Medicaid EPs, the States."

(C) Must specify the EHR reporting period and provide the result of each applicable measure for all patients seen during the EHR reporting period for which a selected measure is applicable.

(ii) Reporting of clinical quality information. For § 495.6(d)(10), "Report ambulatory clinical quality measures to CMS or, in the case of Medicaid EPs, the States," report the ambulatory clinical quality measures selected by CMS electronically to CMS (or in the case of Medicaid EPs, the States) in the manner specified by CMS (or in the case of Medicaid EPs, the States).

(iii) Additional requirements for Medicaid EPs. For Medicaid EPs, if, in accordance with $\S 495.316$ and $\S 495.332$, CMS has approved a State's additional criteria for meaningful use, in addition to meeting paragraphs (a)(2)(i) through (iii), the EP must also demonstrate meeting such additional criteria using the method approved by CMS.

(iv) Exception for Medicaid EPs. If a Medicaid EP has adopted, implemented, or upgrade certified EHR technology in the first payment year, the EP need not demonstrate that it is a meaningful EHR user until the second payment year, as described in $\S 495.6$ and $\S 495.8$ of this subpart.

(3) For all CYs, an EP who practices in multiple physical locations, not all of which have certified EHR technology available, will demonstrate meaningful use using only the locations where the EP has certified EHR technology available. (See also $\$ 495.4$ regarding the definition of meaningful EHR user).

(b) Demonstration by eligible hospitals and CAHs. To successfully demonstrate that it is a meaningful EHR user, an eligible hospital or CAH must the following requirements:

(1) For FY 2011-

(i) Attestation. Attest, through a secure mechanism, in a manner specified by CMS (or for a Medicaid eligible hospital, in a manner specified by the State), that during the EHR reporting period, the eligible hospital or $\mathrm{CAH}-$

(A) Used certified EHR and specify the technology used.

(B) Satisfied the required objectives and associated measures under $\S 495.6$ (f) and § 495.6(g).

(C) Must specify the EHR reporting period and provide the result of each applicable measure for all patients admitted to the inpatient or emergency department (POS 21 or 23) of the hospital during the EHR reporting period for which a selected measure is applicable.

(ii) Additional requirements for Medicaid eligible hospitals. For Medicaid eligible hospitals, if, in accordance with $\S 495.316$ and $\S 495.332$, CMS has approved a State's revised definition for meaningful use, in addition to meeting paragraphs (b)(1)(i) through (ii) of this section, the eligible hospital must also demonstrate meeting the State's revised definition using the method approved by CMS.

(iv) Exception for Medicaid eligible hospitals. If a Medicaid eligible hospital has adopted, implemented or upgraded certified EHR technology in the first payment year, the eligible hospital need not demonstrate meaningful use until the second payment year, as described in $\S 495.6$ and $\S 495.8$ of this subpart.

(2) For FY 2012 and subsequent years-

(i) Attestation. Attest, through a secure mechanism, in a manner specified by CMS (or for a Medicaid eligible hospital, in a manner specified by the State), that during the EHR reporting period, the eligible hospital or $\mathrm{CAH}-$

(A) Used certified EHR and specify the technology used;

(B) Satisfied the required objectives and associated measures under $\S 495.6(\mathrm{f})$ and $\S 495.6(\mathrm{~g})$, except $\S$ 495.6(f)(9) "Report hospital clinical quality measures to CMS or, in the case of Medicaid eligible hospitals, the States;"

(C) Must specify the EHR reporting period and provide the result of each applicable measure for all patients admitted to the inpatient or emergency department (POS 21 or 23) of the hospital during the EHR reporting period for which a selected measure is applicable. (ii) Reporting clinical quality information. For § 495.6(f)(9) "Report hospital clinical quality measures to CMS or, in the case of Medicaid eligible hospitals, the States," report the hospital quality measures selected by CMS electronically to CMS (or in the case of Medicaid eligible hospitals, the States), in the manner specified by CMS (or in the case of Medicaid eligible hospitals, the States).

(iv) Additional requirements for Medicaid eligible hospitals. For Medicaid eligible hospitals if, in accordance with $\S 495.316$ and $\S 495.332$, CMS has approved a State's revised definition for meaningful use, in addition to meeting paragraphs (b)(2)(i) through (iii) of this section, the eligible hospital must also demonstrate meeting the State's revised definition using the method approved by CMS.

(v) Exception for Medicaid eligible hospitals. If a Medicaid eligible hospital has adopted, implemented, or upgraded certified EHR technology in the first payment year, the eligible hospital need not demonstrate that it is a meaningful EHR user until the second payment year, as described in $\S 495.6$ and $\S 495.8$ of this subpart.

(c) Review of meaningful use. (1) CMS (and in the case of Medicaid EPs and eligible hospitals, States) may review an EP, eligible hospital or CAH's demonstration of meaningful use.

(2) All EPs, eligible hospitals, and CAHs must keep documentation supporting their demonstration of meaningful use for 6 years.

\section{$\S 495.10$ Participation requirements for EPs, eligible hospitals, and CAHs.}

(a) An eligible hospital, CAH or EP must submit in a manner specified by CMS the following information in the first payment year:

(1) Name of the EP, eligible hospital or $\mathrm{CAH}$.

(2) National Provider Identifier (NPI).

(3) Business address and phone number.

(4) Such other information as specified by CMS.

(b) In addition to the information submitted under paragraph (a) of this section, an eligible hospital or CAH, must, in the first payment year, submit in a manner specified by CMS its CMS Certification Number (CCN) and its Taxpayer Identification Number (TIN).

(c) Subject to paragraph (f) of this section, in addition to the information submitted under paragraph (a) of this section, an EP must submit in a manner specified by CMS, the Taxpayer Identification Number (TIN) which may be the EP's Social Security Number 
(SSN) to which the EP's incentive payment should be made.

(d) In the event the information specified in paragraphs (a) through (c) of this section as previously submitted to CMS is no longer accurate, the EP, eligible hospital or CAH must provide updated information to CMS or the State on a timely basis in the manner specified by CMS or the State.

(e) An EP that qualifies as both a Medicaid EP and Medicare EP-

(1) Must notify CMS in the manner specified by CMS as to whether he or she elects to participate in the Medicare or the Medicaid EHR incentive program;

(2) After receiving at least one EHR incentive payment, may switch between the two EHR incentive programs only one time, and only for a payment year before 2015

(3) Must, for each payment year, meet all of the applicable requirements, including applicable patient volume requirements, for the program in which he or she chooses to participate

(Medicare or Medicaid);

(4) Is limited to receiving, in total, the maximum payments the EP would receive under the Medicaid EHR program, as described in subpart D of this part; and

(5) Is placed in the payment year the EP would have been in had the EP begun in and remained in the program to which he or she has switched. For example, an EP that begins receiving Medicaid incentive payments in 2011, and then switches to the Medicare program for 2012, is in his or her second payment year in 2012 .

(f) Limitations on incentive payment reassignments. (1) EPs are permitted to reassign their incentive payments to their employer or to an entity with which they have a contractual arrangement allowing the employer or entity to bill and receive payment for the EP's covered professional services.

(2)(i) Assignments in Medicare must be consistent with Section 1842(b)(6)(A) of the Act and 42 CFR part 424 subpart F.

(ii) Medicaid EPs may also assign their incentive payments to a TIN for an entity promoting the adoption of EHR technology, consistent with subpart D of this part.

(3) Each EP may reassign the entire amount of the incentive payment to only one employer or entity.

\section{Subpart B-Requirements Specific to the Medicare Program}

\section{\$495.100 Definitions.}

In this subpart unless otherwise indicated-

Covered professional services means (as specified in section 1848(k)(3) of the
Act) services furnished by an EP for which payment is made under, or is based on, the Medicare physician fee schedule.

Eligible hospital means a hospital subject to the prospective payment system specified in $\S 412.1(a)(1)$ of this chapter, excluding those hospitals specified in $\S 412.23$ of this chapter, and excluding those hospital units specified in $\$ 412.25$ of this chapter.

Eligible professional (EP) means a physician as defined in section 1861(r) of the Act, which includes, with certain limitations, all of the following types of professionals:

(1) A doctor of medicine or osteopathy.

(2) A doctor of dental surgery or medicine.

(3) A doctor of podiatric medicine.

(4) A doctor of optometry.

(5) A chiropractor.

Geographic health professional

shortage area (HPSA) means a

geographic area that is designated by the Secretary under section 332(a)(1)(A) of the PHS Act as of December 31 of the year prior to the payment year as having a shortage of health professionals.

Qualifying $C A H$ means a CAH that is a meaningful EHR user for the EHR reporting period for a cost reporting period beginning during a payment year.

Qualifying eligible professional (qualifying EP) means an EP who is a meaningful EHR user for the EHR reporting period for a payment year and who is not a hospital-based EP, as determined for that payment year.

Qualifying hospital means an eligible hospital that is a meaningful EHR user for the EHR reporting period for a payment year.

\section{\$495.102 Incentive payments to EPs.}

(a) General rules. (1) Subject to paragraph (b) of this section, in addition to the amount otherwise paid under section 1848 of the Act, there must be paid to a qualifying EP (or to an employer or entity in the cases described in section 1842(b)(6)(A) of the Act) for a payment year an amount equal to 75 percent of the estimated allowed charges for covered professional services furnished by the EP during the payment year.

(2) For purposes of this paragraph (a) of this section, the estimated allowed charges for the qualifying EP's covered professional services during the payment year are determined based on claims submitted no later than 2 months after the end of the payment year, and, in the case of a qualifying EP who furnishes covered professional services in more than one practice, are determined based on claims submitted for the EP's covered professional services across all such practices.

(b) Limitations on amounts of incentive payments.

(1) Except as otherwise provided in paragraphs (b)(2) and (c) of this section, the amount of the incentive payment under paragraph (a) of this section for each payment year is limited to the following amounts:

(i) For the first payment year, $\$ 15,000$ (or, if the first payment year for such qualifying EP is 2011 or 2012, $\$ 18,000$ ).

(ii) For the second payment year, $\$ 12,000$.

(iii) For the third payment year, $\$ 8,000$.

(iv) For the fourth payment year, $\$ 4,000$.

(v) For the fifth payment year, $\$ 2,000$.

(vi) For any succeeding payment year for such professional, $\$ 0$.

(2)(i) If the first payment year for a qualifying EP is 2014, then the payment limit for a payment year for the qualifying EP is the same as the amount specified in paragraph (b)(1) of this section for such payment year for a qualifying EP whose first payment year is 2013.

(ii) If the first payment year for a qualifying EP is after 2014, then the payment limit specified in this paragraph for such EP for such year and any subsequent year is $\$ 0$.

(c) Increase in incentive payment limit for EPs who predominantly furnish services in a geographic HPSA. In the case of a qualifying EP who in the year prior to the payment year furnishes more than 50 percent of his or her covered professional services in a geographic HPSA that is designated as of December 31 of such year, the incentive payment limit determined under paragraph (b) of this section is to be increased by 10 percent.

(d) Payment adjustment effective in CY 2015 and subsequent years for nonqualifying EPS.

(1) Subject to paragraph (d)(3) of this section, beginning in 2015, for covered professional services furnished by an EP who is not a qualifying EP or a hospitalbased EP for the year, the payment amount for such services is equal the product of the applicable percent specified in paragraph (d)(2) of this section and the Medicare physician fee schedule amount for such services.

(2) Applicable percent. Applicable percent is as follows:

(i) For 2015, 99 percent if the EP is not subject to the payment adjustment for an EP who is not a successful electronic prescriber under section 1848(a)(5) of the Act, or 98 percent if the $\mathrm{EP}$ is subject to the payment adjustment 
for an EP who is not a successful electronic prescriber under section 1848(a)(5) of the Act).

(ii) For 2016, 98 percent.

(iii) For 2017 and each subsequent year, 97 percent.

(3) Significant hardship exception. (i) The Secretary may, on a case-by-case basis, exempt an EP who is not a qualifying EP from the application of the payment adjustment under paragraph (d)(1) of this section if the Secretary determines that compliance with the requirement for being a meaningful EHR user would result in a significant hardship for the EP.

(ii) The Secretary's determination to grant an EP an exemption under paragraph (d)(3)(i) of this section may be renewed on an annual basis, provided that in no case may an EP be granted an exemption under paragraph (d)(3)(i) of this section for more than 5 years.

\section{$\$ 495.104$ Incentive payments to eligible hospitals.}

(a) General rule. A qualifying hospital (as defined in this subpart) must receive the special incentive payment as determined under the formulas described in paragraph (c) of this section for the period specified in paragraph (b) of this section.

(b) Transition periods. Subject to paragraph (d) of this section and the payment formula specified in paragraph (c) of this section, qualifying hospitals may receive incentive payments during transition periods which comprise the following fiscal years:

(1) Hospitals whose first payment year is FY 2011 may receive such payments for FYs 2011 through 2014.

(2) Hospitals whose first payment year is FY 2012 may receive such payments for FYs 2012 through 2015.

(3) Hospitals whose first payment year is FY 2013 may receive such payments for FYs 2013 through 2016.

(4) Hospitals whose first payment year is FY 2014 may receive such payments for FY 2014 through 2016.

(5) Hospitals whose first payment year is FY 2015 may receive such payments for FY 2015 through 2016.

(c) Payment methodology. (1) The incentive payment for each payment year is calculated as the product of the following:

(i) The initial amount determined under paragraph (c)(3) of this section.

(ii) The Medicare share fraction determined under paragraph (c)(4) of this section.

(iii) The transition factor determined under paragraph (c)(5) of this section.

(2) Interim and final payments. CMS uses data on hospital acute care inpatient discharges, Medicare Part A acute care inpatient-bed-days, Medicare Part C acute care inpatient-bed-days, and total acute care inpatient-bed-days, from the latest submitted 12-month hospital cost report as the basis for making preliminary incentive payments. Final payments are determined at the time of settling the first 12-month hospital cost report for the hospital fiscal year that begins on or after the first day of the payment year, and settled on the basis of data from that cost reporting period.

(3) Initial amount. The initial amount is equal to one of the following:

(i) For each hospital with 1,149 acute care inpatient discharges or fewer, $\$ 2,000,000$.

(ii) For each hospital with at least 1,150 but no more than 23,000 acute care inpatient discharges, $\$ 2,000,000+$ $[\$ 200 \times(n-1,149)]$, where $n$ is the number of discharges for the hospital.

(iii) For each hospital with more than 23,000 acute care inpatient discharges, $\$ 6,370,200$.

(4) Medicare share fraction-(i) General. (A) CMS determines the Medicare share fraction for an eligible hospital by using the number of Medicare Part A, Medicare Part C, and total acute care inpatient-bed-days using data from the Medicare cost report as specified by CMS.

(B) CMS computes the denominator of the Medicare share fraction using the charity care charges reported on the hospital's Medicare cost report.

(ii) The Medicare share fraction is the ratio of-

(A) A numerator which is the sum of-

(1) The number of inpatient-bed-days which are attributable to individuals with respect to whom payment may be made under Part A, including

individuals enrolled in section 1876

Medicare cost plans; and

(2) The number of inpatient-bed-days which are attributable to individuals who are enrolled with a Medicare Advantage organization (as defined in $\S 422.2$ of this chapter).

(B) A denominator which is the product of-

(1) The total number of acute care inpatient-bed-days; and

(2) The total amount of the eligible hospital's charges, not including any charges that are attributable to charity care, divided by the estimated total amount of the hospitals charges.

(5) Transition factor. For purposes of the payment formula, the transition factor is as follows:

(i) For hospitals whose first payment year is FY 2011-

(A) 1 for FY 2011;

(B) $3 / 4$ for FY 2012;
(C) $1 / 2$ for FY 2013; and

(D) $1 / 4$ for FY 2014.

(ii) For hospitals whose first payment year is FY 2012-

(A) 1 for FY 2012;

(B) $3 / 4$ for FY 2013;

(C) $1 / 2$ for FY 2014; and

(D) $1 / 4$ for FY 2015;

(iii) For hospitals whose first payment year is FY 2013-

(A) 1 for FY 2013;

(B) $3 / 4$ for FY 2014;

(C) $1 / 2$ for FY 2015; and

(D) $1 / 4$ for FY 2016.

(iv) For hospitals whose first payment year is FY 2014-

(A) $3 / 4$ for FY 2014;

(B) $1 / 2$ for FY 2015; and

(C) 1/4 for FY 2016.

(v) For hospitals whose first payment year is FY 2015-

(A) $1 / 2$ for FY 2015; and

(B) $1 / 4$ for FY 2016.

(d) No incentive payment for nonqualifying hospitals. After the first payment year, an eligible hospital will not receive an incentive payment for any payment year during which it is not a qualifying hospital.

\$495.106 Incentive payments to CAHs.

(a) Definitions. In this section, unless otherwise indicated-

Payment year means a Federal fiscal year beginning after FY 2010 but before FY 2016.

Qualifying $C A H$ means a CAH that would meet the definition of a meaningful EHR user at $\S 495.4$, if it were an eligible hospital.

Reasonable costs incurred for the purchase of certified EHR technology for a qualifying $\mathrm{CAH}$ means the reasonable acquisition costs incurred for the purchase of depreciable assets as described in part 413 subpart $G$ of this chapter, such as computers and associated hardware and software, necessary to administer certified EHR technology as defined in $\S 495.4$, excluding any depreciation and interest expenses associated with the acquisition.

(b) General rule. A qualifying $\mathrm{CAH}$ receives an incentive payment for its reasonable costs incurred for the purchase of certified EHR technology, as defined in paragraph (a) of this section, in the manner described in paragraph (c) of this section for a cost reporting period beginning during a payment year as defined in paragraph (a) of this section.

(c) Payment methodology. (1) Payment amount. A qualifying $\mathrm{CAH}$ receives an incentive payment amount equal to the product of its reasonable costs incurred for the purchase of certified EHR technology and the Medicare share percentage. 
(2) Calculation of reasonable costs. CMS or its Medicare contractor computes a qualifying CAH's reasonable costs incurred for the purchase of certified EHR technology, as defined in paragraph (a) of this section, as the sum of-

(i) The reasonable costs incurred for the purchase of certified EHR technology during the cost reporting period that begins in a payment year; and

(ii) Any reasonable costs incurred for the purchase of certified EHR technology in cost reporting periods beginning in years prior to the payment year which have not been fully depreciated as of the cost reporting period beginning in the payment year.

(3) Medicare share percentage. Notwithstanding the percentage applicable under $\S 413.70$ (a)(1) of this chapter, the Medicare share percentage equals the lesser of-

(i) 100 percent; or

(ii) The sum of the Medicare share fraction for the CAH as calculated under $\S 495.104$ (c)(4) of this subpart and 20 percentage points.

(d) Incentive payments made to CAHs. (1) The amount of the incentive payment made to a qualifying $\mathrm{CAH}$ under this section represents the expensing and payment of the reasonable costs computed in paragraph (c) of this section in a single payment year and, as specified in $\S 413.70(a)(5)$ of this chapter, such payment is made in lieu of payment that would have been made under $\S 413.70(\mathrm{a})(1)$ of this chapter for the reasonable costs of the purchase of certified EHR technology including depreciation and interest expenses associated with the acquisition.

(2) The amount of the incentive payment made to a qualifying $\mathrm{CAH}$ under this section is paid through a prompt interim payment for the applicable payment year after-

(i) The CAH submits the necessary documentation, as specified by CMS or its Medicare contractors, to support the computation of the incentive payment amount under this section; and

(ii) CMS or its Medicare contractor reviews such documentation and determines the interim amount of the incentive payment.

(3) The interim incentive payment made under this paragraph is subject to a reconciliation process as specified by CMS and the final incentive payment as determined by CMS or its Medicare contractor is considered payment in full for the reasonable costs incurred for the purchase of certified EHR technology in a single payment year.
(4) In no case may an incentive payment be made with respect to a cost reporting period beginning during a payment year before FY 2011 or after FY 2015 and in no case may a CAH receive an incentive payment under this section with respect to more than 4 consecutive payment years.

(e) Reductions in payment to CAHs. For cost reporting periods beginning in FY 2015, if a CAH is not a qualifying $\mathrm{CAH}$ for a payment year, then the payment for inpatient services furnished by a CAH under $\S 413.70$ (a) of this chapter is adjusted by the applicable percentage described in $\S 413.70$ (a)(6) of this chapter unless otherwise exempt from such adjustment.

(f) Administrative or judicial review. There is no administrative or judicial review under sections 1869 or 1878 of the Act, or otherwise, of the-

(1) Methodology and standards for determining the amount of payment, the reasonable cost, and adjustments described in this section including selection of periods for determining, and making estimates or using proxies of, inpatient-bed-days, hospital charges, charity charges, and the Medicare share percentage as described in this section;

(2) Methodology and standards for determining if a $\mathrm{CAH}$ is a qualifying CAH under this section;

(3) Specification of EHR reporting periods, cost reporting periods, paymen years, and fiscal years used to compute the $\mathrm{CAH}$ incentive payment as specified in this section; and

(4) Identification of the reasonable costs used to compute the CAH incentive payment under paragraph (c) of this section including any reconciliation of the $\mathrm{CAH}$ incentive payment amount made under paragraph (d) of this section.

\$495.108 Posting of required information.

(a) CMS posts, on its Internet Web site, the following information regarding EPs, eligible hospitals, and CAHs receiving an incentive payment under subparts $\mathrm{B}$ and $\mathrm{C}$ of this part:

(1) Name.

(2) Business addressee.

(3) Business phone number.

(4) Such other information as specified by CMS.

(b) CMS posts, on its Internet Web site, the following information for qualifying MA organizations that receive an incentive payment under subpart $\mathrm{C}$ of this part-

(1) The information specified in paragraph (a) of this section for each of the qualifying MA organization's MA plan information; and

(2) The information specified in paragraph (a) of this section for each of the qualifying MA organization's MA EPs and MA-affiliated eligible hospitals.

\section{$\$ 495.110$ Preclusion on administrative} and judicial review.

There is no administrative or judicial review under sections 1869 or 1878 of the Act, or otherwise, of the following:

(a) For EPs-

(1) The methodology and standards for determining EP incentive payment amounts;

(2) The methodology and standards for determining the payment adjustments that apply to EPs beginning with 2015 ;

(3) The methodology and standards for determining whether an EP is a meaningful EHR user, including-

(i) The selection of clinical quality measures; and

(ii) The means of demonstrating meaningful EHR use.

(4) The methodology and standards for determining the hardship exception to the payment adjustments;

(5) The methodology and standards for determining whether an EP is hospital-based; and

(6) The specification of the EHR reporting period, as well as whether payment will be made only once, in a single consolidated payment, or in periodic installments.

(b) For eligible hospitals-

(1) The methodology and standards for determining the incentive payment amounts made to eligible hospitals, including-

(i) The estimates or proxies for determining discharges, inpatient-beddays, hospital charges, charity charges, and Medicare share; and

(ii) The period used to determine such estimate or proxy;

(2) The methodology and standards for determining the payment adjustments that apply to eligible hospitals beginning with FY 2015;

(3) The methodology and standards for determining whether an eligible hospital is a meaningful EHR user, including-

(i) The selection of clinical quality measures; and

(ii) The means of demonstrating meaningful EHR use.

(4) The methodology and standards for determining the hardship exception to the payment adjustments; and

(5) The specification of the EHR reporting period, as well as whether payment will be made only once, in a single consolidated payment, or in periodic installments. 


\section{Subpart C-Requirements Specific to Medicare Advantage (MA) Organizations}

\section{\$495.200 Definitions.}

As used in this subpart:

First payment year means with respect to-

(1) Covered professional services furnished by a qualifying MA EP, the first calendar year for which an incentive payment is made for such services under this subsection to a qualifying MA organization.

(2) Qualifying MA-affiliated eligible hospitals, the first fiscal year for which an incentive payment is made for qualifying MA-affiliated eligible hospitals under this section to a qualifying MA organization.

Inpatient-bed-days is defined in the same manner and is used in the same manner as that term is defined and used for purposes of implementing section 4201(a) of the American Recovery and Reinvestment Act of 2009 with respect to the Medicare FFS hospital EHR incentive program in $\S 495.104$ of this part.

Patient care services means health care services for which payment would be made under, or for which payment would be based on, the fee schedule established under Medicare Part B if they were furnished by an EP to a Medicare beneficiary.

Payment year means-

(1) For a qualifying MA EP, a calendar year (CY) beginning with CY 2011 and ending with CY 2016; and

(2) For an eligible hospital, a Federal fiscal year (FY) beginning with FY 2011 and ending with FY 2016.

Qualifying MA-affiliated eligible hospital means an eligible hospital under section 1886(n)(6) of the Act that is under common corporate governance with a qualifying MA organization, for which at least two thirds of the Medicare hospital discharges (or beddays) are of (or for) Medicare individuals enrolled under MA plans, and that is a meaningful user of certified EHR technology as defined by $\S 495.4$ of this part. In the case of a hospital for which at least one-third of whose Medicare bed-days for the year are covered under Part A rather than Part C, payment for that payment year must only be made under section 1886(n) of the Act and not under this section.

Qualifying MA EP means all of the following:

(1) A physician (as described in section 1861(r) of the Act), including a doctor of medicine or osteopathy who is either of the following:

(i) Employed by a qualifying MA organization. (ii) Employed by, or is a partner of, an entity that through a contract with a qualifying MA organization furnishes at least 80 percent of the entity's Medicare patient care services to enrollees of such organization.

(2) Furnishes at least 80 percent of his or her professional services covered under Title XVIII to enrollees of the qualifying MA organization.

(3) Furnishes, on average, at least 20 hours per week of patient care services to enrollees of the qualifying MA organization during the EHR reporting period.

(4) Is a meaningful user of certified EHR technology in accordance with $\S 495.4$ of this part.

(5) Is not a "hospital-based EP" as that term is defined in $\S 495.4$ of this Part.

Qualifying MA organization means a MA organization that is organized as a health maintenance organization (HMO) as defined in section 2791(b)(3) of the Public Health Service (PHS) Act which includes a Federally qualified HMO, an organization recognized as an HMO under State law, or a similar organization regulated for solvency under State law in the same manner and to the same extent as an HMO.

Second, third, fourth, and fifth payment year means with respect to incentive payments for qualifying-

(1) MA EPs to a qualifying MA organization, each successive calendar year immediately following the first payment year for the qualifying MA organization. The first payment year and each successive year immediately following the first payment year, for the qualifying MA organizations, through 2016, is the same for all qualifying MA EPs with respect to any specific qualifying MA organization.

(2) MA-affiliated eligible hospitals to a qualifying MA organization, each successive fiscal year immediately following the first payment year for the qualifying MA organization.

Under common corporate governance means that a qualifying MA organization and a qualifying MAaffiliated eligible hospital have a common parent corporation, that one is a subsidiary of the other, or that the organization and the hospital have a common board of directors.

\$495.202 Identification of qualifying MA organizations, MA-EPs and MA-affiliated eligible hospitals.

(a) Identification of qualifying $M A$ organizations. (1) Beginning with bids due in June 2011 (for plan year 2012), MA organizations seeking reimbursement for qualifying MA EPs and qualifying MA-affiliated eligible hospitals under the MA EHR incentive program are required to identify themselves to CMS in a form and manner specified by CMS, as part of submissions of initial bids under section 1854(a)(1)(A) of the Act.

(2) Qualifying MA organizations offering MA HMO plans, absent evidence to the contrary, are deemed to meet the definition of HMO in 42 U.S.C. 300gg-91(b)(3)—section 2791(b)(3) of the PHS Act.

(3) Qualifying MA organizations offering MA plan types other than HMOs, must attest to the fact that they meet the definition of HMO in 42 U.S.C. 300gg-91(b)(3)—section 2791(b)(3) of the PHS Act.

(4) Beginning with bids due in June 2014 (for plan year 2015), all MA organizations with potentially qualifying MA EPs or potentially qualifying MA-affiliated eligible hospitals under the MA EHR incentive program must identify themselves to CMS in a form and manner specified by CMS, as part of submissions of initial bids under section 1854(a)(1)(A) of the Act. "Potentially qualifying MA EPs" and "potentially qualifying MAaffiliated eligible hospitals" are those EPs and hospitals that meet the respective definitions of "qualifying MA EP" and "qualifying MA-affiliated eligible hospital" in $§ 495.200$ but who (or which) are not meaningful users of certified EHR technology.

(b) Identification of qualifying MA EPS and qualifying $M A$-affiliated eligible hospitals.

(1) A qualifying MA organization, as part of its initial bid starting with plan year 2012, must make a preliminary identification of potentially qualifying MA EPs and potentially qualifying MAaffiliated eligible hospitals for which the organization is seeking incentive payments for the current plan year.

(2) A qualifying MA organization must provide CMS with the following for each MA EP or eligible hospital when reporting under either paragraph (b)(1) or (b)(3) of this section:

(i) The MA EP's or MA-affiliated eligible hospital's name.

(ii) The address of the MA EP's practice or MA-affiliated eligible hospital's location.

(iii) NPI.

(iv) An attestation by MA organization specifying that the MA EP or MAaffiliated eligible hospital meets the eligibility criteria.

(3) Final identification of potentially qualifying MA EP or MA-affiliated eligible hospital must be made within 60 days of the close of the payment year as defined in $\$ 495.200$ for which MA EHR incentive payments are being sought. 
(4) Beginning plan year 2015 and for subsequent plan years, all qualifying MA organizations, as part of their initial bids in June for the following plan year must-

(i) Identify potentially qualifying MA EPs and potentially qualifying MAaffiliated eligible hospitals;

(ii) Include information specified in paragraph (b)(2)(i)(A) through (C) of this section for each professional and hospital.

(iii) Include an attestation that each professional and hospital either meets or does not meet the EHR incentive payment eligibility criteria.

\$495.204 Incentive payments to qualifying MA organizations for MA-EPs and MAaffiliated eligible hospitals.

(a) General rule. A qualifying MA organization receives an incentive payment for its qualifying MA-EPs and its qualifying MA-eligible hospitals. The incentive payment amount paid to a qualifying MA organization for a-

(1) Qualifying MA-EP is the amount determined under paragraph (b) of this section; and

(2) Qualifying MA-eligible hospital is the amount determined under paragraph (c) of this section.

(b) Amount payable to qualifying $M A$ organization for qualifying MA EPS.

(1) CMS substitutes an amount determined to be equivalent to the amount computed under $\S 495.102$ of this part.

(2) The qualifying MA organization must report to CMS within 60 days of the close of the calendar year, the aggregate annual amount of revenue attributable to providing services that would otherwise be covered as professional services under Part B received by each qualifying MA EP for enrollees in MA plans of the MA organization in the payment year.

(3) CMS calculates the incentive amount for the MA organization for each qualifying MA EP as an amount equal to 75 percent of the reported annual revenue specified in paragraph (b)(2) of this section, up to the maximum amounts specified under section 1848(o)(1)(B) of the Act.

(4) For qualifying MA EPs who are compensated on a salaried basis, CMS requires the qualifying MA organization to develop a methodology for estimating the portion of each qualifying MA EP's salary attributable to providing services that would otherwise be covered as professional services under Part B to MA plan enrollees of the MA organization in the payment year. The methodology-

(i) Must be approved by CMS; and

(ii) May include an additional amount related to overhead, where appropriate, estimated to account for the MAenrollee related Part B practice costs of the salaried qualifying MA EP.

(iii) Methodological proposals must be submitted to CMS by June of the payment year and must be auditable by an independent third-party. CMS will review and approve or disapprove such proposals in a timely manner.

(5) For qualifying MA EPs who are not salaried, qualifying MA organizations may obtain attestations from such qualifying MA EPs (or from entities that the MA EPs are employed by or with which they have a partnership interest) as to the amount of compensation received by such EPs for MA plan enrollees of the MA organization. The organizations may submit to CMS compensation information for each such MA EP based on such attestations.

(6) For qualifying MA EPs who are not salaried, qualified MA organizations may have qualifying MA EPs (or from entities that the MA EPs are employed by or with which they have a partnership interest) send MA organization compensation information directly to CMS. CMS will use the information provided in this subparagraph or paragraph (b)(5) of this section for no other purpose than to compute the amount of EHR incentive payment due the MA organization.

(c) Amount payable to qualifying $M A$ organization for qualifying $M A$ affiliated eligible hospitals. (1)(i) CMS substitutes an amount determined to be equivalent to the amount computed under $\S 495.104$, to the extent data are not available to compute payments for qualifying MA-affiliated eligible hospitals under the Medicare FFS EHR hospital incentive program.

(ii) CMS uses the same methodology and defines "inpatient-bed-days"and other terms as used under the Medicare FFS EHR hospital incentive program in $\S 495.104$ of this part in computing amounts due qualifying MA organizations for MA-affiliated eligible hospitals.

(2) To the extent data are available, qualifying MA organizations must receive hospital incentive payments through their affiliated hospitals under the Medicare FFS EHR hospital incentive program, rather than through the MA EHR hospital incentive program.

(d) Payment to qualifying $M A$ organizations. CMS makes payment to qualifying MA organizations for qualifying MA EPs only under the MA EHR incentive program and not under the Medicare FFS EHR incentive program to the extent an EP has earned less than the maximum incentive payment for the same period under the Medicare FFS EHR incentive program.

(e) Payment review under MA. To ensure the accuracy of the incentive payments, CMS conducts selected compliance reviews of qualifying MA organizations to ensure that EPs and eligible hospitals for which such qualifying organizations received incentive payments were meaningful EHR users in accordance with $\S 422.504$ of this chapter.

(1) The reviews include validation of the status of the organization as a qualifying MA organization, verification of meaningful use and review of data used to calculate incentive payments.

(2) MA organizations are required to maintain evidence of their qualification to receive incentive payments and the data necessary to accurately calculate incentive payments.

(3) Documents and records must be maintained for 6 years from the date such payments are made with respect to a given payment year.

(4) Payments that result from incorrect or fraudulent attestations, cost data, or any other submission required to establish eligibility or to qualify for such payment, will be recouped by CMS from the MA organization.

\section{\$495.206 Timeframe for payment to qualifying MA organizations.}

(a) CMS makes payment to qualifying MA organizations for qualifying MA EPs under the MA EHR incentive program after computing incentive payments due under the Medicare FFS EHR incentive program according to $\S 495.102$.

(b) Payments to qualifying MA organizations for qualifying MAaffiliated eligible hospitals under common corporate governance are made under the Medicare FFS EHR incentive program, following the timeline in specified in $\S 495.104$ of this part. To the extent sufficient data do not exist to pay qualifying MA-affiliated eligible hospitals under common corporate governance under the Medicare FFS EHR incentive program, payment is made under the MA EHR incentive program, following the same timeline in $\S 495.104$ of this part.

\section{\$495.208 Avoiding duplicate payment}

(a) Unless a qualifying MA EP is entitled to a maximum payment for a year under the Medicare FFS EHR incentive program, payment for such an individual is only made under the MA EHR incentive program to a qualifying MA organization.

(b) Payment to qualifying MA organizations for a qualifying MAaffiliated eligible hospital under common governance only occurs under 
the MA EHR incentive program to the extent that sufficient data does not exist to pay such hospital under the Medicare FFS hospital incentive program under $\S 495.104$ of this part. In no event are EHR incentive payments made for a hospital for a payment year under this section to the extent they have been made for the same hospital for the same payment year under $\S 495.104$ of this part.

(c) Each qualifying MA organization must ensure that all potentially qualifying MA EPs are enumerated through the NPI system and that other identifying information required under $\S 495.202$ (b) is provided to CMS.

\$495.210 Meaningful EHR user attestation

(a) Qualifying MA organizations are required to attest, in a form and manner specified by CMS, that each qualifying MA EP and qualifying MA-affiliated eligible hospitals is a meaningful EHR user.

(b) Qualifying MA organizations are required to attest within 60 days after the close of a calendar year whether each qualifying MA EP is a meaningful EHR user.

(c) Qualifying MA organizations are required to attest within 60 days after close of the FY whether each qualifying MA-affiliated eligible hospital is a meaningful EHR user.

\section{\$495.212 Limitation on review.}

(a) There is no administrative or judicial review under section 1869 or 1878 of the Act, or otherwise of the methodology and standards for determining payment amounts and payment adjustments under the MA EHR EP incentive program. This includes provisions related to duplication of payment avoidance and rules developed related to the fixed schedule for application of limitation on incentive payments for all qualifying MA EPs related to a specific qualifying MA organization. It also includes the methodology and standards developed for determining qualifying MA EPs and the methodology and standards for determining a meaningful EHR user, including the means of demonstrating meaningful use and the selection of measures.

(b) There is no administrative or judicial review under sections 1869 or 1878 of the Act, or otherwise, of the methodology and standards for determining payment amounts and payment adjustments under the MA EHR hospital incentive program. This includes provisions related to duplication of payment avoidance. It also includes the methodology and standards developed for determining qualifying MA-affiliated eligible hospitals and the methodology and standards for determining a meaningful EHR user, including the means of demonstrating meaningful use and the selection of measures.

\section{Subpart D-Requirements Specific to the Medicaid Program}

\section{\$495.300 Basis and purpose.}

This subpart implements section 4201 of the American Reinvestment and Recovery Act of 2009 and sections 1903(a)(3)(F) and 1903(t) of the Act, which authorize States, at their option, to provide for incentive payments to Medicaid providers for adopting, implementing, or upgrading certified EHR technology or for meaningful use of such technology. This subpart also provides enhanced Federal financial participation (FFP) to States to administer these incentive payments.

\section{§495.302 Definitions.}

As used in this subpart-

Acceptance documents mean written evidence of satisfactory completion of an approved phase of work or contract and acceptance thereof by the State agency.

Acquisition means to acquire health information technology (HIT) equipment or services for the purpose of implementation and administration under this part from commercial sources or from State or local government resources.

Acute care hospital means a health care facility-

(1) Where the average length of patient stay is 25 days or fewer; and

(2) With a CMS certification number (previously known as the Medicare provider number) that has the last four digits in the series $0001-0879$ or 1300 1399

Adopt, implement or upgrade means-

(1) Acquire, purchase, or secure access to certified EHR technology;

(2) Install or commence utilization of certified EHR technology capable of meeting meaningful use requirements; or

(3) Expand the available functionality of certified EHR technology capable of meeting meaningful use requirements at the practice site, including staffing, maintenance, and training, or upgrade from existing EHR technology to certified EHR technology per the ONC EHR certification criteria.

Children's hospital means a separately certified children's hospital, either freestanding or hospital-withinhospital that-

(1) Has a CMS certification number, (previously known as the Medicare provider number), that has the last 4 digits in the series 3300-3399; and

(2) Predominantly treats individuals under 21 years of age.

Entities promoting the adoption of certified electronic health record technology means the State-designated entities that are promoting the adoption of certified EHR technology by enabling oversight of the business, operational and legal issues involved in the adoption and implementation of certified EHR technology or by enabling the exchange and use of electronic clinical and administrative data between participating providers, in a secure manner, including maintaining the physical and organizational relationship integral to the adoption of certified EHR technology by eligible providers.

Health information technology planning advance planning document (HIT PAPD) means a plan of action that requests $\mathrm{FFP}$ and approval to accomplish the planning necessary for a State agency to determine the need for and plan the acquisition of HIT equipment or services or both and to acquire information necessary to prepare a HIT implementation advanced planning document or request for proposal to implement the State Medicaid HIT plan.

HIT implementation advance planning document (HIT IAPD) means a plan of action that requests FFP and approval to acquire and implement the proposed State Medicaid HIT plan services or equipment or both.

Medicaid information technology architecture (MITA) is both an initiative and a framework. It is a national framework to support improved systems development and health care management for the Medicaid enterprise. It is an initiative to establish national guidelines for technologies and processes that enable improved program administration for the Medicaid enterprise. The MITA initiative includes an architecture framework, models, processes, and planning guidelines for enabling State Medicaid enterprises to meet common objectives with the framework while supporting unique local needs.

Medicaid management information system (MMIS) means a mechanized claims processing and information retrieval system-referred to as Medicaid Management Information Systems (MMIS)—that meets specified requirements and that the Department has found (among other things) is compatible with the claims processing and information retrieval systems used in the administration of the Medicare program. The objectives of the MMIS are 
to include claims processing and retrieval of utilization and management information necessary for program administration and audit and must coordinate with other mechanized systems and subsystems that perform other functions, such as eligibility determination.

Needy individuals mean individuals that meet one of following:

(1) Received medical assistance from Medicaid or the Children's Health Insurance Program. (or a Medicaid or CHIP demonstration project approved under section 1115 of the Act).

(2) Were furnished uncompensated care by the provider.

(3) Were furnished services at either no cost or reduced cost based on a sliding scale determined by the individuals' ability to pay.

Patient volume means the minimum participation threshold (as described at $\S 495.304$ (c) through (e)) that is estimated through a numerator and denominator, consistent with the SMHP, and that meets the requirements of $\S 495.306$.

Practices predominantly means an EP for whom the clinical location for over 50 percent of his or her total patient encounters over a period of 6 months in the most recent calendar year occurs at a federally qualified health center or rural health clinic.

Service oriented architecture or service component based architecture means organizing and developing information technology capabilities as collaborating services that interact with each other based on open standards.

State Medicaid health information technology plan (SMHP) means a document that describes the State's current and future HIT activities.

State self-assessment means a process that a State uses to review its strategic goals and objectives, measure its current business processes and capabilities against the (MITA) business capabilities and ultimately develops target capabilities to transform its Medicaid enterprise to be consistent with the MITA principles.

\section{$\$ 495.304$ Medicaid provider scope and eligibility.}

(a) General rule. The following Medicaid providers are eligible to participate in the HIT incentives program:

(1) Medicaid EPs.

(2) Acute care hospitals.

(3) Children's hospitals.

(b) Medicaid EP. The Medicaid professional eligible for an EHR incentive payment is limited to the following when consistent with the scope of practice regulations, as applicable for each professional

(§ 440.50, § 440.60, § 440.100; § 440.165, and $\S 440.166)$ :

(1) A physician.

(2) A dentist.

(3) A certified nurse-midwife.

(4) A nurse practitioner.

(5) A physician assistant practicing in a Federally qualified health center (FQHC) led by a physician assistant or a rural health clinic (RHC), that is so led by a physician assistant.

(c) Additional requirements for the Medicaid EP. To qualify for an EHR incentive payment, a Medicaid EP must, for each year for which the EP seeks an EHR incentive payment, not be hospitalbased as defined at $\S 495.4$ of this subpart, and meet one of the following criteria:

(1) Have a minimum 30 percent patient volume attributable to individuals receiving Medicaid.

(2) Have a minimum 20 percent patient volume attributable to individuals receiving Medicaid, and be a pediatrician.

(3) Practice predominantly in a FQHC or RHC and have a minimum 30 percent patient volume attributable to needy individuals, as defined at $\S 495.302$.

(d) Exception. The hospital-based exclusion in paragraph (c) of this section does not apply to the MedicaidEP qualifying based on practicing predominantly at a FQHC or RHC.

(e) Additional requirement for the eligible hospital. To be eligible for an EHR incentive payment for each year for which the eligible hospital seeks an EHR incentive payment, the eligible hospital must meet the following criteria:

(1) An acute care hospital must have at least a 10 percent Medicaid patient volume for each year for which the hospital seeks an EHR incentive payment.

(2) A children's hospital is exempt from meeting a patient volume threshold.

\section{\$495.306 Establishing patient volume.}

(a) General rule. A Medicaid provider must annually meet patient volume requirements of $\S 495.304$, as these requirements are established through the State's SMHP in accordance with the remainder of this section.

(b) State option(s) through SMHP. A State must submit through the SMHP the option or options it has selected for measuring patient volume. A State must select the methodology described in either paragraph (c) or paragraph (d) of section (or both methodologies). In addition, or as an alternative, a State may select the methodology described in paragraph $(\mathrm{g})$ of this section. (c) Methodology, patient encounter.

(1) EPs. To calculate Medicaid patient volume, an EP must divide:

(i) The total Medicaid patient encounters in any representative, continuous 90-day period in the preceding calendar year; by

(ii) The total patient encounters in the same 90-day period.

(2) Eligible hospitals. To calculate Medicaid patient volume, an eligible hospital must divide-

(i) The total Medicaid encounters in any representative, continuous 90-day period in the preceding fiscal year; by

(ii) The total encounters in the same 90-day period.

(3) Needy individual patient volume. To calculate needy individual patient volume, an EP must divide-

(i) The total needy individual patient encounters in any representative, continuous 90-day period in the preceding calendar year; by

(ii) The total patient encounters in the same 90-day period.

(d) Methodology, patient panel.

(1) EPS. To calculate Medicaid patient volume, an EP must divide:

(i) (A) The total Medicaid patients assigned to the EP's panel in any representative, continuous 90-day period in the preceding calendar year when at least one Medicaid encounter took place with the Medicaid patient in the year prior to the 90-day period; plus

(B) Unduplicated Medicaid encounters in the same 90-day period; by

(ii)(A) The total patients assigned to the provider in that same 90-day period with at least one encounter taking place with the patient during the year prior to the 90-day period; plus

(B) All unduplicated patient encounters in the same 90-day period.

(2) Needy individual patient volume. To calculate needy individual patient volume an EP must divide-

(i)(A) The total Needy Individual patients assigned to the EP's panel in any representative, continuous 90-day period in the preceding calendar year when at least one Needy Individual encounter took place with the Medicaid patient in the year prior to the 90-day period; plus

(B) Unduplicated Needy Individual encounters in the same 90-day period, by

(ii)(A) The total patients assigned to the provider in that same 90-day period with at least one encounter taking place with the patient during the year prior to the 90-day period, plus

(B) All unduplicated patient

encounters in the same 90-day period.

(e) For purposes of this section, the following rules apply: 
(1) For purposes of calculating EP patient volume, a Medicaid encounter means services rendered to an individual on any one day where-

(i) Medicaid (or a Medicaid demonstration project approved under section 1115 of the Act) paid for part or all of the service; or

(ii) Medicaid (or a Medicaid demonstration project approved under section 1115 of the Act) paid all or part of the individual's premiums, copayments, and cost-sharing.

(2) For purposes of calculating hospital patient volume, both of the following definitions in paragraphs (e)(2)(i) and (e)(2)(ii) of this section may apply:

(i) A Medicaid encounter means services rendered to an individual per inpatient discharge where-

(A) Medicaid (or a Medicaid demonstration project approved under section 1115 of the Act) paid for part or all of the service; or

(B) Medicaid (or a Medicaid demonstration project approved under section 1115 of the Act) paid all or part of the individual's premiums, copayments, and/or cost-sharing.

(ii) A Medicaid encounter means services rendered in an emergency department on any one day where-

(A) Medicaid (or a Medicaid demonstration project approved under section 1115 of the Act) paid for part or all of the service; or

(B) Medicaid (or a Medicaid demonstration project approved under section 1115 of the Act) paid all or part of the individual's premiums, copayments, and cost-sharing.

(3) For purposes of calculating needy individual patient volume, a needy patient encounter means services rendered to an individual on any one day where-

(i) Medicaid or CHIP (or a Medicaid or CHIP demonstration project approved under section 1115 of the Act) paid for part or all of the service;

(ii) Medicaid or CHIP (or a Medicaid or CHIP demonstration project approved under section 1115 of the Act) paid all or part of the individual's premiums, copayments, or cost-sharing;

(iii) The services were furnished at no cost; and calculated consistent with $\S 495.310(\mathrm{~h})$; or

(iv) The services were paid for at a reduced cost based on a sliding scale determined by the individual's ability to pay.

(f) Exception. A children's hospital is not required to meet Medicaid patient volume requirements.

(g) Establishing an alternative methodology. A State may submit to CMS for review and approval through the SMHP an alternative from the options included in paragraphs (c) and (d) of this section, so long as it meets the following requirements:

(1) It is submitted consistent with all rules governing the SMHP at $\S 495.332$.

(2) Has an auditable data source.

(3) Has received input from the relevant stakeholder group.

(4) It does not result, in the aggregate, in fewer providers becoming eligible than the methodologies in either paragraphs (c) and (d) of this section.

(h) Group practices. Clinics or group practices will be permitted to calculate patient volume at the group practice/ clinic level, but only in accordance with all of the following limitations:

(1) The clinic or group practice's patient volume is appropriate as a patient volume methodology calculation for the EP.

(2) There is an auditable data source to support the clinic's or group practice's patient volume determination.

(3) All EPs in the group practice or clinic must use the same methodology for the payment year.

(4) The clinic or group practice uses the entire practice or clinic's patient volume and does not limit patient volume in any way.

(5) If an EP works inside and outside of the clinic or practice, then the patient volume calculation includes only those encounters associated with the clinic or group practice, and not the EP's outside encounters.

$\S 495.308$ Net average allowable costs as the basis for determining the incentive payment.

(a) The first year of payment. (1) The incentive is intended to offset the costs associated with the initial adoption, implementation or upgrade of certified electronic health records technology.

(2) The maximum net average allowable costs for the first year are $\$ 25,000$.

(b) Subsequent payment years. (1) The incentive is intended to offset maintenance and operation of certified EHR technology.

(2) The maximum net average allowable costs for each subsequent year are $\$ 10,000$.

\section{\$495.310 Medicaid provider incentive payments.}

(a) Rules for Medicaid EPs. The Medicaid EP's incentive payments are subject to all of the following

limitations:

(1) First payment year. (i) For the first payment year, payment under this subpart may not exceed 85 percent of the maximum threshold of $\$ 25,000$, which equals $\$ 21,250$. (ii) Medicaid EPs are responsible for payment for the remaining 15 percent of the net average allowable cost of certified EHR technology, or $\$ 3,750$ for the first payment year.

(iii) An EP may not begin receiving payments any later than CY 2016.

(2) Subsequent annual payment years.

(i) For subsequent payment years, payment may not exceed 85 percent of the maximum threshold of $\$ 10,000$, which equals $\$ 8,500$.

(ii) Medicaid EPs are responsible for payment for the remaining 15 percent of the net average allowable cost of certified EHR technology, or $\$ 1,500$ per payment year.

(iii) Payments after the first payment year may continue for a maximum of 5 years.

(iv) Medicaid EPs may receive payments on a non-consecutive, annual basis.

(v) No payments may be made after CY 2021.

(3) Maximum incentives. In no case may a Medicaid EP participate for more than a total of 6 years, and in no case will the maximum incentive over a 6year period exceed $\$ 63,750$.

(4) Limitation. For a Medicaid EP who is a pediatrician described in paragraph (b) of this section payment is limited as follows:

(i) The maximum payment in the first payment year is further reduced by twothirds, which equals $\$ 14,167$.

(ii) The maximum payment in subsequent payment years is further reduced by two-thirds, which equals $\$ 5,667$.

(iii) In no case will the maximum incentive payment to a pediatrician under this limitation exceed $\$ 42,500$ over a 6-year period.

(b) Optional exception for pediatricians. A pediatrician described in this paragraph is a Medicaid EP who does not meet the 30 percent patient volume requirements described in $\S 495.304$ and $\S 495.306$, but who meets the 20 percent patient volume requirements described in such sections.

(c) Limitation to only one EHR incentive program. An EP may only receive an incentive payment from either Medicare or Medicaid in a payment year, but not both.

(d) Exception for EPs to switch programs. An EP may change his or her EHR incentive payment program election once, consistent with $\S 495.10$ of this part.

(e) Limitation to one State only. A Medicaid EP or eligible hospital may receive an incentive payment from only one State in a payment year.

(f) Incentive payments to hospitals. Incentive payments to an eligible 
hospital under this subpart are subject to all of the following conditions:

(1) The payment is provided over a minimum of a 3-year period and maximum of a 6-year period.

(2) The total incentive payment received over all payment years of the program is not greater than the aggregate EHR incentive amount, as calculated under paragraph $(\mathrm{g})$ of this section.

(3) No single incentive payment for a payment year may exceed 50 percent of the aggregate EHR hospital incentive amount calculated under paragraph (g) of this section for an individual hospital.

(4) No incentive payments over a 2year period may exceed 90 percent of the aggregate EHR hospital incentive amount calculated under paragraph (g) of this section for an individual hospital.

(5) No hospital may begin receiving incentive payments for any year after FY 2016, and after FY 2016, a hospital may not receive an incentive payment unless it received an incentive payment in the prior fiscal year.

(6) Prior to FY 2016, payments can be made to an eligible hospital on a nonconsecutive, annual basis for the fiscal year.

(7) A multi-site hospital with one CMS Certification Number is considered one hospital for purposes of calculating payment.

(g) Calculation of the aggregate EHR hospital incentive amount. The aggregate EHR hospital incentive amount is calculated as the product of the (overall EHR amount) times (the Medicaid Share).

(1) Overall EHR amount. The overall EHR amount for an eligible hospital is based upon a theoretical 4 years of payment the hospital would receive based, for each of such 4 years, upon the product of the following:

(i) Initial amount. The initial amount is equal to the sum of-

(A) The base amount which is set at $\$ 2,000,000$ for each of the theoretical 4 years; plus

(B) The discharge-related amount for a 12-month period selected by the State, but ending in the Federal fiscal year before the hospital's fiscal year that serves as the first payment year. The discharge-related amount is the sum of the following, with discharges over the 12-month period and based upon the total discharges for the eligible hospital (regardless of any source of payment):

(1) For the first through 1,149th discharge, $\$ 0$.

(2) For the 1,150th through the 23,000 th discharge, $\$ 200$.

(3) For any discharge greater than the 23,000th, \$0.
(C) For purposes of calculating the discharge-related amount under paragraph (g)(1)(i)(B) of this section, for the last 3 of the theoretical 4 years of payment, discharges are assumed to increase by the provider's average annual rate of growth for the most recent 3 years for which data are available per year. Negative rates of growth must be applied as such.

(ii) Medicare share. The Medicare share, which equals 1 .

(iii) Transition factor. The transition factor which equals as follows:

(A) For the first of the theoretical 4 years, 1.

(B) For the second of the theoretical 4 years, $3 / 4$.

(C) For the third of the theoretical 4 years, $1 / 2$.

(D) For the fourth of the theoretical 4 years, $1 / 4$.

(2) Medicaid share. The Medicaid share specified under this paragraph for an eligible hospital is equal to a

fraction-

(i) The numerator of which is the sum (for the 12-month period selected by the State and with respect to the eligible hospital) of-

(A) The estimated number of inpatient-bed-days which are attributable to Medicaid individuals; and

(B) The estimated number of inpatient-bed-days which are attributable to individuals who are enrolled in a managed care organization, a pre-paid inpatient health plan, or a pre-paid ambulatory health plan under part 438 of this chapter; and

(ii) The denominator of which is the product of-

(A) The estimated total number of inpatient-bed-days with respect to the eligible hospital during such period; and

(B) The estimated total amount of the eligible hospital's charges during such period, not including any charges that are attributable to charity care, divided by the estimated total amount of the hospital's charges during such period.

(iii) In computing inpatient-bed-days under paragraph (g)(2)(i) of this section, a State may not include estimated inpatient-bed-days attributable to individuals with respect to whom payment may be made under Medicare Part A, or inpatient-bed-days attributable to individuals who are enrolled with a Medicare Advantage organization under Medicare Part C.

(h) Approximate proxy for charity care. If the State determines that an eligible provider's data are not available on charity care necessary to calculate the portion of the formula specified in paragraph (g)(2)(ii)(B) of this section, the
State may use that provider's data on uncompensated care to determine an appropriate proxy for charity care, but must include a downward adjustment to eliminate bad debt from uncompensated care data. The State must use auditable data sources.

(i) Deeming. In the absence of the data necessary, with respect to an eligible hospital the amount described in paragraph (g)(2)(ii)(B) of this section must be deemed to be 1 . In the absence of data, with respect to an eligible hospital, necessary to compute the amount described in paragraph (g)(2)(i)(B) of this section, the amount under such clause must be deemed to be 0 .

(j) Dual eligibility for incentives payments. A hospital may receive incentive payments from both Medicare and Medicaid if it meets all eligibility criteria in the payment year.

(k) Payments to State-designated entities. Payments to entities promoting the adoption of certified EHR technology as designated by the State must meet the following requirements:

(1) A Medicaid EP may reassign his or her incentive payment to an entity promoting the adoption of certified EHR technology, as defined in $\S 495.302$, and as designated by the State, only under the following conditions:

(i) The State has established a method to designate entities promoting the adoption of EHR technology that comports with the Federal definition in $\S 495.302$.

(ii) The State publishes and makes available to all EPs a voluntary mechanism for reassigning annual payments and includes information about the verification mechanism the State will use to ensure that the reassignment is voluntary and that no more than 5 percent of the annual payment is retained by the entity for costs not related to certified EHR technology.

(2) [Reserved].

\section{\$495.312 Process for payments.}

(a) General rule. States must have a process for making payments consistent with the requirements in subparts $A$ and $\mathrm{D}$ of this part.

(b) Reporting data consistent with this subpart. In order to receive a payment under this part, a provider must report the required data under subpart $\mathrm{A}$ and this subpart within the EHR reporting period described in $\S 495.4$.

(c) State role. The State determines the provider's eligibility for the EHR incentive payment under subpart $A$ and this subpart and approves, processes, and makes timely payments using a process approved by CMS. 
(d) State disbursement. The State disburses an incentive payment to the provider based on the criteria described in subpart $\mathrm{A}$ and this subpart.

(e) Timeframes. Payments are disbursed consistent with the following timeframes for each type of Medicaid eligible provider:

(1) Medicaid EPs. States disburse payments consistent with the calendar year on a rolling basis following verification of eligibility for the payment year.

(2) Medicaid eligible hospitals. States disburse payments consistent with the Federal fiscal year on a rolling basis following verification of eligibility for the payment year.

\section{$\$ 495.314$ Activities required to receive an incentive payment.}

(a) First payment year. (1) In the first payment year, to receive an incentive payment, the Medicaid EP or eligible hospital must meet one of the following:

(i) Demonstrate that during the payment year, it has adopted, implemented, or upgraded certified EHR technology, as defined in $\S 495.302$.

(ii) Demonstrate that during the EHR reporting period for a payment year, it is a meaningful EHR user as defined in $\S 495.4$.

(2) A provider may notify the State of its non-binding intention to participate in the incentives program prior to having fulfilled all of the eligibility criteria

(b) Subsequent payment years. (1) In the second, third, fourth, fifth, and sixth payment years, to receive an incentive payment, the Medicaid EP or eligible hospital must demonstrate that during the EHR reporting period for the applicable payment year, it is a meaningful EHR user, as defined in $\S 495.4$

(2) The automated reporting of the clinical quality measures will be accomplished using certified EHR technology interoperable with the system designated by the State to receive the data.

\section{$\S 495.316$ State monitoring and reporting regarding activities required to receive an incentive payment.}

(a) Subject to $\S 495.332$ the State is responsible for tracking and verifying the activities necessary for a Medicaid EP or eligible hospital to receive an incentive payment for each payment year, as described in $\S 495.314$.

(b) Subject to $\S 495.332$, the State must submit a State Medicaid HIT Plan to CMS that includes-

(1) A detailed plan for monitoring, verifying and periodic auditing of the requirements for receiving incentive payments, as described in $\S 495.314$; and

(2) A description of the how the State will collect and report on provider meaningful use of certified EHR technology.

(c) Subject to $\$ 495.332$ and $\S 495.352$ the State is required to submit to CMS annual reports on the following:

(1) Provider adoption,

implementation, or upgrade of certified EHR technology activities and payments; and

(2) Aggregated, de-identified meaningful use data.

(d)(1) The annual report described in paragraph (c) of this section must include, but is not limited to the following:

(i) The number, type, and practice location(s) of providers who qualified for an incentive payment on the basis of having adopted, implemented, or upgraded certified EHR technology.

(ii) Aggregated data tables

representing the provider adoption, implementation, or upgrade of certified EHR technology.

(iii) The number, type, and practice location(s) of providers who qualified for an incentive payment on the basis of demonstrating that they are meaningful users of certified EHR technology;

(iv) Aggregated data tables representing the provider's clinical quality measures data; and

(v) A description and quantitative data on how its incentive payment program addressed individuals with unique needs such as children.

(2) Subject to § 495.332, the State may propose a revised definition of meaningful use of certified EHR technology, subject to CMS prior approval, but only with respect to the following objectives:

(i) Generate lists of patients by specific conditions to use for quality improvement, reduction of disparities, research or outreach.

(ii) Capability to submit electronic data to immunization registries or immunization information systems and actual submission in accordance with applicable law and practice.

(iii) Capability to provide electronic submission of reportable (as required by State or local law) lab results to public health agencies and actual submission in accordance with applicable law and practice; and

(iv) Capability to provide electronic syndromic surveillance data to public health agencies and actual transmission in accordance with applicable law and practice.

(e) State failure to submit the required reports to CMS may result in discontinued or disallowed funding.
\$495.318 State responsibilities for receiving FFP.

In order to be provided FFP under section 1903(a)(3)(F) of the Act, a State must demonstrate to the satisfaction of HHS, that the State is-

(a) Using the funds provided for the purposes of administering incentive payments to providers under this program, including tracking of meaningful use by Medicaid providers of EHR technology;

(b) Conducting adequate oversight of the program, including routine tracking of meaningful use attestations and reporting mechanisms; and

(c) Is pursuing initiatives to encourage the adoption of certified EHR technology to promote health care quality and the exchange of health care information, subject to applicable laws and regulations governing such exchange.

\section{$\S 495.320$ FFP for payments to Medicaid providers.}

Subject to the requirements outlined in this subpart, FFP is available at 100 percent of State expenditures for payments to Medicaid eligible providers to encourage the adoption and meaningful use of certified EHR technology.

\section{\$495.322 FFP for reasonable administrative expenses.}

Subject to prior approval conditions at $\S 495.324$ of this subpart, FFP is available at 90 percent in State expenditures for administrative activities in support of implementing incentive payments to Medicaid eligible providers.

\section{\$495.324 Prior approval conditions.}

(a) A State must obtain prior written approval as specified in paragraph (b) of this section, when the State plans to initiate planning and implementation activities in support of Medicaid provider incentive payments encouraging the adoption and meaningful use of certified EHR technology with proposed Federal financial participation.

(b) To receive 90 percent match, each State must receive prior approval for all of the following:

(1) The HIT advance planning document and the implementation advance planning document.

(2) A request for proposal and any contract that a State may utilize to complete activities under this subpart, unless specifically exempted by the Department of Health and Human Services, prior to release of the request for proposal or prior to execution of a contract. 
(3) For contract amendments, unless specifically exempted by HHS, before execution of the contract amendment, involving contract cost increases exceeding $\$ 100,000$ or contract time extensions of more than 60 days.

(4) The State Medicaid HIT plan.

(c) Failure to submit any of the information specified in paragraph (b) of this section to the satisfaction of HHS may result in disapproval or suspension of project funding.

(d) A State must obtain prior written approval from HHS of its justification for a sole source acquisition, when it plans to acquire non-competitively from a nongovernmental source HIT equipment or services, with proposed FFP under this subpart if the total State and Federal acquisition cost is more than $\$ 100,000$.

\section{\$495.326 Disallowance of FFP.}

If the HHS finds that any acquisition approved or modified under the provisions of this subpart fails to comply with the criteria, requirements, and other undertakings described in the approved HIT planning advance planning document and HIT implementation advance planning document to the detriment of the proper and efficient operation of the Medicaid program, payment of FFP may be disallowed. In the case of a suspension of approval of a HIT planning advance planning document and HIT implementation advance planning document, suspension would occur in the same manner as 45 CFR 205.37(c) and 307.40(a).

\section{§495.328 Request for reconsideration of adverse determination.}

If CMS disapproves a State request for any elements of a State's advance planning document or State Medicaid HIT Plan under this subpart, or determines that requirements are met for approval on a date later than the date requested, the decision notice includes the following:

(a) The finding of fact upon which the determination was made.

(b) The procedures for appeal of the determination in the form of a request for reconsideration.

\section{\$495.330 Termination of FFP for failure to provide access to information.}

(a) HHS terminates FFP at any time if the Medicaid agency fails to provide State and Federal representatives with full access to records relating to HIT planning and implementation efforts, and the systems used to interoperate with electronic HIT, including on-site inspection.

(b) The Department may request such access at any time to determine whether the conditions in this subpart are being met.

\section{\$495.332 State Medicaid health information technology (HIT) plan requirements.}

Each State Medicaid HIT plan must include all of the following elements:

(a) State systems. For State systems, interoperability, and the current and future visions:

(1) A baseline assessment of the current HIT landscape environment in the State including the inventory of existing HIT in the State. The assessment must include a comprehensive-

(i) Description of the HIT "as-is" landscape;

(ii) Description of the HIT "to-be" landscape; and

(iii) HIT roadmap and strategic plan for the next 5 years.

(2) A description of how the State Medicaid HIT plan will be planned, designed, developed and implemented, including how it will be implemented in accordance with the Medicaid Information Technology Architecture (MITA) principles as described in the Medicaid Information Technology Framework 2.0. The MITA initiative-

(i) Establishes national guidelines for technologies and processes that enable improved program administration for the Medicaid enterprise;

(ii) Includes business, information and technology architectures that provide an overall framework for interoperability, as well as processes and planning guidelines for enabling State Medicaid enterprises to meet common objectives within the framework while supporting unique local needs; and

(iii) Is important to the design and development of State EHR incentive payment systems.

(3) A description of how intrastate systems, including the Medicaid Management Information System (MMIS) and other automated mechanized claims processing and information retrieval systems-

(i) Have been considered in developing a HIT solution; and

(ii) A plan that incorporates the design, development, and implementation phases for interoperability of such State systems with a description of how any planned systems enhancements support overall State and Medicaid goals.

(4) A description of data-sharing components of HIT solutions.

(5) A description of how each State will promote secure data exchange, where permissible under the Health Insurance Portability and
Accountability Act (HIPAA) and other requirements included in ARRA.

(6) A description of how each State will promote the use of data and technical standards to enhance data consistency and data sharing through common data-access mechanisms.

(7) A description of how each State will support integration of clinical and administrative data

(8) A description of the process in place for ensuring improvements in health outcomes, clinical quality, or efficiency resulting from the adoption of certified EHR technology by recipients of Medicaid incentive payments and a methodology for verifying such information.

(9) A description of the process in place for ensuring that any certified EHR technology used as the basis for a payment incentive to Medicaid providers is compatible with State or Federal administrative management systems, including the MMIS or other automated claims processing system or information retrieval system and a methodology for verifying such information.

(10) A description of how each State will adopt national data standards for health and data exchange and open standards for technical solutions as they become available.

(11) A description of how the State intends to address the needs of underserved and vulnerable populations such as children, individuals with chronic conditions, Title IV-E foster care children, individuals in long-term care settings and the aged, blind, and disabled. This description must address the following:

(i) Person centered goals and objectives and shared decision-making;

(ii) Coordination of care across multiple service providers, funding sources, settings, and patient conditions-

(iii) Universal design to ensure access by people with disabilities and older Americans; and

(iv) Institutional discharge planning and diversion activities that are tied to community based service availability.

(b) Eligibility. For eligibility, a description of the process in place for all of the following:

(1) For ensuring that each EP and eligible hospital meets all provider enrollment eligibility criteria upon enrollment and re-enrollment to the Medicaid EHR payment incentive program.

(2) For ensuring patient volume consistent with the criteria in $\S 495.304$ and § 495.306 for each EP who practices predominantly in a FQHC or RHC and for each Medicaid EP who is a 
physician, pediatrician, nurse practitioner, certified nurse midwife or dentist and a methodology in place used to verify such information.

(3) For ensuring that the EP or eligible hospital is a provider who meets patient volume consistent with the criteria in $\S 495.304$ and $\$ 495.306$ and a methodology in place used to verify such information.

(4) For ensuring that each Medicaid $\mathrm{EP}$ is not hospital-based and a methodology in place used to verify such information.

(5) To ensure that a hospital eligible for incentive payments has demonstrated an average length of stay of 25 days or less and a methodology for verifying such information.

(c) Monitoring and validation. For monitoring and validation of information, States must include the following:

(1) A description of the process in place for ensuring that, because of CMS' and the States' oversight responsibilities, all provider information for attestations including meaningful use, efforts to adopt, implement, or upgrade and any information added to the CMS Single Provider Repository including all information related to patient volume, NPI, Tax identification number (TIN), are all true and accurate and that any concealment or falsification of a material fact related to the attestation may result in prosecution under Federal and State laws and a methodology in place used to verify such information.

(2) A description of the process in place for ensuring that the EP or eligible hospital is eligible to receive an incentive payment consistent with the criteria outlined in $\S 495.314$ and a methodology in place used to verify such information.

(3) A description of the process in place for capturing attestations from each EP or eligible hospital that they have meaningfully used certified EHR technology during the EHR reporting period, and that they have adopted, implemented, or upgraded certified EHR technology and a description of the methodology in place used to verify such information.

(4) A description of the process in place for capturing clinical quality data from each EP or eligible hospital and a description of the methodology in place used to verify such information.

(5) A description of the process in place for monitoring the compliance of providers coming onto the program with different requirements depending upon their participation year and a methodology for verifying such information.
(6) A list of the specific actions planned to implement the EHR incentive program, including a description and organizational charts for workgroups within State government including external partners.

(7) A description of the process in place to ensure that no amounts higher than 100 percent of FFP will be claimed by the State for reimbursement of expenditures for State payments to Medicaid eligible providers for the certified EHR technology incentive payment program and a methodology for verifying such information.

(8) A description of the process in place to ensure that no amounts higher than 90 percent of FFP will be claimed by the State for administrative expenses in administering the certified EHR technology incentive payment program and a methodology for verifying such information.

(9) A description of the process and methodology for ensuring and verifying the following:

(i) Amounts received under section 1903(a)(3)(F) of the Act with respect to payments to a Medicaid EP or eligible hospital are paid directly to such provider (or to an employer or facility to which such provider has assigned payments) without any deduction or rebate.

(ii) All incentive payment reassignments to an entity promoting the adoption of certified EHR

technology, as designated by the State, are voluntary for the Medicaid EP involved.

(iii) Entities promoting the adoption of certified EHR technology do not retain more than 5 percent of such payments for costs not related to certified EHR technology (and support services including maintenance and training) that is for, or is necessary for the operation of, such technology.

(10) A description of the process in place for ensuring that each Medicaid EP or eligible hospital that collects an EHR payment incentive has collected a payment incentive from only one State even if the provider is licensed to practice in multiple States and a methodology for verifying such information.

(11)(i) A description of the process in place for ensuring that each EP or eligible hospital that wishes to participate in the EHR incentive payment program will receive a NPI; and

(ii) A description of how the NPI will be used to coordinate with the CMS so that the EP will choose only one program from which to receive the incentive payment and the hospital payments are tracked accordingly.
(12) A description of the process in place for ensuring that each EP or eligible hospital who wishes to participate in the EHR incentive payment program will provide a TIN to the State for purposes of the incentive payment.

(d) Payments. For payments, States must provide descriptions of the following processes that are in place:

(1) The process in place for ensuring that there is no duplication of Medicare and Medicaid incentive payments to EPs and a methodology for verifying such information.

(2) The process in place to ensure that any existing fiscal relationships with providers to disburse the incentive payments through Medicaid managed care plans does not result in payments that exceed 105 percent of the capitation rate, in order to comply with the Medicaid managed care incentive payment rules at $\S 438.6(\mathrm{v})(5)(\mathrm{iii})$ of this chapter and a methodology for verifying such information.

(3) The process in place to ensure that only appropriate funding sources are used to make Medicaid EHR incentive payments and the methodology for verifying such information.

(4) The process in place and the methodology for verifying that information is available in order to ensure that Medicaid EHR incentive payments are made for no more than a total of 6 years; that no EP or eligible hospital begins receiving payments after 2016; that incentive payments cease after 2021; and that an eligible hospital does not receive incentive payments after FY 2016 unless the hospital received an incentive payment in the prior fiscal year.

(5) The process in place to ensure that Medicaid EHR incentive payments are not paid at amounts higher than 85 percent of the net average allowable cost of certified EHR technology and the yearly maximum allowable payment thresholds and a methodology for verifying such information.

(6) The process in place to ensure that all hospital calculations and hospital payment incentives are made consistent with the requirements of this part and a methodology for verifying such information.

(7) The process in place to provide for the timely and accurate payment of incentive payments to EPs and eligible hospitals, including the timeframe specified by the State to meet the timely payment requirement.

(8) The process in place and a methodology for verifying such information to provide that any monies that have been paid inappropriately as an improper payment or otherwise not 
in compliance with this subpart will be recouped and FFP will be repaid.

(9) The process in place and the methodology for verifying that EPs meet their responsibility for 15 percent of the net average allowable cost for certified EHR technology.

(e) For combating fraud and abuse and for provider appeals. (1) A description of the process in place for a provider to appeal consistent with the criteria described in $\S 495.370$ and a methodology for verifying the following related to the EHR incentives payment program:

(i) Incentive payments.

(ii) Provider eligibility

determinations.

(iii) Demonstration of efforts to adopt, implement or upgrade and meaningful use eligibility for incentive payments under this part.

(2) A description of the process in place, and a methodology for verifying such information, to address Federal laws and regulations designed to prevent fraud, waste, and abuse, including, but not limited to applicable provisions of Federal criminal law, the False Claims Act (32 U.S.C. 3729 et seq.), and the anti-kickback statute (section 1128B(b) of the Act).

(f) Optional-proposed alternatives. A State may choose to propose any of the following, but they must be included as an element in the State Medicaid HIT Plan for review and approval:

(1) An alternative methodology for measuring patient volume, consistent with $\S 495.306$ (g).

(2)(i) A revised definition of meaningful use of certified EHR technology consistent with $\S 495.4$ and $\S 495.316(d)(2)$ of this part.

(ii) Any revised definition of meaningful use may not require additional functionality beyond that of certified EHR technology and conform with CMS guidance on Stage 1. See also $\S 495.316(\mathrm{~d})(2)$.

\section{§495.334 [Reserved]}

\$495.336 Health information technology planning advance planning document requirements (HIT PAPD).

Each State's HIT PAPD must contain the following:

(a) A statement of need and objective which clearly state the purpose and objectives of the project to be accomplished and the necessity for the project.

(b) A project management plan which addresses the following:

(1) The planning project organization.

(2) Planning activities and

deliverables.

(3) State and contractor resource needs.
(4) Planning project procurement activities and schedule.

(c) A specific budget for the planning of the project.

(d) An estimated total project cost and a prospective State and Federal cost distribution, including planning and implementation.

(e) A commitment to submit a HIT implementation advance planning document.

(f) A commitment to conduct and complete activities which will result in the production of the State Medicaid HIT plan that includes conduct of the following activities:

(1) A statewide HIT environmental baseline self-assessment.

(2) An assessment of desired HIT future environment.

(3) Development of benchmarks and transition strategies to move from the current environment to the desired future environment.

(g) A commitment to submit the plan to CMS for approval.

\$495.338 Health information technology implementation advance planning document requirements (HIT IAPD).

Each State's HIT IAPD must contain the following:

(a) The results of the activities conducted as a result of the HIT planning advance planning document, including the approved state Medicaid HIT plan.

(b) A statement of needs and objectives.

(c) A statement of alternative considerations.

(d) A personnel resource statement indicating availability of qualified and adequate staff, including a project director to accomplish the project objectives.

(e) A detailed description of the nature and scope of the activities to be undertaken and the methods to be used to accomplish the project.

(f) The proposed activity schedule for the project.

(g) A proposed budget including a consideration of all HIT implementation advance planning document activity costs, including but not limited to the following:

(1) The cost to implement and administer incentive payments.

(2) Procurement or acquisition.

(3) State personnel.

(4) Contractor services.

(5) Hardware, software, and licensing.

(6) Equipment and supplies.

(7) Training and outreach.

(8) Travel.

(9) Administrative operations.

(10) Miscellaneous expenses for the project. (h) An estimate of prospective cost distribution to the various State and Federal funding sources and the proposed procedures for distributing costs including:

(1) Planned annual payment amounts;

(2) Total of planned payment amounts; and

(3) Calendar year of each planned annual payment amount.

(4) A statement setting forth the security and interface requirements to be employed for all State HIT systems, and related systems, and the system failure and disaster recovery procedures available.

\$495.340 As-needed HIT PAPD update and as-needed HIT IAPD update requirements.

Each State must submit a HIT PAPD update or a HIT IAPD no later than 60 days after the occurrence of project changes including but not limited to any of the following:

(a) A projected cost increase of $\$ 100,000$ or more.

(b) A schedule extension of more than 60 days for major milestones.

(c) A significant change in planning approach or implementation approach, or scope of activities beyond that approved in the HIT planning advance planning document or the HIT implementation advance planning document.

(d) A change in implementation concept or a change to the scope of the project.

(e) A change to the approved cost allocation methodology.

\section{\$ 495.342 Annual HIT IAPD requirements.}

Each State's annual HIT IAPD is due 60 days from the HIT IAPD approved anniversary date and must contain the following:

(a) A reference to the approved HIT PAPD/IAPD and all approved changes.

(b) A project activity status which reports the status of the past year's major project tasks and milestones, addressing the degree of completion and tasks/milestones remaining to be completed and discusses past and anticipated problems or delays in meeting target dates in the approved HIT technology PAPD/IAPD and approved changes to it.

(c) A report of all project deliverables completed in the past year and degree of completion for unfinished products.

(d) A project activity schedule for the remainder of the project.

(e) A project expenditure status which consists of a detailed accounting of all expenditures for project development over the past year and an explanation of the differences between projected expenses in the approved HIT PAPD/ 
IAPD and actual expenditures for the past year.

(f) A report of any approved or anticipated changes to the allocation basis in the advance planning document's approved cost methodology.

§495.344 Approval of the State Medicaid HIT plan, the HIT PAPD and update, the HIT IAPD and update, and the annual HIT IAPD.

HHS will not approve the State Medicaid HIT plan, HIT PAPD and update, HIT-IAPD and update, or annual IAPD if any of these documents do not include all of the information required under this subpart.

\$495.346 Access to systems and records.

The State agency must allow HHS access to all records and systems operated by the State in support of this program, including cost records associated with approved administrative funding and incentive payments to Medicaid providers. State records related to contractors employed for the purpose of assisting with implementation or oversight activities or providing assistance, at such intervals as are deemed necessary by the Department to determine whether the conditions for approval are being met and to determine the efficiency, economy, and effectiveness of the program.

\section{\$495.348 Procurement standards.}

(a) General rule. Procurements of HIT equipment and services are subject to the following procurement standards in paragraphs (b) through (f) of this section regardless of any conditions for prior approval. These standards-

(1) Include a requirement for maximum practical open and free competition regardless of whether the procurement is formally advertised or negotiated.

(2) Are established to ensure that such materials and services are obtained in a cost effective manner and in compliance with the provisions of applicable Federal statutes and executive orders.

(3) Apply when the cost of the procurement is treated as a direct cost of an award.

(b) Grantee responsibilities. The standards contained in this section do not relieve the Grantee of the contractual responsibilities arising under its contract(s).

(1) The grantee is the responsible authority, without recourse to the Departmental awarding agency, regarding the settlement and satisfaction of all contractual and administrative issues arising out of procurements entered into in support of an award or other agreement. This includes disputes, claims, and protests of award, source evaluation or other matters of a contractual nature.

(2) Matters concerning violation of statute are to be referred to such Federal, State or local authority as may have proper jurisdiction.

(c) Codes of conduct. The grantee must maintain written standards of conduct governing the performance of its employees engaged in the award and administration of contracts.

(1) No employee, officer, or agent must participate in the selection, award, or administration of a contract supported by Federal funds if a real or apparent conflict of interest would be involved.

(2) Such a conflict would arise when the employee, officer, or agent, or any member of his or her immediate family, his or her partner, or an organization which employs or is about to employ any of the parties indicated herein, has a financial or other interest in the firm selected for an award.

(3) The officers, employees, and agents of the grantee must neither solicit nor accept gratuities, favors, or anything of monetary value from contractors, or parties to sub agreements.

(4) Grantees may set standards for situations in which the financial interest is not substantial or the gift is an unsolicited item of nominal value.

(5) The standards of conduct provide for disciplinary actions to be applied for violations of such standards by officers, employers, or agents of the grantees.

(d) Competition. All procurement transactions must be conducted in a manner to provide, to the maximum extent practical, open and free competition.

(1) The grantee must be alert to organizational conflicts of interest as well as noncompetitive practices among contractors that may restrict or eliminate competition or otherwise restrain trade.

(2) In order to ensure objective contractor performance and eliminate unfair competitive advantage, contractors that develop or draft grant applications, or contract specifications, requirements, statements of work, invitations for bids and requests for proposals must be excluded from competing for such procurements.

(3) Awards must be made to the bidder or offer or whose bid or offer is responsive to the solicitation and is most advantageous to the grantee, price, quality, and other factors considered.

(4) Solicitations must clearly set forth all requirements that the bidder or offer or must fulfill in order for the bid or offer to be evaluated by the grantee.
(5) Any and all bids or offers may be rejected when it is in the grantee's interest to do so.

(e) Procurement procedures. All grantees must establish written procurement procedures. These procedures must provide, at a minimum, the following:

(1) Grantees avoid purchasing unnecessary items.

(2) When appropriate, an analysis is made of lease and purchase alternatives to determine which would be the most economical and practical procurement for the grantee and the Federal government.

(3) Solicitations for goods and services provide for all of the following:

(i) A clear and accurate description of the technical requirements for the material, product or service to be procured. In competitive procurements, such a description must not contain features which unduly restrict competition.

(ii) Requirements which the bidder or offer must fulfill and all other factors to be used in evaluating bids or proposals.

(iii) A description, whenever practicable, of technical requirements in terms of functions to be performed or performance required, including the range of acceptable characteristics or minimum acceptable standards.

(iv) The specific features of brand name or equal descriptions that bidders are required to meet when such items are included in the solicitation.

(v) The acceptance, to the extent practicable and economically feasible, of products and services dimensioned in the metric system of measurement.

(vi) Preference, to the extent practicable and economically feasible, for products and services that conserve natural resources and protect the environment and are energy efficient.

(4) Positive efforts must be made by grantees to utilize small businesses, minority-owned firms, and women's business enterprises, whenever possible. Grantees of Departmental awards must take all of the following steps to further this goal:

(i) Ensure that small businesses, minority-owned firms, and women's business enterprises are used to the fullest extent practicable.

(ii) Make information on forthcoming opportunities available and arrange time frames for purchases and contracts to encourage and facilitate participation by small businesses, minority-owned firms, and women's business enterprises.

(iii) Consider in the contract process whether firms competing for larger contracts intend to subcontract with small businesses, minority-owned firms, and women's business enterprises. 
(iv) Encourage contracting with consortia of small businesses, minorityowned firms and women's business enterprises when a contract is too large for one of these firms to handle individually.

(v) Use the services and assistance, as appropriate, of such organizations as the Small Business Administration and the Department of Commerce's Minority Business Development Agency in the solicitation and utilization of small businesses, minority-owned firms and women's business enterprises.

(5) The type of procuring instruments used (for example, fixed price contracts, cost reimbursable contracts, purchase orders, and incentive contracts) must be determined by the grantee but must be appropriate for the particular procurement and for promoting the best interest of the program or project involved.

(6) The "cost-plus-a-percentage-ofcost" or "percentage of construction cost" methods of contracting must not be used.

(7) Contracts must be made only with responsible contractors who possess the potential ability to perform successfully under the terms and conditions of the proposed procurement.

(8) Consideration must be given to such matters as contractor integrity, record of past performance, financial and technical resources or accessibility to other necessary resources.

(9) In certain circumstances, contracts with certain parties are restricted by agencies' implementation of Executive Orders 12549 and 12689, "Debarment and Suspension" as described in 2 CFR part 376.

(10) Some form of cost or price analysis must be made and documented in the procurement files in connection with every procurement action.

(11) Price analysis may be accomplished in various ways, including the comparison of price quotations submitted, market prices, and similar indicia, together with discounts.

(12) Cost analysis is the review and evaluation of each element of cost to determine reasonableness, allocability, and allowability.

(13) Procurement records and files for purchases in excess of the simplified acquisition threshold must include the following at a minimum:

(i) Basis for contractor selection.

(ii) Justification for lack of competition when competitive bids or offers are not obtained.

(iii) Basis for award cost or price.

(f) Contract administration. A system for contract administration must be maintained to ensure contractor conformance with the terms, conditions and specifications of the contract and to ensure adequate and timely follow up of all purchases. Grantees must evaluate contractor performance and document, as appropriate, whether contractors have met the terms, conditions, and specifications of the contract.

(g) Additional contract requirements. The grantee must include, in addition to provisions to define a sound and complete agreement, the following provisions in all contracts, which must also be applied to subcontracts:

(1) Contracts in excess of the simplified acquisition threshold must contain contractual provisions or conditions that allow for administrative, contractual, or legal remedies in instances in which a contractor violates or breaches the contract terms, and provide for such remedial actions as may be appropriate.

(2) All contracts in excess of the simplified acquisition threshold (currently \$100,000) must contain suitable provisions for termination by the grantee, including the manner by which termination must be effected and the basis for settlement.

(h) Conditions for default or termination. Such contracts must describe conditions under which the contract may be terminated for default as well as conditions where the contract may be terminated because of circumstances beyond the control of the contractor.

(i) Access to contract materials and staff. All negotiated contracts (except those for less than the simplified acquisition threshold) awarded by grantees must include a provision to the effect that the grantee, the Departmental awarding agency, the U.S. Comptroller General, or any of their duly authorized representatives, must have access to any books, documents, papers and records and staff of the contractor which are directly pertinent to a specific program for the purpose of making audits, examinations, excerpts and transcriptions.

\section{$\S 495.350$ State Medicaid agency attestations.}

(a) The State must provide assurances to HHS that amounts received with respect to sums expended that are attributable to payments to a Medicaid provider for the adoption of EHR are paid directly to such provider, or to an employer or facility to which such provider has assigned payments, without any deduction or rebate.

\section{§495.352 Reporting requirements.}

Each State must submit to HHS on a quarterly basis a progress report documenting specific implementation and oversight activities performed during the quarter, including progress in implementing the State's approved Medicaid HIT plan.

\section{\$495.354 Rules for charging equipment.}

Equipment acquired under this subpart is subject to the public assistance program requirements concerning the computation of claims for Federal financial participation in accordance with the provisions of 45 CFR part 95, subpart G.

\section{§495.356 Nondiscrimination requirements.}

State agencies and any other recipients or subrecipients of Federal financial assistance provided under this subpart are subject to the nondiscrimination requirements in 45 CFR parts 80, 84, and 91.

(a) These regulations in 45 CFR parts 80,84 , and 91 prohibit individuals from being excluded from participation in, being denied the benefits of, or being otherwise subjected to discrimination under any program or activity which received Federal financial assistance.

(b) Specifically, 45 CFR part 80 prohibits discrimination on the basis of race, color, or national origin; $45 \mathrm{CFR}$ part 84 prohibits discrimination on the basis of disability; and 45 CFR part 91 prohibits discrimination on the basis of age.

\section{\$495.358 Cost allocation plans.}

State agencies that acquire HIT equipment and services under this subpart are subject to cost allocation plan requirements in 45 CFR part 95

\$495.360 Software and ownership rights.

(a) General rule. The State or local government must include a clause in all procurement instruments that provides that the State or local government will have all ownership rights in software or modifications thereof and associated documentation designed, developed or installed with FFP under this Subpart.

(b) Federal license. HHS reserves a royalty-free, non-exclusive, and irrevocable license to reproduce, publish or otherwise use and to authorize others to use for Federal government purposes, the software, modifications, and documentation designed, developed or installed with FFP under this Subpart.

(c) Proprietary software. Proprietary operating/vendor software packages such as software that is owned and licensed for use by third parties, which are provided at established catalog or market prices and sold or leased to the general public must not be subject to the 
ownership provisions in paragraphs (a) and (b) of this section.

(d) Limitation. Federal financial participation is not available for proprietary applications software developed specifically for the public assistance programs covered under this subpart.

\$495.362 Retroactive approval of FFP with an effective date of February 18, 2009.

For administrative activities performed by a State, without obtaining prior approval, which are in support of planning for incentive payments to providers, a State may request consideration of FFP by recorded request in a HIT advance planning document or implementation advance planning document update. In such a consideration, the agency takes into consideration overall Federal interests which may include any of the following:

(a) The acquisition must not be before

February 18, 2009.

(b) The acquisition must be reasonable, useful, and necessary.

(c) The acquisition must be attributable to payments for reasonable administrative expenses under section 1903(a)(3)(F)(ii) of the Act.

\$495.364 Review and assessment of administrative activities and expenses of Medicaid provider health information technology adoption and operation.

(a) CMS conducts periodic reviews on an as needed basis to assess the State's progress described in its approved HIT planning advance planning document and health information technology implementation advance planning document.

(b) During planning, development, and implementation, these reviews will generally be limited to the overall progress, work performance, expenditure reports, project deliverables, and supporting documentation.

(c) CMS assesses the State's overall compliance with the approved advance planning document and provide technical assistance and information sharing from other State projects.

(d) CMS will, on a continuing basis, review, assess and inspect the planning, design, development, implementation, and operation of activities and payments for reasonable administrative expenses related to the administration of payment for Medicaid provider HIT adoption and operation payments to determine the extent to which such activities meet the following:

(1) All requirements of this subpart.

(2) The goals and objectives stated in the approved HIT implementation advance planning document and State Medicaid HIT plan.

(3) The schedule, budget, and other conditions of the approved HIT implementation advance planning document and State Medicaid HIT plan.

\section{\$495.366 Financial oversight and monitoring of expenditures.}

(a) General rule. (1) The State must have a process in place to estimate expenditures for the Medicaid EHR payment incentive program using the Medicaid Budget Expenditure System.

(2) The State must have a process in place to report actual expenditures for the Medicaid EHR payment incentive program using the Medicaid Budget Expenditure System.

(3) The State must have an automated payment and information retrieval mechanized system, (Medicaid Management Information System) to make EHR payment incentives, to ensure Medicaid provider eligibility, to ensure the accuracy of payment incentives, and to identify potential improper payments.

(b) Provider eligibility as basis for making payment. Subject to $§ 495.332$, the State must do all of the following:

(1) Collect and verify basic information on Medicaid providers to assure provider enrollment eligibility upon enrollment or re-enrollment to the Medicaid EHR payment incentive program

(2) Collect and verify basic information on Medicaid providers to assure patient volume.

(3) Collect and verify basic information on Medicaid providers to assure that EPs are not hospital-based including the determination that substantially all health care services are not furnished in a hospital setting, either inpatient or outpatient.

(4) Collect and verify basic information on Medicaid providers to assure that EPs are practicing predominantly in a Federally-qualified health center or rural health clinic.

(5) Have a process in place to assure that Medicaid providers who wish to participate in the EHR incentive payment program has or will have a NPI and will choose only one program from which to receive the incentive payment using the NPI, a TIN, and CMS' national provider election database.

(c) Meaningful use and efforts to adopt, implement, or upgrade to certified electronic health record technology to make payment. Subject to $\S 495.312$, 495.314, and $\S 495.332$, the State must annually collect and verify information regarding the efforts to adopt, implement, or upgrade certified EHR technology and the meaningful use of said technology before making any payments to providers. (d) Claiming Federal reimbursement for State expenditures. Subject to $\S 495.332$, the State must do the following:

(1) Assure that State expenditures are claimed in accordance with, including but not limited to, applicable Federal laws, regulations, and policy guidance.

(2) Have a process in place to assure that expenditures for administering the Medicaid EHR incentive payment program will not be claimed at amounts higher than 90 percent of the cost of such administration.

(3) Have a process in place to assure that expenditures for payment of Medicaid EHR incentive payments will not be claimed at amounts higher than 100 percent of the cost of such payments to Medicaid providers.

(e) Improper Medicaid electronic health record payment incentives.

(1) Subject to $\$ 495.332$, the State must have a process in place to assure that no duplicate Medicaid EHR payment incentives are paid between the Medicare and Medicaid programs, or paid by more than one State even if the provider is licensed to practice in multiple States, or paid within more than one area of a State.

(2) Subject to $\S 495.332$, the State must have a process in place to assure that Medicaid EHR incentive payments are made without reduction or rebate, have been paid directly to an eligible provider or to an employer, a facility, or an eligible third-party entity to which the Medicaid eligible provider has assigned payments.

(3) Subject to $\S 495.332$, the State must have a process in place to assure that that Medicaid EHR incentive payments are made for no more than 6 years; that no EP or eligible hospital begins receiving payments after 2016; that incentive payments cease after 2021; and that an eligible hospital does not receive incentive payments after FY 2016 unless the hospital received an incentive payment in the prior fiscal year.

(4) Subject to $\S 495.332$, the State must have a process in place to assure that only appropriate funding sources are used to make Medicaid EHR incentive payments.

(5) Subject to $\S 495.332$, the State must have a process in place to assure that Medicaid EHR incentive payments are not paid at amounts higher than 85 percent of the net average allowable cost of certified EHR technology and the yearly maximum allowable payment thresholds.

(6) Subject to $\S 495.332$, the State must have a process in place to assure that for those entities promoting the adoption of EHR technology, the 
Medicaid EHR incentive payments are paid on a voluntary basis and that these entities do not retain more than 5 percent of such payments for costs not related to certified EHR technology.

(7) Subject to $\S 495.332$, the State must have a process in place to assure that any existing fiscal relationships with providers to disburse the incentive through Medicaid managed care plans does not exceed 105 percent of the capitation rate, in order to comply with the Medicaid managed care incentive payment rules at $\S 438.6$ (c)(5)(iii) of this chapter and a methodology for verifying such information.

(8) The State must not request reimbursement for Federal financial participation unless all requirements of this subpart have been satisfied.

\section{\$495.368 Combating fraud and abuse.}

(a) General rule. (1) The State must comply with Federal requirements to-

(i) Ensure the qualifications of the providers who request Medicaid EHR incentive payments;

(ii) Detect improper payments; and

(iii) In accordance with $\S 455.15$ and $\S 455.21$ of this chapter, refer suspected cases of fraud and abuse to the Medicaid Fraud Control Unit.

(2) The State must take corrective action in the case of improper EHR payment incentives to Medicaid providers.

(b) Providers' statements regarding submission of documentation containing falsification or concealment of a material fact on EHR incentive payment documentation. For any forms on which a provider submits information necessary to the determination of eligibility to receive EHR payments, the State must obtain a statement that meets the following requirements:

(1) Is signed by the provider and contains the following statement: "This is to certify that the foregoing information is true, accurate, and complete. I understand that Medicaid EHR incentive payments submitted under this provider number will be from Federal funds, and that any falsification, or concealment of a material fact may be prosecuted under Federal and State laws."

(2) Appears directly above the claimant's signature, or if it is printed on the reverse of the form, a reference to the statements must appear immediately preceding the provider's signature.

(3) Is resubmitted upon a change in provider representative.

(4) Is updated as needed.

(c) Overpayments. States must repay to CMS all Federal financial participation received by providers identified as an overpayment regardless of recoupment from such providers, within 60 days of discovery of the overpayment, in accordance with sections 1903(a)(1), (d)(2), and (d)(3) of the Act and part 433 subpart $F$ of the regulations.

(d) Complying with Federal laws and regulations. States must comply with all Federal laws and regulations designed to prevent fraud, waste, and abuse, including, but not limited to applicable provisions of Federal criminal law, the False Claims Act (32 U.S.C. 3729 et seq.), and the anti-kickback statute (section 1128B(b) of the Act).

$\$ 495.370$ Appeals process for a Medicaid provider receiving electronic health record incentive payments.

(a) The State must have a process in place consistent with the requirements established in $\S 447.253(\mathrm{e})$ of this chapter for a provider or entity to appeal the following issues related to the HIT incentives payment program:

(1) Incentive payments.

(2) Incentive payment amounts.

(3) Provider eligibility determinations.

(4) Demonstration of adopting, implementing, and upgrading, and meaningful use eligibility for incentives under this subpart.

(b) Subject to paragraph (a) of this section, the State's process must ensure the following:

(1) That the provider (whether an individual or an entity) has an opportunity to challenge the State's determination under this Part by submitting documents or data or both to support the provider's claim.

(2) That such process employs methods for conducting an appeal that are consistent with the State's Administrative Procedure law(s).

(c) The State must provide that the provider (whether individual or entity) is also given any additional appeals rights that would otherwise be available under procedures established by the State.

Authority: Catalog of Federal Domestic Assistance Program No. 93.773, MedicareHospital Insurance; and Program No. 93.774, Medicare-Supplementary Medical Insurance Program, Program No. 93.778, Medical Assistance Program.

Dated: June 16, 2010.

Marilyn Tavenner,

Acting Administrator, Centers for Medicare $\&$ Medicaid Services.

Approved: July 9, 2010.

Kathleen Sebelius,

Secretary.

[FR Doc. 2010-17207 Filed 7-13-10; 8:45 am]

BILLING CODE 4120-01-P 